Prepared in cooperation with the Georgia Department of Natural Resources, Environmental Protection Division

\title{
Water Use in Georgia by County for 2010 and Water-Use Trends, 1985-2010
}
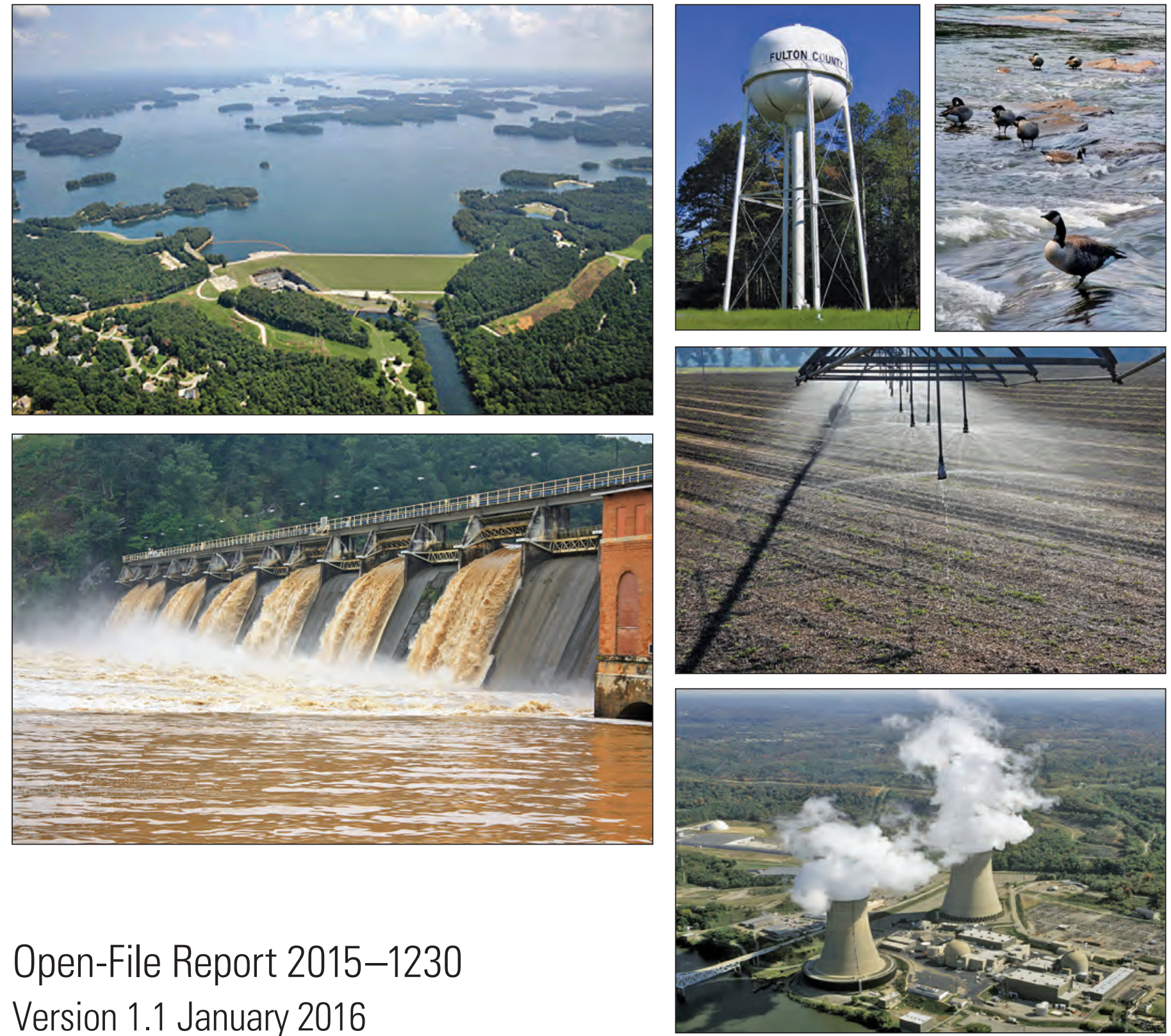

Open-File Report 2015-1230

Version 1.1 January 2016

U.S. Department of the Interior

U.S. Geological Survey 

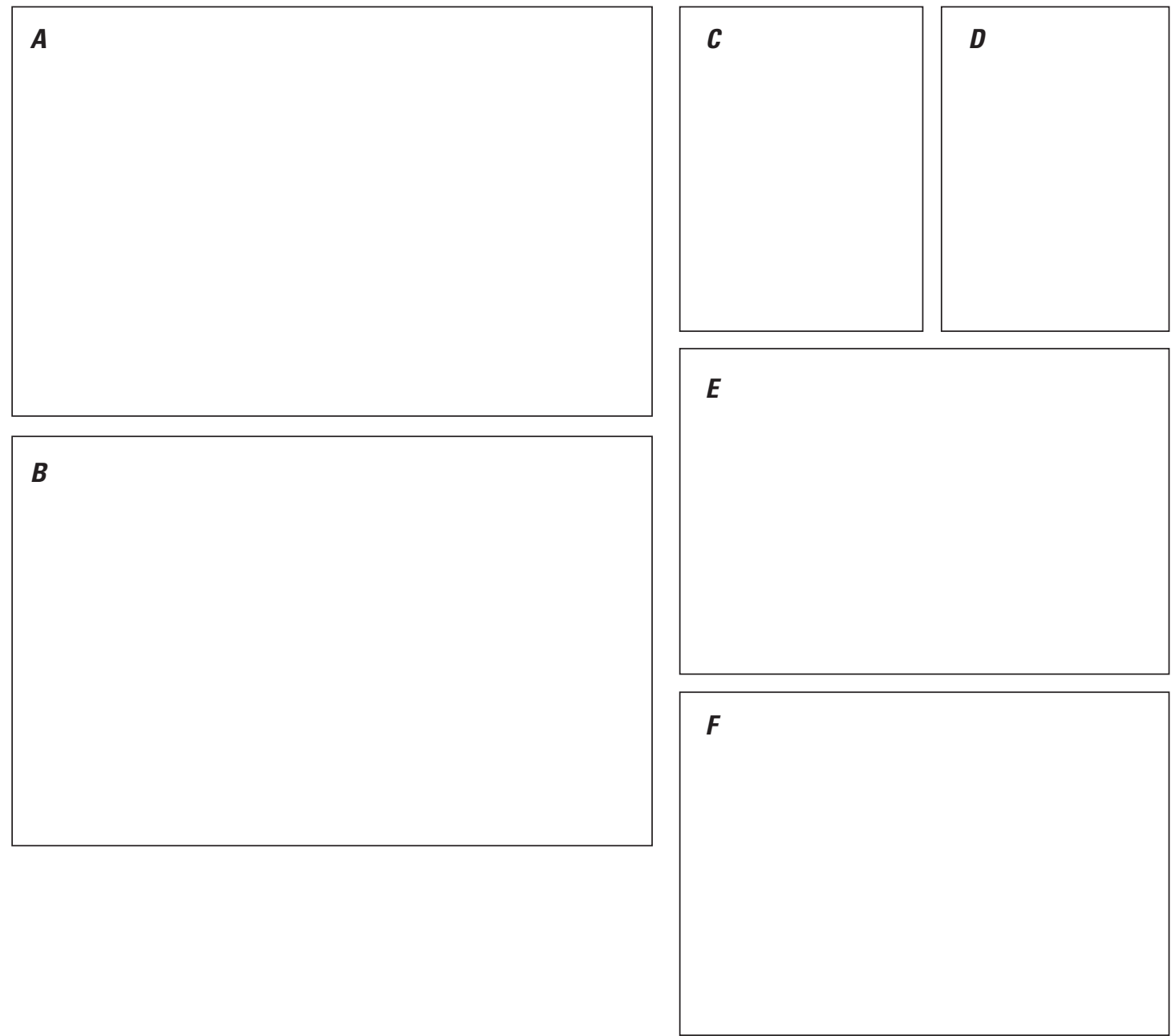

Front cover. Photo collage of water use and supply. A, Lake Sidney Lanier (Lake Lanier Convention and Visitors Bureau). B, Morgan Falls Dam (Alan M. Cressler, U.S. Geological Survey). C, Water tower (Nancy L. Barber, U.S. Geological Survey). D, Canada geese, Chattahoochee River (Alan M. Cressler, U.S. Geological Survey). E, Center-pivot irrigation, Albany (Alan M. Cressler, U.S. Geological Survey). F, Cooling towers (U.S. Nuclear Regulatory Commission). 


\section{Water Use in Georgia by County for 2010 and Water-Use Trends, 1985-2010}

By Stephen J. Lawrence

Prepared in cooperation with the Georgia Department of Natural Resources,

Environmental Protection Division

Open-File Report 2015-1230

Version 1.1, January 2016 


\title{
U.S. Department of the Interior SALLY JEWELL, Secretary
}

\section{U.S. Geological Survey Suzette M. Kimball, Director}

\author{
U.S. Geological Survey, Reston, Virginia \\ First release: 2015 \\ Revised: January 2016 (ver. 1.1)
}

\begin{abstract}
For more information on the USGS - the Federal source for science about the Earth, its natural and living resources, natural hazards, and the environment-visit http://www.usgs.gov or call 1-888-ASK-USGS.

For an overview of USGS information products, including maps, imagery, and publications, visit http://www.usgs.gov/pubprod/.
\end{abstract}

Any use of trade, firm, or product names is for descriptive purposes only and does not imply endorsement by the U.S. Government.

Although this information product, for the most part, is in the public domain, it also may contain copyrighted materials as noted in the text. Permission to reproduce copyrighted items must be secured from the copyright owner.

Suggested citation:

Lawrence, S.J., 2016, Water use in Georgia by county for 2010 and water-use trends, 1985-2010 (ver. 1.1, January 2016): U.S. Geological Survey Open-File Report 2015-1230, 206 p., http://dx.doi.org/10.3133/ofr20151230.

ISSN 2331-1258 (online) 


\section{Contents}

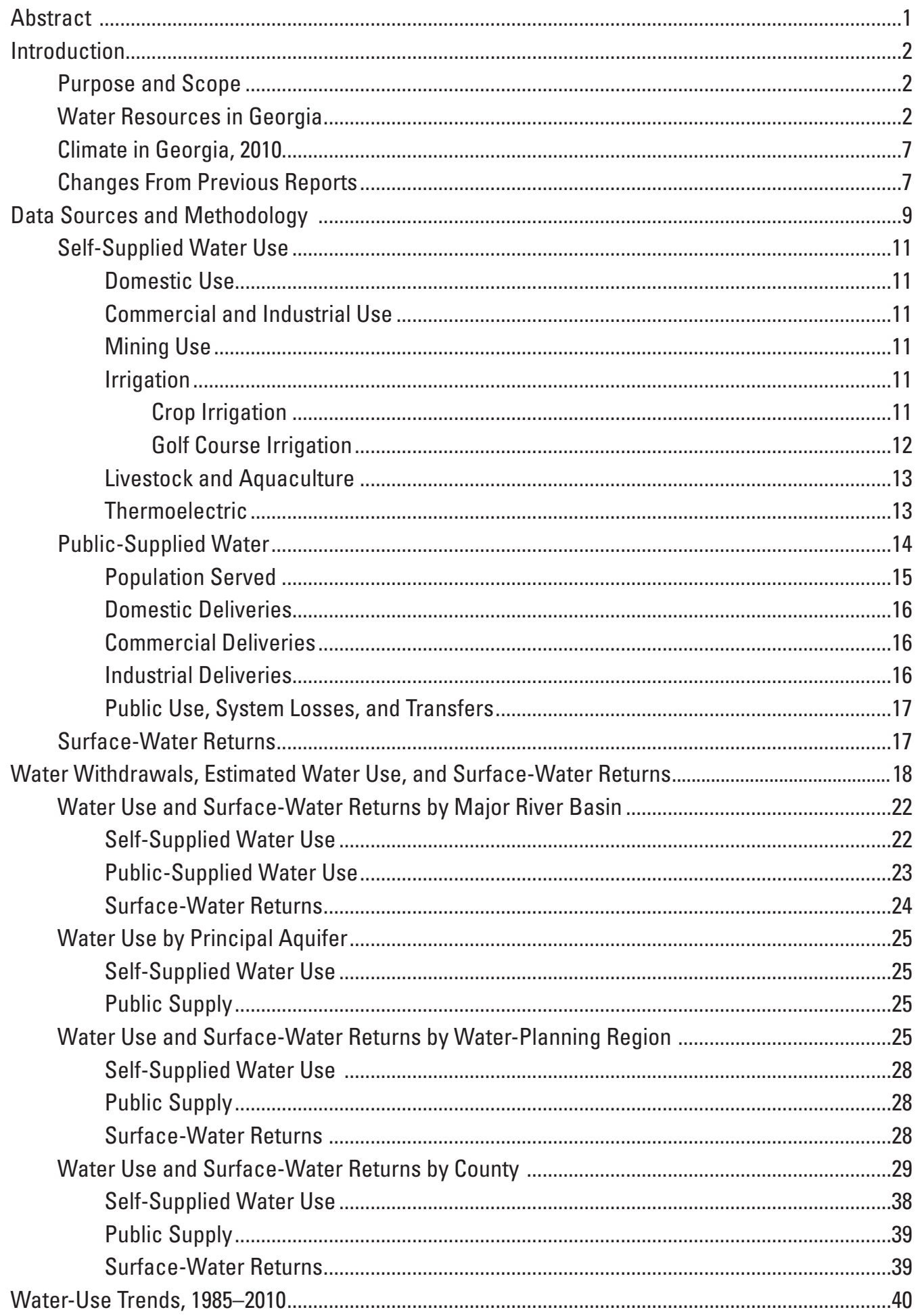




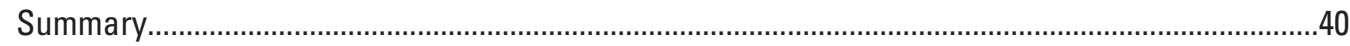

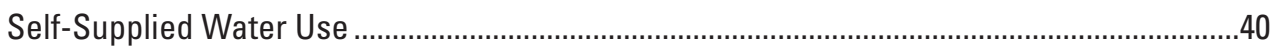

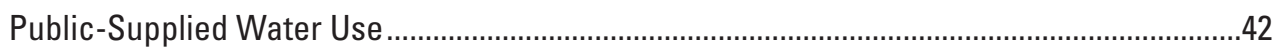

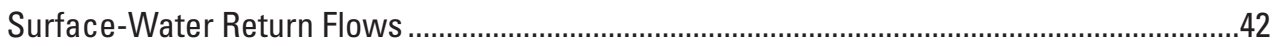

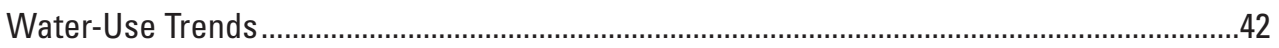

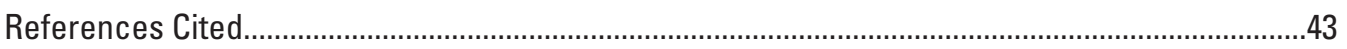

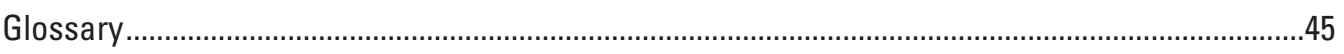

Appendix 1. North American Industrial Classification Codes ...........................................................46

Appendix 2. Population, Water Withdrawals, and Water Use by Source of Water for Each County in Georgia, 2010 .......................................................................................

\section{Figures}

1. Map showing the State of Georgia showing all 159 counties and select cities ................3

2. Map showing physiographic provinces and area of principal aquifers in Georgia ..........4

3. Map showing major river basins and streams in Georgia .............................................

4. Map showing water-planning regions in Georgia .......................................................

5. Graphs showing the 2010 average monthly precipitation departures from normal precipitation by climate division in Georgia ..................................................8

6. Schematic diagram of public and self-supplied water withdrawals and components of total water use for a typical county in Georgia..........................................

7. Maps showing surface-water and groundwater withdrawals by county

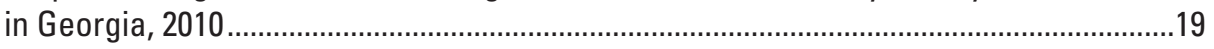

8. Pie diagrams showing water withdrawals by source and water-use category

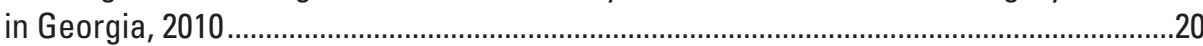

9. Maps showing percentage of total water withdrawals by county and source in Georgia, 2010 ............................................................................................................21

10. Graph showing water-withdrawal trends by water source in Georgia, 1985-2010 ........41

11. Graph showing water-withdrawal trends by water-use category in Georgia, 1985-2010

12. Graph showing surface-water return trends in Georgia, 1985-2010 .............................41 


\section{Tables}

1. Water-withdrawal, surface-water returns, and water-use data sources

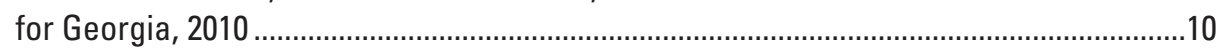

2. 2010 population, and water withdrawals by source and water-use category in Georgia, 2010.

3. Permitted commercial and industrial water withdrawals by major North American Industrial Classification code in Georgia, 2010.

4. Water withdrawals and public-supply deliveries by water-use category, for major river basins in Georgia, 2010.

5. Surface-water returns by water-use category for major river basins in Georgia, 2010...

6. Groundwater withdrawals and public-supply deliveries by use category for the principal aquifers in Georgia, 2010.

7. Water withdrawals and public-supply deliveries by water-use category for water-planning regions in Georgia, 2010.

8. Surface-water returns by water-use category for water planning regions in Georgia, 2010

9. Water withdrawals, water use, and surface water returns by county in Georgia, 2010

10. Population served and public-supply deliveries by county in Georgia, 2010 .34 


\section{Conversion Factors}

Inch/Pound to International System of Units

\begin{tabular}{|c|c|c|}
\hline Multiply & By & To obtain \\
\hline \multicolumn{3}{|c|}{ Length } \\
\hline inch (in.) & 2.54 & centimeters $(\mathrm{cm})$ \\
\hline foot $(\mathrm{ft})$ & 0.3048 & meter $(\mathrm{m})$ \\
\hline mile (mi) & 1.609 & kilometer (km) \\
\hline \multicolumn{3}{|c|}{ Area } \\
\hline acre & 4,047 & square meter $\left(\mathrm{m}^{2}\right)$ \\
\hline acre & 0.4047 & hectare (ha) \\
\hline acre & 0.004047 & square kilometer $\left(\mathrm{km}^{2}\right)$ \\
\hline square mile $\left(\mathrm{mi}^{2}\right)$ & 259.0 & hectare (ha) \\
\hline square mile $\left(\mathrm{mi}^{2}\right)$ & 2.590 & square kilometer $\left(\mathrm{km}^{2}\right)$ \\
\hline \multicolumn{3}{|c|}{ Volume } \\
\hline gallon (gal) & 3.785 & liter (L) \\
\hline gallon (gal) & 0.003785 & cubic meter $\left(\mathrm{m}^{3}\right)$ \\
\hline million gallons (Mgal) & 3,785 & cubic meter $\left(\mathrm{m}^{3}\right)$ \\
\hline cubic foot $\left(\mathrm{ft}^{3}\right)$ & 0.02832 & cubic meter $\left(\mathrm{m}^{3}\right)$ \\
\hline acre-foot (acre-ft) & 0.325851 & million gallons per day (Mgal/d) \\
\hline acre-foot (acre-ft) & 0.001233 & cubic hectometer $\left(\mathrm{hm}^{3}\right)$ \\
\hline \multicolumn{3}{|c|}{ Flow rate } \\
\hline gallon per day (gal/d) & 0.003785 & cubic meter per day $\left(\mathrm{m}^{3} / \mathrm{d}\right)$ \\
\hline million gallons per day (Mgal/d) & 0.04381 & cubic meter per second $\left(\mathrm{m}^{3} / \mathrm{s}\right)$ \\
\hline
\end{tabular}

\section{Datum}

Horizontal coordinate information is referenced to the North American Datum of 1983 (NAD 83). 


\section{Abbreviations}

$\begin{array}{ll}\text { AWUDS } & \text { Aggregate Water Use Database System } \\ \text { BMPs } & \text { best management practices } \\ \text { CAFR } & \text { Comprehensive Annual Financial Audit Report } \\ \text { DMR } & \text { discharge monitoring report } \\ \text { GaEPD } & \text { Georgia Department of Natural Resources, Environmental Protection Division } \\ \text { GaSWCC } & \text { Georgia Soil and Water Conservation Commission } \\ \text { GGCSA } & \text { Georgia Golf Course Superintendents Association } \\ \text { GIS } & \text { geographic information system } \\ \text { GWUDS } & \text { Georgia Water-Use Database System } \\ \text { MNGWPD } & \text { Metropolitan North Georgia Water Planning District } \\ \text { NASS } & \text { National Agricultural Statistics Service } \\ \text { NPDES } & \text { National Pollutant Discharge Elimination System } \\ \text { NWIS } & \text { National Water Information System } \\ \text { NWUIP } & \text { National Water Use Information Program } \\ \text { SPI } & \text { standardized precipitation index } \\ \text { SWUDS } & \text { Site Specific Water Use Database System } \\ \text { USGS } & \text { U.S. Geological Survey }\end{array}$




\section{Acknowledgments}

This compilation of water use for Georgia in 2010 would not be possible without the assistance and data provided by the Georgia Environmental Protection Division, local water supply (public and private) and water management agencies in Georgia, and the Georgia Power Company. The author acknowledges the Georgia Power Company for providing water-use data and patiently explaining how a thermoelectric-power generation facility operates. The author also thanks Nancy Barber, Kristin Linsey, and Melissa Harris with the U.S. Geological Survey, and Cliff Lewis with the Georgia Environmental Protection Division for their thorough review of the manuscript, tables, and figures in this report. 


\title{
Water Use in Georgia by County for 2010 and Water-Use Trends, 1985-2010
}

\author{
By Stephen J. Lawrence
}

\begin{abstract}
Water use and water withdrawals and returns in 2010 are estimated for each major river basin, principal aquifer, waterplanning region, and county in Georgia using data obtained from various Federal and State agencies and local sources. Offstream water use in 2010 is estimated for the categories of public supply, domestic, commercial, industrial, mining, irrigation, livestock, aquaculture, and thermoelectric power. Water-use trends for 1985 to 2010 are also shown.

The period between 2007 and 2010 was a challenging time economically and climatologically in Georgia. During that period, the United States was in the midst of a major recession, resulting in decreases in the manufacturing and construction industries and large increases in unemployment. During 2007, 2008, and the latter half of 2010, precipitation in Georgia was substantially below the 30 -year norm.

According to the 2010 Census of Population and Housing, nearly 9.7 million people lived in Georgia. The water for about 85 percent of that population was provided by public water suppliers. Estimated total water withdrawals from groundwater and surface-water sources were about 4,670 million gallons per day (Mgal/d) in 2010, about a 15-percent reduction from 2005 (5,471 Mgal/d). In 2010, thermoelectric-power facilities (2,046 Mgal/d) and public-supply uses (1,121 Mgal/d) accounted for 68 percent of all water withdrawn in Georgia. Surface-water withdrawals were greatest for thermoelectricpower generation (2,043 Mgal/d), whereas irrigation used the largest amount of groundwater $(599 \mathrm{Mgal} / \mathrm{d})$. Surface water provided 78 percent of the $1,121 \mathrm{Mgal} /$ day withdrawn for public supply in 2010. Typically, counties in northern Georgia withdraw a larger percentage of water from surface water than groundwater sources; whereas, counties in the southern part of the State withdraw more water from groundwater sources.

Historically, water withdrawals in Georgia were highest in $1980(6,725 \mathrm{Mgal} / \mathrm{d})$. By 1990, water use had decreased by 20 percent to $5,353 \mathrm{Mgal} / \mathrm{d}$, but increased to $6,487 \mathrm{Mgal} / \mathrm{d}$ in 2000. By 2005, water use had decreased to an estimated $5,471 \mathrm{Mgal} / \mathrm{d}$, and declined further to 4,670 Mgal/d
\end{abstract}

in 2010 - a 30-percent decrease since 1980. This decline was evident across all water-use categories, but was greatest for surface-water withdrawals by thermoelectric-power facilities. The estimated total water use per capita in 1985 (total withdrawals for all categories divided by total population) was about 850 gallons per day (gal/d), steadily decreasing to about $798 \mathrm{gal} / \mathrm{d}$ in 2000 , and decreasing further to $460 \mathrm{gal} / \mathrm{d}$ in 2010. Although water use declined among all use categories during that 10-year period, most of the decline in per capita water use was caused by the large decrease in water used for thermoelectric-power generation.

Throughout 1985-2010 water withdrawn for thermoelectric-power generation has constituted the largest volume of offstream water use in Georgia. Total withdrawals for thermoelectric-power generation declined about 37 percent between 2000 and 2010, mostly due to the decommissioning of power plants in the State. Also during this period, several power plants were shut down and re-tooled to use natural gaspowered generators; thus, water withdrawals for cooling were substantially reduced.

The decline in water withdrawals and use between 2005 and 2010 can probably be attributed to several factors working together during this period: (1) water conservation laws and policies along with advances in water-conservation technology; (2) the onset of a major recession in 2007; and (3) below average rainfall in 2007, 2008, and the latter half of 2010. Because of these factors, water withdrawn by public suppliers decreased by 4.8 percent (despite a nearly 11 -percent increase in population served) and per capita use decreased by 19 percent between 2005 and 2010 .

About 2,225 Mgal/d of water was returned to Georgia streams and lakes in 2010 under the National Pollutant Discharge Elimination System program administered by the Georgia Environmental Protection Division. This amount is about 48 percent of the total water withdrawn from all sources in 2010. Water returns declined 39 percent between 1995 and 2010, mirroring the decline in water withdrawals during that period. In addition, land applications of treated wastewater increased steadily between 1995 and 2010. 


\section{Introduction}

Among the natural resources in Georgia, water has the greatest potential to affect the future prosperity of Georgia's citizens. Credible knowledge of the quantity of water withdrawn from available water resources and how that water is used is necessary for managing the water resources of Georgia. The term "water use" is broadly applied to describe the movement of water from its natural source, through its interaction with human activities, and its subsequent return to the natural environment. This water-use model consists of various components such as (1) water withdrawal; (2) offstream treatment, distribution and delivery (including conveyance or system losses), and consumptive use; (3) land application of treated wastewater; (4) surface-water returns; and (5) instream use (such as hydroelectric- power generation). These components can be further subdivided into categories such as publicsupplied water, domestic use, commercial use, industrial use, mining use, irrigation use (crop and golf course), livestock use (including aquaculture), and thermoelectric-power generation.

Estimates of how much water is consumed and no longer available for use helps quantify the amount of water available to downstream users. In addition to water withdrawals, surface-water returns, and consumptive use, water-use trends can provide a basis for estimating future water needs. By examining historical water-use patterns and current water-use practices, water managers can improve projections of water needs and identify management and infrastructure needs.

To provide estimates of water withdrawal, water use, and return flows to surface water needed for water management, the U.S. Geological Survey (USGS), in cooperation with Georgia Department of Natural Resources, Environmental Protection Division (GaEPD), developed the Georgia WaterUse Program in 1979 (Fanning, 1985). This ongoing program focuses on collecting, compiling, and disseminating water-use information for the State. In the past, these data were stored in a central database known as the Georgia Water-Use Database System (GWUDS) and the Aggregate Water Use Database System (AWUDS) maintained by the USGS. For the 2010 water-use compilation, GWUDS was replaced by the Site Specific Water Use Database System (SWUDS), a subsystem of the USGS National Water Information System (NWIS). In Georgia, SWUDS is maintained at the USGS South Atlantic Water Science Center.

Although water use in Georgia has been estimated since 1970 (Carter and Johnson, 1974), this report for 2010 is the eighth in a bi-decadal series of reports describing water use by county in Georgia. Previous reports from the Georgia Water-Use Program were published as companions to the USGS series "Estimated use of water in the United States" and include summaries for 1980 (Pierce and others, 1982), 1985 (Turlington and others, 1987), 1987 (Trent and others, 1990), 1990 (Fanning and others, 1992), 1995 (Fanning, 1997), 2000 (Fanning, 2003), and 2005 (Fanning and Trent, 2009). This 2010 report is the first report in the series to include county summaries of surface-water returns.

\section{Purpose and Scope}

The purpose of this report is to present a compilation of water withdrawals, surface-water returns (treated and untreated water), and water-use estimates for nine water-use categories in Georgia during 2010. These categories include public supply, domestic, commercial, industrial, mining, irrigation (subdivided into crop and golf-course irrigation), livestock, aquaculture, and thermoelectric power. Water withdrawals for public supply are allocated among the use categories of domestic, commercial, and industrial based on information about the customer base and water sales of public-supply systems. Sales to other public-supply systems (for example, public use for services such as recreation and fire suppression, and losses in the delivery system) are also estimated and compiled. Water-use estimates and water withdrawals and surface-water return flows for each use category are reported by major river basin, principal aquifer, waterplanning region, and county in Georgia. Water-use trends during 1985-2010 are presented as well. Data are presented in tabular form, on maps showing surface-water and groundwater withdrawals in each county, and graphs showing 25-year wateruse trends and the 20-year statewide trend in return flows.

\section{Water Resources in Georgia}

The water resources in Georgia consist of 14 major river basins and multiple aquifer systems; several of these basins and aquifers extend into adjacent States. Climate, geology, and landforms control the amount, distribution, and availability of Georgia's water resources. Georgia is considered a 'headwaters' State, in which the rivers originate in Georgia and flow into other States. Eleven of the 14 rivers that originate in Georgia flow into Alabama, Florida, South Carolina, or Tennessee. As of 2015, Georgia, Florida, and Alabama are involved in litigation regarding the amount and distribution of surface water withdrawn from the ApalachicolaChattahoochee-Flint River Basin.

Georgia encompasses parts of 5 physiographic provinces, 8 principal aquifers, 14 major river basins, 11 water-planning regions, and 159 counties (figs. 1-4). The physiographic provinces are the Southern Valley and Ridge, Appalachian Plateau, Blue Ridge, and Piedmont in north Georgia, and the Coastal Plain in south Georgia (fig. 2). The major aquifers in Georgia include the Claiborne aquifer, Clayton aquifer, Cretaceous aquifer system, crystalline-rock aquifers, Floridan aquifer system, Brunswick aquifer, and Paleozoicrock aquifers (fig. 2). The major river basins in Georgia are the Savannah, Ogeecheee, Oconee, Ocmulgee, Altamaha, Satilla and St. Mary's, Suwannee and Aucila, Ochlockonee, Apalachicola, Coosa and Tallapoosa, and Tennessee (fig. 3). To facilitate water planning in Georgia, the Georgia Statewide Water Plan, identified 11 water-planning regions, each consisting of 9 to 20 counties (Georgia Environmental Protection Division, 2008; fig. 4). 


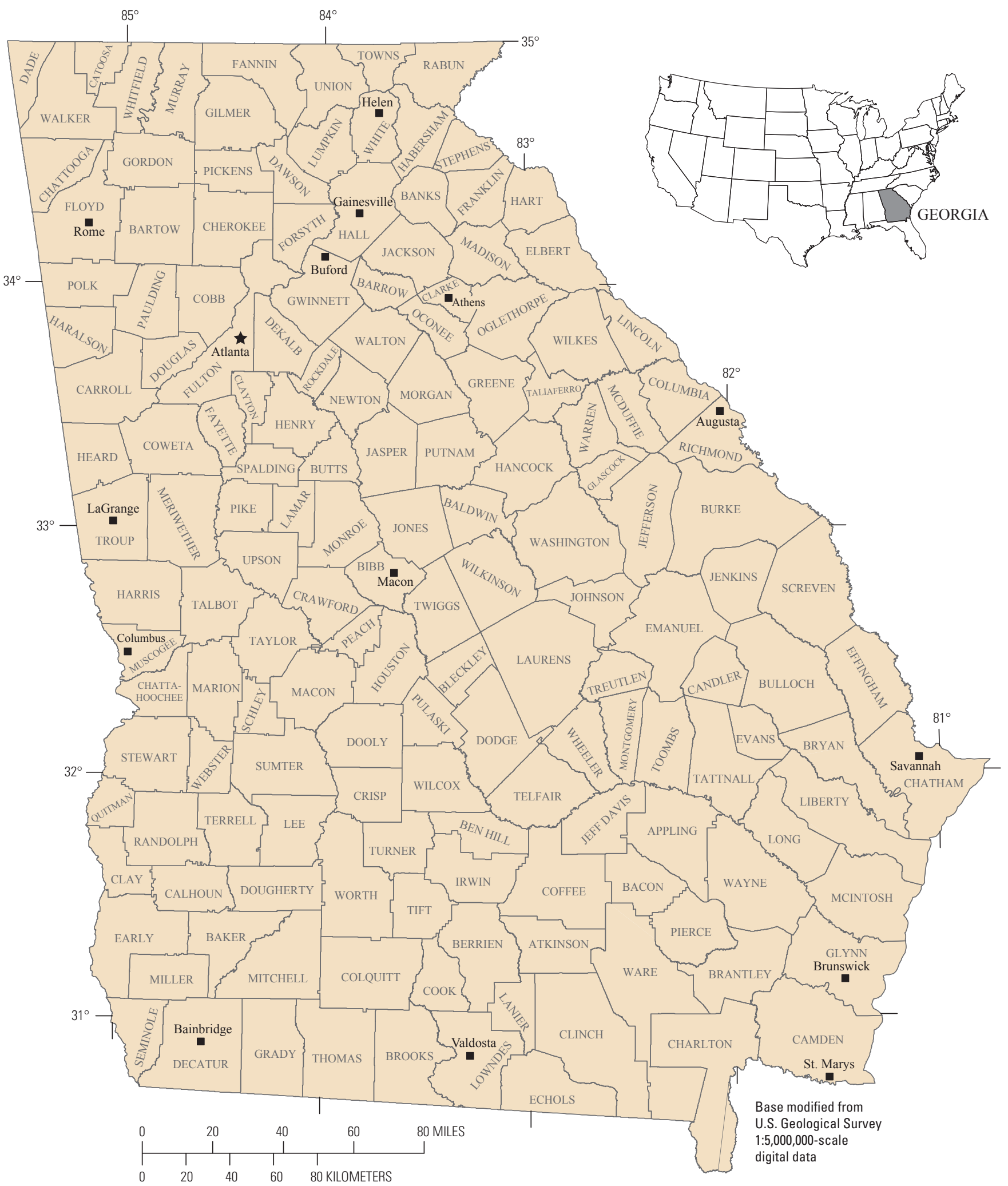

Figure 1. The State of Georgia showing all 159 counties and select cities. 


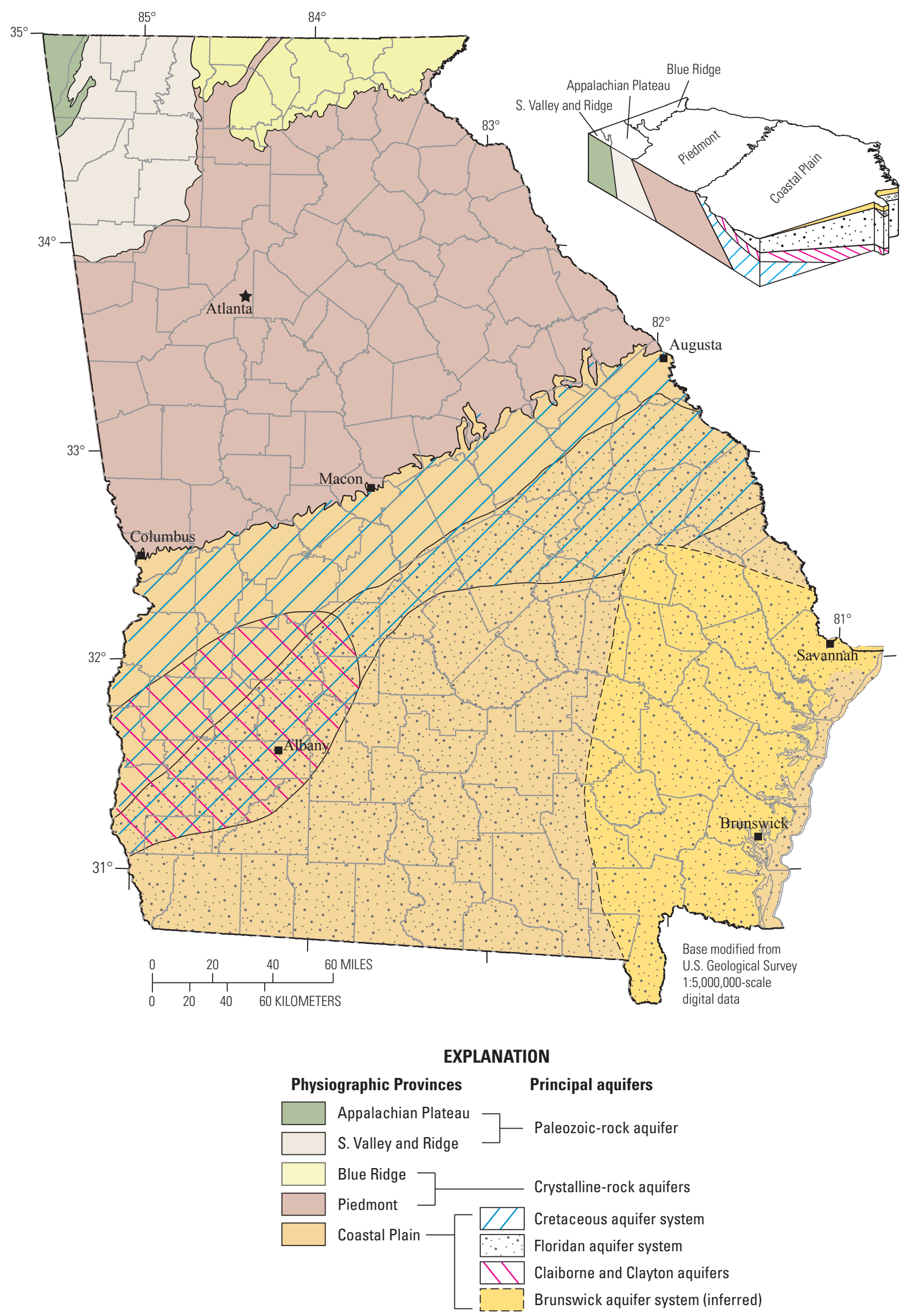

Figure 2. Physiographic provinces and area of principal aquifers in Georgia (modified from Peck and others, 2013). 


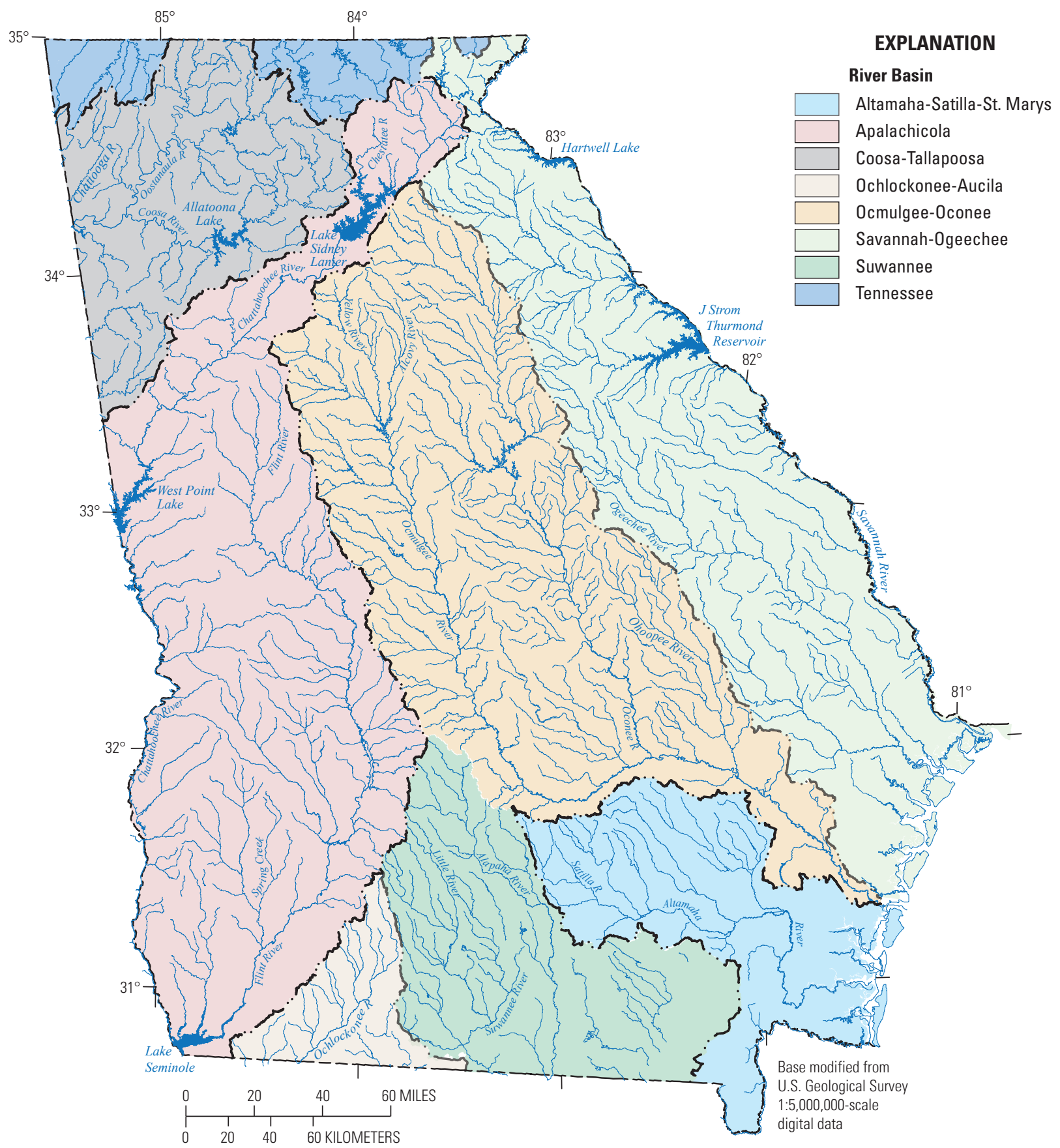

Figure 3. Major river basins and streams in Georgia. 


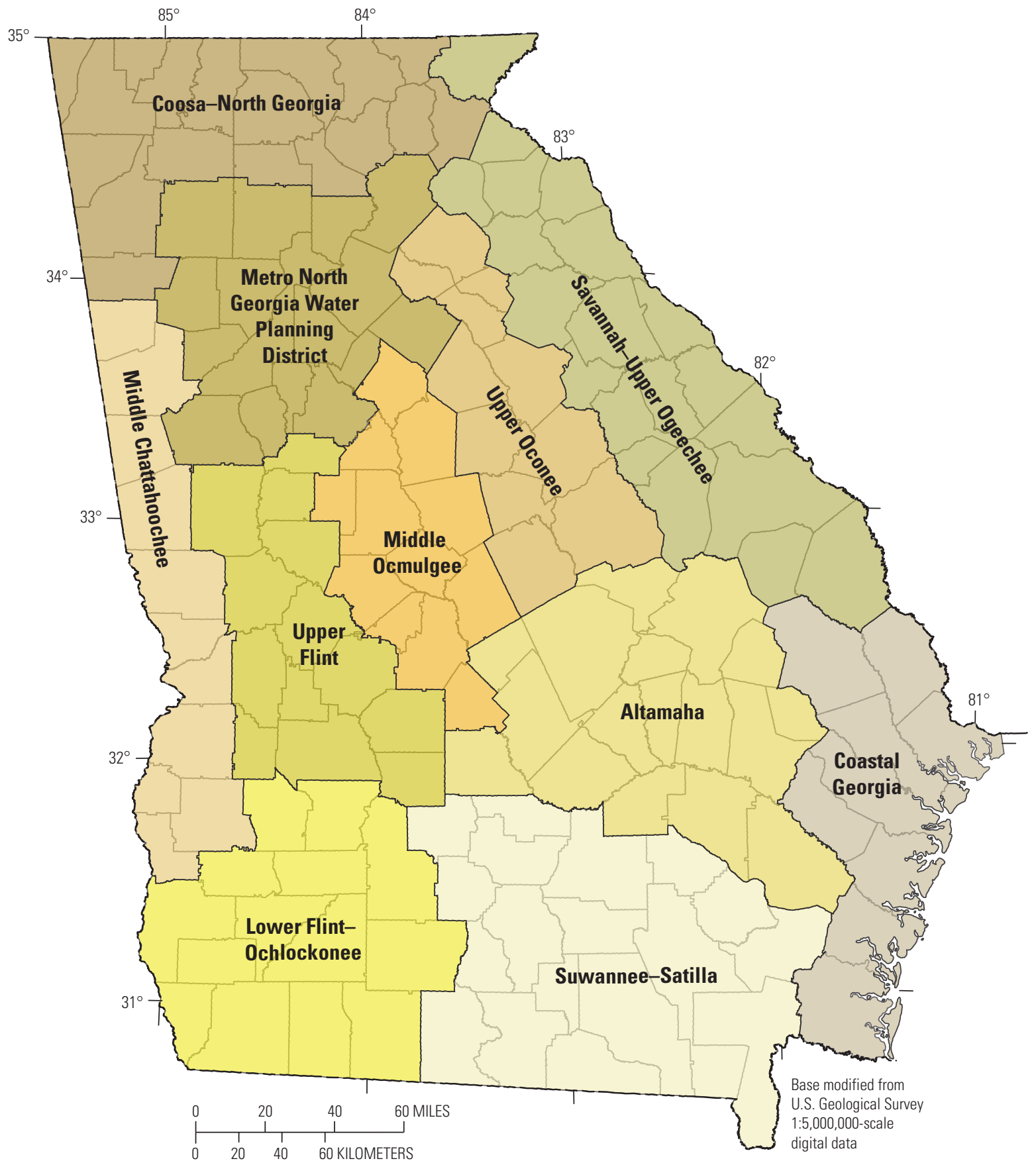

Figure 4. Water-planning regions in Georgia. 
Georgia is a "headwaters" State, with most of the rivers beginning as springs or small streams in northern Georgia and flowing generally southward into southern Georgia and Florida. In north-central Georgia, however, streams in the Tennessee River Basin flow northward into southern Tennessee. In addition, streams in the Coosa and Tallapoosa Basins (in northwestern Georgia) flow southwest into northeastern Alabama.

In northern Georgia, surface water from reservoirs or reservoir-augmented streamflow is the primary source of water; groundwater availability is limited in northern Georgia because storage capacity is typically low in the underlying fractured crystalline- and Paleozoic-rock aquifers. Most of the groundwater withdrawn in Georgia happens in southern Georgia, where aquifers, such as the Upper Floridan aquifer, are highly productive.

\section{Climate in Georgia, 2010}

The climate of northern Georgia typically is slightly wetter than that of southern Georgia. The long-term average annual rainfall (1925-2010) is 51.7 inches (in.) for northern Georgia (north of the Fall Line and 48.7 in. for southern Georgia (National Oceanic and Atmospheric Administration, 2014). A monthly standardized precipitation index (SPI) was utilized to identify 2010 average monthly precipitation departures from the 30-year monthly normal precipitation across Georgia (National Oceanic and Atmospheric Administration, 2011). The SPI is an index based on the probability of recording a given amount of precipitation, and the probabilities are standardized so that an index of zero indicates the median (normal) precipitation amount (half of the historical precipitation amounts are below the median and half are above). The index is negative for dry conditions and positive for wet conditions. As the dry or wet conditions become more severe, the index becomes more negative or more positive, respectively.

Following the record amounts of precipitation in the third and fourth quarters of 2009, above normal precipitation continued into the first half of 2010 (fig. 5). Beginning in July 2010, precipitation was below normal with most areas of Georgia considered dry according to the monthly SPI. By December, north Georgia was moderately dry (nearly 2 in. below the 30-year monthly normal precipitation), whereas south Georgia was very dry to extremely dry (3 to nearly 4 in. below the 30-year monthly average precipitation; fig. 5). The lack of rainfall in south Georgia, especially during the growing season, resulted in increased crop irrigation and livestock water use. In the urban areas of Georgia, however, increases in water use were tempered by outdoor watering restrictions in place since 2007.

\section{Changes From Previous Reports}

Consumptive use and hydroelectric power generation were presented in previous reports from the Georgia WaterUse Program, but are not provided in this report; however, return flows to surface water are included. Estimates of consumptive use are not included in this report because water conservation laws and policies along with advances in water-conservation technology have changed the way water is used and reused in Georgia. As a result, the consumptive-use coefficients given in previous water-use reports for Georgia may not be appropriate for 2010. Research is currently needed to develop more accurate consumptive-use coefficients for the domestic, commercial, and industrial categories. In addition, the amount of water used to generate hydroelectric power could not be obtained from all hydroelectric facilities; therefore, this category is not included in this 2010 report. 

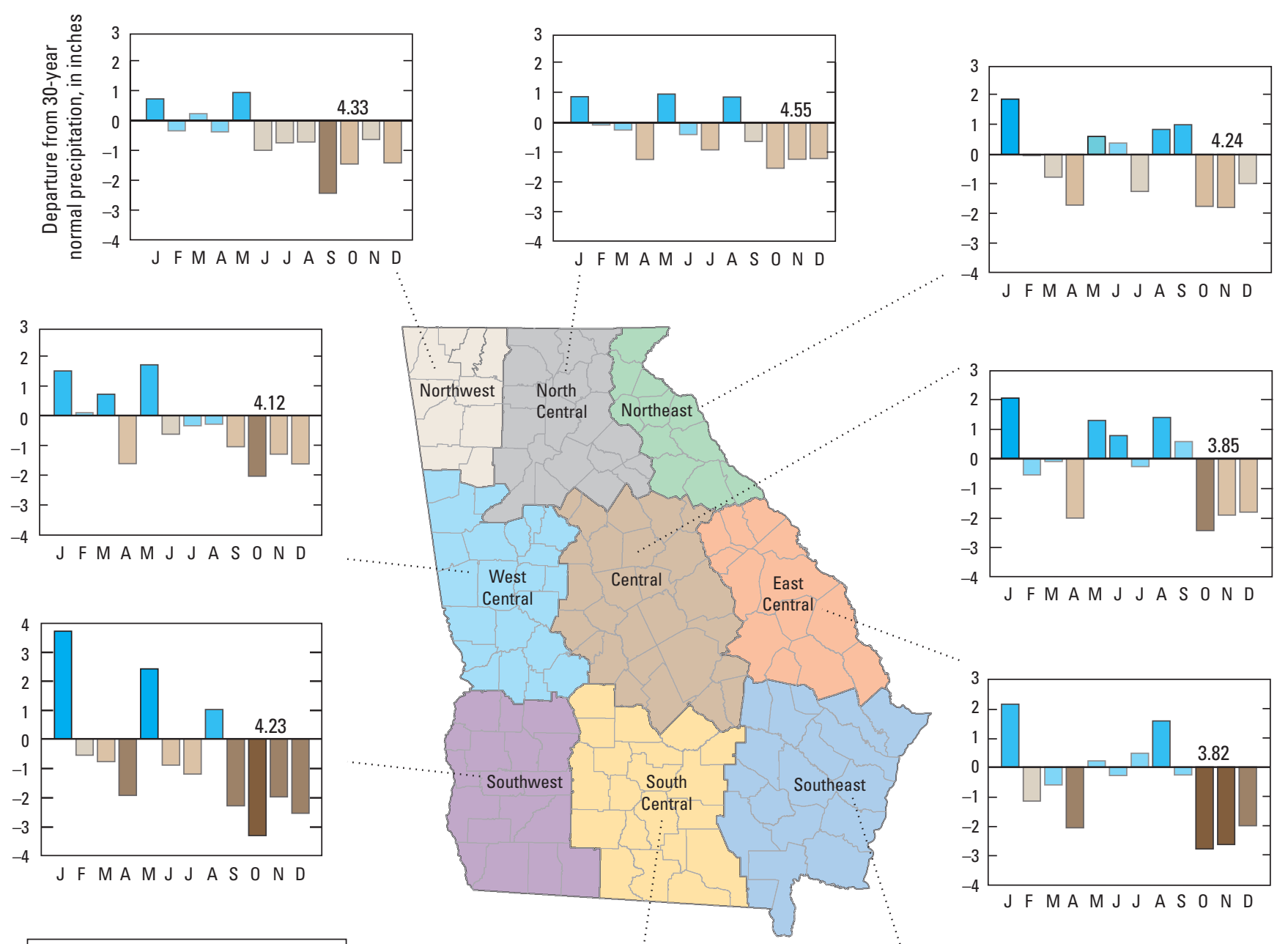

JFMAMJJASOND

\begin{tabular}{|c|}
\hline EXPLANATION \\
Standardized Precipitation Index \\
(Western Regional Climate Center, 2014) \\
$\square$ Very wet \\
Moderately wet \\
Near normal \\
Dry \\
Moderately dry \\
Very dry \\
Extremely dry \\
4.55 30-year normal monthly \\
precipitation, in inches \\
(1981-2010)
\end{tabular}

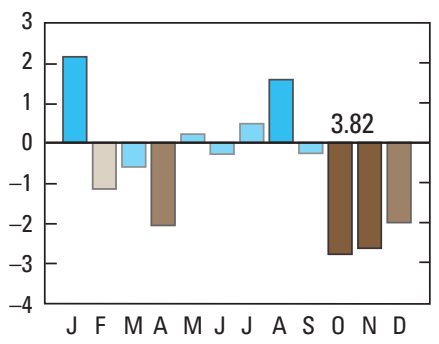

Figure 5. The 2010 average monthly precipitation departures from normal precipitation (1981-2010) by climate division in Georgia. Standardized precipitation index based on climate normals for 1895-2010. 


\section{Data Sources and Methodology}

A generalized snapshot of water use for a typical county in Georgia and outlines for the types of data acquired and used to summarize water use in Georgia for 2010 are shown in figure 6. A variety of Federal, State, local, private, and online sources were used to gather surface-water and groundwater withdrawal, surface-water return flows, and water-use data for 2010 (table 1). Permitted withdrawal data for selfsupplied commercial, industrial, mining, and thermoelectric power uses and public water suppliers were obtained from GaEPD's nonfarm withdrawal permit program. Georgia law (the Georgia Groundwater Use Act [OCGA 12-5-90] and the Georgia Water Supply Act [OCGA 12-5-170]) requires a withdrawal permit for any city, industry, or other water user that withdraws more than 100,000 gallons per day (gal/d; monthly average) and to report monthly withdrawals to the GaEPD each year.

Surface-water return flow data were obtained from discharge monitoring reports (DMRs) supplied by GaEPD. The GaEPD administers the National Pollutant Discharge Elimination System (NPDES) program in Georgia which requires that all wastewater or other water discharges with the potential to carry pollutants to surface water must be permitted regardless of the volume discharged (U.S. Environmental Protection Agency, 2014). All NPDES permit holders are required to report monthly surface-water discharges to the GaEPD. All reported and estimated withdrawal, public-supplied wholesale water sales, and surfacewater return data for 2010 were entered into the USGS SWUDS before the data were analyzed and summarized for this report.

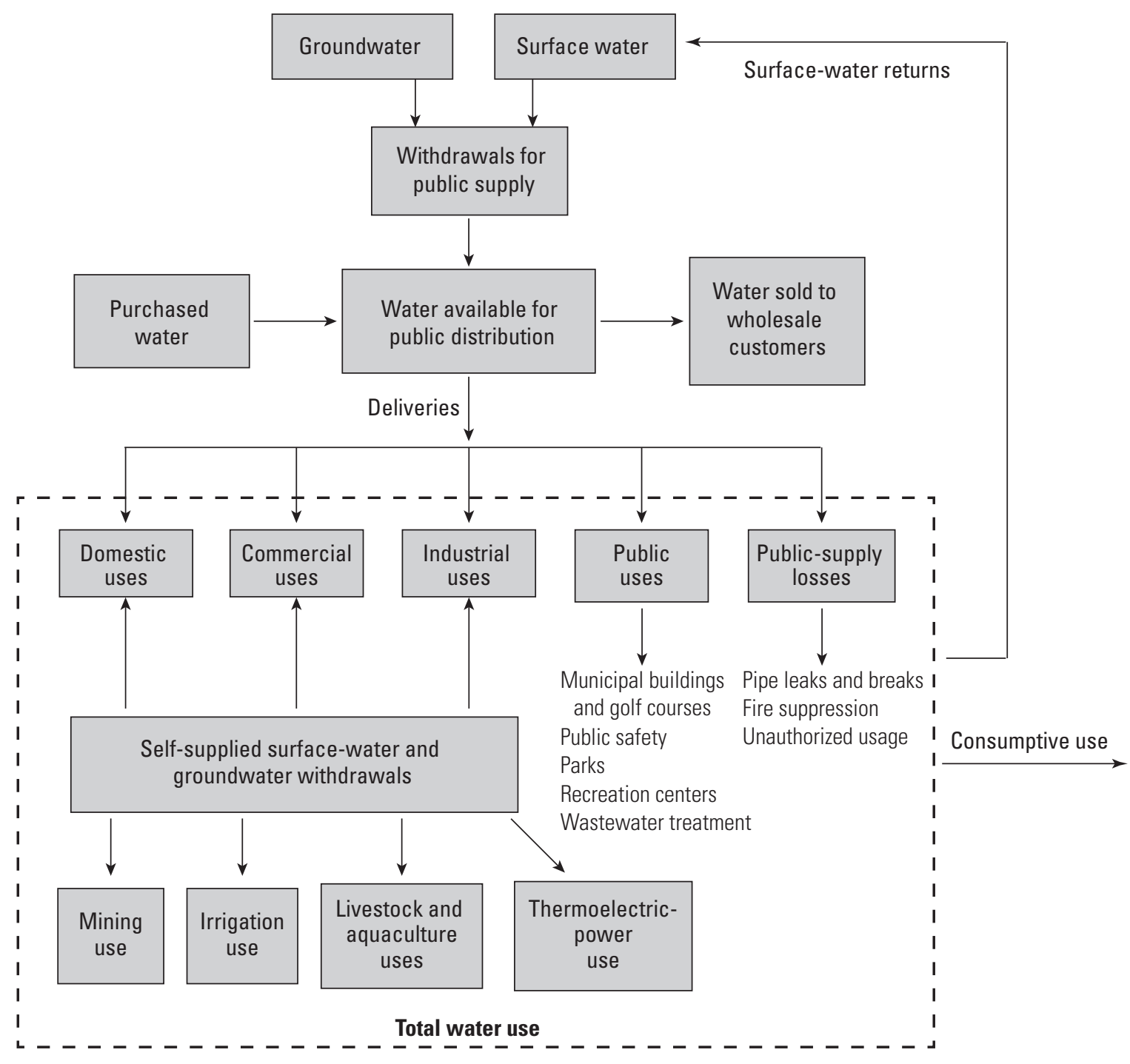

Figure 6. Schematic diagram of public and self-supplied water withdrawals and components of total water use for a typical county in Georgia. 
Table 1. Water-withdrawal, surface-water returns, and water-use data sources for Georgia, 2010.

[GaEPD, Georgia Environmental Protection Division, Watershed Protection Branch; USGS, U.S. Geological Survey]

\begin{tabular}{|c|c|c|}
\hline Water-use category & Type of data & Data source \\
\hline \multirow[t]{5}{*}{ Public supply } & $\begin{array}{l}\text { Reported groundwater and surface-water } \\
\text { withdrawals by permitted users }\end{array}$ & $\begin{array}{l}\text { GaEPD Drinking Water and Water Withdrawal Program, } \\
\text { groundwater and surface-water permitting units }\end{array}$ \\
\hline & $\begin{array}{l}\text { Population served, wholesale and retail water sales, } \\
\text { and wholesale purchases }\end{array}$ & Mail survey to operators of municipal water systems \\
\hline & $\begin{array}{l}\text { Total number of accounts; in some cases number } \\
\text { of residential, commercial, and industrial ac- } \\
\text { counts and amount of water billed }\end{array}$ & $\begin{array}{l}\text { City and County 2010/2011 Comprehensive Financial } \\
\text { Audit reports }\end{array}$ \\
\hline & Primarily water service area and zoning maps & $\begin{array}{l}\text { City and County Comprehensive Planning documents, } \\
\text { maps, and Web pages }\end{array}$ \\
\hline & $\begin{array}{l}\text { Population served by small public suppliers } \\
\text { (subdivisions and mobile home parks) }\end{array}$ & $\begin{array}{l}\text { GaEPD Drinking Water and Water Withdrawal Program, } \\
\text { Drinking Water permit unit }\end{array}$ \\
\hline Domestic & $\begin{array}{l}\text { County, city, and census block populations, } \\
\text { average household size, and housing unit } \\
\text { occupancy percentage }\end{array}$ & U.S. Census Bureau (2011a, 2011b, 2011c) \\
\hline Commercial & $\begin{array}{l}\text { Reported groundwater and surface-water } \\
\text { withdrawals by permitted users }\end{array}$ & $\begin{array}{l}\text { GaEPD Drinking Water and Water Withdrawal Program, } \\
\text { groundwater and surface-water permitting units }\end{array}$ \\
\hline Industrial & $\begin{array}{l}\text { Reported groundwater and surface-water } \\
\text { withdrawals by permitted users }\end{array}$ & $\begin{array}{l}\text { GaEPD Drinking Water and Water Withdrawal Program, } \\
\text { groundwater and surface-water permitting units }\end{array}$ \\
\hline \multirow[t]{2}{*}{ Mining } & $\begin{array}{l}\text { Reported groundwater and surface-water } \\
\text { withdrawals by permitted users }\end{array}$ & $\begin{array}{l}\text { GaEPD Drinking Water and Water Withdrawal Program, } \\
\text { groundwater and surface-water permitting units }\end{array}$ \\
\hline & Withdrawal estimates by county & $\begin{array}{l}\text { John Lovelace, USGS, written commun., } 2011 \text { (estimated } \\
\text { from USGS Minerals Information Team data) }\end{array}$ \\
\hline $\begin{array}{r}\text { Livestock and } \\
\text { aquaculture }\end{array}$ & $\begin{array}{l}\text { Livestock water-use estimates by county, water- } \\
\text { planning region, and 8-digit hydrologic unit }\end{array}$ & $\begin{array}{l}\text { Georgia State Water Plan (Georgia Environmental } \\
\text { Protection Division, 2008); Masters (2009) }\end{array}$ \\
\hline \multirow[t]{2}{*}{ Irrigation-Crops } & $\begin{array}{l}\text { Withdrawal estimates and irrigated acreage } \\
\text { by county }\end{array}$ & $\begin{array}{l}\text { Georgia State-Wide Water Plan (Georgia Environmental } \\
\text { Protection Division, 2008); Hook (2010) }\end{array}$ \\
\hline & $\begin{array}{l}\text { Metered agricultural withdrawals and estimated } \\
\text { withdrawals in non-metered counties in } \\
\text { south Georgia }\end{array}$ & $\begin{array}{l}\text { Georgia Soil and Water Conservation Commission; } \\
\text { USGS; Torak and Painter (2011) }\end{array}$ \\
\hline \multirow[t]{2}{*}{$\begin{array}{l}\text { Irrigation-Golf } \\
\text { courses }\end{array}$} & $\begin{array}{l}\text { Reported groundwater and surface-water } \\
\text { withdrawals by permitted users }\end{array}$ & $\begin{array}{l}\text { GaEPD Drinking Water and Water Withdrawal Program, } \\
\text { groundwater and surface-water permitting units }\end{array}$ \\
\hline & Acreage, water application rates & $\begin{array}{l}\text { Georgia State-Wide Water Plan (Georgia Environmental } \\
\text { Protection Division, 2008); Lewis (2010) }\end{array}$ \\
\hline $\begin{array}{l}\text { Thermoelectric } \\
\text { power }\end{array}$ & $\begin{array}{l}\text { Reported groundwater and surface-water } \\
\text { withdrawals by permitted users }\end{array}$ & $\begin{array}{l}\text { GaEPD Drinking Water and Water Withdrawal Program, } \\
\text { groundwater and surface-water permitting units, The } \\
\text { Georgia Power Company; U.S. Energy Information Admin- } \\
\text { istration's (2011) annual electric generator report (form } \\
\text { EIA-923; U.S. Energy Information Administration, 2011) }\end{array}$ \\
\hline $\begin{array}{l}\text { Surface-water } \\
\text { return flows }\end{array}$ & $\begin{array}{l}\text { Reported surface-water discharges by } \\
\text { permitted users }\end{array}$ & $\begin{array}{l}\text { GaEPD Drinking and Wastewater Regulatory Program, } \\
\text { Municipal and Industrial permitting units }\end{array}$ \\
\hline
\end{tabular}


Because water withdrawals, surface-water returns, and water use in any given county or public- supply system commonly occur from and in more than one river basin, aquifer, or county, those amounts had to be apportioned among the appropriate river basins, aquifers, or counties. This apportionment was especially crucial for computing publicsupply deliveries to domestic, commercial, and industrial customers; and for self-supplied domestic use, crop irrigation, and livestock use. A geographic information system (GIS) was used to spatially locate water intakes (surface-water withdrawal points) and outfalls (surface-water return points), groundwater wells, public-supply water service areas, irrigated acres, and livestock use within river basins. The percentage of water withdrawals from the various aquifers in Georgia was determined using location information on withdrawal and agricultural permits, lithology at the well using drillers logs and borehole geophysics, and aquifer tests (Lester Williams, Hydrologist, U.S. Geological Survey, written comm., 2013). A GIS coverage was created using these data and known locations and depths of the principal aquifers.

\section{Self-Supplied Water Use}

Self-supplied water is surface water or groundwater that is withdrawn, treated (drinking water use), and used onsite within a facility. Many self-supplied commercial, industrial, mining, and thermoelectric power facilities hold surface-water or groundwater (or both) withdrawal permits.

\section{Domestic Use}

Self-supplied groundwater withdrawals for domestic uses are commonly less than $100,000 \mathrm{gal} / \mathrm{d}$ and typically occur at individual dwellings in rural or semi-rural areas where access to public-supplied water is limited or non-existent. Surface water is generally not a source for self-supplied domestic uses in Georgia. The amount of water withdrawn and used for domestic purposes was calculated as the self-supplied population multiplied by a per capita coefficient of 75 gallons per day (gal/d) per person. The self-supplied domestic population was computed as the difference between the total population and the public-supplied population.

\section{Commercial and Industrial Use}

The 2010 annual and monthly withdrawal amounts for large self-supplied commercial and industrial establishments were reported to GaEPD (table 1) and provided to USGS for this report. Small, self-supplied commercial establishments such as campgrounds, marinas, State or county parks, restaurants, convenience stores, recreation areas, and recreational vehicle parks do not withdraw enough water to require a withdrawal permit, but do have drinking water permits and withdraw and treat water for drinking and other indoor or outdoor uses. Water use by these small commercial establishments are not included in the 2010 estimates because the amount of water withdrawn and used could not be determined. Omitting those data in this report will likely have little effect on the water withdrawal and use estimates because these small establishments use relatively small amounts of water.

\section{Mining Use}

The majority of water withdrawn during mining operations is used for the extraction of minerals, quarrying, milling, and other typical preparations at a mine site. Granite, kaolin, fuller's earth, marble, and sand and gravel are mined extensively in the State, but these mining operations do not require large quantities of water. Although large amounts of water may be pumped to dewater mine pits and quarries, this water is not withdrawn for a beneficial use and is not included in the mining water-use estimates, but is included as a surfacewater return if the water is discharged to surface water. Kaolin and fuller's earth operations use large amounts of water to transport and process the clay after extraction. Although some mining operations (especially kaolin and fuller's earth) had water withdrawal permits that indicated they withdrew more than 100,000 gal/d, these withdrawals were included in the industrial-use category rather than the mining category because the water is not used in the extraction of the clay, but in the processing of it.

The 2010 water-use estimates for mining operations that withdrew less than $100,000 \mathrm{gal} / \mathrm{d}$, which is the threshold for a withdrawal permit, were provided for each Georgia county by the USGS National Water Use Program (Lovelace, 2009a). A detailed description of the methods used for estimating water use during mining operations are given in Lovelace (2009a). Mine production data were used with a water-use coefficient to compute water use for mining in Georgia. Water-use coefficients for specific minerals are not presented in this 2010 water-use compilation to avoid potential disclosure of confidential production data provided by mining operations to the USGS.

\section{Irrigation}

Surface-water and groundwater withdrawals for crop and golf course irrigation in 2010 were estimated using a variety of data sources (table 1). A few public suppliers provided metered irrigation usage data on the 2010 water-use questionnaire.

\section{Crop Irrigation}

Two different datasets were used to estimate crop irrigation: (1) modeled estimates for north Georgia and nonmetered irrigation in some parts of south Georgia computed by the GaEPD for the Georgia Statewide Water Plan, and (2) metered data in south Georgia supplied by the Georgia 
Soil and Water Conservation Commission (GaSWCC). The modeled estimates used for north Georgia and unmetered irrigated fields were those forecasted for 2011 because estimates for 2010 were not available.

Detailed methods used to estimate crop irrigation forecasts (irrigated acres, amount of water applied, and irrigation type such as center pivot, drip, and micro for the Georgia Statewide Water Plan (Georgia Environmental Protection Division, 2008) and links to datasets are available (Georgia Environmental Protection Division, 2010). Crop irrigation water use for the Georgia Statewide Water Plan was computed for average, above average, and below average precipitation during the growing season. Because most of the 2010 growing season in Georgia was dry to very dry, a mean of the estimated crop irrigation use for below-average and average precipitation conditions was computed for this 2010 water-use report.

For the Georgia Statewide Water Plan, withdrawal quantities for non-metered irrigation were computed for each county or drainage area as the product of three values:

- Projected irrigated area for a crop, in acres.

- Predicted monthly irrigation application depth, in inches.

- Proportion of irrigation water derived from a source.

The annualized withdrawal for each county, in million gallons per day, was the product of total acres irrigated and the total amount of water applied, in inches, converted to million gallons and divided by 365 days. Initial irrigated areas were measured on 2007-8 aerial imagery by the Georgia Department of Natural Resources (GaDNR; GaDNR Agricultural Lands, Danna Betts, Georgia Department of Natural Resources, written commun., May 2012) using fields that had been identified as irrigated and were assumed to represent irrigated areas in 2010; this identification was facilitiated by interviewing farmers, estimates by the GaEPD Agriculture Water Permitting Unit, data and ground-truthing by the GaSWCC Agriculture Meter Program, and estimates by University of Georgia Agricultural Water Demand GIS.

Estimates of metered and unmetered crop irrigation in south Georgia were based on geostatistical modeling of metered groundwater pumping data supplied by the GaSWCC (Torak and Painter, 2011). Datasets were created containing a meter identification number, acres associated with each meter, annually reported withdrawals (acre-inches converted to million gallons per day) supplied by the GaSWCC, and water source (groundwater, surface water, well-to-pond). Irrigated acres were assigned to a meter using acreages determined for the Georgia Statewide Water Plan and 2010 aerial imagery (GaDNR Agricultural Lands, Danna Betts, Georgia Department of Natural Resources, written commun., May 2010). Metered data were not used if a meter was not assigned an acreage, or if irrigation depth was less than 2 in. (field not irrigated) or greater than 62 in. (malfunctioning meter or rollback was occurring). Rollback occurs when pumping ends, water in the pipes reverses and returns to the well, then spins the meter impeller and continues to increment the amount recorded during pumping.

Three statistical regions were identified in south Georgia. The metered usage within each of these regions was used to develop a geostatistical model from which to compute irrigation depth over the region. When the geostatistical models were completed, irrigation depths for unmetered fields were computed using the model to spatially interpolate irrigation depth. The model outputs were brought into a GIS for linkage to agricultural fields. Within the GIS, the centroid for each field polygon was determined and used as a feature to represent each unmetered field. The estimated irrigation depths from the model were assigned to each field centroid. Irrigation demand in acre-inches was computed by multiplying irrigated acres by irrigation depth. Irrigation usage for fields that were not assigned to a meter represented the unmetered irrigation demand. Irrigation demand in acre-inches was converted to million gallons per day using a conversion factor and dividing by 365 to annualize the demand. Hot-spot, cluster, and outlier analyses were used to identify erroneous or incomplete data and data that represented different populations of metered usage.

\section{Golf Course Irrigation}

Surface-water and groundwater withdrawals for golf course irrigation in 2010 were estimated using data from two sources: (1) agricultural water withdrawal permits and (2) nonfarm withdrawal permits, both managed by the GaEPD. Under the Georgia Groundwater Use Act, the definition of farm use includes the statement "the irrigation of recreational turf, except in Bryan, Chatham, Effingham, and Glynn counties." In addition, the 1988 amendments to the Georgia Water Quality Control Act state that the irrigation of recreational turf in the Chattahoochee River Basin above the Peachtree Creek confluence is not a farm use. Thus, in those areas where the irrigation of recreational turf is considered a nonfarm use, water withdrawals greater than $100,000 \mathrm{gal} / \mathrm{d}$ are permitted under Georgia's nonfarm water withdrawal permit program.

The GaEPD partnered with the Georgia Golf Course Superintendents Association (GGCSA) and a University of Georgia researcher to estimate irrigation demand at golf courses for the Georgia Statewide Water Plan. Golf course irrigation demand was computed using the acres listed on agricultural water withdrawal permits and annual irrigation depths determined for well-managed golf courses that were supplied by Dr. Clint Waltz (University of Georgia, Griffin, Georgia, written commun., 2010). Using these data, golf course irrigation demand was computed for above-average, average, and below-average rainfall conditions (Lewis, 2010).

According to Lewis (2010), assumptions were made to estimate water use on agricultural permitted golf courses in 
Georgia. Permitted acreages for golf courses in Georgia were obtained from the GaEPD agricultural permit database. These permitted acreages represent the most reliable source of irrigated acreage currently available to GaEPD. No comparison was made between area permitted and as-installed area of tees, greens, fairways, and other irrigated landscapes within those golf courses.

The recommended irrigation depth for turfgrass is $1 \mathrm{in}$. per week without rainfall (Clint Waltz, University of Georgia, Griffin, Georgia, written commun., 2008). Because Georgia has a 30-week growing season (April 1 to October 31), 30 in. of water is the recommended golf course irrigation rate in Georgia for a dry year (Clint Waltz, University of Georgia, Griffin, Georgia, written commun., 2008). The average irrigation depth reported in GGCSA best management practices (BMPs) program was 14.06 in. per year for 2004 to 2007 (Clint Waltz, University of Georgia, Griffin, Georgia, written commun., 2008). This average depth of 14.06 in. was considered the amount of water needed to irrigate golf courses in an average rainfall year. For each GaEPD agricultural permitted golf course, 14.06 in. was multiplied by the permitted acreage to determine the amount of water needed to irrigate golf courses in an average rainfall year. Likewise, the amount of water needed to irrigate golf courses in a year without rainfall was calculated by multiplying the total number of permitted acreage by $30 \mathrm{in}$. The irrigated water in acre-inches was converted to million gallons and divided by 365 to annualize the water use in terms of million gallons per day. Because the growing season in 2010 was dry, a mean of the estimated golf irrigation use for below-average and average precipitation conditions was computed for this 2010 water-use report.

Golf course irrigation in Bryan, Chatham, Effingham, and Glynn Counties and in the Chattahoochee River Basin above Peachtree Creek, is not considered a farm use and water withdrawals are permitted under the GaEPD nonfarm (municipal/industrial) permitting program. Under this permit program, 52 golf courses were permitted to withdraw water in 2010. The reported 2010 withdrawals for these golf courses were summed by county, river basin, water-planning region, and major aquifer and added to the estimates from the agricultural permitting program as described above. Estimates of golf course irrigation in each major river basin were computed by disaggregating the golf course irrigation in a county by the proportion of golf courses in a river basin within the county.

\section{Livestock and Aquaculture}

The water-use estimates for the livestock category were supplied by Mark Masters, Albany State University, Flint River Water Policy Center for the Georgia Statewide Water Plan (Georgia Environmental Protection Division, 2008; Masters, 2010). The most current data on the amount of water used for each livestock category in gallons per day per head were compiled in 2008; whereas animal census data by county were obtained from the 2011 Farm Gate report for 2010 (University of Georgia, 2011). The total daily water use for each livestock category was computed by multiplying the estimated daily water use per animal by the number of animals. This daily water use was multiplied by 365 to annualize the water use and then converted to million gallons per day. The annual livestock use for each county, river basin, water-planning region, and major aquifer was the sum of the annual water use for each livestock category. Estimates of livestock water use for each major river basin were computed by disaggregating the livestock water use in a county by the proportion of livestock in a river basin within the county.

In 2010, catfish and trout production were the predominant aquaculture enterprises in Georgia (University of Georgia, 2011). The 2010 water-use estimates for aquaculture in each Georgia county were computed by the USGS National Water Use Information Program (NWUIP). A detailed description of the methods used for commercial and non-commercial aquaculture operations are given in Lovelace (2009b), in which county-level data on the number of farms using particular sources of water (groundwater, surface water, and so forth), pond acreage, the number of raceways and raceway flow rates, and the number of recirculating and nonrecirculating tanks and their volumes were obtained from the National Agricultural Statistics Service (NASS; U.S. Department of Agriculture, 2009). Estimates of aquaculture water use for each major river basin were computed by first plotting known aquaculture operations in GIS, then disaggregating the aquaculture water use in a county by the proportion of aquaculture operations in a river basin within the county.

\section{Thermoelectric}

Most of the water withdrawn for thermoelectric-power generation is used for cooling. Twenty-one thermoelectric power facilities (19 in Georgia, 1 each in Alabama and South Carolina with intakes in Georgia) were permitted to withdraw water in 2010. Of the 21 facilities, 15 use fossil fuels (such as oil, coal, or natural gas), 4 use biomass (wood waste), and 2 use nuclear energy to generate electricity. The 2010 water withdrawal data for thermoelectric facilities in Georgia were obtained from three sources (table 1): (1) the Georgia Power Company provided monthly surface-water, water discharge, and consumptive use data for the thermoelectric facilities they own or operate; (2) permitted groundwater withdrawals were provided by the GaEPD under their groundwater withdrawal permit program; and (3) water withdrawal data for small, independent facilities were obtained from the U.S. Energy Information Administration's (2011) annual electric generator report (form EIA-923) database as reported by facility operators (U.S. Energy Information Administration, 2011). 


\section{Water Use in Georgia by County for 2010 and Water-Use Trends, 1985-2010}

\section{Public-Supplied Water}

Public-supplied water is withdrawn, treated, and delivered to domestic (residential), commercial, and industrial customers by public water suppliers. Any water supplier (municipal or private entity) that regularly serves at least 25 people or has at least 15 water connections is considered a public water system under Georgia law (OCGA 12-5-172-11). This definition includes large municipal, private, county, and small community water systems.

Small community water systems commonly withdraw less than 100,000 gal/d (monthly average) of water and typically are not required to obtain a withdrawal permit, but may be required to obtain a permit or certificate of use to deliver drinking water to customers. Small community water systems include incorporated and unincorporated towns and cities, subdivisions, mobile home parks, and apartment or condominium complexes.

Compiling the public-supplied water use for 2010 was a multitiered effort. Surface-water and groundwater withdrawals were summarized for permitted public water suppliers by county using data reported to the GaEPD under their nonfarm permit program (table 1). A list of entities with a drinking water permit under the GaEPD Drinking Water Program was used to identify small community water systems that withdraw less than 100,000 gal/d (monthly average threshold) from surface-water or groundwater sources.

Small-community water systems and small commercial establishments that typically withdraw less than 100,000 gal/d (monthly average) of water are not required to obtain a withdrawal permit, but may be required to obtain a drinking water permit from the GaEPD under the Georgia Safe Drinking Water Act of 1977 (OCGA 12-5-70). Small community water systems include incorporated and unincorporated towns and cities, subdivisions, mobile home parks, and apartment or condominium complexes. Small commercial establishments include campgrounds, marinas, parks, restaurants, convenience stores, recreation areas, and recreational vehicle parks.

In 2011, the GaEPD sent a water-use questionnaire to most of the public water suppliers in Georgia. All public water suppliers with withdrawal permits were surveyed. Many of the public water suppliers that withdraw a monthly average of less than 100,000 gal/d, but are permitted under the drinking water program were also surveyed. The questionnaire requested the following information for the 2010 calendar year:

- Amount of water withdrawn

- Amount of water purchased, if any, and the entity selling the water

- Total number of service connections

- Population served
- Amount of water or percentage of water delivered and number of connections for customers in the residential, commercial, industrial, and non-revenue categories.

- Amount of water sold and names of wholesale customers

- Three largest industrial customers and the amount of water delivered to each customer

Information on deliveries to customer groups and on wholesale sales was obtained from the 2010 wateruse questionnaires and a variety of other public sources (table 1). About 21 percent of the water-use questionnaires were returned; many of the largest public water suppliers did not return a questionnaire. In order to acquire water-use information for those water suppliers that did not return a questionnaire, a number of different sources of information (table 1) were investigated using the following sequence:

- Statistical section of 2010-11 Comprehensive Annual Financial Audit Reports (CAFR) for total customers and amount of water billed to residential, commercial, industrial, and public customers (municipal buildings, parks and so forth).

- Comprehensive Planning Documents for service area maps, number of customers for 2010, if available, and proportion of residential, commercial, and industrial customers.

- Public-supplier internet Web sites for total customers and service area.

- 2005 water-use survey by the GaEPD (proportion of residential, commercial, and industrial customers).

- Internet business directories and Google Earth ${ }^{\circledR}$ for the number of commercial and industrial businesses within a service area, omitting those with a water withdrawal permit.

- 2010 census-block population and housing data for the population within a service area boundary (U.S. Census Bureau, 2011b, 2011c).

Surface-water and groundwater withdrawals were estimated for small community water systems that did not return a questionnaire. The population served by small community systems was provided by the GaEPD, but the populations served by the largest small community systems were checked against the 2010 census block data (U.S. Census Bureau, 2011b, 2011c) for Georgia using GIS. Water withdrawals were estimated using population served times a water-use coefficient of $75 \mathrm{gal} / \mathrm{d}$ per person plus 5 percent for distribution losses. The 5 percent distribution loss acknowledges that all distribution systems leak in proportion to the length of watersupply pipeage (U.S. Environmental Protection Agency, 2009) 
and that the extent of water-supply pipeage for small community water systems is short, typically less than 5 miles long. The per capita water use was derived from USGS surveys in 1983 of self-supplied domestic users near Athens, Georgia (Julia Fanning, U.S. Geological Survey, written commun., 1983). Among 86 public water suppliers who returned the 2010 water use questionnaire, the median domestic per capita use was $76 \mathrm{gal} / \mathrm{d}$. Moreover, the Metropolitan North Georgia Water Planning District (2011) listed the 2009 per capita water use as $61 \mathrm{gal} / \mathrm{d}$.

Several public-supply systems convey water withdrawn from a river or stream to a storage reservoir from which water is withdrawn for treatment and distribution. The reservoir may be used only when streamflow is too low to support direct withdrawals, or may always be used as the direct source for the treatment plant. In some instances, water is withdrawn from a river or stream in one county and conveyed to a reservoir in a different county. When the withdrawal point and storage reservoir were within the same county, only water withdrawn from the reservoir was counted as a withdrawal for this compilation; however, when the withdrawal point and storage reservoir were located in different counties, the amount withdrawn from each county was computed in the following way.

- If the amount of water withdrawn from the reservoir was less than the amount conveyed from the river or stream for a given month, then the amount withdrawn from the reservoir was attributed to the county where the withdrawal point was located on the river or stream.

- For example, if $5 \mathrm{Mgal} / \mathrm{d}$ of water was withdrawn from the Ocmulgee River in Bibb County and conveyed to Town Creek Reservoir in Jones County from which $2 \mathrm{Mgal} / \mathrm{d}$ was withdrawn for treatment and distribution, then the $5 \mathrm{Mgal} / \mathrm{d}$ was counted as a withdrawal from the Ocmulgee River in Bibb County.

- If the water withdrawn from the reservoir exceeded the amount conveyed from the river or stream, then the difference was attributed to the county where the reservoir was located and the remainder attributed to the county where the conveyance from the river or stream occurred.

- For example, if $5 \mathrm{Mgal} / \mathrm{d}$ of water was withdrawn from the Ocmulgee River in Bibb County and conveyed to Town Creek Reservoir in Jones County from which $8 \mathrm{Mgal} / \mathrm{d}$ was withdrawn for treatment and distribution, then $3 \mathrm{Mgal} / \mathrm{d}$ was counted as a withdrawal from Jones County and $5 \mathrm{Mgal} / \mathrm{d}$ counted as a withdrawal from Bibb County.

\section{Population Served}

The population served by public suppliers within a defined water service area was computed from 2010 census block data (U.S. Census Bureau, 2011a, 2011b, 2011c). Water service-area maps and census block data were imported as layers into a GIS (hereafter referred to as "census map"). The population served for each public water supplier was estimated in the following manner.

- If the number of residential connections was available for a public supplier and the service area extent was the legal limits of a city or town, then the population served was the 2010 census population for the city or town.

- If the number of residential connections was available for a public supplier and the service area included and extended beyond the legal limits of a city or town, then the service area was outlined as a layer in the census map. The population served by public water suppliers was the summed census block population within the service area. When census blocks were split by service area boundaries, the proportion of the census block population in the service area was added to the population served. Prison populations were not included in population served estimates because prison water use is accounted for in the commercial use category.

- If the number of residential connections was available for a public supplier and the service area was identified by the extent of major water lines (commonly county water systems), then those water lines were added as a layer to the census map and census blocks closest to the water lines and with the greatest population density were selected. The occupied housing units in the selected census blocks were then summed until they equaled the known number of residential connections. The population served was the summed population in the census blocks.

- If the number of residential connections was available for a public supplier but the number was larger than the number of occupied housing units of the incorporated city or town, and the service area was unknown, then the service area was estimated. Beginning with the census blocks within the city or town boundaries, census blocks with the greatest number of housing units surrounding the city or town were added to those within the city boundary until the number of residential connections equaled the number of occupied housing units; these census blocks became the estimated service area. The population served was the 2010 census population within the estimated service area. 
- Initial population served numbers for small community water systems were provided by the GaEPD Drinking Water Permit program. These data were used primarily for most of the subdivisions, apartment complexes, and mobile home parks in each county and river basin. These small community water systems were numerous and in many instances their locations and areal extent were not easily obtained. Nevertheless, the service areas for the largest of these small community systems were identified on the census map and the computed 2010 population was compared to the population served numbers obtained from the GaEPD. Typically, the GaEPD data were accurate for subdivisions with less than 100 households, but tended to be over or under estimated by 2-25 percent in subdivisions with more than 100 households. In some cases (primarily small towns), the population served on the drinking water permit was larger than the 2010 census population. In these instances, Google $\operatorname{Earth}^{\circledR}$ was then used to identify dwellings beyond the town limits that could be served by the town's water system. In most cases, the city or town was surrounded by farmland or a water body and the reported population served on the drinking water permit was overestimated. In these cases the reported population served was adjusted to equal the 2010 census population of the city or town.

\section{Domestic Deliveries}

Domestic water use is water used for typical household purposes such as drinking, food preparation, bathing, washing clothes and dishes, flushing toilets, washing cars, and watering lawns and gardens. In Georgia, most of the water for domestic uses is provided by public suppliers; however, households in rural areas typically are supplied by individual water wells. These are termed self-supplied domestic water users.

Domestic deliveries from public supply are estimated using the percentage of water delivered to residential connections given in the 2010 water-use questionnaire. If a questionnaire was not returned, or deliveries were missing from the questionnaire, then the data were acquired using the sources and in the sequence listed previously. When the population served was determined, this population was multiplied by a domestic water-use coefficient of $75 \mathrm{gal} / \mathrm{d}$ per person. This coefficient reflects the annual average per capita domestic water use each day, as discussed in Self-supplied Water Use, Domestic Use section in this report.

\section{Commercial Deliveries}

Commercial establishments include motels, hotels, restaurants, medical centers, hospitals, military installations, public safety facilities (police stations, fire houses and so forth), correctional institutions, retail stores, educational facilities (public and private), campgrounds, and recreational vehicle parks. The amount of water delivered to commercial establishments by public water suppliers was summarized from the 2010 water-use questionnaire, 2010/2011 CAFR, the proportion stated in the 2005 water-use questionnaire, or was estimated using water-use coefficients. In some instances, the number of possible commercial customers in a known service area was determined by using internet business directories and Google Earth ${ }^{\circledR}$.

Commercial water-use coefficients were computed using the amount of water delivered divided by the number of commercial connections from 2010 water-use questionnaires or 2010/2011 CAFRs. Among 73 public water suppliers who returned a correctly completed water-use questionnaire or listed commercial deliveries in a 2010/2011 CAFR, the median water delivery per commercial customer was $540 \mathrm{gal} / \mathrm{d}$. The populations served for these 73 water suppliers ranged from 360 to 753,000 . Water-use coefficients were used when the number of commercial customers was known, but the amount of water delivered was unknown. Commercial deliveries were computed as the number of commercial customers multiplied by the water-use coefficient. A coefficient of $200 \mathrm{gal} / \mathrm{d}$ per commercial customer was used for public suppliers serving fewer than 2,000 people and with commercial customers consisting primarily of small downtown businesses and gas station/convenience stores (as determined from Google Earth ${ }^{\circledR}$ ), whereas a public supply delivery of $550 \mathrm{gal} / \mathrm{d}$ per commercial customer was used for all other public suppliers.

\section{Industrial Deliveries}

Industrial establishments include those involved with the production of chemical, food, textile, paper, wood, petroleum and allied products, metal fabrication, and machinery. The amount of water delivered to industries by public water suppliers in 2010 is summarized from the 2010 water-use questionnaire, 2010/2011 CAFR, the proportion of industrial deliveries given in the 2005 water-use questionnaire, or was estimated using water-use coefficients. In some instances, the number of possible industrial customers in a known service area was determined by using internet business directories, 
Google Earth ${ }^{\circledR}$, and the Dun and Bradstreet Hoovers database (Dun and Bradstreet, Inc., 2011).

A water-use coefficient was used when the number of industrial connections or customers was known but the amount of water delivered was unknown. Industrial deliveries were computed as the number of industrial customers multiplied by the water-use coefficient. Among 81 public water suppliers who returned a correctly completed water-use questionnaire or listed industrial deliveries in a 2010-11 CAFR, the median water delivery per industrial customer was $2,000 \mathrm{gal} / \mathrm{d}$ and ranged from 100 to $280,000 \mathrm{gal} / \mathrm{d}$. This median value of 2,000 $\mathrm{gal} / \mathrm{d}$ was used as the industrial water-use coefficient.

\section{Public Use, System Losses, and Transfers}

Non-revenue deliveries include unbilled authorized water use, unauthorized water use, meter inaccuracies and data errors, and storage and system losses (also known as distribution or conveyance losses) due to leakage (U.S. Environmental Protection Agency, 2009, p. 2-2). Unbilled authorized deliveries include water deliveries to churches, charitable organizations, schools, and fire suppression; public connections such as government office buildings, vehicle garages and maintenance buildings, and public recreation areas such as parks, recreation complexes, athletic fields, and public golf courses. Unauthorized water deliveries typically represent theft of water. Nonrevenue deliveries by privately owned water suppliers typically are restricted to system losses and unauthorized water use.

System losses reported on 2010 water-use questionnaires or in 2010-11 CAFRs ranged from about 4 to 32 percent of the total water entering the distribution system. If the actual system loss was not available, then the average system loss was estimated at 15 percent for public water suppliers and 5 percent for subdivisions. The 15 percent loss was about the average for all water losses reported on the 2010 questionnaires or in the 2010-11 CAFRs and was similar to that reported by U.S. Environmental Protection Agency (2009, p. B-2, B-3) and by a consulting firm (CH2M HILL, 2007). Non-revenue deliveries were inconsistently reported on the 2010 water-use questionnaire and in some cases were reported as the difference between withdrawals plus purchases and residential, commercial, and industrial deliveries. Water transfers refer to water purchased and water sold on a wholesale basis between water suppliers. The amount of water purchased or sold in 2010 was taken from the 2010 water-use questionnaire, from 2010/2011 CAFRs (if reported), or from the 2005 questionnaire.

\section{Surface-Water Returns}

The amounts of treated and untreated water discharged to rivers, streams, and reservoirs in Georgia (surface-water returns) during 2010 were provided by the GaEPD and the Georgia Power Company. Each entity with a discharge permit under Georgia's NPDES wastewater permit program is required to report average monthly discharges to the GaEPD. These discharge monitoring reports were the source of surface-water return data. These data were then summarized and compiled for each county, major river basin, and waterplanning region in Georgia. 
Water Withdrawals, Estimated Water Use, and Surface-Water Returns

The period between 2007 and 2010 was a challenging time economically and climatologically in Georgia. During that period, major recession affected the United States, including Georgia, resulting in large increases in unemployment and decreases in manufacturing, construction, and other industrial activity (Glassman, 2014).

According to the 2010 census, nearly 9.7 million people lived in Georgia (U.S. Census Bureau, 2011a; table 2). The water for 85 percent of that population was provided by public water suppliers. On a county basis, total water withdrawals ranged from less than 5 to 975 million gallons per day (Mgal/d) in 2010 (fig. 7). Estimated total withdrawals from groundwater and surface-water sources were about 4,670 Mgal/d in 2010 (table 2; fig. 8), a nearly 15 percent reduction from $2005(5,471 \mathrm{Mgal} / \mathrm{d})$. In 2010, thermoelectric power facilities $(2,046 \mathrm{Mgal} / \mathrm{d})$ and public supply systems $(1,121 \mathrm{Mgal} / \mathrm{d})$ withdrew nearly 68 percent of all water withdrawn in Georgia.

In 2010, surface-water withdrawals $(3,481 \mathrm{Mgal} / \mathrm{d})$ were nearly three times greater than groundwater withdrawals (1,189 Mgal/d) in Georgia (table 2; fig. 8). Public suppliers, self-supplied industrial, golf course irrigation, livestock, aquaculture, and thermoelectric power facilities withdrew more surface water than groundwater. Surface-water withdrawals were greatest for thermoelectric-power generation (2,043 Mgal/d) and public supply (873 Mgal/d), accounting for nearly 84 percent of surface-water withdrawals in 2010 . Agricultural and industrial withdrawals accounted for

Table 2. 2010 population, and water withdrawals by source and water-use category in Georgia, 2010.

[Mgal/d, million gallons per day; -, not applicable]

Population: 9,687,660

Population served by public supply: $8,255,250$

Population using groundwater: $1,656,800$

Population using surface water: 6,598,450

Self-supplied population (groundwater): 1,432,410

Total per capita use $\mathrm{e}^{\mathrm{a}}$ : 460 gallons per person per day

Acres irrigated: $1,303,050$

\begin{tabular}{|c|c|c|c|c|c|c|}
\hline \multirow[t]{2}{*}{ Category } & \multicolumn{3}{|c|}{$\begin{array}{l}\text { Water withdrawals } \\
\text { (Mgal/d) }\end{array}$} & \multirow{2}{*}{$\begin{array}{l}\text { Deliveries from } \\
\text { public supply } \\
\text { (Mgal/d) }\end{array}$} & \multirow{2}{*}{$\begin{array}{l}\text { Total use }^{c} \\
\text { (Mgal/d) }\end{array}$} & \multirow{2}{*}{$\begin{array}{l}\text { Surface- } \\
\text { water returns } \\
\text { (Mgal/d) }\end{array}$} \\
\hline & Groundwater & Surface water & Total & & & \\
\hline Public supply & 247.8 & 872.9 & 1,121 & - & - & - \\
\hline Domestic & 107.4 & 0.00 & 107.4 & 623.5 & 730.9 & - \\
\hline Commercial/public use ${ }^{\mathrm{d}}$ & 2.08 & 0.58 & 2.66 & 209.2 & 211.9 & 1.21 \\
\hline Industrial & 206.5 & $288.5^{\mathrm{e}}$ & 495.1 & 107.9 & 603.0 & 405.4 \\
\hline Public supply system losses ${ }^{\mathrm{f}}$ & - & - & - & 181.8 & 181.8 & - \\
\hline Public wastewater treatment & - & - & - & - & - & 711.4 \\
\hline Mining & 16.67 & 0.29 & 16.96 & - & 16.96 & 24.10 \\
\hline Irrigation-Crop & $577.5^{\mathrm{g}}$ & 169.8 & 747.4 & - & 747.4 & - \\
\hline Irrigation-Golf courses & 21.50 & 33.12 & 54.62 & 3.14 & 57.76 & - \\
\hline Livestock and aquaculture & 6.36 & 72.85 & 79.22 & - & 79.22 & 1.95 \\
\hline Thermoelectric power & 2.92 & $2,043^{\mathrm{d}}$ & 2,046 & - & 2,046 & 1,081 \\
\hline Total & 1,189 & 3,481 & 4,670 & 1,125 & 4,675 & 2,225 \\
\hline
\end{tabular}

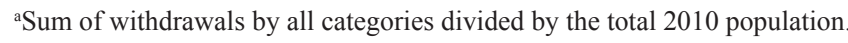

${ }^{\mathrm{b}} \mathrm{A}$ net transfer of about $6.7 \mathrm{Mgal} / \mathrm{d}$ of public-supplied water occurred from Alabama and Tennessee to Georgia in 2010.

${ }^{\mathrm{c}}$ Total use is total withdrawal plus public supply deliveries.

${ }^{d}$ Public use includes self-supplied withdrawals and unbilled public water delivered to municipal buildings, parks, golf courses, schools, churches, and so forth.

eIncludes withdrawals from saline water.

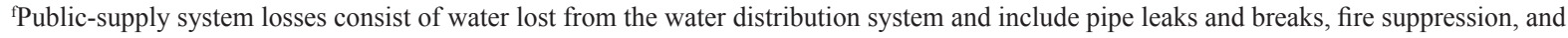
unauthorized use.

gIncludes permitted well-to-pond systems. 

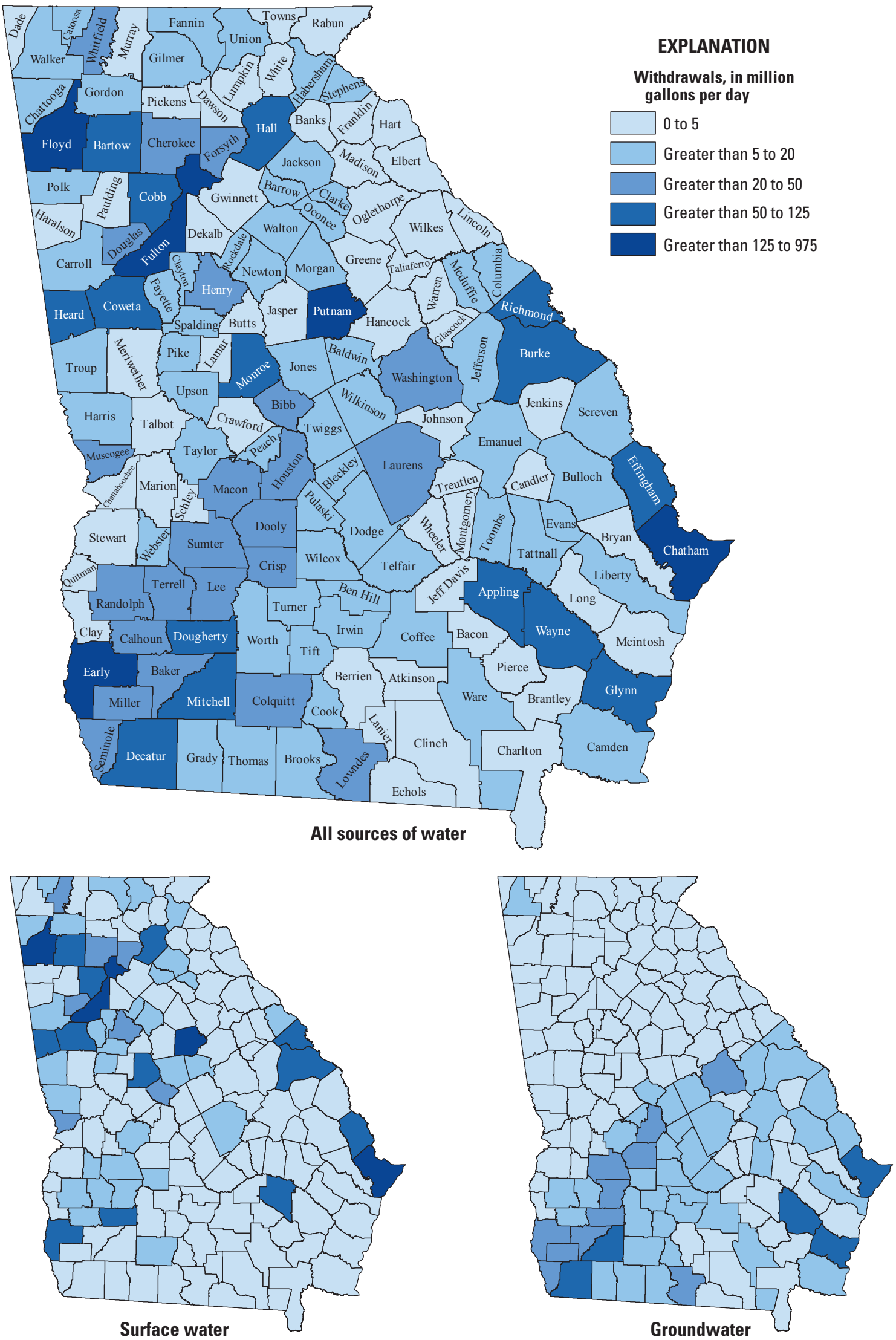

Figure 7. Surface-water and groundwater withdrawals by county in Georgia, 2010. 

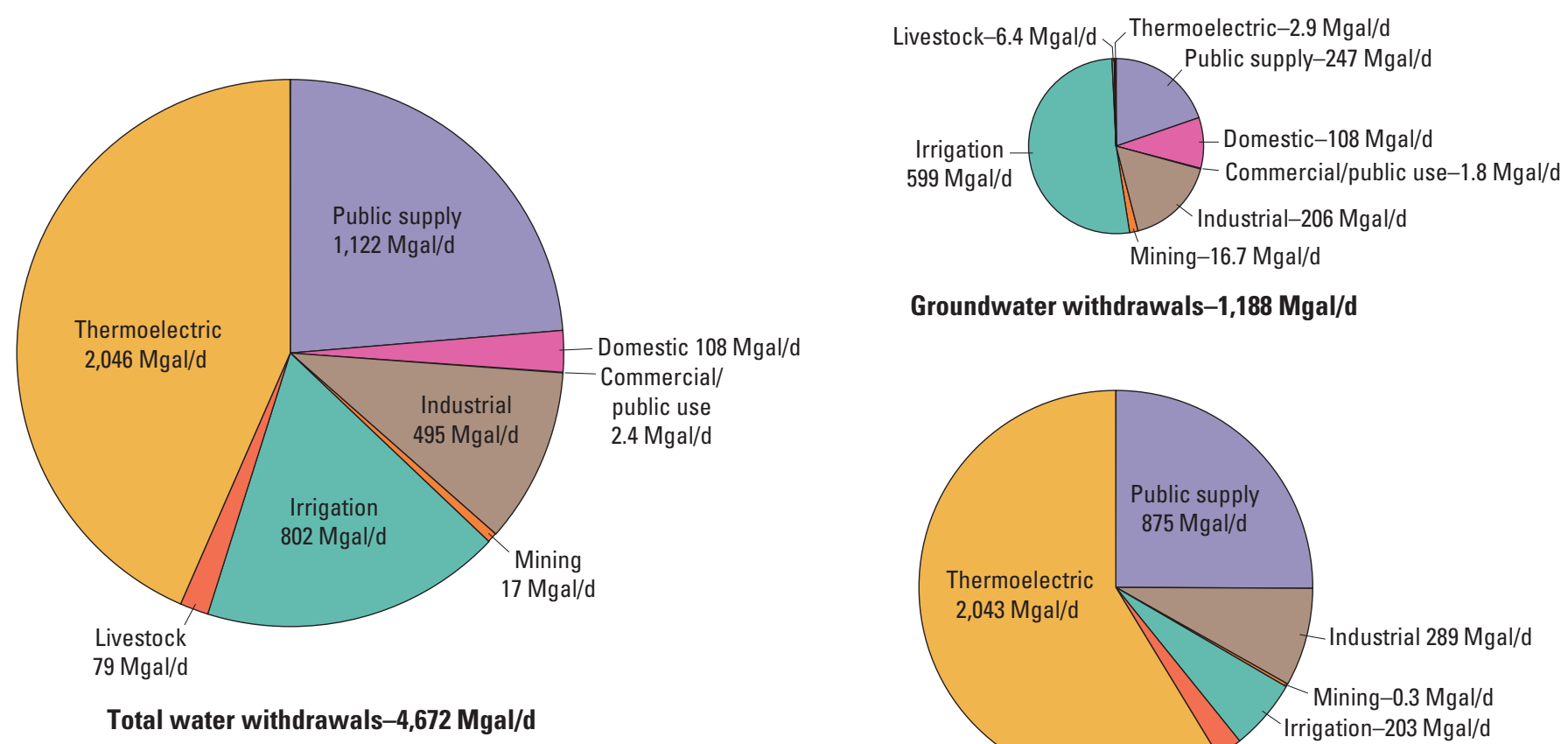

Groundwater withdrawals-1,188 Mgal/d

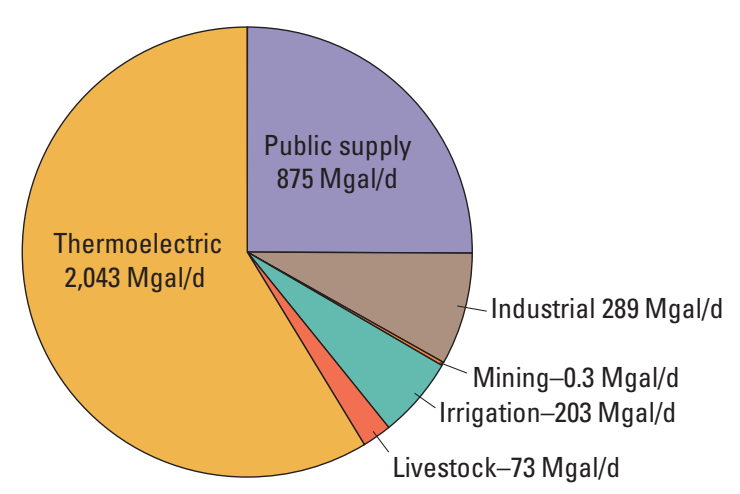

Surface-water withdrawals-3,484 Mgal/d

Figure 8. Water withdrawals by source and water-use category in Georgia, 2010. [Mgal/d, million gallons per day]

15 percent of surface-water withdrawals, whereas commercial, mining, and golf course irrigation accounted for the remaining 1 percent. Surface water provided 78 percent of the 1,121 Mgal/day withdrawn for public supply in 2010.

Self-supplied domestic, commercial, mining, and crop irrigation uses withdrew more groundwater than surface water in 2010 (table 2; fig. 8). Crop irrigation withdrew the largest amount of groundwater (578 Mgal/d). Thermoelectric-power and commercial uses withdrew the least amount of groundwater. Typically, counties in northern Georgia withdraw a larger percentage of water from surface water than groundwater sources, whereas counties in the southern Georgia withdraw more water from groundwater than surface-water sources (figs. 7 and 9).

Self-supplied industries withdrew about $495 \mathrm{Mgal} / \mathrm{d}$ in 2010 , of which 58 percent was surface water (table 2). Paper and pulp facilities withdrew 78 percent of all water withdrawn by self-supplied industries ( $387 \mathrm{Mgal} / \mathrm{d})$, of which $255 \mathrm{Mgal} / \mathrm{d}$ was surface water (table 3 ). In contrast, the kaolin and fuller's earth industries (primarily located in middle Georgia, south of the Fall Line) withdrew 8.7 percent of all water withdrawn by self-supplied industries (43.1 Mgal/d), of which $34.7 \mathrm{Mgal} / \mathrm{d}$ was groundwater. Chemical manufacturing facilities (appendix 1) used 7.8 percent of the total groundwater $(16.3 \mathrm{Mgal} / \mathrm{d})$ and 5.5 percent of the total surface water $(15.8 \mathrm{Mgal} / \mathrm{d})$ withdrawn by all industries in Georgia during 2010 (table 3).
In 2010, total water use in Georgia is estimated at $4,675 \mathrm{Mgal} / \mathrm{d}$ (table 2). This amount includes $1,125 \mathrm{Mgal} / \mathrm{d}$ of water delivered by public suppliers to domestic, commercial and public use, and industrial customers, and publicsupply losses. About 6.7 Mgal/d of water was delivered from public suppliers in Alabama and Tennessee to customers in Georgia. Thermoelectric-power generation accounted for about 44 percent of all water used in Georgia during 2010. Crop irrigation and domestic categories each accounted for nearly 16 percent of the total water use in 2010 , whereas the industrial and commercial/public use categories accounted for nearly 13 and 4.5 percent, respectively. Public-supply losses accounted for 3.9 percent and mining, golf course irrigation, and livestock collectively accounted for 3.3 percent of the total water use in Georgia.

About 2,225 Mgal/d of water was returned to surfacewater bodies in Georgia and represents about 48 percent of the water withdrawn in 2010 (table 2). Nearly 49 percent of all surface-water returns were from thermoelectric-power facilities using once-through cooling processes. About 32 percent of all surface-water returns was treated wastewater from public treatment facilities $(711 \mathrm{Mgal} / \mathrm{d})$, while industrial facilities discharged $405 \mathrm{Mgal} / \mathrm{d}$ or 18 percent of the total returns. Mining, aquaculture, and commercial facilities discharged about 1 percent of the water returned to surface water in 2010. 

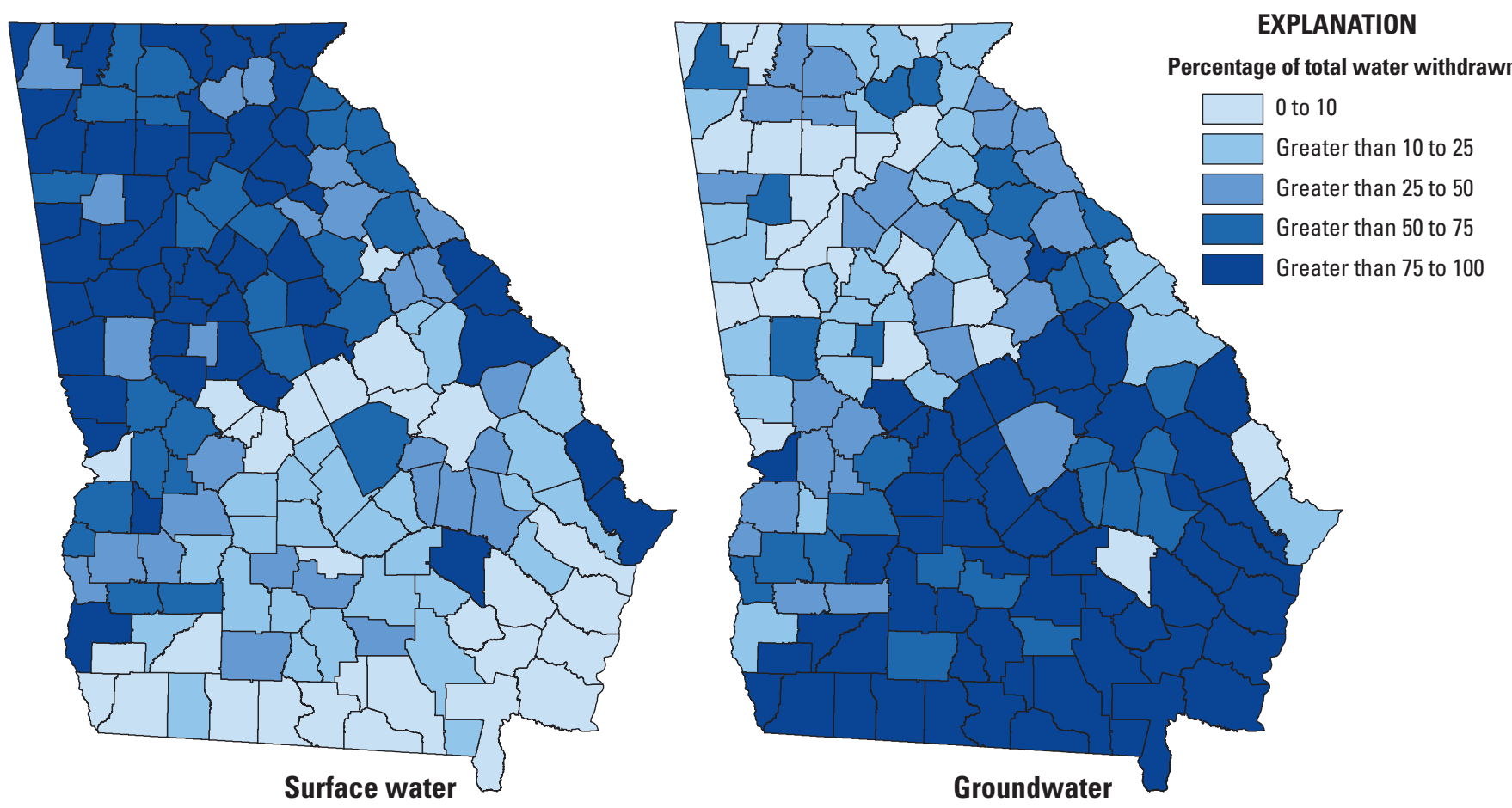

Figure 9. Percentage of total water withdrawals by county and source in Georgia, 2010.

Table 3. Permitted commercial and industrial water withdrawals by major North American Industrial Classification code (NAICS, appendix 1) in Georgia, 2010.

\begin{tabular}{|lccc|}
\hline \multicolumn{1}{c}{$\begin{array}{c}\text { NAICS } \\
\text { (Appendix 1) }\end{array}$} & \multicolumn{3}{c}{$\begin{array}{c}\text { Withdrawals } \\
\text { (million gallons per day) }\end{array}$} \\
\cline { 2 - 4 } & Groundwater & Surface water & Total \\
\hline 322-Paper, pulp & 132.3 & 254.8 & 387.1 \\
\hline 212-Kaolin and ball clay & 34.72 & 8.43 & 43.15 \\
\hline 325-Chemical products & 16.25 & 15.81 & 32.06 \\
\hline 311-Food & 9.22 & 3.16 & 12.38 \\
\hline 313, 314-Textiles & 3.16 & 6.14 & 9.30 \\
221-Electrical utility & 0.89 & 0.00 & 0.89 \\
\hline 327-Stone, clay & 2.68 & 0.10 & 2.78 \\
\hline 312-Beverage manufacturing & 2.85 & 0.00 & 2.85 \\
\hline 321-Lumber and wood products & 2.43 & 0.00 & 2.45 \\
\hline 331-Primary metals & 0.44 & 0.12 & 0.56 \\
\hline 339-Miscellaneous manufacturing & 0.53 & 0.00 & 0.53 \\
\hline 326-Rubber, plastic & 0.23 & 0.00 & 0.23 \\
\hline 332-Metal fabrication & 0.05 & 0.00 & 0.05 \\
\hline Total & $\mathbf{2 0 5 . 8}$ & $\mathbf{2 8 8 . 6}$ & $\mathbf{4 9 4 . 4}$ \\
\hline All other industries & 0.70 & 0.00 & 0.70 \\
\hline
\end{tabular}




\section{Water Use and Surface-Water Returns by Major River Basin}

Of the $2,624 \mathrm{Mgal} / \mathrm{d}$ of water withdrawn for non-thermoelectric uses in 2010, about 57 percent was self-supplied water (1,503 Mgal/d; table 2). Non-thermoelectric water withdrawals were greatest in the Apalachicola River Basin (1,250 Mgal/d) and smallest in the Ochlockonee River Basin (26 Mgal/d; table 4). Per capita water use (public supply, domestic, commercial, and industrial water use per person) ranged from $81 \mathrm{gal} / \mathrm{d}$ in the Ocmulgee River Basin to $553 \mathrm{gal} / \mathrm{d}$ in the Altamaha River Basin (table 4).

\section{Self-Supplied Water Use}

Self-supplied water use is water withdrawn directly from a groundwater or a surface-water source by a user, in contrast to water that is delivered by a public supplier. Self-supplied domestic withdrawals amounted to $107 \mathrm{Mgal} / \mathrm{d}$ and 56 percent of that use was in the Apalachicola (26.2 Mgal/d), Ocmulgee (20.7 Mgal/d), and Savannah River Basins (12.4 Mgal/d; table 4). In the Coosa and Tallapoosa River Basins, only 6.5 percent of the population relied on domestic wells for their water supply (table 4). In contrast, nearly 47 percent of the population in the Ochlockonee River Basin and 57 percent of the population in the Altamaha River Basin relied on domestic wells for their water (table 4).

About $515 \mathrm{Mgal} / \mathrm{d}$ was withdrawn by self-supplied commercial, industrial, and mining users in 2010 (table 4). Self-supplied commercial and industrial use was greatest in the Apalachicola (134 Mgal/d) and Savannah River (130 Mgal/d) Basins. In contrast, self-supplied commercial and industrial use was lowest $(0.81 \mathrm{Mgal} / \mathrm{d}$ each $)$ in the Ochlockonee and Tennessee River Basins. Mining withdrawals were greatest in the Apalachicola (6.1 Mgal/d), Ocmulgee (2.6 Mgal/d), and Savannah River Basins (2.5 Mgal/d), but lowest in the Satilla and St. Mary's River (0.15 Mgal/d), Tennessee River (0.20 Mgal/d), and the Suwannee and Aucilla River Basins (0.22 Mgal/d).

Crop irrigation, livestock management, and aquaculture are the primary agricultural users of water in Georgia. Crop irrigation accounts for about 90 percent of all water withdrawn for agriculture in Georgia (table 2). Agricultural activities

Table 4. Water withdrawals and public-supply deliveries by water-use category, for major river basins in Georgia, 2010.

$[-$, not applicable $]$

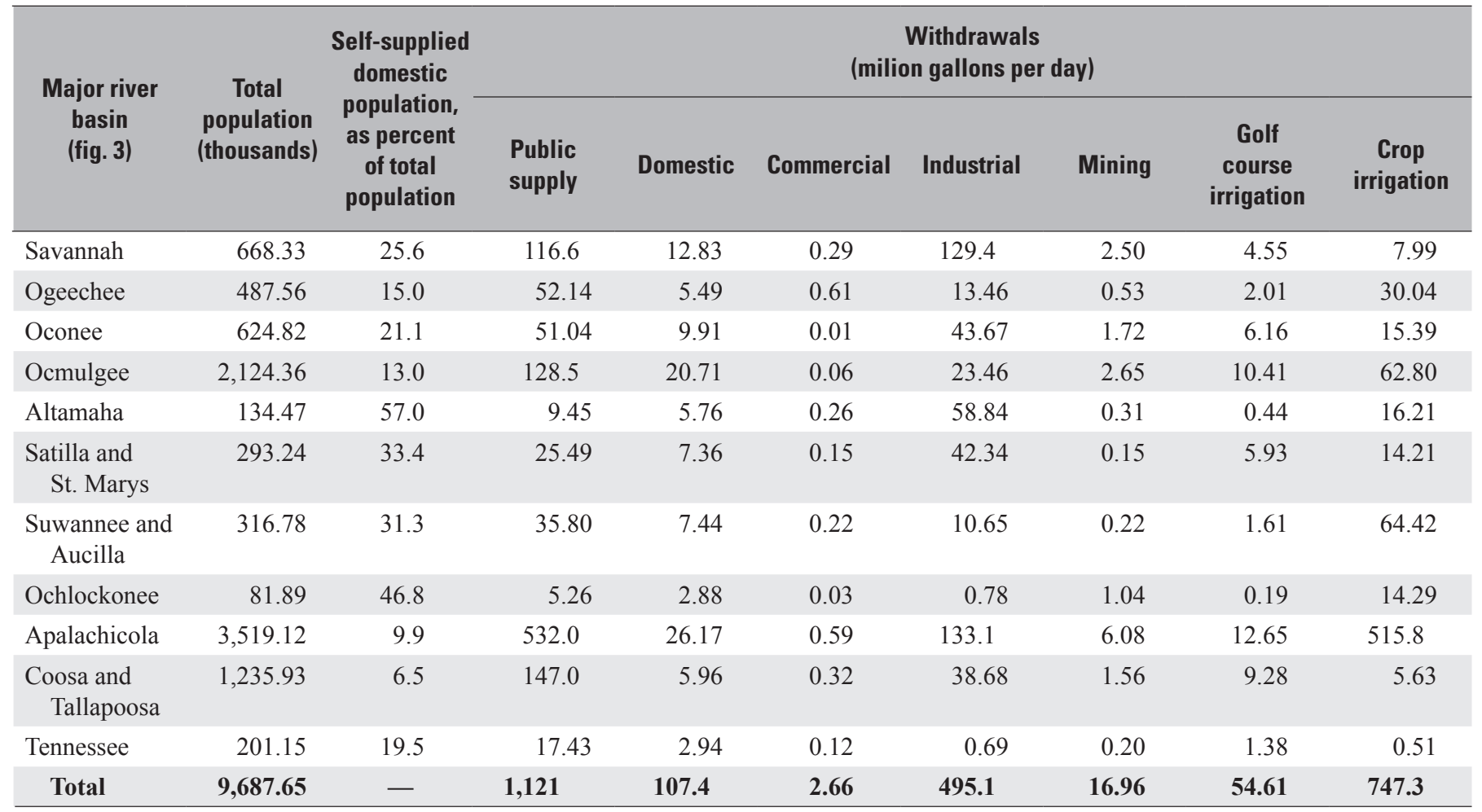

aPer capita use based on public-supply, domestic, commercial, and industrial withdrawals divided by the total population in the basin.

bPublic-supply system losses include water lost from the water distribution system and includes pipe leaks and breaks, fire suppression, and unauthorized use.

cAbout $6.7 \mathrm{Mgal} / \mathrm{d}$ of water was delivered into and used in Georgia by public water suppliers in Alabama and Tennessee in 2010.

Includes $213 \mathrm{Mgal} / \mathrm{d}$ of saline surface water. 
withdrew about $827 \mathrm{Mgal} / \mathrm{d}$ of water in 2010 and 65 percent of these withdrawals were in the Apalachicola River Basin (table 4). Agricultural withdrawals in the Apalachicola Basin were 8-times higher than those in the Suwannee and Aucilla River Basins (67.2 Mgal/d), the basins with the next largest withdrawals. In contrast, agricultural withdrawals were lowest in the Coosa and Tallapoosa River Basins (14.7 Mgal/d) and Ochlockonee River Basin (15.7 Mgal/d), and the Tennessee River Basin (15.8 Mgal/d).

Nearly $55 \mathrm{Mgal} / \mathrm{d}$ of water was withdrawn to irrigate golf courses in 2010 (table 2). Of that total, 61 percent was surface water. Golf course irrigation was highest in the Apalachicola River and Ocmulgee River Basins (respectively, 12.7 and $10.4 \mathrm{Mgal} / \mathrm{d}$; table 4). Water withdrawals for golf course irrigation were lowest in the Ochlockonee River Basin (0.19 Mgal/d).

Surface-water withdrawals for thermoelectric-power generation accounted for 59 percent of all surface-water withdrawals in 2010 (table 2). The thermoelectric-power generation from Plants Arkwright, Branch, Hatch, and Scherer in the Ocmulgee and Oconee River Basins withdrew 1,008 Mgal/d in 2010, representing about 49 percent of the surface-water withdrawals for thermoelectric power in Georgia. Within the Coosa and Tallapoosa River Basins, Plant Bowen and Plant Hammond withdrew $480 \mathrm{Mgal} / \mathrm{d}$ (23 percent) of surface water in 2010. In the Savannah River Basin, Plant McIntosh, Plant Vogtle and Plant Wentworth (Kraft) withdrew $342 \mathrm{Mgal} / \mathrm{d}$ (17 percent) of surface water in $2010,213 \mathrm{Mgal} / \mathrm{d}$ of which was saline surface water.

\section{Public-Supplied Water Use}

Any entity (public or private) that regularly delivers water to at least 25 people or has at least 15 water connections is considered a public water system (public supplier) under Georgia law (OCGA 12-5-172-11). In 2010, public suppliers withdrew about 1,121 Mgal/d in Georgia. Included in this report is public-supplied water delivered from public suppliers in Alabama, South Carolina, and Tennessee and used in Georgia. Water delivered from public suppliers in Georgia to Alabama, South Carolina, or Tennessee is counted as a public-supply delivery to water users in those States, but is not included in water-use calculations for Georgia because that water is not used in Georgia.

Table 4. Water withdrawals and public-supply deliveries by water-use category, for major river basins in Georgia, 2010.—Continued $[-$, not applicable $]$

\begin{tabular}{|c|c|c|c|c|c|c|c|c|c|c|}
\hline \multicolumn{3}{|c|}{$\begin{array}{l}\text { Withdrawals-Continued } \\
\text { (milion gallons per day) }\end{array}$} & \multirow{2}{*}{$\begin{array}{c}\text { Per } \\
\text { capita }^{\text {use }} \\
\text { usallons }^{\text {(gay) }}\end{array}$} & \multicolumn{6}{|c|}{$\begin{array}{l}\text { Public supply deliveries } \\
\text { (milion gallons per day) }\end{array}$} & \multirow[b]{2}{*}{$\begin{array}{c}\text { System loss, } \\
\text { as a percent } \\
\text { of total } \\
\text { deliveries }\end{array}$} \\
\hline $\begin{array}{c}\text { Livestock } \\
\text { and } \\
\text { aquaculture }\end{array}$ & $\begin{array}{l}\text { Thermo- } \\
\text { electric }\end{array}$ & Totals & & Domestic & $\begin{array}{c}\text { Commercial } \\
\text { and public } \\
\text { use }\end{array}$ & Industrial & Irrigation & $\begin{array}{l}\text { System } \\
\text { losses }^{b}\end{array}$ & Total' & \\
\hline 6.08 & $341.9^{\mathrm{d}}$ & 622.1 & 388 & 39.37 & 22.49 & 8.47 & 0.00 & 11.63 & 81.97 & 14.2 \\
\hline 9.50 & 952.4 & 1,090 & 167 & 28.57 & 14.66 & 5.12 & 0.00 & 7.97 & 56.32 & 14.2 \\
\hline 3.21 & 55.23 & 307.0 & 81 & 133.5 & 45.99 & 7.95 & 0.00 & 24.33 & 211.8 & 11.5 \\
\hline 2.12 & 56.89 & 150.3 & 553 & 4.32 & 1.73 & 0.73 & 0.00 & 1.18 & 7.96 & 14.8 \\
\hline 3.27 & 8.09 & 107.0 & 257 & 14.99 & 5.04 & 1.36 & 0.00 & 3.23 & 24.62 & 13.1 \\
\hline 23.68 & 151.4 & 1,402 & 197 & 271.1 & 79.62 & 35.03 & 3.14 & 92.95 & 481.8 & 19.3 \\
\hline 9.05 & 480.1 & 697.6 & 155 & 61.72 & 19.54 & 18.63 & 0.00 & 17.75 & 117.6 & 15.1 \\
\hline 15.30 & 0.29 & 38.86 & 105 & 12.95 & 2.35 & 0.34 & 0.00 & 3.10 & 18.74 & 16.5 \\
\hline 79.18 & 2,046 & 4,670 & - & 623.5 & 209.2 & 107.9 & 3.14 & 181.8 & 1,125 & 16.2 \\
\hline
\end{tabular}


Of the $1,125 \mathrm{Mgal} / \mathrm{d}$ of water delivered by public suppliers, 55 percent was used for domestic (residential; $623 \mathrm{Mgal} / \mathrm{d}$ ) purposes in Georgia (table 2). The domestic use of water is directly related to the population served by public water suppliers and inversely related to the self-supplied populations; thus, public-supplied domestic water use was greatest in the Apalachicola River Basin, which has the highest publicsupplied population and lowest in the Ochlockonee River Basin, which has the lowest public-supplied population.

Nearly 19 percent of public-supplied water was delivered to commercial customers (including public-use connections) in 2010 (table 2). Public-supply deliveries for commercial and public uses were greatest in the Apalachicola ( $80 \mathrm{Mgal} / \mathrm{d}$ ) and Ocmulgee River Basins (46 Mgal/d). In contrast, commercial deliveries were lowest in the Ochlockonee (0.43 Mgal/d) and the Altamaha (1.7 Mgal/d) River Basins (table 4).

Nearly 9.6 percent of public-supplied water was delivered to industrial customers in 2010 (tables 2 and 4). Industrial uses of public-supplied water were greatest in the Apalachicola (35 Mgal/d) and Ogeechee (27 Mgal/d) River Basins (table 4). In contrast, industrial deliveries were lowest in the Tennessee (0.34 Mgal/d) and Ochlockonee (0.05 Mgal/d) River Basins.

Water lost in the public-supply distribution system (system or public losses) is typically the result of pipe leakage and breaks. System losses are related to the total length of the pipeage, age of the pipes, and water pressure (U.S. Environmental Protection Agency, 2009). Thus, the largest and oldest public-supply systems will lose the greatest amount of water. In 2010, an estimated $182 \mathrm{Mgal} / \mathrm{d}$ of water was lost in the public-supply distribution system (table 2). Average public-supply losses were estimated at 16.1 percent of the water delivered in 2010 and ranged from 11.4 percent in the Ocmulgee River Basin to 19.3 percent in the Apalachicola River Basin (table 4).

\section{Surface-Water Returns}

In basins where there is a greater reliance on groundwater than surface water for water supplies, surface-water returns may exceed surface-water withdrawals because once used, that groundwater enters sanitary sewer systems for treatment and discharge to surface water. About 2,225 Mgal/d of water was discharged to surface water by public (municipal and privately owned) wasterwater treatment facilities, commercial, industrial, mining, aquaculture, and thermoelectric power facilities in 2010 (table 2). Thermoelectric power facilities returned nearly 49 percent of all water discharged to surface water, followed by public wastewater facilities ( 32 percent), and combined commercial and industrial discharges (19 percent).

River basins with the greatest populations returned the greatest amount of publicly-treated wastewater to surface water; thus, the Apalachicola and Ocmulgee River Basins received the greatest amount of public wastewater (respectively, 341 and $111 \mathrm{Mgal} / \mathrm{d}$, table 5). In contrast, public wastewater discharges were lowest in the Tennessee $(2.44 \mathrm{Mgal} / \mathrm{d})$ and the Altamaha (7.3 Mgal/d) River Basins in 2010 .

About 19 percent of surface-water returns were contributed by the commercial, industrial, mining, and aquaculture sectors in Georgia (table 5). Surface-water returns from commercial facilities were greatest in the Altamaha

Table 5. Surface-water returns by water-use category for major river basins in Georgia, 2010.

\begin{tabular}{|c|c|c|c|c|c|c|c|}
\hline \multirow{2}{*}{$\begin{array}{l}\text { Major river basin } \\
\text { (fig. 3) }\end{array}$} & \multicolumn{7}{|c|}{$\begin{array}{l}\text { Surface-water returns }{ }^{a} \\
\text { (million gallons per day) }\end{array}$} \\
\hline & $\begin{array}{c}\text { Public } \\
\text { wastewater }\end{array}$ & Commercial & Industrial & Mining & Aquaculture & $\begin{array}{l}\text { Thermo- } \\
\text { electric }\end{array}$ & Totals \\
\hline Savannah & 75.02 & 0.03 & 140.7 & 4.83 & 0.00 & 205.0 & 425.6 \\
\hline Ogeechee & 18.47 & 0.00 & 9.15 & 0.00 & 0.00 & 0.00 & 27.59 \\
\hline Ocmulgee & 111.0 & 0.00 & 5.37 & 4.35 & 0.00 & 0.00 & 120.7 \\
\hline Altamaha & 7.31 & 0.82 & 53.88 & 0.00 & 0.00 & 0.00 & 62.01 \\
\hline Satilla and St. Marys & 23.06 & 0.00 & 40.05 & 0.00 & 0.00 & 8.10 & 71.21 \\
\hline Suwannee and Aucilla & 22.14 & 0.01 & 0.02 & 0.00 & 0.00 & 0.00 & 22.16 \\
\hline Tennessee & 2.44 & 0.00 & 0.93 & 0.00 & 1.95 & 0.00 & 5.29 \\
\hline Total & 711.4 & 1.21 & 405.4 & 24.10 & 1.95 & 1,081 & 2,225 \\
\hline
\end{tabular}

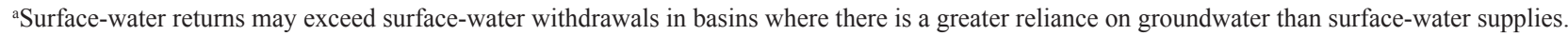


$(0.82 \mathrm{Mgal} / \mathrm{d})$ and the Ochlockonee $(0.27 \mathrm{Mgal} / \mathrm{d})$ River Basins; however, seven basins - Ogeechee, Ocmulgee, Satilla and St. Mary's, Coosa and Tallapoosa, and Tennessee - did not receive any return flows from commercial facilities in 2010. Surface-water returns from industrial facilities were greatest in the Savannah (141 Mgal/d) and Coosa and Tallapoosa (91 Mgal/d) River Basins, whereas the Ochlockonee $(0.60 \mathrm{Mgal} / \mathrm{d})$ and the Suwannee and Aucilla $(0.02 \mathrm{Mgal} / \mathrm{d})$ River Basins received the lowest return flows from industrial facilities in 2010. Surface-water returns from mining activities were greatest in the Apalachicola River Basin (6.7 Mgal/d) followed by the Savannah and Oconee River Basins (4.8 Mgal/d each). Federal and State fish hatcheries discharged nearly $2 \mathrm{Mgal} / \mathrm{d}$ of water to surface water in the Tennessee Basin, the only basin with reported surface-water returns from aquaculture facilities.

Surface-water returns from thermoelectric-power facilities are primarily from facilities that utilize once-through cooling processes. Within the Coosa and Tallapoosa Basins, Plant Hammond returned $448 \mathrm{Mgal} / \mathrm{d}$ (41 percent of all returns in Georgia) to surface water in 2010, whereas Plant Arkwright and Plant Branch, and the Crisp County plant in the Oconee River Basin returned $369 \mathrm{Mgal} / \mathrm{d}$ (34 percent; table 5). In the Savannah River Basin, Plant McIntosh returned $205 \mathrm{Mgal} / \mathrm{d}$ to the lower Savannah River in 2010.

\section{Water Use by Principal Aquifer}

About 1,189 Mgal/d of groundwater were withdrawn from the principal aquifers in Georgia during 2010 (tables 2 and 6). About 68 percent of all groundwater withdrawals in 2010 came from the Floridan aquifer system, followed by the Cretaceous aquifer system (15 percent), the crystalline-rock aquifers (5.3 percent), and the Claiborne aquifer (5.0 percent). The surficial aquifer system supplied the least amount of groundwater in 2010, only 0.1 percent of total groundwater withdrawals.

In 2010, nearly 3.1 million people or 32 percent of the 2010 Georgia population used groundwater for domestic purposes (tables 2 and 6). Of that population, about 1.4 million people relied on small domestic wells for their water (table 2). The Floridan aquifer system supplied about 56 percent of the population using groundwater, followed by the the Cretaceous aquifer system (22 percent), and the crystalline-rock and Paleozoic-rock aquifers (15.4 percent). The Claiborne and Clayton aquifers supplied about 5 percent of the population using groundwater in 2010. Only about 1.6 percent of the groundwater population used water from the Brunswick aquifer or surficial aquifer system.

\section{Self-Supplied Water Use}

Of groundwater used in 2010, 79 percent was withdrawn by self-supplied users (table 6). Of the 1,189 Mgal/d of groundwater withdrawn in 2010, 50 percent was used for crop and golf course irrigation (599 Mgal/d), 17 percent was used by industry ( $206 \mathrm{Mgal} / \mathrm{d}), 9$ percent for domestic use (107 Mgal/d), and 2.3 percent for mining, livestock and aquaculture, thermoelectric power generation, and commercial uses (table 6). Except for mining, the Floridan aquifer system supplied the majority of the water used in the other seven use categories. Of the groundwater used for mining, 75 percent was withdrawn from the crystalline-rock aquifers (7.3 Mgal/d) and the Cretaceous aquifer system (5.3 Mgal/d). About 2.7 percent of the self-supplied groundwater used for irrigation and industry came from the Brunswick aquifer.

\section{Public Supply}

Only 21 percent of groundwater withdrawn in 2010 was used for public supply (table 6). Nearly 1.7 million people used groundwater delivered by public water suppliers for domestic purposes in 2010. The two principal aquifers in southern Georgia (south of the Fall Line), the Floridan and the Cretaceous aquifer systems, provided 58 and 22 percent, respectively, of the groundwater used for public supply (table 6). The crystalline-rock and the Paleozoic-rock aquifers provided nearly all of the groundwater used in northern Georgia. Public-supply withdrawals from the crystalline-rock and the Paleozoic-rock aquifers accounted for, respectively, 6.9 and 4.9 percent of all public-supplied groundwater withdrawn in Georgia in 2010.

In 2010, nearly all groundwater withdrawals for public supply were delivered to users in Georgia. About $0.4 \mathrm{Mgal} / \mathrm{d}$ was delivered to users in Alabama, South Carolina, and Tennessee. Domestic uses accounted for nearly 57 percent of all public-supply deliveries of groundwater in Georgia (table 6). Commercial and public uses accounted for 17 percent and industrial uses accounted for about 10 percent of public-supplied groundwater delivered in Georgia.

\section{Water Use and Surface-Water Returns by Water-Planning Region}

Total water withdrawals and public-supply deliveries for each of the 11 water-planning regions recognized by the State of Georgia are listed in table 7. A total of 1,228 Mgal/d of water was withdrawn for public supply and by self-supplied domestic users in 2010 (table 7). Water-planning regions with the greatest populations withdrew the greatest amount of water. Excluding withdrawals for thermoelectric-power generation, water withdrawals were greatest in the Lower Flint-Ochlockonee region (612 Mgal/d) followed by the Metropolitan North Georgia Water Planning District (hereafter referred to as the Metro North Georgia District; $591 \mathrm{Mgal} / \mathrm{d}$ ). Water withdrawals were lowest in the Suwannee-Satilla region (116 Mgal/d). 
Table 6. Groundwater withdrawals and public-supply deliveries by use category for the principal aquifers in Georgia, 2010.

\begin{tabular}{|c|c|c|c|c|c|c|c|c|c|c|}
\hline \multirow{2}{*}{$\begin{array}{c}\text { Aquifer } \\
\text { name } \\
\text { (fig. 2) }\end{array}$} & \multirow{2}{*}{$\begin{array}{l}\text { Population }^{a} \\
\text { (thousands) }\end{array}$} & \multicolumn{9}{|c|}{$\begin{array}{c}\text { Withdrawals } \\
\text { (million gallons per day) }\end{array}$} \\
\hline & & $\begin{array}{l}\text { Public } \\
\text { supply }\end{array}$ & Domestic & Commercial & Industrial & Mining & Irrigation & $\begin{array}{l}\text { Livestock and } \\
\text { aquaculture }\end{array}$ & $\begin{array}{l}\text { Thermo- } \\
\text { electric }\end{array}$ & Total \\
\hline \multicolumn{11}{|c|}{ Floridan aquifer system (S400FLORDN) } \\
\hline $\begin{array}{r}\text { Floridan } \\
\text { system } \\
\end{array}$ & $1,452.03$ & 148.3 & 57.22 & 1.50 & 142.4 & 2.95 & 445.9 & 2.90 & 1.82 & 803.0 \\
\hline \multicolumn{11}{|c|}{ Surficial aquifer system (S100SURFCL) } \\
\hline Brunswick & 27.84 & 2.87 & 1.04 & 0.06 & 8.93 & 0.00 & 23.26 & 0.00 & 0.00 & 36.16 \\
\hline Surficial & 2.68 & 0.28 & 0.12 & 0.00 & 0.10 & 0.00 & 0.76 & 0.00 & 0.03 & 1.29 \\
\hline \multicolumn{11}{|c|}{ Southeastern Coastal Plain aquifer system (S100SECSLP) } \\
\hline Claiborne & 120.51 & 7.63 & 0.07 & 0.04 & 1.77 & 0.37 & 48.68 & 0.51 & 0.00 & $\mathbf{5 9 . 0 7}$ \\
\hline $\begin{array}{l}\text { Crystalline } \\
\text { rock }\end{array}$ & 808.31 & 17.22 & 22.40 & 0.20 & 3.91 & 7.39 & 11.17 & 1.25 & 0.06 & 63.60 \\
\hline \multicolumn{11}{|c|}{ Valley and ridge aquifer (N500VLYRDG) } \\
\hline $\begin{array}{l}\text { Paleozoic } \\
\text { rock }\end{array}$ & 139.29 & 12.06 & 5.21 & 0.00 & 2.65 & 0.66 & 0.42 & 0.33 & 0.00 & 21.33 \\
\hline Total & $3,089.21$ & 247.8 & 107.4 & 2.08 & 206.5 & 16.67 & $\mathbf{5 9 9 . 0}$ & 6.36 & 2.92 & 1,189 \\
\hline
\end{tabular}

\begin{tabular}{|c|c|c|c|c|c|c|}
\hline \multirow{2}{*}{$\begin{array}{l}\text { Aquifer name } \\
\text { (fig. 2) }\end{array}$} & \multirow{2}{*}{$\begin{array}{l}\text { Population }^{a} \\
\text { (thousands) }\end{array}$} & \multicolumn{5}{|c|}{$\begin{array}{l}\text { Public-supply deliveries } \\
\text { (million gallons per day) }\end{array}$} \\
\hline & & Domestic & $\begin{array}{c}\text { Commercial } \\
\text { and } \\
\text { public use }\end{array}$ & Industrial & Losses & Total $^{\mathrm{b}}$ \\
\hline \multicolumn{7}{|c|}{ Floridan aquifer system (S400FLORDN) } \\
\hline Floridan system & $1,452.03$ & 79.05 & 25.79 & 21.15 & 23.77 & 149.8 \\
\hline \multicolumn{7}{|c|}{ Surficial aquifer system (S100SURFCL) } \\
\hline Brunswick & 27.84 & 1.79 & 0.49 & 0.13 & 0.51 & 2.92 \\
\hline Surficial & 2.68 & 0.14 & 0.08 & 0.02 & 0.04 & 0.28 \\
\hline \multicolumn{7}{|c|}{ Southeastern Coastal Plain aquifer system (S100SECSLP) } \\
\hline Claiborne & 120.51 & 4.20 & 1.64 & 0.52 & 1.42 & 7.78 \\
\hline Clayton & 40.81 & 2.82 & 1.25 & 0.25 & 0.97 & 5.29 \\
\hline Cretaceous system & 497.74 & 30.66 & 9.90 & 3.03 & 9.00 & 52.59 \\
\hline \multicolumn{7}{|c|}{ Piedmont and Blue Ridge crystalline rock aquifers (N400PDMBRX) } \\
\hline Crystalline rock & 808.31 & 13.14 & 1.92 & 0.38 & 1.85 & 17.29 \\
\hline \multicolumn{7}{|c|}{ Valley and ridge aquifer (N500VLYRDG) } \\
\hline Paleozoic rock & 139.29 & 8.52 & 0.89 & 0.66 & 1.75 & 11.82 \\
\hline Total & $3,089.21$ & 140.3 & 41.97 & 25.81 & 39.34 & 247.4 \\
\hline
\end{tabular}

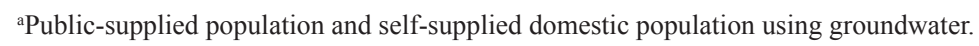

${ }^{\text {b}}$ The difference between the amount of water withdrawn and the amount of water delivered represents deliveries from water suppliers in Alabama and Tennessee to water users in Georgia. 
Table 7. Water withdrawals and public-supply deliveries by water-use category for water-planning regions in Georgia, 2010.

[Mgal/d, million gallons per day; —, not applicable]

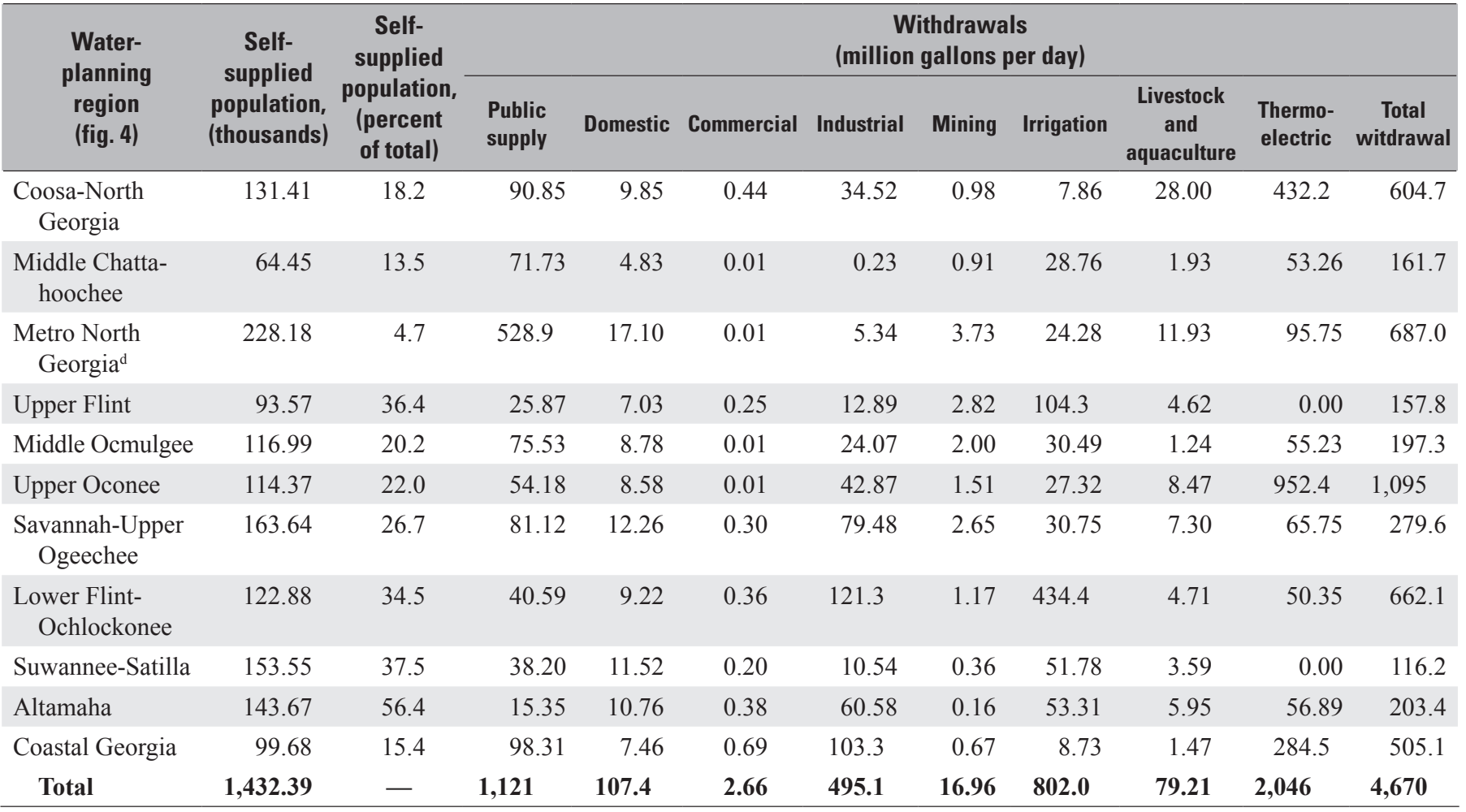

\begin{tabular}{|c|c|c|c|c|c|c|c|}
\hline \multirow{2}{*}{$\begin{array}{l}\text { Water-planning region } \\
\text { (fig. 4) }\end{array}$} & \multirow{2}{*}{$\begin{array}{c}\text { Public- } \\
\text { supplied } \\
\text { population } \\
\text { (thousands) }\end{array}$} & \multicolumn{6}{|c|}{$\begin{array}{l}\text { Public-supply deliveries } \\
\text { (million gallons per day) }\end{array}$} \\
\hline & & Domestic & $\begin{array}{l}\text { Commercial } \\
\text { and } \\
\text { public use }\end{array}$ & Industrial & Irrigation ${ }^{\mathrm{a}}$ & $\begin{array}{l}\text { System } \\
\text { losses }^{b}\end{array}$ & $\begin{array}{c}\text { Total } \\
\text { deliveries }^{c}\end{array}$ \\
\hline Coosa-North Georgia & 614.85 & 48.99 & 18.63 & 16.24 & 0.00 & 15.76 & 99.62 \\
\hline Middle Chattahoochee & 406.39 & 34.14 & 11.96 & 10.10 & 0.00 & 10.22 & 66.42 \\
\hline Metro North Georgia ${ }^{d}$ & $4,604.56$ & 322.5 & 94.87 & 27.68 & 3.14 & 84.13 & 532.3 \\
\hline Upper Flint & 155.75 & 12.88 & 3.64 & 1.10 & 0.00 & 5.99 & 23.61 \\
\hline Middle Ocmulgee & 450.74 & 42.50 & 12.10 & 3.85 & 0.00 & 10.75 & 69.20 \\
\hline Upper Oconee & 438.18 & 30.80 & 14.75 & 5.25 & 0.00 & 9.97 & 60.77 \\
\hline Savannah-Upper Ogeechee & 446.57 & 36.79 & 21.68 & 7.98 & 0.00 & 13.34 & 79.79 \\
\hline Lower Flint-Ochlockonee & 233.55 & 21.39 & 8.54 & 3.27 & 0.00 & 7.37 & 40.57 \\
\hline Suwannee-Satilla & 253.03 & 22.33 & 7.46 & 2.92 & 0.00 & 5.49 & 38.20 \\
\hline Altamaha & 111.09 & 8.88 & 3.00 & 1.00 & 0.00 & 2.49 & 15.37 \\
\hline Coastal Georgia & 540.54 & 42.25 & 12.59 & 28.54 & 0.00 & 16.32 & 99.70 \\
\hline Total & $8,255.25$ & 623.5 & 209.2 & 107.9 & 3.14 & 181.8 & 1,125 \\
\hline
\end{tabular}

aRepresents public-supplied water measured by irrigation-only meters.

bPublic-supply system losses is water lost from the water distribution system and includes pipe leaks and breaks, fire suppression, and unauthorized use. About $3 \mathrm{Mgal} / \mathrm{d}$ of public-supply deliveries are unaccountable and may be additional system losses.

'Public-supply withdrawals may not equal public-supply deliveries because of interregion and interstate transfers.

${ }^{\mathrm{d} M e t r o p o l i t a n}$ North Georgia Water Planning District. 


\section{Self-Supplied Water Use}

About 1,500 Mgal/d of water was withdrawn by selfsupplied users, excluding thermoelectric power facilities, in 2010 (table 7). The percentage of the population that was self-supplied in each planning region ranged from 4.7 in the Metro North Georgia District to 56 in the Altamaha planning region. Although the self-supplied population in the Metro North Georgia District represented only 4.7 percent of the population, the self-supplied domestic withdrawals were greatest (17.3 Mgal/d) among the water-planning regions. Self-supplied domestic withdrawals were lowest in the Middle Chattahoochee water-planning region (4.8 Mgal/d). Self-supplied commercial withdrawals accounted for less than 0.2 percent of all self-supplied water withdrawn in 2010; most of the commercial withdrawals were in the Coastal Georgia, Coosa-North Georgia, and Altamaha water-planning regions.

Of the $495 \mathrm{Mgal} / \mathrm{d}$ of water withdrawn by self-supplied industrial users in 2010, 25 percent was withdrawn in the Lower Flint-Ochlockonee water-planning region and 21 percent in the Coastal Georgia water-planning region. Of the $17 \mathrm{Mgal} / \mathrm{d}$ withdrawn for mining, the greatest withdrawals were in the Metro North Georgia District (22 percent), followed by the Upper Flint (17 percent) and SavannahUpper Ogeechee (16 percent) water-planning regions. In 2010, about $802 \mathrm{Mgal} / \mathrm{d}$ was withdrawn for irrigation and the greatest withdrawals were in the Lower Flint-Ochlockonee (54 percent) and Upper Flint (13 percent) water-planning regions. Of 79.2 Mgal/d withdrawn for livestock and aquaculture uses, the greatest withdrawals were in the CoosaNorth Georgia water-planning region (35 percent), followed by the Metro North Georgia District (15 percent); in these two regions, poultry and beef production, and public and private fish hatcheries used the majority of water withdrawn for livestock and aquaculture. About 2,046 Mgal/d of water was withdrawn for thermoelectric-power generation and the greatest withdrawals were in the Upper Oconee waterplanning region (46 percent), followed by the Coosa-North Georgia (21 percent), and Coastal Georgia (14 percent) waterplanning regions.

\section{Public Supply}

In 2010, public water suppliers withdrew 1,121 Mgal/d of water and delivered 1,125 Mgal/d of water to customers in Georgia (table 7). About $0.4 \mathrm{Mgal} / \mathrm{d}$ was delivered to wholesale customers in Alabama, Tennessee, and South Carolina; about 6.7 Mgal/d was delivered from public suppliers in Alabama and Tennessee to customers and wholesale suppliers in Georgia.
Domestic use accounted for 55 percent of the publicsupply deliveries, followed by commercial and public uses at nearly 19 percent. About 9.6 percent of public-supplied water was delivered to industrial users. Estimated system losses during deliveries amounted to $182 \mathrm{Mgal} / \mathrm{d}$ or about 16 percent of public-supply deliveries (tables 2 and 7). Most public suppliers did not report their system losses on the water-use questionnaire; therefore, a coefficient of 15 percent was used to estimate unreported losses.

Of the water withdrawn by public suppliers, 47 percent was withdrawn in the Metro North Georgia District, followed by the Coastal Georgia and the Coosa-North Georgia waterplanning regions at, respectively, 8.8 and 8.1 percent of the total public-supply withdrawals. These three water-planning regions had the highest public-supplied populations in 2010 (table 7).

\section{Surface-Water Returns}

Public wastewater discharges accounted for 32 percent of the $2,225 \mathrm{Mgal} / \mathrm{d}$ of water returned to surface water and 62 percent of all non-thermoelectric returns (table 8). Four water-planning regions discharged 73 percent of all public wastewater returned to surface water in 2010. The Metro North Georgia District discharged $364 \mathrm{Mgal} / \mathrm{d}$ of publicly-treated wastewater to surface water, the greatest amount in Georgia by a large margin. The Coastal Georgia, Savannah-Upper Ogeechee, and Middle Ocmulgee water-planning regions each discharged publicly-treated wastewater at a rate 7 times lower than the discharges in the Metro North Georgia District.

Water discharges from commercial and industrial facilities accounted for about 18 percent and mining facilities accounted for 1.1 percent of all surface-water returns (table 8). Of the nearly $407 \mathrm{Mgal} / \mathrm{d}$ of water discharged to surface water by commercial and industrial facilities, 34 percent was discharged in the Coastal Georgia and 22 percent in the Coosa-North Georgia water-planning regions (table 8 ). Commercial and industrial discharges were lowest in the Upper Flint (0.39 Mgal/d) and Suwannee-Satilla (0.09 Mgal/d) water-planning regions. Mining activities discharged $24 \mathrm{Mgal} / \mathrm{d}$ of water to surface water and nearly 30 percent of that occurred in the Metro North Georgia District, whereas 20 percent of the mining discharges were in the Savannah-Upper Ogeechee water-planning region and 17 percent in the Middle Ocmulgee water-planning region (table 8). Surface-water returns from mining activities were not recorded in the Suwannee-Satilla, Altamaha, and Coastal Georgia water-planning regions in 2010. The dewatering of mine pits during sand and gravel extraction is the primary source of these surface-water returns. 
Table 8. Surface-water returns by water-use category for water planning regions in Georgia, 2010.

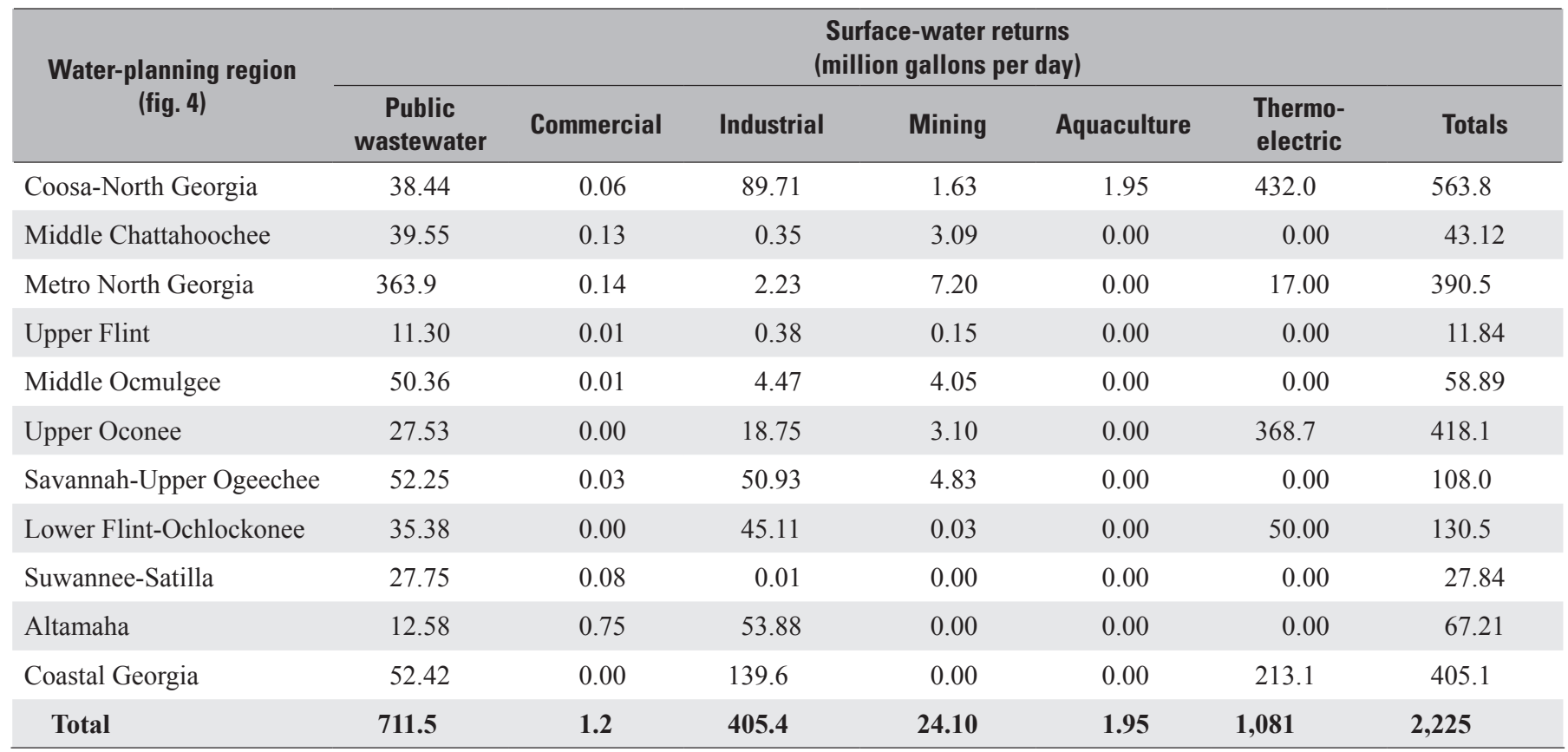

\section{Water Use and Surface-Water Returns by County}

Total water withdrawals, water use, and surfacewater returns by use category are listed in table 9 and in appendix 2 for each of the 159 Georgia counties. In 2010, 6 of the 159 counties in Georgia (Fulton, Gwinnett, Dekalb, Cobb, Clayton, and Chatham) provided water to 43 percent of the nearly 8.3 million people receiving public-supplied water in Georgia. At least 70 percent of the total population in 10 Georgia counties were self-supplied with water from domestic wells; however, in Brantley County, 87 percent of the population was self-supplied (table 10). In contrast, less than 1 percent of the 2010 population in Baldwin, Clayton, Dekalb, Gwinnett, and Muscogee Counties used self-supplied water. 
Table 9. Water withdrawals, water use, and surface water returns by county in Georgia, 2010.

[Mgal/d, million gallons per day]

\begin{tabular}{|c|c|c|c|c|c|c|c|c|c|c|c|}
\hline \multirow[b]{2}{*}{$\begin{array}{l}\text { County } \\
\text { (fig. 1) }\end{array}$} & \multicolumn{9}{|c|}{ Withdrawals, in Mgal/d } & \multirow[b]{2}{*}{$\begin{array}{c}\text { Total } \\
\text { use }^{\mathrm{b}} \\
\text { (Mgal/d) }\end{array}$} & \multirow[b]{2}{*}{$\begin{array}{c}\text { Surface- } \\
\text { water } \\
\text { returns } \\
\text { (Mgal/d) }\end{array}$} \\
\hline & $\begin{array}{l}\text { Public } \\
\text { supply }\end{array}$ & Domestic & $\begin{array}{l}\text { Commer- } \\
\text { cial and } \\
\text { public } \\
\text { use }^{\mathrm{a}}\end{array}$ & Industrial & Mining & Irrigation & $\begin{array}{l}\text { Livestock } \\
\text { and } \\
\text { aqua- } \\
\text { culture }\end{array}$ & $\begin{array}{l}\text { Thermo- } \\
\text { electric }\end{array}$ & Totals & & \\
\hline Appling & 0.91 & 0.94 & 0.00 & 0.00 & 0.00 & 1.76 & 0.27 & 56.90 & 60.78 & 60.78 & 0.93 \\
\hline Atkinson & 0.44 & 0.34 & 0.00 & 0.40 & 0.00 & 0.44 & 0.99 & 0.00 & 2.61 & 2.61 & 0.27 \\
\hline Baldwin & 6.58 & 0.03 & 0.00 & 0.01 & 0.08 & 0.08 & 0.12 & 0.00 & 6.90 & 8.96 & 4.44 \\
\hline Banks & 2.54 & 0.56 & 0.00 & 0.00 & 0.05 & 0.00 & 0.53 & 0.00 & 3.68 & 2.08 & 0.10 \\
\hline Barrow & 4.60 & 0.51 & 0.00 & 0.39 & 0.05 & 0.79 & 0.19 & 0.00 & 6.53 & 9.59 & 0.51 \\
\hline Bartow & 54.87 & 1.21 & 0.00 & 1.55 & 0.45 & 2.72 & 0.45 & 47.92 & 109.2 & 69.93 & 27.21 \\
\hline Bleckley & 0.74 & 0.59 & 0.12 & 0.00 & 0.00 & 6.32 & 0.08 & 0.00 & 7.85 & 7.85 & 0.33 \\
\hline Brantley & 0.25 & 1.20 & 0.00 & 0.00 & 0.00 & 0.03 & 0.04 & 0.00 & 1.52 & 1.52 & 0.00 \\
\hline Brooks & 0.94 & 0.72 & 0.01 & 0.06 & 0.00 & 5.61 & 0.42 & 0.00 & 7.76 & 7.76 & 0.00 \\
\hline Bryan & 2.57 & 0.21 & 0.00 & 0.02 & 0.00 & 0.08 & 0.37 & 0.00 & 3.25 & 3.25 & 0.97 \\
\hline Bulloch & 4.89 & 1.52 & 0.30 & 0.00 & 0.00 & 2.25 & 0.27 & 0.00 & 9.23 & 9.23 & 3.92 \\
\hline Burke & 1.06 & 1.05 & 0.01 & 0.23 & 0.00 & 10.06 & 0.29 & 65.41 & 78.11 & 78.11 & 1.04 \\
\hline Butts & 2.59 & 0.21 & 0.00 & 0.00 & 0.00 & 0.19 & 0.03 & 0.00 & 3.02 & 2.90 & 0.60 \\
\hline Calhoun & 0.64 & 0.24 & 0.00 & 0.00 & 0.02 & 27.40 & 0.18 & 0.00 & 28.48 & 28.48 & 0.47 \\
\hline Camden & 4.72 & 0.86 & 0.00 & 0.05 & 0.00 & 0.76 & 0.00 & 0.00 & 6.39 & 6.39 & 3.15 \\
\hline Chattooga & 3.32 & 0.42 & 0.00 & 5.31 & 0.02 & 0.00 & 1.65 & 0.00 & 10.72 & 10.24 & 7.67 \\
\hline Cherokee & 17.93 & 0.52 & 0.00 & 3.18 & 0.11 & 2.40 & 0.18 & 0.00 & 24.32 & 26.48 & 10.78 \\
\hline Clarke & 12.77 & 1.09 & 0.00 & 0.00 & 0.00 & 1.23 & 0.02 & 0.00 & 15.11 & 17.09 & 12.38 \\
\hline Clay & 0.29 & 0.11 & 0.00 & 0.00 & 0.00 & 4.10 & 0.08 & 0.00 & 4.58 & 4.58 & 0.09 \\
\hline Clayton & 9.35 & 0.04 & 0.00 & 0.00 & 0.25 & 0.16 & 0.01 & 0.00 & 9.81 & 27.64 & 2.20 \\
\hline Clinch & 0.48 & 0.22 & 0.00 & 0.05 & 0.00 & 0.00 & 0.01 & 0.00 & 0.76 & 0.76 & 0.26 \\
\hline Cobb & 43.84 & 3.22 & 0.00 & 0.39 & 0.00 & 1.97 & 0.02 & 5.25 & 54.69 & 82.72 & 79.42 \\
\hline Coffee & 3.48 & 1.59 & 0.00 & 0.00 & 0.00 & 3.48 & 0.58 & 0.00 & 9.13 & 9.13 & 2.33 \\
\hline Colquitt & 3.64 & 1.67 & 0.00 & 1.14 & 0.00 & 16.27 & 0.65 & 0.00 & 23.37 & 23.37 & 3.10 \\
\hline Columbia & 16.19 & 1.36 & 0.00 & 0.03 & 0.40 & 1.06 & 0.04 & 0.00 & 19.08 & 19.08 & 8.85 \\
\hline Cook & 1.80 & 0.53 & 0.00 & 0.00 & 0.21 & 5.18 & 0.16 & 0.00 & 7.88 & 7.88 & 1.26 \\
\hline Coweta & 7.73 & 0.90 & 0.00 & 0.00 & 0.12 & 1.72 & 0.07 & 42.58 & 53.12 & 55.58 & 3.59 \\
\hline Crawford & 0.35 & 0.67 & 0.00 & 0.00 & 0.39 & 1.23 & 0.22 & 0.00 & 2.86 & 2.86 & 0.12 \\
\hline Crisp & 2.23 & 0.60 & 0.00 & 0.12 & 0.00 & 15.72 & 0.07 & 0.00 & 18.74 & 18.74 & 2.88 \\
\hline Dade & 2.09 & 0.09 & 0.00 & 0.00 & 0.00 & 0.00 & 0.10 & 0.00 & 2.28 & 2.28 & 0.28 \\
\hline Dawson & 1.70 & 0.45 & 0.00 & 0.00 & 0.00 & 0.30 & 0.30 & 0.00 & 2.75 & 2.86 & 0.00 \\
\hline
\end{tabular}


Table 9. Water withdrawals, water use, and surface water returns by county in Georgia, 2010.—Continued

[Mgal/d, million gallons per day]

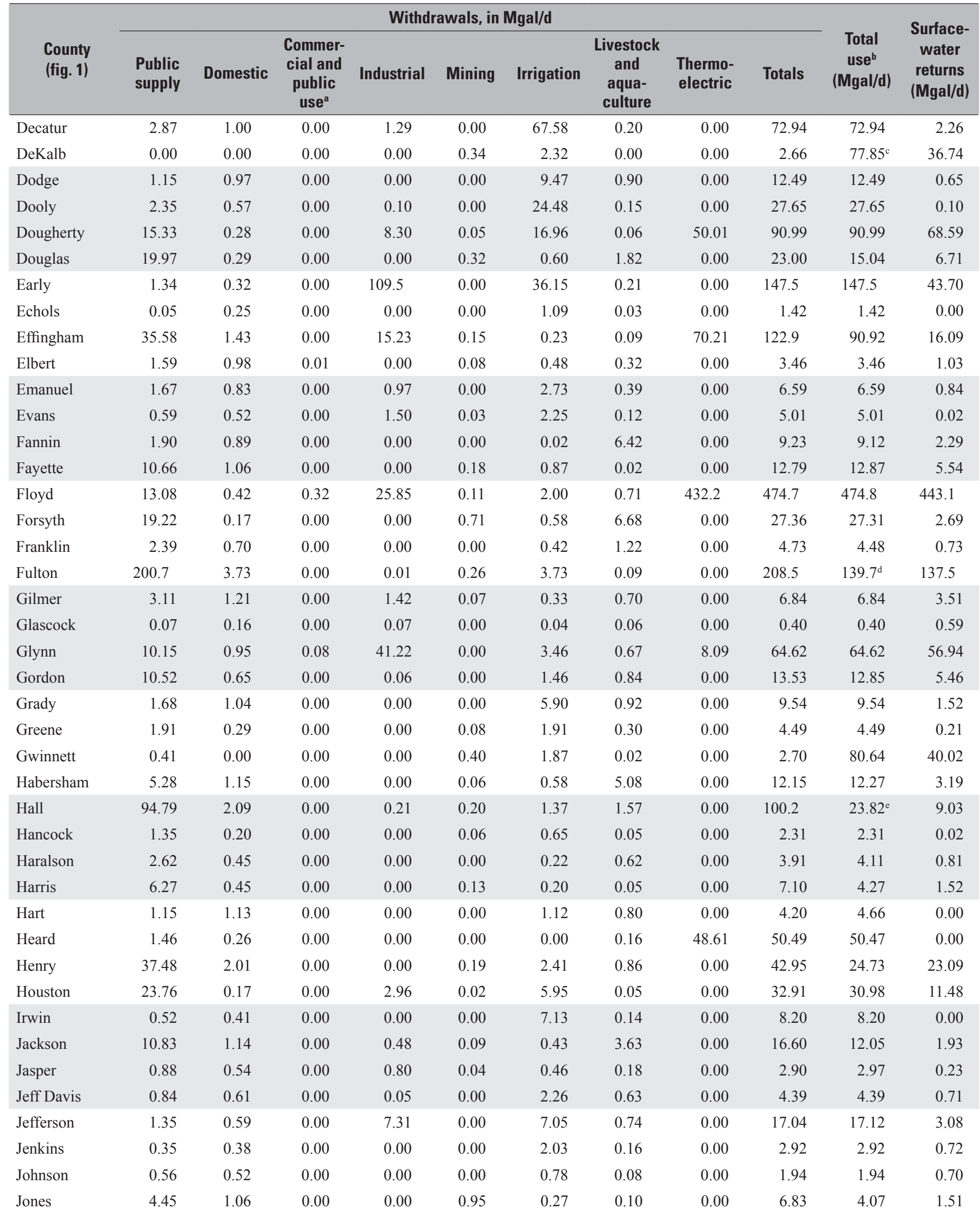


Table 9. Water withdrawals, water use, and surface water returns by county in Georgia, 2010._-Continued

[Mgal/d, million gallons per day]

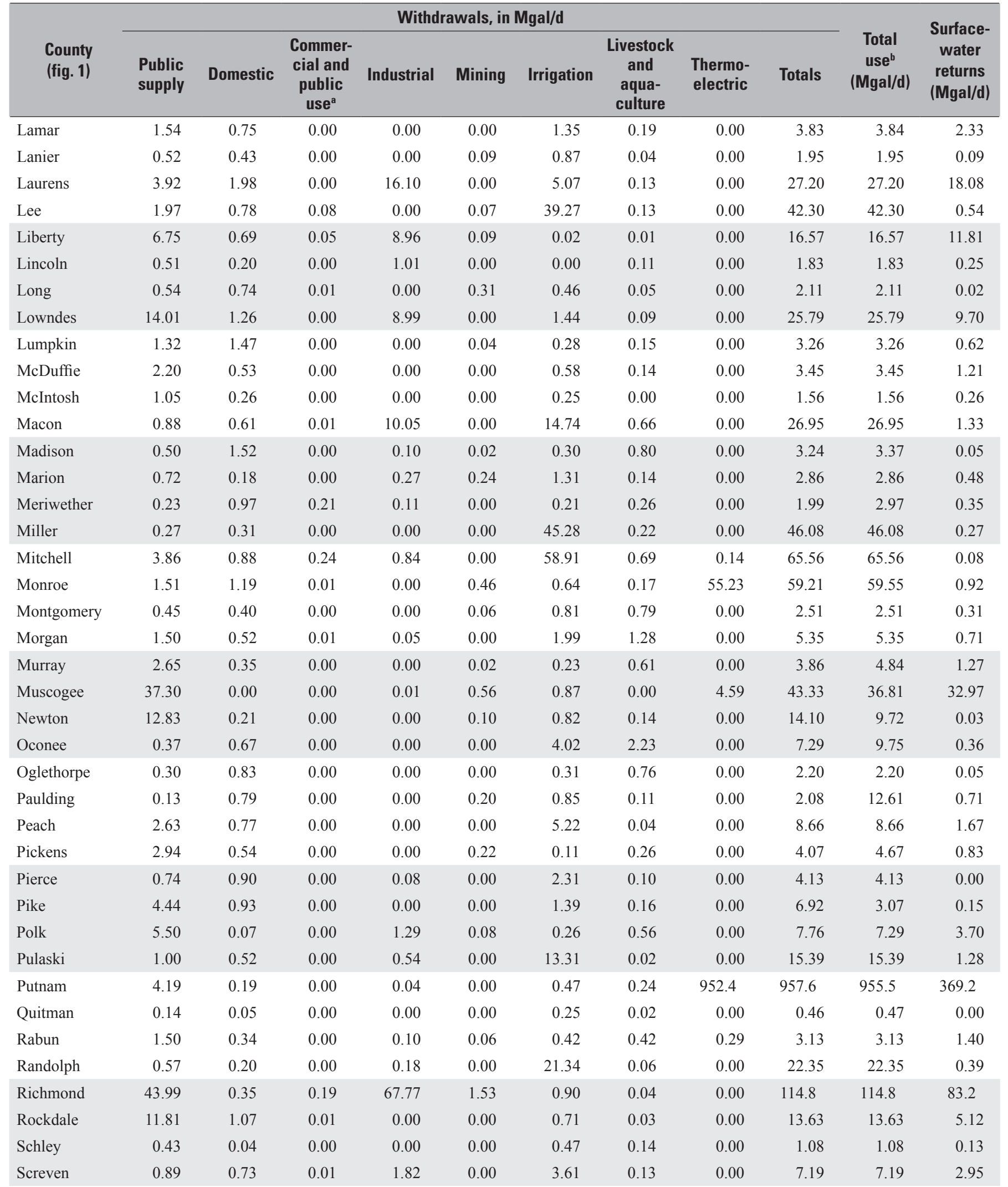


Table 9. Water withdrawals, water use, and surface water returns by county in Georgia, 2010.—Continued

[Mgal/d, million gallons per day]

\begin{tabular}{|c|c|c|c|c|c|c|c|c|c|c|c|}
\hline \multirow[b]{2}{*}{$\begin{array}{l}\text { County } \\
\text { (fig. 1) }\end{array}$} & \multicolumn{9}{|c|}{ Withdrawals, in Mgal/d } & \multirow[b]{2}{*}{$\begin{array}{c}\text { Total } \\
\text { use }^{\mathrm{b}} \\
\text { (Mgal/d) }\end{array}$} & \multirow[b]{2}{*}{$\begin{array}{c}\text { Surface- } \\
\text { water } \\
\text { returns } \\
\text { (Mgal/d) }\end{array}$} \\
\hline & $\begin{array}{l}\text { Public } \\
\text { supply }\end{array}$ & Domestic & $\begin{array}{c}\text { Commer- } \\
\text { cial and } \\
\text { public } \\
\text { use }^{\mathrm{a}}\end{array}$ & Industrial & Mining & Irrigation & $\begin{array}{l}\text { Livestock } \\
\text { and } \\
\text { aqua- } \\
\text { culture }\end{array}$ & $\begin{array}{l}\text { Thermo- } \\
\text { electric }\end{array}$ & Totals & & \\
\hline Seminole & 0.58 & 0.37 & 0.00 & 0.00 & 0.00 & 44.22 & 0.07 & 0.00 & 45.24 & 45.24 & 0.43 \\
\hline Spalding & 5.22 & 0.54 & 0.00 & 0.00 & 0.08 & 0.42 & 0.02 & 0.00 & 6.28 & 7.55 & 0.86 \\
\hline Sumter & 2.78 & 0.93 & 0.02 & 0.26 & 1.60 & 35.40 & 0.54 & 0.00 & 41.53 & 41.53 & 2.63 \\
\hline Talbot & 1.22 & 0.18 & 0.00 & 0.00 & 0.81 & 0.01 & 0.30 & 0.00 & 2.52 & 1.86 & 0.15 \\
\hline Taliaferro & 0.05 & 0.08 & 0.00 & 0.00 & 0.00 & 0.00 & 0.00 & 0.00 & 0.13 & 0.13 & 0.05 \\
\hline Tattnall & 1.11 & 1.32 & 0.22 & 0.03 & 0.01 & 5.03 & 1.66 & 0.00 & 9.38 & 9.38 & 1.88 \\
\hline Thomas & 5.49 & 1.03 & 0.03 & 0.19 & 1.03 & 3.01 & 0.29 & 0.00 & 11.07 & 11.07 & 4.06 \\
\hline Tift & 5.62 & 0.86 & 0.17 & 0.00 & 0.00 & 9.04 & 0.12 & 0.00 & 15.81 & 15.81 & 4.80 \\
\hline Toombs & 2.92 & 0.64 & 0.00 & 0.00 & 0.06 & 6.94 & 0.14 & 0.00 & 10.70 & 10.70 & 1.40 \\
\hline Towns & 1.37 & 0.05 & 0.00 & 0.00 & 0.01 & 0.17 & 2.88 & 0.00 & 4.48 & 4.48 & 0.29 \\
\hline Treutlen & 0.45 & 0.33 & 0.00 & 0.19 & 0.00 & 1.03 & 0.02 & 0.00 & 2.02 & 1.98 & 0.43 \\
\hline Troup & 10.52 & 1.08 & 0.01 & 0.02 & 0.11 & 0.13 & 0.10 & 0.00 & 11.97 & 12.09 & 5.87 \\
\hline Turner & 0.70 & 0.24 & 0.02 & 0.00 & 0.00 & 8.15 & 0.50 & 0.00 & 9.61 & 9.61 & 1.03 \\
\hline Twiggs & 0.39 & 0.52 & 0.00 & 5.16 & 0.03 & 0.41 & 0.06 & 0.00 & 6.57 & 6.57 & 1.93 \\
\hline Union & 1.90 & 0.28 & 0.00 & 0.00 & 0.08 & 0.39 & 5.36 & 0.00 & 8.01 & 8.01 & 0.29 \\
\hline Wayne & 1.73 & 1.18 & 0.02 & 57.84 & 0.00 & 1.09 & 0.09 & 0.00 & 61.95 & 61.95 & 55.91 \\
\hline Webster & 0.13 & 0.15 & 0.00 & 0.00 & 0.00 & 7.55 & 1.11 & 0.00 & 8.94 & 8.94 & 0.00 \\
\hline Wheeler & 0.18 & 0.42 & 0.00 & 0.00 & 0.00 & 2.02 & 0.10 & 0.00 & 2.72 & 2.72 & 0.37 \\
\hline White & 1.50 & 0.99 & 0.00 & 0.00 & 0.01 & 0.05 & 0.34 & 0.00 & 2.89 & 2.89 & 0.32 \\
\hline Whitfield & 20.68 & 0.41 & 0.00 & 0.00 & 0.21 & 0.59 & 0.69 & 0.00 & 22.58 & 26.47 & 87.79 \\
\hline Wilcox & 0.27 & 0.45 & 0.02 & 0.00 & 0.00 & 6.33 & 0.56 & 0.00 & 7.63 & 7.63 & 1.99 \\
\hline Wilkes & 1.02 & 0.37 & 0.00 & 0.00 & 0.00 & 0.08 & 0.43 & 0.00 & 1.90 & 1.90 & 0.62 \\
\hline Wilkinson & 0.67 & 0.30 & 0.00 & 13.78 & 0.01 & 0.05 & 0.02 & 0.00 & 14.83 & 14.83 & 2.87 \\
\hline Worth & 1.21 & 0.91 & 0.00 & 0.00 & 0.00 & 15.64 & 0.21 & 0.20 & 18.17 & 18.17 & 3.96 \\
\hline Total & 1,121 & 107.4 & 2.66 & 495.1 & 16.96 & 802.0 & 79.21 & 2,046 & 4,670 & 4,675 & 2,225 \\
\hline
\end{tabular}

aPublic use is water delivered by a public supplier to municipal buildings and golf courses, parks, and public safety buildings and areas.

${ }^{b}$ Total use includes public-supplied deliveries for domestic, commercial, industrial, and public uses; public supply losses (table 10); and self-supplied domestic, commercial, industrial, minning, irrigation, livestock and aquaculture, and thermoelectric uses. If total withdrawals exceed total use, then water was delivered out of the county. If total withdrawal is less than total use, then water was delivered into the county.

'Includes $0.12 \mathrm{Mgal} / \mathrm{d}$ metered irrigation deliveries.

${ }^{\mathrm{d}}$ Includes $2.77 \mathrm{Mgal} / \mathrm{d}$ metered irrigation deliveries.

'Includes $0.25 \mathrm{Mgal} / \mathrm{d}$ metered irrigation deliveries. 
Table 10. Population served and public-supply deliveries by county in Georgia, 2010.

[Mgal/d, million gallons per day; <, less than]

\begin{tabular}{|c|c|c|c|c|c|c|c|c|c|c|c|c|}
\hline \multirow{2}{*}{$\begin{array}{l}\text { County } \\
\text { (fig. 1) }\end{array}$} & \multicolumn{2}{|c|}{$\begin{array}{l}\text { Population } \\
\text { (thousands) }\end{array}$} & \multirow{2}{*}{$\begin{array}{l}\text { Percentage } \\
\text { of popu- } \\
\text { lation } \\
\text { self } \\
\text { supplied }\end{array}$} & \multicolumn{5}{|c|}{$\begin{array}{l}\text { Public-supply deliveries }{ }^{b} \\
\text { (million gallons per day) }\end{array}$} & \multicolumn{4}{|c|}{$\begin{array}{c}\text { Percentage of } \\
\text { public-supply deliveries }\end{array}$} \\
\hline & $\begin{array}{l}\text { Public } \\
\text { supplied }\end{array}$ & $\begin{array}{c}\text { Self- } \\
\text { supplied } \\
\text { domestic }^{\text {a }}\end{array}$ & & Domestic & $\begin{array}{l}\text { Commercial } \\
\text { and } \\
\text { public use }\end{array}$ & Industrial & Irrigation & $\begin{array}{l}\text { System } \\
\text { losses }^{c}\end{array}$ & Domestic & $\begin{array}{l}\text { Commercial } \\
\text { and public } \\
\text { use }\end{array}$ & Industrial & $\begin{array}{l}\text { System } \\
\text { losses }\end{array}$ \\
\hline Appling & 5.70 & 12.54 & 68.7 & 0.64 & 0.04 & 0.05 & 0.00 & 0.18 & 70.3 & 4.4 & 5.5 & 19.8 \\
\hline Atkinson & 3.90 & 4.48 & 53.4 & 0.25 & 0.01 & 0.12 & 0.00 & 0.06 & 56.8 & 2.3 & 27.3 & 13.6 \\
\hline Baker & 0.87 & 2.58 & 74.8 & 0.10 & 0.00 & 0.00 & 0.00 & 0.02 & 83.3 & 0.0 & 0.0 & 16.7 \\
\hline Baldwin & 45.29 & 0.43 & 0.9 & 2.61 & 4.00 & 0.78 & 0.00 & 1.25 & 30.2 & 46.3 & 9.0 & 14.5 \\
\hline Banks & 10.94 & 7.46 & 40.5 & 0.73 & 0.06 & 0.01 & 0.00 & 0.14 & 77.7 & 6.4 & 1.1 & 14.9 \\
\hline Barrow & 62.63 & 6.74 & 9.7 & 4.76 & 0.87 & 0.78 & 0.00 & 1.25 & 62.1 & 11.4 & 10.2 & 16.3 \\
\hline Bartow & 84.03 & 16.13 & 16.1 & 8.16 & 1.90 & 2.47 & 0.00 & 3.10 & 52.2 & 12.2 & 15.8 & 19.8 \\
\hline Bleckley & 5.22 & 7.84 & 60.0 & 0.32 & 0.15 & 0.00 & 0.00 & 0.27 & 42.6 & 21.4 & 0.0 & 36.0 \\
\hline Brantley & 2.38 & 16.03 & 87.1 & 0.18 & 0.05 & 0.00 & 0.00 & 0.02 & 72.0 & 20.0 & 0.0 & 8.0 \\
\hline Brooks & 6.67 & 9.57 & 58.9 & 0.61 & 0.21 & 0.01 & 0.00 & 0.11 & 64.9 & 22.3 & 1.1 & 11.7 \\
\hline Bryan & 27.38 & 2.85 & 9.4 & 2.31 & 0.16 & 0.00 & 0.00 & 0.10 & 89.9 & 6.2 & 0.0 & 3.9 \\
\hline Bulloch & 49.98 & 20.24 & 28.8 & 3.08 & 1.07 & 0.20 & 0.00 & 0.54 & 63.0 & 21.9 & 4.1 & 11.0 \\
\hline Burke & 9.36 & 13.96 & 59.9 & 0.62 & 0.10 & 0.14 & 0.00 & 0.20 & 58.5 & 9.4 & 13.2 & 18.9 \\
\hline Butts & 20.81 & 2.84 & 12.0 & 1.62 & 0.36 & 0.21 & 0.00 & 0.28 & 65.6 & 14.6 & 8.5 & 11.3 \\
\hline Calhoun & 3.47 & 3.22 & 48.2 & 0.32 & 0.22 & 0.00 & 0.00 & 0.10 & 50.0 & 34.4 & 0.0 & 15.6 \\
\hline Camden & 39.00 & 11.51 & 22.8 & 3.13 & 0.73 & 0.18 & 0.00 & 0.68 & 66.3 & 15.5 & 3.8 & 14.4 \\
\hline Chattooga & 20.45 & 5.57 & 21.4 & 1.68 & 0.25 & 0.27 & 0.00 & 0.64 & 59.2 & 8.8 & 9.5 & 22.5 \\
\hline Cherokee & 207.38 & 6.97 & 3.2 & 14.95 & 1.33 & 0.59 & 0.00 & 3.22 & 74.4 & 6.6 & 2.9 & 16.0 \\
\hline Clarke & 102.12 & 14.59 & 12.5 & 7.15 & 3.07 & 2.30 & 0.00 & 2.23 & 48.5 & 20.8 & 15.6 & 15.1 \\
\hline Clay & 1.70 & 1.48 & 46.6 & 0.23 & 0.03 & 0.00 & 0.00 & 0.03 & 79.3 & 10.3 & 0.0 & 10.3 \\
\hline Clayton & 258.90 & 0.52 & 0.2 & 19.34 & 2.06 & 1.65 & 0.00 & 4.13 & 71.2 & 7.6 & 6.1 & 15.2 \\
\hline Clinch & 3.84 & 2.96 & 43.5 & 0.28 & 0.07 & 0.07 & 0.00 & 0.06 & 58.3 & 14.6 & 14.6 & 12.5 \\
\hline Cobb & 645.08 & 43.00 & 6.2 & 34.81 & 23.54 & 3.02 & 0.00 & 10.50 & 48.4 & 32.8 & 4.2 & 14.6 \\
\hline Coffee & 21.14 & 21.22 & 50.1 & 1.72 & 0.97 & 0.32 & 0.00 & 0.47 & 49.4 & 27.9 & 9.2 & 13.5 \\
\hline Colquitt & 23.27 & 22.23 & 48.9 & 1.76 & 0.75 & 0.08 & 0.00 & 1.05 & 48.4 & 20.6 & 2.2 & 28.8 \\
\hline Columbia & 105.91 & 18.14 & 14.6 & 10.61 & 4.02 & 0.16 & 0.00 & 1.40 & 65.5 & 24.8 & 1.0 & 8.6 \\
\hline Cook & 10.20 & 7.01 & 40.7 & 1.13 & 0.36 & 0.03 & 0.00 & 0.28 & 62.8 & 20.0 & 1.7 & 15.6 \\
\hline Coweta & 115.29 & 12.03 & 9.4 & 6.88 & 1.14 & 0.85 & 0.00 & 1.32 & 67.5 & 11.2 & 8.3 & 13.0 \\
\hline Crawford & 3.76 & 8.87 & 70.2 & 0.26 & 0.04 & 0.00 & 0.00 & 0.05 & 74.3 & 11.4 & 0.0 & 14.3 \\
\hline Crisp & 15.46 & 7.98 & 34.0 & 1.20 & 0.62 & 0.01 & 0.00 & 0.40 & 53.8 & 27.8 & 0.4 & 17.9 \\
\hline Dade & 15.47 & 1.16 & 7.0 & 1.21 & 0.53 & 0.04 & 0.00 & 0.31 & 57.9 & 25.4 & 1.9 & 14.8 \\
\hline Dawson & 16.37 & 5.96 & 26.7 & 1.14 & 0.29 & 0.02 & 0.00 & 0.36 & 63.0 & 16.0 & 1.1 & 19.9 \\
\hline
\end{tabular}


Table 10. Population served and public-supply deliveries by county in Georgia, 2010.-Continued

[Mgal/d, million gallons per day; <, less than]

\begin{tabular}{|c|c|c|c|c|c|c|c|c|c|c|c|c|}
\hline \multirow{2}{*}{$\begin{array}{l}\text { County } \\
\text { (fig. 1) }\end{array}$} & \multicolumn{2}{|c|}{$\begin{array}{l}\text { Population } \\
\text { (thousands) }\end{array}$} & \multirow{2}{*}{$\begin{array}{l}\text { Percentage } \\
\text { of popu- } \\
\text { lation } \\
\text { self } \\
\text { supplied }\end{array}$} & \multicolumn{5}{|c|}{$\begin{array}{l}\text { Public-supply deliveries } \\
\text { (million gallons per day) }\end{array}$} & \multicolumn{4}{|c|}{$\begin{array}{c}\text { Percentage of } \\
\text { public-supply deliveries }\end{array}$} \\
\hline & $\begin{array}{l}\text { Public } \\
\text { supplied }\end{array}$ & $\begin{array}{c}\text { Self- } \\
\text { supplied } \\
\text { domestic }^{\text {a }}\end{array}$ & & Domestic & $\begin{array}{l}\text { Commercial } \\
\text { and } \\
\text { public use }\end{array}$ & Industrial & Irrigation & $\begin{array}{l}\text { System } \\
\text { losses }^{c}\end{array}$ & Domestic & $\begin{array}{l}\text { Commercial } \\
\text { and public } \\
\text { use }\end{array}$ & Industrial & $\begin{array}{l}\text { System } \\
\text { losses }\end{array}$ \\
\hline Decatur & 14.49 & 13.35 & 48.0 & 2.24 & 0.36 & 0.00 & 0.00 & 0.27 & 78.0 & 12.5 & 0.0 & 9.4 \\
\hline DeKalb & 691.89 & 0.00 & 0.0 & 38.97 & 20.49 & 5.29 & 0.12 & 10.32 & 51.8 & 27.3 & 7.0 & 13.7 \\
\hline Dooly & 7.29 & 7.63 & 51.1 & 0.76 & 0.51 & 0.52 & 0.00 & 0.56 & 32.3 & 21.7 & 22.1 & 23.8 \\
\hline Dougherty & 90.87 & 3.69 & 3.9 & 8.23 & 4.09 & 0.71 & 0.00 & 2.30 & 53.7 & 26.7 & 4.6 & 15.0 \\
\hline Douglas & 128.54 & 3.86 & 2.9 & 8.20 & 1.36 & 0.41 & 0.00 & 2.04 & 68.3 & 11.3 & 3.4 & 17.0 \\
\hline Early & 6.79 & 4.22 & 38.3 & 0.84 & 0.27 & 0.00 & 0.00 & 0.22 & 63.2 & 20.3 & 0.0 & 16.5 \\
\hline Echols & 0.76 & 3.27 & 81.2 & 0.05 & 0.00 & 0.00 & 0.00 & 0.00 & 100.0 & 0.0 & 0.0 & 0.0 \\
\hline Evans & 4.12 & 6.88 & 62.5 & 0.40 & 0.10 & 0.02 & 0.00 & 0.07 & 67.8 & 16.9 & 3.4 & 11.9 \\
\hline Fannin & 11.82 & 11.86 & 50.1 & 1.09 & 0.39 & 0.04 & 0.00 & 0.27 & 60.9 & 21.8 & 2.2 & 15.1 \\
\hline Fayette & 92.43 & 14.14 & 13.3 & 7.88 & 0.92 & 0.46 & 0.00 & 1.42 & 73.8 & 8.6 & 4.3 & 13.3 \\
\hline Floyd & 90.71 & 5.61 & 5.8 & 6.72 & 3.08 & 1.15 & 0.00 & 2.27 & 50.8 & 23.3 & 8.7 & 17.2 \\
\hline Forsyth & 173.29 & 2.22 & 1.3 & 11.50 & 3.11 & 2.14 & 0.00 & 2.42 & 60.0 & 16.2 & 11.2 & 12.6 \\
\hline Franklin & 12.72 & 9.36 & 42.4 & 1.13 & 0.42 & 0.40 & 0.00 & 0.19 & 52.8 & 19.6 & 18.7 & 8.9 \\
\hline Fulton & 870.81 & 49.77 & 5.4 & 84.18 & 9.61 & 3.53 & 2.77 & 31.80 & 63.8 & 7.3 & 2.7 & 24.1 \\
\hline Gilmer & 12.15 & 16.14 & 57.1 & 0.65 & 0.46 & 1.51 & 0.00 & 0.49 & 20.9 & 14.8 & 48.6 & 15.8 \\
\hline Glascock & 0.90 & 2.18 & 70.8 & 0.07 & 0.00 & 0.00 & 0.00 & 0.00 & 100.0 & 0.0 & 0.0 & 0.0 \\
\hline Hall & 151.82 & 27.86 & 15.5 & 8.43 & 2.16 & 4.85 & 0.25 & 2.69 & 45.9 & 11.8 & 26.4 & 14.6 \\
\hline Hancock & 6.80 & 2.63 & 27.9 & 0.51 & 0.62 & 0.00 & 0.00 & 0.22 & 37.8 & 45.9 & 0.0 & 16.3 \\
\hline Haralson & 22.75 & 6.03 & 21.0 & 1.65 & 0.47 & 0.05 & 0.00 & 0.65 & 58.5 & 16.7 & 1.8 & 23.0 \\
\hline Harris & 25.98 & 6.04 & 18.9 & 2.17 & 0.12 & 0.94 & 0.00 & 0.21 & 63.1 & 3.5 & 27.3 & 6.1 \\
\hline Hart & 10.10 & 15.11 & 59.9 & 0.98 & 0.35 & 0.04 & 0.00 & 0.24 & 60.9 & 21.7 & 2.5 & 14.9 \\
\hline Heard & 8.37 & 3.46 & 29.3 & 0.60 & 0.55 & 0.00 & 0.00 & 0.31 & 41.1 & 37.7 & 0.0 & 21.2 \\
\hline Henry & 177.08 & 26.84 & 13.2 & 13.87 & 2.10 & 0.49 & 0.00 & 2.80 & 72.0 & 10.9 & 2.5 & 14.5 \\
\hline Houston & 137.57 & 2.33 & 1.7 & 14.47 & 2.56 & 1.15 & 0.00 & 3.65 & 66.3 & 11.7 & 5.3 & 16.7 \\
\hline Irwin & 4.03 & 5.51 & 57.7 & 0.26 & 0.15 & 0.04 & 0.00 & 0.07 & 50.0 & 28.8 & 7.7 & 13.5 \\
\hline Jackson & 45.34 & 15.15 & 25.0 & 3.61 & 1.21 & 0.45 & 0.00 & 1.01 & 57.5 & 19.3 & 7.2 & 16.1 \\
\hline Jasper & 6.70 & 7.20 & 51.8 & 0.60 & 0.20 & 0.04 & 0.00 & 0.11 & 63.2 & 21.1 & 4.2 & 11.6 \\
\hline Jeff Davis & 6.96 & 8.11 & 53.8 & 0.52 & 0.07 & 0.05 & 0.00 & 0.20 & 61.9 & 8.3 & 6.0 & 23.8 \\
\hline Jefferson & 9.06 & 7.87 & 46.5 & 0.91 & 0.27 & 0.03 & 0.00 & 0.14 & 67.4 & 20.0 & 2.2 & 10.4 \\
\hline Jenkins & 3.34 & 5.00 & 60.0 & 0.22 & 0.06 & 0.00 & 0.00 & 0.07 & 62.9 & 17.1 & 0.0 & 20.0 \\
\hline Johnson & 3.06 & 6.92 & 69.3 & 0.20 & 0.28 & 0.00 & 0.00 & 0.08 & 35.7 & 50.0 & 0.0 & 14.3 \\
\hline Jones & 14.55 & 14.12 & 49.2 & 1.29 & 0.14 & 0.00 & 0.00 & 0.26 & 76.3 & 8.3 & 0.0 & 15.4 \\
\hline
\end{tabular}


Table 10. Population served and public-supply deliveries by county in Georgia, 2010.-Continued

[Mgal/d, million gallons per day; <, less than]

\begin{tabular}{|c|c|c|c|c|c|c|c|c|c|c|c|c|}
\hline \multirow{2}{*}{$\begin{array}{l}\text { County } \\
\text { (fig. 1) }\end{array}$} & \multicolumn{2}{|c|}{$\begin{array}{l}\text { Population } \\
\text { (thousands) }\end{array}$} & \multirow{2}{*}{$\begin{array}{l}\text { Percentage } \\
\text { of popu- } \\
\text { lation } \\
\text { self } \\
\text { supplied }\end{array}$} & \multicolumn{5}{|c|}{$\begin{array}{l}\text { Public-supply deliveries } \\
\text { (million gallons per day) }\end{array}$} & \multicolumn{4}{|c|}{$\begin{array}{c}\text { Percentage of } \\
\text { public-supply deliveries }\end{array}$} \\
\hline & $\begin{array}{l}\text { Public } \\
\text { supplied }\end{array}$ & $\begin{array}{c}\text { Self- } \\
\text { supplied } \\
\text { domestic }^{\text {a }}\end{array}$ & & Domestic & $\begin{array}{l}\text { Commercial } \\
\text { and } \\
\text { public use }\end{array}$ & Industrial & Irrigation & $\begin{array}{l}\text { System } \\
\text { losses }^{c}\end{array}$ & Domestic & $\begin{array}{l}\text { Commercial } \\
\text { and public } \\
\text { use }\end{array}$ & Industrial & $\begin{array}{l}\text { System } \\
\text { losses }\end{array}$ \\
\hline Lamar & 8.33 & 9.99 & 54.5 & 1.12 & 0.18 & 0.01 & 0.00 & 0.23 & 72.7 & 11.7 & 0.6 & 14.9 \\
\hline Lanier & 4.36 & 5.72 & 56.7 & 0.33 & 0.12 & 0.00 & 0.00 & 0.07 & 63.5 & 23.1 & 0.0 & 13.5 \\
\hline Liberty & 54.20 & 9.25 & 14.6 & 3.34 & 0.68 & 1.79 & 0.00 & 0.94 & 49.5 & 10.1 & 26.5 & 13.9 \\
\hline Lincoln & 5.28 & 2.72 & 34.0 & 0.38 & 0.06 & 0.01 & 0.00 & 0.06 & 74.5 & 11.8 & 2.0 & 11.8 \\
\hline Long & 4.56 & 9.90 & 68.5 & 0.32 & 0.14 & 0.00 & 0.00 & 0.08 & 59.3 & 25.9 & 0.0 & 14.8 \\
\hline Lowndes & 92.40 & 16.83 & 15.4 & 7.66 & 3.08 & 1.14 & 0.00 & 2.13 & 54.7 & 22.0 & 8.1 & 15.2 \\
\hline Macon & 6.57 & 8.17 & 55.4 & 0.48 & 0.08 & 0.15 & 0.00 & 0.17 & 54.5 & 9.1 & 17.0 & 19.3 \\
\hline Madison & 7.84 & 20.28 & 72.1 & 0.42 & 0.05 & 0.01 & 0.00 & 0.15 & 66.7 & 7.9 & 1.6 & 23.8 \\
\hline Marion & 6.29 & 2.45 & 28.0 & 0.47 & 0.06 & 0.05 & 0.00 & 0.14 & 65.3 & 8.3 & 6.9 & 19.4 \\
\hline Meriwether & 9.08 & 12.91 & 58.7 & 0.72 & 0.24 & 0.01 & 0.00 & 0.24 & 59.5 & 19.8 & 0.8 & 19.8 \\
\hline Miller & 1.97 & 4.16 & 67.8 & 0.15 & 0.07 & 0.00 & 0.00 & 0.05 & 55.6 & 25.9 & 0.0 & 18.5 \\
\hline Mitchell & 11.79 & 11.71 & 49.8 & 1.01 & 0.68 & 1.77 & 0.00 & 0.40 & 26.2 & 17.6 & 45.9 & 10.4 \\
\hline Monroe & 10.58 & 15.84 & 60.0 & 1.33 & 0.22 & 0.02 & 0.00 & 0.28 & 71.9 & 11.9 & 1.1 & 15.1 \\
\hline Montgomery & 3.73 & 5.39 & 59.1 & 0.37 & 0.07 & 0.00 & 0.00 & 0.01 & 82.2 & 15.6 & 0.0 & 2.2 \\
\hline Morgan & 10.94 & 6.93 & 38.8 & 0.63 & 0.46 & 0.23 & 0.00 & 0.18 & 42.0 & 30.7 & 15.3 & 12.0 \\
\hline Peach & 17.42 & 10.28 & 37.1 & 1.53 & 0.52 & 0.09 & 0.00 & 0.49 & 58.2 & 19.8 & 3.4 & 18.6 \\
\hline Pickens & 22.26 & 7.17 & 24.4 & 2.53 & 0.36 & 0.10 & 0.00 & 0.55 & 71.5 & 10.2 & 2.8 & 15.5 \\
\hline Pierce & 6.76 & 12.00 & 64.0 & 0.54 & 0.07 & 0.05 & 0.00 & 0.08 & 73.0 & 9.5 & 6.8 & 10.8 \\
\hline Pike & 5.49 & 12.38 & 69.3 & 0.44 & 0.09 & 0.00 & 0.00 & 0.06 & 74.6 & 15.3 & 0.0 & 10.2 \\
\hline Polk & 40.52 & 0.95 & 2.3 & 3.20 & 0.51 & 0.45 & 0.00 & 0.87 & 63.6 & 10.1 & 8.9 & 17.3 \\
\hline Pulaski & 5.08 & 6.93 & 57.7 & 0.59 & 0.17 & 0.01 & 0.00 & 0.23 & 59.0 & 17.0 & 1.0 & 23.0 \\
\hline Putnam & 18.66 & 2.56 & 12.1 & 0.93 & 0.33 & 0.05 & 0.00 & 0.78 & 44.5 & 15.8 & 2.4 & 37.3 \\
\hline Quitman & 1.86 & 0.65 & 26.0 & 0.14 & 0.00 & 0.00 & 0.00 & $<0.01$ & 100.0 & 0.0 & 0.0 & 0.0 \\
\hline Rabun & 11.68 & 4.60 & 28.2 & 0.90 & 0.04 & 0.00 & 0.00 & 0.56 & 60.0 & 2.7 & 0.0 & 37.3 \\
\hline Randolph & 5.09 & 2.63 & 34.1 & 0.39 & 0.08 & 0.00 & 0.00 & 0.10 & 68.4 & 14.0 & 0.0 & 17.5 \\
\hline Richmond & 195.88 & 4.67 & 2.3 & 14.85 & 14.73 & 5.64 & 0.00 & 8.77 & 33.8 & 33.5 & 12.8 & 19.9 \\
\hline Rockdale & 70.92 & 14.30 & 16.8 & 5.45 & 3.01 & 1.27 & 0.00 & 2.08 & 46.1 & 25.5 & 10.8 & 17.6 \\
\hline Schley & 4.53 & 0.48 & 9.6 & 0.32 & 0.03 & 0.01 & 0.00 & 0.07 & 74.4 & 7.0 & 2.3 & 16.3 \\
\hline Screven & 4.87 & 9.72 & 66.6 & 0.39 & 0.25 & 0.05 & 0.00 & 0.20 & 43.8 & 28.1 & 5.6 & 22.5 \\
\hline
\end{tabular}


Table 10. Population served and public-supply deliveries by county in Georgia, 2010.—Continued

[Mgal/d, million gallons per day; <, less than]

\begin{tabular}{|c|c|c|c|c|c|c|c|c|c|c|c|c|}
\hline \multirow{2}{*}{$\begin{array}{l}\text { County } \\
\text { (fig. 1) }\end{array}$} & \multicolumn{2}{|c|}{$\begin{array}{l}\text { Population } \\
\text { (thousands) }\end{array}$} & \multirow{2}{*}{$\begin{array}{l}\text { Percentage } \\
\text { of popu- } \\
\text { lation } \\
\text { self } \\
\text { supplied }\end{array}$} & \multicolumn{5}{|c|}{$\begin{array}{l}\text { Public-supply deliveries } \\
\text { (million gallons per day) }\end{array}$} & \multicolumn{4}{|c|}{$\begin{array}{c}\text { Percentage of } \\
\text { public-supply deliveries }\end{array}$} \\
\hline & $\begin{array}{l}\text { Public } \\
\text { supplied }\end{array}$ & $\begin{array}{c}\text { Self- } \\
\text { supplied } \\
\text { domestic }^{\text {a }}\end{array}$ & & Domestic & $\begin{array}{c}\text { Commercial } \\
\text { and } \\
\text { public use }\end{array}$ & Industrial & Irrigation & $\begin{array}{l}\text { System } \\
\text { losses }^{c}\end{array}$ & Domestic & $\begin{array}{c}\text { Commercial } \\
\text { and public } \\
\text { use }\end{array}$ & Industrial & $\begin{array}{l}\text { System } \\
\text { losses }\end{array}$ \\
\hline Seminole & 3.80 & 4.93 & 56.5 & 0.41 & 0.08 & 0.01 & 0.00 & 0.08 & 70.7 & 13.8 & 1.7 & 13.8 \\
\hline Spalding & 56.87 & 7.20 & 11.2 & 3.09 & 0.73 & 0.22 & 0.00 & 2.45 & 47.6 & 11.2 & 3.4 & 37.8 \\
\hline Stephens & 24.04 & 2.14 & 8.2 & 1.84 & 0.43 & 0.31 & 0.00 & 0.43 & 61.1 & 14.3 & 10.3 & 14.3 \\
\hline Stewart & 4.26 & 1.80 & 29.7 & 0.32 & 0.04 & 0.00 & 0.00 & 0.04 & 80.0 & 10.0 & 0.0 & 10.0 \\
\hline Sumter & 20.47 & 12.35 & 37.6 & 1.68 & 0.46 & 0.03 & 0.00 & 0.61 & 60.4 & 16.5 & 1.1 & 21.9 \\
\hline Talbot & 4.51 & 2.36 & 34.3 & 0.38 & 0.09 & 0.00 & 0.00 & 0.09 & 67.9 & 16.1 & 0.0 & 16.1 \\
\hline Taliaferro & 0.67 & 1.05 & 61.0 & 0.05 & 0.00 & 0.00 & 0.00 & 0.00 & 100.0 & 0.0 & 0.0 & 0.0 \\
\hline Tattnall & 7.90 & 17.62 & 69.0 & 0.57 & 0.16 & 0.20 & 0.00 & 0.18 & 51.4 & 14.4 & 18.0 & 16.2 \\
\hline Taylor & 3.76 & 5.15 & 57.8 & 0.56 & 0.08 & 0.00 & 0.00 & 0.12 & 73.7 & 10.5 & 0.0 & 15.8 \\
\hline Telfair & 7.83 & 8.67 & 52.5 & 0.73 & 0.37 & 0.06 & 0.00 & 0.27 & 51.0 & 25.9 & 4.2 & 18.9 \\
\hline Terrell & 6.70 & 2.62 & 28.1 & 0.54 & 0.17 & 0.28 & 0.00 & 0.60 & 34.0 & 10.7 & 17.6 & 37.7 \\
\hline Thomas & 30.94 & 13.78 & 30.8 & 2.46 & 1.21 & 0.36 & 0.00 & 1.46 & 44.8 & 22.0 & 6.6 & 26.6 \\
\hline Tift & 28.61 & 11.51 & 28.7 & 3.77 & 1.01 & 0.05 & 0.00 & 0.79 & 67.1 & 18.0 & 0.9 & 14.1 \\
\hline Toombs & 18.72 & 8.50 & 31.2 & 1.45 & 0.52 & 0.44 & 0.00 & 0.51 & 49.7 & 17.8 & 15.1 & 17.5 \\
\hline Towns & 9.75 & 0.72 & 6.9 & 1.00 & 0.14 & 0.01 & 0.00 & 0.22 & 73.0 & 10.2 & 0.7 & 16.1 \\
\hline Treutlen & 2.48 & 4.41 & 64.0 & 0.26 & 0.07 & 0.00 & 0.00 & 0.07 & 65.0 & 17.5 & 0.0 & 17.5 \\
\hline Troup & 52.62 & 14.42 & 21.5 & 4.33 & 2.47 & 2.15 & 0.00 & 1.57 & 41.2 & 23.5 & 20.4 & 14.9 \\
\hline Turner & 5.67 & 3.26 & 36.5 & 0.44 & 0.12 & 0.02 & 0.00 & 0.12 & 62.9 & 17.1 & 2.9 & 17.1 \\
\hline Twiggs & 2.12 & 6.90 & 76.5 & 0.22 & 0.12 & 0.00 & 0.00 & 0.05 & 56.4 & 30.8 & 0.0 & 12.8 \\
\hline Union & 17.56 & 3.80 & 17.8 & 1.12 & 0.23 & 0.19 & 0.00 & 0.36 & 58.9 & 12.1 & 10.0 & 18.9 \\
\hline Upson & 14.59 & 12.56 & 46.3 & 2.67 & 0.65 & 0.10 & 0.00 & 1.06 & 59.6 & 14.5 & 2.2 & 23.7 \\
\hline Walker & 63.98 & 4.78 & 6.9 & 4.67 & 1.60 & 0.40 & 0.00 & 1.98 & 54.0 & 18.5 & 4.6 & 22.9 \\
\hline Walton & 72.94 & 10.83 & 12.9 & 4.03 & 1.10 & 0.12 & 0.00 & 1.25 & 62.0 & 16.9 & 1.8 & 19.2 \\
\hline Ware & 31.16 & 5.15 & 14.2 & 2.00 & 0.51 & 0.46 & 0.00 & 0.40 & 59.3 & 15.1 & 13.6 & 11.9 \\
\hline Warren & 2.61 & 3.22 & 55.3 & 0.23 & 0.04 & 0.02 & 0.00 & 0.05 & 67.6 & 11.8 & 5.9 & 14.7 \\
\hline Washington & 9.83 & 11.36 & 53.6 & 0.81 & 1.42 & 0.05 & 0.00 & 0.39 & 30.3 & 53.2 & 1.9 & 14.6 \\
\hline Wayne & 14.30 & 15.80 & 52.5 & 1.03 & 0.49 & 0.00 & 0.00 & 0.21 & 59.5 & 28.3 & 0.0 & 12.1 \\
\hline Webster & 0.84 & 1.96 & 70.0 & 0.11 & 0.00 & 0.00 & 0.00 & 0.02 & 84.6 & 0.0 & 0.0 & 15.4 \\
\hline Wheeler & 1.77 & 5.65 & 76.1 & 0.12 & 0.03 & 0.00 & 0.00 & 0.03 & 66.7 & 16.7 & 0.0 & 16.7 \\
\hline White & 14.00 & 13.14 & 48.4 & 0.83 & 0.48 & 0.03 & 0.00 & 0.16 & 55.3 & 32.0 & 2.0 & 10.7 \\
\hline Whitfield & 97.18 & 5.42 & 5.3 & 7.40 & 6.17 & 8.14 & 0.00 & 2.86 & 30.1 & 25.1 & 33.1 & 11.6 \\
\hline Wilcox & 3.19 & 6.07 & 65.5 & 0.22 & 0.03 & 0.00 & 0.00 & 0.03 & 78.6 & 10.7 & 0.0 & 10.7 \\
\hline Wilkes & 5.61 & 4.98 & 47.0 & 0.48 & 0.11 & 0.29 & 0.00 & 0.14 & 47.1 & 10.8 & 28.4 & 13.7 \\
\hline Wilkinson & 5.57 & 3.99 & 41.8 & 0.46 & 0.09 & 0.06 & 0.00 & 0.06 & 68.7 & 13.4 & 9.0 & 9.0 \\
\hline Worth & 9.54 & 12.14 & 56.0 & 0.71 & 0.18 & 0.04 & 0.00 & 0.28 & 58.7 & 14.9 & 3.3 & 23.1 \\
\hline Total & $8,255.25$ & $1,432.40$ & $36.7^{\mathrm{a}}$ & 623.5 & 209.2 & 107.9 & 3.14 & 181.8 & $60.9^{d}$ & $14.9^{\mathrm{d}}$ & $2.9^{\mathrm{d}}$ & $14.9^{\mathrm{d}}$ \\
\hline
\end{tabular}

aSelf-supplied domestic population is the difference between the total county population and the population served by public water suppliers.

${ }^{b}$ Not included is $0.4 \mathrm{Mgal} / \mathrm{d}$ of water delivered to Alabama, South Carolina, and Tennessee.

'Public-supply system losses include water lost from the water distribution system and includes pipe leaks and breaks, fire suppression, and unauthorized use. About $3 \mathrm{Mgal} / \mathrm{d}$ of public-supply deliveries are unaccountable and may be additional system losses.

${ }^{\mathrm{d}}$ Median. 


\section{Self-Supplied Water Use}

Self-supplied water is water withdrawn from a groundwater or surface-water source by a user other than a public supplier. Self-supplied users include rural households on individual wells, commercial and industrial businesses, mining operations, irrigated crop land and golf courses (irrigation), livestock and aquaculture production, and thermoelectricpower generation. Although the self-supplied populations in Fulton County and Cobb County were less than 6.5 percent of the 2010 Georgia population (table 10), they had the highest self-supplied domestic withdrawals, $3.7 \mathrm{Mgal} / \mathrm{d}$ in Fulton County and $3.2 \mathrm{Mgal} / \mathrm{d}$ in Cobb County, among Georgia counties in 2010 (table 9).

Estimated self-supplied commercial, industrial, and mining withdrawals amounted to $2.7,495$, and $17 \mathrm{Mgal} / \mathrm{d}$, respectively, in 2010 (table 2). About 78 percent of commercial withdrawals was groundwater. The highest commercial withdrawals were in Floyd County $(0.32 \mathrm{Mgal} / \mathrm{d})$, Bulloch County $(0.30 \mathrm{Mgal} / \mathrm{d})$, and Chatham County $(0.25 \mathrm{Mgal} / \mathrm{d}$; table 9). About 58 percent of industrial withdrawals was surface water (table 2). The highest industrial withdrawals occurred in Early County (109 Mgal/d), followed by Richmond (68 Mgal/d) and Wayne Counties (58 Mgal/d). Mining withdrawals represent water used in the actual extraction of the raw mineral or rock complex. About 98 percent of mining withdrawals were groundwater in 2010 . The counties with the highest withdrawals for mining were Sumter (1.6 Mgal/d), Richmond (1.5 Mgal/d), and Washington (1.1 Mgal/d; table 9).

Estimated self-supplied withdrawals for irrigation were $802 \mathrm{Mgal} / \mathrm{d}$ in 2010 (table 2). Crop irrigation accounted for 93 percent of the total water withdrawn for irrigation; golf course irrigation accounted for the remaining 7 percent. About 77 percent of water for crop irrigation was groundwater, whereas groundwater accounted for 39 percent of water used to irrigate golf courses. Withdrawals for crop irrigation (mostly groundwater) were highest in Decatur County (68 Mgal/d), followed by Mitchell County $(59 \mathrm{Mgal} / \mathrm{d})$ and Miller County (45 Mgal/d; table 9). Ninety-seven Georgia counties used a greater percentage of groundwater than surface water to irrigate crops, whereas 38 Georgia counties used a greater percentage of surface water than groundwater to irrigate crops in 2010. Surface-water withdrawals for irrigation were highest in Calhoun County at $17 \mathrm{Mgal} / \mathrm{d}$. Two counties, Fannin and Clay, used an equal percentage of surface water and groundwater for crop irrigation. In addition, 21 counties withdrew less than $9,000 \mathrm{gal} / \mathrm{d}$ for crop irrigation.
Water withdrawals for golf course irrigation were nearly $55 \mathrm{Mgal} / \mathrm{d}$ in 2010; about 61 percent of these withdrawals were from surface water (table 2). Withdrawals were highest in Fulton County (3.7 Mgal/d, mostly surface water), followed by Glynn County (3.44 Mgal/d, mostly groundwater), Henry County and Stephens County (each $2.3 \mathrm{Mgal} / \mathrm{d}$, mostly groundwater), and Dekalb County (2.3 Mgal/d, mostly surface water).

Water withdrawals for livestock and aquaculture production totaled $79 \mathrm{Mgal} / \mathrm{d}$ in 2010, of which 92 percent was surface water (table 2). Of that $79 \mathrm{Mgal} / \mathrm{d}$, livestock used nearly 37 percent and aquaculture about 63 percent. Water withdrawals for livestock and aquaculture were highest in Forsyth (6.7 Mgal/d), Fannin (6.4 Mgal/d), Union (5.4 Mgal/d), and Habersham (5.1 Mgal/d) Counties (table 9), with 90 to 99 percent of the use attributed to high aquaculture water use associated with Federal and State gamefish hatcheries, private baitfish hatcheries, and private trout farms. Water use for livestock production was highest in Franklin County (1.2 Mgal/d, primarily poultry production), followed by Gordon, Carroll, Hart, Madison, and Oglethorpe Counties ( $0.8 \mathrm{Mgal} / \mathrm{d}$ each, primarily poultry and beef production).

At least one thermoelectric-power generation facility was present in 17 Georgia counties in 2010 . About 99.8 percent of the 2,046 Mgal/d of water withdrawn for thermoelectricpower generation was surface water and about 52 percent of that water was returned to surface water in 2010 (table 2). The thermoelectric facilities in seven counties use once-through cooling, which means that water is withdrawn, conveyed through the facility for cooling, and discharged back to surface water. Facilities that use once-through cooling return about 99 percent of the amount withdrawn back to surface water. Once-through cooling facilities include Plant Branch in Putnam County (952 Mgal/d), Plant Hammond in Floyd County (432 Mgal/d), Plant Kraft in Chatham County (206 Mgal/d of saline surface water), Plant McIntosh in Effingham County (70 Mgal/d), Plant Mitchell in Dougherty County (50 Mgal/d), Plant McManus in Glynn County (8.1 Mgal/d of saline surface water), and Crisp County Power in Worth County $(0.2 \mathrm{Mgal} / \mathrm{d}$; table 9$)$.

The thermoelectric-power facilities in 10 counties use a recirculating cooling system, which means that water is withdrawn from a source and discharged into a storage reservoir, where the water is conveyed through the facility to cooling towers, and the remaining water is returned to the storage reservoir. Typically, most of the water withdrawn is lost through evaporation in the cooling towers (consumptive use). About $343 \mathrm{Mgal} / \mathrm{d}$ was withdrawn by thermoelectric-power 
facilities using recirculating cooling systems. Two of those facilities, one in Burke County (65 Mgal/d) and one in Heard County (49 Mgal/d), withdrew the most water, followed by facilities in Appling, Monroe, and Dougherty Counties (table 9).

\section{Public Supply}

About 1,121 Mgal/d of water was withdrawn for public supply in 2010 and 78 percent of that amount was surface water (table 2). Public suppliers in Fulton County withdrew $201 \mathrm{Mgal} / \mathrm{d}$ of water (mostly surface water), the highest among all counties in Georgia (table 9). This amount represents 18 percent of all public-supply withdrawals and 23 percent of all surface-water withdrawals for public supply in 2010. After Fulton County, public-supply withdrawals in Hall County ( $95 \mathrm{Mgal} / \mathrm{d}$ ) were the next highest in Georgia. In contrast, public-supply withdrawals were only $0.05 \mathrm{Mgal} / \mathrm{d}$ in Taliaferro County and $0.13 \mathrm{Mgal} / \mathrm{d}$ in Webster County, the two least populated counties in Georgia.

In 2010, public water suppliers delivered about $1,125 \mathrm{Mgal} / \mathrm{d}$ to their customers. Approximately $0.4 \mathrm{Mgal} / \mathrm{d}$ of water was delivered to public suppliers in Alabama, South Carolina, and Tennessee. In Georgia, 60.9 percent (median) of public water was delivered to domestic customers, with a range of 6.1 percent in Chattahoochee County to 100 percent in Echols, Glascock, Quitman, and Taliaferro Counties (table 10). About 14.9 percent (median) of public water was delivered to commercial and public use customers, with a range of 0 percent in seven counties to 53 percent in Washington County. In addition, 2.9 percent (median) of public water was delivered to industrial customers, with a range of 0 percent in 40 counties to about 92 percent in Chattahoochee County (table 10). An estimated 14.9 percent (median) of public-supplied water was lost during delivery, with a range of less than 0.1 percent in Echols, Glascock, Quitman, and Taliaferro Counties to 38 percent in Terrell County. The amount of water lost from public distribution systems is typically a result of the age and extent (length in miles) of the pipeage (U.S. Environmental Protection Agency, 2009). Thus, small and revenue-poor public suppliers commonly have distribution systems that are at or exceed the life expectancy of the distribution pipeage, resulting in a greater percentage of water lost than where the public distribution system is newer.

\section{Surface-Water Returns}

About 2,225 Mgal/d of water was returned to Georgia streams and lakes in 2010 (tables 2 and 9). This amount is about 48 percent of the total water withdrawn from all sources in 2010. Surface-water returns declined 39 percent between 1995 and 2010, mirroring the decline in water withdrawals during that period. In addition, land applications of treated wastewater have increased steadily between 1995 and 2010 . Surface-water returns ranged from zero in 14 counties to $443 \mathrm{Mgal} / \mathrm{d}$ in Floyd County (table 9). Counties with the greatest returns-Floyd, Putnam, Chatham - were those with the greatest returns from thermoelectric-power facilities using once-through cooling.

About $711 \mathrm{Mgal} / \mathrm{d}$ of treated wastewater was returned to Georgia's surface waters by public wastewater treatment facilities in 2010 (table 2). The median amount of public wastewater discharged to surface water by county was $0.73 \mathrm{Mgal} / \mathrm{d}$ and discharges were less than $5 \mathrm{Mgal} / \mathrm{d}$ in 84 percent of Georgia counties. Public wastewater discharges were greatest in Fulton County (136 Mgal/d) and Cobb County ( $77 \mathrm{Mgal} / \mathrm{d}$; appendix 2). The minimum reporting level for surface-water returns in this report is $5,000 \mathrm{gal} / \mathrm{d}$; returns of less than 5,000 gal/d are recorded as zero. Wastewater discharges in rural counties with small towns and other facilities that rely on sewage lagoons may not exceed the reporting level. Sewage lagoons commonly discharge wastewater intermittently. In addition, onsite septic systems are the primary means of wastewater disposal in many counties resulting in no surface-water returns.

Commercial, industrial, and mining facilities returned $431 \mathrm{Mgal} / \mathrm{d}$ of water to Georgia's surface waters in 2010 (table 2). Commercial and industrial facilities reported surface-water discharges in 41 Georgia counties (26 percent) in 2010. Commercial and industrial discharges were less than $2 \mathrm{Mgal} / \mathrm{d}$ in 25 counties and greater than $15 \mathrm{Mgal} / \mathrm{d}$ in 8 counties. Commercial and industrial discharges were greatest in Whitfield County ( $88 \mathrm{Mgal} / \mathrm{d})$ and Chatham County (77 Mgal/d; appendix 2). Mining facilities reported surfacewater returns in 32 counties totaling $24 \mathrm{Mgal} / \mathrm{d}$. Surfacewater returns from mining were greatest in Muscogee County (2.6 Mgal/d) and Warren County (2.2 Mgal/d; appendix 2). In Muscogee County, quarry dewatering was the source of surface-water returns, whereas kaolin mining was the major source of returns in Warren County. 


\section{Water-Use Trends, 1985-2010}

Statewide water withdrawals reached an all-time high of 6,725 Mgal/d in 1980. By 1990, water use had decreased by 20 percent to $5,353 \mathrm{Mgal} / \mathrm{d}$, but increased to $6,487 \mathrm{Mgal} / \mathrm{d}$ in 2000. By 2005, water use had decreased to an estimated $5,471 \mathrm{Mgal} / \mathrm{d}$, and declined further to 4,670 Mgal/d in 2010 . Between 2000 and 2010, water use declined by 28 percent and between 2005 and 2010 had declined by 15 percent (fig. 10). The decrease in water withdrawals between 2005 and 2010 was driven by declines in surface-water withdrawals. In contrast, groundwater withdrawals increased slightly during this period. This decline was evident across most water-use categories (fig. 10). The estimated total water use per capita in 1985 was about $850 \mathrm{gal} / \mathrm{d}$, steadily decreasing to about $798 \mathrm{gal} / \mathrm{d}$ in 2000 , and decreasing rapidly to $460 \mathrm{gal} / \mathrm{d}$ between 2000 and 2010 (fig. 11). The decline in water withdrawals and use between 2005 and 2010 can probably be attributed to several factors working together during this period: (1) water conservation laws and policies along with advances in waterconservation technology; (2) the onset of a major recession in 2007; and (3) below average rainfall in 2007 and 2008, and in the latter half of 2010. Because of these factors, water withdrawn by public suppliers decreased by 4.8 percent (despite a nearly 11-percent increase in population served) and per capita use decreased by 19 percent between 2005 and 2010 .

Domestic and commercial water use increased slightly between 2005 and 2010 (fig. 11); however, because selfsupplied domestic withdrawals are computed using an estimated self-served population, this increase is probably due to population increases in some rural areas of Georgia. Irrigation withdrawals also increased between 2005 and 2010 but are probably a response to the very dry conditions during most of the growing season in central and south Georgia.

Throughout 1985-2010, water withdrawn for thermoelectric-power generation has constituted the largest volume of offstream water use in Georgia (fig. 11). Total withdrawals for thermoelectric-power generation declined about 37 percent between 2000 and 2010, mostly due to the decommissioning or re-tooling of power plants in the State. During this period, several of the plants operated by the Georgia Power Company were re-tooled to use natural gas-powered generators. Surfacewater returns declined 39 percent between 1995 and 2010, mirroring the decline in water withdrawals during that period (fig. 12). In addition, land applications of treated wastewater increased steadily between 1995 and 2010.

\section{Summary}

The period between 2007 and 2010 was a challenging time economically and climatologically in Georgia. During that period, the United States was in the midst of a major recession, resulting in decreases in the manufacturing and construction industries and large increases in unemployment.
During 2007, 2008, and the latter half of 2010, precipitation in Georgia was substantially below the 30 -year norm.

According to the 2010 census, nearly 9.7 million people lived in Georgia. The water for about 85 percent of that population (8.3 million) was provided by public water suppliers. Estimated total water withdrawals from groundwater and surface-water sources were about 4,670 million gallons per day (Mgal/d) in 2010, a nearly 15-percent reduction from 2005 (5,471 Mgal/d). In 2010, surface-water withdrawals $(3,481 \mathrm{Mgal} / \mathrm{d})$ were nearly three times greater than groundwater withdrawals $(1,189 \mathrm{Mgal} / \mathrm{d})$ in Georgia.

In 2010, thermoelectric-power facilities $(2,046 \mathrm{Mgal} / \mathrm{d})$ and public-supply uses $(1,121 \mathrm{Mgal} / \mathrm{d})$ accounted for 68 percent of all water withdrawn in Georgia. Surface-water withdrawals were greatest for thermoelectric-power generation $(2,043 \mathrm{Mgal} / \mathrm{d})$, whereas irrigation used the largest amount of groundwater (599 Mgal/d). Surface water provided 78 percent of the 1,121 Mgal/day withdrawn for public supply in 2010 . Typically, counties in northern Georgia withdraw a larger percentage of water from surface-water than groundwater sources, whereas counties in the southern part of the State withdraw more water from groundwater sources.

\section{Self-Supplied Water Use}

Self-supplied domestic withdrawals amounted to $107 \mathrm{Mgal} / \mathrm{d}$ and 56 percent of that use was in the Apalachicola (26.2 Mgal/d), Ocmulgee (20.7 Mgal/d), and Savannah River Basins (12.4 Mgal/d). In the Coosa and Tallapoosa River Basins, only 6.5 percent of the population relied on domestic wells for their water supply. In contrast, nearly 47 percent of the population in the Ochlockonee River Basin and 57 percent of the population in the Altamaha River Basin relied on domestic wells for their water.

About $515 \mathrm{Mgal} / \mathrm{d}$ was withdrawn by self-supplied commercial, industrial, and mining users in 2010. Self-supplied industries withdrew about $495 \mathrm{Mgal} / \mathrm{d}$ in 2010, of which 58 percent was surface water. Paper and pulp facilities withdrew 78 percent of all water withdrawn by self-supplied industries (387 Mgal/d), of which $255 \mathrm{Mgal} / \mathrm{d}$ was surface water. Selfsupplied commercial and industrial use was greatest in the Apalachicola (134 Mgal/d) and Savannah River (130 Mgal/d) Basins. Mining withdrawals also were greatest in the Apalachicola (6.1 Mgal/d) and Savannah River Basins (2.5 Mgal/d).

Crop irrigation, livestock management, and aquaculture are the primary agricultural users of water in Georgia. Estimated self-supplied withdrawals for irrigation were $802 \mathrm{Mgal} / \mathrm{d}$ in 2010. Crop irrigation accounted for 93 percent of the total water withdrawn for irrigation and golf course irrigation accounted for the remaining 7 percent. About 77 percent of water for crop irrigation was groundwater, whereas groundwater accounted for 39 percent of water used to irrigate golf courses. About 65 percent of the irrigation withdrawals were in the Apalachicola River Basin. Golf course irrigation was highest in the Apalachicola River and Ocmulgee River Basins (respectively, 12.7 and 10.4 Mgal/d). 


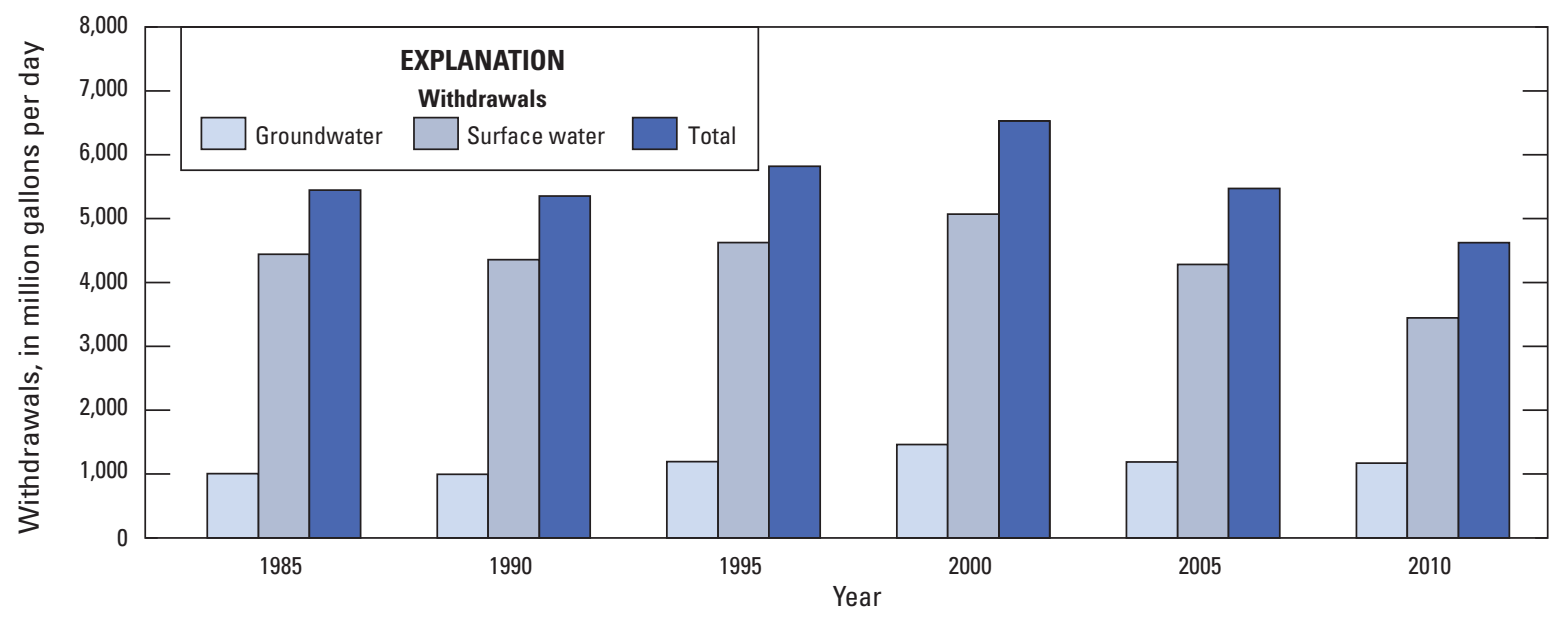

Figure 10. Water-withdrawal trends by water source in Georgia, 1985-2010.

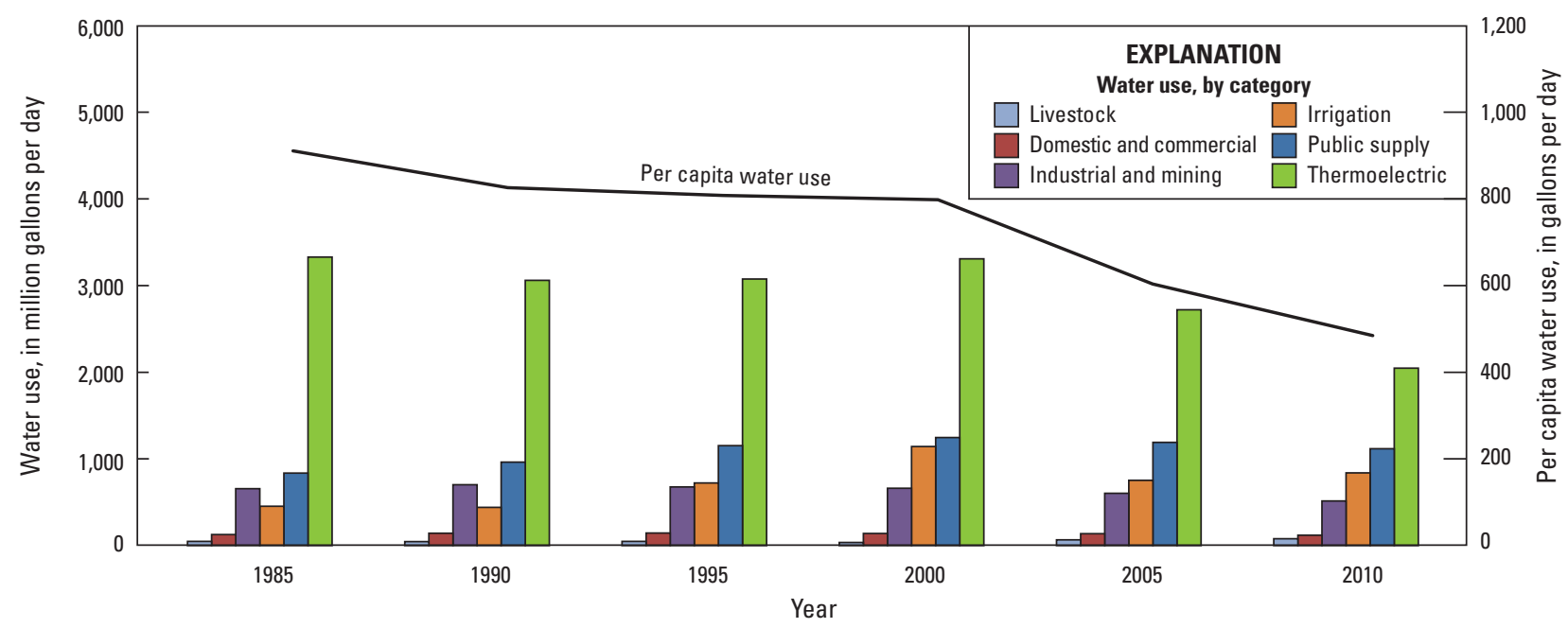

Figure 11. Water-withdrawal trends by water-use category in Georgia, 1985-2010.

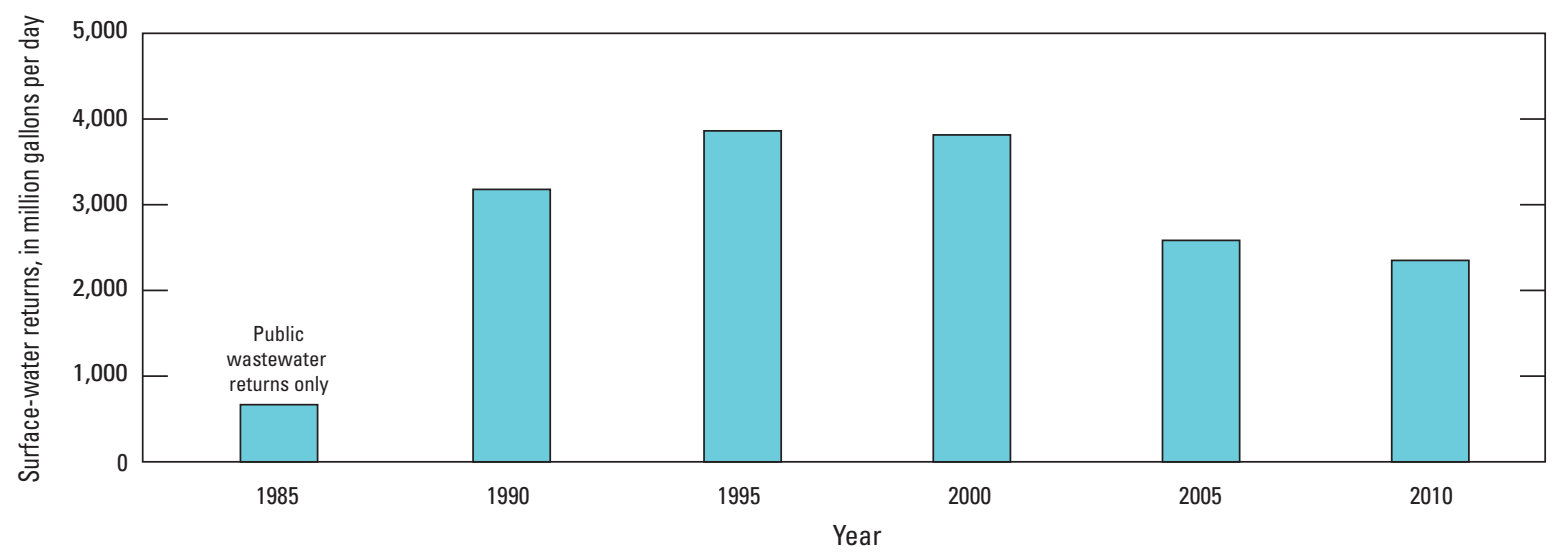

Figure 12. Surface-water return trends in Georgia, 1985-2010. 
Withdrawals for crop irrigation (mostly groundwater) were highest in Decatur County (68 Mgal/d), followed by Mitchell County (59 Mgal/d) and Miller County (45 Mgal/d).

Water withdrawals for livestock and aquaculture production totaled $79 \mathrm{Mgal} / \mathrm{d}$ in 2010, of which 92 percent was surface water. Of that $79 \mathrm{Mgal} / \mathrm{d}$, livestock used nearly 37 percent and aquaculture about 63 percent. Water withdrawals for livestock and aquaculture were highest in Forsyth (6.7 Mgal/d), Fannin (6.4 Mgal/d), Union (5.4 Mgal/d), and Habersham (5.1 Mgal/d) Counties; 90 to 99 percent of this use is for aquaculture at Federal and State gamefish hatcheries, private baitfish hatcheries, and private trout farms. Water use for livestock production was highest in Franklin County (1.2 Mgal/d, primarily poultry production).

\section{Public-Supplied Water Use}

About 1,121 Mgal/d of water was withdrawn for public supply in 2010 and 78 percent of that amount was surface water. About 1,125 Mgal/d of water was delivered to customers in Georgia. About $0.4 \mathrm{Mgal} / \mathrm{d}$ was delivered from Georgia to wholesale customers in Alabama, Tennessee, and South Carolina; about 6.7 Mgal/d was delivered from public suppliers in Alabama and Tennessee to customers and wholesale suppliers in Georgia. Of the $1,125 \mathrm{Mgal} / \mathrm{d}$ of water delivered by public suppliers, 55 percent was used for domestic (residential; $623 \mathrm{Mgal} / \mathrm{d}$ ) purposes in Georgia. Nearly 19 percent of public-supplied water was delivered to commercial customers (including public-use connections) and nearly 9.6 percent was delivered to industrial customers in 2010. An estimated $182 \mathrm{Mgal} / \mathrm{d}$ of water was lost in the public-supply distribution system in 2010. Average public-supply losses were estimated at 16.1 percent of the water delivered.

In 2010, nearly 3.1 million people or 32 percent of the 2010 Georgia population used groundwater for domestic purposes. Of that population, about 1.4 million people relied on small domestic wells for their water. About 1,189 Mgal/d of groundwater was withdrawn from the principal aquifers in Georgia during 2010. About 68 percent of all groundwater withdrawals in 2010 came from the Floridan aquifer system. The two principal aquifers in southern Georgia (south of the Fall Line), the Floridan and the Cretaceous aquifer systems provided 56 and 22 percent, respectively, of the groundwater used for public supply. The crystalline-rock and the Paleozoicrock aquifers provided nearly all of the groundwater used in northern Georgia.

In 2010, public suppliers in 6 of the 159 counties in Georgia (Fulton, Gwinnett, Dekalb, Cobb, Clayton, and Chatham) provided water to 43 percent of the nearly 8.3 million people receiving public-supplied water in Georgia. Public suppliers in Fulton County withdrew $201 \mathrm{Mgal} / \mathrm{d}$ of water mostly surface water), the highest among all counties in Georgia. This amount represents 18 percent of all public-supply withdrawals and 23 percent of all surface-water withdrawals for public supply.

\section{Surface-Water Return Flows}

In basins where there is a greater reliance on groundwater than surface water for water supplies, surface-water returns may exceed surface-water withdrawals because once used, that groundwater enters sanitary sewer systems for treatment and discharge to surface water. About $2,225 \mathrm{Mgal} / \mathrm{d}$ of water was discharged to surface water by public (municipal and privately owned) wasterwater treatment facilities, commercial, industrial, mining, aquaculture, and thermoelectric power facilities in 2010 (table 2). Thermoelectric power facilities returned nearly 49 percent of all water discharged to surface water, followed by public wastewater facilities ( 32 percent), and combined commercial and industrial discharges (19 percent).

Surface-water returns from thermoelectric-power facilities are primarly from facilities that utilize once-through cooling processes. Within the Coosa and Tallapoosa Basins, Plant Hammond returned $448 \mathrm{Mgal} / \mathrm{d}$ (41 percent of all returns in the basin) to surface water in 2010, whereas Plant Arkwright and Plant Branch, and the Crisp County plant in the Oconee River Basin returned $369 \mathrm{Mgal} / \mathrm{d}$ (34 percent). River basins with the greatest populations returned the greatest amount of publicly-treated wastewater to surface water; thus, the Apalachicola and Ocmulgee River Basins received the greatest amount of public wastewater (respectively, 341 and $111 \mathrm{Mgal} / \mathrm{d})$.

\section{Water-Use Trends}

Historically, water withdrawals in Georgia were highest in $1980(6,725 \mathrm{Mgal} / \mathrm{d})$. By 1990, water use had decreased by 20 percent to $5,353 \mathrm{Mgal} / \mathrm{d}$, but increased to $6,487 \mathrm{Mgal} / \mathrm{d}$ in 2000. By 2005, water use had decreased to an estimated 5,471 Mgal/d, and declined further to 4,670 Mgal/d in 2010 a 30-percent decrease since 1980. This decline was evident across all water-use categories, but was greatest for surfacewater withdrawals by thermoelectric-power facilities. The estimated total water use per capita in 1985 (total withdrawals for all categories divided by total population ) was about 850 gallons per day (gal/d), steadily decreasing to about $798 \mathrm{gal} / \mathrm{d}$ in 2000, and decreasing further to $460 \mathrm{gal} / \mathrm{d}$ in 2010 . Although water use declined among all use categories during that 10 -year period, most of the decline in per capita water use was caused by the large decrease in water used for thermoelectric-power generation.

Throughout 1985-2010 water withdrawn for thermoelectric-power generation constituted the largest volume of offstream water use in Georgia. Total withdrawals for thermoelectric-power generation declined about 37 percent between 2000 and 2010, mostly due to the decommissioning of power plants in the State. Also during this period, several power plants were shut down and re-tooled to use natural gaspowered generators; thus, water withdrawals for cooling were substantially reduced. 
The decline in water withdrawals and use between 2005 and 2010 can probably be attributed to several factors working together during this period: (1) water conservation laws and policies along with advances in water-conservation technology; (2) the onset of a major recession in 2007; and (3) below average rainfall in 2007, 2008, and the latter half of 2010. Because of these factors, water withdrawn by public suppliers decreased by 4.8 percent (despite a nearly 11 -percent increase in population served) and per capita use decreased by 19 percent between 2005 and 2010 .

\section{References Cited}

Carter, R.F., and Johnson, A.M.F., 1974, Use of water in Georgia, 1970, with projections to 1990: Georgia Geologic Survey Hydrologic Report 2, 74 p.

CH2M HILL, 2007, Georgia water use and conservation profiles, TM-2 data analysis: Technical memorandum prepared for Georgia Environmental Protection Division, 40 p.

Dun and Bradstreet, Inc., 2011, Hoover's Relationship Manager: Dun and Bradstreet data base: accessed November 10, 2011, at http://www.hoovers.com.

Fanning, J.L., 1985, The Georgia water-use program: U.S. Geological Survey Open-File Report 85-481, pamphlet.

Fanning, J.L., 1997, Water use in Georgia by county for 1995: Georgia Geologic Survey Information Circular 101, 95 p.

Fanning, J.L., 2003, Water use in Georgia, 2000; and trends, 1950-2000 in Kathryn J. Hatcher (ed.), Proceedings of the 2003 Georgia Water Resources Conference, April 23-24, 2003, University of Georgia, Athens, Georgia, accessed October 29, 2014, at http://ga.water.usgs.gov/ publications/other/gwrc2003/pdf/Fanning-GWRC2003.pdf.

Fanning, J.L., Doonan, G.A., and Montgomery, L.T., 1992, Water use in Georgia by county for 1990: Georgia Geologic Survey Information Circular 90, 98 p.

Fanning, J.L. and Trent, V.P., 2009, Water use in Georgia by county for 2005; and water-use trends, 1980-2005: U.S. Geological Survey Scientific Investigations Report 2009-5002, 186 p., accessed June 25, 2014, at http://pubs.usgs.gov/sir/2009/5002/.

Georgia Environmental Protection Division, 2008, Georgia comprehensive state-wide water management plan: Atlanta, Georgia, Georgia Water Council, 39 p. [Also available online at http://www.georgiawaterplanning.org/.]
Georgia Environmental Protection Division, 2010, Agricultural irrigation water demand: Atlanta, Georgia, Georgia Department of Natural Resources, accessed June 25, 2014, at http://www.nespal.org/sirp/waterinfo/State/awd/ agwaterdemand.htm.

Glassman, J.E., 2014, Regional perspectives - Georgia economic outlook: JP Morgan Chase and Company, accessed October 30, 2014, at https://www.chase.com/ content/dam/chasecom/en/commercial-bank/documents/ georgia-economy.pdf.

Lewis, Clifford, 2010, Water use for Georgia agricultural withdrawal permitted golf courses: Atlanta, Georgia, Georgia Department of Natural Resources, Environmental Protection Division, accessed October 30, 2014, at http:/www.nespal.org/ sirp/waterinfo/State/awd/AgWaterDemand_GolfCourses.htm.

Lovelace, J.K., 2009a, Methods for estimating water withdrawals for mining in the United States, 2005: U.S. Geological Survey Scientific Investigations Report 2009-5053, 7 p., accessed June 26, 2014, at http://pubs.usgs.gov/ sir/2009/5053/.

Lovelace, J.K., 2009b, Methods for estimating water withdrawals for aquaculture in the United States, 2005: U.S. Geological Survey Scientific Investigations Report 2009-5042, 13 p., accessed June 26, 2014, at http://pubs.usgs.gov/sir/2009/5042/.

Masters, Mark, 2010, Water for Georgia’s livestock: Albany, Georgia, Flint River Policy Center, Albany State University, accessed June 26, 2014, at http://www.georgiawaterplanning.org/pages/forecasting/agricultural_water_use.php.

Metropolitan North Georgia Water Planning District, 2011, Water metrics report: Atlanta, Georgia, Metropolitan North Georgia Water Planning District, accessed October 29, 2014, at http://documents.northgeorgiawater. org/2010_Water_Metrics_Report_FINAL\%281\%29.pdf.

National Oceanic and Atmospheric Administration, 2011, U.S. monthly climate normals (1981-2010): National Climatic Data Center, NESDIS, NOAA, U.S. Department of Commerce, dataset C00822, accessed May 24, 2014, at http://www.ncdc.noaa.gov/land-based-station-data/climatenormals/1981-2010-normals-data.

National Oceanic and Atmospheric Administration, 2014, Climate at a glance-Time series: National Climatic Data Center, NESDIS, NOAA, U.S. Department of Commerce, dataset C00822, accessed May 24, 2014, at http://www. ncdc.noaa.gov/cag/time-series. 
Peck, M.F., Gordon, D.W., and Painter, J.A., 2013, Groundwater conditions in Georgia, 2010-2011: U.S. Geological Survey Scientific Investigations Report 2013-5084, 63 p., accessed May 24, 2015, at http://pubs.usgs.gov/ $\operatorname{sir} / 2013 / 5084 /$.

Pierce, R.R., Barber, N.L., and Stiles, H.R., 1982, Water use in Georgia by county for 1980: Georgia Geologic Survey Information Circular 59, 180 p.

Torak, L.J. and Painter, J.A., 2011, Summary of the Georgia Agricultural Water Conservation and Metering Program and evaluation of methods used to collect and analyze irrigation data in the middle and lower Chattahoochee and Flint River Basins, 2004-2010: U.S. Geological Survey Scientific Investigations Report 2011-5126, 25 p., accessed June 24, 2014, at http://pubs.usgs.gov/sir/2011/5126/.

Trent, V.P., Fanning, J.L., and Doonan, G.A., 1990, Water use in Georgia by county for 1987: Georgia Geologic Survey Information Circular 85, 112 p.

Turlington, M.C., Fanning, J.L., and Doonan, G.A., 1987, Water use in Georgia by county for 1985: Georgia Geologic Survey Information Circular 81, 110 p.

University of Georgia, 2011, 2010 Georgia farm gate value report: Athens, Georgia, The Center for Agribusiness and Economic Development, AR-11-01, 180 p., accessed June 26, 2014, at http://www.caes.uga.edu/center/caed/ pubs/2011/documents/AR-11-01.pdf.

U.S. Census Bureau, 2011a, Table GCT-PL2-Population and Housing Occupancy Status, 2010: Washington, D.C., U.S. Department of Commerce. accessed November 1, 2011, at http://www.census.gov/2010census/popmap/ipmtext. php?fl=13.

U.S. Census Bureau, 2011b, 2010 Census Summary File 1, Total population in occupied housing units, Table H10: Using American FactFinder, Washington, D.C., U.S. Department of Commerce. accessed November 1, 2011, at http://factfinder2.census.gov.
U.S. Census Bureau, 2011c, MAF/TIGER database extract2010 Census Population and Housing Unit Counts-Blocks: Washington, D.C., U.S. Department of Commerce. Census block shape files accessed February 13, 2013, at http://www.census.gov/geo/maps-data/data/tiger.html.

U.S. Census Bureau, 2012, North American Industry Classification System: Washington, D.C., U.S. Department of Commerce, U.S. Census Bureau, accessed June 26, 2014, at http://www.census.gov/eos/www/naics/.

U.S. Department of Agriculture, 2009, 2007 Census of agriculture, United States, Summary and State data, Vol. 1, Chapter 2, County level data - Georgia: Washington, D.C., National Agricultural Statistics Service AC-07-A-51, accessed June 26, 2014, at http:/www.agcensus.usda.gov/ Publications/2007/Full_Report/.

U.S. Energy Information Administration, 2011, Electricity: Washington, D.C., U.S. Department of Energy, U.S. Energy Information Administration, accessed October 30, 2014, at http://www.eia.gov/electricity.

U.S. Environmental Protection Agency, 2009, Control and mitigation of drinking water losses in distribution systems: Office of Water, U.S. Environmental Protection Agency EPA 816-D-09-001, 128 p.

U.S. Environmental Protection Agency, 2014, NPDES permit program basics, water permitting, accessed October 29, 2014, at http://water.epa.gov/polwaste/ npdes/basics/upload/101pape.pdf.

Western Regional Climate Center, 2014, 1-month standardized precipitation index: Reno, Nevada, Desert Research Institute, accessed May 28, 2014, at http:/www.wrcc.dri.edu/. 


\section{Glossary}

aquaculture water use Water used for the offstream farming of water organisms such as finfish and shellfish in ponds and fish hatcheries.

census block U.S. census blocks are statistical areas bounded by visible features such as roads, streets, water bodies, railroad tracks, city, town, county, township, or school district boundaries.

commercial water use Public or self-supplied water used for motels, hotels, restaurants, medical centers, hospitals, military installations, public safety, correctional institutions, educational facilities (public and private), campgrounds, and recreational vehicle parks.

domestic water use Water used for indoor household purposes such as drinking, food preparation, bathing, washing clothes and dishes, flushing toilets, and outdoor purposes such as watering lawns and gardens.

freshwater Water that contains less than 1,000 milligrams per liter of dissolved solids.

groundwater All subsurface water, distinct from surface water. Specifically, the part of the subsurface water in the saturated zone, which is a zone where all voids are filled with water.

industrial water use Water used by self-supplied industries for the fabrication, processing, washing, and cooling in industries associated with the fabrication of machinery and steel products, and the production of chemical, food, paper, wood, petroleum, and their allied products.

irrigation water use Water used for the production of fruit, vegetable, and nut crops and pasture, hay, turf, flower, and ornamental horticulture and nurseries.

livestock water use Water used for the production of animals for food or hobby and raised in feedlots, dairy operations, corrals, barns, or pens; in this report, includes aquaculture operations.

mining water use Water used in the extraction of minerals and includes quarrying, and milling, but does not include dewatering of quarries or mine pits.

offstream water use Water withdrawn from a surface-water source and used in areas not within the surface-water channel or basin.

per capita water use The average amount of water used per person during a standard time period, generally per day. Per capita use may be calculated based on total water use, public-supply water use, self-supplied domestic water use, or domestic deliveries from public supply. public-supply withdrawals The withdrawal of water by a public supplier.

public water supplier Any water supplier (municipal or private entity) that regularly serves at least 25 people or has at least 15 water connections is considered a public water system under Georgia Law (OCGA 12-5-172-11).

public water use Water provided by a public supply for such uses as firefighting, street washing, water treatment, municipal buildings, parks, and swimming pools.

raw water Water that has not been filtered or treated before use.

saline water Water that contains more than 1,000 milligrams per liter of dissolved solids.

self-supplied domestic Surface water or groundwater withdrawn from a private intake or well at a single residence for domestic uses.

self-supplied water Water that is withdrawn directly from a groundwater or surface-water source by a user, as opposed to water that is delivered by a public supplier.

surface water An open body of water, such as a creek, canal, river, stream, lake, pond, or reservoir.

surface-water return flows Raw or treated water discharge to a river, stream, pond, lake, or reservoir.

thermoelectric-power use Water used to generate electricity with steam-driven turbine generators. Water is cooled using three different strategies: recirculating, once-through, and a mixture of the two. Recirculating systems consume the greatest amount of water.

water use Water used for a beneficial purpose, such as domestic activities (drinking, washing), industrial processes, agriculture, and thermoelectric- and hydroelectric-power generation.

water-use coefficient A factor or ratio used to estimate a quantity of water used based on a related quantity. Examples of water-use coefficients include daily per capita water use, consumptive crop irrigation requirements, livestock water requirements, per employee water use, and per unit of product water use.

water withdrawal The removal of water from a surfacewater body or an aquifer that is conveyed to a storage tank or reservoir, or a place of use such as an agricultural field, water treatment or commercial, industrial, or thermoelectric facility. 


\section{Appendix 1. North American Industrial Classification Codes}

Industrial water use is given by industry type, classified by a North American Industrial Classification System Code. The following is a brief description of the codes used in this publication (U.S. Census Bureau, 2012).

212-Mining and processing of kaolin and fuller's earth. This major group includes establishments engaged in the mining and processing of kaolin and fuller's clay. Water washing is a processing method in which water and chemical dispersants are added to the mined clay to produce a slurry. The slurry is then transported through pipes to the processing facility.

311 - Food and kindred products. This major group includes establishments that manufacture or process foods and beverages for human consumption, and certain related products, such as manufactured ice, chewing gum, vegetable oils and animal fats, and prepared feeds for animals and fowls.

312-Beverage manufacturing. The industrial group includes establishments such as breweries, distilleries, wineries, and those that produce soft drinks and bottled water.

313, 314-Textile mills and textile product mills. This major group includes establishments engaged in performing any of the following operations: (1) preparation of fiber and subsequent manufacturing of yarn, thread, braids, twine, and cordage; (2) manufacturing broad woven fabric, narrow woven fabric, knit fabric, and carpets and rugs from yarn; (3) dyeing and finishing fiber, yarn, fabric, and knit apparel; (4) coating, waterproofing, or otherwise treating fabric; (5) the integrated manufacture of knit apparel and other finished articles from yarn; and (6) the manufacture of felt goods, lace goods, nonwoven fabrics, and miscellaneous textiles.

321 - Wood product manufacturing, except furniture. This major group includes sawmills, lathe mills, shingle mills, cooperage stock mills, planing mills, and pulpwood mills and veneer mills engaged in producing veneers, plywood, engineered wood products, millwork, and wood door and window manufacturing.

322 - Paper, pulp, and allied products. This major group includes the manufacture of pulps from wood and other cellulose fibers and from rags; the manufacture of paper and paperboard; and the manufacture of paper and paperboard into converted products such as paper coated off the paper machine, paper bags, paper boxes, and envelopes.

325-Chemicals and allied products. This major group includes establishments producing basic chemicals and those manufacturing products by predominantly chemical processes. Establishments classified in this major group manufacture three general classes of products: (1) basic chemicals such as acids, alkalies, salts, and organic chemicals; (2) chemical products to be used in further manufacture such as synthetic fibers, plastics materials, dry colors, and pigments; and (3) finished chemical products to be used for ultimate consumption such as drugs, cosmetics, and soaps, or to be used as materials or supplies in other industries such as paints, fertilizers, and explosives.

326-Rubber and miscellaneous plastics products. This major group includes establishments manufacturing from natural, synthetic, or reclaimed rubber; rubber products such as tires, rubber footwear, mechanical rubber goods, heels and soles, flooring, and rubber sundries; plastics such as pipes, pipe fittings, plastic bags, sheets, packaging material, polystyrene, and urethane foam products.

327-Stone, clay, glass, and concrete products. This major group includes establishments engaged in manufacturing flat glass and other glass products, cement, structural clay products, pottery, ceramics, concrete and gypsum products, cut stone, abrasive and asbestos products, and so forth, from materials taken principally from the earth in the form of stone, clay, and sand.

331-Primary metal industries. This major group includes establishments engaged in the smelting and refining of ferrous and nonferrous metals from ore, pig, or scrap; in the rolling, drawing, and alloying of ferrous and non-ferrous metals; in the manufacture of castings and other basic products of ferrous and nonferrous metals; and in the manufacture of aluminum sheet, foil, extruded products, pipes, tubes, nails, spikes, and insulated wire and cable.

332-Fabricated metal products, except machinery and transportation equipment. This major group includes establishments engaged in fabricating ferrous and nonferrous metal products such as metal cans, tinware, hand tools, cutlery, general hardware, non-electric heating apparatus, fabricated structural metal products, metal forging, metal stamping, ordnance (except vehicles and guided missiles), and a variety of metal and wire products not elsewhere classified.

339-Miscellaneous manufacturing. This major group includes establishments engaged in manufacturing medical equipment such as surgical, dental, and medical instruments and supplies; laboratory apparatus and furniture; manufacturing of jewelry and silverware; and a variety of other goods such as toys, sporting goods and athletic equipment, office supplies, musical instruments, signs, fasteners, needles, buttons, and caskets. 


\title{
Appendix 2. Population, Water Withdrawals, and Water Use by Source of Water for Each County in Georgia, 2010
}

\author{
Abbreviations
}

$\begin{array}{ll}\text { Ala. } & \text { Alabama } \\ \text { Ga. } & \text { Georgia } \\ \text { Mgal/d } & \text { million gallons per day } \\ \text { NAICS } & \text { North American Industrial Classification System code } \\ < & \text { less than } \\ - & \text { not applicable }\end{array}$

\section{Terms}

Total use is total withdrawal plus public supply deliveries and losses.

Net transfers/losses for a county is the sum of all water purchased by public suppliers in a county from public-water suppliers in a different county minus the sum of all public-supplied water sold to public suppliers or delivered to customers in a different county and public-supply losses.

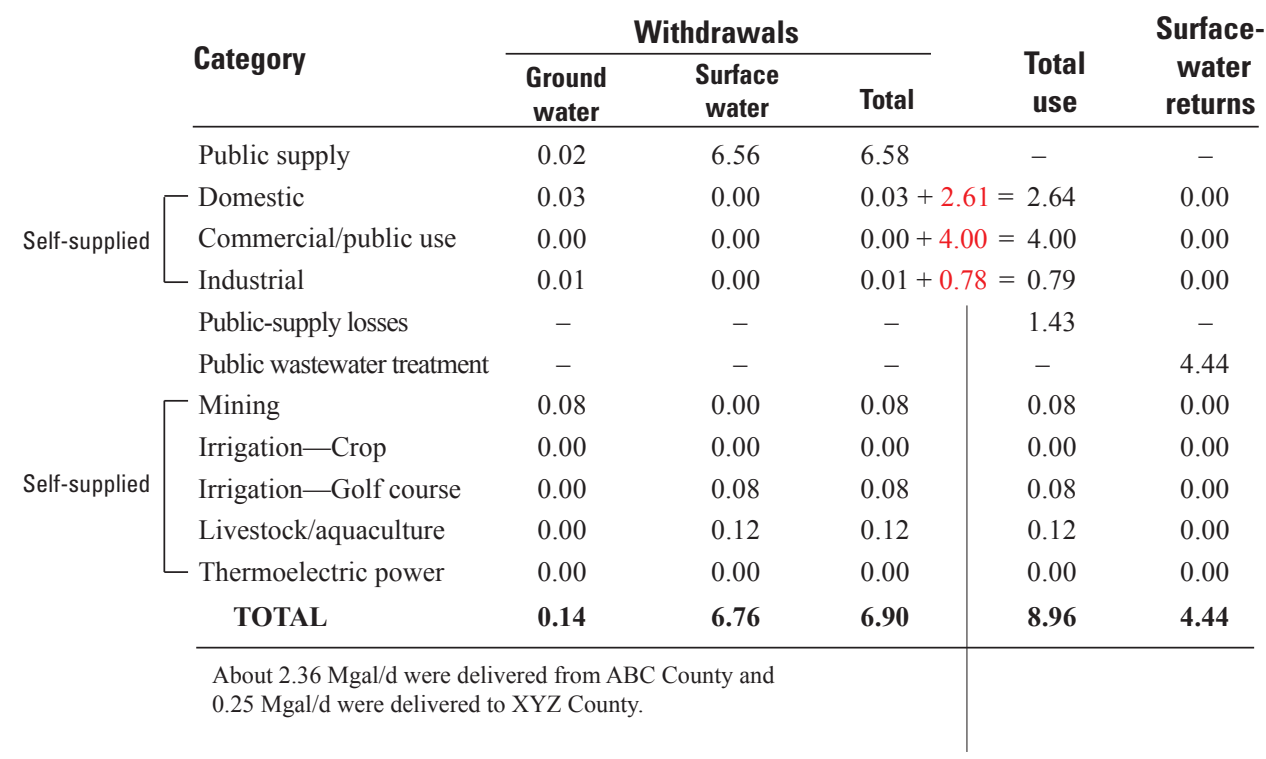

Public-Supply Deliveries' by Use Category

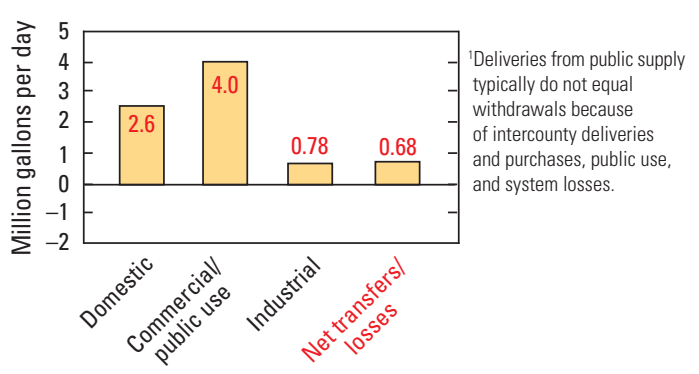




\section{APPLING COUNTY}

Population

18,236

Population served by public supply_Groundwater $\quad 5,700$

Population served by public supply-Surface water $\quad 0$

Acres irrigated

7,260

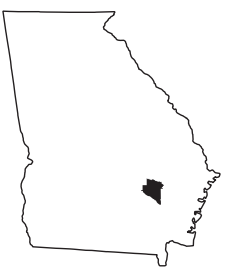

2010 WATER WITHDRAWALS AND ESTIMATED USE, IN MILLION GALLONS PER DAY

$[-$, not applicable $]$

\begin{tabular}{|c|c|c|c|c|c|c|c|c|}
\hline \multirow{3}{*}{ Category } & \multicolumn{3}{|c|}{ Withdrawals } & \multirow{3}{*}{$\begin{array}{l}\text { Total } \\
\text { use }^{1}\end{array}$} & \multirow{3}{*}{$\begin{array}{c}\text { Surface- } \\
\text { water } \\
\text { returns } \\
\end{array}$} & \multirow{2}{*}{\multicolumn{3}{|c|}{$\begin{array}{l}\text { Withdrawals by Major Industrial Groups } \\
\text { [NAICS, North American Industrial Classification System code] }\end{array}$}} \\
\hline & \multirow{2}{*}{$\begin{array}{c}\text { Ground- } \\
\text { water }\end{array}$} & \multirow{2}{*}{$\begin{array}{c}\text { Surface } \\
\text { water }\end{array}$} & \multirow[b]{2}{*}{ Total } & & & & & \\
\hline & & & & & & NAICS & Groundwater & Surface water \\
\hline Public supply & 0.91 & 0.00 & 0.91 & - & - & None & - & - \\
\hline Domestic & 0.94 & 0.00 & 0.94 & 1.58 & 0.00 & Nonte & & \\
\hline Commercial/public use & 0.00 & 0.00 & 0.00 & 0.04 & 0.00 & & & \\
\hline Industrial & 0.00 & 0.00 & 0.00 & 0.05 & 0.00 & & & \\
\hline Public-supply losses & - & - & - & 0.18 & - & & & \\
\hline Public wastewater treatment & - & - & - & - & 0.93 & Withdrawals & r Public Supp & \\
\hline Mining & 0.00 & 0.00 & 0.00 & 0.00 & 0.00 & Name & Groundwater & Surface water \\
\hline Irrigation-Crop & 1.31 & 0.45 & 1.76 & 1.76 & 0.00 & City of Baxley & 0.83 & 0.00 \\
\hline Irrigation-Golf course & 0.00 & 0.00 & 0.00 & 0.00 & 0.00 & & & \\
\hline Livestock and aquaculture & 0.05 & 0.22 & 0.27 & 0.27 & 0.00 & & & \\
\hline Thermoelectric power & 0.21 & 56.69 & 56.90 & 56.90 & 0.00 & & & \\
\hline TOTAL & 3.42 & $\mathbf{5 7 . 3 6}$ & 60.78 & 60.78 & 0.93 & & & \\
\hline
\end{tabular}

Total use is total withdrawal plus public supply deliveries and losses.

\section{Withdrawals by Source}
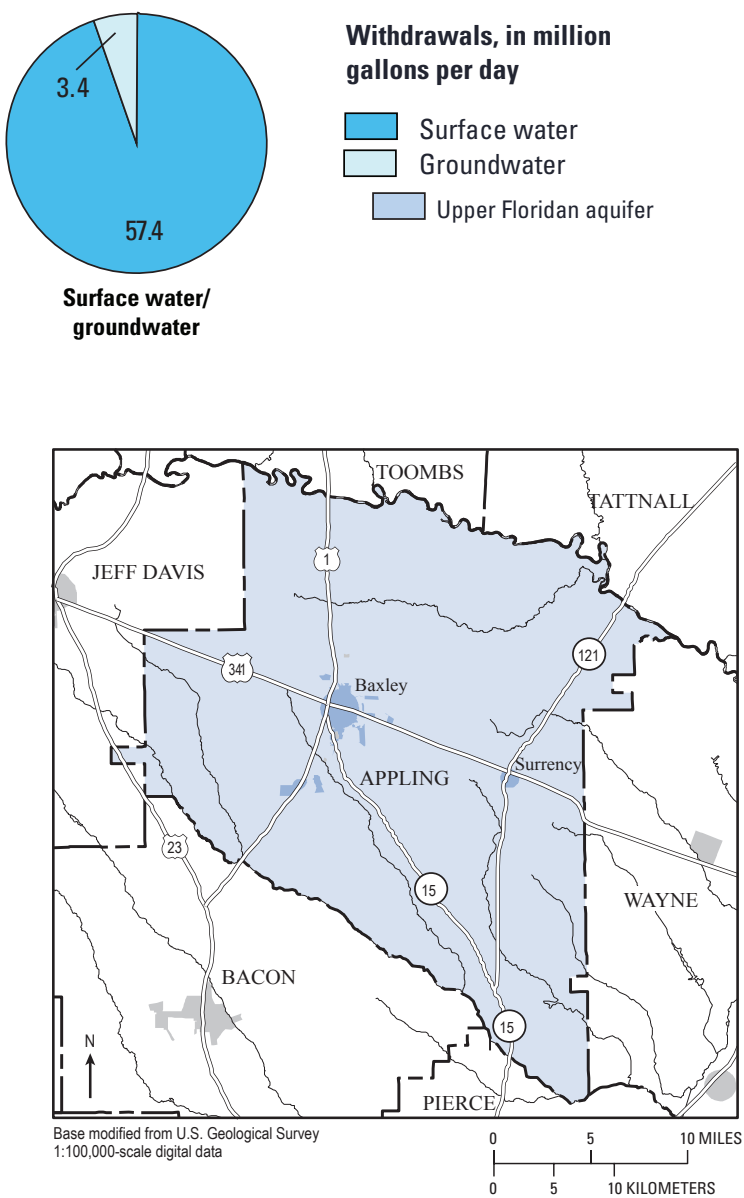

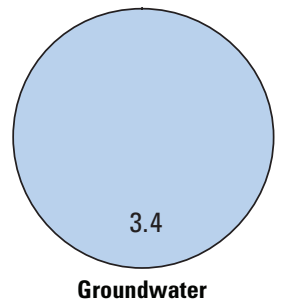

Public-Supply Deliveries by Use Category

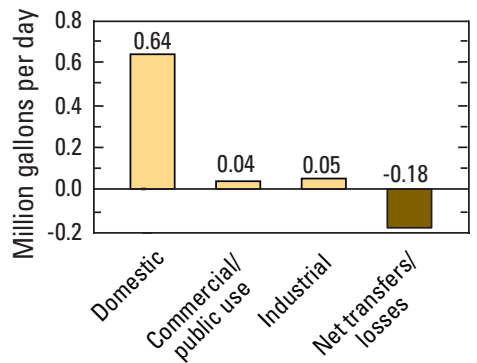

Surface-Water Withdrawals by Year

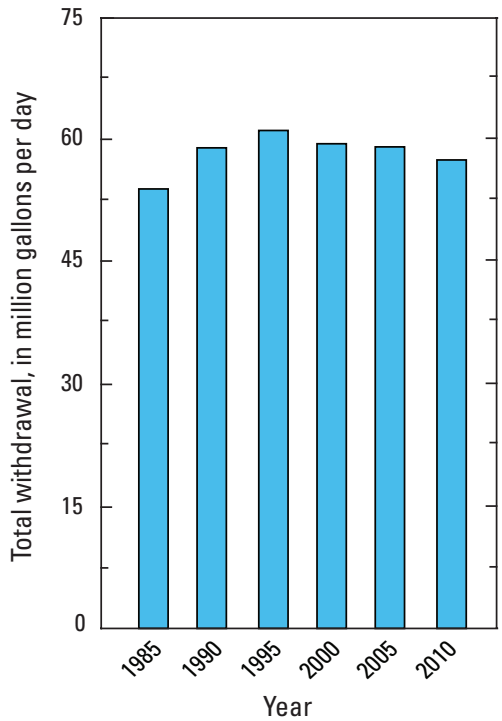

Groundwater Withdrawals by Year

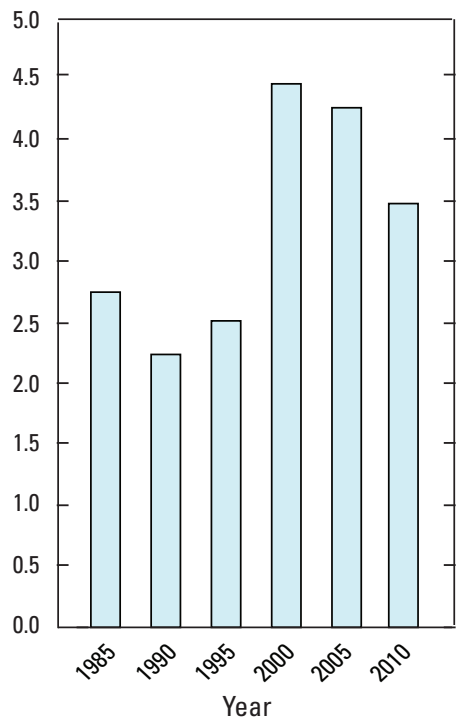




\section{ATKINSON COUNTY}

Population

Population served by public supply-Groundwater

Population served by public supply-Surface water

Acres irrigated

6,690

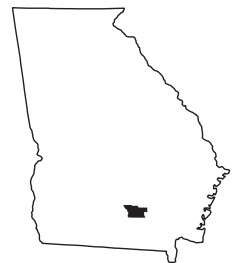

2010 WATER WITHDRAWALS AND ESTIMATED USE, IN MILLION GALLONS PER DAY

\begin{tabular}{|c|c|c|c|c|c|}
\hline \multirow[b]{2}{*}{ Category } & \multicolumn{3}{|c|}{ Withdrawals } & \multirow[b]{2}{*}{$\begin{array}{l}\text { Total } \\
\text { use }^{1}\end{array}$} & \multirow{2}{*}{$\begin{array}{c}\text { Surface- } \\
\text { water } \\
\text { returns }\end{array}$} \\
\hline & $\begin{array}{c}\text { Ground- } \\
\text { water }\end{array}$ & $\begin{array}{c}\text { Surface } \\
\text { water }\end{array}$ & Total & & \\
\hline Public supply & 0.44 & 0.00 & 0.44 & - & - \\
\hline Domestic & 0.34 & 0.00 & 0.34 & 0.59 & 0.00 \\
\hline Commercial/public use & 0.00 & 0.00 & 0.00 & 0.01 & 0.00 \\
\hline Industrial & 0.40 & 0.00 & 0.40 & 0.52 & 0.01 \\
\hline Public-supply losses & - & - & - & 0.06 & - \\
\hline Public wastewater treatment & - & - & - & - & 0.26 \\
\hline Mining & 0.00 & 0.00 & 0.00 & 0.00 & 0.00 \\
\hline Irrigation-Crop & 0.40 & 0.04 & 0.44 & 0.44 & 0.00 \\
\hline Irrigation-Golf course & 0.00 & 0.00 & 0.00 & 0.00 & 0.00 \\
\hline Livestock and aquaculture & 0.10 & 0.89 & 0.99 & 0.99 & 0.00 \\
\hline Thermoelectric power & 0.00 & 0.00 & 0.00 & 0.00 & 0.00 \\
\hline TOTAL & 1.68 & 0.93 & 2.61 & 2.61 & 0.27 \\
\hline
\end{tabular}

Withdrawals by Major Industrial Groups

[NAICS, North American Industrial Classification System code] NAICS Groundwater Surface water

\begin{tabular}{lll}
\hline 321 -Wood & 0.40 & 0.00 \\
\hline
\end{tabular}

Withdrawals by Major Public Suppliers

\begin{tabular}{lcc}
\hline Name & Groundwater & Surface water \\
\hline City of Pearson & 0.22 & 0.00 \\
City of Willacoochee & 0.20 & 0.00 \\
\hline
\end{tabular}

Total use is total withdrawals plus public supply deliveries and losses.

\section{Withdrawals by Source}
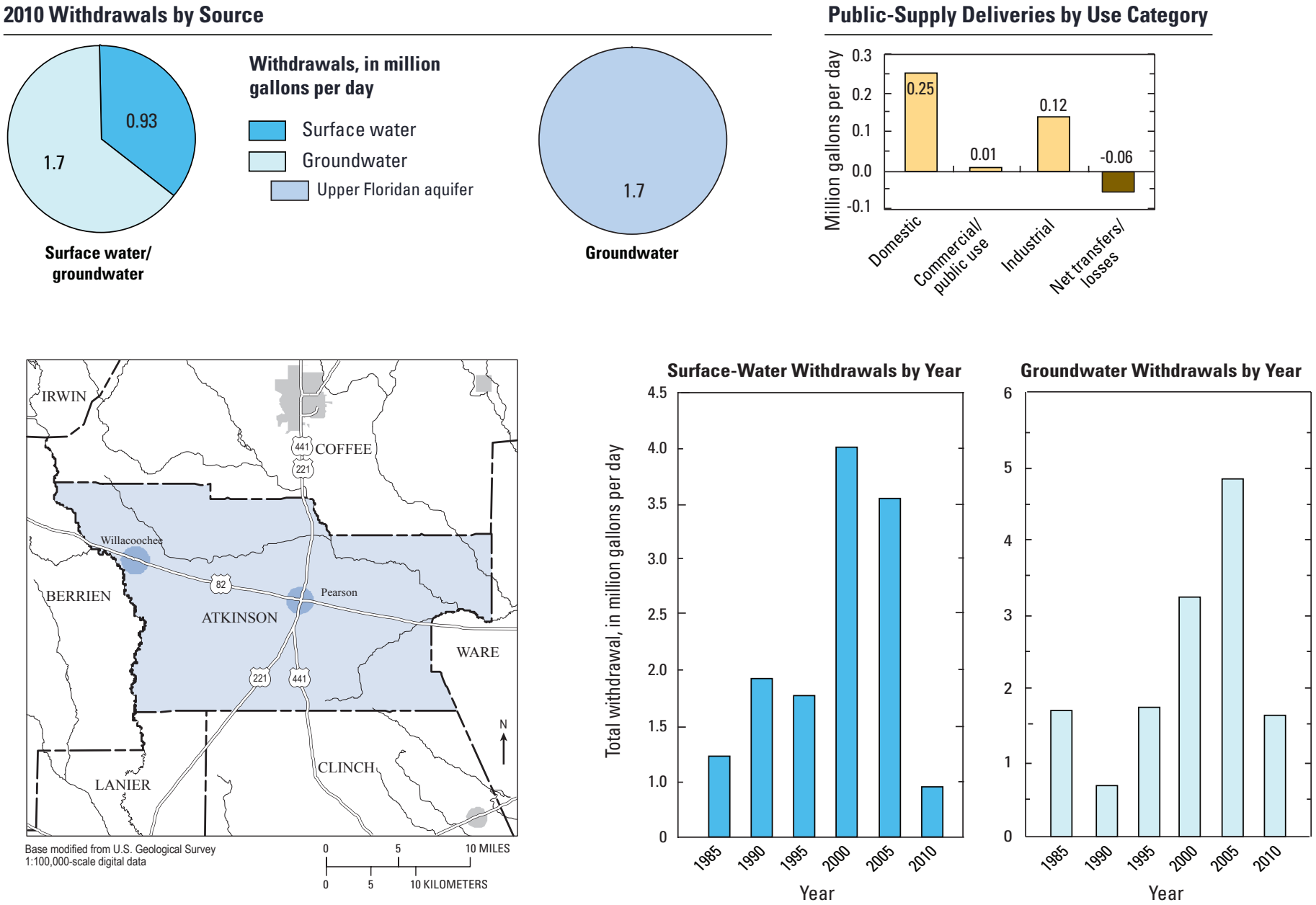


\section{BACON COUNTY}

Population

Population served by public supply-Groundwater

Population served by public supply_-Surface water

Acres irrigated
11,096

4,860

6,210

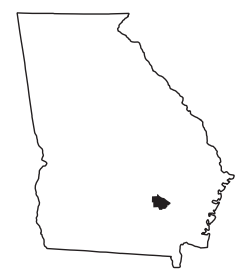

\section{WATER WITHDRAWALS AND ESTIMATED USE, IN MILLION GALLONS PER DAY}

[-, not applicable]

\begin{tabular}{|c|c|c|c|c|c|c|c|c|}
\hline \multirow{3}{*}{ Category } & \multicolumn{3}{|c|}{ Withdrawals } & \multirow{3}{*}{$\begin{array}{l}\text { Total } \\
\text { use }^{1}\end{array}$} & \multirow{3}{*}{$\begin{array}{c}\text { Surface- } \\
\text { water } \\
\text { returns } \\
\end{array}$} & \multirow{2}{*}{\multicolumn{3}{|c|}{$\begin{array}{l}\text { Withdrawals by Major Industrial Groups } \\
\text { [NAICS, North American Industrial Classification System code] }\end{array}$}} \\
\hline & \multirow{2}{*}{$\begin{array}{c}\text { Ground- } \\
\text { water }\end{array}$} & \multirow{2}{*}{$\begin{array}{c}\text { Surface } \\
\text { water }\end{array}$} & \multirow[b]{2}{*}{ Total } & & & & & \\
\hline & & & & & & NAICS & Groundwater & Surface water \\
\hline Public supply & 0.59 & 0.00 & 0.59 & - & - & 313 - Textiles & 030 & 000 \\
\hline Domestic & 0.47 & 0.00 & 0.47 & 0.89 & 0.00 & $313-1$ ext1les & 0.50 & 0.00 \\
\hline Commercial/public use & 0.00 & 0.00 & 0.00 & 0.06 & 0.00 & & & \\
\hline Industrial & 0.30 & 0.00 & 0.30 & 0.32 & 0.00 & & & \\
\hline Public-supply losses & - & - & - & 0.09 & - & & & \\
\hline Public wastewater treatment & - & - & - & - & 0.71 & Withdrawals & or Public Sup & \\
\hline Mining & 0.00 & 0.00 & 0.00 & 0.00 & 0.00 & Name & Groundwater & Surface water \\
\hline Irrigation-Crop & 0.60 & 0.21 & 0.81 & 0.81 & 0.00 & City of Alma & 0.58 & 0.00 \\
\hline Irrigation-Golf course & 0.00 & 0.00 & 0.00 & 0.00 & 0.00 & & & \\
\hline Livestock/aquaculture & 0.01 & 0.07 & 0.08 & 0.08 & 0.00 & & & \\
\hline Thermoelectric power & 0.00 & 0.00 & 0.00 & 0.00 & 0.00 & & & \\
\hline TOTAL & 1.97 & 0.28 & 2.25 & 2.25 & 0.71 & & & \\
\hline
\end{tabular}

${ }^{1}$ Total use is total withdrawal plus public supply deliveries and losses.

\section{Withdrawals by Source}
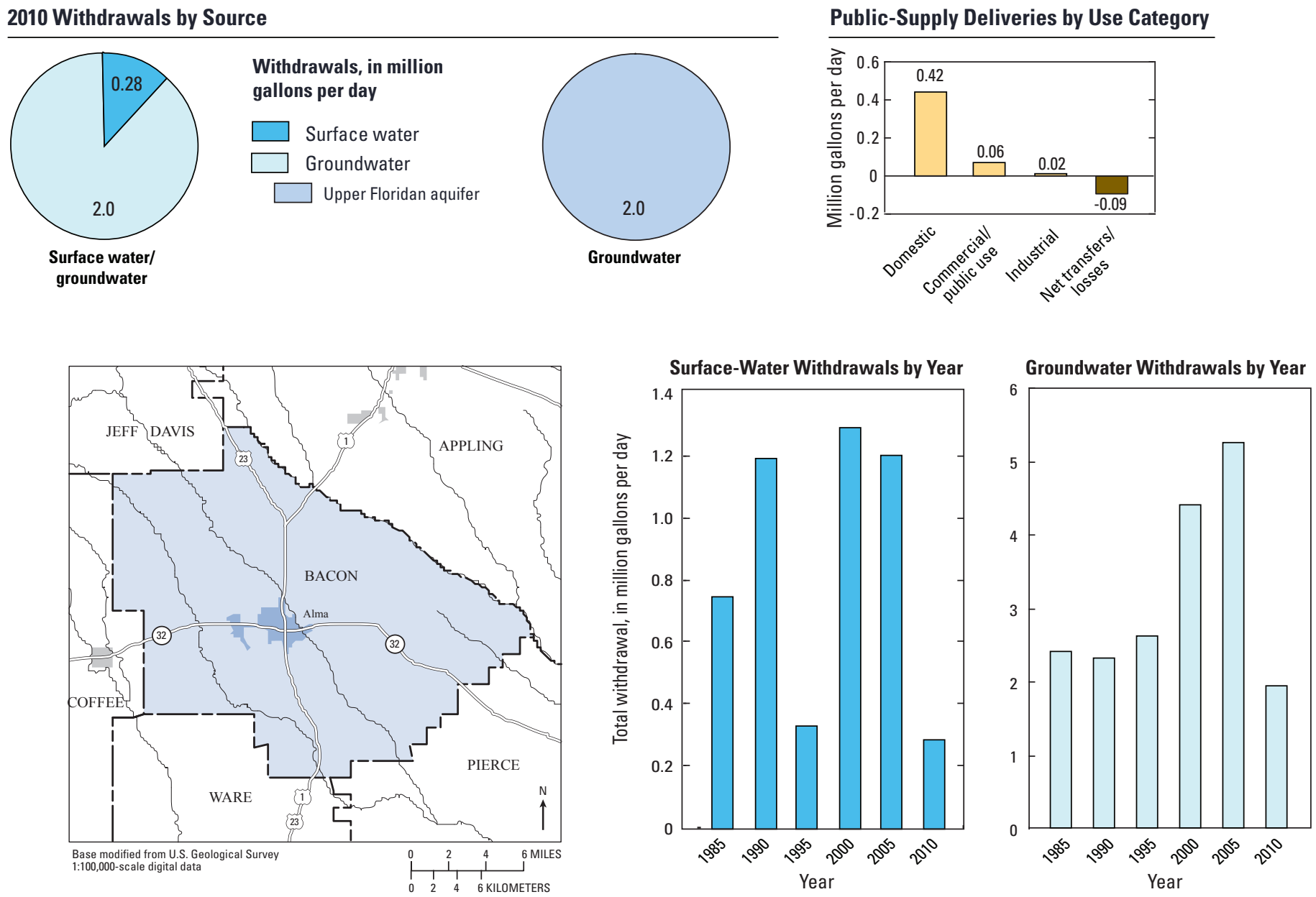
BAKER COUNTY

Population

Population served by public supply-Groundwater

Population served by public supply-Surface water Acres irrigated

43,010

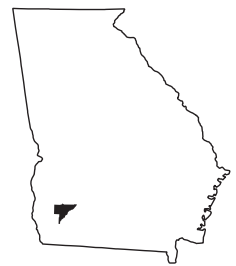

2010 WATER WITHDRAWALS AND ESTIMATED USE, IN MILLION GALLONS PER DAY

$[-$, not applicable; $<$, less than

\begin{tabular}{|c|c|c|c|c|c|}
\hline \multirow[b]{2}{*}{ Category } & \multicolumn{3}{|c|}{ Withdrawals } & \multirow[b]{2}{*}{$\begin{array}{l}\text { Total } \\
\text { use }^{1}\end{array}$} & \multirow{2}{*}{$\begin{array}{c}\text { Surface- } \\
\text { water } \\
\text { returns }\end{array}$} \\
\hline & $\begin{array}{c}\text { Ground- } \\
\text { water }\end{array}$ & $\begin{array}{c}\text { Surface } \\
\text { water }\end{array}$ & Total & & \\
\hline Public supply & 0.12 & 0.00 & 0.12 & - & - \\
\hline Domestic & 0.19 & 0.00 & 0.19 & 0.29 & 0.00 \\
\hline Commercial/public use & 0.00 & 0.00 & 0.00 & $<0.01$ & 0.00 \\
\hline Industrial & 0.00 & 0.00 & 0.00 & 0.00 & 0.00 \\
\hline Public-supply losses & - & - & - & 0.02 & - \\
\hline Public wastewater treatment & - & - & - & - & 0.00 \\
\hline Mining & 0.00 & 0.00 & 0.00 & 0.00 & 0.00 \\
\hline Irrigation-Crop & 31.00 & 3.99 & 34.99 & 34.99 & 0.00 \\
\hline Irrigation-Golf course & 0.00 & 0.00 & 0.00 & 0.00 & 0.00 \\
\hline Livestock/aquaculture & 0.00 & 0.72 & 0.72 & 0.72 & 0.00 \\
\hline Thermoelectric power & 0.00 & 0.00 & 0.00 & 0.00 & 0.00 \\
\hline TOTAL & 31.32 & 4.71 & 36.02 & 36.02 & 0.00 \\
\hline
\end{tabular}

Withdrawals by Major Industrial Groups

[NAICS, North American Industrial Classification System code]

NAICS Groundwater Surface water

None

Withdrawals by Major Public Suppliers

Name Groundwater Surface water

$\begin{array}{lll}\text { City of Newton } & 0.12 & 0.00\end{array}$

\section{Withdrawals by Source}

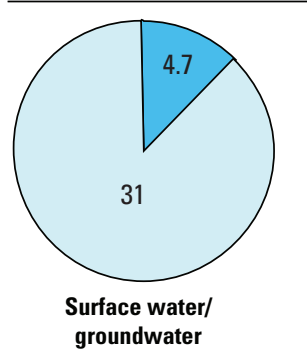

Withdrawals, in million gallons per day

Surface water Groundwater

$\square$ Upper Floridan aquifer $\square$ Claiborne aquifer groundwater

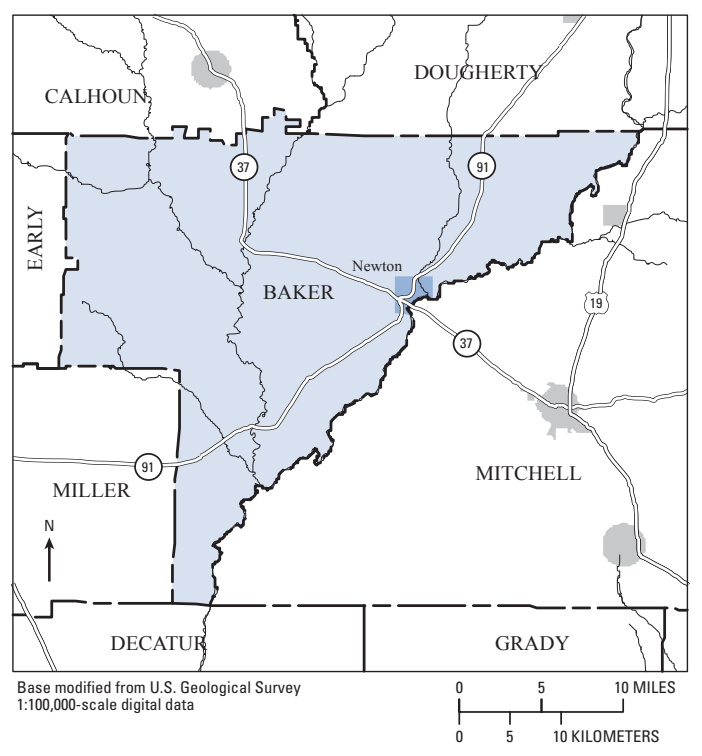

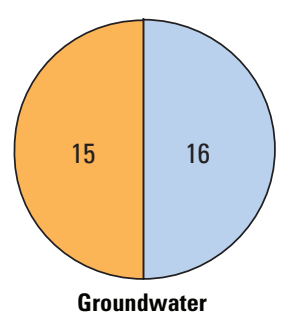

Public-Supply Deliveries by Use Category

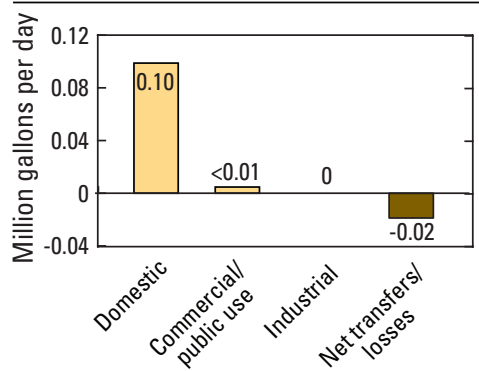

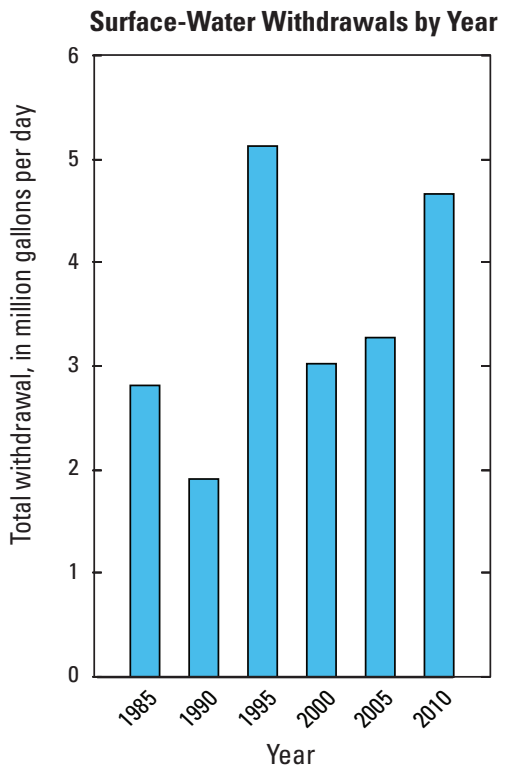

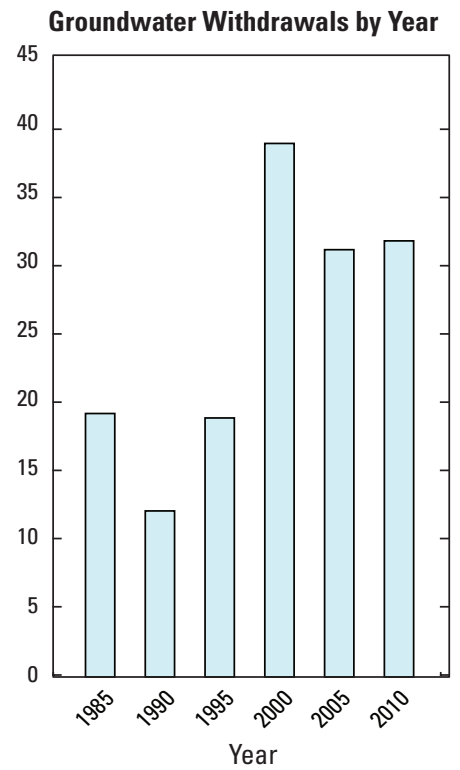




\section{BALDWIN COUNTY}

Population

45,720

Population served by public supply-Groundwater

Population served by public supply-Surface water

Acres irrigated

50

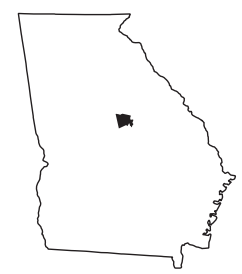

2010 WATER WITHDRAWALS AND ESTIMATED USE, IN MILLION GALLONS PER DAY

[-, not applicable; Mgal/d, million gallons per day]

\begin{tabular}{|c|c|c|c|c|c|c|c|c|}
\hline \multirow{3}{*}{ Category } & \multicolumn{3}{|c|}{ Withdrawals } & \multirow{3}{*}{$\begin{array}{l}\text { Total } \\
\text { use }^{1}\end{array}$} & \multirow{3}{*}{$\begin{array}{c}\text { Surface- } \\
\text { water } \\
\text { returns } \\
\end{array}$} & \multirow{2}{*}{\multicolumn{3}{|c|}{$\begin{array}{l}\text { Withdrawals by Major Industrial Groups } \\
\text { [NAICS, North American Industrial Classification System code] }\end{array}$}} \\
\hline & \multirow{2}{*}{$\begin{array}{c}\text { Ground- } \\
\text { water }\end{array}$} & \multirow{2}{*}{$\begin{array}{c}\text { Surface } \\
\text { water }\end{array}$} & \multirow{2}{*}{ Total } & & & & & \\
\hline & & & & & & NAICS & Groundwater & Surface water \\
\hline Public supply & 0.02 & 6.56 & 6.58 & - & - & 321-Wood & 0.01 & 0.00 \\
\hline Domestic & 0.03 & 0.00 & 0.03 & 2.64 & 0.00 & & & \\
\hline Commercial/public use & 0.00 & 0.00 & 0.00 & 4.00 & 0.00 & & & \\
\hline Industrial & 0.01 & 0.00 & 0.01 & 0.79 & 0.00 & & & \\
\hline Public-supply losses & - & - & - & 1.43 & - & & & \\
\hline Public wastewater treatment & - & - & - & - & 4.44 & Withdrawals by Ma & r Public Supp & \\
\hline Mining & 0.08 & 0.00 & 0.08 & 0.08 & 0.00 & Name & Groundwater & Surface water \\
\hline Irrigation-Crop & 0.00 & 0.00 & 0.00 & 0.00 & 0.00 & City of Milledgeville & 0.00 & 6.56 \\
\hline Irrigation-Golf course & 0.00 & 0.08 & 0.08 & 0.08 & 0.00 & & & \\
\hline Livestock/aquaculture & 0.00 & 0.12 & 0.12 & 0.12 & 0.00 & & & \\
\hline Thermoelectric power & 0.00 & 0.00 & 0.00 & 0.00 & 0.00 & & & \\
\hline TOTAL & 0.14 & 6.76 & 6.90 & 8.96 & 4.44 & & & \\
\hline
\end{tabular}

'Total use is total withdrawal plus public supply deliveries and losses. An average of $2.11 \mathrm{Mgal} / \mathrm{d}$ was delivered from Putnam County, Georgia to Baldwin County in 2010.

\section{Withdrawals by Source}
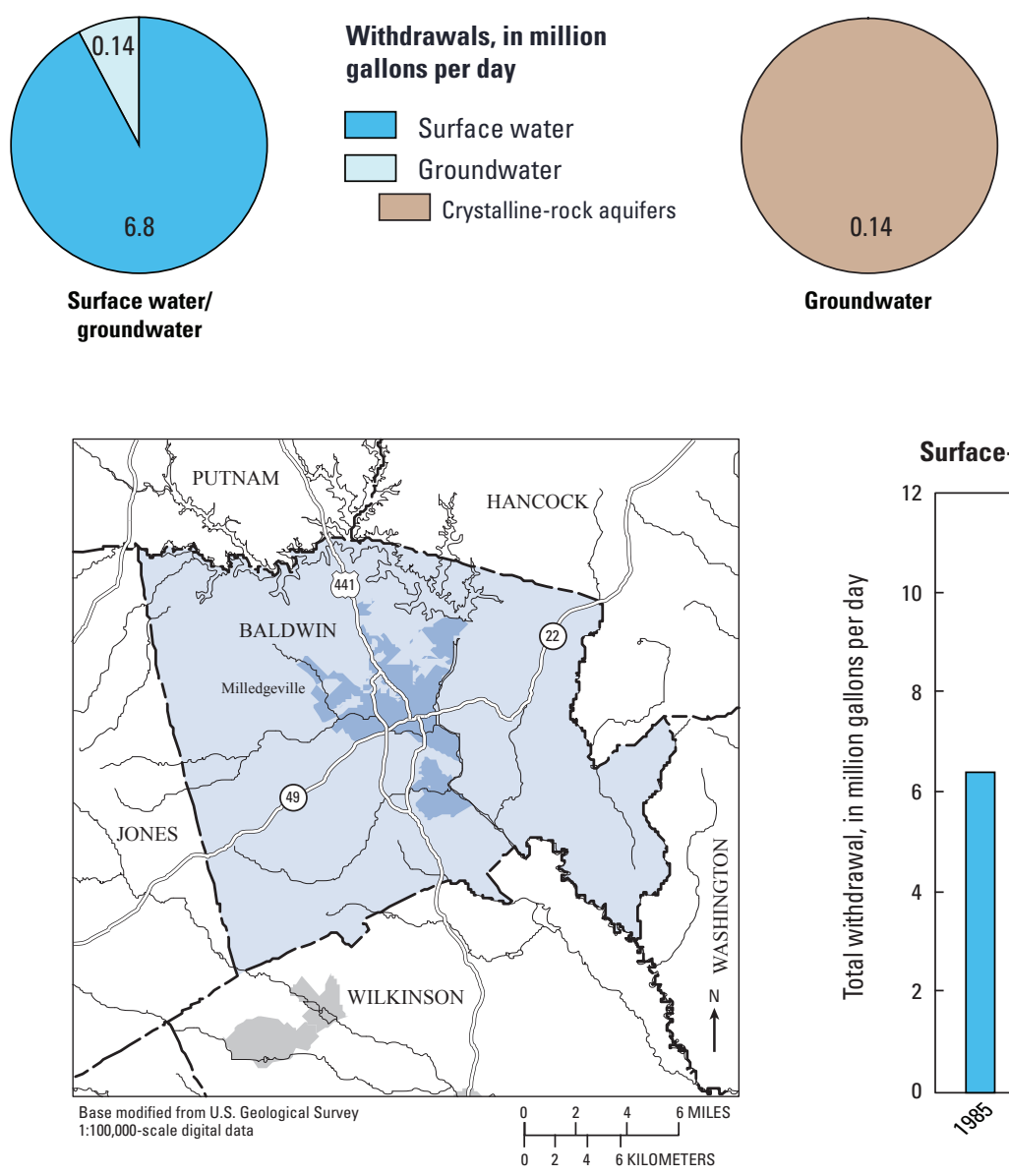

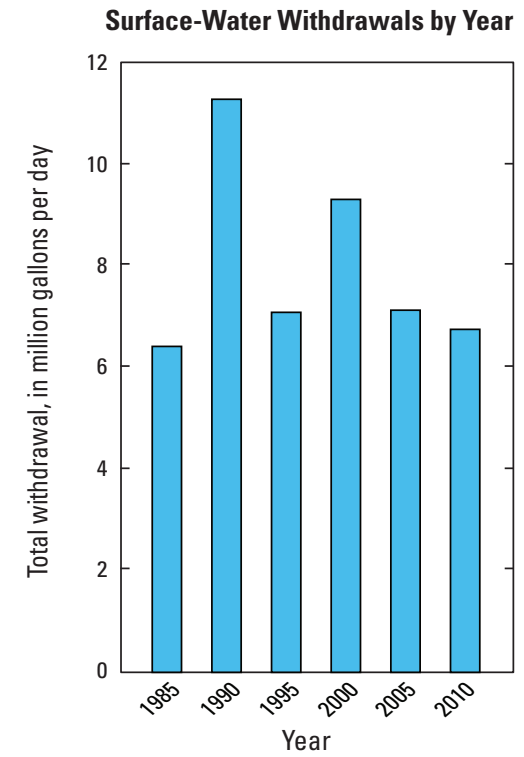

Groundwater Withdrawals by Year

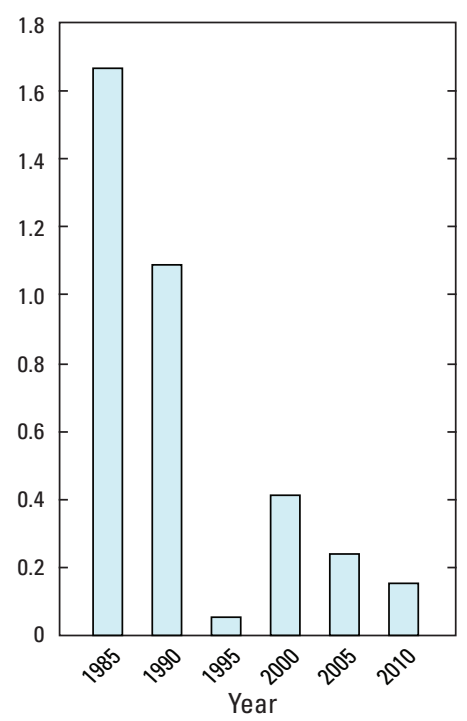




\section{BANKS COUNTY}

Population

8,395

2,320

8,620

130

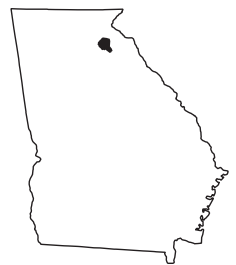

2010 WATER WITHDRAWALS AND ESTIMATED USE, IN MILLION GALLONS PER DAY

$[-$, not applicable; Mgal/d, million gallons per day]

\begin{tabular}{|c|c|c|c|c|c|}
\hline \multirow{2}{*}{ Category } & \multicolumn{3}{|c|}{ Withdrawals } & \multirow[b]{2}{*}{$\begin{array}{l}\text { Total } \\
\text { use }^{1}\end{array}$} & \multirow{2}{*}{$\begin{array}{c}\text { Surface- } \\
\text { water } \\
\text { returns }\end{array}$} \\
\hline & $\begin{array}{l}\text { Ground- } \\
\text { water }\end{array}$ & $\begin{array}{c}\text { Surface } \\
\text { water }\end{array}$ & Total & & \\
\hline Public supply & 0.14 & 2.40 & 2.54 & - & - \\
\hline Domestic & 0.56 & 0.00 & 0.56 & 1.29 & 0.00 \\
\hline Commercial/public use & 0.00 & 0.00 & 0.00 & 0.06 & 0.00 \\
\hline Industrial & 0.00 & 0.00 & 0.00 & 0.01 & 0.00 \\
\hline Public-supply losses & - & - & - & 0.14 & - \\
\hline Public wastewater treatment & - & - & - & - & 0.10 \\
\hline Mining & 0.05 & 0.00 & 0.05 & 0.05 & 0.00 \\
\hline Irrigation-Crop & 0.00 & 0.00 & 0.00 & 0.00 & 0.00 \\
\hline Irrigation-Golf course & 0.00 & 0.00 & 0.00 & 0.00 & 0.00 \\
\hline Livestock/aquaculture & 0.00 & 0.53 & 0.53 & 0.53 & 0.00 \\
\hline Thermoelectric power & 0.00 & 0.00 & 0.00 & 0.00 & 0.00 \\
\hline TOTAL & 0.75 & 2.93 & 3.68 & 2.08 & 0.10 \\
\hline
\end{tabular}

'Total use is total withdrawal plus public supply deliveries and losses. An average of $1.69 \mathrm{Mgal} / \mathrm{d}$ was delivered to Jackson County, Georgia and $0.04 \mathrm{Mgal} / \mathrm{d}$ to Franklin County, Georgia from Banks County in 2010.

\section{Withdrawals by Source}
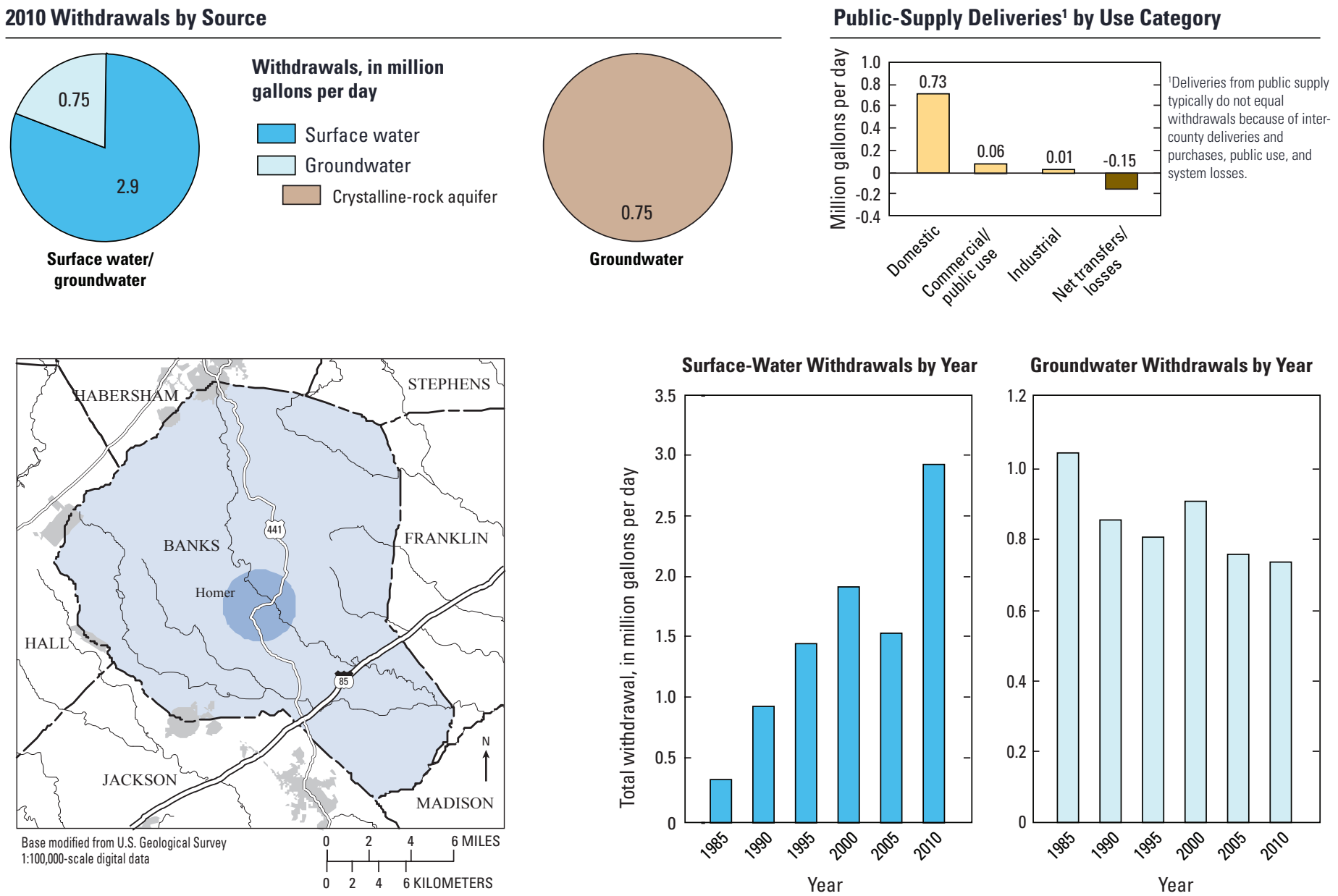

Groundwater Withdrawals by Year

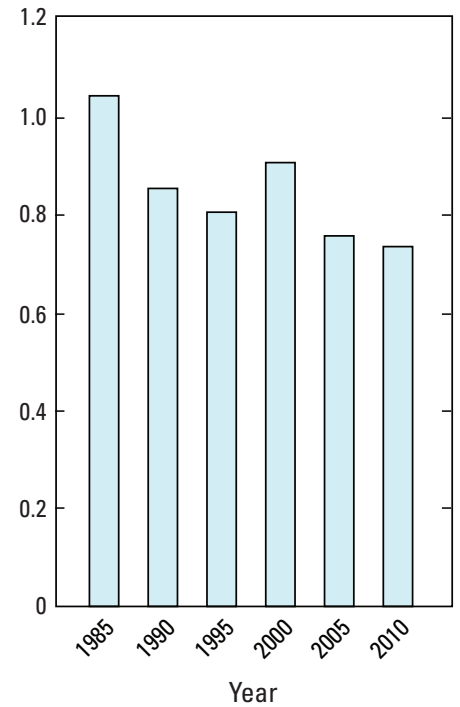

Withdrawals by Major Public Suppliers

\begin{tabular}{lcc}
\hline Name & Groundwater & Surface water \\
\hline City of Commerce & 0.00 & ${ }^{1} 1.74$ \\
$\begin{array}{l}\text { Banks County Board of } \\
\text { Commissioners }\end{array}$ & 0.00 & 0.66 \\
\hline \multicolumn{2}{l}{ 'Water withdrawn was used in Jackson County, Georgia. }
\end{tabular}

Withdrawals by Major Industrial Groups

\begin{tabular}{lcc}
\hline NAICS & Groundwater & Surface water \\
\hline None & - & -
\end{tabular}




\section{BARROW COUNTY}

Population

69,367

Population served by public supply-Groundwater

Population served by public supply-Surface water

61,920

Acres irrigated

480

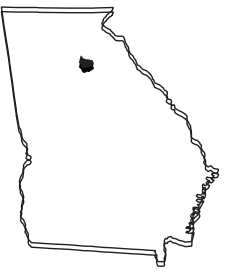

2010 WATER WITHDRAWALS AND ESTIMATED USE, IN MILLION GALLONS PER DAY

[-, not applicable; Mgal/d, million gallons per day]

\begin{tabular}{|c|c|c|c|c|c|}
\hline \multirow[b]{2}{*}{ Category } & \multicolumn{3}{|c|}{ Withdrawals } & \multirow[b]{2}{*}{$\begin{array}{l}\text { Total } \\
\text { use }^{1} \\
\end{array}$} & \multirow{2}{*}{$\begin{array}{c}\text { Surface- } \\
\text { water } \\
\text { returns } \\
\end{array}$} \\
\hline & $\begin{array}{c}\text { Ground- } \\
\text { water }\end{array}$ & $\begin{array}{c}\text { Surface } \\
\text { water }\end{array}$ & Total & & \\
\hline Public supply & 0.06 & 4.54 & 4.60 & - & - \\
\hline Domestic & 0.51 & 0.00 & 0.51 & 5.27 & 0.00 \\
\hline Commercial/public use & 0.00 & 0.00 & 0.00 & 0.87 & 0.00 \\
\hline Industrial & 0.39 & 0.00 & 0.39 & 1.17 & 0.00 \\
\hline Public-supply losses & - & - & - & 1.25 & - \\
\hline Public wastewater treatment & - & - & - & - & 0.51 \\
\hline Mining & 0.05 & 0.00 & 0.05 & 0.05 & 0.00 \\
\hline Irrigation-Crop & 0.00 & 0.00 & 0.00 & 0.00 & 0.00 \\
\hline Irrigation-Golf course & 0.25 & 0.54 & 0.79 & 0.79 & 0.00 \\
\hline Livestock/aquaculture & 0.00 & 0.19 & 0.19 & 0.19 & 0.00 \\
\hline Thermoelectric power & 0.00 & 0.00 & 0.00 & 0.00 & 0.00 \\
\hline TOTAL & 1.26 & 5.27 & 6.53 & 9.59 & 0.51 \\
\hline
\end{tabular}

Withdrawals by Major Industrial Groups

[NAICS, North American Industrial Classification System code]

NAICS Groundwater Surface water

\begin{tabular}{lll}
\hline 311 -Food & 0.39 & 0.00
\end{tabular}

Total use is total withdrawal plus public supply deliveries and losses. An average of $2.17 \mathrm{Mgal} / \mathrm{d}$ was delivered from Jackson County, Georgia to Barrow County in 2010

\section{Withdrawals by Source}

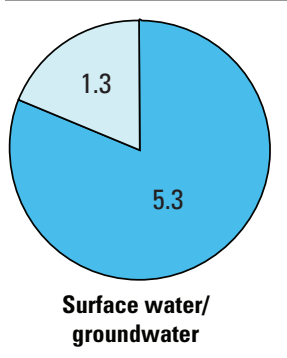

Withdrawals, in million gallons per day

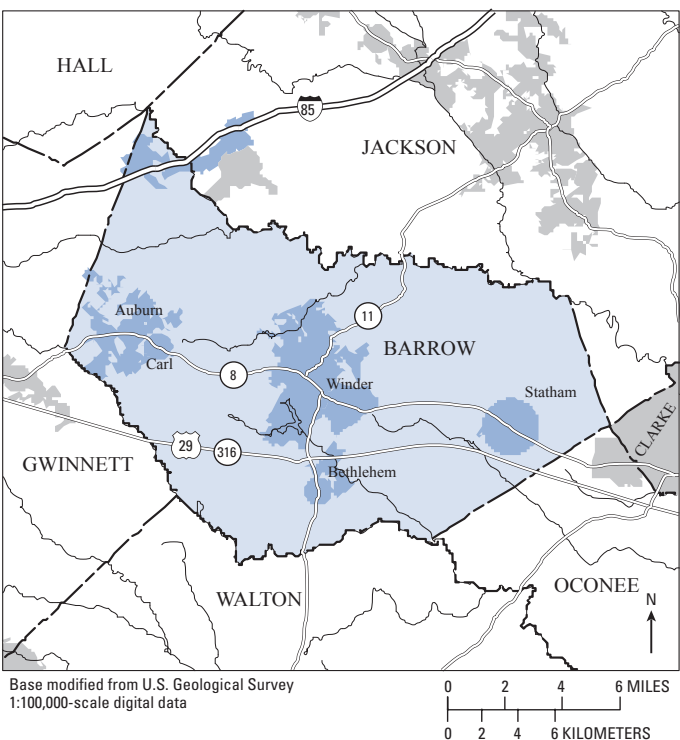

Surface water
Groundwater
$\square$ Crystalline-rock aquifer

$\square$ Crystalline-rock aquifer

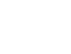

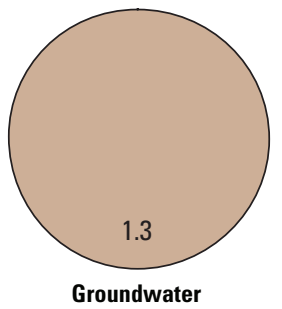

Public-Supply Deliveries ${ }^{1}$ by Use Category

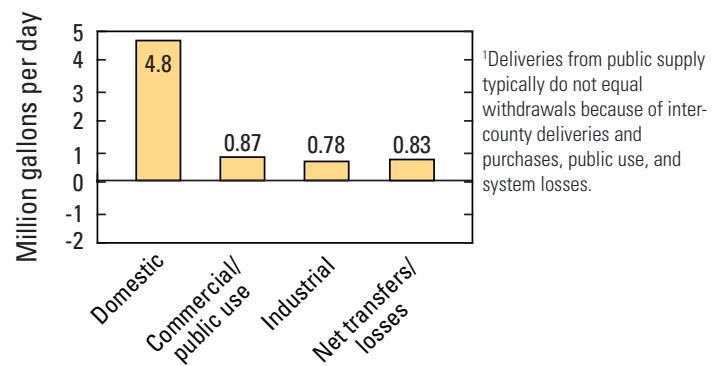

Withdrawals by Major Public Suppliers

\begin{tabular}{lcc}
\hline Name & Groundwater & Surface water \\
\hline City of Winder & 0.00 & 4.19 \\
City of Statham & 0.00 & 0.35 \\
\hline
\end{tabular}




\section{BARTOW COUNTY}

Population

100,157

1,900

82,130

2,140

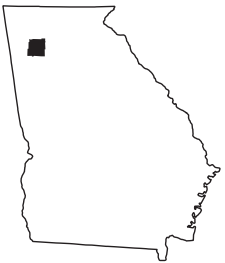

2010 WATER WITHDRAWALS AND ESTIMATED USE, IN MILLION GALLONS PER DAY

[-, not applicable; Mgal/d, million gallons per day]

\begin{tabular}{|c|c|c|c|c|c|}
\hline \multirow[b]{2}{*}{ Category } & \multicolumn{3}{|c|}{ Withdrawals } & \multirow[b]{2}{*}{$\begin{array}{l}\text { Total } \\
\text { use }^{1}\end{array}$} & \multirow{2}{*}{$\begin{array}{c}\text { Surface- } \\
\text { water } \\
\text { returns }\end{array}$} \\
\hline & $\begin{array}{c}\text { Ground- } \\
\text { water }\end{array}$ & $\begin{array}{c}\text { Surface } \\
\text { water }\end{array}$ & Total & & \\
\hline Public supply & 0.17 & 54.70 & 54.87 & - & - \\
\hline Domestic & 1.21 & 0.00 & 1.21 & 9.37 & 0.00 \\
\hline Commercial/public use & 0.00 & 0.00 & 0.00 & 1.90 & 0.00 \\
\hline Industrial & 1.14 & 0.41 & 1.55 & 4.02 & 0.96 \\
\hline Public-supply losses & - & - & - & 3.10 & - \\
\hline Public wastewater treatment & - & - & - & - & 9.65 \\
\hline Mining & 0.44 & 0.01 & 0.45 & 0.45 & 0.60 \\
\hline Irrigation-Crop & 0.15 & 2.21 & 2.36 & 2.36 & 0.00 \\
\hline Irrigation-Golf course & 0.00 & 0.36 & 0.36 & 0.36 & 0.00 \\
\hline Livestock/aquaculture & 0.02 & 0.43 & 0.45 & 0.45 & 0.00 \\
\hline Thermoelectric power & 0.00 & 47.92 & 47.92 & 47.92 & 16.00 \\
\hline TOTAL & 3.13 & 106.04 & 109.17 & 69.93 & 27.21 \\
\hline
\end{tabular}

'Total use is total withdrawals plus public supply deliveries and losses. An average of $40 \mathrm{Mgal} / \mathrm{d}$ was withdrawn in Bartow County and delivered to Cobb County, Georgia; whereas, $0.61 \mathrm{Mgal} / \mathrm{d}$ was delivered to Floyd County, Georgia in 2010.

\section{Withdrawals by Source}
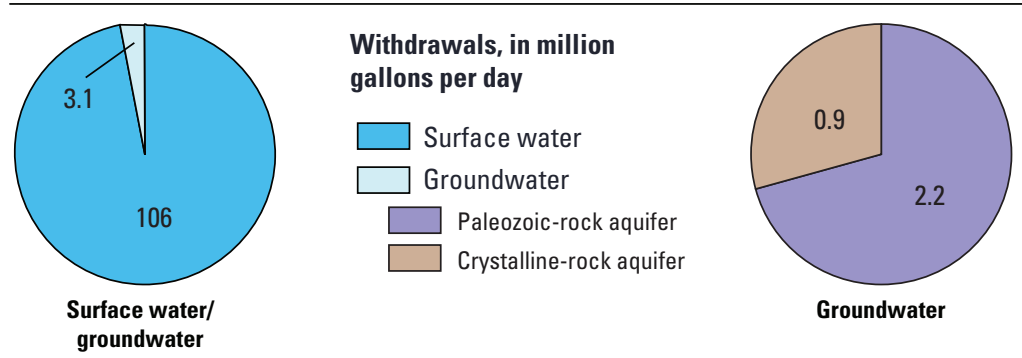

Withdrawals by Major Industrial Groups

[NAICS, North American Industrial Classification System code]

NAICS Groundwater Surface water

325 - Chemical products $0.98 \quad 0.00$

Withdrawals by Major Public Suppliers

Name

Groundwater Surface water

Cobb Co.-Marietta

Water Authority

${ }^{1} 40.07$

City of Cartersville

0.00

11.18

City of Adairsville

0.00

2.65

Bartow County

0.00

0.52

City of Emerson

0.00

0.28

'Delivered and used in Cobb County, Georgia.

Public-Supply Deliveries ${ }^{1}$ by Use Category
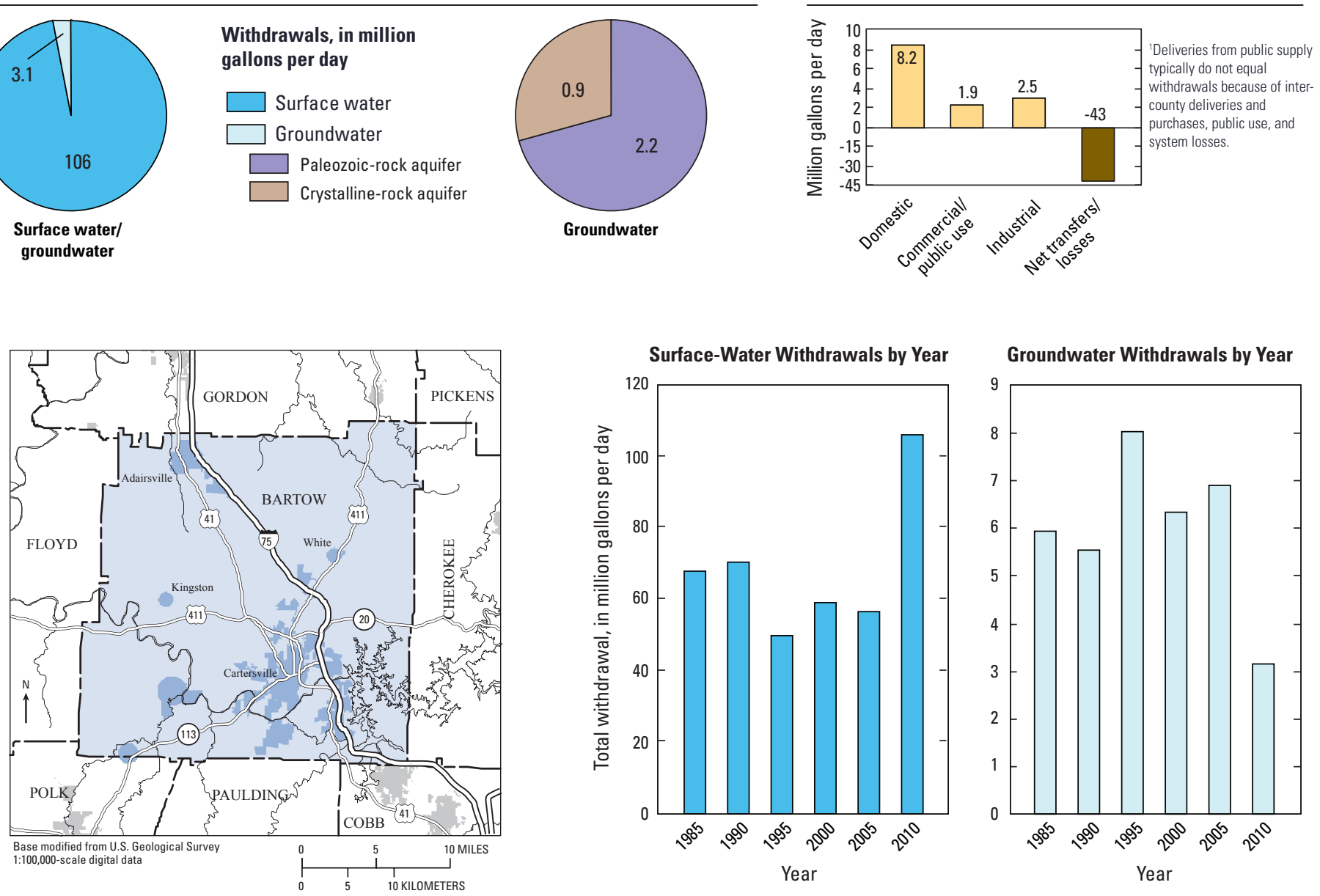

Groundwater Withdrawals by Year

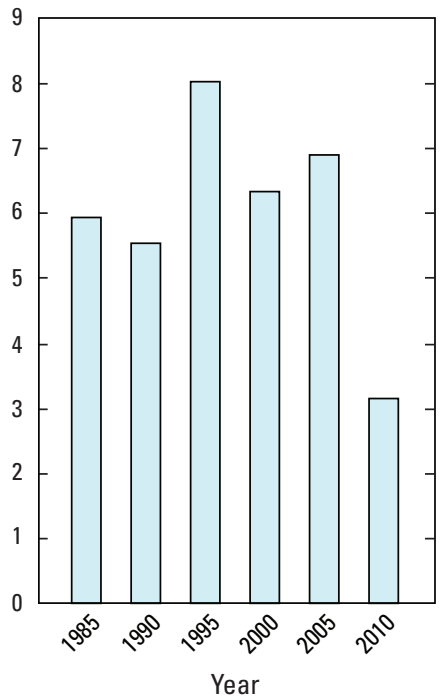




\section{BEN HILL COUNTY}

Population

17,634

Population served by public supply_-Groundwater $\quad 13,730$

Population served by public supply-Surface water $\quad 0$

Acres irrigated

7,280

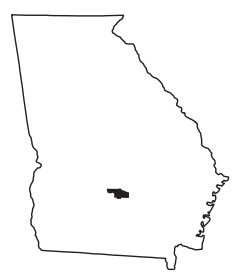

2010 WATER WITHDRAWALS AND ESTIMATED USE, IN MILLION GALLONS PER DAY

$[-$, not applicable $]$

\begin{tabular}{lcccccc}
\hline \multirow{2}{*}{ Category } & \multicolumn{3}{c}{ Withdrawals } & & Total & $\begin{array}{c}\text { Surface- } \\
\text { water } \\
\text { returns }\end{array}$ \\
\cline { 2 - 4 } & $\begin{array}{c}\text { Ground- } \\
\text { water }\end{array}$ & $\begin{array}{c}\text { Surface } \\
\text { water }\end{array}$ & Total & & - & - \\
use & Public supply & 3.23 & 0.00 & 3.23 & - & 0.00 \\
Domestic & 0.29 & 0.00 & 0.29 & 1.91 & 0.00 \\
Commercial/public use & 0.00 & 0.00 & 0.00 & 0.45 & 0.00 \\
Industrial & 0.00 & 0.00 & 0.00 & 0.58 & - \\
Public-supply losses & - & - & - & 0.58 & 2.65 \\
Public wastewater treatment & - & - & - & - & 0.00 \\
Mining & 0.00 & 0.00 & 0.00 & 0.00 & 0.00 \\
Irrigation-Crop & 1.50 & 0.50 & 2.00 & 2.00 & 0.00 \\
Irrigation-Golf course & 0.07 & 0.00 & 0.07 & 0.07 & 0.00 \\
Livestock/aquaculture & 0.01 & 0.06 & 0.07 & 0.07 & 0.00 \\
Thermoelectric power & 0.00 & 0.00 & 0.00 & 0.00 & $\mathbf{2 . 6 5}$ \\
\multicolumn{1}{c}{ TOTAL } & $\mathbf{5 . 1 0}$ & $\mathbf{0 . 5 6}$ & $\mathbf{5 . 6 6}$ & $\mathbf{5 . 6 6}$ & $\mathbf{2 0}$ \\
\hline
\end{tabular}

Withdrawals by Major Industrial Groups

[NAICS, North American Industrial Classification System code] NAICS Groundwater Surface water

None

Withdrawals by Major Public Suppliers

\begin{tabular}{lcc}
\hline Name & Groundwater & Surface water \\
\hline $\begin{array}{l}\text { Fitzgerald Water, Land, } \\
\text { and Bond Commission }\end{array}$ & 3.23 & 0.00 \\
\hline
\end{tabular}

Total use is total withdrawal plus public supply deliveries and losses.

\section{Withdrawals by Source}
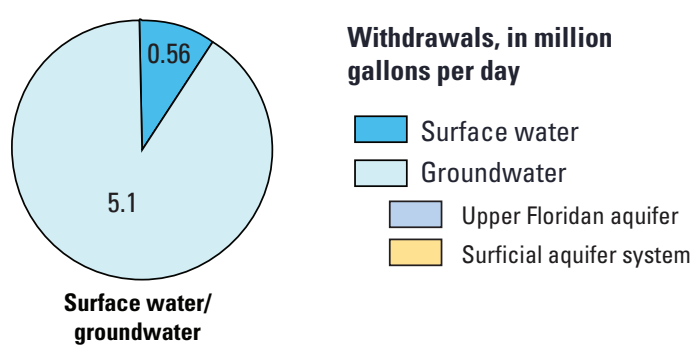

groundwater

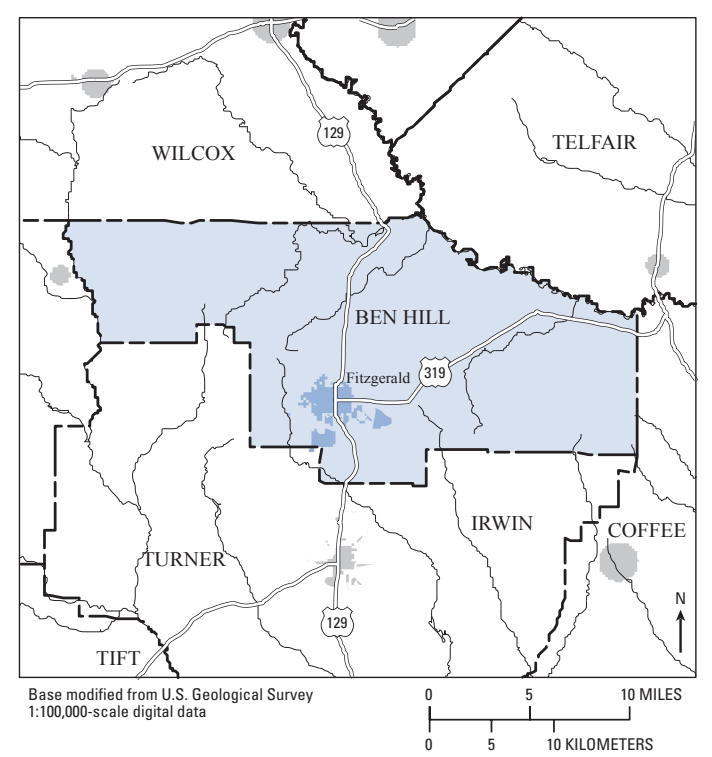

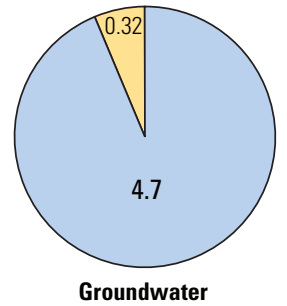

Public-Supply Deliveries by Use Category

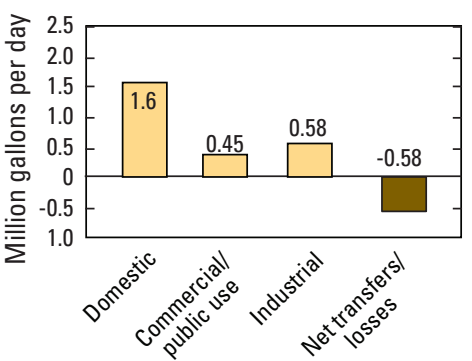

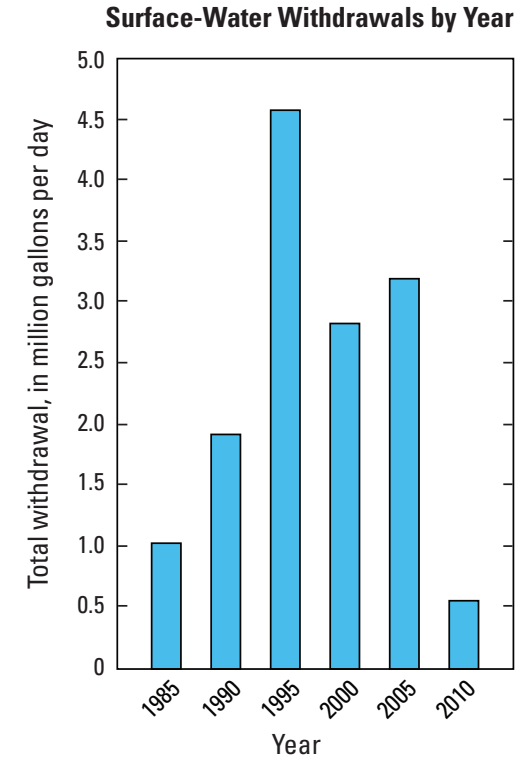

Groundwater Withdrawals by Year

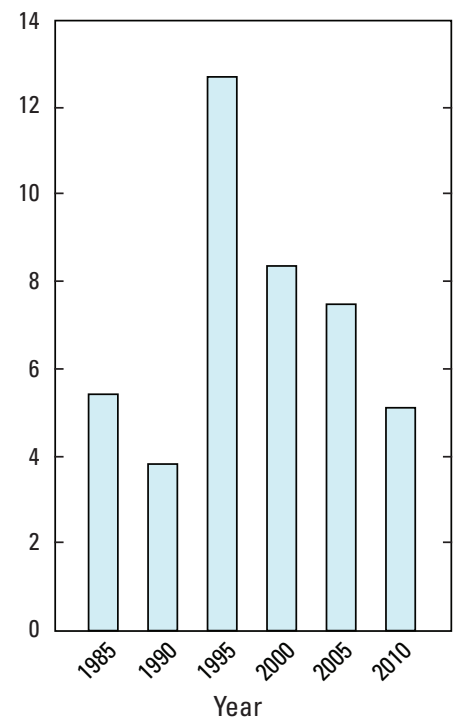




\section{BERRIEN COUNTY}

Population

19,286

Population served by public supply-Groundwater

7,660

Population served by public supply-Surface water 0

Acres irrigated

14,320

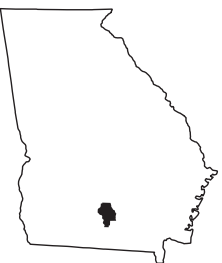

2010 WATER WITHDRAWALS AND ESTIMATED USE, IN MILLION GALLONS PER DAY

[-, not applicable]

\begin{tabular}{|c|c|c|c|c|c|c|c|c|}
\hline \multirow{3}{*}{ Category } & \multicolumn{3}{|c|}{ Withdrawals } & \multirow{3}{*}{$\begin{array}{l}\text { Total } \\
\text { use }^{1}\end{array}$} & \multirow{3}{*}{$\begin{array}{c}\text { Surface- } \\
\text { water } \\
\text { returns } \\
\end{array}$} & \multirow{2}{*}{\multicolumn{3}{|c|}{$\begin{array}{l}\text { Withdrawals by Major Industrial Groups } \\
\text { [NAICS, North American Industrial Classification System code] }\end{array}$}} \\
\hline & \multirow{2}{*}{$\begin{array}{c}\text { Ground- } \\
\text { water }\end{array}$} & \multirow{2}{*}{$\begin{array}{c}\text { Surface } \\
\text { water }\end{array}$} & \multirow[b]{2}{*}{ Total } & & & & & \\
\hline & & & & & & NAICS & Groundwater & Surface water \\
\hline Public supply & 0.83 & 0.00 & 0.83 & - & - & 313-Textiles & 0.02 & 0.00 \\
\hline Domestic & 0.87 & 0.00 & 0.87 & 1.45 & 0.00 & ग1J-1CXIICs & 0.02 & 0.00 \\
\hline Commercial/public use & 0.00 & 0.00 & 0.00 & 0.15 & 0.00 & & & \\
\hline Industrial & 0.02 & 0.00 & 0.02 & 0.03 & 0.00 & & & \\
\hline Public-supply losses & - & - & - & 0.09 & - & & & \\
\hline Public wastewater treatment & - & - & - & - & 0.17 & Withdrawals by & r Public Supp & \\
\hline Mining & 0.00 & 0.00 & 0.00 & 0.00 & 0.00 & Name & Groundwater & Surface water \\
\hline Irrigation-Crop & 2.00 & 0.85 & 2.85 & 2.85 & 0.00 & City of Nashville & 0.62 & 0.00 \\
\hline Irrigation-Golf course & 0.00 & 0.00 & 0.00 & 0.00 & 0.00 & Cily or Nasivine & 0.02 & 0.00 \\
\hline Livestock/aquaculture & 0.02 & 0.08 & 0.10 & 0.10 & 0.00 & & & \\
\hline Thermoelectric power & 0.00 & 0.00 & 0.00 & 0.00 & 0.00 & & & \\
\hline TOTAL & 3.74 & 0.93 & 4.67 & 4.67 & 0.17 & & & \\
\hline
\end{tabular}

'Total use is total withdrawal plus public supply deliveries and losses.

\section{Withdrawals by Source}
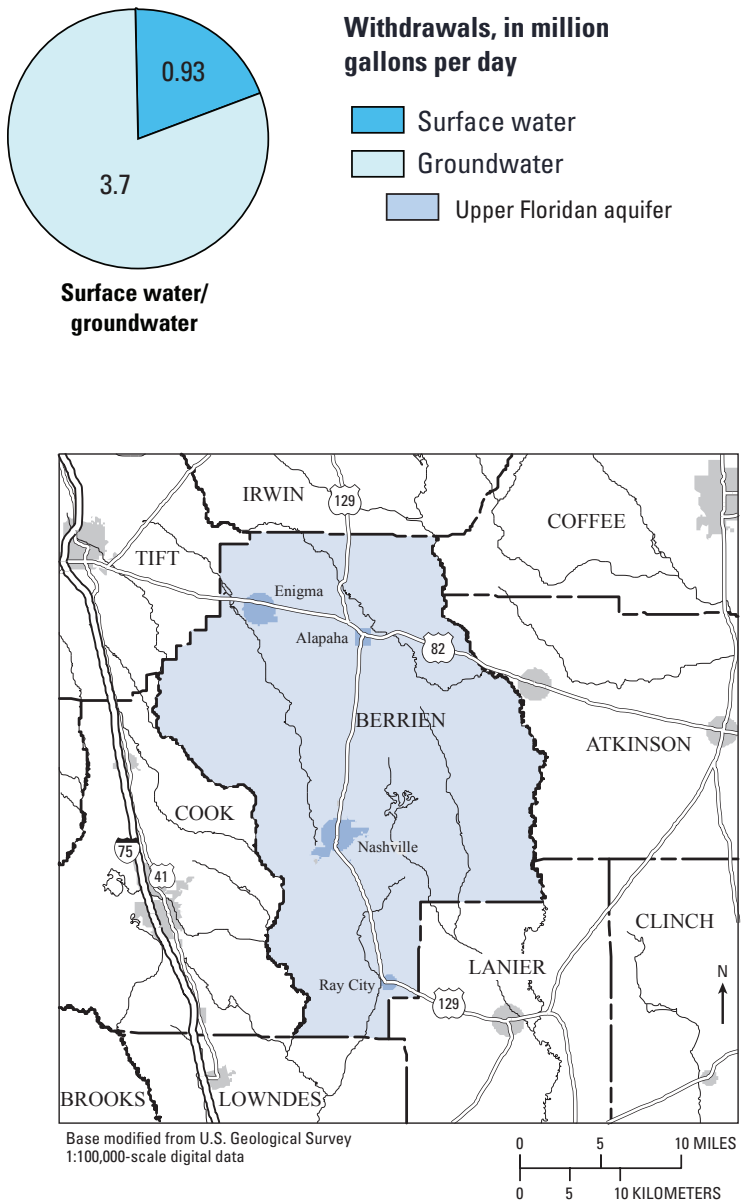

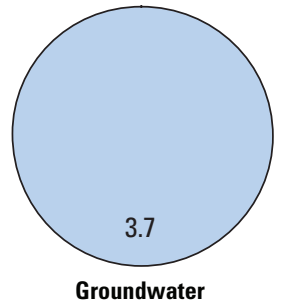

Public-Supply Deliveries by Use Category

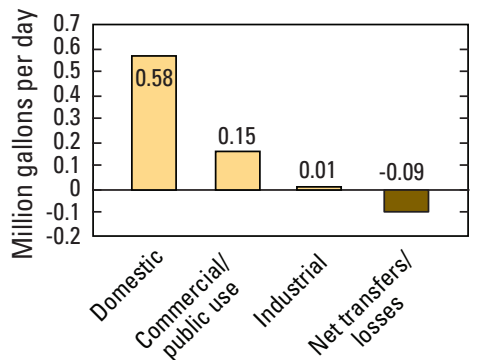

Surface-Water Withdrawals by Year

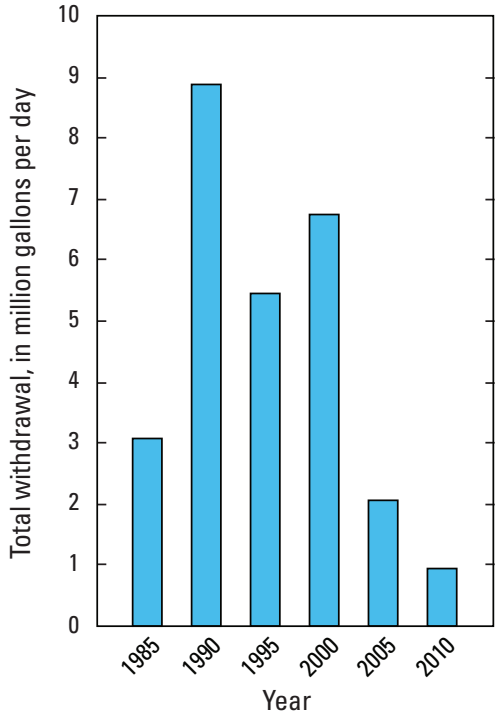

Groundwater Withdrawals by Year

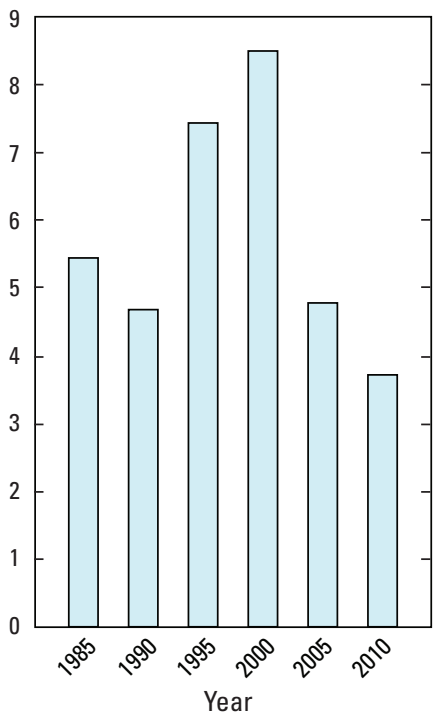




\section{BIBB COUNTY}

Population

Population served by public supply-Groundwater $\quad 1,770$

Population served by public supply-Surface water 124,900

Acres irrigated

347

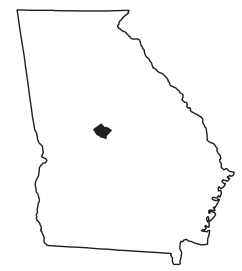

2010 WATER WITHDRAWALS AND ESTIMATED USE, IN MILLION GALLONS PER DAY

[-, not applicable; Mgal/d, million gallons per day]

\begin{tabular}{|c|c|c|c|c|c|c|c|c|}
\hline \multirow{3}{*}{ Category } & \multicolumn{3}{|c|}{ Withdrawals } & \multirow{3}{*}{$\begin{array}{l}\text { Total } \\
\text { use }^{1}\end{array}$} & \multirow{3}{*}{$\begin{array}{l}\text { Surface- } \\
\text { water } \\
\text { returns } \\
\end{array}$} & \multirow{2}{*}{\multicolumn{3}{|c|}{$\begin{array}{l}\text { Withdrawals by Major Industrial Groups } \\
\text { [NAICS, North American Industrial Classification System code] }\end{array}$}} \\
\hline & \multirow{2}{*}{$\begin{array}{c}\text { Ground- } \\
\text { water }\end{array}$} & \multirow{2}{*}{$\begin{array}{c}\text { Surface } \\
\text { water }\end{array}$} & \multirow[b]{2}{*}{ Total } & & & & & \\
\hline & & & & & & NAICS & Groundwater & Surface water \\
\hline Public supply & 0.14 & 23.46 & 23.60 & - & - & 322-Paper, pulp & 0.41 & 12.89 \\
\hline Domestic & 2.17 & 0.00 & 2.17 & 15.90 & 0.00 & 321 -Wood & & \\
\hline Commercial/public use & 0.00 & 0.00 & 0.00 & 6.59 & 0.00 & 321 -Wood & 1.08 & 0.00 \\
\hline Industrial & 1.72 & 12.89 & 14.61 & 16.50 & 2.86 & 326-Rubber, plastics & 0.23 & 0.00 \\
\hline
\end{tabular}

Public-supply losses

$\begin{array}{lllll}- & - & - & & 3.84\end{array}$

Public wastewater treatment

$-$

Mining

$$
0.00
$$

0.33

0.21

0.01

0.00

4.58

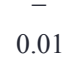

0.03

0.08

\subsection{1}

0.36

0.29

0.03

0.04

0.00

0.00

36.50

41.08 $\begin{array}{cr}3.84 & - \\ - & 33.28\end{array}$

0.01

0.36

0.29

0.04

0.00

43.53
33.28
0.65

0.00

0.00

0.00

0.00

36.79

${ }^{1}$ Total use is total withdrawal plus public supply deliveries and losses. An average of $0.23 \mathrm{Mgal} / \mathrm{d}$ was delivered from Bibb County to Monroe County, Georgia in 2010

\section{Withdrawals by Source}

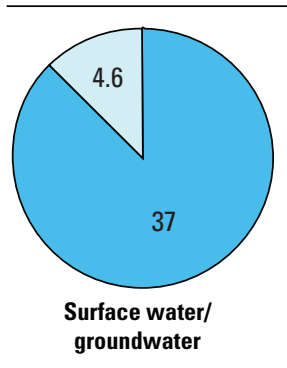

Withdrawals, in million gallons per day
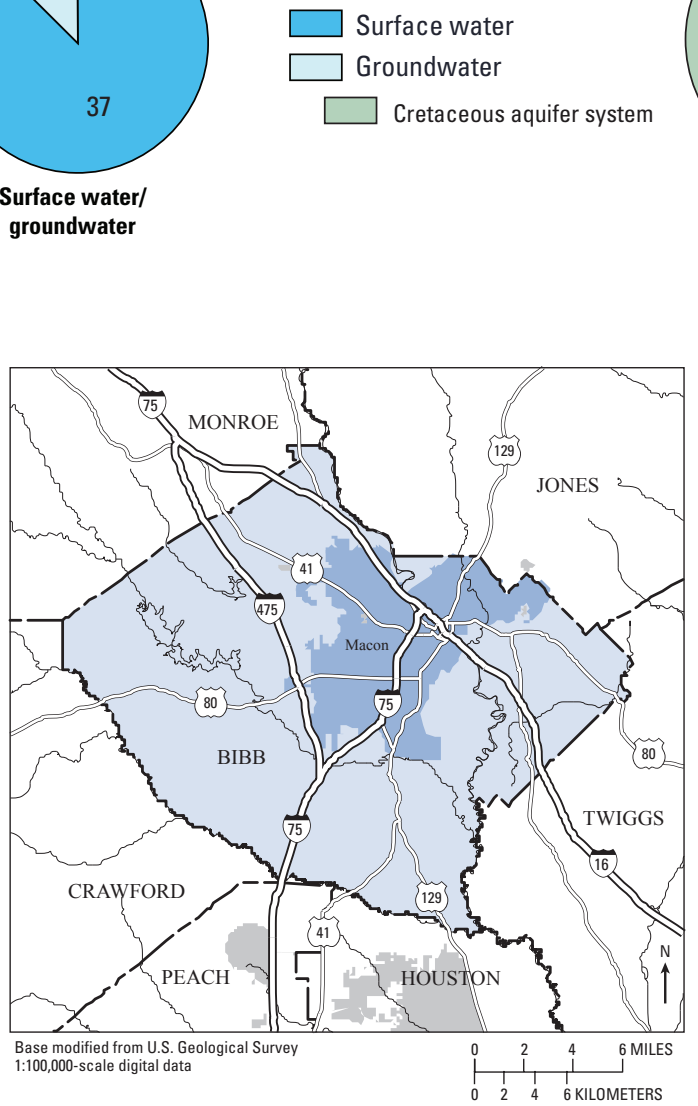

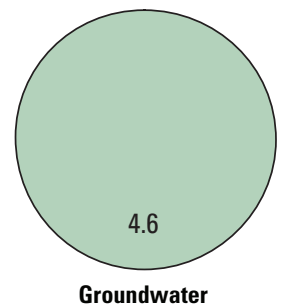

Public-Supply Deliveries' by Use Category

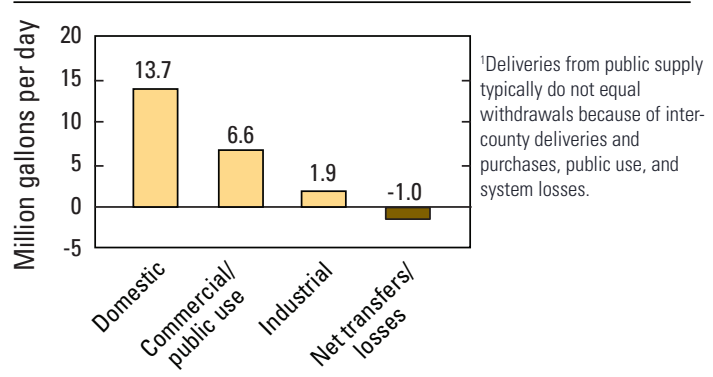

Withdrawals by Major Public Suppliers

[Mgal/d, million gallons per day]

\begin{tabular}{lcc}
\hline Name & Groundwater & Surface water \\
\hline Macon Water Authority & 0.00 & 123.46 \\
\hline $\begin{array}{l}\text { 'An average of } 3.1 \mathrm{Mgal} / \mathrm{d} \text { was withdrawn in Jones County, Georgia and delivered to Bibb } \\
\text { County, Georgia in 2010. }\end{array}$
\end{tabular}




\section{BLECKLEY COUNTY}

Population

13,063

$\begin{array}{lr}\text { Population served by public supply-Groundwater } & 5,220 \\ \text { Population served by public supply-Surface water } & 0\end{array}$ Acres irrigated

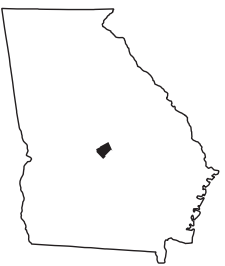

2010 WATER WITHDRAWALS AND ESTIMATED USE, IN MILLION GALLONS PER DAY $[-$, not applicable $]$

\begin{tabular}{lccccc}
\hline & \multicolumn{3}{c}{ Withdrawals } & & Turface- \\
\cline { 2 - 4 } Category & $\begin{array}{c}\text { Ground- } \\
\text { water }\end{array}$ & $\begin{array}{c}\text { Surface } \\
\text { water }\end{array}$ & Total & $\begin{array}{c}\text { Total } \\
\text { use }\end{array}$ & $\begin{array}{c}\text { water } \\
\text { returns }\end{array}$ \\
\hline Public supply & 0.22 & 0.00 & 0.22 & - & - \\
Domestic & 0.59 & 0.00 & 0.59 & 0.70 & 0.00 \\
Commercial/public use & 0.12 & 0.00 & 0.12 & 0.18 & 0.00 \\
Industrial & 0.00 & 0.00 & 0.00 & 0.00 & 0.00 \\
Public-supply losses & - & - & - & 0.05 & - \\
Public wastewater treatment & - & - & - & - & 0.33 \\
Mining & 0.00 & 0.00 & 0.00 & 0.00 & 0.00 \\
Irrigation-Crop & 4.58 & 1.23 & 5.81 & 5.81 & 0.00 \\
Irrigation-Golf course & 0.25 & 0.26 & 0.51 & 0.51 & 0.00 \\
Livestock/aquaculture & 0.02 & 0.06 & 0.08 & 0.08 & 0.00 \\
Thermoelectric power & 0.00 & 0.00 & 0.00 & 0.00 & 0.00 \\
\multicolumn{1}{c}{ TOTAL } & $\mathbf{5 . 7 8}$ & $\mathbf{1 . 5 5}$ & $\mathbf{7 . 3 3}$ & $\mathbf{7 . 3 3}$ & $\mathbf{0 . 3 3}$ \\
\hline
\end{tabular}

Withdrawals by Major Industrial Groups

[NAICS, North American Industrial Classification System code]

NAICS Groundwater Surface water

None

Withdrawals by Major Public Suppliers

Name Groundwater Surface water

\begin{tabular}{lll}
\hline City of Cochran & 0.22 & 0.00
\end{tabular}

${ }^{1}$ Total use is total withdrawal plus public supply deliveries and losses.

\section{Withdrawals by Source}
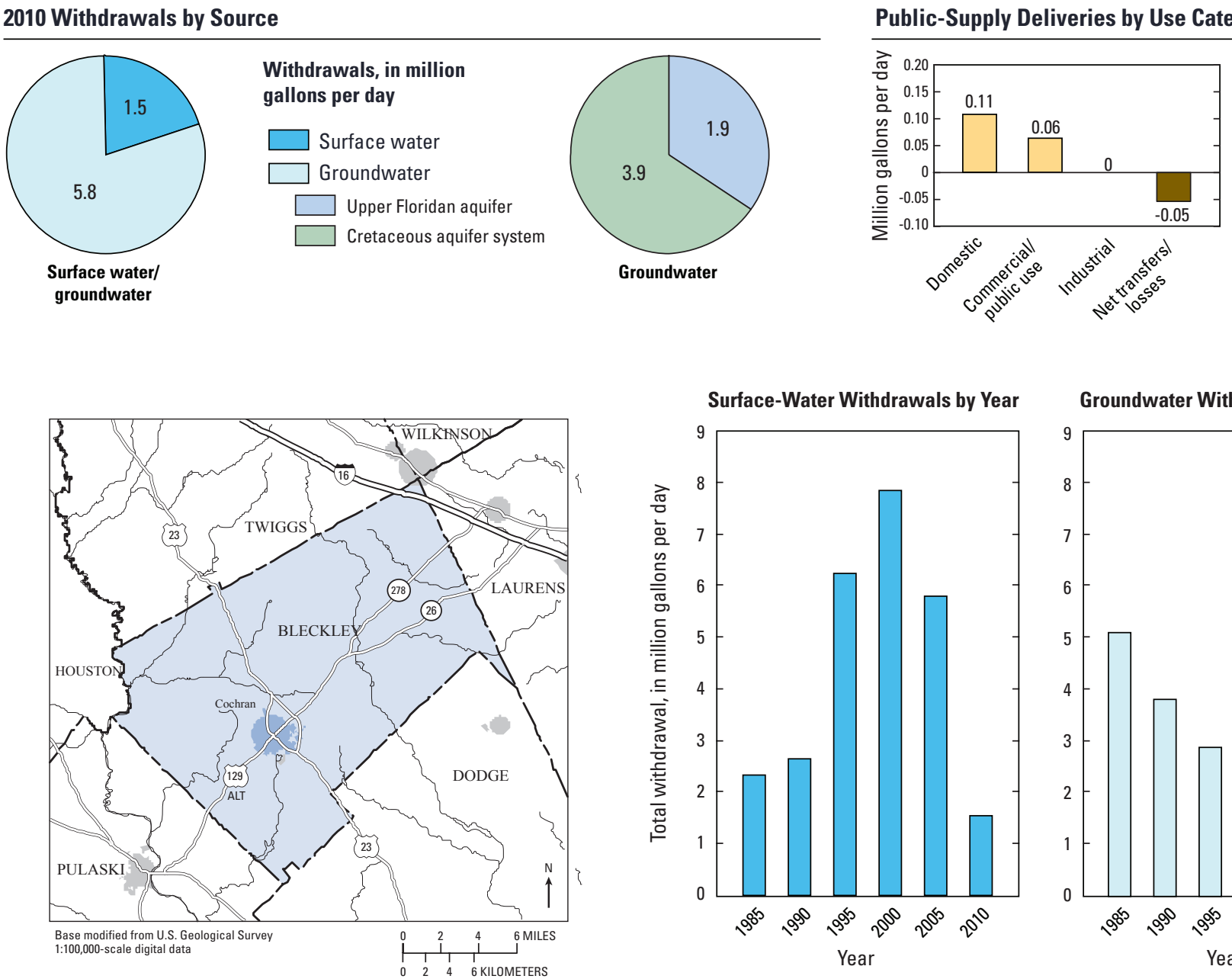

Surface-Water Withdrawals by Year

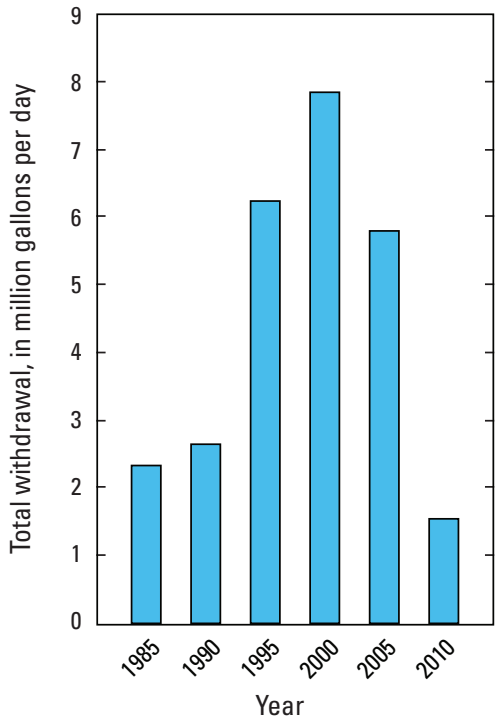

Groundwater Withdrawals by Year

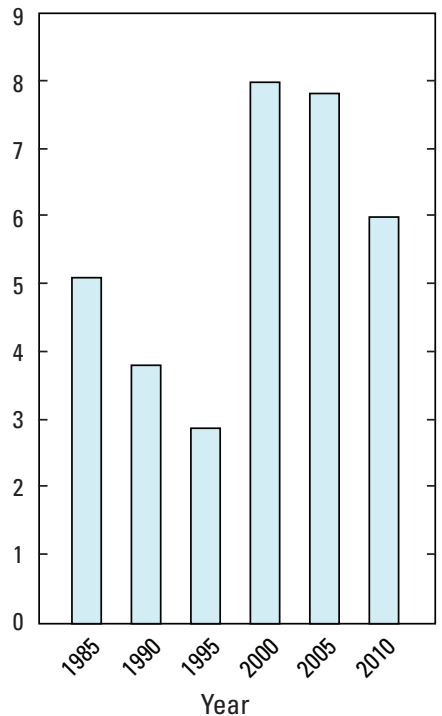




\section{BRANTLEY COUNTY}

Population

18,411

Population served by public supply-Groundwater

Population served by public supply-Surface water

Acres irrigated

720

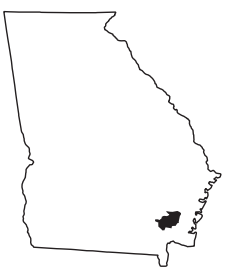

2010 WATER WITHDRAWALS AND ESTIMATED USE, IN MILLION GALLONS PER DAY

$[-$, not applicable $]$

\begin{tabular}{|c|c|c|c|c|c|}
\hline \multirow[b]{2}{*}{ Category } & \multicolumn{3}{|c|}{ Withdrawals } & \multirow[b]{2}{*}{$\begin{array}{l}\text { Total } \\
\text { use }^{1}\end{array}$} & \multirow{2}{*}{$\begin{array}{r}\text { Surface- } \\
\text { water } \\
\text { returns }\end{array}$} \\
\hline & $\begin{array}{c}\text { Ground- } \\
\text { water }\end{array}$ & $\begin{array}{c}\text { Surface } \\
\text { water }\end{array}$ & Total & & \\
\hline Public supply & 0.25 & 0.00 & 0.25 & - & - \\
\hline Domestic & 1.20 & 0.00 & 1.20 & 1.38 & 0.00 \\
\hline Commercial/public use & 0.00 & 0.00 & 0.00 & 0.05 & 0.00 \\
\hline Industrial & 0.00 & 0.00 & 0.00 & 0.00 & 0.00 \\
\hline Public-supply losses & - & - & - & 0.02 & - \\
\hline Public wastewater treatment & - & - & - & - & 0.00 \\
\hline Mining & 0.00 & 0.00 & 0.00 & 0.00 & 0.00 \\
\hline Irrigation-Crop & 0.00 & 0.03 & 0.03 & 0.03 & 0.00 \\
\hline Irrigation-Golf course & 0.00 & 0.00 & 0.00 & 0.00 & 0.00 \\
\hline Livestock/aquaculture & 0.01 & 0.03 & 0.04 & 0.04 & 0.00 \\
\hline Thermoelectric power & 0.00 & 0.00 & 0.00 & 0.00 & 0.00 \\
\hline TOTAL & 1.46 & 0.06 & 1.52 & 1.52 & 0.00 \\
\hline
\end{tabular}

Withdrawals by Major Industrial Groups

[NAICS, North American Industrial Classification System code]

NAICS Groundwater Surface water

None

Withdrawals by Major Public Suppliers

\begin{tabular}{lcc} 
Name & Groundwater & Surface water \\
\hline City of Nahunta & 0.14 & 0.00 \\
\hline
\end{tabular}

${ }^{1}$ Total use is total withdrawal plus public supply deliveries and losses.

\section{Withdrawals by Source}

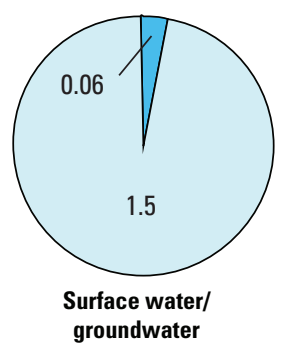

Withdrawals, in million gallons per day

$\square$ Surface water

$\square$ Groundwater

$\square$ Upper Floridan aquifer groundwater

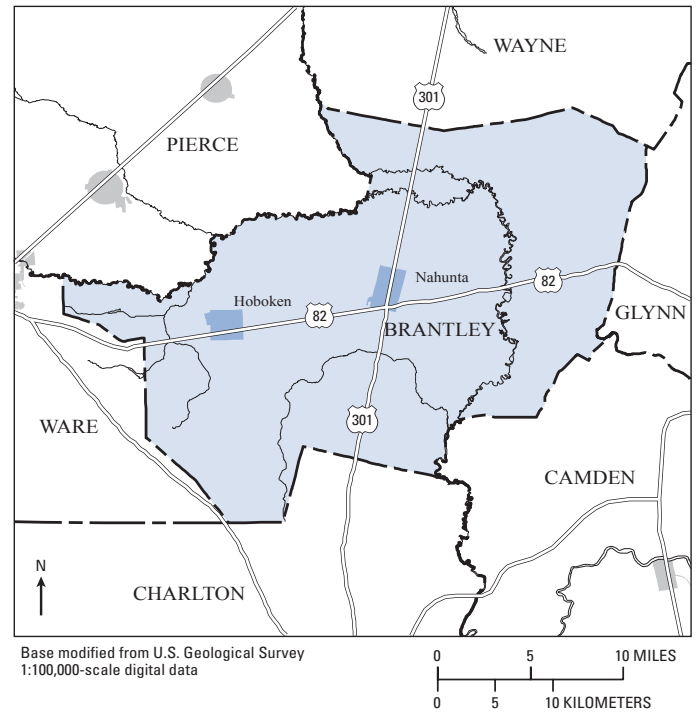

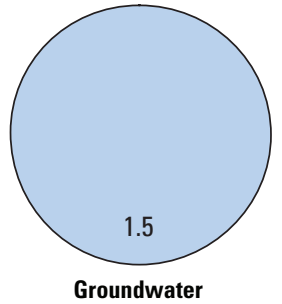

Public-Supply Deliveries by Use Category

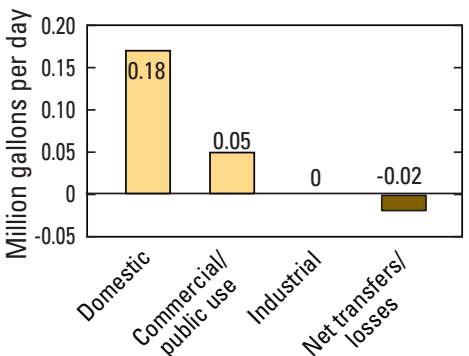

Surface-Water Withdrawals by Year

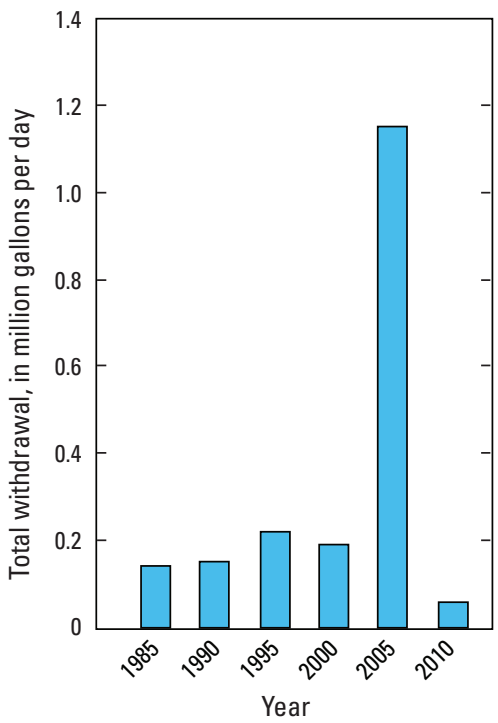

Groundwater Withdrawals by Year

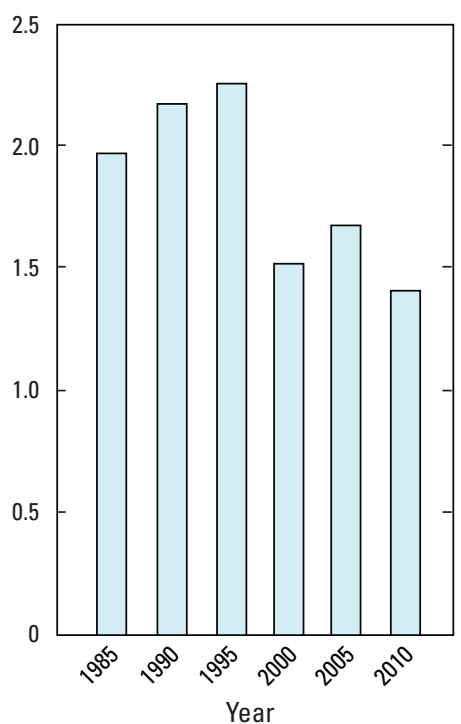




\section{BROOKS COUNTY}

Population

16,243

Population served by public supply-Groundwater Population served by public supply-Surface water Acres irrigated
6,670

0

15,480

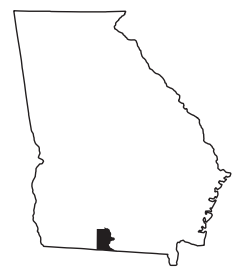

2010 WATER WITHDRAWALS AND ESTIMATED USE, IN MILLION GALLONS PER DAY $[-$, not applicable $]$

\begin{tabular}{|c|c|c|c|c|c|}
\hline \multirow[b]{2}{*}{ Category } & \multicolumn{3}{|c|}{ Withdrawals } & \multirow[b]{2}{*}{$\begin{array}{l}\text { Total } \\
\text { use }^{1}\end{array}$} & \multirow{2}{*}{$\begin{array}{c}\text { Surface- } \\
\text { water } \\
\text { returns }\end{array}$} \\
\hline & $\begin{array}{c}\text { Ground- } \\
\text { water }\end{array}$ & $\begin{array}{c}\text { Surface } \\
\text { water }\end{array}$ & Total & & \\
\hline Public supply & 0.94 & 0.00 & 0.94 & - & - \\
\hline Domestic & 0.72 & 0.00 & 0.72 & 1.33 & 0.00 \\
\hline Commercial/public use & 0.01 & 0.00 & 0.01 & 0.22 & 0.00 \\
\hline Industrial & 0.06 & 0.00 & 0.06 & 0.07 & 0.00 \\
\hline Public-supply losses & - & - & - & 0.11 & - \\
\hline Public wastewater treatment & - & - & - & - & 0.00 \\
\hline Mining & 0.00 & 0.00 & 0.00 & 0.00 & 0.00 \\
\hline Irrigation-Crop & 5.53 & 0.08 & 5.61 & 5.61 & 0.00 \\
\hline Irrigation-Golf course & 0.00 & 0.00 & 0.00 & 0.00 & 0.00 \\
\hline Livestock/aquaculture & 0.08 & 0.34 & 0.42 & 0.42 & 0.00 \\
\hline Thermoelectric power & 0.00 & 0.00 & 0.00 & 0.00 & 0.00 \\
\hline TOTAL & 7.34 & 0.42 & 7.76 & 7.76 & 0.00 \\
\hline
\end{tabular}

Withdrawals by Major Industrial Groups

[NAICS, North American Industrial Classification System code] NAICS Groundwater Surface water

321-Wood $0.06 \quad 0.00$

Withdrawals by Major Public Suppliers

Name Groundwater Surface water

\begin{tabular}{lll}
\hline City of Quitman & 0.73 & 0.00
\end{tabular}

'Total use is total withdrawal plus public supply deliveries and losses.

\section{Withdrawals by Source}
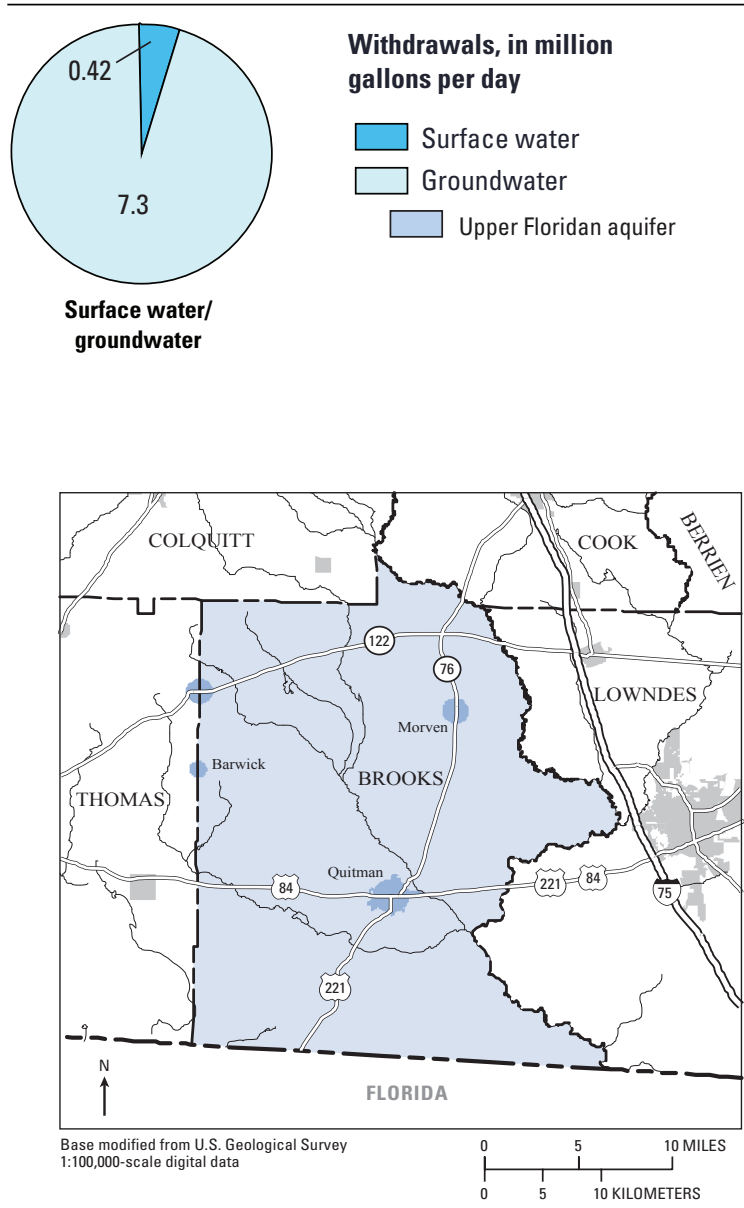

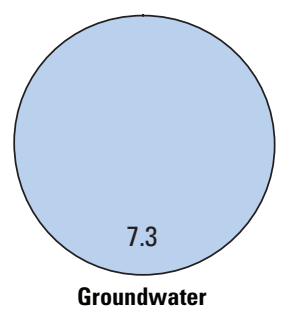

Public-Supply Deliveries by Use Category

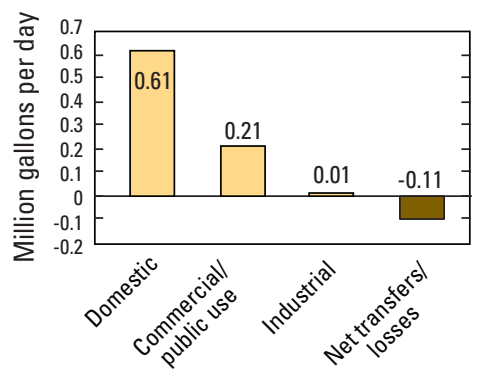

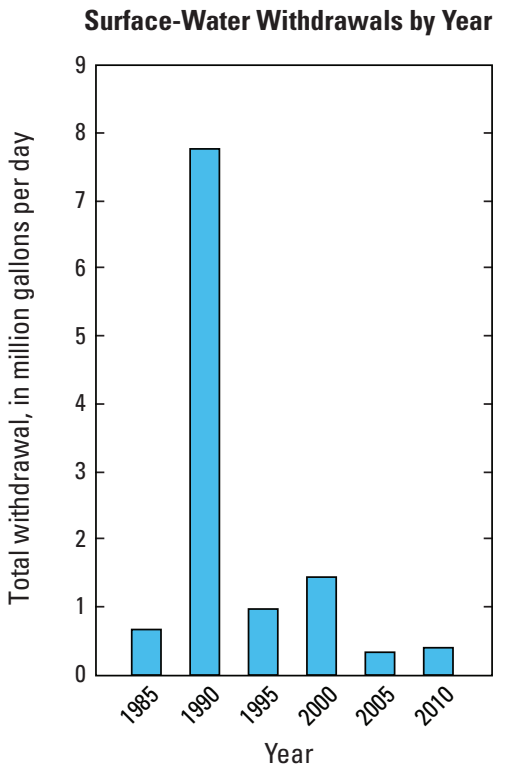

Groundwater Withdrawals by Year

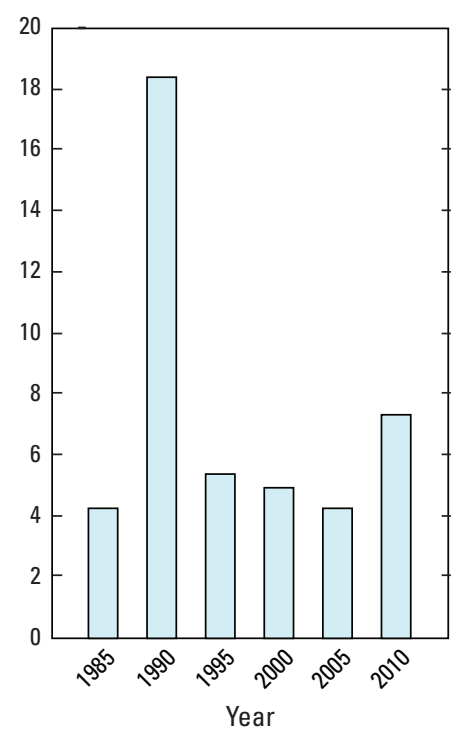




\section{BRYAN COUNTY}

Population

30,233

Population served by public supply-Groundwater

Population served by public supply-Surface water

27,380

Acres irrigated

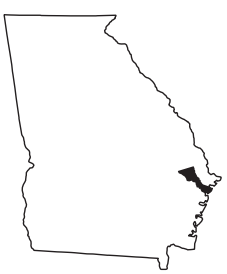

2010 WATER WITHDRAWALS AND ESTIMATED USE, IN MILLION GALLONS PER DAY

$[-$, not applicable $]$

\begin{tabular}{|c|c|c|c|c|c|c|c|c|}
\hline \multirow{3}{*}{ Category } & \multicolumn{3}{|c|}{ Withdrawals } & \multirow{3}{*}{$\begin{array}{l}\text { Total } \\
\text { use }^{1}\end{array}$} & \multirow{3}{*}{$\begin{array}{l}\text { Surface- } \\
\text { water } \\
\text { returns } \\
\end{array}$} & \multirow{2}{*}{\multicolumn{3}{|c|}{$\begin{array}{l}\text { Withdrawals by Major Industrial Groups } \\
\text { [NAICS, North American Industrial Classification System code] }\end{array}$}} \\
\hline & \multirow{2}{*}{$\begin{array}{c}\text { Ground- } \\
\text { water }\end{array}$} & \multirow{2}{*}{$\begin{array}{c}\text { Surface } \\
\text { water }\end{array}$} & \multirow[b]{2}{*}{ Total } & & & & & \\
\hline & & & & & & NAICS G & Groundwater & Surface water \\
\hline Public supply & 2.57 & 0.00 & 2.57 & - & - & 339_-Misc manufacturing & 0.02 & 0.00 \\
\hline Domestic & 0.21 & 0.00 & 0.21 & 2.52 & 0.00 & & & \\
\hline Commercial/public use & 0.00 & 0.00 & 0.00 & 0.16 & 0.00 & & & \\
\hline Industrial & 0.02 & 0.00 & 0.02 & 0.02 & 0.00 & & & \\
\hline Public-supply losses & - & - & - & 0.10 & - & & & \\
\hline Public wastewater treatment & - & - & - & - & 0.97 & Withdrawals by Major $\mathrm{P}$ & Public Supp & \\
\hline $\begin{array}{l}\text { Mining } \\
\text { Irrigation-Crop }\end{array}$ & $\begin{array}{l}0.00 \\
0.04\end{array}$ & $\begin{array}{l}0.00 \\
0.03\end{array}$ & $\begin{array}{l}0.00 \\
0.07\end{array}$ & $\begin{array}{l}0.00 \\
0.07\end{array}$ & $\begin{array}{l}0.00 \\
0.00\end{array}$ & Name G & Groundwater & Surface water \\
\hline Irrigation-Golf course & 0.01 & 0.00 & 0.01 & 0.01 & 0.00 & City of Richmond Hill & 1.41 & 0.00 \\
\hline Livestock/aquaculture & 0.03 & 0.34 & 0.37 & 0.37 & 0.00 & Water Utility Management & 0.40 & 0.00 \\
\hline Thermoelectric power & 0.00 & 0.00 & 0.00 & 0.00 & 0.00 & Middle Georgia Water Co. & 0.25 & 0.00 \\
\hline TOTAL & 2.88 & 0.37 & 3.25 & 3.25 & 0.97 & City of Pembroke & 0.22 & 0.00 \\
\hline
\end{tabular}

${ }^{1}$ Total use is total withdrawal plus public supply deliveries and losses.

\section{Withdrawals by Source}
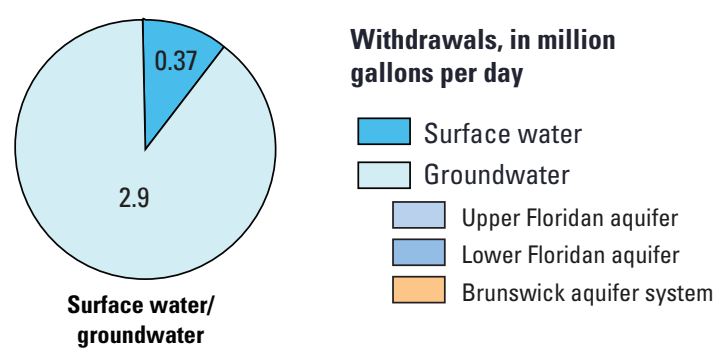
groundwater

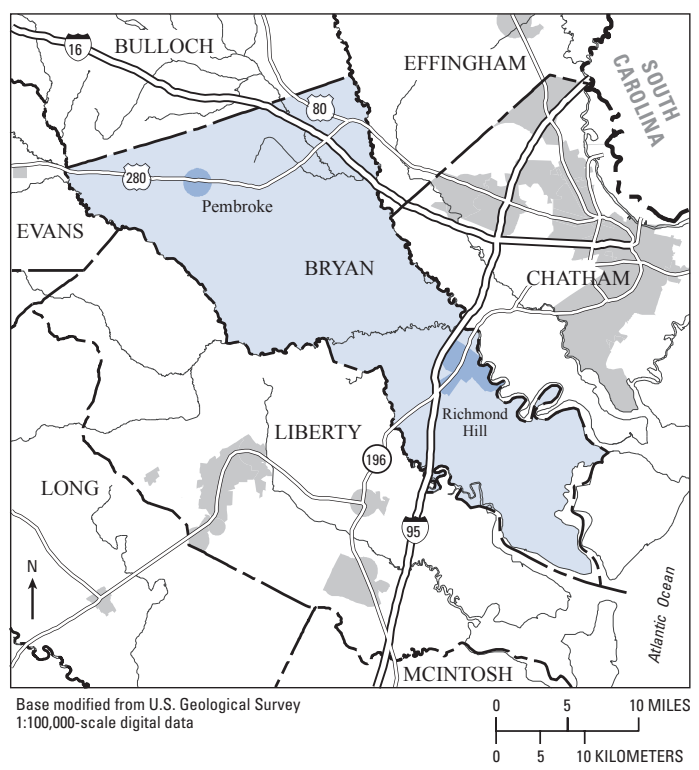

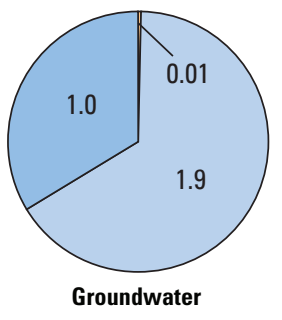

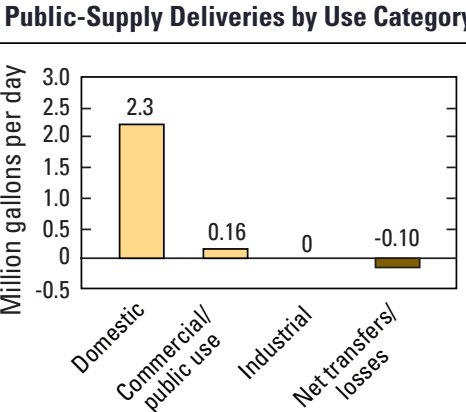

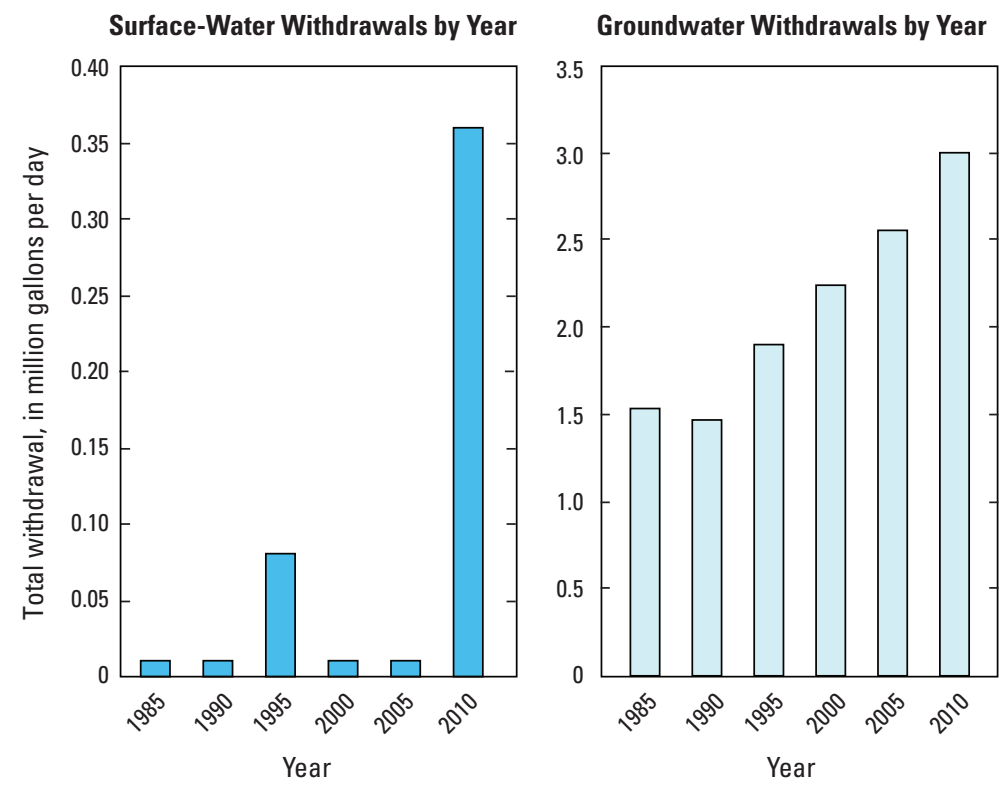




\section{BULLOCH COUNTY}

Population

70,217

Population served by public supply-Groundwater Population served by public supply-Surface water Acres irrigated
49,980

0

15,970

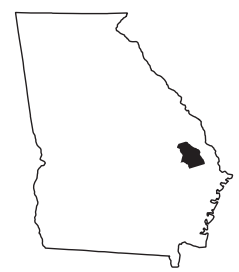

2010 WATER WITHDRAWALS AND ESTIMATED USE, IN MILLION GALLONS PER DAY $[-$, not applicable $]$

\begin{tabular}{|c|c|c|c|c|c|c|c|c|}
\hline \multirow{3}{*}{ Category } & \multicolumn{3}{|c|}{ Withdrawals } & \multirow{3}{*}{$\begin{array}{l}\text { Total } \\
\text { use }^{1}\end{array}$} & \multirow{3}{*}{$\begin{array}{l}\text { Surface- } \\
\text { water } \\
\text { returns } \\
\end{array}$} & \multirow{2}{*}{\multicolumn{3}{|c|}{$\begin{array}{l}\text { Withdrawals by Major Industrial Groups } \\
\text { [NAICS, North American Industrial Classification System code] }\end{array}$}} \\
\hline & \multirow{2}{*}{$\begin{array}{c}\text { Ground- } \\
\text { water }\end{array}$} & \multirow{2}{*}{$\begin{array}{c}\text { Surface } \\
\text { water }\end{array}$} & \multirow[b]{2}{*}{ Total } & & & & & \\
\hline & & & & & & NAICS & Groundwater & Surface water \\
\hline Public supply & 4.89 & 0.00 & 4.89 & - & - & None & - & - \\
\hline Domestic & 1.52 & 0.00 & 1.52 & 4.60 & 0.00 & None & - & - \\
\hline Commercial/public use & 0.30 & 0.00 & 0.30 & 1.37 & 0.00 & & & \\
\hline Industrial & 0.00 & 0.00 & 0.00 & 0.20 & 0.00 & & & \\
\hline Public-supply losses & - & - & - & 0.54 & - & & & \\
\hline Public wastewater treatment & - & - & - & - & 3.92 & Withdrawals by & r Public Supp & \\
\hline Mining & 0.00 & 0.00 & 0.00 & 0.00 & 0.00 & Name & Groundwater & Surface water \\
\hline Irrigation - Crop & 0.62 & 1.45 & 2.07 & 2.07 & 0.00 & City of Statesboro & 3.27 & 0.00 \\
\hline Irrigation-Golf course & 0.18 & 0.00 & 0.18 & 0.18 & 0.00 & City of Brooklet & & \\
\hline Livestock/aquaculture & 0.03 & 0.24 & 0.27 & 0.27 & 0.00 & City of Brooklet & 0.18 & 0.00 \\
\hline
\end{tabular}

Thermoelectric power

$\begin{array}{lllll}0.00 & 0.00 & 0.00 & 0.00 & 0.00 \\ \mathbf{7 . 5 4} & \mathbf{1 . 6 9} & \mathbf{9 . 2 3} & \mathbf{9 . 2 3} & \mathbf{3 . 9 2}\end{array}$

TOTAL

7.54

1.69

3.92

'Total use is total withdrawal plus public supply deliveries and losses.

\section{Withdrawals by Source}
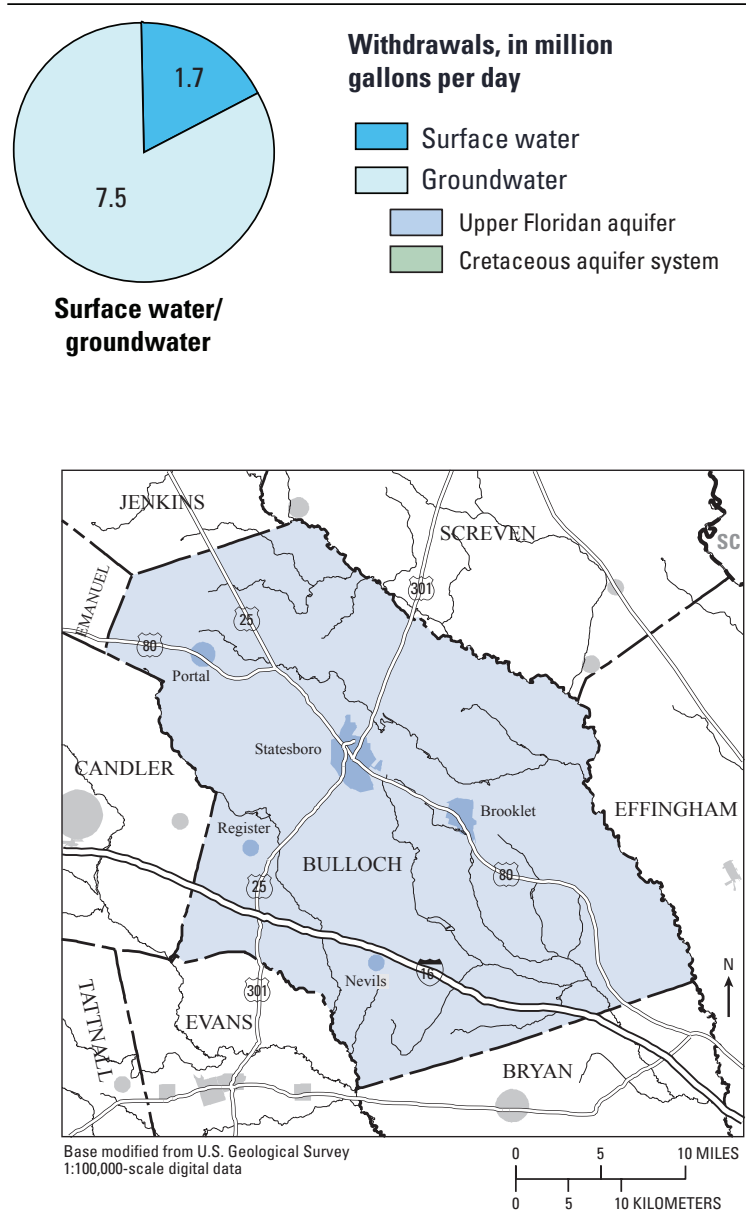
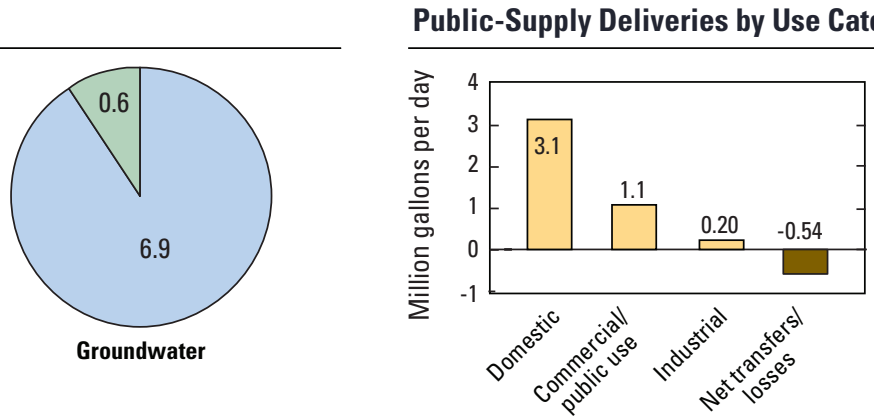

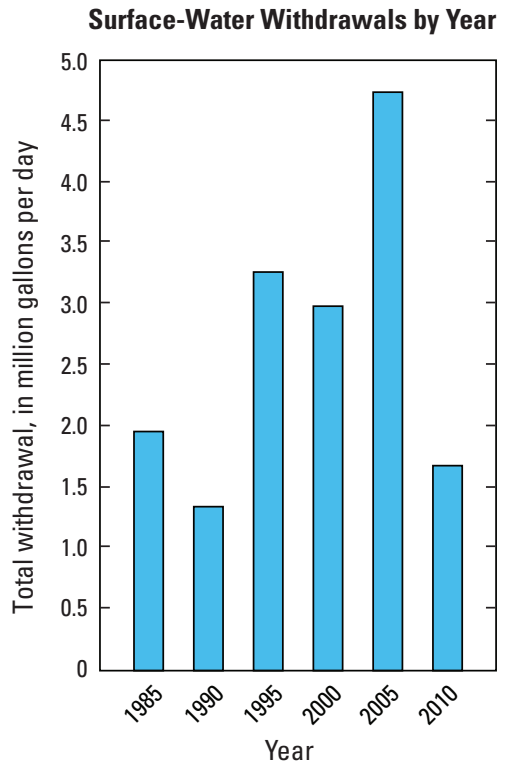

Groundwater Withdrawals by Year

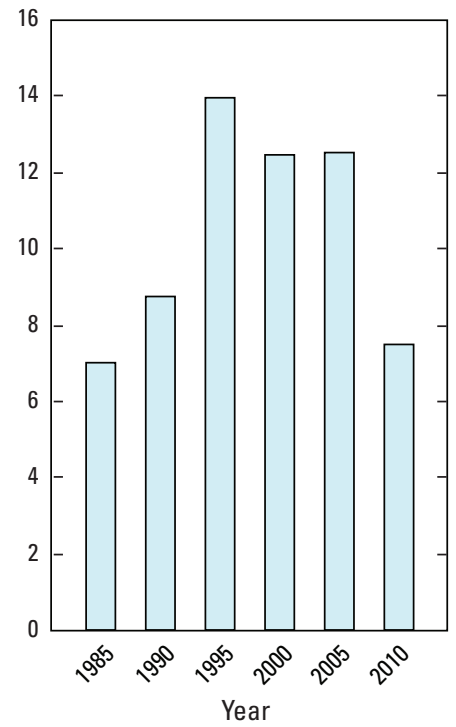




\section{BURKE COUNTY}

Population

$\begin{array}{lr}\text { Population served by public supply-Groundwater } & 8,990 \\ \text { Population served by public supply-Surface water } & 370\end{array}$

Acres irrigated

23,230

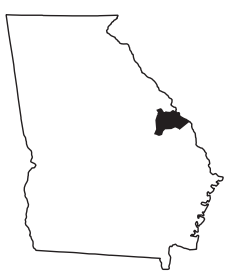

2010 WATER WITHDRAWALS AND ESTIMATED USE, IN MILLION GALLONS PER DAY

$[-$, not applicable $]$

\begin{tabular}{|c|c|c|c|c|c|c|c|c|}
\hline \multirow{3}{*}{ Category } & \multicolumn{3}{|c|}{ Withdrawals } & \multirow{3}{*}{$\begin{array}{l}\text { Total } \\
\text { use }^{1}\end{array}$} & \multirow{3}{*}{$\begin{array}{c}\text { Surface- } \\
\text { water } \\
\text { returns } \\
\end{array}$} & \multirow{2}{*}{\multicolumn{3}{|c|}{$\begin{array}{l}\text { Withdrawals by Major Industrial Groups } \\
\text { [NAICS, North American Industrial Classification System code] }\end{array}$}} \\
\hline & \multirow{2}{*}{$\begin{array}{c}\text { Ground- } \\
\text { water }\end{array}$} & \multirow{2}{*}{$\begin{array}{c}\text { Surface } \\
\text { water }\end{array}$} & \multirow[b]{2}{*}{ Total } & & & & & \\
\hline & & & & & & NAICS & Groundwater & Surface water \\
\hline Public supply & 1.01 & 0.05 & 1.06 & - & & 322-Paper, pulp & 0.23 & 0.00 \\
\hline Domestic & 1.05 & 0.00 & 1.05 & 1.67 & 0.00 & & & \\
\hline Commercial/public use & 0.01 & 0.00 & 0.01 & 0.11 & 0.00 & & & \\
\hline Industrial & 0.23 & 0.00 & 0.23 & 0.37 & 0.00 & & & \\
\hline Public-supply losses & - & - & - & 0.20 & - & & & \\
\hline Public wastewater treatment & - & - & - & - & 1.04 & Withdrawals by M & Public Supp & \\
\hline Mining & 0.00 & 0.00 & 0.00 & 0.00 & 0.00 & Name & Groundwater & Surface water \\
\hline Irrigation-Crop & 6.84 & 3.22 & 10.06 & 10.06 & 0.00 & & & \\
\hline Irrigation-Golf course & 0.00 & 0.00 & 0.00 & 0.00 & 0.00 & City of Waynesboro & 0.82 & 0.05 \\
\hline Livestock/aquaculture & 0.06 & 0.23 & 0.29 & 0.29 & 0.00 & & & \\
\hline Thermoelectric power & 1.06 & 64.35 & 65.41 & 65.41 & 0.00 & & & \\
\hline TOTAL & 10.26 & 67.85 & 78.11 & 78.11 & 1.04 & & & \\
\hline
\end{tabular}

'Total use is total withdrawal plus public supply deliveries and losses.

\section{Withdrawals by Source}

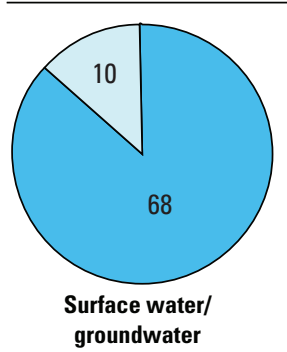

Withdrawals, in million gallons per day

$\square$ Surface water
$\square$ Groundwater
$\square$ Floridan aquifer system
$\square$ Cretaceous aquifer system

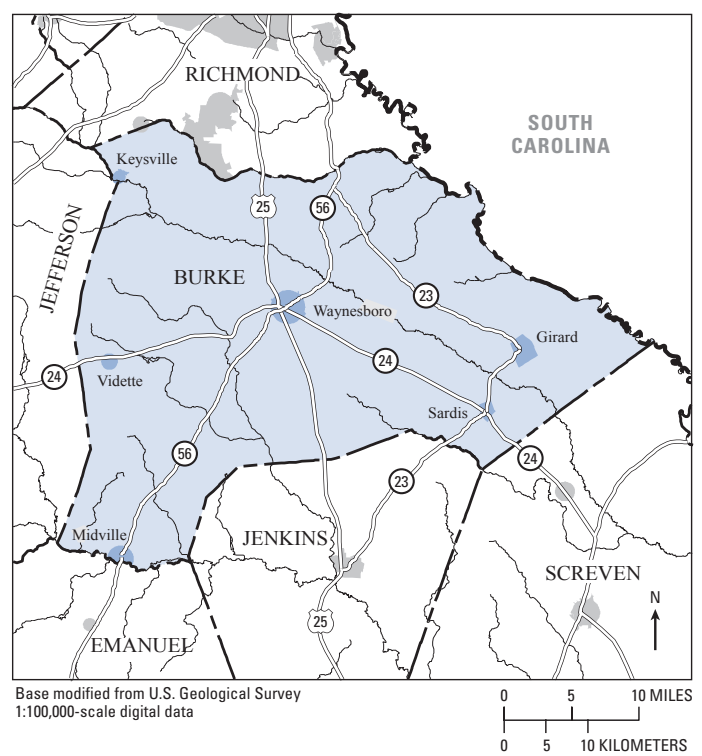

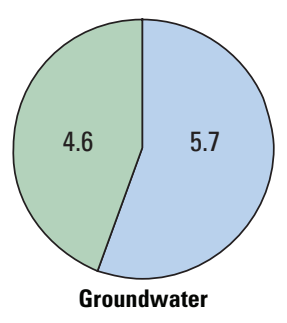

Public-Supply Deliveries by Use Category

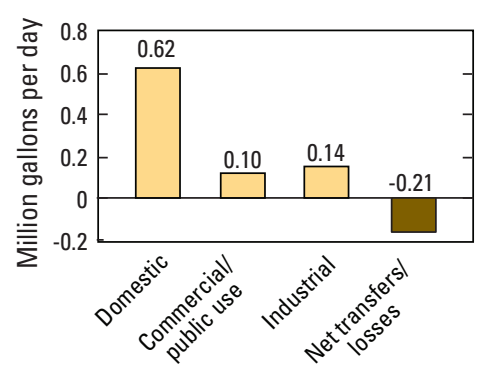

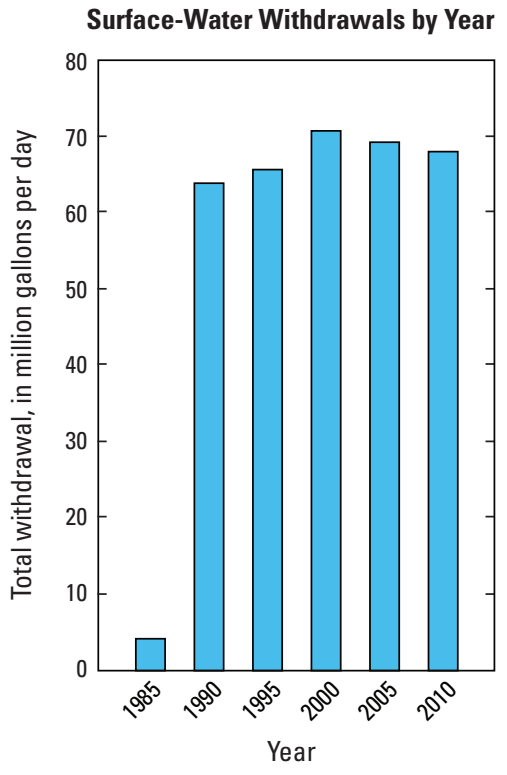

Groundwater Withdrawals by Year

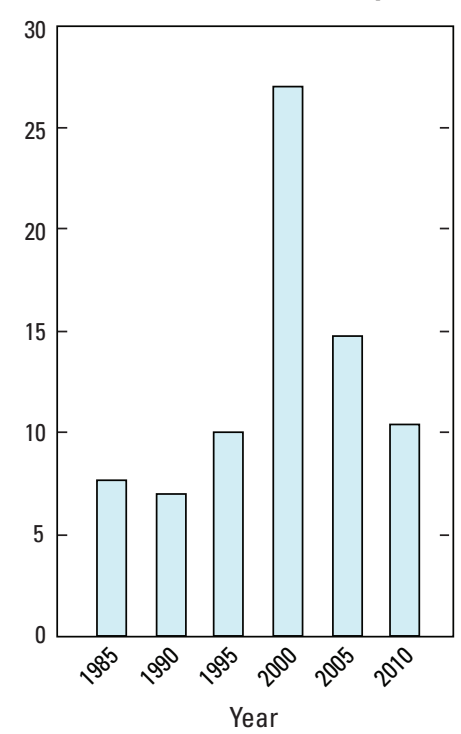




\section{BUTTS COUNTY}

Population

23,655

1,780

19,030

110

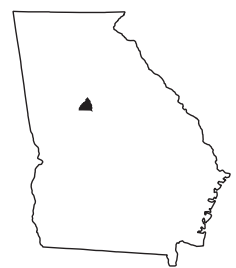

2010 WATER WITHDRAWALS AND ESTIMATED USE, IN MILLION GALLONS PER DAY

$[-$, not applicable; Mgal/d, million gallons per day $]$

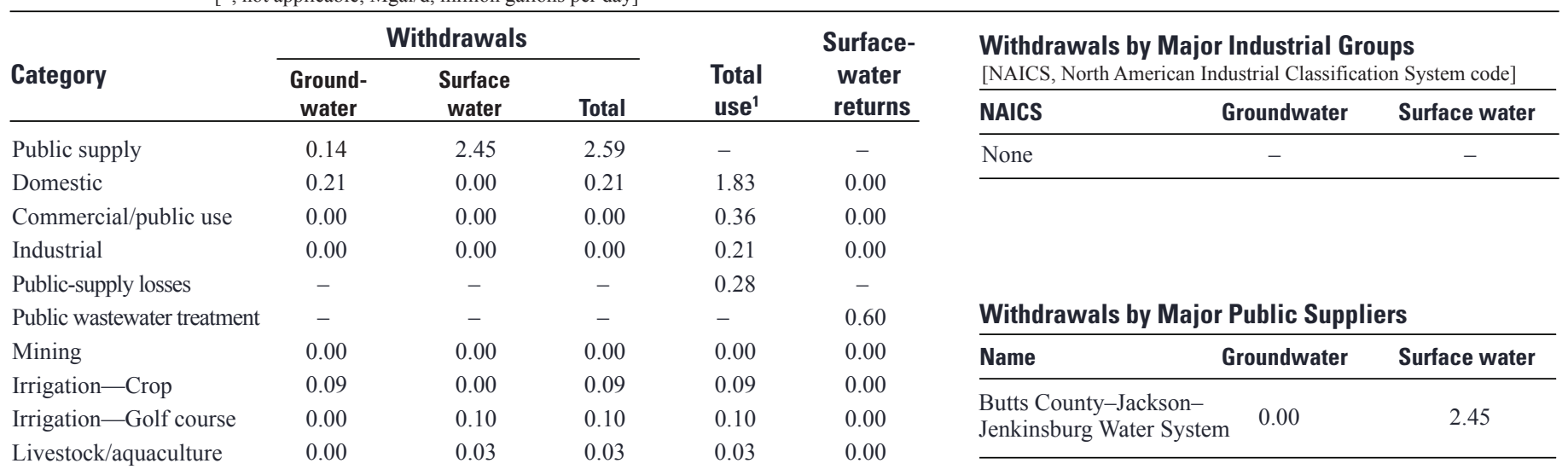

$\begin{array}{llllll}\text { Thermoelectric power } & 0.00 & 0.00 & 0.00 & 0.00 & 0.00\end{array}$

TOTAL

0.44

2.58

3.02

2.90

0.60

'Total use is total withdrawal plus public supply deliveries and losses. An average of $0.01 \mathrm{Mgal} / \mathrm{d}$ was delivered to Butts County, Georgia from

Spalding County, Georgia and about $0.13 \mathrm{Mgal} / \mathrm{d}$ delivered from Butts County to Monroe County, Georgia in 2010.

\section{Withdrawals by Source}

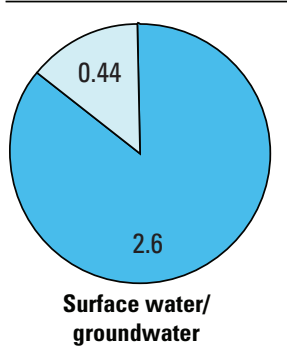

Withdrawals, in million gallons per day

$\square$ Surface water
$\square$ Groundwater

$\square$ Cretaceous aquifer system

groundwath

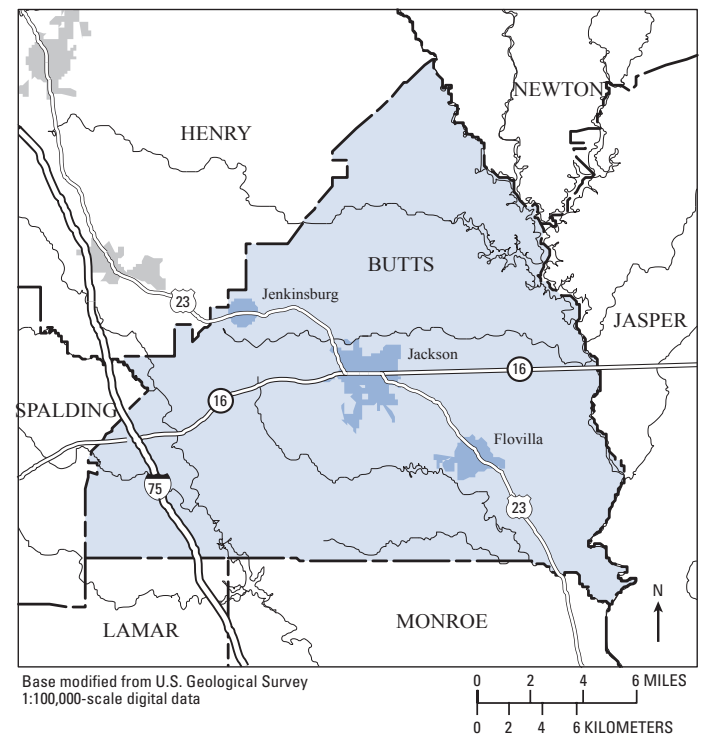

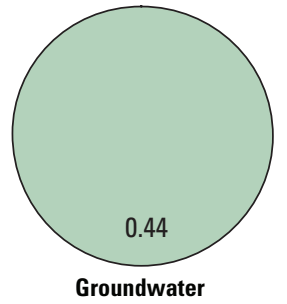

Public-Supply Deliveries' by Use Category

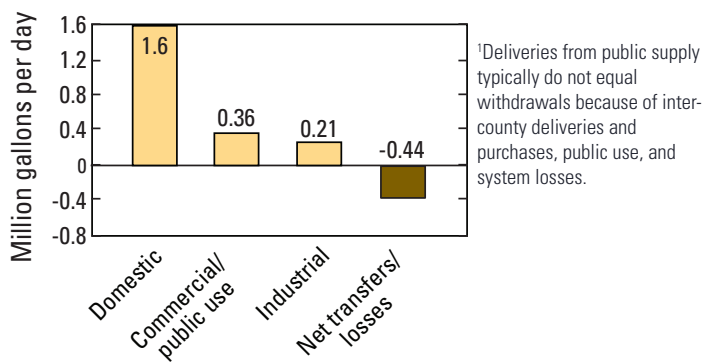

Surface-Water Withdrawals by Year

Groundwater Withdrawals by Year
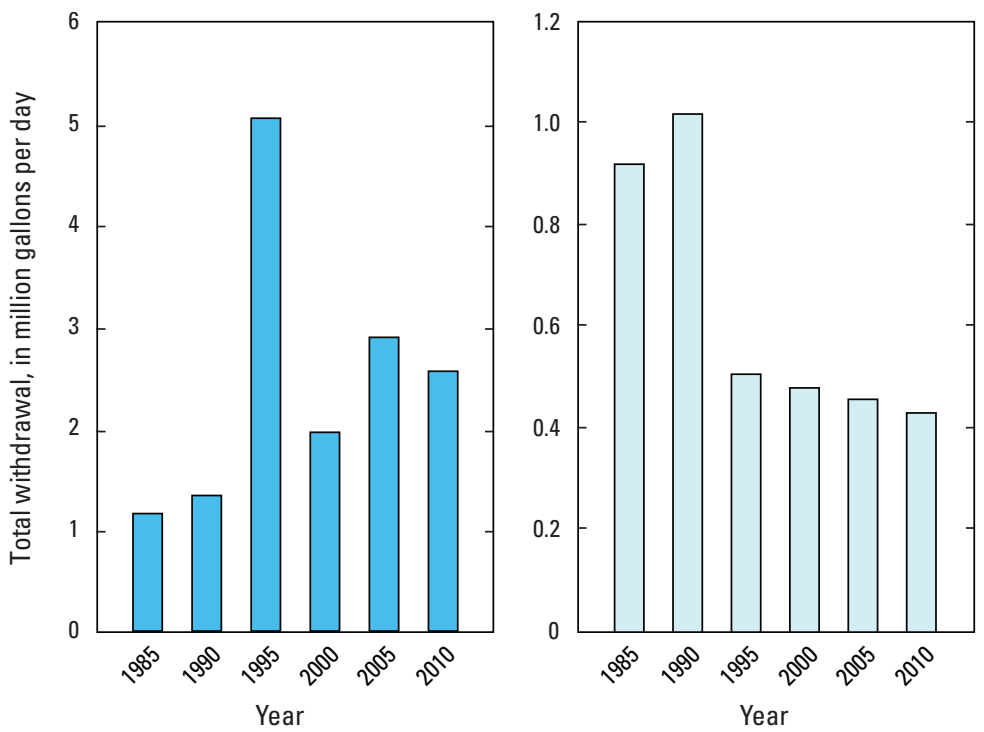


\section{CALHOUN COUNTY}

Population

Population served by public supply_-Groundwater

Population served by public supply-Surface water Acres irrigated

30,170

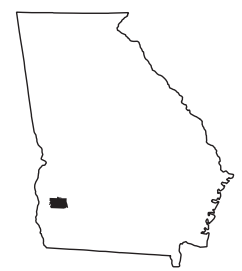

2010 WATER WITHDRAWALS AND ESTIMATED USE, IN MILLION GALLONS PER DAY

$[-$, not applicable $]$

\begin{tabular}{|c|c|c|c|c|c|c|c|c|}
\hline \multirow{3}{*}{ Category } & \multicolumn{3}{|c|}{ Withdrawals } & \multirow{3}{*}{$\begin{array}{l}\text { Total } \\
\text { use }^{1}\end{array}$} & \multirow{3}{*}{$\begin{array}{l}\text { Surface- } \\
\text { water } \\
\text { returns } \\
\end{array}$} & \multirow{2}{*}{\multicolumn{3}{|c|}{$\begin{array}{l}\text { Withdrawals by Major Industrial Groups } \\
\text { [NAICS, North American Industrial Classification System code] }\end{array}$}} \\
\hline & \multirow{2}{*}{$\begin{array}{c}\text { Ground- } \\
\text { water }\end{array}$} & \multirow{2}{*}{$\begin{array}{c}\text { Surface } \\
\text { water }\end{array}$} & \multirow[b]{2}{*}{ Total } & & & & & \\
\hline & & & & & & NAICS & Groundwater & Surface water \\
\hline Public supply & 0.64 & 0.00 & 0.64 & - & - & None & - & - \\
\hline Domestic & 0.24 & 0.00 & 0.24 & 0.56 & 0.00 & & & \\
\hline Commercial/public use & 0.00 & 0.00 & 0.00 & 0.22 & 0.00 & & & \\
\hline Industrial & 0.00 & 0.00 & 0.00 & 0.00 & 0.00 & & & \\
\hline Public-supply losses & - & - & - & 0.10 & - & & & \\
\hline Public wastewater treatment & - & - & - & - & 0.47 & Withdrawals by & r Public Supp & \\
\hline Mining & 0.02 & 0.00 & 0.02 & 0.02 & 0.00 & Name & Groundwater & Surface water \\
\hline Irrigation-Crop & 10.43 & 16.97 & 27.40 & 27.40 & 0.00 & & & \\
\hline Irrigation-Golf course & 0.00 & 0.00 & 0.00 & 0.00 & 0.00 & Town of Morgan & 0.27 & 0.00 \\
\hline Livestock/aquaculture & 0.05 & 0.13 & 0.18 & 0.18 & 0.00 & City of Edison & 0.16 & $\begin{array}{l}0.00 \\
0.00\end{array}$ \\
\hline Thermoelectric power & 0.00 & 0.00 & 0.00 & 0.00 & 0.00 & City of Arlington & $\begin{array}{l}0.12 \\
0.09\end{array}$ & $\begin{array}{l}0.00 \\
0.00\end{array}$ \\
\hline TOTAL & 11.28 & 17.10 & 28.48 & 28.48 & 0.47 & ceary & 0.09 & 0.00 \\
\hline
\end{tabular}

${ }^{1}$ Total use is total withdrawal plus public supply deliveries and losses.

\section{Withdrawals by Source}
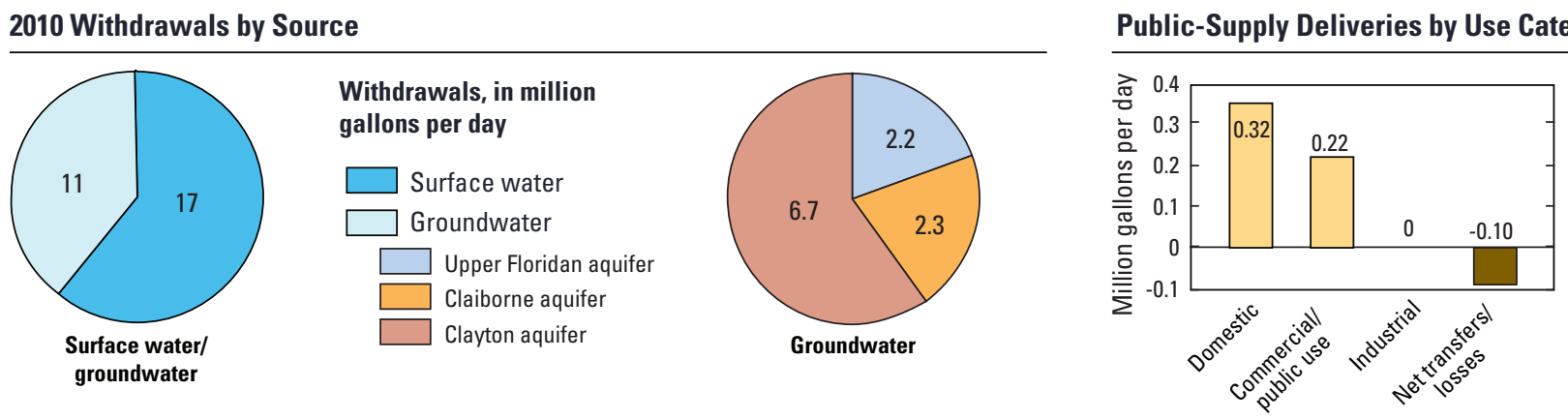
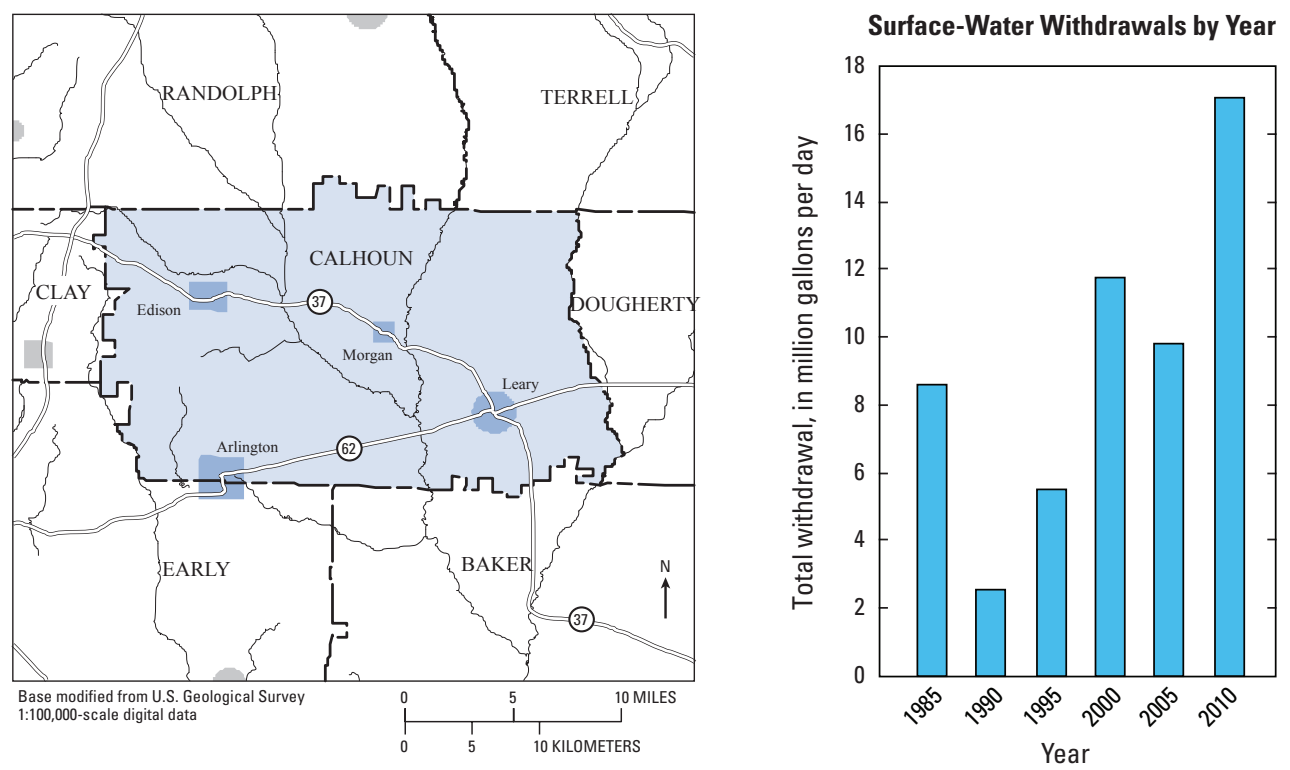

Groundwater Withdrawals by Year

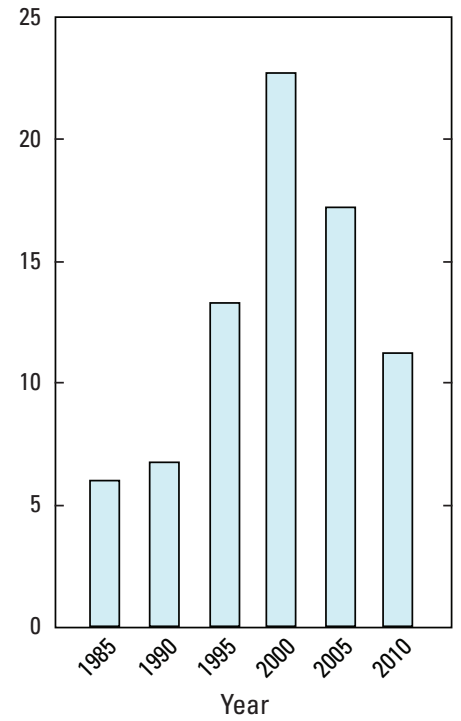




\section{CAMDEN COUNTY}

Population

50,513

Population served by public supply-Groundwater

Population served by public supply-Surface water

Acres irrigated
39,000

0

440

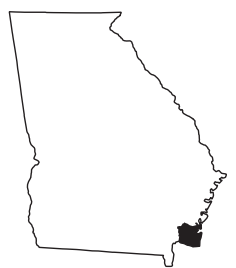

2010 WATER WITHDRAWALS AND ESTIMATED USE, IN MILLION GALLONS PER DAY

$[-$, not applicable $]$

\begin{tabular}{|c|c|c|c|c|c|c|c|c|}
\hline \multirow{3}{*}{ Category } & \multicolumn{3}{|c|}{ Withdrawals } & \multirow{3}{*}{$\begin{array}{l}\text { Total } \\
\text { use }^{1}\end{array}$} & \multirow{3}{*}{$\begin{array}{l}\text { Surface- } \\
\text { water } \\
\text { returns } \\
\end{array}$} & \multirow{2}{*}{\multicolumn{3}{|c|}{$\begin{array}{l}\text { Withdrawals by Major Industrial Groups } \\
\text { [NAICS, North American Industrial Classification System code] }\end{array}$}} \\
\hline & \multirow{2}{*}{$\begin{array}{c}\text { Ground- } \\
\text { water }\end{array}$} & \multirow{2}{*}{$\begin{array}{c}\text { Surface } \\
\text { water }\end{array}$} & \multirow[b]{2}{*}{ Total } & & & & & \\
\hline & & & & & & NAICS & Groundwater & Surface water \\
\hline Public supply & 4.72 & 0.00 & 4.72 & - & - & & 0.05 & 0.00 \\
\hline Domestic & 0.86 & 0.00 & 0.86 & 3.99 & 0.00 & 325 - Chemical products & 0.05 & 0.00 \\
\hline Commercial/public use & 0.00 & 0.00 & 0.00 & 0.73 & 0.00 & & & \\
\hline Industrial & 0.05 & 0.00 & 0.05 & 0.23 & 0.00 & & & \\
\hline Public-supply losses & - & - & - & 0.68 & - & & & \\
\hline Public wastewater treatment & - & - & - & - & 3.15 & Withdrawals by Major & r Public Supp & \\
\hline $\begin{array}{l}\text { Mining } \\
\text { Irrigation-Crop }\end{array}$ & $\begin{array}{l}0.00 \\
0.00\end{array}$ & $\begin{array}{l}0.00 \\
0.03\end{array}$ & $\begin{array}{l}0.00 \\
0.03\end{array}$ & $\begin{array}{l}0.00 \\
0.03\end{array}$ & $\begin{array}{l}0.00 \\
0.00\end{array}$ & Name & Groundwater & Surface water \\
\hline $\begin{array}{l}\text { Irrigation-Crop } \\
\text { Irrigation-Golf course }\end{array}$ & $\begin{array}{l}0.00 \\
0.48\end{array}$ & $\begin{array}{l}0.03 \\
0.25\end{array}$ & $\begin{array}{l}0.03 \\
0.73\end{array}$ & $\begin{array}{l}0.03 \\
0.73\end{array}$ & 0.00 & City of Kingsland & 1.79 & 0.00 \\
\hline Livestock/aquaculture & 0.00 & 0.00 & 0.00 & 0.00 & 0.00 & City of St. Mary’s & 1.54 & 0.00 \\
\hline Thermoelectric power & 0.00 & 0.00 & 0.00 & 0.00 & 0.00 & U.S. Navy-Kings Bay & 1.12 & 0.00 \\
\hline TOTAL & 6.11 & 0.28 & 6.39 & 6.39 & 3.15 & W \& D Utilities & 0.27 & 0.00 \\
\hline
\end{tabular}

'Total use is total withdrawal plus public supply deliveries and losses.

\section{Withdrawals by Source}
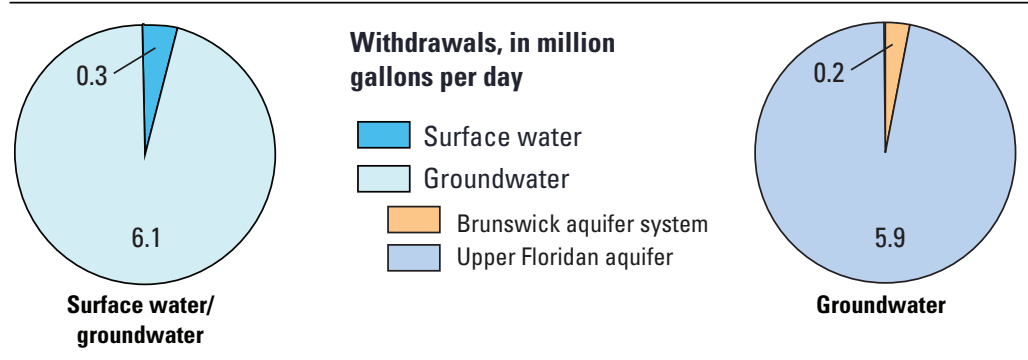

Groundwater

\section{Public-Supply Deliveries by Use Category}

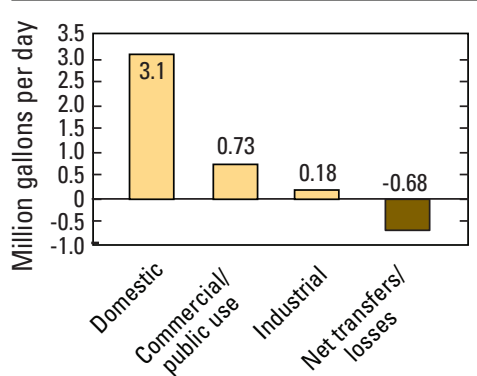

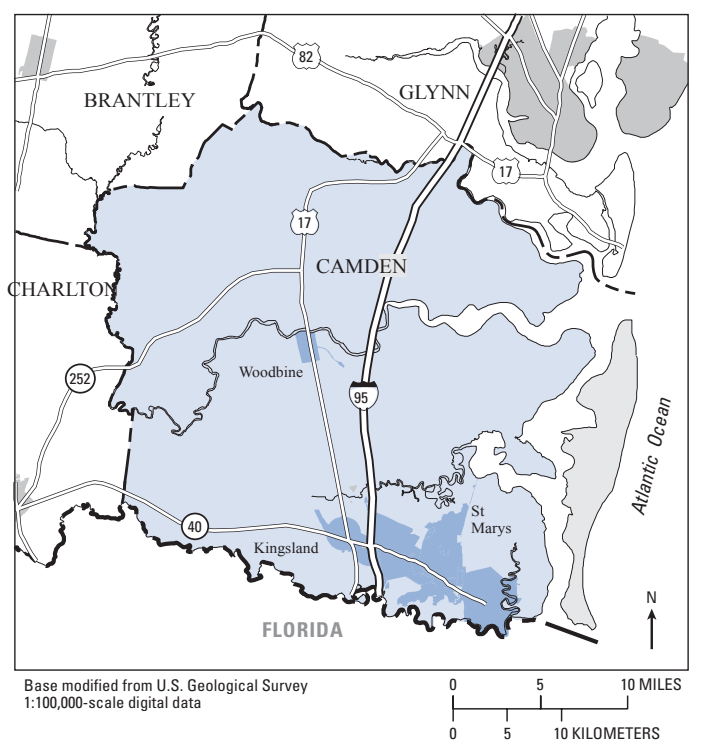

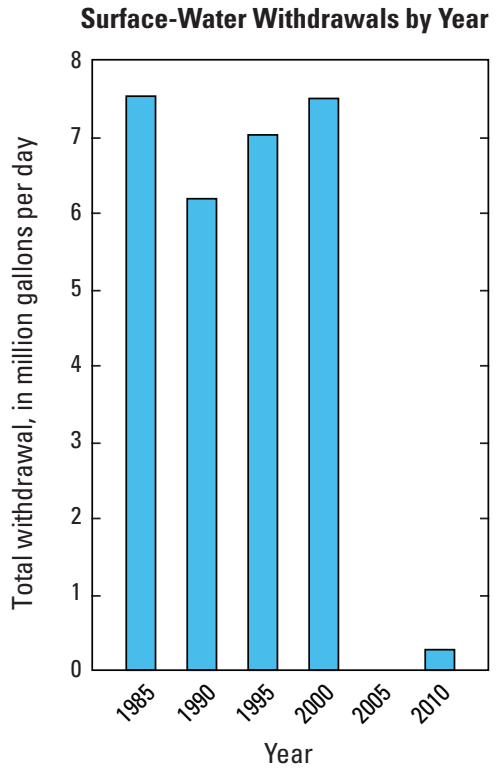

Groundwater Withdrawals by Year

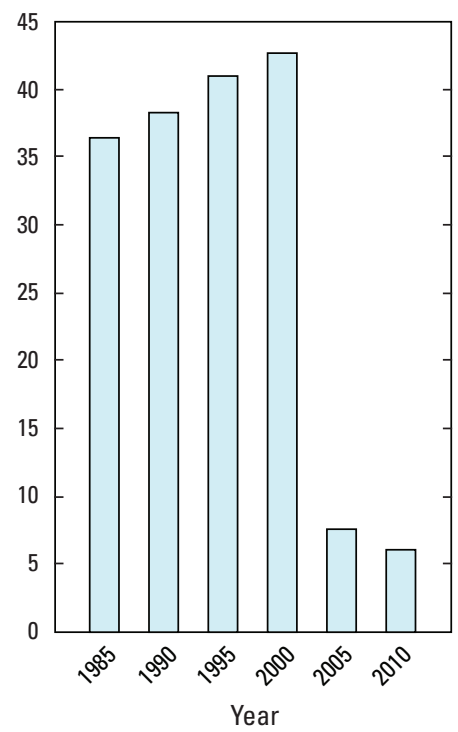




\section{CANDLER COUNTY}

Population

10,998

Population served by public supply-Groundwater $\quad 5,740$

Population served by public supply_-Surface water Acres irrigated
0

4,825

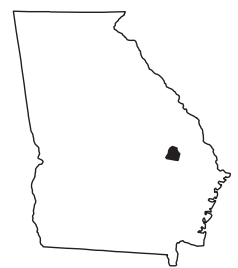

2010 WATER WITHDRAWALS AND ESTIMATED USE, IN MILLION GALLONS PER DAY

$[-$, not applicable]

\begin{tabular}{|c|c|c|c|c|c|}
\hline \multirow[b]{2}{*}{ Category } & \multicolumn{3}{|c|}{ Withdrawals } & \multirow[b]{2}{*}{$\begin{array}{l}\text { Total } \\
\text { use }^{1}\end{array}$} & \multirow{2}{*}{$\begin{array}{c}\text { Surface- } \\
\text { water } \\
\text { returns }\end{array}$} \\
\hline & $\begin{array}{c}\text { Ground- } \\
\text { water }\end{array}$ & $\begin{array}{c}\text { Surface } \\
\text { water }\end{array}$ & Total & & \\
\hline Public supply & 0.48 & 0.00 & 0.48 & - & - \\
\hline Domestic & 0.39 & 0.00 & 0.39 & 0.76 & 0.00 \\
\hline Commercial/public use & 0.00 & 0.00 & 0.00 & 0.05 & 0.00 \\
\hline Industrial & 0.00 & 0.00 & 0.00 & 0.00 & 0.00 \\
\hline Public-supply losses & - & - & - & 0.06 & - \\
\hline Public wastewater treatment & - & - & - & - & 0.00 \\
\hline Mining & 0.00 & 0.00 & 0.00 & 0.00 & 0.00 \\
\hline Irrigation-Crop & 0.51 & 0.65 & 1.16 & 1.16 & 0.00 \\
\hline Irrigation-Golf course & 0.07 & 0.11 & 0.18 & 0.18 & 0.00 \\
\hline Livestock/aquaculture & 0.01 & 0.05 & 0.06 & 0.06 & 0.00 \\
\hline Thermoelectric power & 0.00 & 0.00 & 0.00 & 0.00 & 0.00 \\
\hline TOTAL & 1.46 & 0.81 & 2.27 & 2.27 & 0.00 \\
\hline
\end{tabular}

Withdrawals by Major Industrial Groups

[NAICS, North American Industrial Classification System code]

NAICS Groundwater Surface water

None

\section{Withdrawals by Major Public Suppliers}

\begin{tabular}{lcc}
\hline Name & Groundwater & Surface water \\
\hline City of Metter & 0.43 & 0.00
\end{tabular}

'Total use is total withdrawal plus public supply deliveries and losses.

\section{Withdrawals by Source}
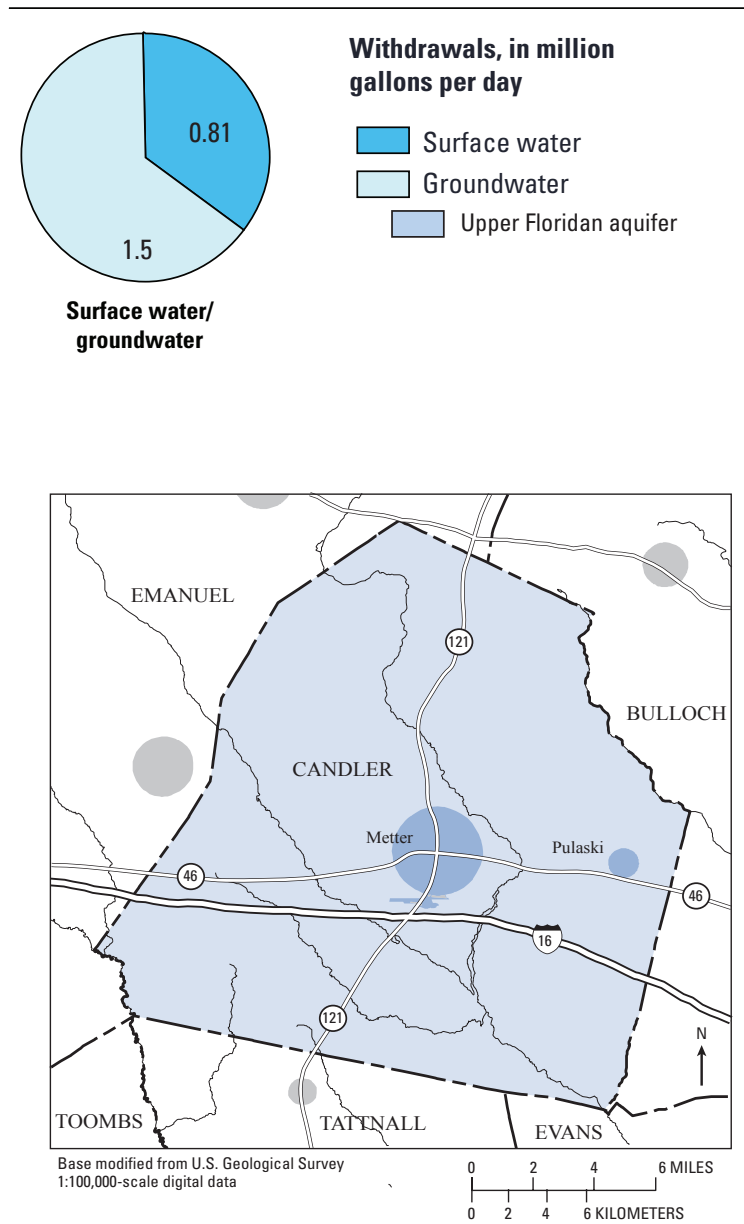

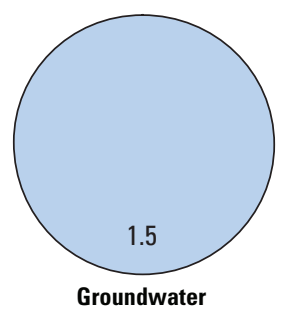

Public-Supply Deliveries by Use Category

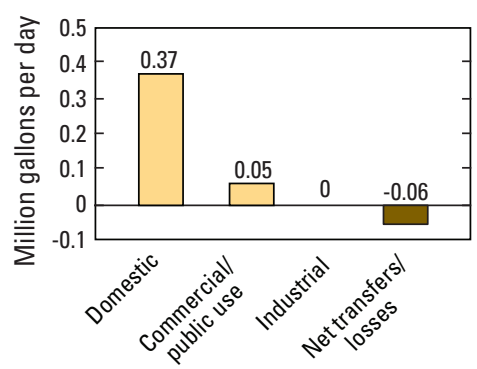

Surface-Water Withdrawals by Year

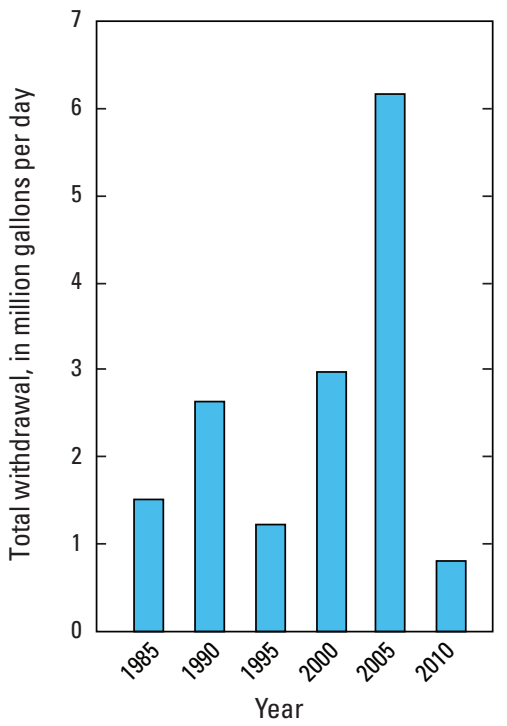

Groundwater Withdrawals by Year

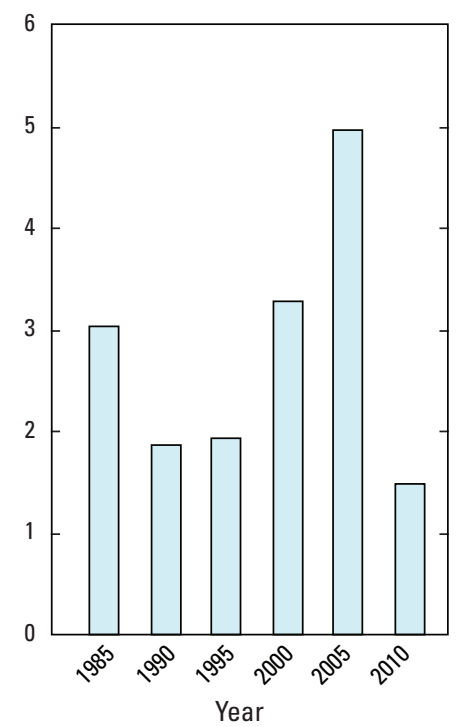




\section{CARROLL COUNTY}

Population

110,527

$\begin{array}{lr}\text { Population served by public supply-Groundwater } & 4,530 \\ \text { Population served by public supply-Surface water } & 85,230\end{array}$

Acres irrigated

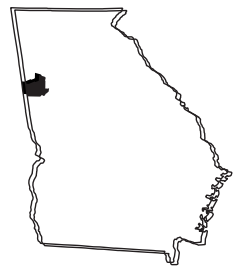

2010 WATER WITHDRAWALS AND ESTIMATED USE, IN MILLION GALLONS PER DAY

$[-$, not applicable $]$

\begin{tabular}{|c|c|c|c|c|c|c|c|c|}
\hline \multirow{3}{*}{ Category } & \multicolumn{3}{|c|}{ Withdrawals } & \multirow{3}{*}{$\begin{array}{l}\text { Total } \\
\text { use }^{1}\end{array}$} & \multirow{3}{*}{$\begin{array}{l}\text { Surface- } \\
\text { water } \\
\text { returns } \\
\end{array}$} & \multirow{2}{*}{\multicolumn{3}{|c|}{$\begin{array}{l}\text { Withdrawals by Major Industrial Groups } \\
\text { [NAICS, North American Industrial Classification System code] }\end{array}$}} \\
\hline & \multirow{2}{*}{$\begin{array}{c}\text { Ground- } \\
\text { water }\end{array}$} & \multirow{2}{*}{$\begin{array}{c}\text { Surface } \\
\text { water }\end{array}$} & \multirow[b]{2}{*}{ Total } & & & & & \\
\hline & & & & & & NAICS & Groundwater & Surface water \\
\hline Public supply & 0.49 & 11.33 & 11.82 & - & - & & 0.00 & 0.02 \\
\hline Domestic & 1.56 & 0.00 & 1.56 & 8.26 & 0.00 & 331 -Primary metals & 0.00 & \\
\hline Commercial/public use & 0.00 & 0.00 & 0.00 & 1.73 & 0.00 & & & \\
\hline Industrial & 0.00 & 0.02 & 0.02 & 1.39 & 0.35 & & & \\
\hline Public-supply losses & - & - & - & 2.02 & - & & & \\
\hline Public wastewater treatment & - & - & - & - & 0.75 & & & \\
\hline Mining & 0.11 & 0.00 & 0.11 & 0.11 & 0.00 & Withdrawals by Ma & Public Supp & \\
\hline Irrigation-Crop & 0.09 & 0.12 & 0.21 & 0.21 & 0.00 & Name & Groundwater & Surface water \\
\hline Irrigation-Golf course & 0.00 & 0.48 & 0.48 & 0.48 & 0.00 & City of Carrollton & 0.00 & 5.04 \\
\hline Livestock/aquaculture & 0.02 & 0.80 & 0.82 & 0.82 & 0.00 & Carroll County & 0.00 & 4.32 \\
\hline Thermoelectric power & 0.00 & 0.00 & 0.00 & 0.00 & 0.00 & City of Villa Rica & 0.00 & 1.31 \\
\hline TOTAL & 2.27 & 12.75 & 15.02 & 15.02 & 1.10 & City of Bowdon & 0.00 & 0.65 \\
\hline
\end{tabular}

${ }^{1}$ Total use is total withdrawal plus public supply deliveries and losses.

\section{Withdrawals by Source}
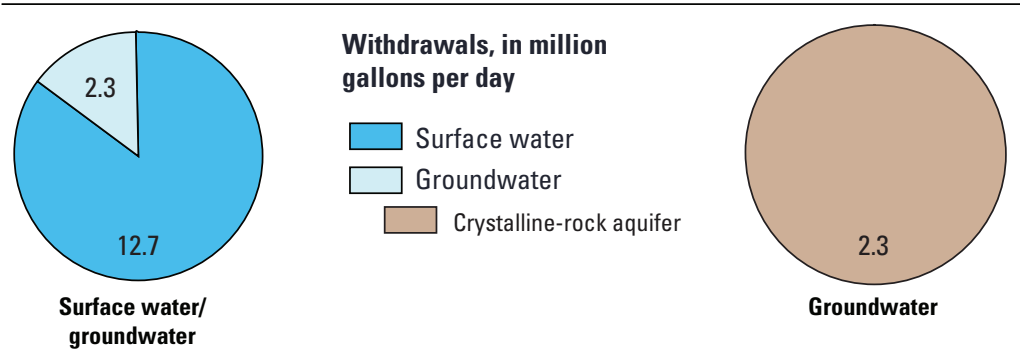

\section{Public-Supply Deliveries by Use Category}

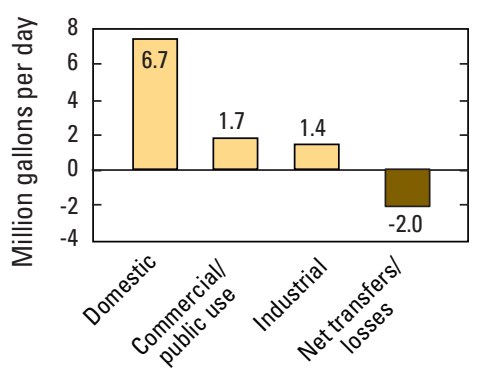

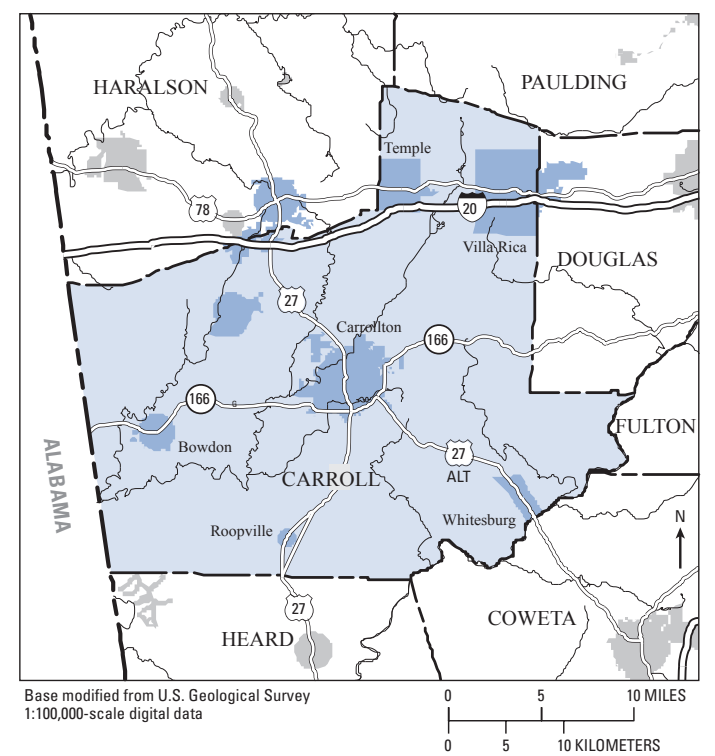

Surface-Water Withdrawals by Year

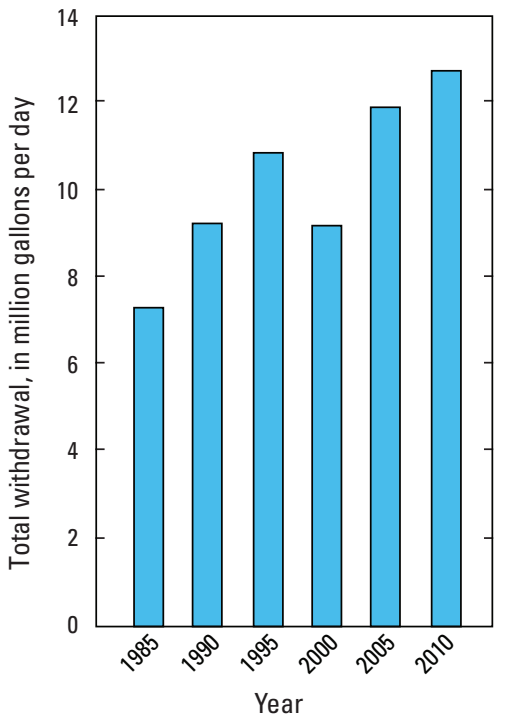

Groundwater Withdrawals by Year

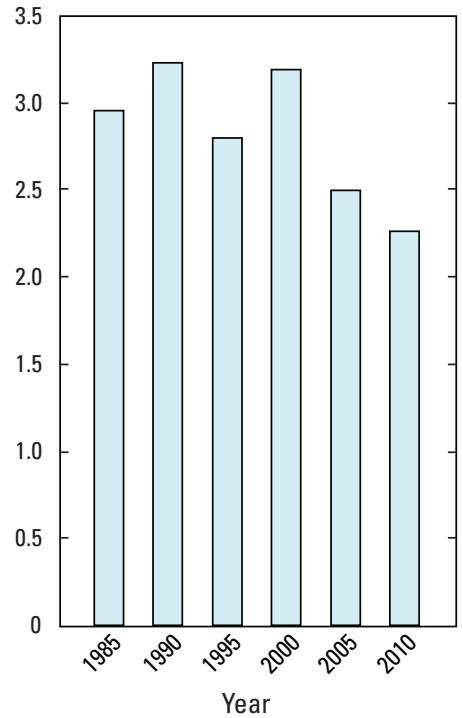




\section{CATOOSA COUNTY}

Population

Population served by public supply-Groundwater

Population served by public supply-Surface water Acres irrigated
63,942

63,210

420

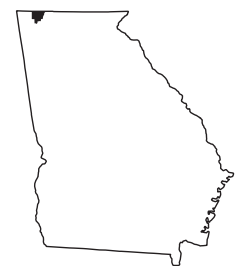

2010 WATER WITHDRAWALS AND ESTIMATED USE, IN MILLION GALLONS PER DAY

$[-$, not applicable; Mgal/d, million gallons per day $]$

\begin{tabular}{|c|c|c|c|c|c|}
\hline \multirow[b]{2}{*}{ Category } & \multicolumn{3}{|c|}{ Withdrawals } & \multirow[b]{2}{*}{$\begin{array}{l}\text { Total } \\
\text { use }^{1}\end{array}$} & \multirow{2}{*}{$\begin{array}{c}\text { Surface- } \\
\text { water } \\
\text { returns }\end{array}$} \\
\hline & $\begin{array}{c}\text { Ground- } \\
\text { water }\end{array}$ & $\begin{array}{c}\text { Surface } \\
\text { water }\end{array}$ & Total & & \\
\hline Public supply & 0.00 & 5.66 & 5.66 & - & - \\
\hline Domestic & 0.05 & 0.00 & 0.05 & 5.97 & 0.00 \\
\hline Commercial/public use & 0.00 & 0.00 & 0.00 & 0.55 & 0.00 \\
\hline Industrial & 0.00 & 0.00 & 0.00 & 0.07 & 0.00 \\
\hline Public-supply losses & - & - & - & 1.47 & - \\
\hline Public wastewater treatment & - & - & - & - & 0.00 \\
\hline Mining & 0.05 & 0.00 & 0.05 & 0.05 & 0.00 \\
\hline Irrigation-Crop & 0.09 & 0.24 & 0.33 & 0.33 & 0.00 \\
\hline Irrigation-Golf course & 0.33 & 0.41 & 0.74 & 0.74 & 0.00 \\
\hline Livestock/aquaculture & 0.01 & 0.15 & 0.16 & 0.16 & 0.00 \\
\hline Thermoelectric power & 0.00 & 0.00 & 0.00 & 0.00 & 0.00 \\
\hline TOTAL & 0.53 & 6.46 & 6.99 & 9.34 & 0.00 \\
\hline
\end{tabular}

Total use is total withdrawal plus public supply deliveries and losses. An average of $1.71 \mathrm{Mgal} / \mathrm{d}$ was delivered from Hamilton

County, Tennessee and $0.28 \mathrm{Mgal} / \mathrm{d}$ from Walker County, Georgia to Catoosa County, Georgia; whereas, $0.08 \mathrm{Mgal} / \mathrm{d}$ from Catoosa to Walker

County, Georgia in 2010.

\section{Withdrawals by Source}
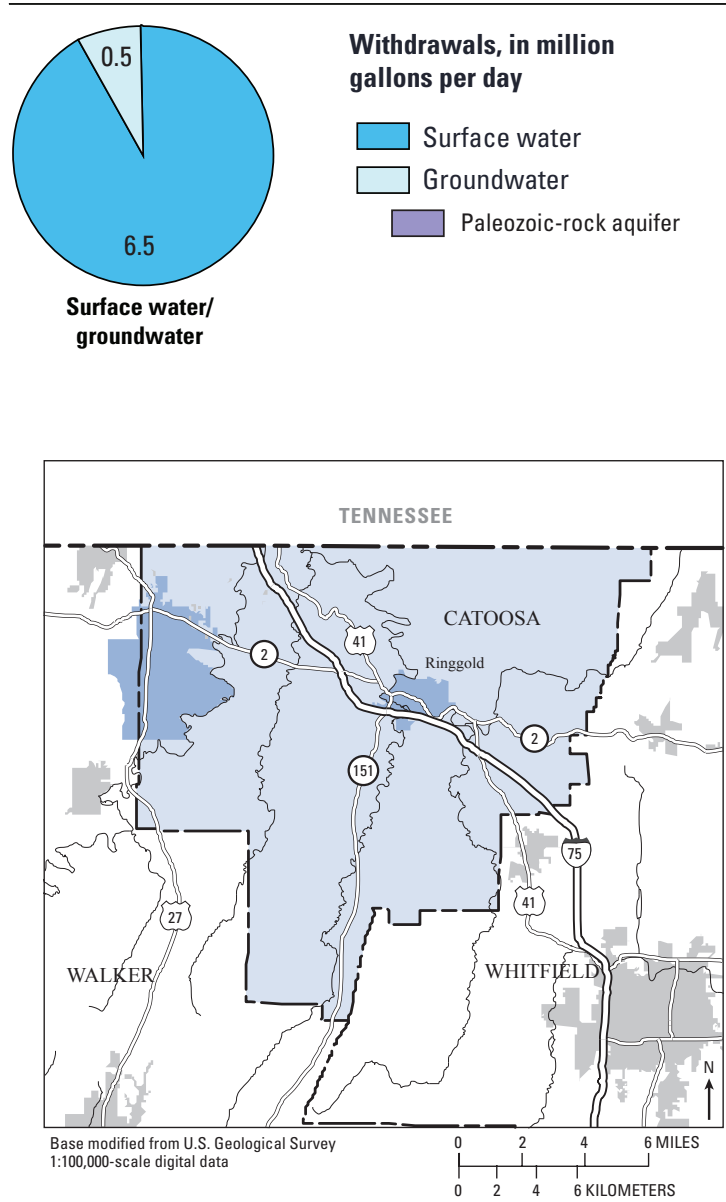

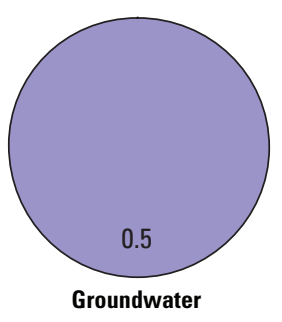

Surface-Water Withdrawals by Year

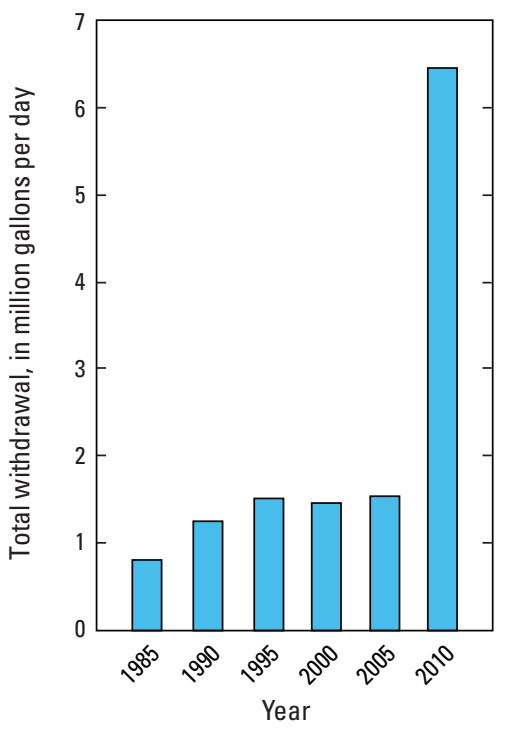

Groundwater Withdrawals by Year

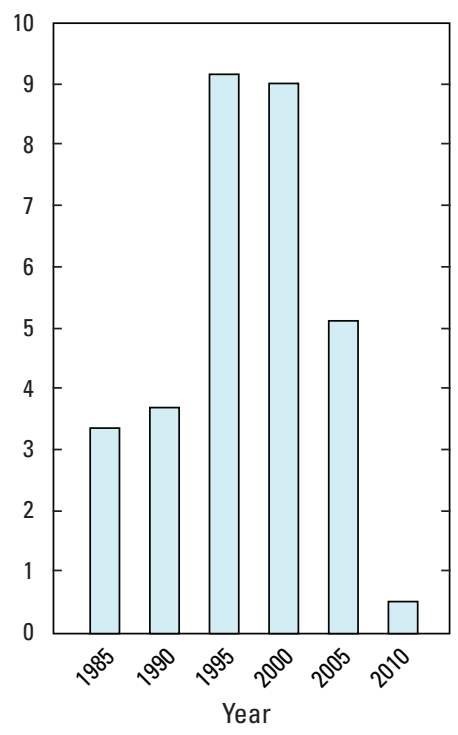

Public-Supply Deliveries' by Use Category

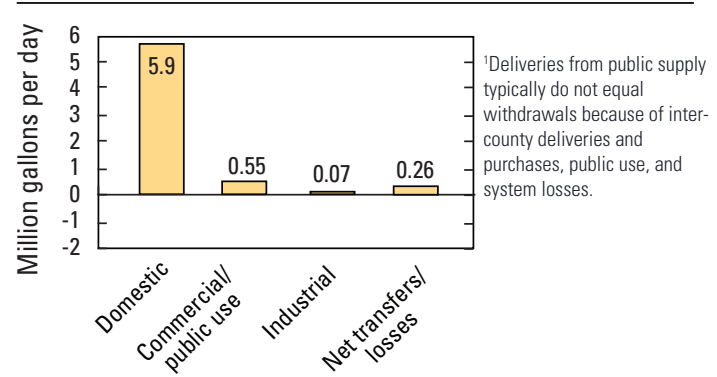

Withdrawals by Major Public Suppliers

\begin{tabular}{lcc}
\hline Name & Groundwater & Surface water \\
\hline Catoosa Utility District & 0.00 & 5.34 \\
Authority & 0.00 & 0.32 \\
City of Ringgold &
\end{tabular}




\section{CHARLTON COUNTY}

Population

12,171

4,900

Population served by public supply-Groundwater

Population served by public supply-Surface water Acres irrigated

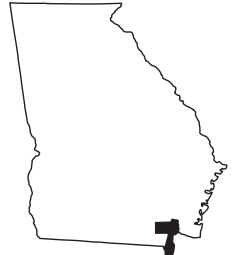

2010 WATER WITHDRAWALS AND ESTIMATED USE, IN MILLION GALLONS PER DAY $[-$, not applicable $]$

\begin{tabular}{|c|c|c|c|c|c|}
\hline \multirow[b]{2}{*}{ Category } & \multicolumn{3}{|c|}{ Withdrawals } & \multirow[b]{2}{*}{$\begin{array}{c}\text { Total } \\
\text { use }^{1}\end{array}$} & \multirow{2}{*}{$\begin{array}{c}\text { Surface- } \\
\text { water } \\
\text { returns } \\
\end{array}$} \\
\hline & $\begin{array}{c}\text { Ground- } \\
\text { water }\end{array}$ & $\begin{array}{c}\text { Surface } \\
\text { water }\end{array}$ & Total & & \\
\hline Public supply & 0.63 & 0.00 & 0.63 & - & - \\
\hline Domestic & 0.55 & 0.00 & 0.55 & 1.04 & 0.00 \\
\hline Commercial/public use & 0.00 & 0.00 & 0.00 & 0.07 & 0.00 \\
\hline Industrial & 0.00 & 0.00 & 0.00 & 0.00 & 0.00 \\
\hline Public-supply losses & - & - & - & 0.07 & - \\
\hline Public wastewater treatment & - & - & - & - & 0.48 \\
\hline Mining & 0.06 & 0.00 & 0.06 & 0.06 & 0.00 \\
\hline Irrigation-Crop & 0.00 & 0.00 & 0.00 & 0.00 & 0.00 \\
\hline Irrigation-Golf course & 0.00 & 0.00 & 0.00 & 0.00 & 0.00 \\
\hline Livestock/aquaculture & 0.00 & 0.03 & 0.03 & 0.03 & 0.00 \\
\hline Thermoelectric power & 0.00 & 0.00 & 0.00 & 0.00 & 0.00 \\
\hline TOTAL & 1.24 & 0.03 & 1.27 & 1.27 & 0.48 \\
\hline
\end{tabular}

Withdrawals by Major Industrial Groups

[NAICS, North American Industrial Classification System code]

NAICS Groundwater Surface water

None

Withdrawals by Major Public Suppliers

Name Groundwater Surface water

\begin{tabular}{lll}
\hline City of Folkston & 0.56 & 0.00
\end{tabular}

\section{Withdrawals by Source}
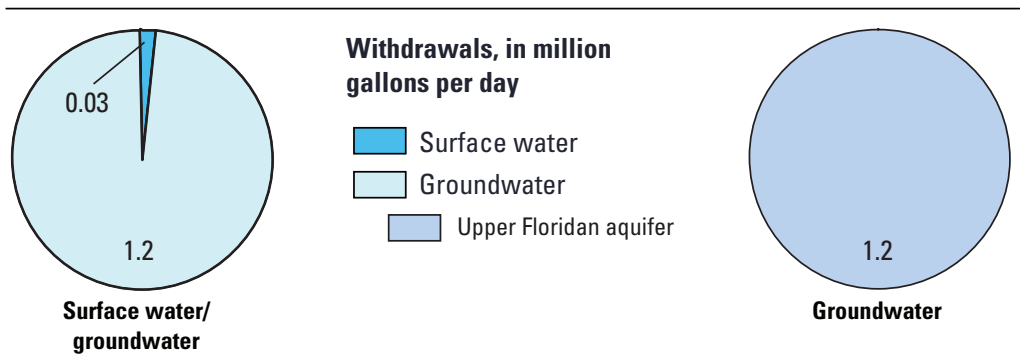

Public-Supply Deliveries by Use Category
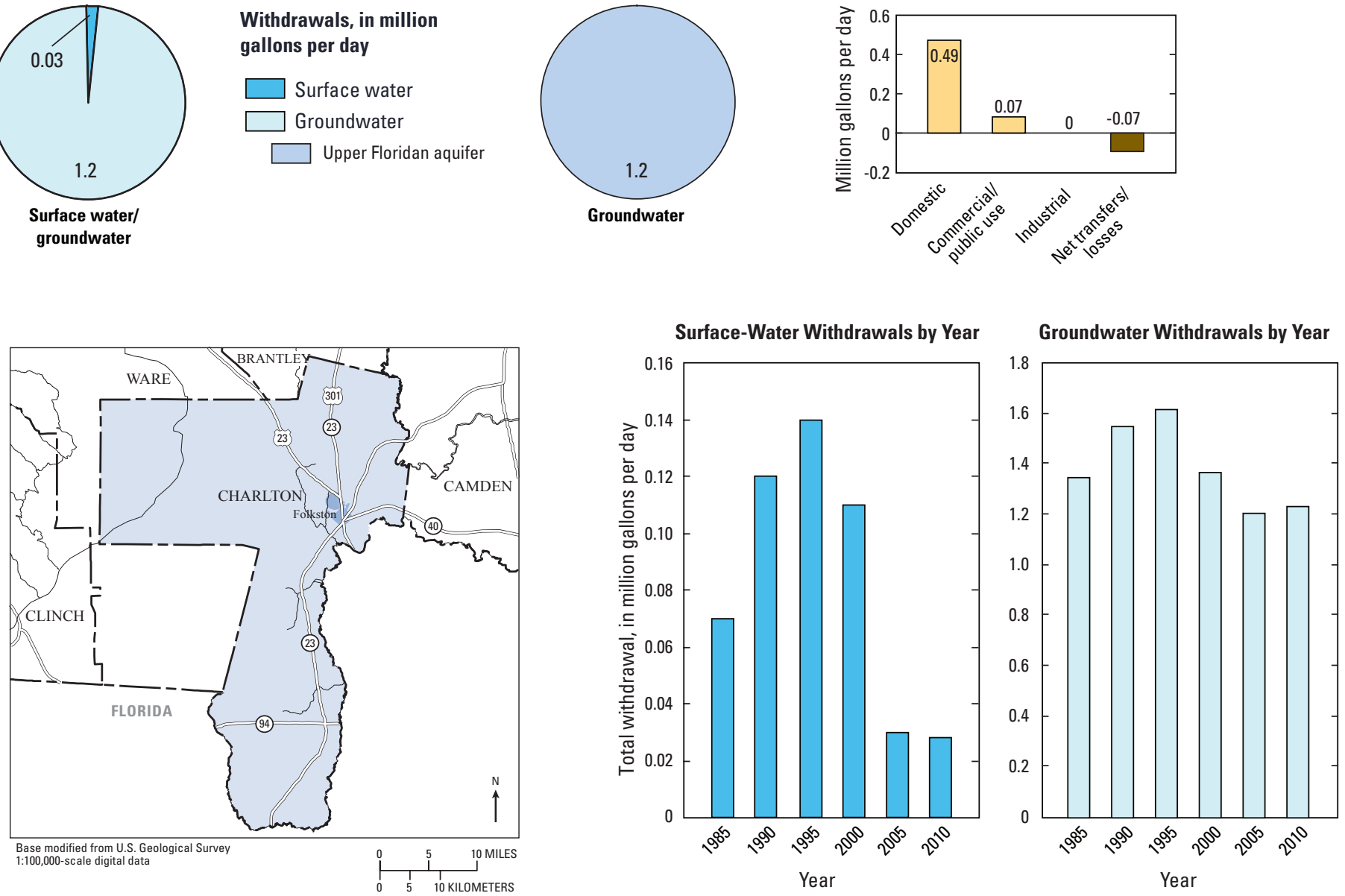


\section{CHATHAM COUNTY}

Population

265,128

Population served by public supply-Groundwater $\quad 159,770$

Population served by public supply-Surface water $\quad 94,680$

Acres irrigated

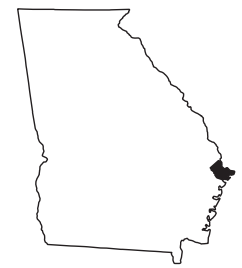

2010 WATER WITHDRAWALS AND ESTIMATED USE, IN MILLION GALLONS PER DAY

$[-$, not applicable; Mgal/d, million gallons per day]

\begin{tabular}{|c|c|c|c|c|c|c|c|c|}
\hline \multirow{3}{*}{ Category } & \multicolumn{3}{|c|}{ Withdrawals } & \multirow{3}{*}{$\begin{array}{l}\text { Total } \\
\text { use }^{1}\end{array}$} & \multirow{3}{*}{$\begin{array}{c}\text { Surface- } \\
\text { water } \\
\text { returns } \\
\end{array}$} & \multirow{2}{*}{\multicolumn{3}{|c|}{$\begin{array}{l}\text { Withdrawals by Major Industrial Groups } \\
\text { [NAICS, North American Industrial Classification System code] }\end{array}$}} \\
\hline & \multirow{2}{*}{$\begin{array}{c}\text { Ground- } \\
\text { water }\end{array}$} & \multirow{2}{*}{$\begin{array}{c}\text { Surface } \\
\text { water }\end{array}$} & \multirow[b]{2}{*}{ Total } & & & & & \\
\hline & & & & & & NAICS & Groundwater & Surface water \\
\hline Public supply & 32.06 & 0.00 & 32.06 & - & - & 322-Paper, pulp & 15.24 & 13.44 \\
\hline Domestic & 0.80 & 0.00 & 0.80 & 21.64 & 0.00 & 325 -Chemicals & 2.45 & 5.63 \\
\hline Commercial/public use & 0.25 & 0.00 & 0.25 & 7.20 & 0.00 & $311-$ Food & 0.73 & 0.00 \\
\hline Industrial & 18.72 & 19.07 & 37.79 & 63.59 & 77.08 & 321 -Wood & 0.25 & 0.00 \\
\hline
\end{tabular}

Public-supply losses

(1)

wastewater treatment

0.12

0.21

0.75

0.00

1.40

$\mathbf{5 4 . 3 1}$

$-$

$\begin{array}{ll}- & 11.85\end{array}$

Irrigation-Crop

Irrigation-Golf cours

Livestock/aquaculture

Thermoelectric power

TOTAL

$$
0.00
$$

$$
0.12
$$

0.00

0.26

0.01

204.83

224.17

\subsection{1}

1.01

0.01

206.23

278.48

'Total use is total withdrawal plus public supply deliveries and losses. An average of $2.1 \mathrm{Mgal} / \mathrm{d}$ was delivered from Chatham to Effingham County, Georgia and $23.8 \mathrm{Mgal} / \mathrm{d}$ from Effingham County to Chatham County, Georgia in 2010.

2010 Withdrawals by Source
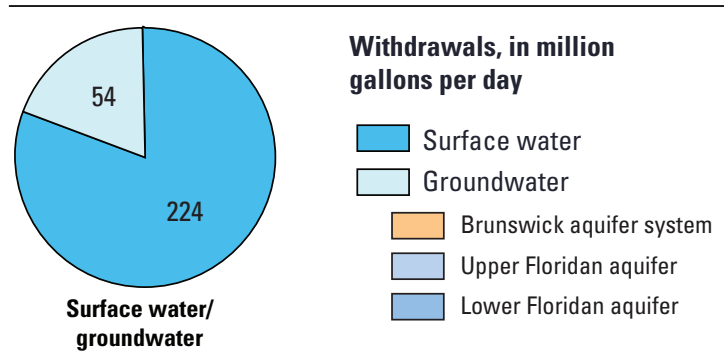

groundwater

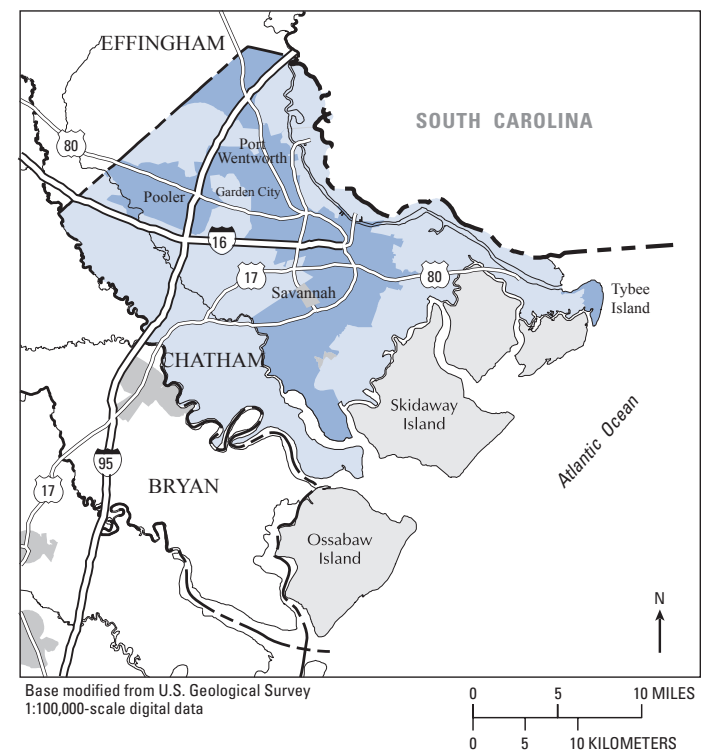

29.73

Withdrawals by Major Public Suppliers

\begin{tabular}{lcc}
\hline Name & Groundwater & Surface water \\
\hline City of Savannah & 23.35 & 0.00 \\
Skidaway Island Utilities & 1.71 & 0.00 \\
City of Garden City & 1.08 & 0.00 \\
Hunter Army Airfield & 1.04 & 0.00 \\
City of Tybee Island & 0.85 & 0.00 \\
Chatham County & 0.65 & 0.00 \\
City of Pooler & 0.57 & 0.00 \\
Consolidated Utilities, Inc. & 0.52 & 0.00 \\
Water Utility Management & 0.50 & 0.00
\end{tabular}

Public-Supply Deliveries ${ }^{1}$ by Use Category

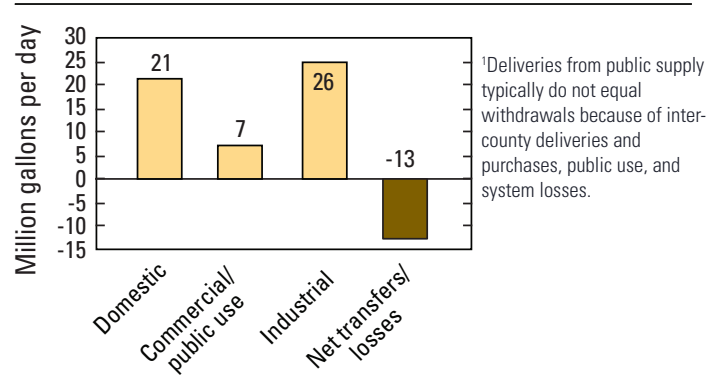

Surface-Water Withdrawals by Year
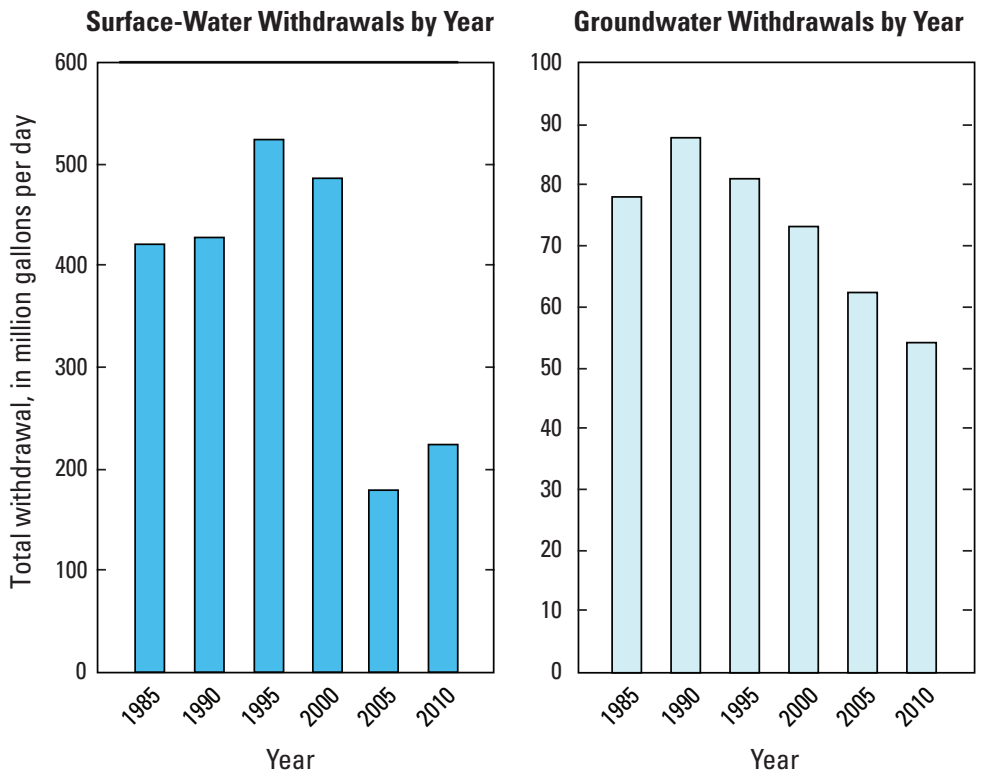
CHATTAHOOCHEE COUNTY

Population

Population served by public supply-Groundwater

Population served by public supply-Surface water

Acres irrigated

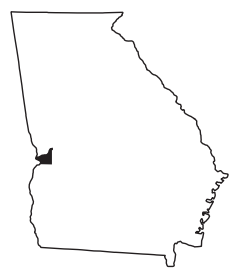

2010 WATER WITHDRAWALS AND ESTIMATED USE, IN MILLION GALLONS PER DAY

$[-$, not applicable; Mgal/d, million gallons per day]

\begin{tabular}{|c|c|c|c|c|c|c|c|c|}
\hline \multirow{3}{*}{ Category } & \multicolumn{3}{|c|}{ Withdrawals } & \multirow{3}{*}{$\begin{array}{l}\text { Total } \\
\text { use }^{1}\end{array}$} & \multirow{3}{*}{$\begin{array}{c}\text { Surface- } \\
\text { water } \\
\text { returns } \\
\end{array}$} & \multirow{2}{*}{\multicolumn{3}{|c|}{$\begin{array}{l}\text { Withdrawals by Major Industrial Groups } \\
\text { [NAICS, North American Industrial Classification System code] }\end{array}$}} \\
\hline & \multirow{2}{*}{$\begin{array}{c}\text { Ground- } \\
\text { water }\end{array}$} & \multirow{2}{*}{$\begin{array}{c}\text { Surface } \\
\text { water }\end{array}$} & \multirow[b]{2}{*}{ Total } & & & & & \\
\hline & & & & & & NAICS & Groundwater & Surface water \\
\hline Public supply & 0.34 & 0.00 & 0.34 & - & - & None & - & - \\
\hline Domestic & 0.54 & 0.00 & 0.54 & 0.82 & 0.00 & & & \\
\hline Commercial/public use & 0.00 & 0.00 & 0.00 & 0.01 & 0.00 & & & \\
\hline Industrial & 0.00 & 0.00 & 0.00 & 3.84 & 0.00 & & & \\
\hline Public-supply losses & - & - & - & 0.05 & - & & & \\
\hline Public wastewater treatment & - & - & - & - & 0.00 & Withdrawals by Majo & r Public Supp & \\
\hline Mining & 0.00 & 0.00 & 0.00 & 0.00 & 0.00 & Name & Groundwater & Surface water \\
\hline Irrigation-Crop & 0.00 & 0.00 & 0.00 & 0.00 & 0.00 & & & \\
\hline Irrigation-Golf course & 0.00 & 0.00 & 0.00 & 0.00 & 0.00 & Water System & 0.34 & 0.00 \\
\hline Livestock/aquaculture & 0.00 & 0.00 & 0.00 & 0.00 & 0.00 & & & \\
\hline
\end{tabular}

$\begin{array}{llllll}\text { Thermoelectric power } & 0.00 & 0.00 & 0.00 & 0.00 & 0.00\end{array}$

$$
\text { TOTAL }
$$

0.88

0.00

0.88

4.72

0.00

'Total use is total withdrawal plus public supply deliveries and losses. About $4 \mathrm{Mgal} / \mathrm{d}$ was delivered from Muscogee County, Georgia to

Chattahoochee County in 2010.

\section{Withdrawals by Source}
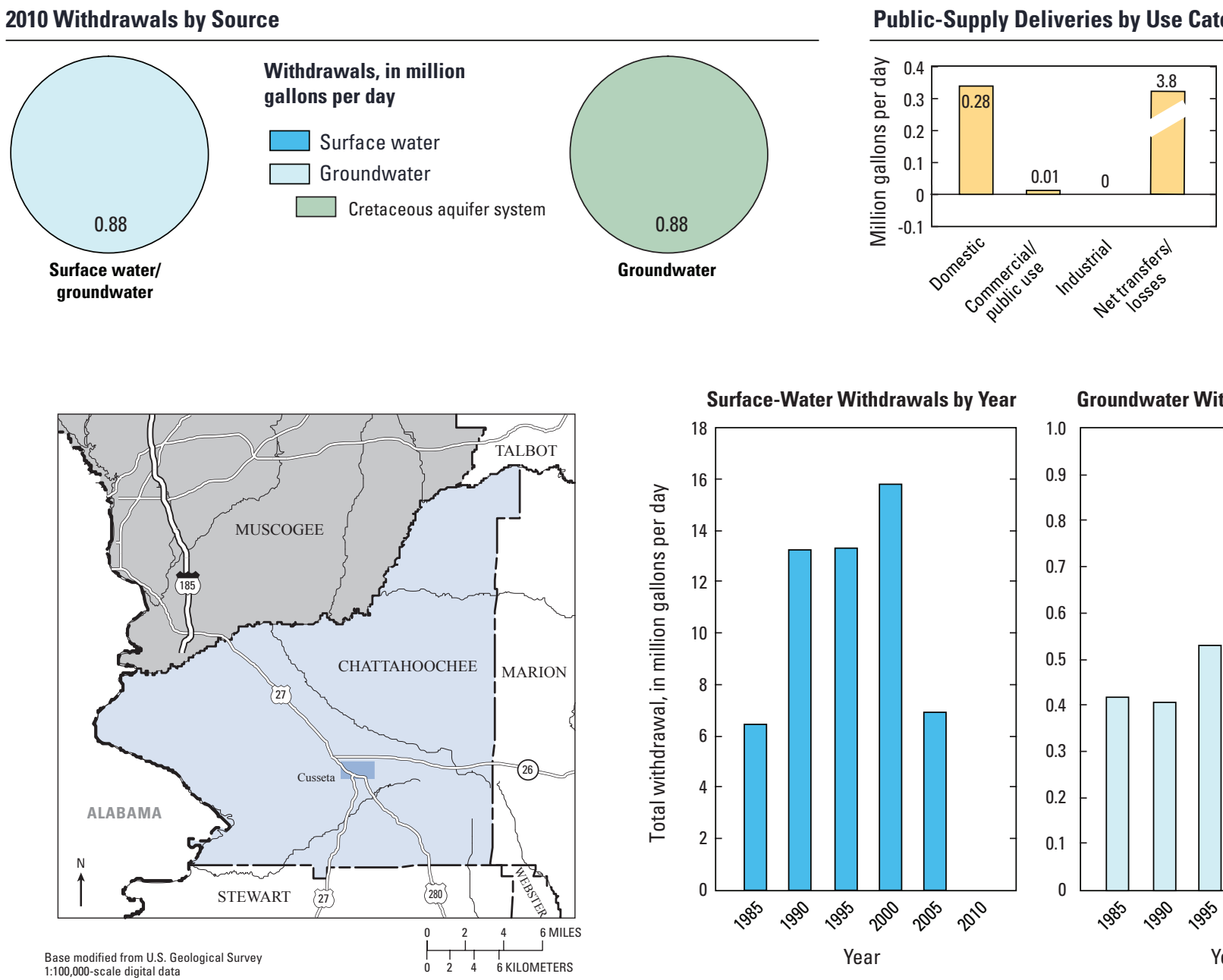

Groundwater Withdrawals by Year

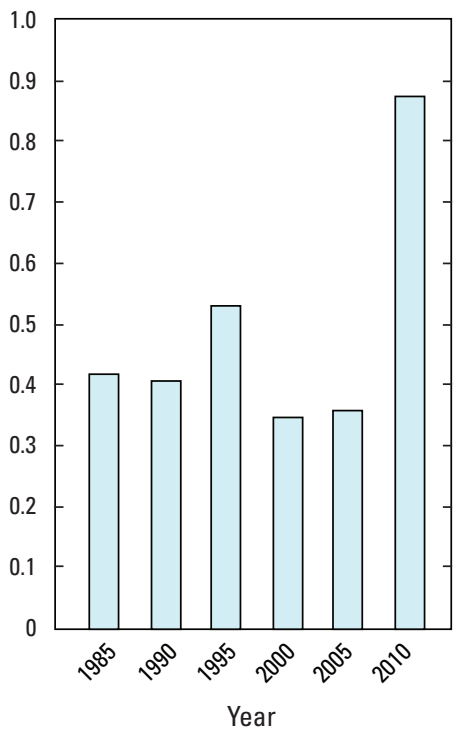




\section{CHATTOOGA COUNTY}

Population

Population served by public supply-Groundwater $\quad 8,540$

Population served by public supply-Surface water $\quad 11,910$

Acres irrigated

0

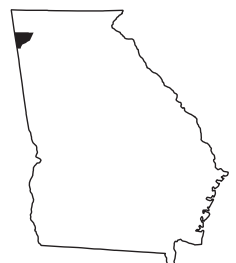

2010 WATER WITHDRAWALS AND ESTIMATED USE, IN MILLION GALLONS PER DAY

$[-$, not applicable; Mgal/d, million gallons per day $]$

\begin{tabular}{|c|c|c|c|c|c|c|c|c|}
\hline \multirow{3}{*}{ Category } & \multicolumn{3}{|c|}{ Withdrawals } & \multirow{3}{*}{$\begin{array}{l}\text { Total } \\
\text { use }^{1}\end{array}$} & \multirow{3}{*}{$\begin{array}{l}\text { Surface- } \\
\text { water } \\
\text { returns } \\
\end{array}$} & \multirow{2}{*}{\multicolumn{3}{|c|}{$\begin{array}{l}\text { Withdrawals by Major Industrial Groups } \\
\text { [NAICS, North American Industrial Classification System code] }\end{array}$}} \\
\hline & \multirow{2}{*}{$\begin{array}{c}\text { Ground- } \\
\text { water }\end{array}$} & \multirow{2}{*}{$\begin{array}{c}\text { Surface } \\
\text { water }\end{array}$} & \multirow[b]{2}{*}{ Total } & & & & & \\
\hline & & & & & & NAICS & Groundwater & Surface water \\
\hline Public supply & 0.81 & 2.51 & 3.32 & - & - & 313-Textile Mills & 0.00 & 5.31 \\
\hline Domestic & 0.42 & 0.00 & 0.42 & 2.10 & 0.00 & & & \\
\hline Commercial/public use & 0.00 & 0.00 & 0.00 & 0.25 & 0.00 & & & \\
\hline Industrial & 0.00 & 5.31 & 5.31 & 5.58 & 1.32 & & & \\
\hline Public-supply losses & - & - & - & 0.64 & - & & & \\
\hline Public wastewater treatment & - & - & - & - & 6.30 & & & \\
\hline Mining & 0.02 & 0.00 & 0.02 & 0.02 & 0.00 & Withdrawals by $\mathbf{M}$ & r Public Supp & \\
\hline Irrigation-Crop & 0.00 & 0.00 & 0.00 & 0.00 & 0.00 & Name & Groundwater & Surface water \\
\hline Irrigation-Golf course & 0.00 & 0.00 & 0.00 & 0.00 & 0.00 & & & \\
\hline Livestock/aquaculture & 0.42 & 1.23 & 1.65 & 1.65 & 0.00 & City of Summerville & 0.00 & 2.51 \\
\hline Thermoelectric power & 0.00 & 0.00 & 0.00 & 0.00 & 0.00 & Chattooga County & 0.63 & 0.00 \\
\hline TOTAL & 1.67 & 9.05 & 10.72 & 10.24 & 7.67 & City of Lyerly & 0.14 & 0.00 \\
\hline
\end{tabular}

${ }^{1}$ Total use is total withdrawal plus public supply deliveries and losses. An average of $0.41 \mathrm{Mgal} / \mathrm{d}$ was delivered to an unidentified county in 2010

\section{Withdrawals by Source}
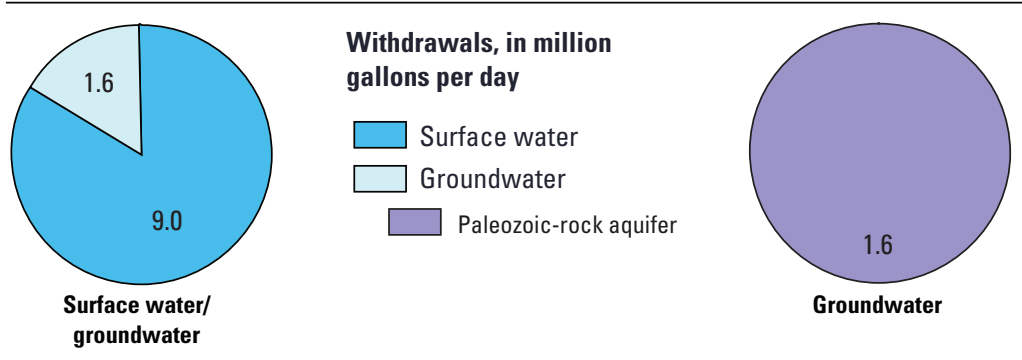

Public-Supply Deliveries ${ }^{1}$ by Use Category

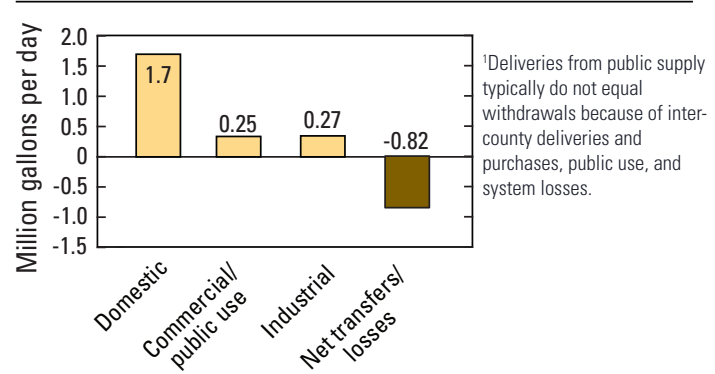

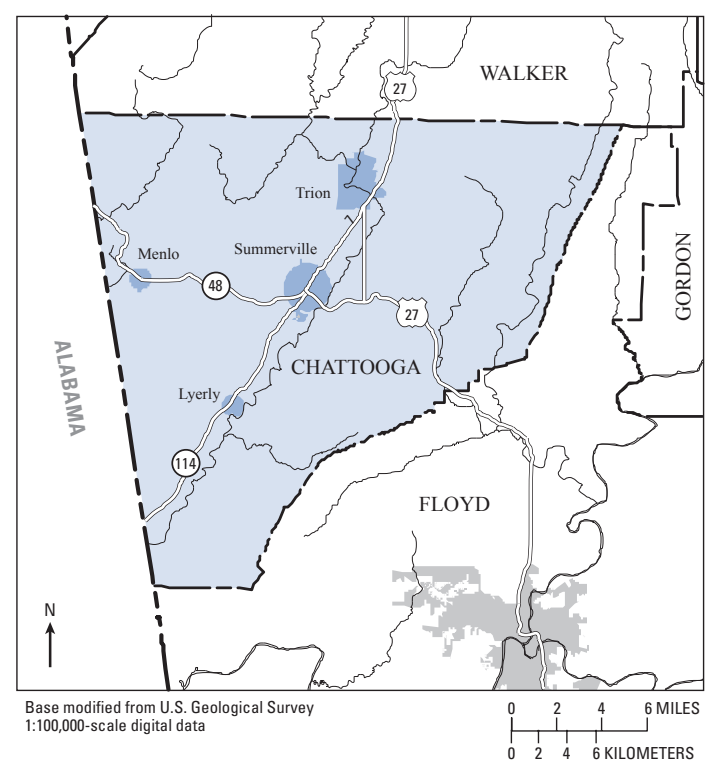

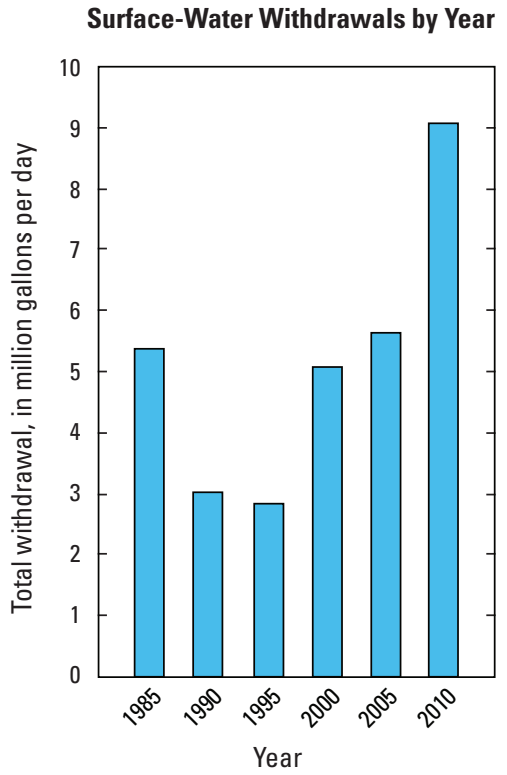

Groundwater Withdrawals by Year

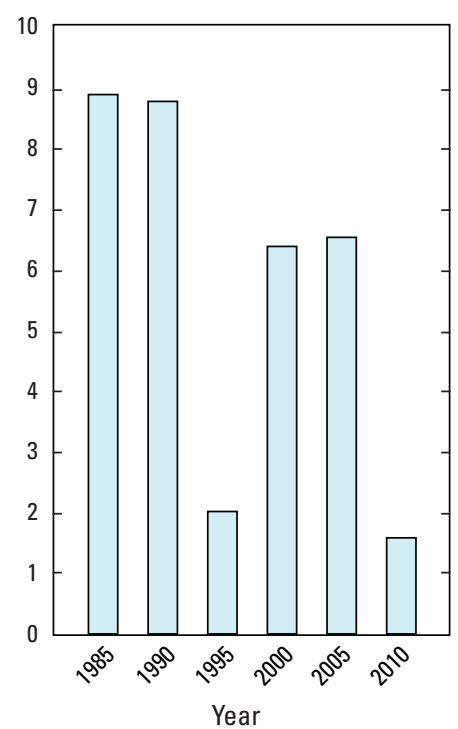




\section{CHEROKEE COUNTY}

Population

214,346

Population served by public supply-Groundwater $\quad 4,560$

Population served by public supply-Surface water 202,820

Acres irrigated

670

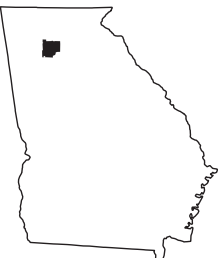

2010 WATER WITHDRAWALS AND ESTIMATED USE, IN MILLION GALLONS PER DAY

$[-$, not applicable; Mgal/d, million gallons per day]

\begin{tabular}{|c|c|c|c|c|c|c|c|c|}
\hline \multirow{3}{*}{ Category } & \multicolumn{3}{|c|}{ Withdrawals } & \multirow{3}{*}{$\begin{array}{l}\text { Total } \\
\text { use }^{1}\end{array}$} & \multirow{3}{*}{$\begin{array}{l}\text { Surface- } \\
\text { water } \\
\text { returns }\end{array}$} & \multirow{2}{*}{\multicolumn{3}{|c|}{$\begin{array}{l}\text { Withdrawals by Major Industrial Groups } \\
\text { [NAICS, North American Industrial Classification System code] }\end{array}$}} \\
\hline & \multirow{2}{*}{$\begin{array}{c}\text { Ground- } \\
\text { water }\end{array}$} & \multirow{2}{*}{$\begin{array}{c}\text { Surface } \\
\text { water }\end{array}$} & \multirow[b]{2}{*}{ Total } & & & & & \\
\hline & & & & & & NAICS & Groundwater & Surface water \\
\hline Public supply & 0.39 & 17.54 & 17.93 & - & - & $311-$ Food & 0.02 & 3.16 \\
\hline Domestic & 0.52 & 0.00 & 0.52 & 15.47 & 0.00 & & & \\
\hline Commercial/public use & 0.00 & 0.00 & 0.00 & 1.33 & 0.00 & & & \\
\hline Industrial & 0.02 & 3.16 & 3.18 & 3.77 & 0.13 & & & \\
\hline Public-supply losses & - & - & - & 3.22 & - & & & \\
\hline Public wastewater treatment & - & - & - & - & 10.21 & Withdrawals by MajoI & Public Supp & \\
\hline Mining & 0.11 & 0.00 & 0.11 & 0.11 & 0.44 & Name & Groundwater & Surface water \\
\hline Irrigation-Crop & 0.16 & 0.11 & 0.27 & 0.27 & 0.00 & & & \\
\hline Irrigation-Golf course & 0.63 & 1.50 & 2.13 & 2.13 & 0.00 & Cherokee County WSA & 0.00 & 14.74 \\
\hline Livestock/aquaculture & 0.01 & 0.17 & 0.18 & 0.18 & 0.00 & City of Canton & 0.00 & 2.87 \\
\hline Thermoelectric power & 0.00 & 0.00 & 0.00 & 0.00 & 0.00 & Lake Arrowhead Utility & 0.17 & 0.00 \\
\hline TOTAL & 1.84 & 22.48 & 24.32 & 26.48 & 10.78 & City of Ball Ground & 0.17 & 0.00 \\
\hline
\end{tabular}

${ }^{1}$ Total use is total withdrawal plus public supply deliveries and losses. An average of $1.4 \mathrm{Mgal} / \mathrm{d}$ was delivered from Cobb and Pickens

Counties, Georgia to Cherokee County, Georgia and about 0.62 Mgal/d from Cherokee County to Bartow, Pickens, and Dawson Counties,

Georgia, collectively, in 2010 .

\section{Withdrawals by Source}
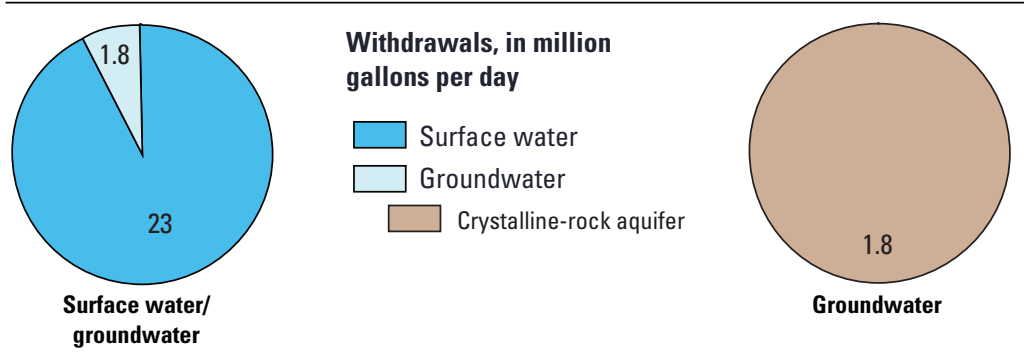

Public-Supply Deliveries' by Use Category
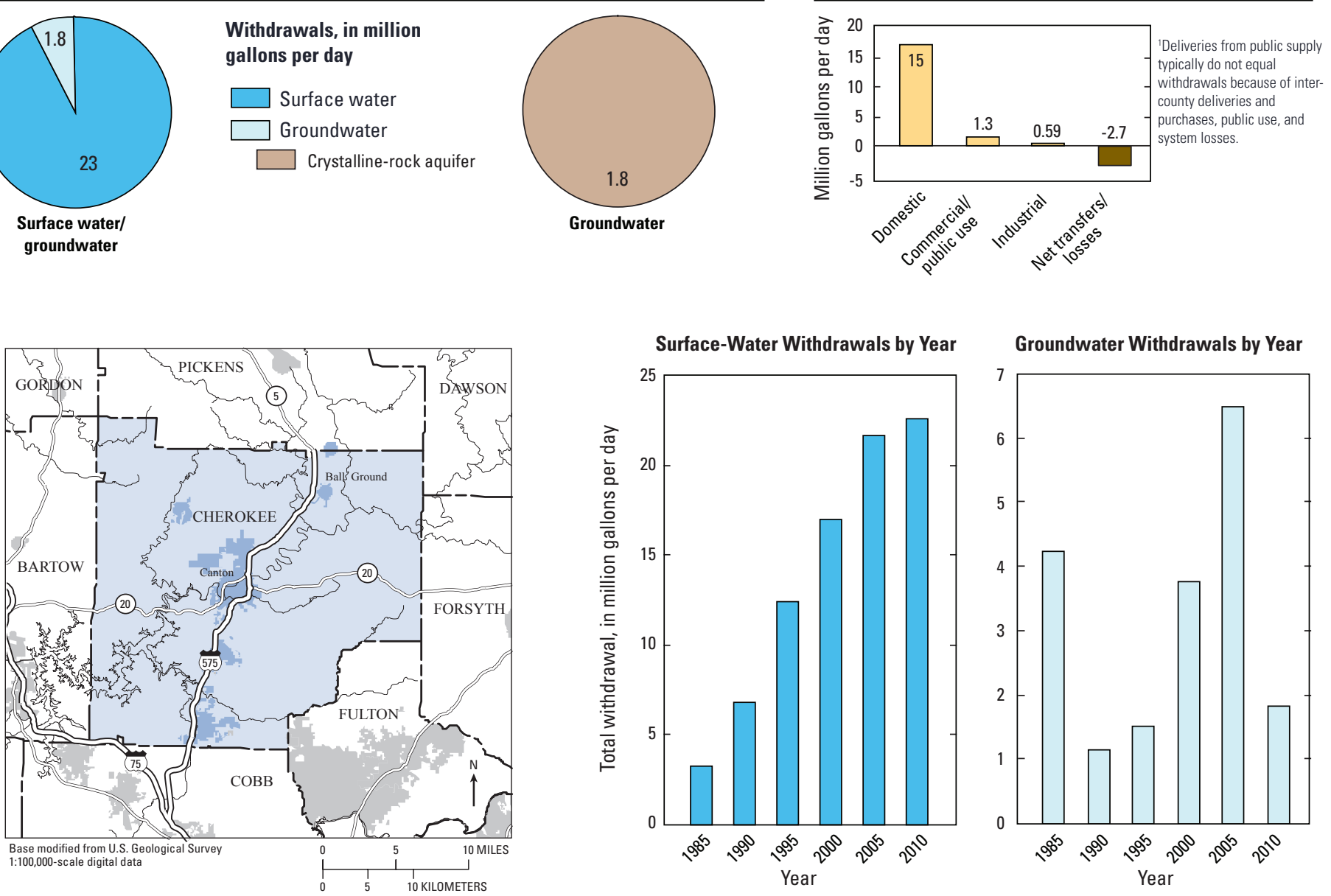

Groundwater Withdrawals by Year

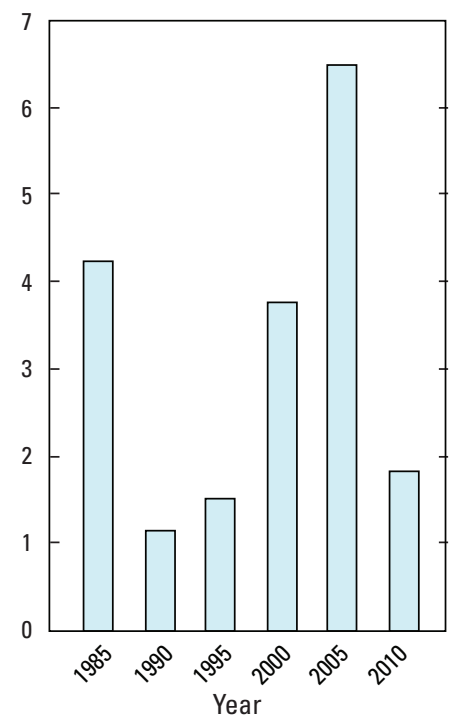




\section{CLARKE COUNTY}

Population

116,714

Population served by public supply-Groundwater

Population served by public supply-Surface water 100,240

Acres irrigated

410

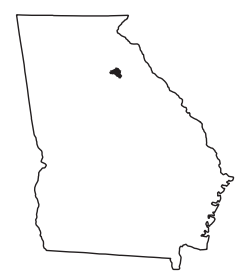

2010 WATER WITHDRAWALS AND ESTIMATED USE, IN MILLION GALLONS PER DAY

$[-$, not applicable; Mgal/d, million gallons per day $]$

\begin{tabular}{|c|c|c|c|c|c|c|c|c|}
\hline \multirow{3}{*}{ Category } & \multicolumn{3}{|c|}{ Withdrawals } & \multirow{3}{*}{$\begin{array}{l}\text { Total } \\
\text { use }^{1}\end{array}$} & \multirow{3}{*}{$\begin{array}{l}\text { Surface- } \\
\text { water } \\
\text { returns } \\
\end{array}$} & \multirow{2}{*}{\multicolumn{3}{|c|}{$\begin{array}{l}\text { Withdrawals by Major Industrial Groups } \\
\text { [NAICS, North American Industrial Classification System code] }\end{array}$}} \\
\hline & \multirow{2}{*}{$\begin{array}{c}\text { Ground- } \\
\text { water }\end{array}$} & \multirow{2}{*}{$\begin{array}{c}\text { Surface } \\
\text { water }\end{array}$} & \multirow[b]{2}{*}{ Total } & & & & & \\
\hline & & & & & & NAICS & Groundwater & Surface water \\
\hline Public supply & 0.15 & 12.62 & 12.77 & - & - & None & - & - \\
\hline Domestic & 1.09 & 0.00 & 1.09 & 8.24 & 0.00 & & & \\
\hline Commercial/public use & 0.00 & 0.00 & 0.00 & 3.07 & 0.00 & & & \\
\hline Industrial & 0.00 & 0.00 & 0.00 & 2.30 & 0.00 & & & \\
\hline Public-supply losses & - & - & - & 2.23 & - & & & \\
\hline Public wastewater treatment & - & - & - & - & 12.38 & Withdrawals by Majc & r Public Supp & \\
\hline Mining & 0.00 & 0.00 & 0.00 & 0.00 & 0.00 & Name & Groundwater & Surface water \\
\hline Irrigation-Crop & 0.32 & 0.06 & 0.38 & 0.38 & 0.00 & & & \\
\hline Irrigation-Golf course & 0.05 & 0.80 & 0.85 & 0.85 & 0.00 & $\begin{array}{l}\text { Athens-Clarke County } \\
\text { Water System }\end{array}$ & 0.00 & 12.62 \\
\hline Livestock/aquaculture & 0.00 & 0.02 & 0.02 & 0.02 & 0.00 & & & \\
\hline
\end{tabular}

$\begin{array}{llllll}\text { Thermoelectric power } & 0.00 & 0.00 & 0.00 & 0.00 & 0.00\end{array}$

\begin{tabular}{llllll} 
TOTAL & 1.61 & 13.50 & 15.11 & 17.09 & 12.38 \\
\hline
\end{tabular}

'Total use is total withdrawal plus public supply deliveries and losses. An average of $2.2 \mathrm{Mgal} / \mathrm{d}$ was withdrawn in Jackson County, Georgia and delivered to Clark County, Georgia in 2010.

\section{Withdrawals by Source}

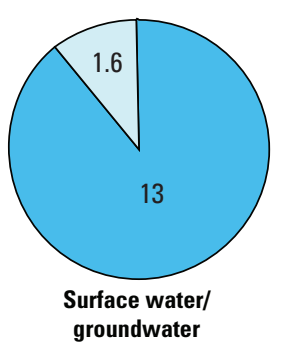

Withdrawals, in million gallons per day

Surface water
Groundwater
$\square$ Crystalline-rock aquifer

groundwater

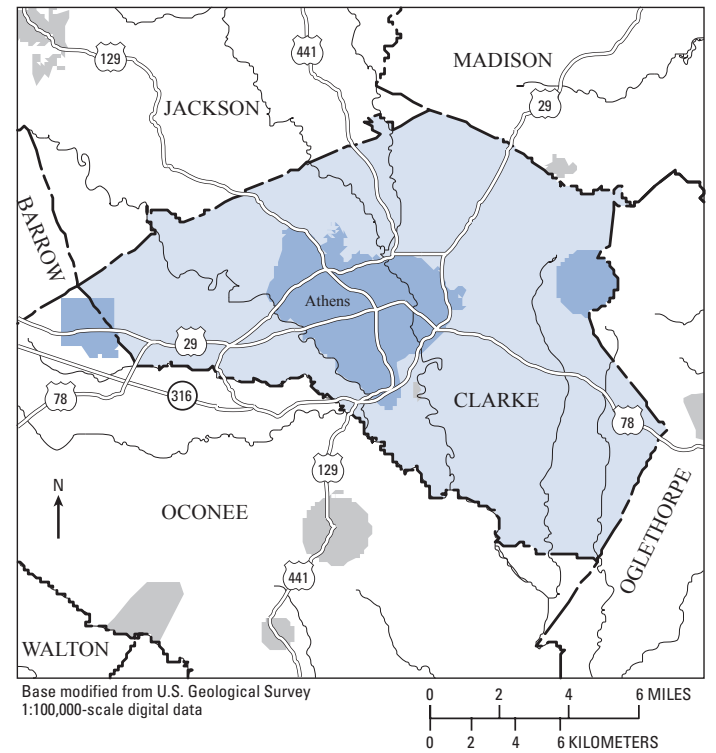

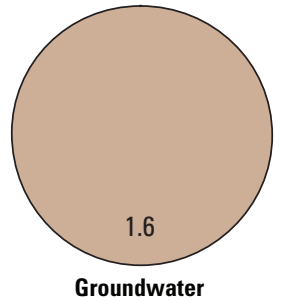

Public-Supply Deliveries ${ }^{1}$ by Use Category

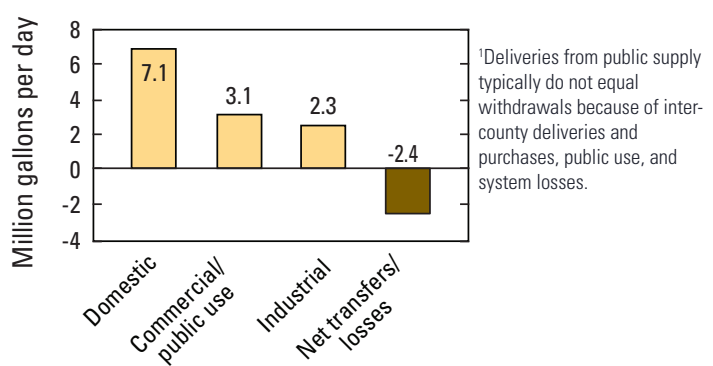

Surface-Water Withdrawals by Year

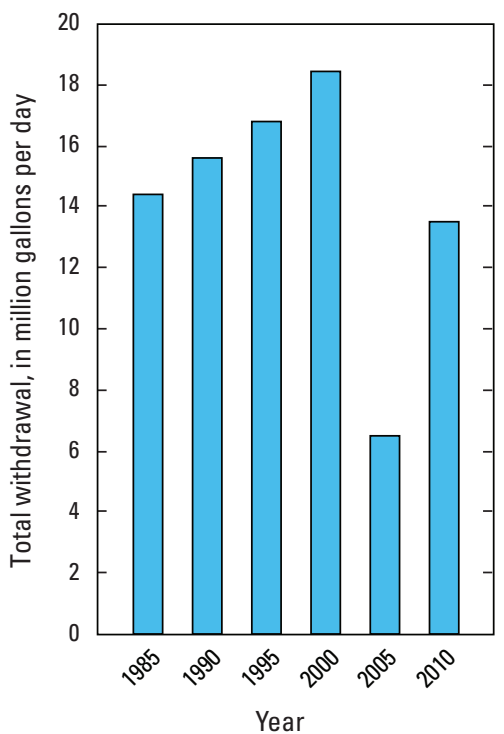

Groundwater Withdrawals by Year

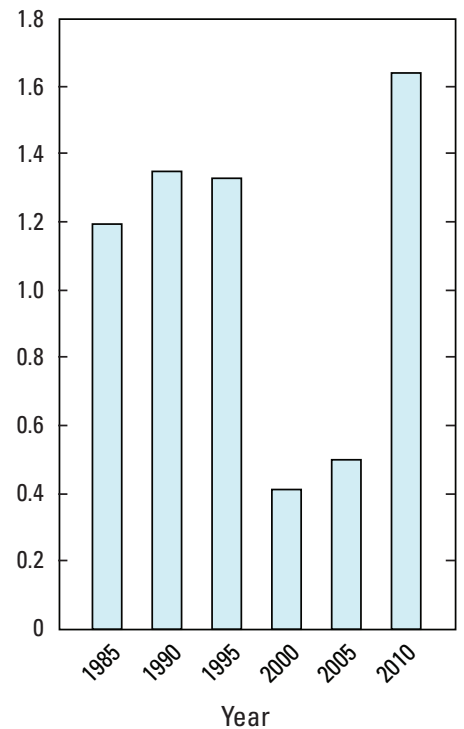




\section{CLAY COUNTY}

Population

3,183

Population served by public supply-Groundwater

Population served by public supply-Surface water Acres irrigated
1,700

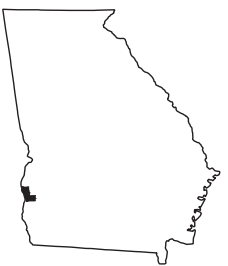

2010 WATER WITHDRAWALS AND ESTIMATED USE, IN MILLION GALLONS PER DAY

$[-$, not applicable $]$

\begin{tabular}{|c|c|c|c|c|c|c|c|c|}
\hline \multirow{3}{*}{ Category } & \multicolumn{3}{|c|}{ Withdrawals } & \multirow{3}{*}{$\begin{array}{l}\text { Total } \\
\text { use }^{1}\end{array}$} & \multirow{3}{*}{$\begin{array}{l}\text { Surface- } \\
\text { water } \\
\text { returns } \\
\end{array}$} & \multirow{2}{*}{\multicolumn{3}{|c|}{$\begin{array}{l}\text { Withdrawals by Major Industrial Groups } \\
\text { [NAICS, North American Industrial Classification System code] }\end{array}$}} \\
\hline & \multirow{2}{*}{$\begin{array}{c}\text { Ground- } \\
\text { water }\end{array}$} & \multirow{2}{*}{$\begin{array}{c}\text { Surface } \\
\text { water }\end{array}$} & \multirow[b]{2}{*}{ Total } & & & & & \\
\hline & & & & & & NAICS & Groundwater & Surface water \\
\hline Public supply & 0.29 & 0.00 & 0.29 & - & - & None & - & - \\
\hline Domestic & 0.11 & 0.00 & 0.11 & 0.34 & 0.00 & & & \\
\hline Commercial/public use & 0.00 & 0.00 & 0.00 & 0.03 & 0.00 & & & \\
\hline Industrial & 0.00 & 0.00 & 0.00 & 0.00 & 0.00 & & & \\
\hline Public-supply losses & - & - & - & 0.03 & - & & & \\
\hline Public wastewater treatment & - & - & - & - & 0.09 & Withdrawals by Major & Public Supp & \\
\hline Mining & 0.00 & 0.00 & 0.00 & 0.00 & 0.00 & Name & Groundwater & Surface water \\
\hline Irrigation-Crop & 1.91 & 1.86 & 3.77 & 3.77 & 0.00 & City of Fort Gaines & 0.25 & 0.00 \\
\hline Irrigation-Golf course & 0.33 & 0.00 & 0.33 & 0.33 & 0.00 & City of Fort Gaines & 0.25 & 0.00 \\
\hline Livestock/aquaculture & 0.04 & 0.04 & 0.08 & 0.08 & 0.00 & Clay County Water System & 0.04 & 0.00 \\
\hline
\end{tabular}

Thermoelectric power

1.90

4.58

0.09

${ }^{1}$ Total use is total withdrawal plus public supply deliveries and losses.
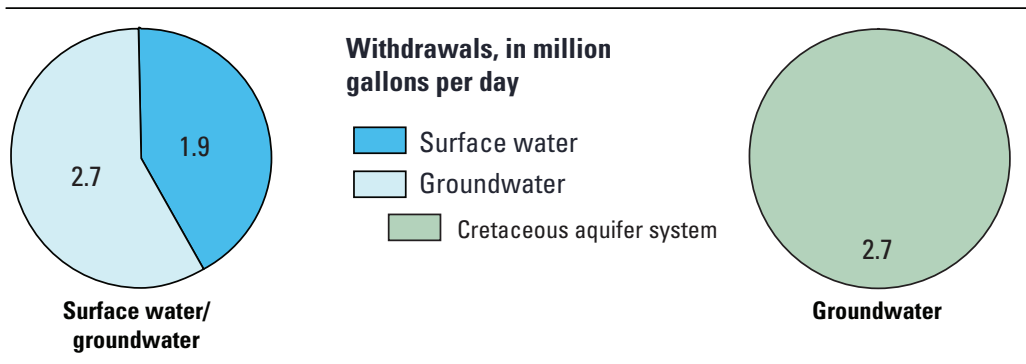

Public-Supply Deliveries by Use Category
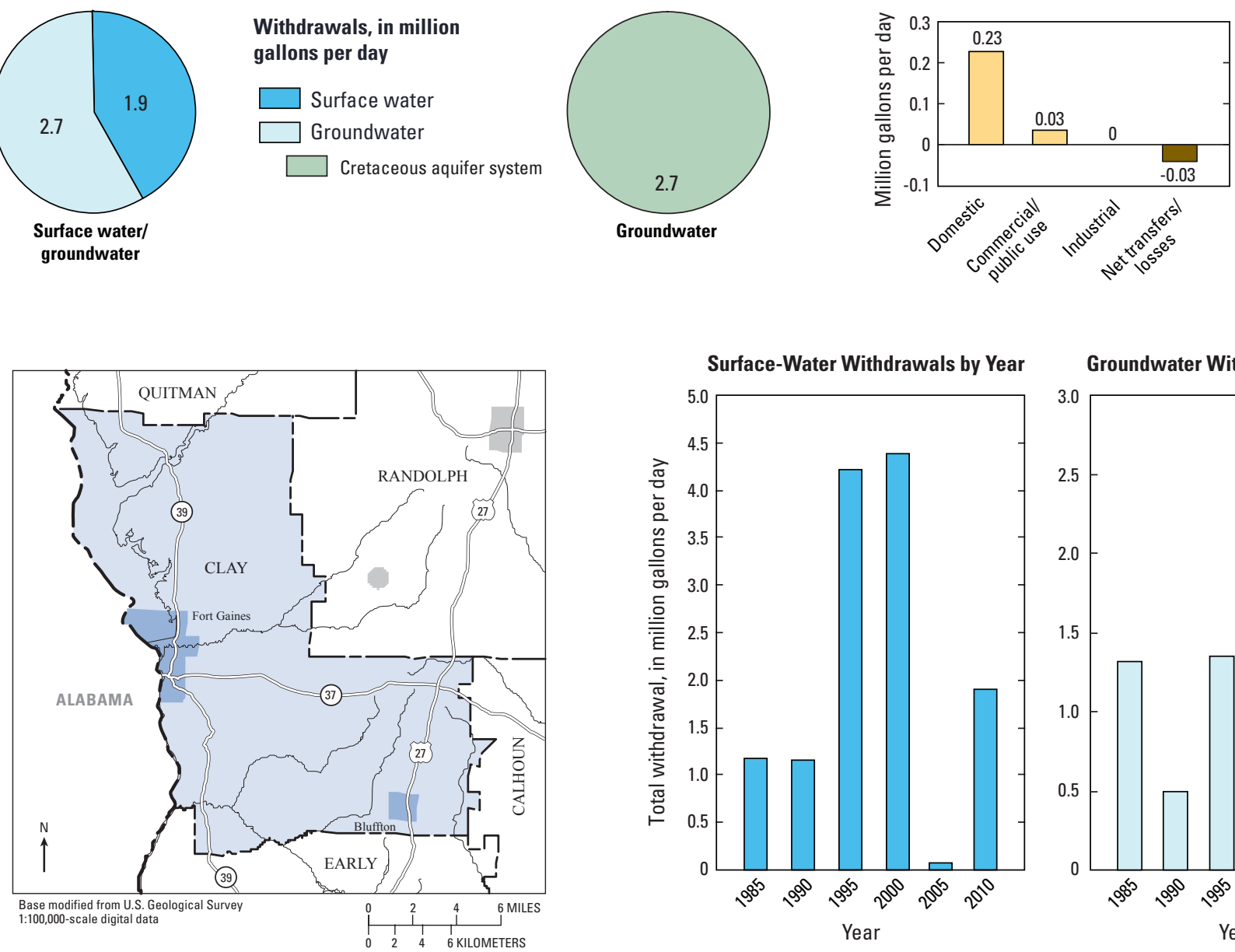

Surface-Water Withdrawals by Year

Groundwater Withdrawals by Year
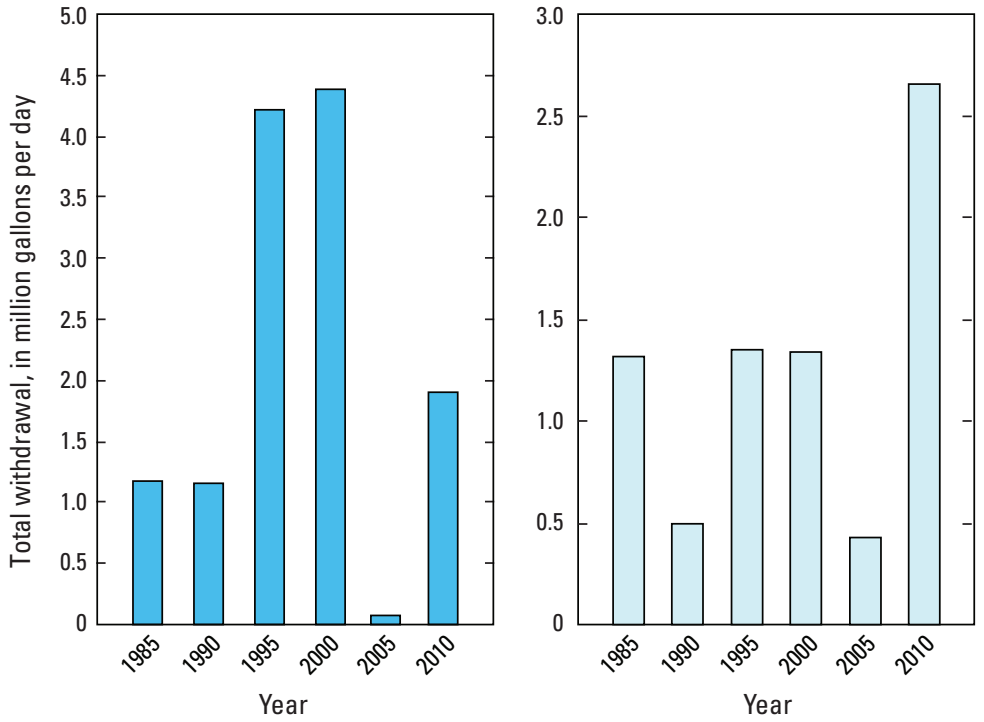


\section{CLAYTON COUNTY}

Population

259,424

Population served by public supply-Groundwater

Population served by public supply-Surface water 258,820

Acres irrigated

60

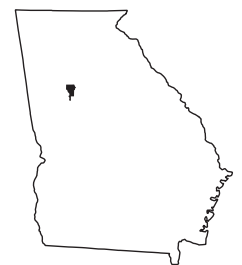

2010 WATER WITHDRAWALS AND ESTIMATED USE, IN MILLION GALLONS PER DAY (Mgal/d)

[-, not applicable; Mgal/d, million gallons per day]

\begin{tabular}{|c|c|c|c|c|c|}
\hline \multirow[b]{2}{*}{ Category } & \multicolumn{3}{|c|}{ Withdrawals } & \multirow[b]{2}{*}{$\begin{array}{l}\text { Total } \\
\text { use }^{1} \\
\end{array}$} & \multirow{2}{*}{$\begin{array}{c}\text { Surface- } \\
\text { water } \\
\text { returns }\end{array}$} \\
\hline & $\begin{array}{c}\text { Ground- } \\
\text { water }\end{array}$ & $\begin{array}{c}\text { Surface } \\
\text { water }\end{array}$ & Total & & \\
\hline Public supply & 0.05 & 9.30 & 9.35 & - & - \\
\hline Domestic & 0.04 & 0.00 & 0.04 & 19.38 & 0.00 \\
\hline Commercial/public use & 0.00 & 0.00 & 0.00 & 2.06 & 0.00 \\
\hline Industrial & 0.00 & 0.00 & 0.00 & 1.65 & 0.00 \\
\hline Public-supply losses & - & - & - & 4.13 & - \\
\hline Public wastewater treatment & - & - & - & - & 1.73 \\
\hline Mining & 0.23 & 0.02 & 0.25 & 0.25 & 0.00 \\
\hline Irrigation-Crop & 0.00 & 0.00 & 0.00 & 0.00 & 0.00 \\
\hline Irrigation-Golf course & 0.00 & 0.16 & 0.16 & 0.16 & 0.00 \\
\hline Livestock/aquaculture & 0.01 & 0.00 & 0.01 & 0.01 & 0.00 \\
\hline Thermoelectric power & 0.00 & 0.00 & 0.00 & 0.00 & 0.00 \\
\hline TOTAL & 0.33 & 9.48 & 9.81 & 27.64 & 2.20 \\
\hline
\end{tabular}

${ }^{1}$ Total use is total withdrawal plus public supply deliveries and losses. A net average of $0.06 \mathrm{Mgal} / \mathrm{d}$ was delivered from Fulton County, Georgi to Clayton County, Georgia in 2010.

\section{Withdrawals by Source}
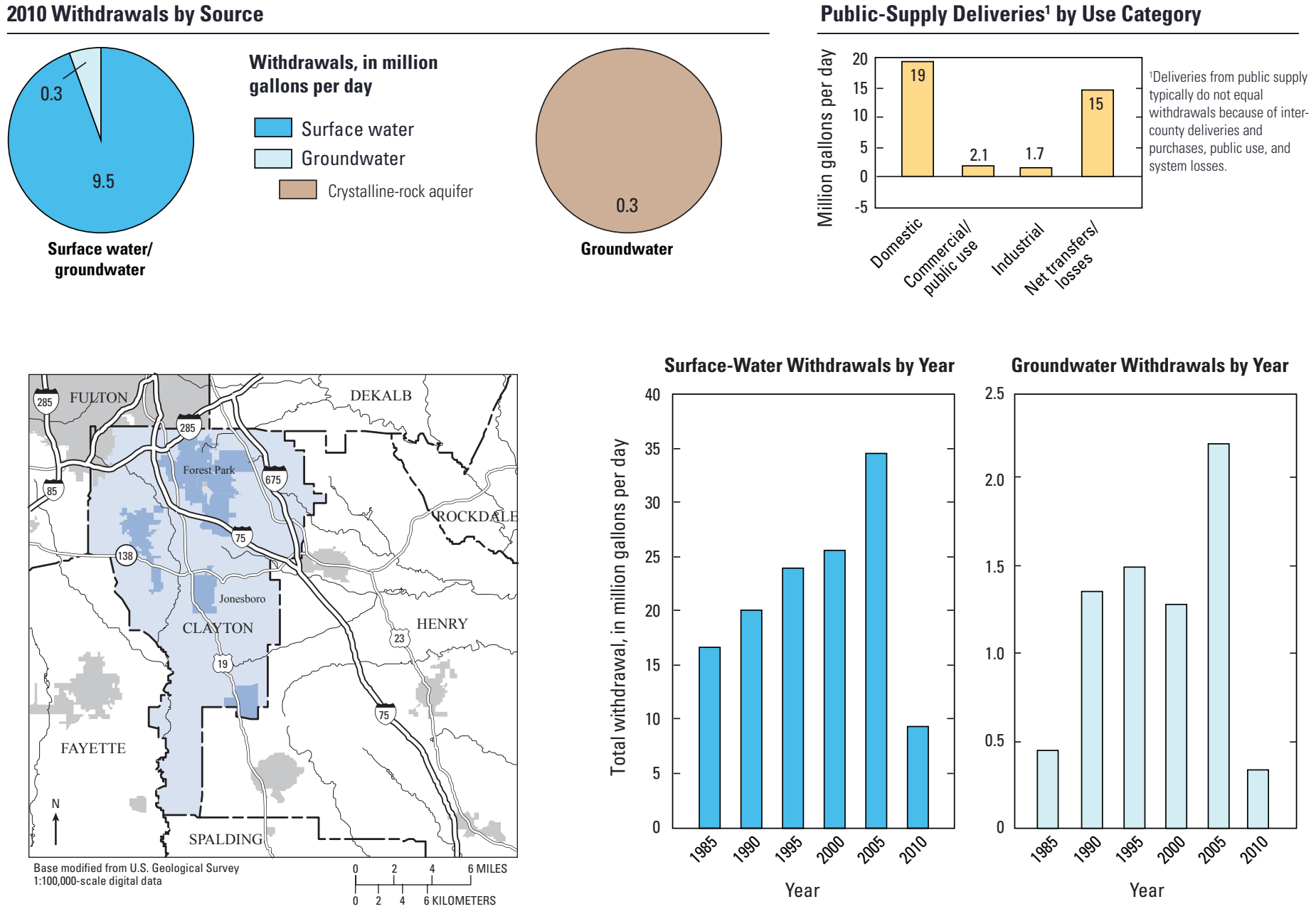

Groundwater Withdrawals by Year

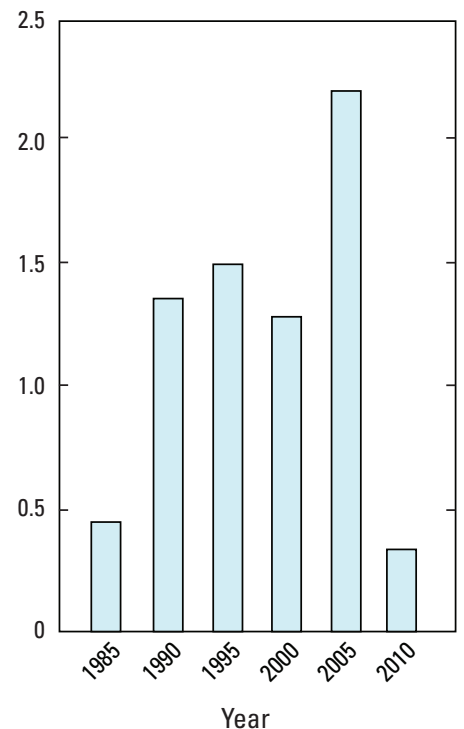

Withdrawals by Major Public Suppliers

\begin{tabular}{lcc}
\hline Name & Groundwater & Surface water \\
\hline $\begin{array}{lc}\text { Clayton County Water } \\
\text { Authority }^{1}\end{array}$ & 0.05 & 9.30 \\
\hline${ }^{1}$ An average of $18.1 \mathrm{Mgal} / \mathrm{d}$ of additional surface water was withdrawn from Henry County,
\end{tabular}

Georgia and delivered to Clayton County, Georgia in 2010.

[NAICS, North American Industrial Classification System code]

\begin{tabular}{lcc}
\hline NAICS & Groundwater & Surface water \\
\hline None & - & - \\
\hline
\end{tabular}

Public-Supply Deliveries' by Use Category 


\section{CLINCH COUNTY}

Population

6,798

Population served by public supply-Groundwater

Population served by public supply-Surface water Acres irrigated
3,840

2,840

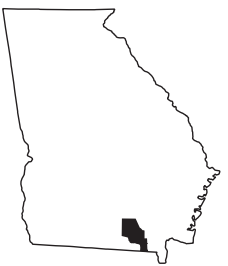

2010 WATER WITHDRAWALS AND ESTIMATED USE, IN MILLION GALLONS PER DAY

$[-$, not applicable $]$

\begin{tabular}{|c|c|c|c|c|c|c|c|c|}
\hline \multirow{3}{*}{ Category } & \multicolumn{3}{|c|}{ Withdrawals } & \multirow{3}{*}{$\begin{array}{l}\text { Total } \\
\text { use }^{1}\end{array}$} & \multirow{3}{*}{$\begin{array}{l}\text { Surface- } \\
\text { water } \\
\text { returns } \\
\end{array}$} & \multirow{2}{*}{\multicolumn{3}{|c|}{$\begin{array}{l}\text { Withdrawals by Major Industrial Groups } \\
\text { [NAICS, North American Industrial Classification System code] }\end{array}$}} \\
\hline & \multirow{2}{*}{$\begin{array}{c}\text { Ground- } \\
\text { water }\end{array}$} & \multirow{2}{*}{$\begin{array}{c}\text { Surface } \\
\text { water }\end{array}$} & \multirow[b]{2}{*}{ Total } & & & & & \\
\hline & & & & & & NAICS & Groundwater & Surface water \\
\hline Public supply & 0.48 & 0.00 & 0.48 & - & - & 332 -Fabrication & 0.05 & 0.00 \\
\hline Domestic & 0.22 & 0.00 & 0.22 & 0.50 & 0.00 & $532-$ rabricalion & 0.05 & 0.00 \\
\hline Commercial/public use & 0.00 & 0.00 & 0.00 & 0.07 & 0.00 & & & \\
\hline Industrial & 0.05 & 0.00 & 0.05 & 0.12 & 0.00 & & & \\
\hline Public-supply losses & - & - & - & 0.06 & - & & & \\
\hline Public wastewater treatment & - & - & - & - & 0.26 & Withdrawals by I & r Public Supp & \\
\hline Mining & 0.00 & 0.00 & 0.00 & 0.00 & 0.00 & Name & Groundwater & Surface water \\
\hline Irrigation-Crop & 0.00 & 0.00 & 0.00 & 0.00 & 0.00 & & 0.43 & 0.00 \\
\hline Irrigation-Golf course & 0.00 & 0.00 & 0.00 & 0.00 & 0.00 & City of Homerville & 0.43 & 0.00 \\
\hline Livestock/aquaculture & 0.00 & 0.01 & 0.01 & 0.01 & 0.00 & & & \\
\hline Thermoelectric power & 0.00 & 0.00 & 0.00 & 0.00 & 0.00 & & & \\
\hline TOTAL & 0.75 & 0.01 & 0.76 & 0.76 & 0.26 & & & \\
\hline
\end{tabular}

\section{Withdrawals by Source}
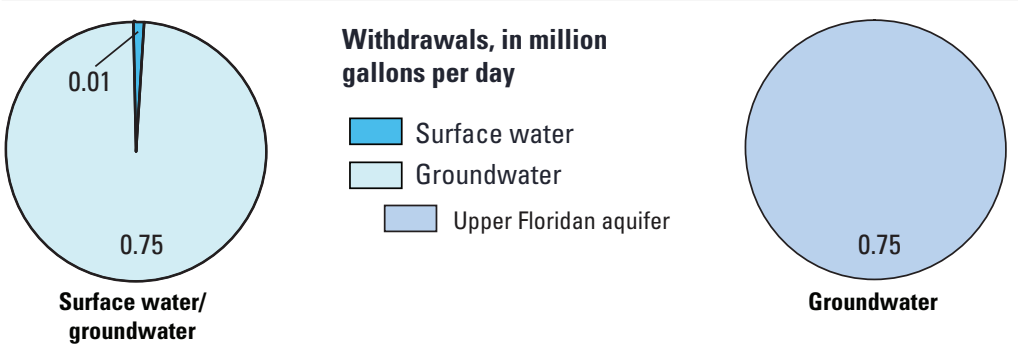

Public-Supply Deliveries by Use Category
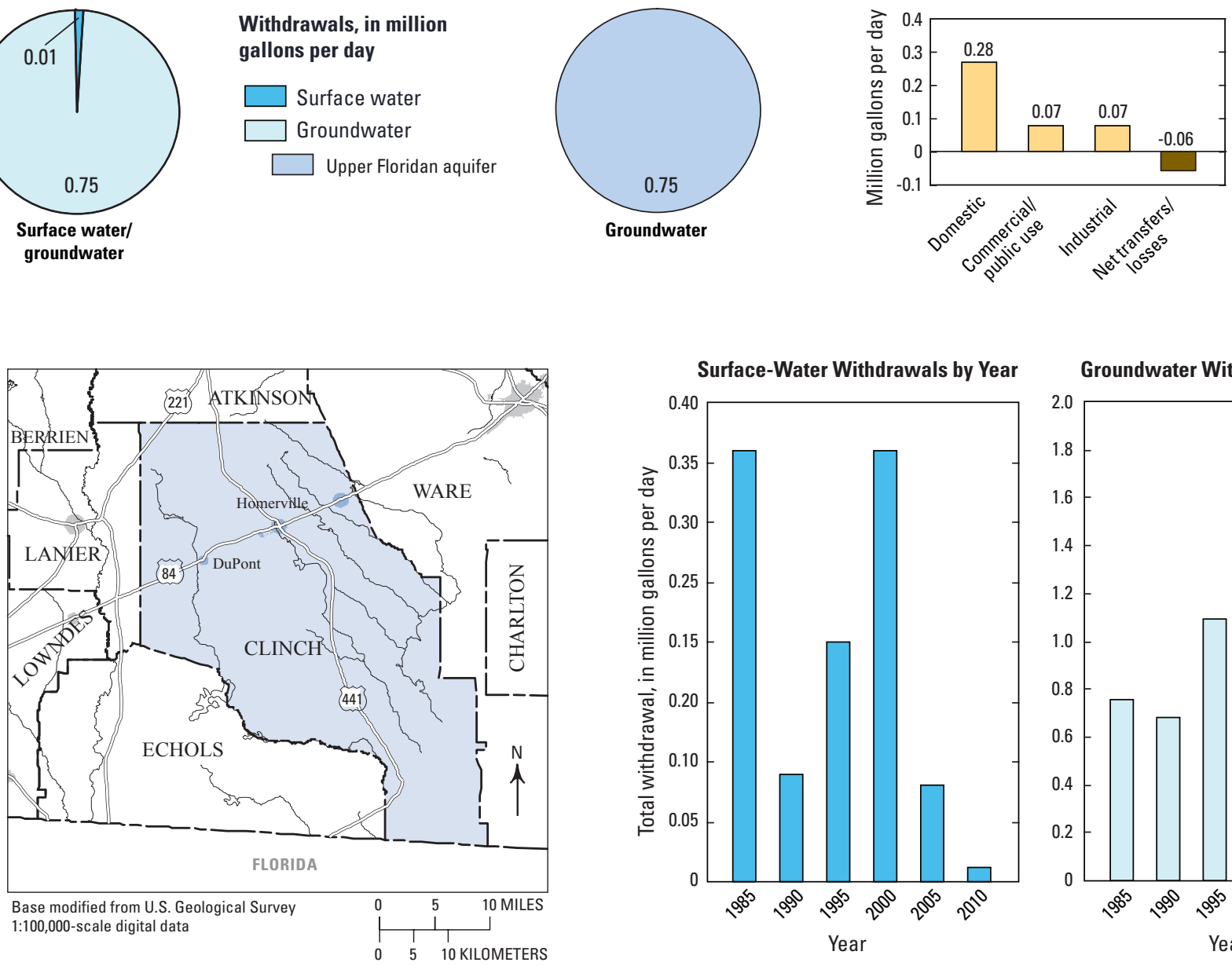

Groundwater Withdrawals by Year

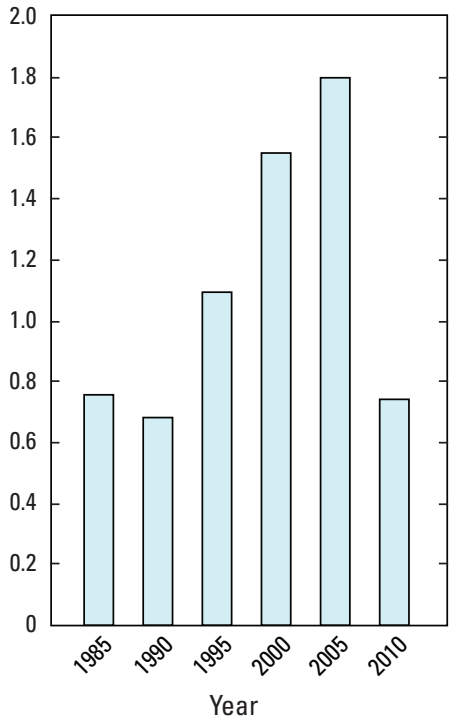




\section{COBB COUNTY}

Population

688,078

Population served by public supply-Groundwater

Population served by public supply-Surface water 645,080

Acres irrigated

680

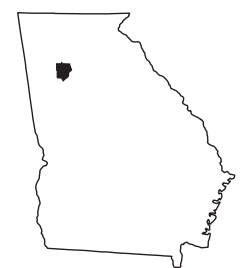

2010 WATER WITHDRAWALS AND ESTIMATED USE, IN MILLION GALLONS PER DAY (Mgal/d)

[-, not applicable; Mgal/d, million gallons per day]

\begin{tabular}{|c|c|c|c|c|c|}
\hline \multirow[b]{2}{*}{ Category } & \multicolumn{3}{|c|}{ Withdrawals } & \multirow[b]{2}{*}{$\begin{array}{l}\text { Total } \\
\text { use }^{1}\end{array}$} & \multirow{2}{*}{$\begin{array}{c}\text { Surface- } \\
\text { water } \\
\text { returns }\end{array}$} \\
\hline & $\begin{array}{c}\text { Ground- } \\
\text { water }\end{array}$ & $\begin{array}{c}\text { Surface } \\
\text { water }\end{array}$ & Total & & \\
\hline Public supply & 0.57 & 43.27 & 43.84 & - & - \\
\hline Domestic & 3.22 & 0.00 & 3.22 & 38.03 & 0.00 \\
\hline Commercial/public use & 0.00 & 0.00 & 0.00 & 23.54 & 0.00 \\
\hline Industrial & 0.00 & 0.39 & 0.39 & 3.41 & 0.00 \\
\hline Public-supply losses & - & - & - & 10.50 & - \\
\hline Public wastewater treatment & - & - & - & - & 77.44 \\
\hline Mining & 0.00 & 0.00 & 0.00 & 0.00 & 0.92 \\
\hline Irrigation-Crop & 0.15 & 0.00 & 0.15 & 0.15 & 0.00 \\
\hline Irrigation-Golf course & 0.34 & 1.48 & 1.82 & 1.82 & 0.00 \\
\hline Livestock/aquaculture & 0.00 & 0.02 & 0.02 & 0.02 & 0.00 \\
\hline Thermoelectric power & 0.00 & 5.25 & 5.25 & 5.25 & 1.00 \\
\hline TOTAL & 4.28 & 50.41 & 54.69 & 82.72 & 79.42 \\
\hline
\end{tabular}

Withdrawals by Major Industrial Groups

[NAICS, North American Industrial Classification System code]

\begin{tabular}{lcc}
\hline NAICS & Groundwater & Surface water \\
\hline $322-$ Paper, pulp & 0.00 & 0.39 \\
\hline
\end{tabular}

Withdrawals by Major Public Suppliers

\begin{tabular}{lcc}
\hline Name & Groundwater & Surface water \\
\hline Cobb County-Marietta & 0.00 & 43.28 \\
Water Authority $^{1}$ & & 0.00 \\
Cobb County Water System & 0.57 &
\end{tabular}

${ }^{1}$ An average of $40.1 \mathrm{Mgal} / \mathrm{d}$ was withdrawn from Bartow County, Georgia and delivered to Cobb County, Georgia in 2010.

\section{Public-Supply Deliveries ${ }^{1}$ by Use Category}
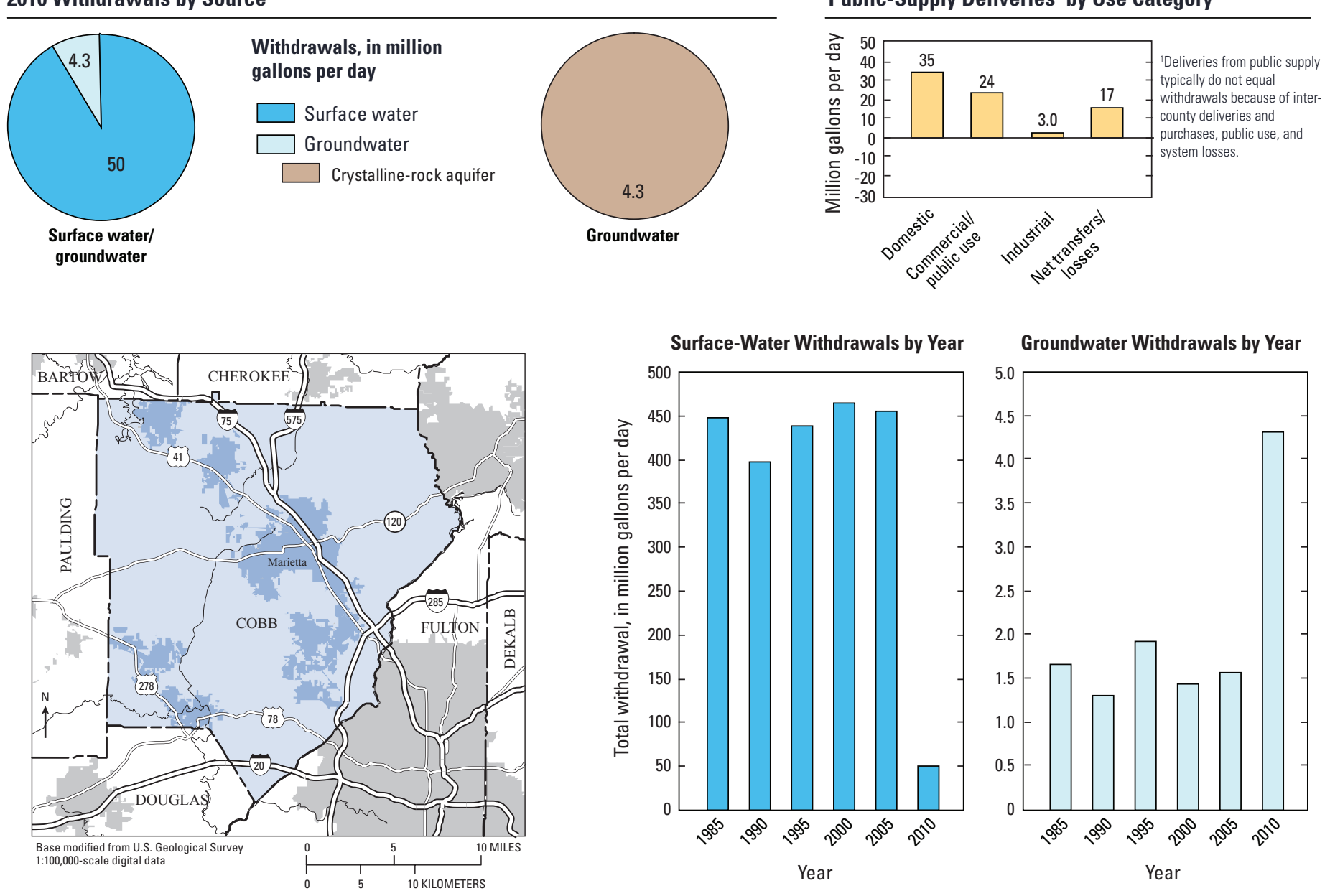

Groundwater Withdrawals by Year

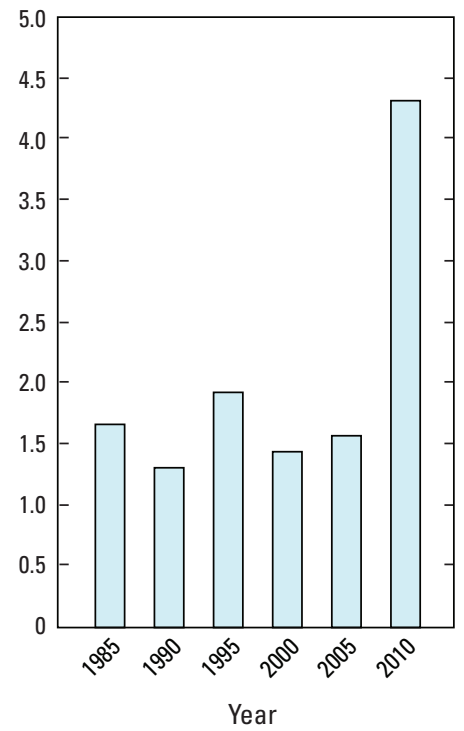


COFFEE COUNTY

Population

Population served by public supply-Groundwater

Population served by public supply-Surface water

Acres irrigated
42,356

21,140

23,000

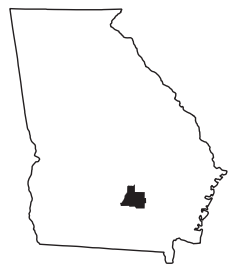

2010 WATER WITHDRAWALS AND ESTIMATED USE, IN MILLION GALLONS PER DAY

[-, not applicable]

\begin{tabular}{|c|c|c|c|c|c|c|c|c|}
\hline \multirow{3}{*}{ Category } & \multicolumn{3}{|c|}{ Withdrawals } & \multirow{3}{*}{$\begin{array}{l}\text { Total } \\
\text { use }^{1}\end{array}$} & \multirow{3}{*}{$\begin{array}{l}\text { Surface- } \\
\text { water } \\
\text { returns } \\
\end{array}$} & \multirow{2}{*}{\multicolumn{3}{|c|}{$\begin{array}{l}\text { Withdrawals by Major Industrial Groups } \\
\text { [NAICS, North American Industrial Classification System code] }\end{array}$}} \\
\hline & \multirow{2}{*}{$\begin{array}{c}\text { Ground- } \\
\text { water }\end{array}$} & \multirow{2}{*}{$\begin{array}{c}\text { Surface } \\
\text { water }\end{array}$} & \multirow[b]{2}{*}{ Total } & & & & & \\
\hline & & & & & & NAICS & Groundwater & Surface water \\
\hline Public supply & 3.48 & 0.00 & 3.48 & - & - & None & - & - \\
\hline Domestic & 1.59 & 0.00 & 1.59 & 3.31 & 0.00 & & & \\
\hline Commercial/public use & 0.00 & 0.00 & 0.00 & 0.97 & 0.00 & & & \\
\hline Industrial & 0.00 & 0.00 & 0.00 & 0.32 & 0.00 & & & \\
\hline Public-supply losses & - & - & - & 0.47 & - & & & \\
\hline Public wastewater treatment & - & - & - & - & 2.33 & & & \\
\hline Mining & 0.00 & 0.00 & 0.00 & 0.00 & 0.00 & Withdrawals b & Public Supp & \\
\hline Irrigation-Crop & 1.91 & 0.87 & 2.78 & 2.78 & 0.00 & Name & Groundwater & Surface water \\
\hline $\begin{array}{l}\text { Irrigation-Golf course } \\
\text { Livestock/aquaculture }\end{array}$ & $\begin{array}{l}0.27 \\
0.06\end{array}$ & $\begin{array}{l}0.43 \\
0.52\end{array}$ & $\begin{array}{l}0.70 \\
0.58\end{array}$ & $\begin{array}{l}0.70 \\
0.58\end{array}$ & $\begin{array}{l}0.00 \\
0.00\end{array}$ & City of Douglas & 2.48 & 0.00 \\
\hline Thermoelectric power & 0.00 & 0.00 & 0.00 & 0.00 & 0.00 & City of Nicholls & 0.37 & 0.00 \\
\hline TOTAL & 7.31 & 1.82 & 9.13 & 9.13 & 2.33 & City of Broxton & 0.14 & 0.00 \\
\hline
\end{tabular}

${ }^{1}$ Total use is total withdrawal plus public supply deliveries and losses.

\section{Withdrawals by Source}

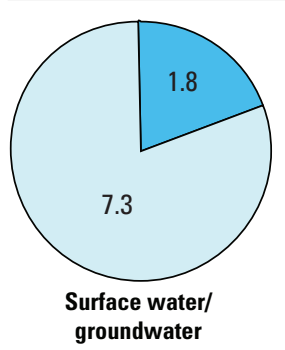

Withdrawals, in million gallons per day

Surface wate

Groundwater

Upper Floridan aquifer

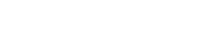

groundwater

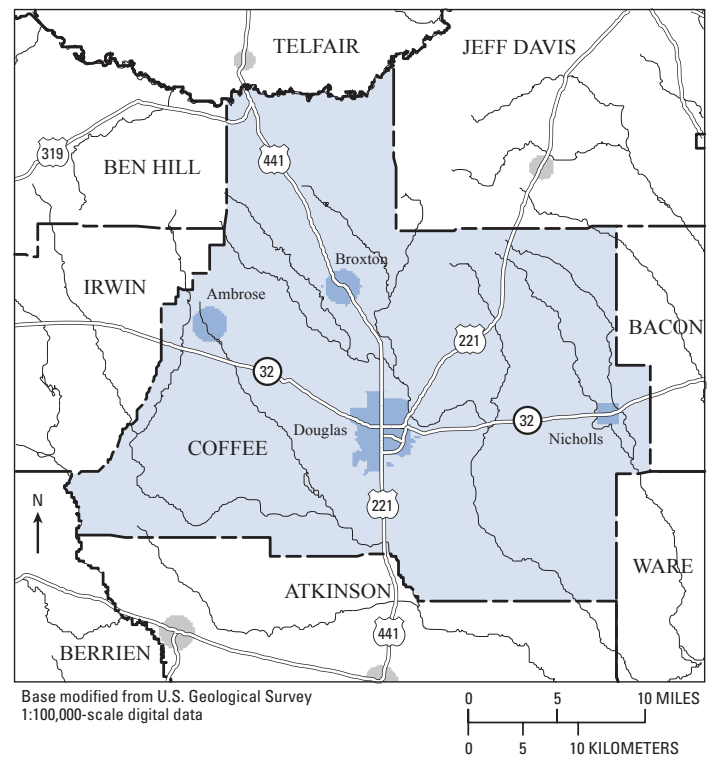

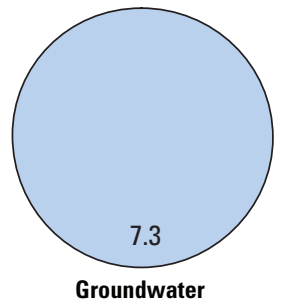

Public-Supply Deliveries by Use Category

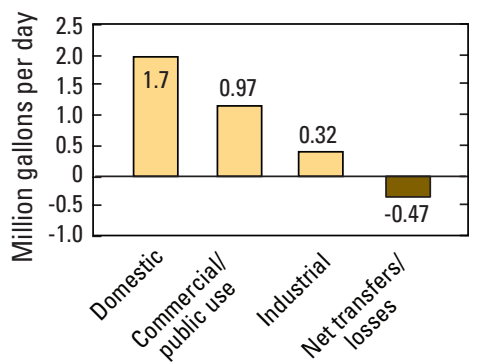

Surface-Water Withdrawals by Year

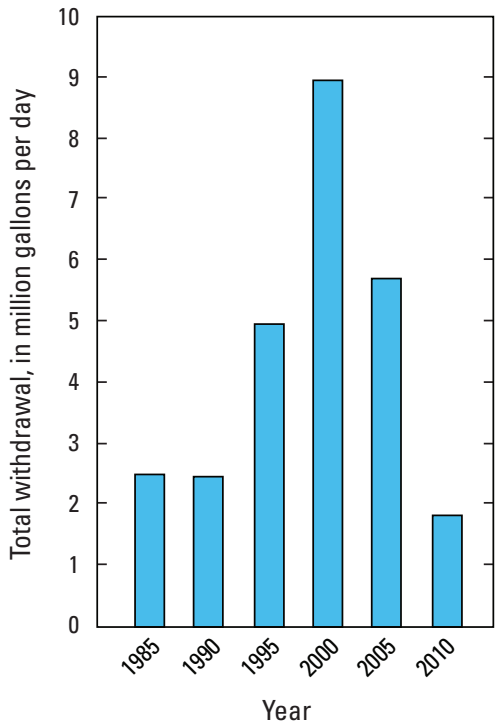

Groundwater Withdrawals by Year

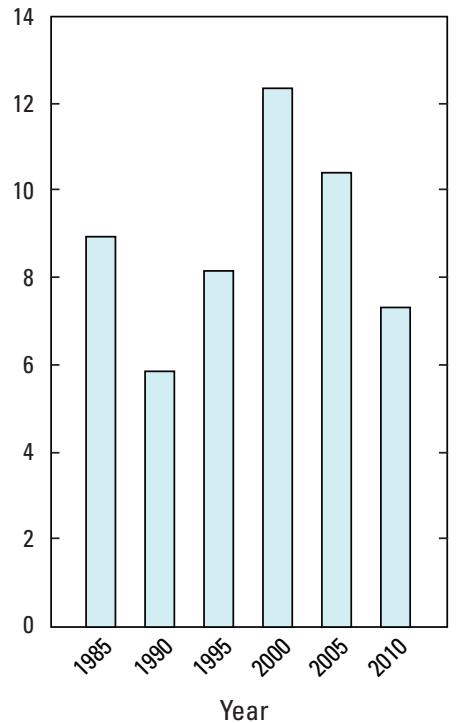




\section{COLOUITT COUNTY}

Population

Population served by public supply-Groundwater

Population served by public supply-Surface water

Acres irrigated
45,498

23,270

44,920

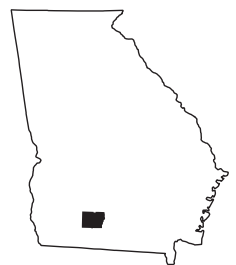

2010 WATER WITHDRAWALS AND ESTIMATED USE, IN MILLION GALLONS PER DAY

[-, not applicable]

\begin{tabular}{|c|c|c|c|c|c|c|c|c|}
\hline \multirow{3}{*}{ Category } & \multicolumn{3}{|c|}{ Withdrawals } & \multirow{3}{*}{$\begin{array}{l}\text { Total } \\
\text { use }^{1}\end{array}$} & \multirow{3}{*}{$\begin{array}{l}\text { Surface- } \\
\text { water } \\
\text { returns } \\
\end{array}$} & \multirow{2}{*}{\multicolumn{3}{|c|}{$\begin{array}{l}\text { Withdrawals by Major Industrial Groups } \\
\text { [NAICS, North American Industrial Classification System code] }\end{array}$}} \\
\hline & \multirow{2}{*}{$\begin{array}{c}\text { Ground- } \\
\text { water }\end{array}$} & \multirow{2}{*}{$\begin{array}{c}\text { Surface } \\
\text { water }\end{array}$} & \multirow[b]{2}{*}{ Total } & & & & & \\
\hline & & & & & & NAICS & Groundwater & Surface water \\
\hline Public supply & 3.64 & 0.00 & 3.64 & - & - & 311-Food & 1.14 & 0.00 \\
\hline Domestic & 1.67 & 0.00 & 1.67 & 3.43 & 0.00 & $311-\mathrm{F} 00 \mathrm{~d}$ & 1.14 & 0.00 \\
\hline Commercial/public use & 0.00 & 0.00 & 0.00 & 0.75 & 0.00 & & & \\
\hline Industrial & 1.14 & 0.00 & 1.14 & 1.22 & 0.00 & & & \\
\hline Public-supply losses & - & - & - & 1.05 & - & & & \\
\hline Public wastewater treatment & - & - & - & - & 3.10 & Withdrawals by Major & Public Supp & \\
\hline Mining & 0.00 & 0.00 & 0.00 & 0.00 & 0.00 & & Groundwater & Surface water \\
\hline Irrigation-Crop & 7.80 & 8.47 & 16.27 & 16.27 & 0.00 & Name & Grounawater & \\
\hline Irrigation-Golf course & 0.00 & 0.00 & 0.00 & 0.00 & 0.00 & City of Moultrie & 2.98 & 0.00 \\
\hline Livestock/aquaculture & 0.13 & 0.52 & 0.65 & 0.65 & 0.00 & City of Norman Park & 0.18 & 0.00 \\
\hline Thermoelectric power & 0.00 & 0.00 & 0.00 & 0.00 & 0.00 & Water Service Company & & \\
\hline TOTAL & 14.38 & 8.99 & 23.37 & 23.37 & 3.10 & of Georgia, Inc. & 0.16 & 0.00 \\
\hline
\end{tabular}

${ }^{1}$ Total use is total withdrawal plus public supply deliveries and losses.

\section{Withdrawals by Source}

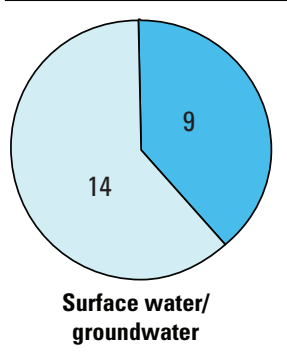

Withdrawals, in million gallons per day

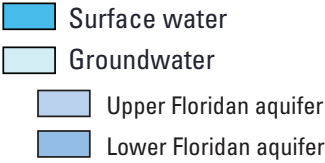
groundwater

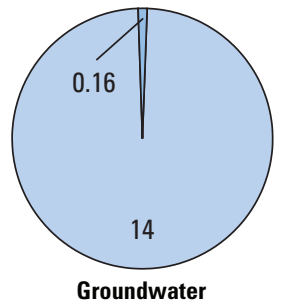

Public-Supply Deliveries by Use Category

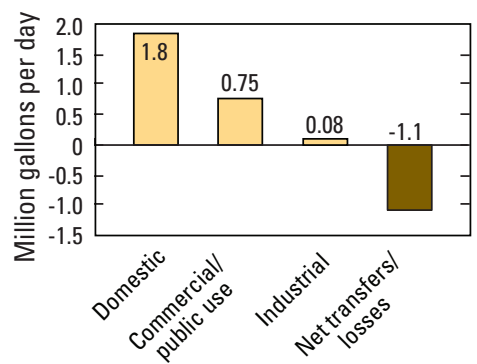

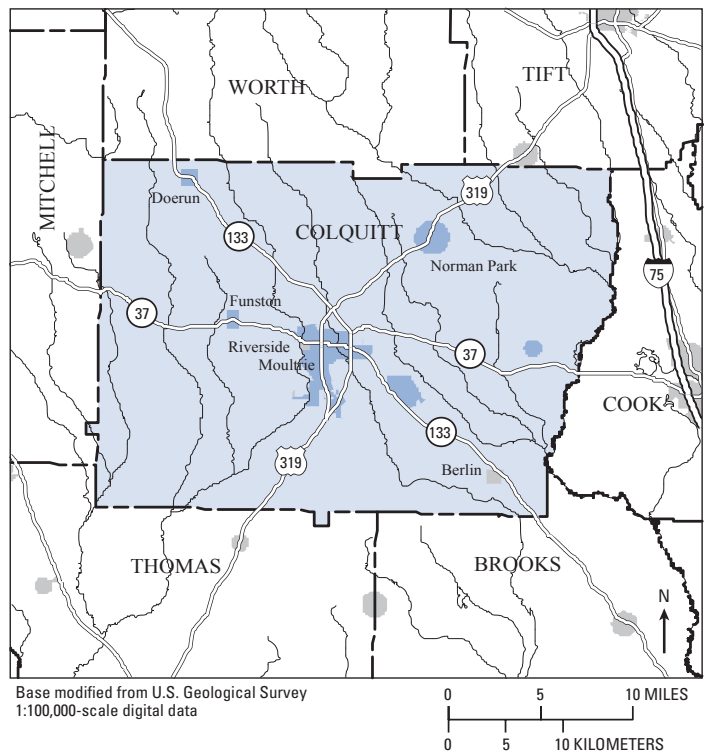

Surface-Water Withdrawals by Year

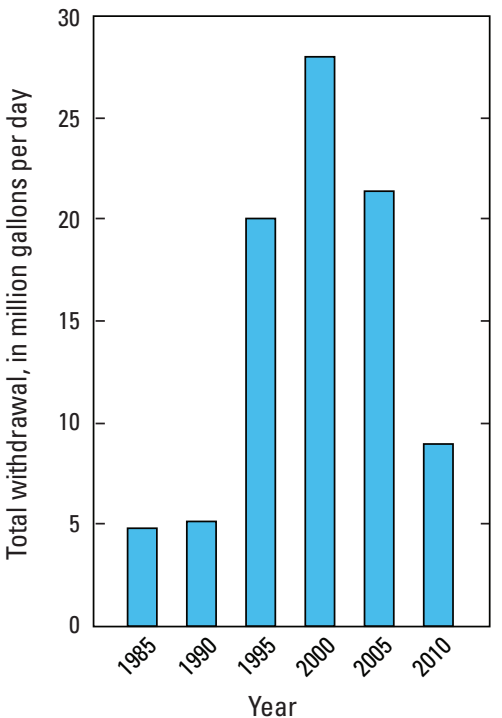

Groundwater Withdrawals by Year

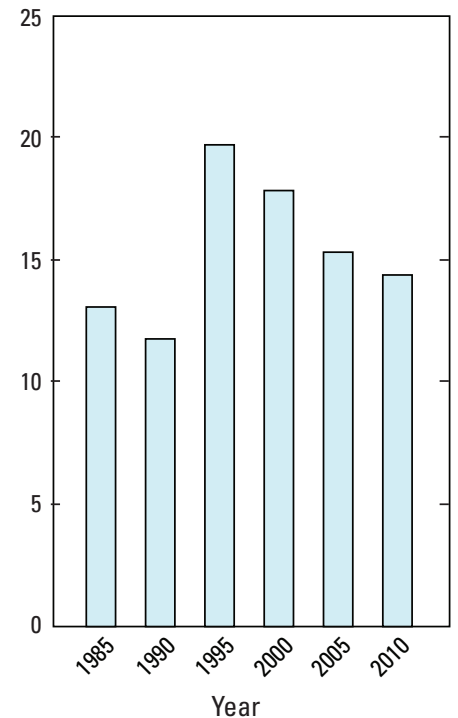


COLUMBIA COUNTY

Population

124,053

Population served by public supply-Groundwater $\quad 1,380$

Population served by public supply-Surface water 104,530

Acres irrigated
600

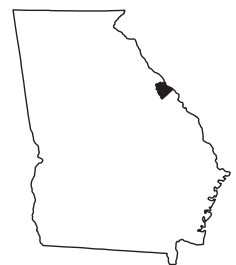

2010 WATER WITHDRAWALS AND ESTIMATED USE, IN MILLION GALLONS PER DAY

$[-$, not applicable $]$

\begin{tabular}{|c|c|c|c|c|c|c|c|c|}
\hline \multirow{3}{*}{ Category } & \multicolumn{3}{|c|}{ Withdrawals } & \multirow{3}{*}{$\begin{array}{l}\text { Total } \\
\text { use }^{1}\end{array}$} & \multirow{3}{*}{$\begin{array}{c}\text { Surface- } \\
\text { water } \\
\text { returns } \\
\end{array}$} & \multirow{2}{*}{\multicolumn{3}{|c|}{$\begin{array}{l}\text { Withdrawals by Major Industrial Groups } \\
\text { [NAICS, North American Industrial Classification System code] }\end{array}$}} \\
\hline & \multirow{2}{*}{$\begin{array}{c}\text { Ground- } \\
\text { water }\end{array}$} & \multirow{2}{*}{$\begin{array}{c}\text { Surface } \\
\text { water }\end{array}$} & \multirow[b]{2}{*}{ Total } & & & & & \\
\hline & & & & & & NAICS & Groundwater & Surface water \\
\hline Public supply & 0.13 & 16.06 & 16.19 & - & - & 312 - Beverages & 0.03 & 0.00 \\
\hline Domestic & 1.36 & 0.00 & 1.36 & 11.97 & 0.00 & & & \\
\hline Commercial/public use & 0.00 & 0.00 & 0.00 & 4.02 & 0.00 & & & \\
\hline Industrial & 0.03 & 0.00 & 0.03 & 0.19 & 0.00 & & & \\
\hline Public-supply losses & - & - & - & 1.40 & - & & & \\
\hline Public wastewater treatment & - & - & - & - & 7.57 & Withdrawals by I & r Public Supp & \\
\hline Mining & 0.40 & 0.00 & 0.40 & 0.40 & 1.28 & Name & Groundwater & Surface water \\
\hline Irrigation-Crop & 0.10 & 0.06 & 0.16 & 0.16 & 0.00 & & & \\
\hline Irrigation-Golf course & 0.13 & 0.77 & 0.90 & 0.90 & 0.00 & Columbia County & 0.00 & 16.06 \\
\hline Livestock/aquaculture & 0.00 & 0.04 & 0.04 & 0.04 & 0.00 & City of Groveland & 0.09 & 0.00 \\
\hline Thermoelectric power & 0.00 & 0.00 & 0.00 & 0.00 & 0.00 & City of Harlem & 0.02 & 0.00 \\
\hline
\end{tabular}

'Total use is total withdrawal plus public supply deliveries and losses.

\section{Withdrawals by Source}
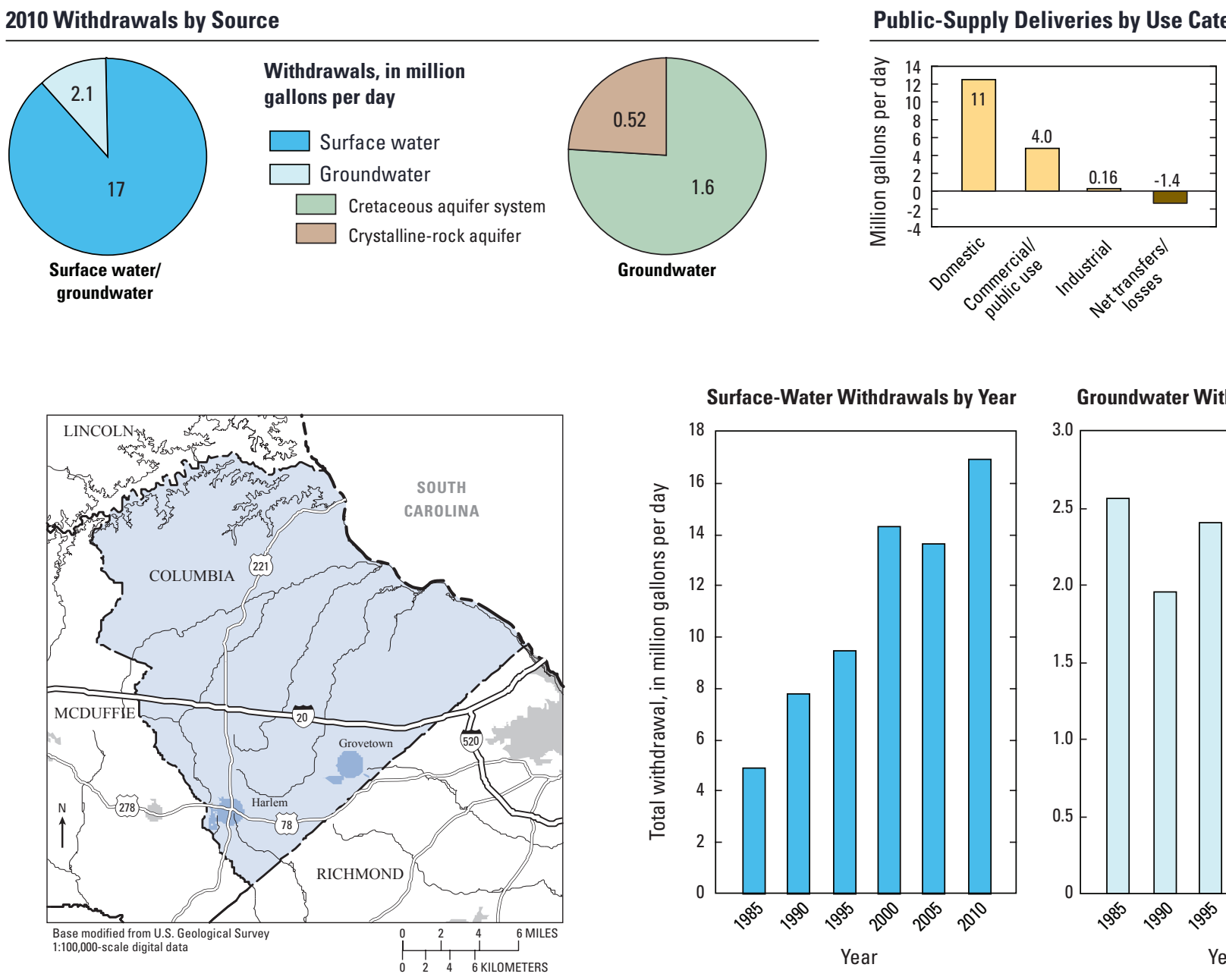

Groundwater Withdrawals by Year

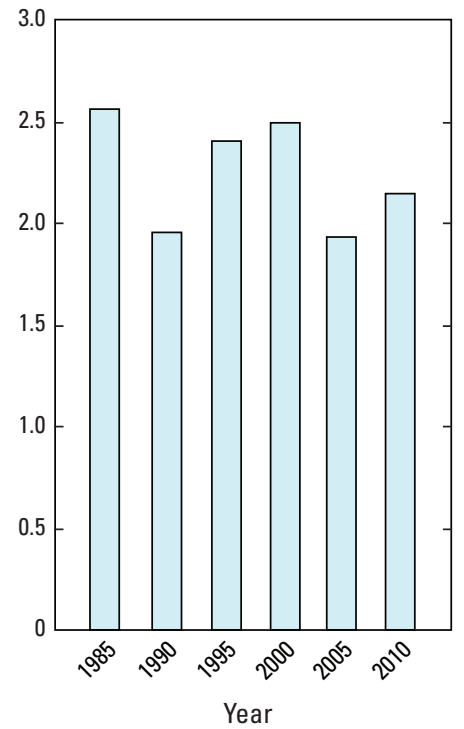




\section{COOK COUNTY}

Population

17,212

Population served by public supply-Groundwater

Population served by public supply-Surface water

Acres irrigated
10,200

10,460

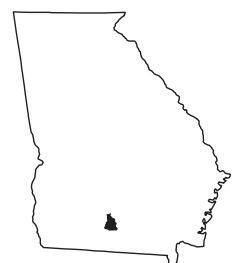

2010 WATER WITHDRAWALS AND ESTIMATED USE, IN MILLION GALLONS PER DAY

$[-$, not applicable $]$

\begin{tabular}{|c|c|c|c|c|c|}
\hline \multirow[b]{2}{*}{ Category } & \multicolumn{3}{|c|}{ Withdrawals } & \multirow[b]{2}{*}{$\begin{array}{l}\text { Total } \\
\text { use }^{1}\end{array}$} & \multirow{2}{*}{$\begin{array}{c}\text { Surface- } \\
\text { water } \\
\text { returns }\end{array}$} \\
\hline & $\begin{array}{c}\text { Ground- } \\
\text { water }\end{array}$ & $\begin{array}{c}\text { Surface } \\
\text { water }\end{array}$ & Total & & \\
\hline Public supply & 1.80 & 0.00 & 1.80 & - & - \\
\hline Domestic & 0.53 & 0.00 & 0.53 & 1.66 & 0.00 \\
\hline Commercial/public use & 0.00 & 0.00 & 0.00 & 0.36 & 0.01 \\
\hline Industrial & 0.00 & 0.00 & 0.00 & 0.03 & 0.00 \\
\hline Public-supply losses & - & - & - & 0.28 & - \\
\hline Public wastewater treatment & - & - & - & - & 1.25 \\
\hline Mining & 0.21 & 0.00 & 0.21 & 0.21 & 0.00 \\
\hline Irrigation-Crop & 4.10 & 0.88 & 4.98 & 4.98 & 0.00 \\
\hline Irrigation-Golf course & 0.00 & 0.20 & 0.20 & 0.20 & 0.00 \\
\hline Livestock/aquaculture & 0.03 & 0.13 & 0.16 & 0.16 & 0.00 \\
\hline Thermoelectric power & 0.00 & 0.00 & 0.00 & 0.00 & 0.00 \\
\hline TOTAL & 6.67 & 1.21 & 7.88 & 7.88 & 1.26 \\
\hline
\end{tabular}

Withdrawals by Major Industrial Groups

[NAICS, North American Industrial Classification System code]

NAICS

Groundwater

Surface water

None

Withdrawals by Major Public Suppliers

\begin{tabular}{lcc}
\hline Name & Groundwater & Surface water \\
\hline City of Adel & 1.26 & 0.00 \\
City of Sparks & 0.42 & 0.00 \\
\hline
\end{tabular}

Total use is total withdrawal plus public supply deliveries and losses.

\section{Withdrawals by Source}
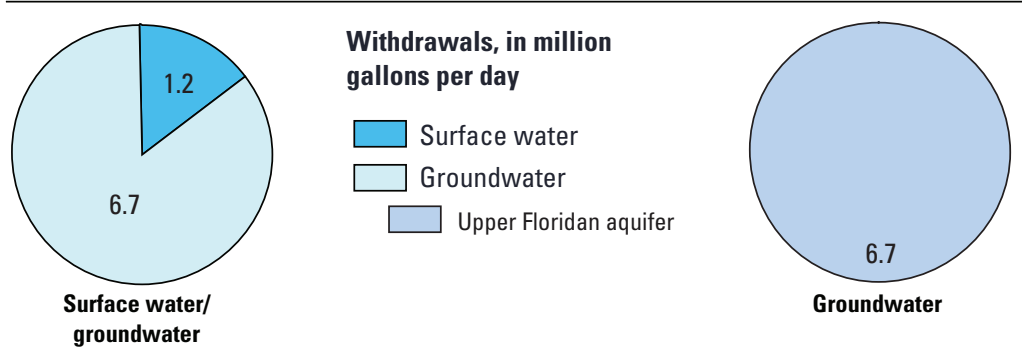

Public-Supply Deliveries by Use Category
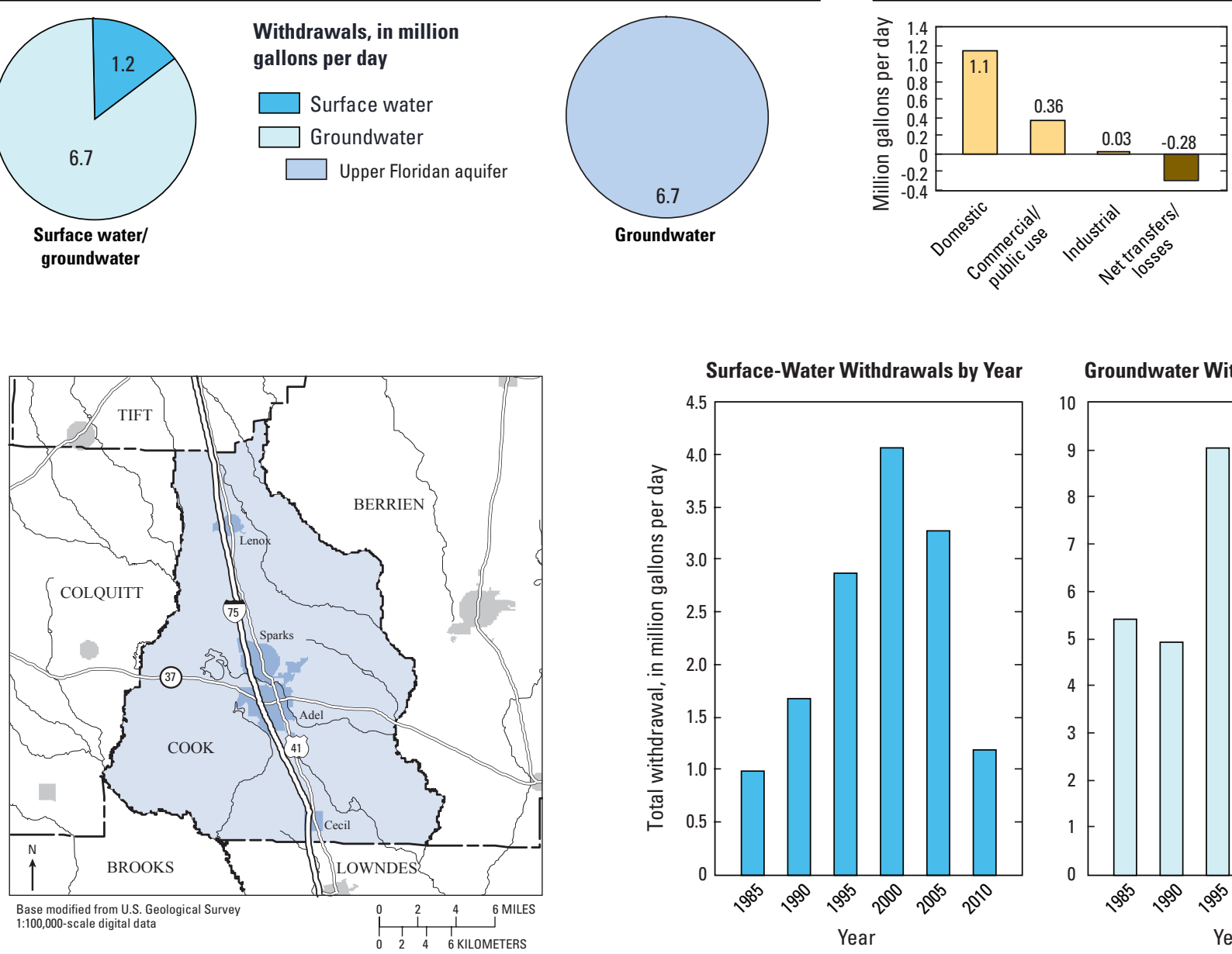

Groundwater Withdrawals by Year

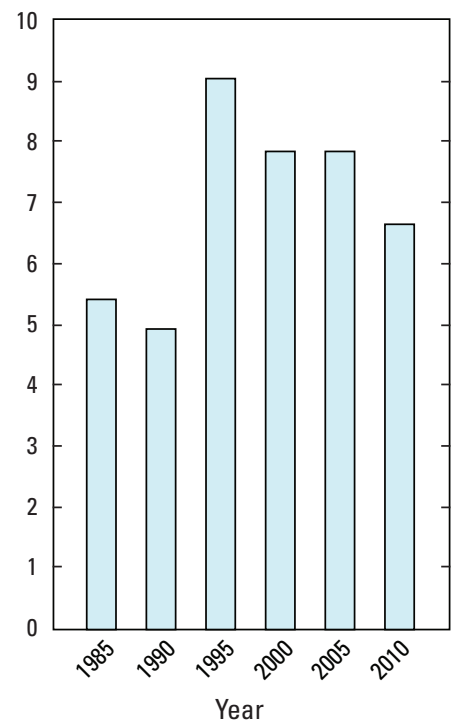




\section{COWETA COUNTY}

Population

127,317

Population served by public supply-Groundwater $\quad 6,990$

Population served by public supply-Surface water 108,300

Acres irrigated
810

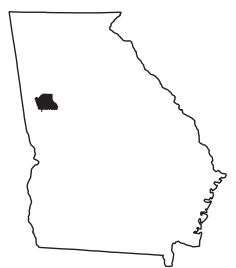

2010 WATER WITHDRAWALS AND ESTIMATED USE, IN MILLION GALLONS PER DAY

$[-$, not applicable; Mgal/d, million gallons per day]

\begin{tabular}{|c|c|c|c|c|c|c|c|c|}
\hline \multirow{3}{*}{ Category } & \multicolumn{3}{|c|}{ Withdrawals } & \multirow{3}{*}{$\begin{array}{l}\text { Total } \\
\text { use }^{1}\end{array}$} & \multirow{3}{*}{$\begin{array}{c}\text { Surface- } \\
\text { water } \\
\text { returns } \\
\end{array}$} & \multirow{2}{*}{\multicolumn{3}{|c|}{$\begin{array}{l}\text { Withdrawals by Major Industrial Groups } \\
\text { [NAICS, North American Industrial Classification System code] }\end{array}$}} \\
\hline & \multirow{2}{*}{$\begin{array}{l}\text { Ground- } \\
\text { water }\end{array}$} & \multirow{2}{*}{$\begin{array}{c}\text { Surface } \\
\text { water }\end{array}$} & \multirow[b]{2}{*}{ Total } & & & & & \\
\hline & & & & & & NAICS & Groundwater & Surface water \\
\hline Public supply & 0.38 & 7.35 & 7.73 & - & - & None & - & - \\
\hline Domestic & 0.90 & 0.00 & 0.90 & 7.78 & 0.00 & Pvoll & & \\
\hline Commercial/public use & 0.00 & 0.00 & 0.00 & 1.14 & 0.00 & & & \\
\hline Industrial & 0.00 & 0.00 & 0.00 & 0.85 & 0.00 & & & \\
\hline Public-supply losses & - & - & - & 1.32 & - & Withdrawals by Maj & r Public Supp & \\
\hline Public wastewater treatment & - & - & - & - & 3.03 & Name & Groundwater & Surface water \\
\hline Mining & 0.12 & 0.00 & 0.12 & 0.12 & 0.00 & City of Newnan & 0.00 & 6.56 \\
\hline Irrigation-Crop & 0.02 & 0.11 & 0.13 & 0.13 & 0.00 & Coweta County Water & & \\
\hline Irrigation-Golf course & 0.00 & 1.59 & 1.59 & 1.59 & 0.00 & \& Sewer Authority & 0.03 & 0.60 \\
\hline Livestock/aquaculture & 0.00 & 0.07 & 0.07 & 0.07 & 0.00 & City of Senoia & 0.06 & 0.18 \\
\hline Thermoelectric power & 0.00 & 42.58 & 42.58 & 42.58 & 0.00 & Georgia Community & 0.13 & 0.00 \\
\hline TOTAL & 1.42 & 51.70 & 53.12 & 55.58 & 3.59 & Water, Inc. & 0.15 & \\
\hline
\end{tabular}

'Total use is total withdrawal plus public supply deliveries and losses. An average of $2.43 \mathrm{Mgal} / \mathrm{d}$ was delivered to Coweta County, Georgia

from Spalding County, Georgia and 0.036 Mgal/d was delivered from Fulton County, Georgia in 2010.

\section{Withdrawals by Source}

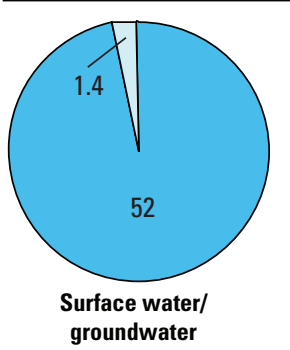

Withdrawals, in million gallons per day

$\square$ Surface water
$\square$ Groundwater
$\square$ Crystalline-rock aquife

groundwater

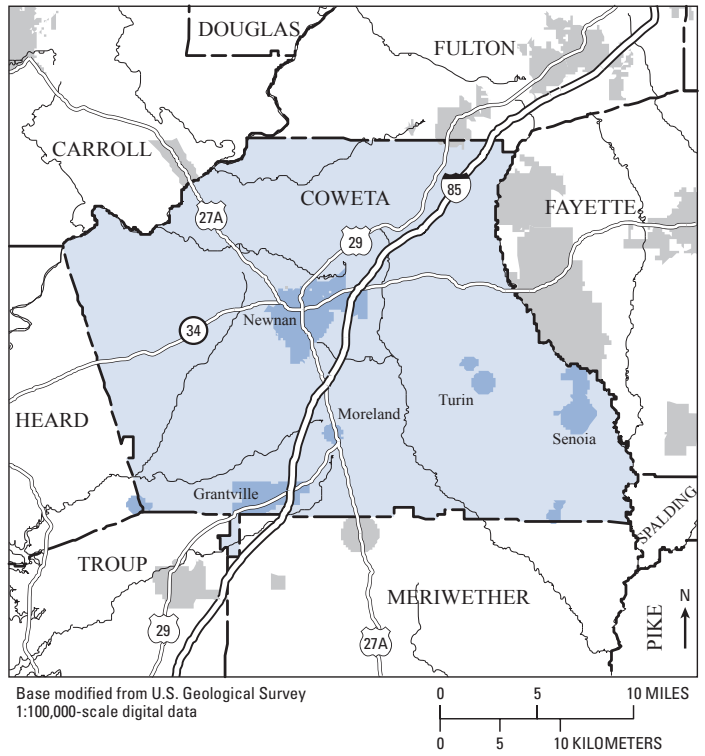

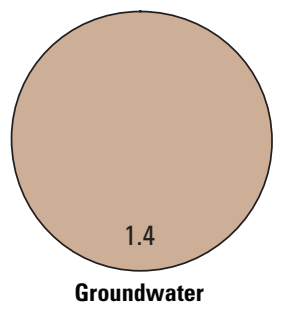

Public-Supply Deliveries' by Use Category

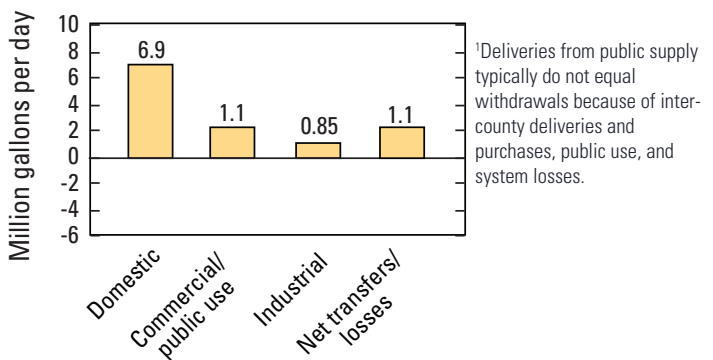

Surface-Water Withdrawals by Year

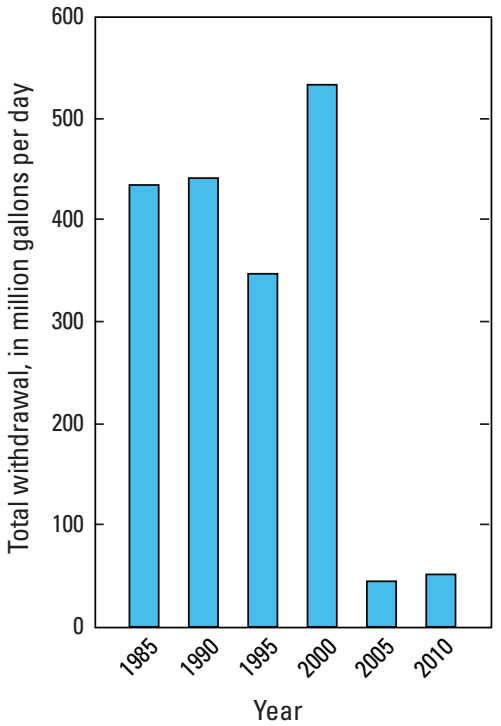

Groundwater Withdrawals by Year

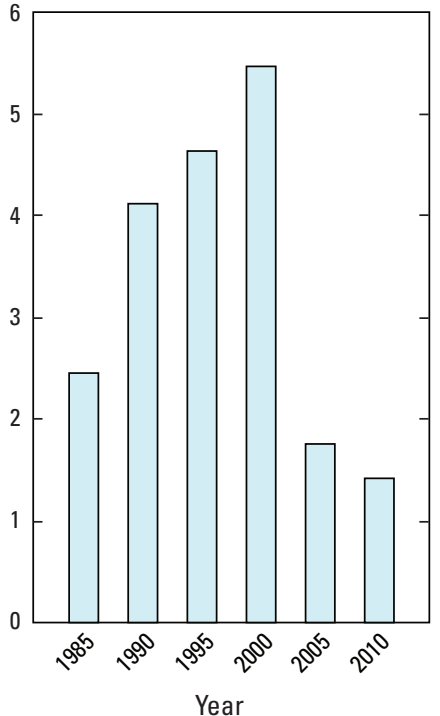




\section{CRAWFORD COUNTY}

Population

Population served by public supply-Groundwater $\quad 3,760$

Population served by public supply-Surface water 0

Acres irrigated

2,980

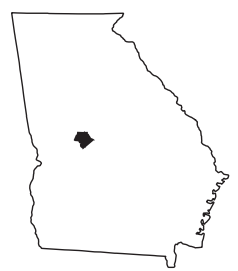

2010 WATER WITHDRAWALS AND ESTIMATED USE, IN MILLION GALLONS PER DAY

[-, not applicable]

\begin{tabular}{|c|c|c|c|c|c|c|c|c|}
\hline \multirow{3}{*}{ Category } & \multicolumn{3}{|c|}{ Withdrawals } & \multirow{3}{*}{$\begin{array}{l}\text { Total } \\
\text { use }^{1}\end{array}$} & \multirow{3}{*}{$\begin{array}{c}\text { Surface- } \\
\text { water } \\
\text { returns } \\
\end{array}$} & \multirow{2}{*}{\multicolumn{3}{|c|}{$\begin{array}{l}\text { Withdrawals by Major Industrial Groups } \\
\text { [NAICS, North American Industrial Classification System code] }\end{array}$}} \\
\hline & \multirow{2}{*}{$\begin{array}{l}\text { Ground- } \\
\text { water }\end{array}$} & \multirow{2}{*}{$\begin{array}{c}\text { Surface } \\
\text { water }\end{array}$} & \multirow[b]{2}{*}{ Total } & & & & & \\
\hline & & & & & & NAICS & Groundwater & Surface water \\
\hline Public supply & 0.35 & 0.00 & 0.35 & - & - & None & - & - \\
\hline Domestic & 0.67 & 0.00 & 0.67 & 0.93 & 0.00 & 18010 & & \\
\hline Commercial/public use & 0.00 & 0.00 & 0.00 & 0.04 & 0.00 & & & \\
\hline Industrial & 0.00 & 0.00 & 0.00 & 0.00 & 0.00 & & & \\
\hline Public-supply losses & - & - & - & 0.05 & - & & & \\
\hline Public wastewater treatment & - & - & - & - & 0.12 & Withdrawals by & r Public Supp & \\
\hline Mining & 0.38 & 0.01 & 0.39 & 0.39 & 0.00 & Name & Groundwater & Surface water \\
\hline Irrigation-Crop & 1.23 & 0.00 & 1.23 & 1.23 & 0.00 & Crawford County & & \\
\hline Irrigation-Golf course & 0.00 & 0.00 & 0.00 & 0.00 & 0.00 & $\begin{array}{l}\text { Crawford County } \\
\text { Water System }\end{array}$ & 0.14 & 0.00 \\
\hline Livestock/aquaculture & 0.00 & 0.22 & 0.22 & 0.22 & 0.00 & City of Roberta & 0.18 & 0.00 \\
\hline Thermoelectric power & 0.00 & 0.00 & 0.00 & 0.00 & 0.00 & & & \\
\hline
\end{tabular}

2.63

0.23

2.86

2.86

0.12

${ }^{1}$ Total use is total withdrawal plus public supply deliveries and losses.

\section{Withdrawals by Source}
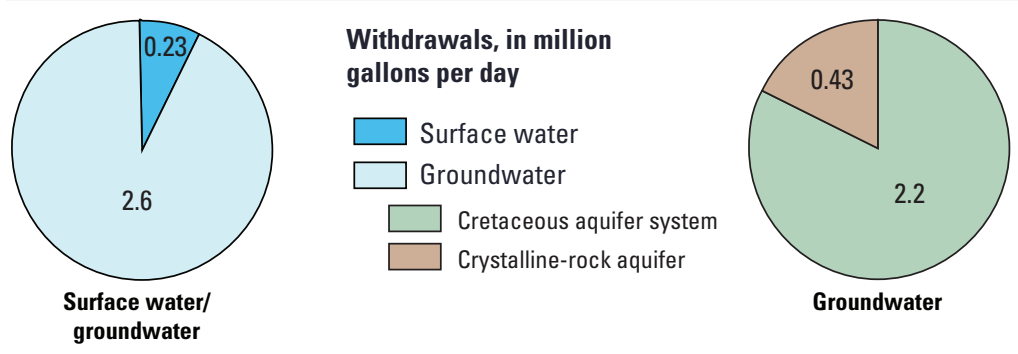

Groundwater
Public-Supply Deliveries by Use Category

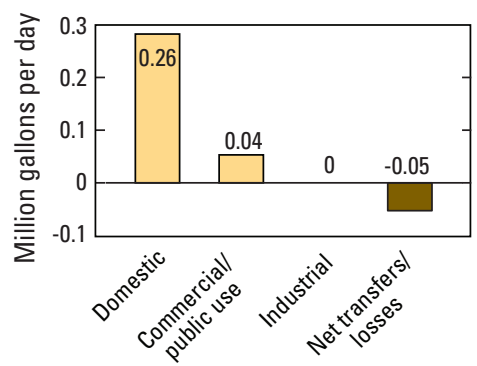

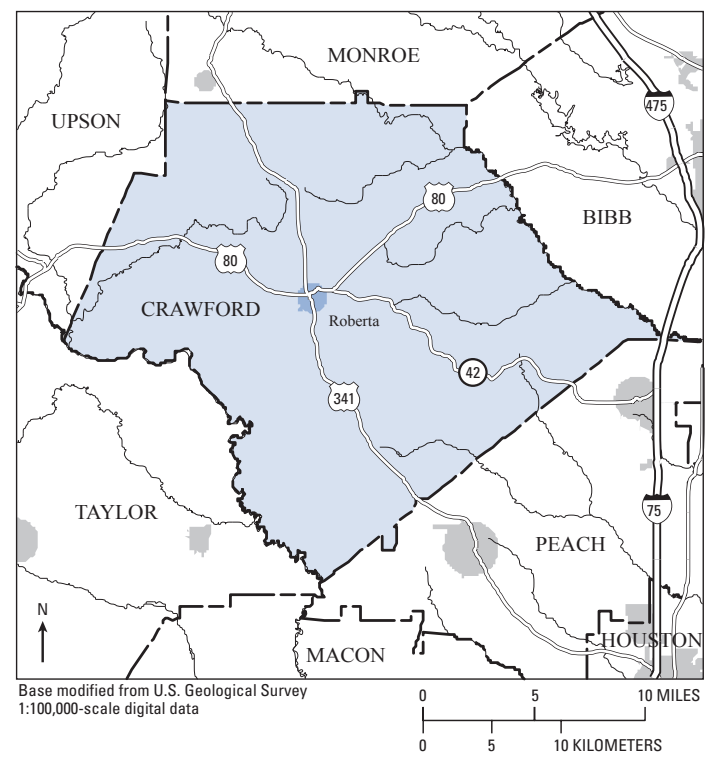

Surface-Water Withdrawals by Year

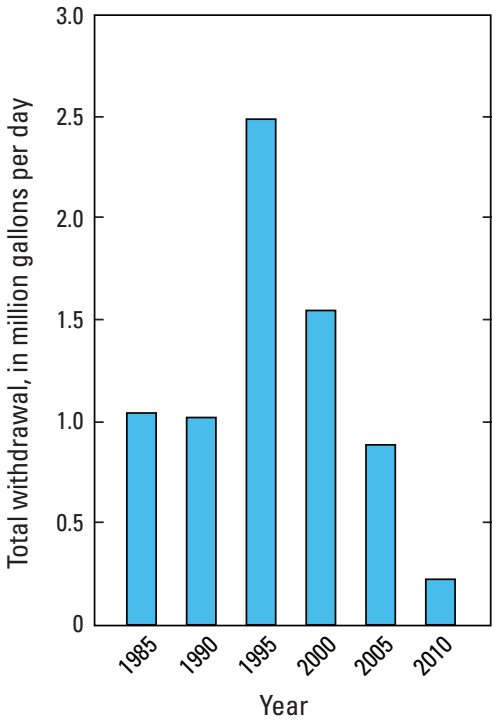

Groundwater Withdrawals by Year

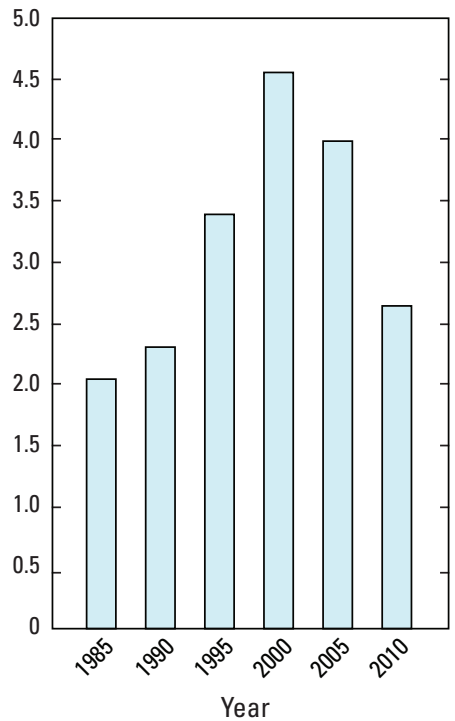




\section{CRISP COUNTY}

Population

23,439

Population served by public supply-Groundwater

Population served by public supply-Surface water

Acres irrigated
15,460

28,790

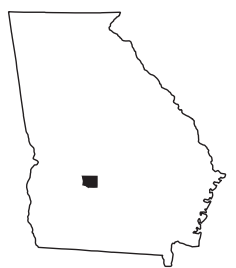

2010 WATER WITHDRAWALS AND ESTIMATED USE, IN MILLION GALLONS PER DAY

$[-$, not applicable $]$

\begin{tabular}{|c|c|c|c|c|c|c|c|c|}
\hline \multirow{3}{*}{ Category } & \multicolumn{3}{|c|}{ Withdrawals } & \multirow{3}{*}{$\begin{array}{l}\text { Total } \\
\text { use }^{1}\end{array}$} & \multirow{3}{*}{$\begin{array}{c}\text { Surface- } \\
\text { water } \\
\text { returns } \\
\end{array}$} & \multirow{2}{*}{\multicolumn{3}{|c|}{$\begin{array}{l}\text { Withdrawals by Major Industrial Groups } \\
\text { [NAICS, North American Industrial Classification System code] }\end{array}$}} \\
\hline & \multirow{2}{*}{$\begin{array}{c}\text { Ground- } \\
\text { water }\end{array}$} & \multirow{2}{*}{$\begin{array}{c}\text { Surface } \\
\text { water }\end{array}$} & \multirow[b]{2}{*}{ Total } & & & & & \\
\hline & & & & & & NAICS & Groundwater & Surface water \\
\hline Public supply & 2.23 & 0.00 & 2.23 & - & - & $321-$ Wood & 0.11 & 0.00 \\
\hline Domestic & 0.60 & 0.00 & 0.60 & 1.80 & 0.00 & $321-$ wood & & \\
\hline Commercial/public use & 0.00 & 0.00 & 0.00 & 0.62 & 0.00 & & & \\
\hline Industrial & 0.12 & 0.00 & 0.12 & 0.13 & 0.00 & & & \\
\hline Public-supply losses & - & - & - & 0.40 & - & & & \\
\hline Public wastewater treatment & - & - & - & - & 2.88 & & & \\
\hline Mining & 0.00 & 0.00 & 0.00 & 0.00 & 0.00 & Withdrawals by Major & Public Supp & \\
\hline Irrigation-Crop & 10.49 & 4.90 & 15.39 & 15.39 & 0.00 & Name & Groundwater & Surface water \\
\hline Irrigation-Golf course & 0.33 & 0.00 & 0.33 & 0.33 & 0.00 & & & \\
\hline Livestock/aquaculture & 0.02 & 0.05 & 0.07 & 0.07 & 0.00 & City of Cordele & 1.84 & 0.00 \\
\hline Thermoelectric power & 0.00 & 0.00 & 0.00 & 0.00 & 0.00 & Crisp County Waterworks & 0.29 & 0.00 \\
\hline TOTAL & 13.79 & 4.95 & 18.74 & 18.74 & 2.88 & & & \\
\hline
\end{tabular}

TOTAL

'Total use is total withdrawal plus public supply deliveries and losses.

\section{Withdrawals by Source}

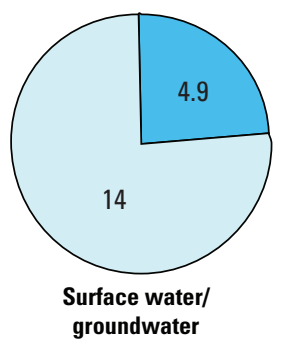

Withdrawals, in million gallons per day

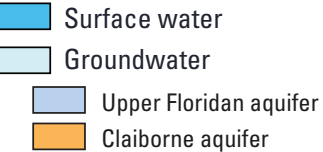

Claiborne aquifer groundwater

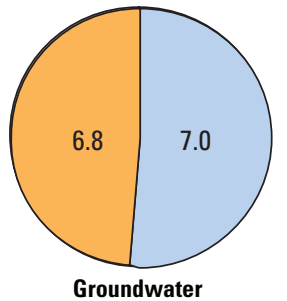

Groundwate
Public-Supply Deliveries by Use Category

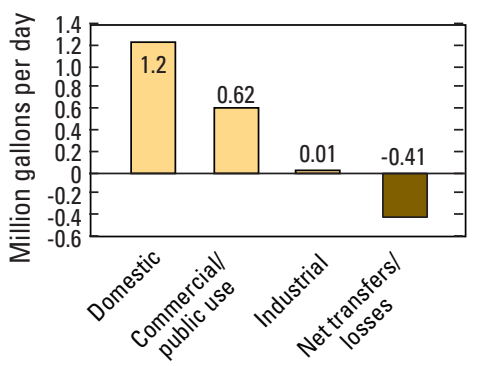

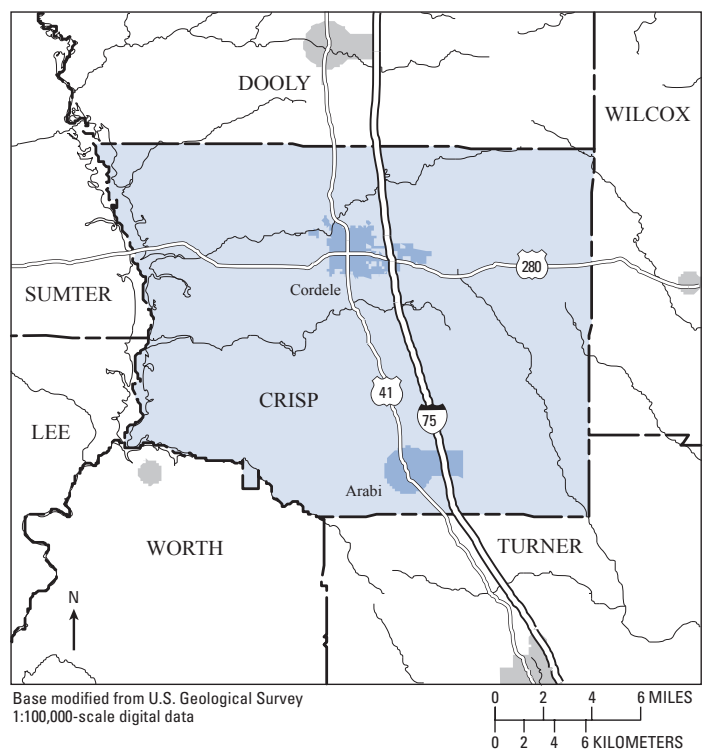

Surface-Water Withdrawals by Year

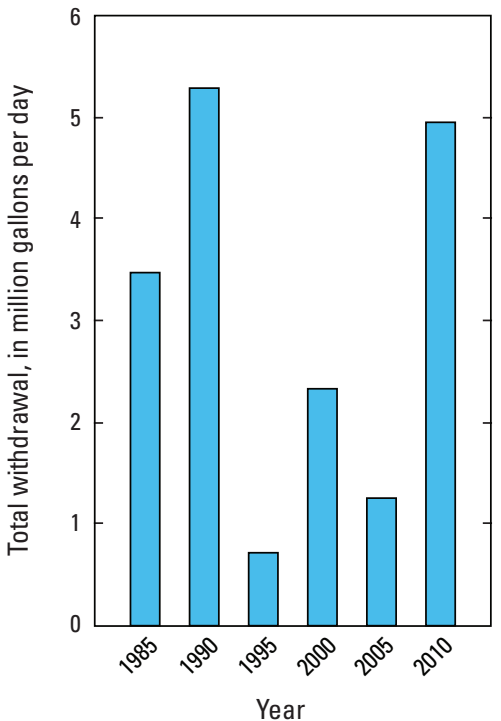

Groundwater Withdrawals by Year

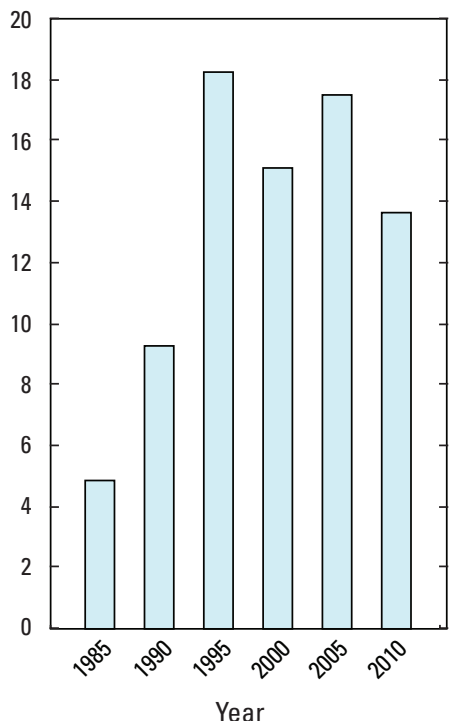




\section{DADE COUNTY}

Population

Population served by public supply-Groundwater

Population served by public supply-Surface water $\quad 15,470$

Acres irrigated

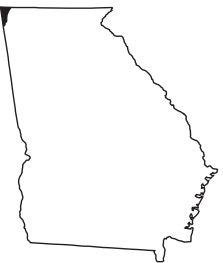

2010 WATER WITHDRAWALS AND ESTIMATED USE, IN MILLION GALLONS PER DAY

$[-$, not applicable $]$

\begin{tabular}{|c|c|c|c|c|c|}
\hline \multirow[b]{2}{*}{ Category } & \multicolumn{3}{|c|}{ Withdrawals } & \multirow[b]{2}{*}{$\begin{array}{l}\text { Total } \\
\text { use }^{1}\end{array}$} & \multirow{2}{*}{$\begin{array}{c}\text { Surface- } \\
\text { water } \\
\text { returns }\end{array}$} \\
\hline & $\begin{array}{c}\text { Ground- } \\
\text { water }\end{array}$ & $\begin{array}{c}\text { Surface } \\
\text { water }\end{array}$ & Total & & \\
\hline Public supply & 0.00 & 2.09 & 2.09 & - & - \\
\hline Domestic & 0.09 & 0.00 & 0.09 & 1.30 & 0.00 \\
\hline Commercial/public use & 0.00 & 0.00 & 0.00 & 0.53 & 0.00 \\
\hline Industrial & 0.00 & 0.00 & 0.00 & 0.04 & 0.00 \\
\hline Public-supply losses & - & - & - & 0.31 & - \\
\hline Public wastewater treatment & - & - & - & - & 0.28 \\
\hline Mining & 0.00 & 0.00 & 0.00 & 0.00 & 0.00 \\
\hline Irrigation-Crop & 0.00 & 0.00 & 0.00 & 0.00 & 0.00 \\
\hline Irrigation-Golf course & 0.00 & 0.00 & 0.00 & 0.00 & 0.00 \\
\hline Livestock/aquaculture & 0.01 & 0.09 & 0.10 & 0.10 & 0.00 \\
\hline Thermoelectric power & 0.00 & 0.00 & 0.00 & 0.00 & 0.00 \\
\hline TOTAL & 0.10 & 2.18 & 2.28 & 2.28 & 0.28 \\
\hline
\end{tabular}

Withdrawals by Major Industrial Groups

[NAICS, North American Industrial Classification System code]

\begin{tabular}{lcc}
\hline NAICS & Groundwater & Surface water \\
\hline None & - & - \\
\hline
\end{tabular}

Total use is total withdrawal plus public supply deliveries and losses

\section{Withdrawals by Source}

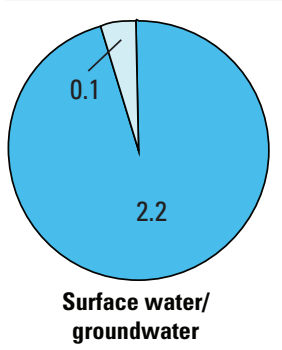

Withdrawals, in million gallons per day

Surface water

Groundwater

$\square$ Paleozoic-rock aquifer groundwater

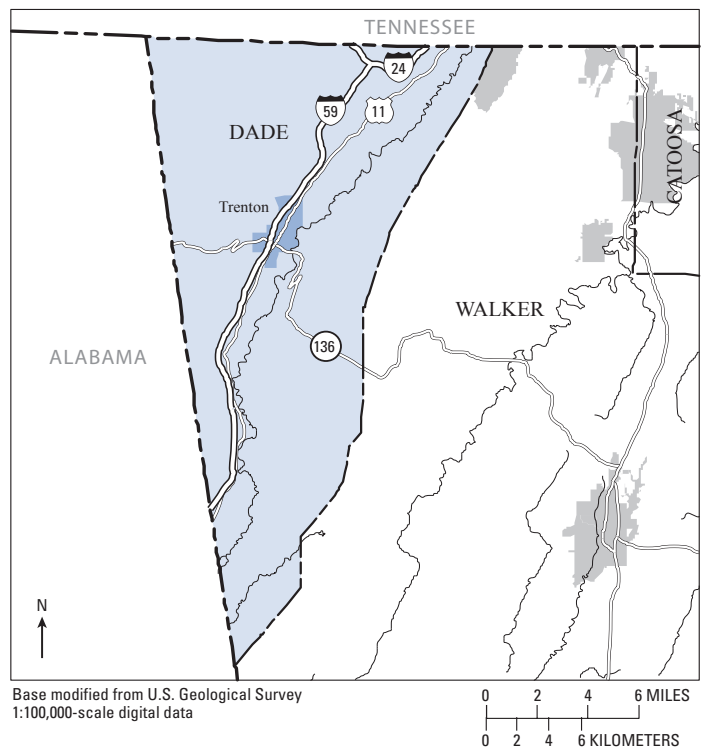

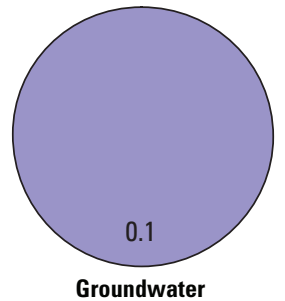

Public-Supply Deliveries by Use Category

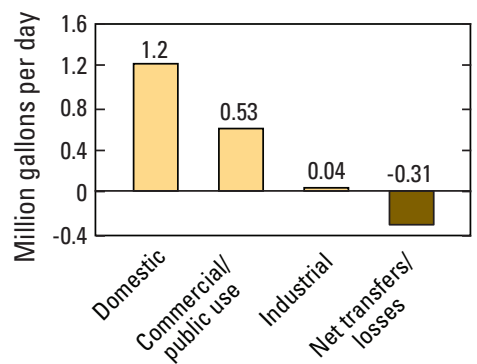

Withdrawals by Major Public Suppliers

\begin{tabular}{lcc}
\hline Name & Groundwater & Surface water \\
\hline $\begin{array}{l}\text { Dade County Water } \\
\text { Authority }\end{array}$ & 0.00 & 2.09 \\
\hline
\end{tabular}




\section{DAWSON COUNTY}

Population

22,330

Population served by public supply-Groundwater

Population served by public supply-Surface water Acres irrigated
15,900

300

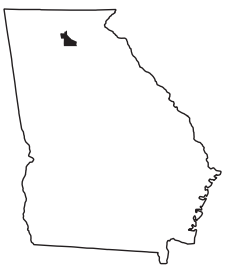

2010 WATER WITHDRAWALS AND ESTIMATED USE, IN MILLION GALLONS PER DAY

[-, not applicable; Mgal/d, million gallons per day]

\begin{tabular}{|c|c|c|c|c|c|c|c|c|}
\hline \multirow{3}{*}{ Category } & \multicolumn{3}{|c|}{ Withdrawals } & \multirow{3}{*}{$\begin{array}{l}\text { Total } \\
\text { use }^{1}\end{array}$} & \multirow{3}{*}{$\begin{array}{c}\text { Surface- } \\
\text { water } \\
\text { returns } \\
\end{array}$} & \multirow{2}{*}{\multicolumn{3}{|c|}{$\begin{array}{l}\text { Withdrawals by Major Industrial Groups } \\
\text { [NAICS, North American Industrial Classification System code] }\end{array}$}} \\
\hline & \multirow{2}{*}{$\begin{array}{c}\text { Ground- } \\
\text { water }\end{array}$} & \multirow{2}{*}{$\begin{array}{c}\text { Surface } \\
\text { water }\end{array}$} & \multirow[b]{2}{*}{ Total } & & & & & \\
\hline & & & & & & NAICS & Groundwater & Surface water \\
\hline Public supply & 0.45 & 1.25 & 1.70 & - & - & None & - & - \\
\hline Domestic & 0.45 & 0.00 & 0.45 & 1.59 & 0.00 & None & - & \\
\hline Commercial/public use & 0.00 & 0.00 & 0.00 & 0.29 & 0.00 & & & \\
\hline Industrial & 0.00 & 0.00 & 0.00 & 0.02 & 0.00 & & & \\
\hline Public-supply losses & - & - & - & 0.36 & - & & & \\
\hline Public wastewater treatment & - & - & - & - & 0.00 & Withdrawals by Majo & r Public Supp & \\
\hline Mining & 0.00 & 0.00 & 0.00 & 0.00 & 0.00 & Name & Groundwater & Surface water \\
\hline Irrigation-Crop & 0.00 & 0.09 & 0.09 & 0.09 & 0.00 & Ftowah Water \& Sewer & & \\
\hline Irrigation-Golf course & 0.00 & 0.21 & 0.21 & 0.21 & 0.00 & $\begin{array}{l}\text { Etowan water \& Sewer } \\
\text { Authority }\end{array}$ & 0.00 & 1.25 \\
\hline Livestock/aquaculture & 0.00 & 0.30 & 0.30 & 0.30 & 0.00 & City of Dawsonville & 0.42 & 000 \\
\hline Thermoelectric power & 0.00 & 0.00 & 0.00 & 0.00 & 0.00 & City of Dawsonville & 0.42 & 0.00 \\
\hline
\end{tabular}

$\begin{array}{lllll}0.90 & 1.85 & 2.75 & 2.86 & 0.00\end{array}$

${ }^{1}$ Total use is total withdrawal plus public supply deliveries and losses. An average of $0.06 \mathrm{Mgal} / \mathrm{d}$ was delivered to Dawson County, Georgia from Cherokee County, Georgia and $0.05 \mathrm{Mgal} / \mathrm{d}$ from Forsyth County, Georgia in 2010.

\section{Withdrawals by Source}
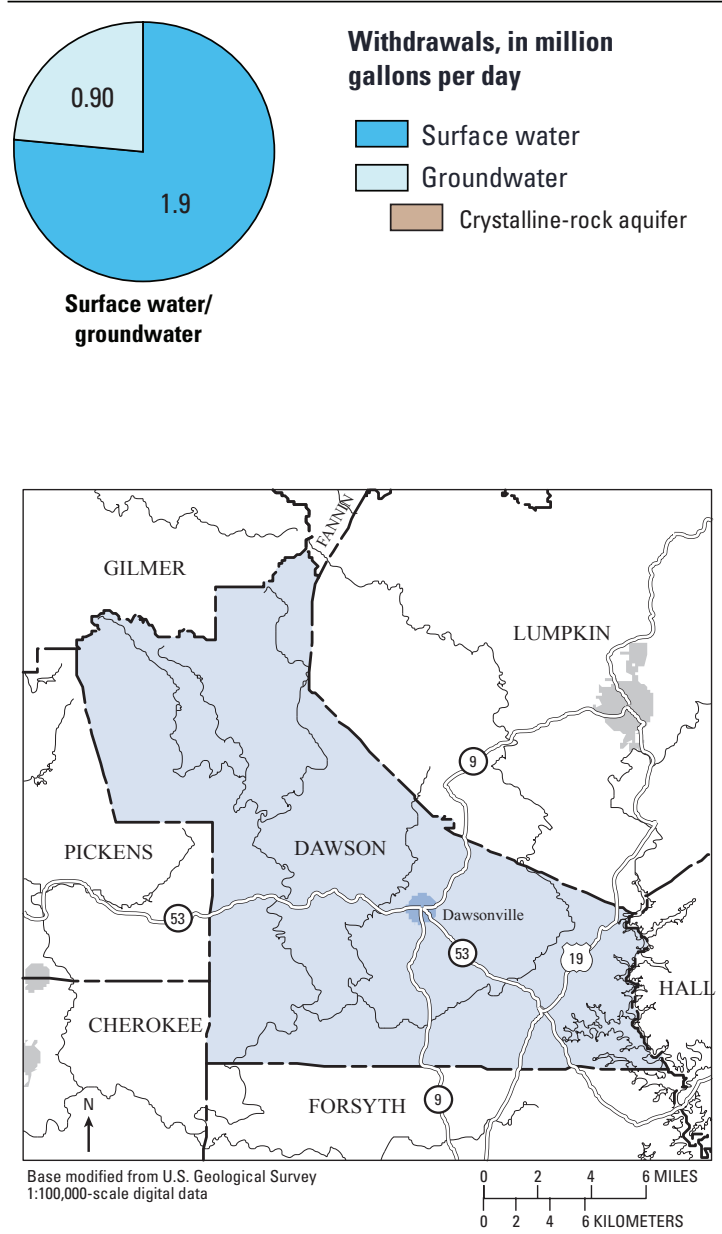

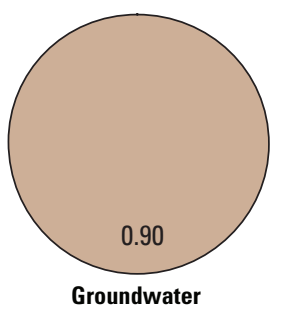

Public-Supply Deliveries' by Use Category

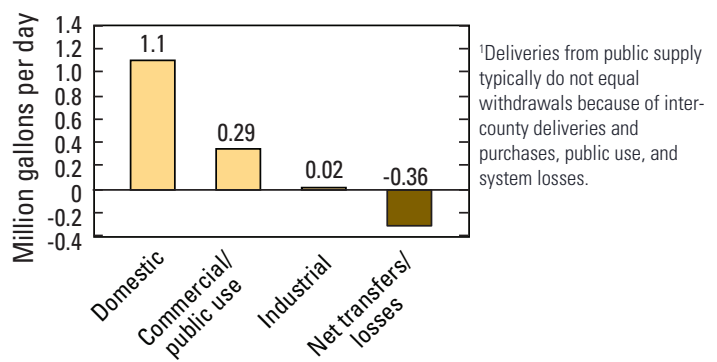

Surface-Water Withdrawals by Year

Groundwater Withdrawals by Year
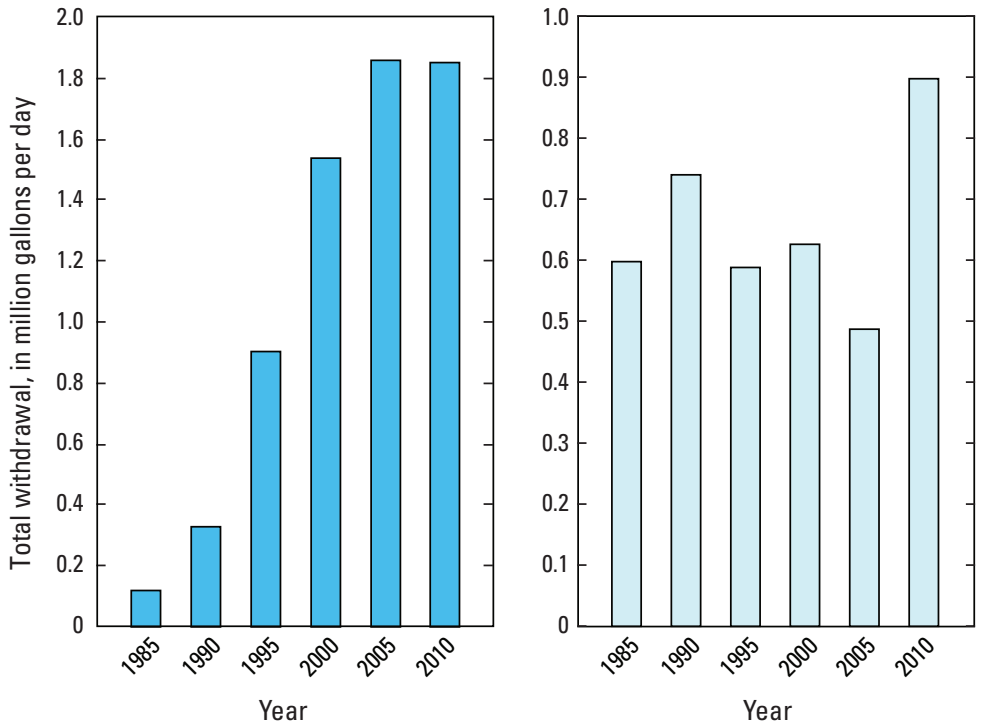


\section{DECATUR COUNTY}

Population

27,842

Population served by public supply-Groundwater

Population served by public supply-Surface water

Acres irrigated

69,010

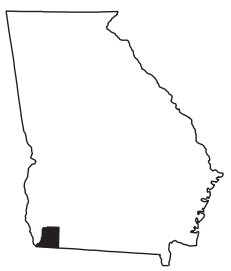

2010 WATER WITHDRAWALS AND ESTIMATED USE, IN MILLION GALLONS PER DAY

$[-$, not applicable $]$

\begin{tabular}{|c|c|c|c|c|c|c|c|c|}
\hline \multirow{3}{*}{ Category } & \multicolumn{3}{|c|}{ Withdrawals } & \multirow{3}{*}{$\begin{array}{l}\text { Total } \\
\text { use }^{1}\end{array}$} & \multirow{3}{*}{$\begin{array}{l}\text { Surface- } \\
\text { water } \\
\text { returns } \\
\end{array}$} & \multirow{2}{*}{\multicolumn{3}{|c|}{$\begin{array}{l}\text { Withdrawals by Major Industrial Groups } \\
\text { [NAICS, North American Industrial Classification System code] }\end{array}$}} \\
\hline & \multirow{2}{*}{$\begin{array}{c}\text { Ground- } \\
\text { water }\end{array}$} & \multirow{2}{*}{$\begin{array}{c}\text { Surface } \\
\text { water }\end{array}$} & \multirow[b]{2}{*}{ Total } & & & & & \\
\hline & & & & & & NAICS & Groundwater & Surface water \\
\hline Public supply & 2.87 & 0.00 & 2.87 & - & - & & & \\
\hline Domestic & 1.00 & 0.00 & 1.00 & 3.24 & 0.00 & 327 -Stone, clay & 0.17 & 0.58 \\
\hline Commercial/public use & 0.00 & 0.00 & 0.00 & 0.36 & 0.00 & 339-Misc manufacturing & 0.30 & 0.00 \\
\hline Industrial & 0.71 & 0.58 & 1.29 & 1.29 & 0.57 & 313-Textiles & 0.20 & 0.00 \\
\hline
\end{tabular}

Public-supply losses

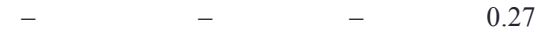

Public wastewater treatment

$$
-
$$

Mining

\subsection{0}

$-$

$-$

Irrigation-Crop

66.51

0.00

0.00

$$
\begin{aligned}
& 0.27 \\
& -
\end{aligned}
$$

Irrigation-Golf course

0.07

1.00

67.51

0.00

1.69

Livestock/aquaculture

0.15

0.00

67.51

Thermoelectric power

\section{Withdrawals by Source}
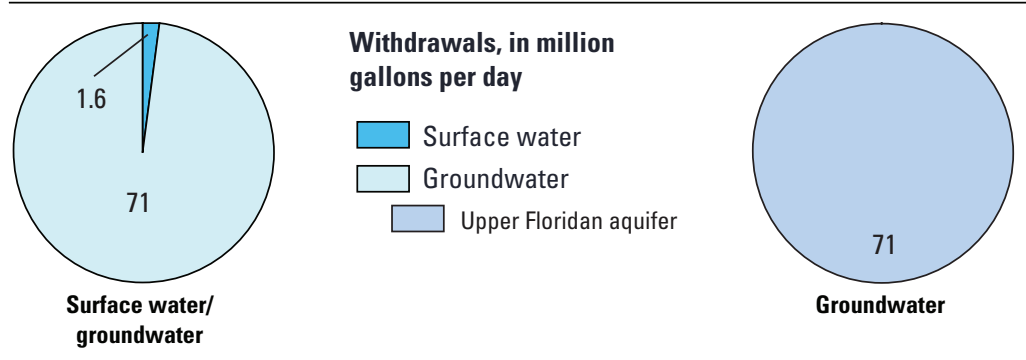

Public-Supply Deliveries by Use Category
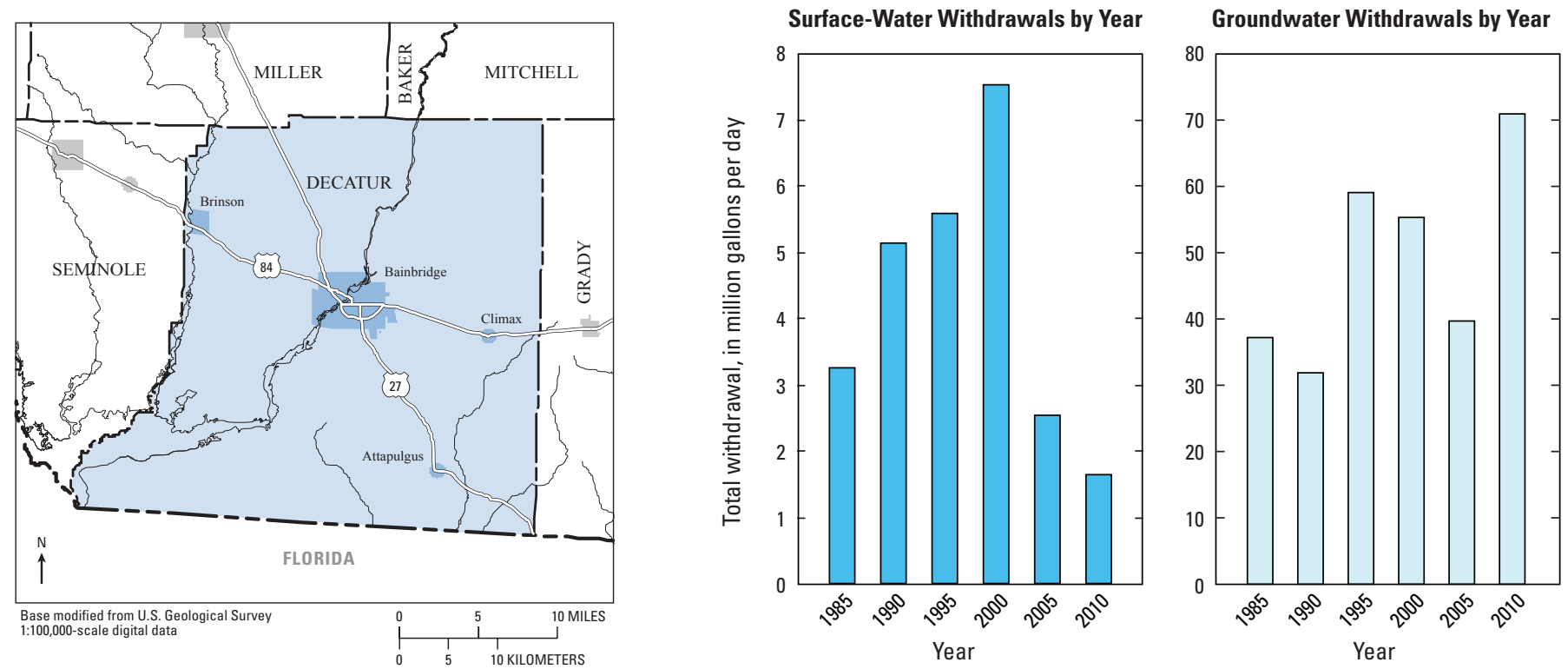

Withdrawals by Major Public Suppliers

\begin{tabular}{lcc}
\hline Name & Groundwater & Surface water \\
\hline City of Bainbridge & 2.75 & 0.00 \\
City of Attapulgus & 0.04 & 0.00 \\
\hline
\end{tabular}

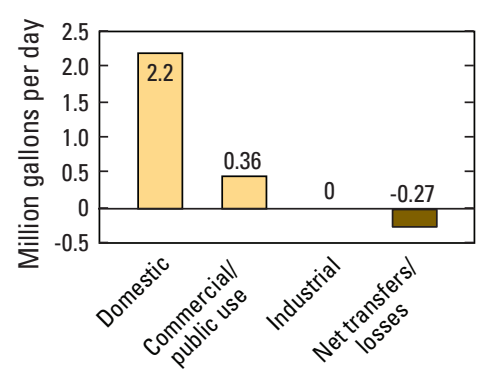




\section{DEKALB COUNTY}

Population

Population served by public supply-Groundwater

Population served by public supply-Surface water

Acres irrigated
691,890

0

691,890

900

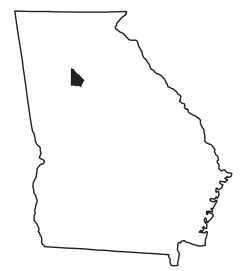

2010 WATER WITHDRAWALS AND ESTIMATED USE, IN MILLION GALLONS PER DAY

[-, not applicable; Mgal/d, million gallons per day]

\begin{tabular}{|c|c|c|c|c|c|c|c|}
\hline \multirow{3}{*}{ Category } & \multicolumn{3}{|c|}{ Withdrawals } & \multirow{3}{*}{$\begin{array}{l}\text { Total } \\
\text { use }^{1}\end{array}$} & \multirow{3}{*}{$\begin{array}{l}\text { Surface- } \\
\text { water } \\
\text { returns } \\
\end{array}$} & \multirow{2}{*}{\multicolumn{2}{|c|}{$\begin{array}{l}\text { Withdrawals by Major Industrial Groups } \\
\text { [NAICS, North American Industrial Classification System code] }\end{array}$}} \\
\hline & \multirow{2}{*}{$\begin{array}{c}\text { Ground- } \\
\text { water }\end{array}$} & \multirow{2}{*}{$\begin{array}{c}\text { Surface } \\
\text { water }\end{array}$} & \multirow{2}{*}{ Total } & & & & \\
\hline & & & & & & Groundwater & Surface water \\
\hline Public supply & 0.00 & 0.00 & 0.00 & - & - & & \\
\hline Domestic & 0.00 & 0.00 & 0.00 & 38.97 & 0.00 & None & - \\
\hline Commercial/public use & 0.00 & 0.00 & 0.00 & 20.49 & 0.00 & & \\
\hline Industrial & 0.00 & 0.00 & 0.00 & 5.29 & 0.00 & & \\
\hline Public-supply losses & - & - & - & 10.32 & - & & \\
\hline Public wastewater treatment & - & - & - & - & 36.34 & Withdrawals by Major Public Supp & \\
\hline Mining & 0.34 & 0.00 & 0.34 & 0.34 & 0.40 & Groundwater & Surface water \\
\hline Irrigation-Crop & 0.00 & 0.00 & 0.00 & 0.00 & 0.00 & & \\
\hline Irrigation-Golf course & 0.59 & 1.73 & 2.32 & 2.44 & 0.00 & None-DeKalb County withdraws wate & Fulton \\
\hline Livestock/aquaculture & 0.00 & 0.00 & 0.00 & 0.00 & 0.00 & County & \\
\hline
\end{tabular}

Thermoelectric power

0.00

0.00

0.00

0.00

0.00

TOTAL

$\begin{array}{llll}1.73 & 2.66 & 77.85 & 36.74\end{array}$

${ }^{1}$ Total use is total withdrawal plus public supply deliveries and losses. An average of $74.95 \mathrm{Mgal} / \mathrm{d}$ was withdrawn in Fulton County, Georgia and delivered to DeKalb County and 0.1 Mgal/d from DeKalb County to Henry County, Georgia in 2010. About 4.2 Mgal/d were delivered to customers within the City of Atlanta water service area in DeKalb County.

\section{Withdrawals by Source}
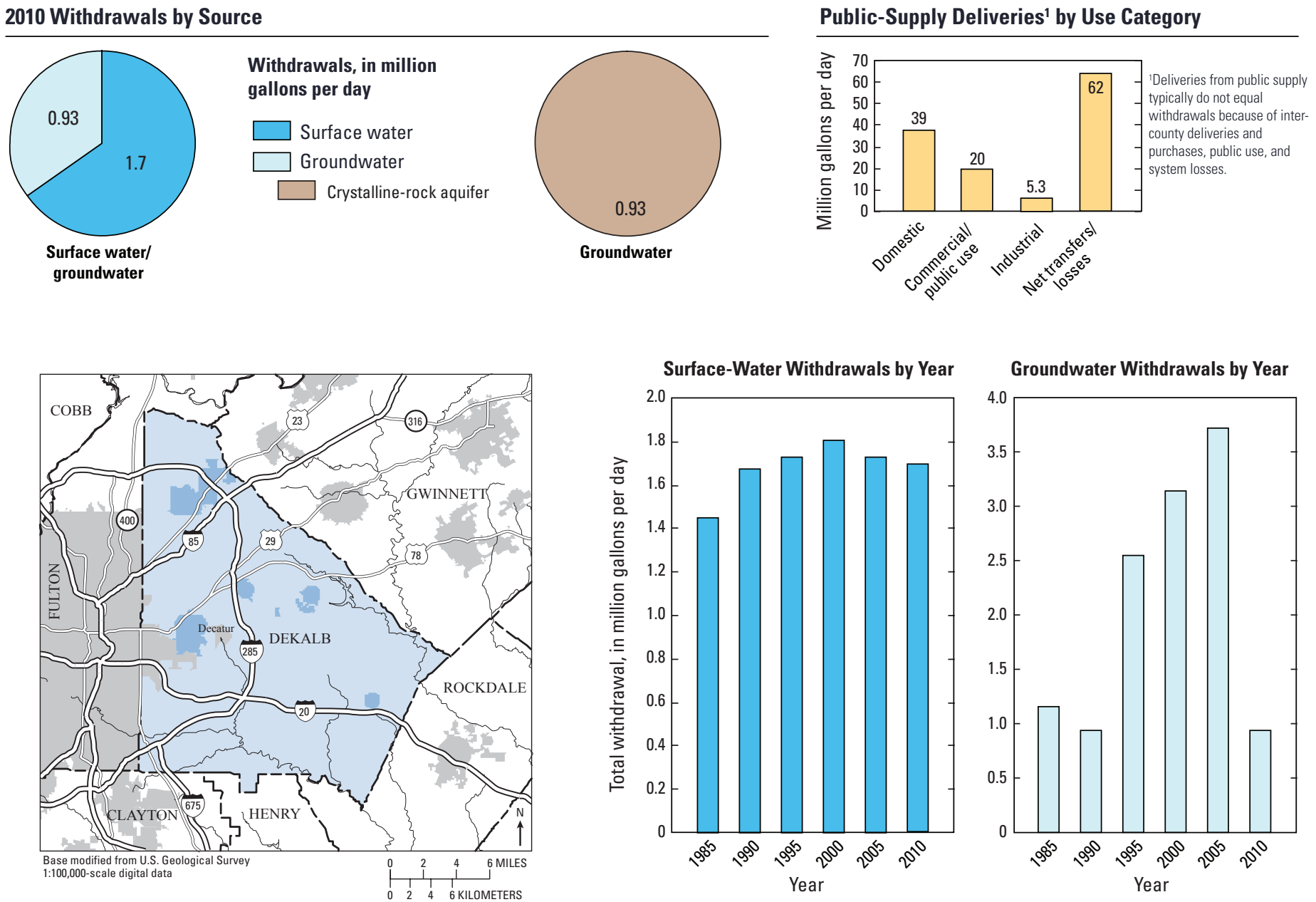

Groundwater Withdrawals by Year

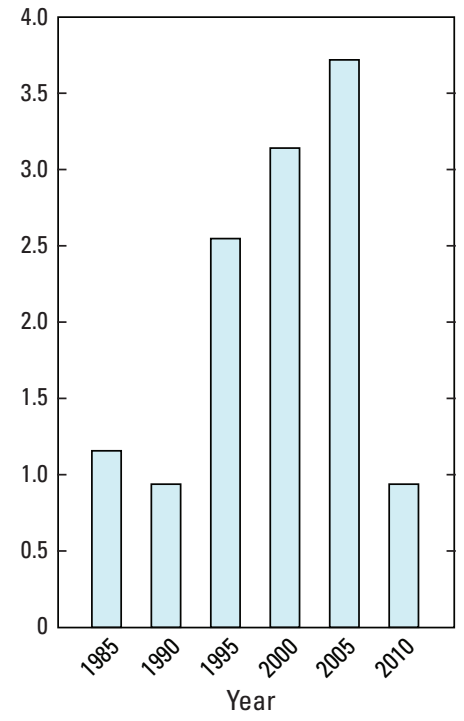


DODGE COUNTY

Population

Population served by public supply-Groundwater $\quad 8,800$

Population served by public supply-Surface water 0

Acres irrigated

14,320

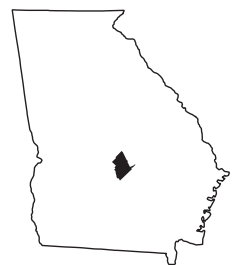

2010 WATER WITHDRAWALS AND ESTIMATED USE, IN MILLION GALLONS PER DAY

$[-$, not applicable $]$

\begin{tabular}{|c|c|c|c|c|c|}
\hline \multirow[b]{2}{*}{ Category } & \multicolumn{3}{|c|}{ Withdrawals } & \multirow[b]{2}{*}{$\begin{array}{c}\text { Total } \\
\text { use }^{1}\end{array}$} & \multirow{2}{*}{$\begin{array}{c}\text { Surface- } \\
\text { water } \\
\text { returns }\end{array}$} \\
\hline & $\begin{array}{c}\text { Ground- } \\
\text { water }\end{array}$ & $\begin{array}{c}\text { Surface } \\
\text { water }\end{array}$ & Total & & \\
\hline Public supply & 1.15 & 0.00 & 1.15 & - & - \\
\hline Domestic & 0.97 & 0.00 & 0.97 & 1.59 & 0.00 \\
\hline Commercial/public use & 0.00 & 0.00 & 0.00 & 0.29 & 0.00 \\
\hline Industrial & 0.00 & 0.00 & 0.00 & 0.09 & 0.00 \\
\hline Public-supply losses & - & - & - & 0.15 & - \\
\hline Public wastewater treatment & - & - & - & - & 0.65 \\
\hline Mining & 0.00 & 0.00 & 0.00 & 0.00 & 0.00 \\
\hline Irrigation-Crop & 6.29 & 3.02 & 9.31 & 9.31 & 0.00 \\
\hline Irrigation-Golf course & 0.16 & 0.00 & 0.16 & 0.16 & 0.00 \\
\hline Livestock/aquaculture & 0.76 & 0.14 & 0.90 & 0.90 & 0.00 \\
\hline Thermoelectric power & 0.00 & 0.00 & 0.00 & 0.00 & 0.00 \\
\hline TOTAL & 9.33 & 3.16 & 12.49 & 12.49 & 0.65 \\
\hline
\end{tabular}

Withdrawals by Major Industrial Groups

[NAICS, North American Industrial Classification System code]

\begin{tabular}{lcc}
\hline NAICS & Groundwater & Surface water \\
\hline None & - & - \\
\hline
\end{tabular}

Withdrawals by Major Public Suppliers

\begin{tabular}{lcc}
\hline Name & Groundwater & Surface water \\
\hline City of Eastman & 1.01 & 0.00 \\
Town of Chester & 0.08 & 0.00 \\
\hline
\end{tabular}

${ }^{1}$ Total use is total withdrawal plus public supply deliveries and losses.

\section{Withdrawals by Source}
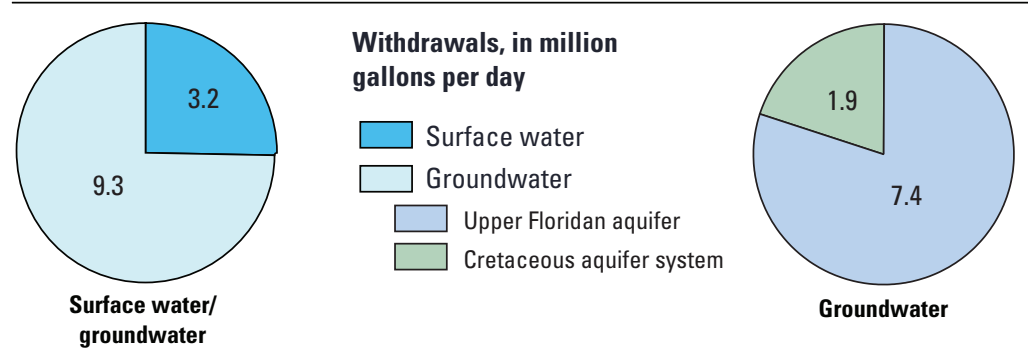

Public-Supply Deliveries by Use Category
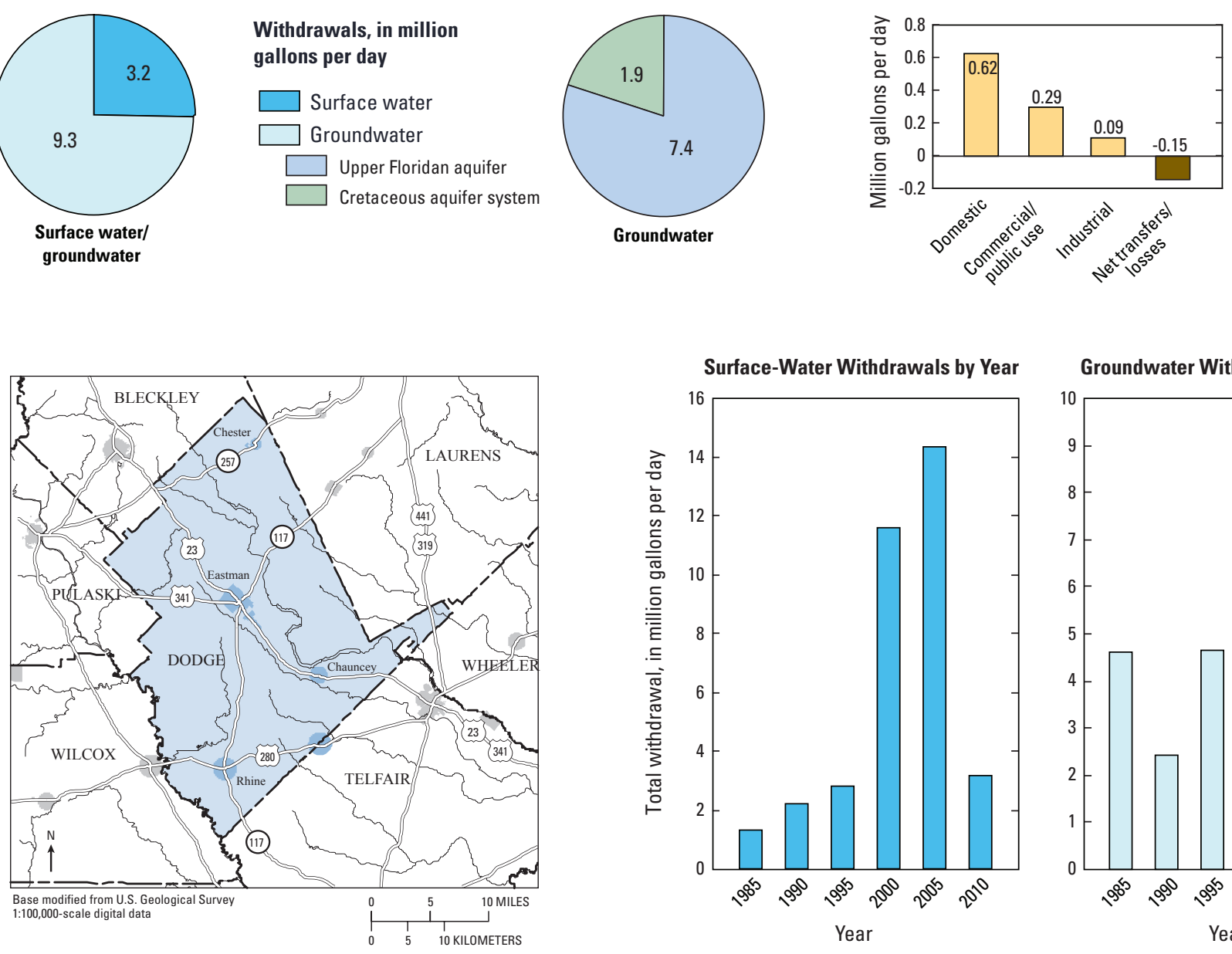

Groundwater Withdrawals by Year

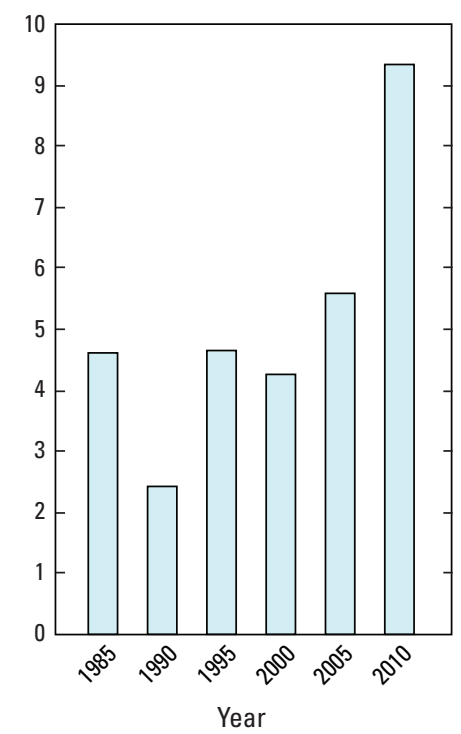




\section{DOOLY COUNTY}

Population

14,918

Population served by public supply-Groundwater

Population served by public supply-Surface water Acres irrigated
7,290

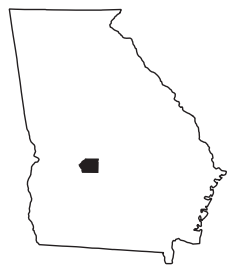

2010 WATER WITHDRAWALS AND ESTIMATED USE, IN MILLION GALLONS PER DAY

$[-$, not applicable $]$

\begin{tabular}{|c|c|c|c|c|c|c|c|c|}
\hline \multirow{3}{*}{ Category } & \multicolumn{3}{|c|}{ Withdrawals } & \multirow{3}{*}{$\begin{array}{l}\text { Total } \\
\text { use }^{1}\end{array}$} & \multirow{3}{*}{$\begin{array}{l}\text { Surface- } \\
\text { water } \\
\text { returns } \\
\end{array}$} & \multirow{2}{*}{\multicolumn{3}{|c|}{$\begin{array}{l}\text { Withdrawals by Major Industrial Groups } \\
\text { [NAICS, North American Industrial Classification System code] }\end{array}$}} \\
\hline & \multirow{2}{*}{$\begin{array}{c}\text { Ground- } \\
\text { water }\end{array}$} & \multirow{2}{*}{$\begin{array}{c}\text { Surface } \\
\text { water }\end{array}$} & \multirow[b]{2}{*}{ Total } & & & & & \\
\hline & & & & & & NAICS & Groundwater & Surface water \\
\hline Public supply & 2.35 & 0.00 & 2.35 & - & - & \multirow{2}{*}{321 -Wood products } & \multirow[t]{2}{*}{0.10} & \multirow{2}{*}{0.00} \\
\hline Domestic & 0.57 & 0.00 & 0.57 & 1.33 & 0.00 & & & \\
\hline Commercial/public use & 0.00 & 0.00 & 0.00 & 0.51 & 0.00 & & & \\
\hline Industrial & 0.10 & 0.00 & 0.10 & 0.62 & 0.00 & & & \\
\hline Public-supply losses & - & - & - & 0.56 & - & & & \\
\hline Public wastewater treatment & - & - & - & - & 0.10 & \multicolumn{3}{|c|}{ Withdrawals by Major Public Suppliers } \\
\hline Mining & 0.00 & 0.00 & 0.00 & 0.00 & 0.00 & Name & \multirow{2}{*}{ Groundwater } & Surface water \\
\hline $\begin{array}{l}\text { Irrigation-Crop } \\
\text { Irrigation-Golf course }\end{array}$ & $\begin{array}{r}19.95 \\
0.00\end{array}$ & $\begin{array}{l}4.53 \\
0.00\end{array}$ & $\begin{array}{r}24.48 \\
0.00\end{array}$ & $\begin{array}{r}24.48 \\
0.00\end{array}$ & $\begin{array}{l}0.00 \\
0.00\end{array}$ & City of Vienna & & 0.00 \\
\hline Livestock/aquaculture & 0.02 & 0.13 & 0.15 & 0.15 & 0.00 & City of Unadilla & 0.63 & 0.00 \\
\hline Thermoelectric power & 0.00 & 0.00 & 0.00 & 0.00 & 0.00 & City of Pinehurst & 0.10 & 0.00 \\
\hline TOTAL & 22.99 & 4.66 & 27.65 & 27.65 & 0.10 & & & \\
\hline
\end{tabular}

'Total use is total withdrawal plus public supply deliveries and losses.

\section{Withdrawals by Source}
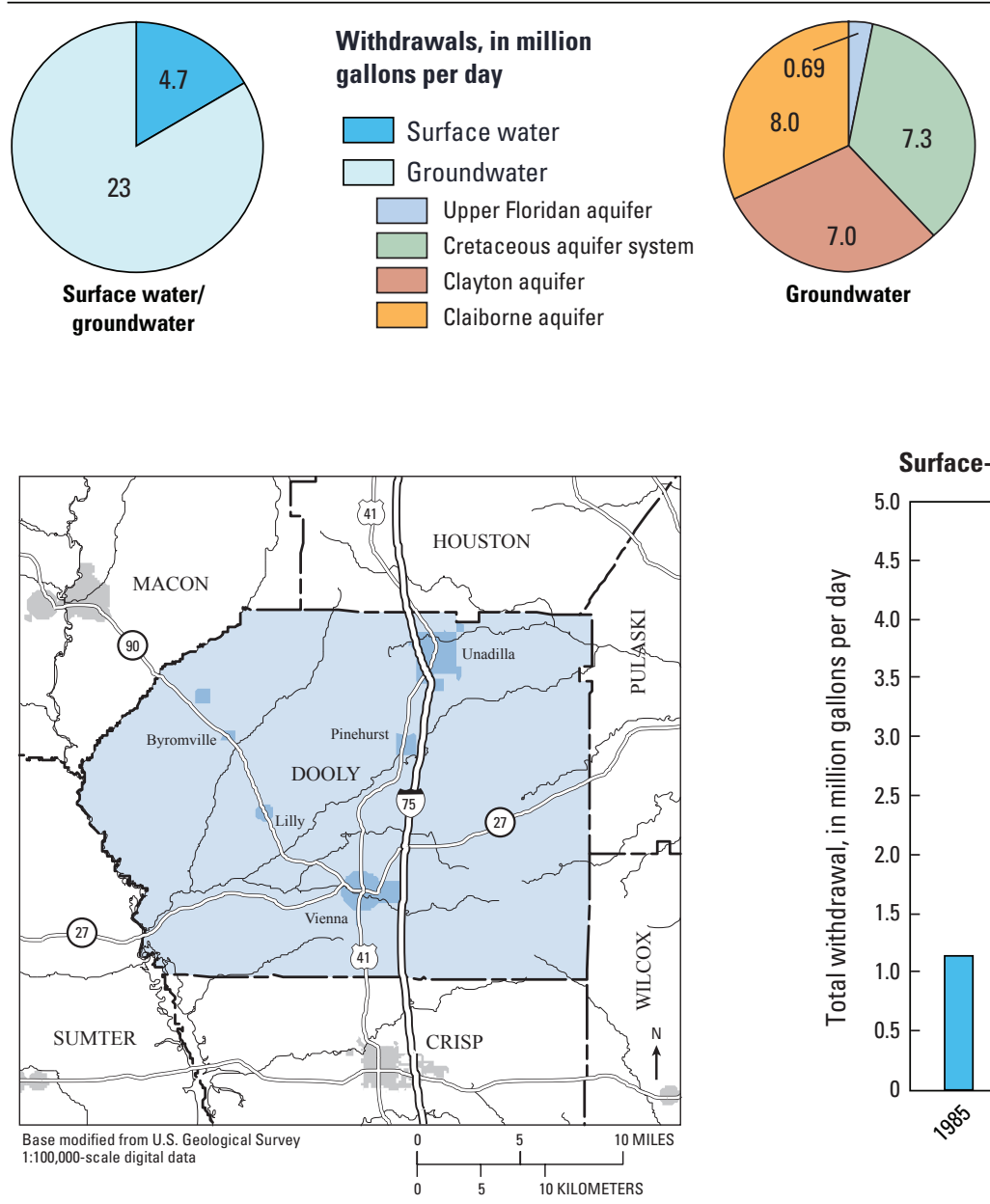

Public-Supply Deliveries by Use Category

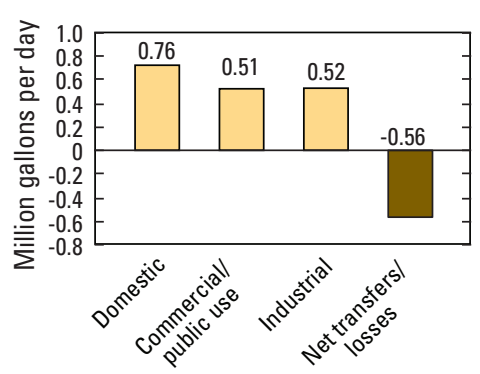

Surface-Water Withdrawals by Year

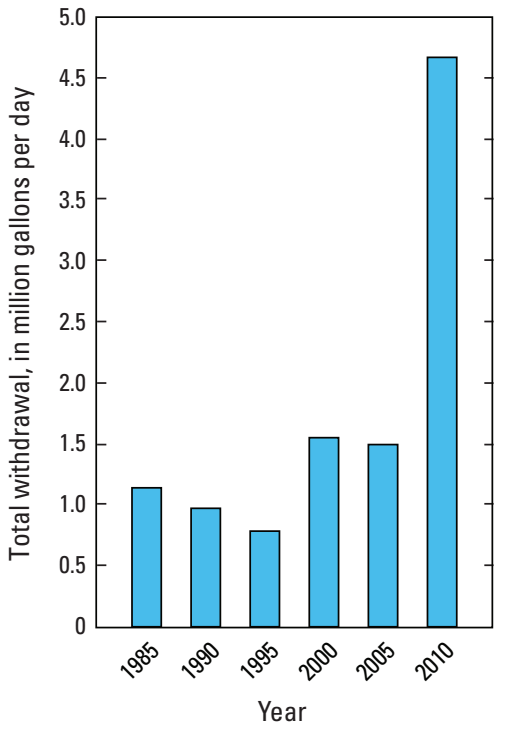

Groundwater Withdrawals by Year

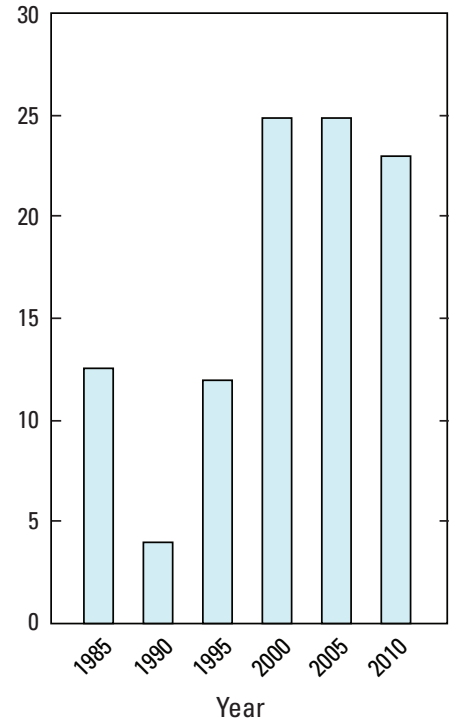




\section{DOUGHERTY COUNTY}

Population

94,565

Population served by public supply-Groundwater $\quad 90,870$

Population served by public supply-Surface water 0

Acres irrigated

19,350

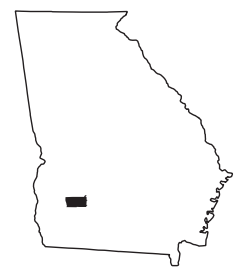

2010 WATER WITHDRAWALS AND ESTIMATED USE, IN MILLION GALLONS PER DAY

$[-$, not applicable $]$

\begin{tabular}{|c|c|c|c|c|c|c|c|c|}
\hline \multirow{3}{*}{ Category } & \multicolumn{3}{|c|}{ Withdrawals } & \multirow{3}{*}{$\begin{array}{l}\text { Total } \\
\text { use }^{1}\end{array}$} & \multirow{3}{*}{$\begin{array}{l}\text { Surface- } \\
\text { water } \\
\text { returns } \\
\end{array}$} & \multirow{2}{*}{\multicolumn{3}{|c|}{$\begin{array}{l}\text { Withdrawals by Major Industrial Groups } \\
\text { [NAICS, North American Industrial Classification System code] }\end{array}$}} \\
\hline & \multirow{2}{*}{$\begin{array}{c}\text { Ground- } \\
\text { water }\end{array}$} & \multirow{2}{*}{$\begin{array}{c}\text { Surface } \\
\text { water }\end{array}$} & \multirow[b]{2}{*}{ Total } & & & & & \\
\hline & & & & & & NAICS & Groundwater & Surface water \\
\hline Public supply & 15.33 & 0.00 & 15.33 & - & - & & & \\
\hline Domestic & 0.28 & 0.00 & 0.28 & 8.51 & 0.00 & 322 -Paper, pulp & 5.37 & 0.00 \\
\hline Commercial/public use & 0.00 & 0.00 & 0.00 & 4.09 & 0.00 & 312-Beverages & 2.83 & 0.00 \\
\hline Industrial & 8.30 & 0.00 & 8.30 & 9.01 & 1.99 & & & \\
\hline
\end{tabular}

Public-supply losses

$\begin{array}{llllll}- & - & - & 2.30\end{array}$

Public wastewater treatment

Mining $\quad 0.05$

Irrigation-Crop $\quad 15.53$

Irrigation-Golf course $\quad 1.09$

Livestock/aquaculture

0.04

$\begin{array}{ll}- & -\end{array}$

Thermoelectric power

0.01

0.00
0.21

0.05

$-$

16.60

TOTAL

40.63

0.13

15.74

0.05

15.74

0.00

0.00

0.02

1.22

1.22

0.00

0.00

$50.01 \quad 50.00$

'Total use is total withdrawal plus public supply deliveries and losses.

\section{Withdrawals by Source}
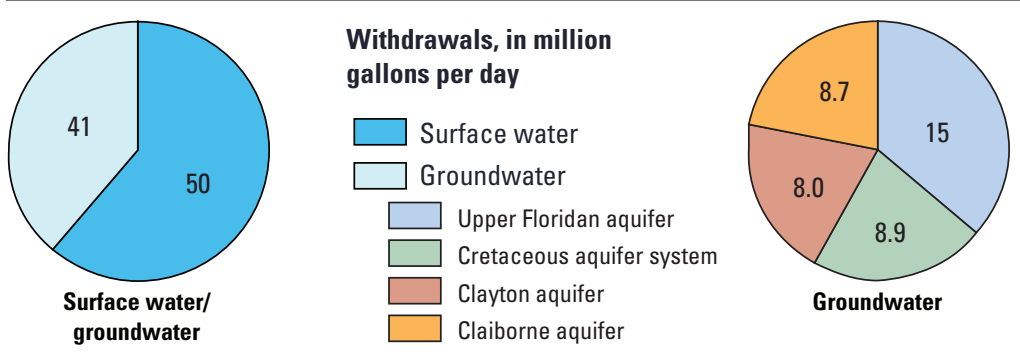

Public-Supply Deliveries by Use Category
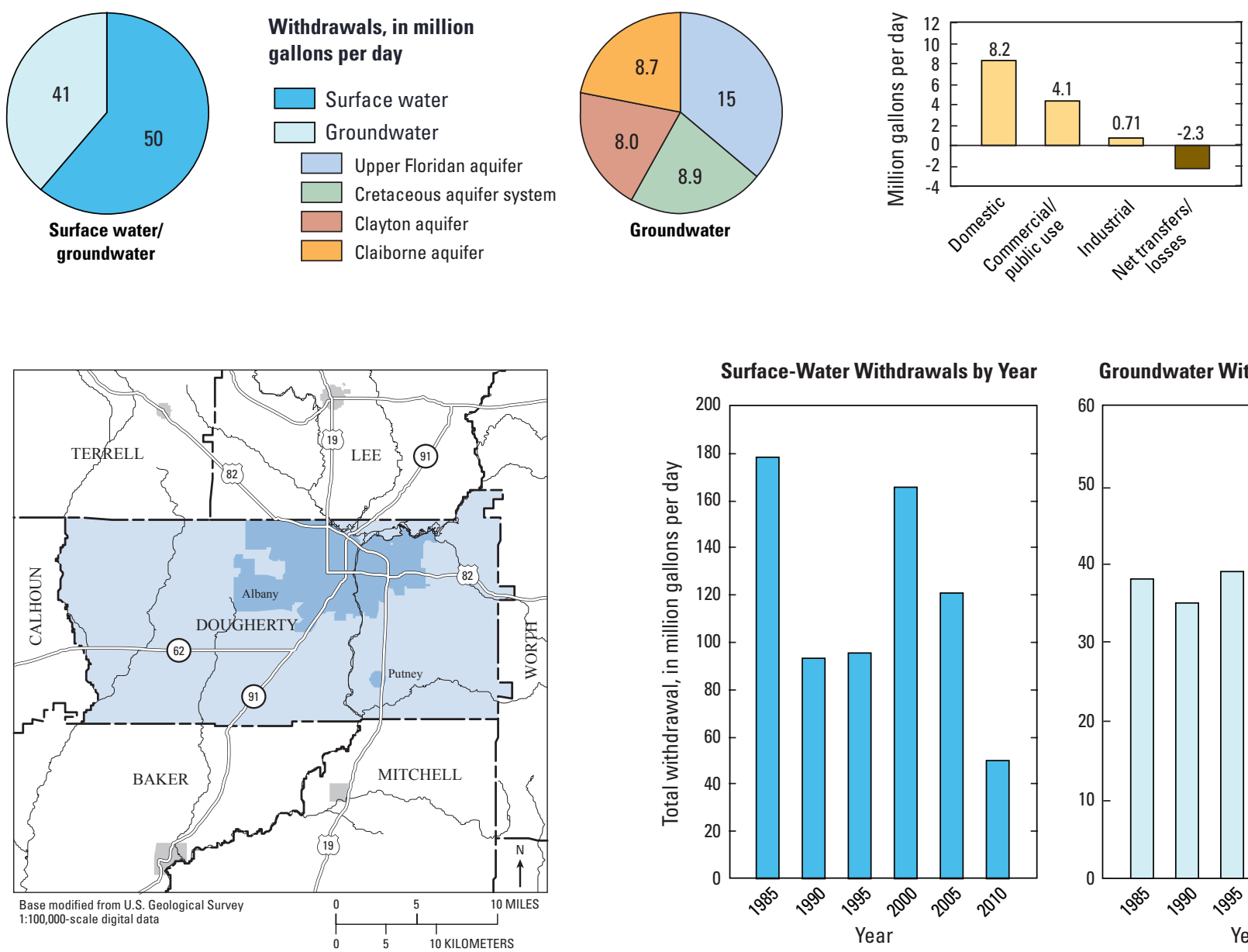

Groundwater Withdrawals by Year

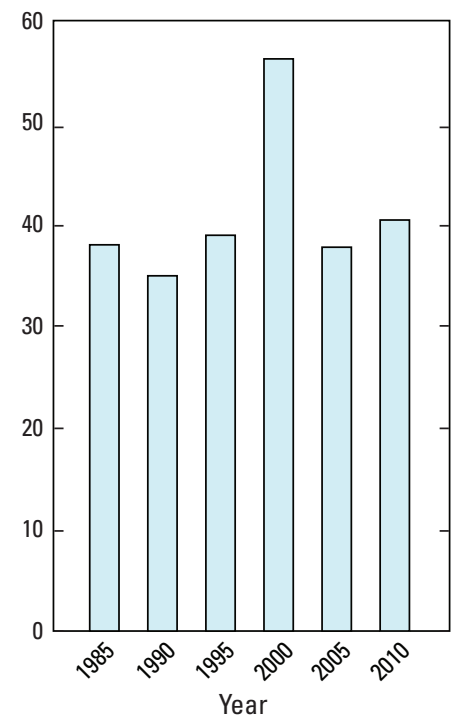


DOUGLAS COUNTY

Population

132,403

540

Population served by public supply-Groundwater

Population served by public supply-Surface water 128,000

Acres irrigated

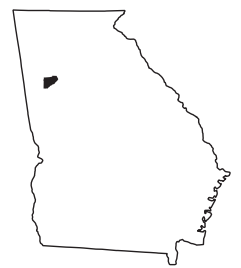

2010 WATER WITHDRAWALS AND ESTIMATED USE, IN MILLION GALLONS PER DAY

$[-$, not applicable; Mgal/d, million gallons per day $]$

\begin{tabular}{|c|c|c|c|c|c|c|c|c|}
\hline \multirow{3}{*}{ Category } & \multicolumn{3}{|c|}{ Withdrawals } & \multirow{3}{*}{$\begin{array}{l}\text { Total } \\
\text { use }^{1}\end{array}$} & \multirow{3}{*}{$\begin{array}{l}\text { Surface- } \\
\text { water } \\
\text { returns } \\
\end{array}$} & \multirow{2}{*}{\multicolumn{3}{|c|}{$\begin{array}{l}\text { Withdrawals by Major Industrial Groups } \\
\text { [NAICS, North American Industrial Classification System code] }\end{array}$}} \\
\hline & \multirow{2}{*}{$\begin{array}{c}\text { Ground- } \\
\text { water }\end{array}$} & \multirow{2}{*}{$\begin{array}{c}\text { Surface } \\
\text { water }\end{array}$} & \multirow[b]{2}{*}{ Total } & & & & & \\
\hline & & & & & & NAICS & Groundwater & Surface water \\
\hline Public supply & 0.04 & 19.93 & 19.97 & - & - & & & \\
\hline Domestic & 0.29 & 0.00 & 0.29 & 8.49 & 0.00 & None & - & - \\
\hline Commercial/public use & 0.00 & 0.00 & 0.00 & 1.36 & 0.00 & & & \\
\hline Industrial & 0.00 & 0.00 & 0.00 & 0.41 & 0.00 & & & \\
\hline Public-supply losses & - & - & - & 2.04 & - & & & \\
\hline Public wastewater treatment & - & - & - & - & 6.29 & Withdrawals by Major & Public Supp & \\
\hline Mining & 0.32 & 0.00 & 0.32 & 0.32 & 0.42 & Name & Groundwater & Surface water \\
\hline Irrigation-Crop & 0.00 & 0.00 & 0.00 & 0.00 & 0.00 & & & . \\
\hline Irrigation-Golf course & 0.00 & 0.60 & 0.60 & 0.60 & 0.00 & Douglasville-Douglas Co. & 0.00 & 11.95 \\
\hline Livestock/aquaculture & 0.16 & 1.66 & 1.82 & 1.82 & 0.00 & & & \\
\hline Thermoelectric power & 0.00 & 0.00 & 0.00 & 0.00 & 0.00 & City of East Point & 0.00 & 17.97 \\
\hline TOTAL & 0.81 & 22.19 & 23.00 & 15.04 & 6.71 & and use & Fulton & \\
\hline
\end{tabular}

'Total use is total withdrawal plus public supply deliveries and losses. An average of $7.97 \mathrm{Mgal} / \mathrm{d}$ were withdrawn from Douglas County and delivered to Fulton County, Georgia in 2010.

\section{Withdrawals by Source}

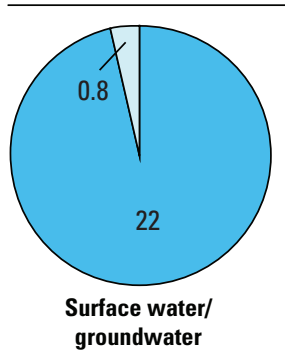

Withdrawals, in million gallons per day

\section{Surface water \\ Groundwater \\ Crystalline-rock aquifer} groundwater

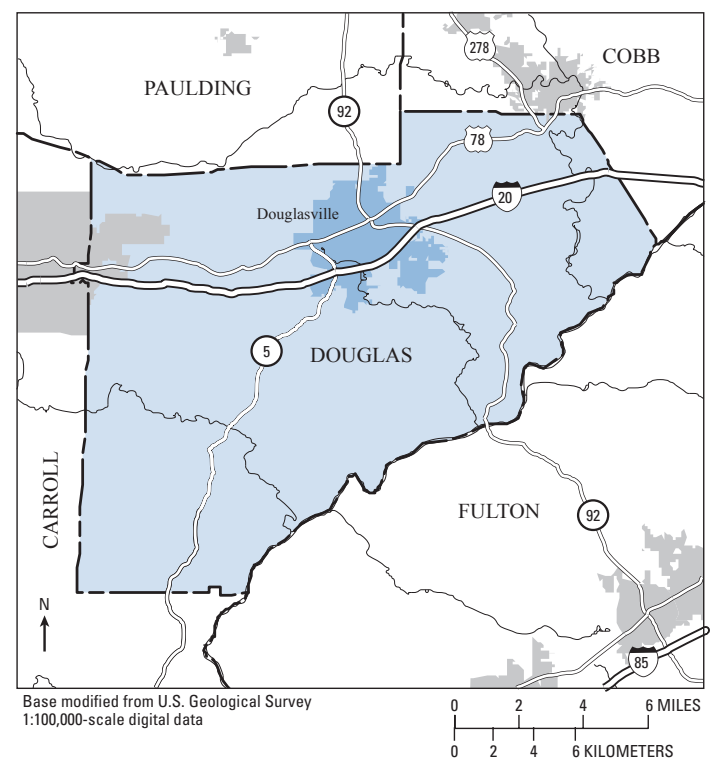

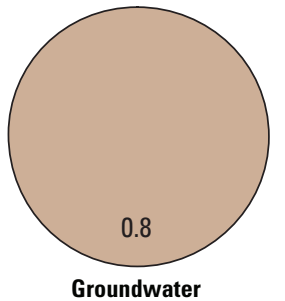

Public-Supply Deliveries ${ }^{1}$ by Use Category

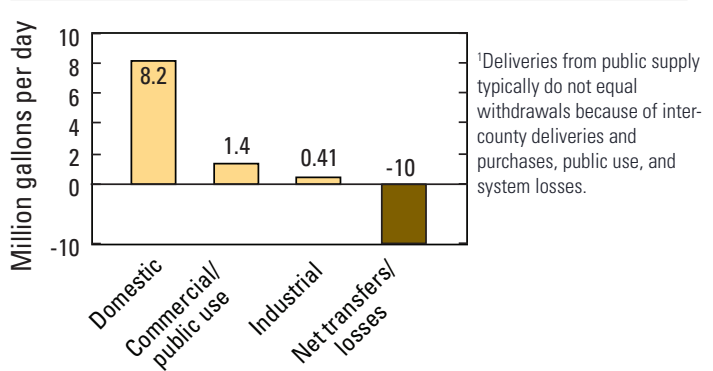

Surface-Water Withdrawals by Year

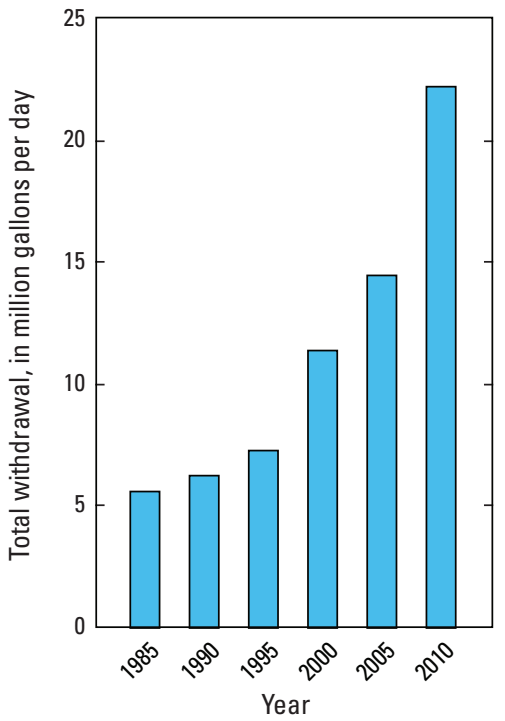

Groundwater Withdrawals by Year

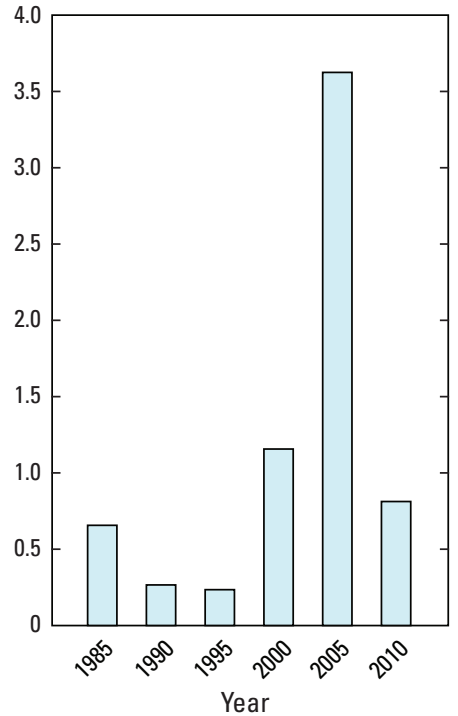




\section{EARLY COUNTY}

Population

Population served by public supply-Groundwater

Population served by public supply-Surface water

Acres irrigated

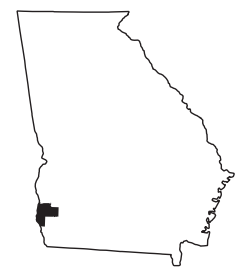

2010 WATER WITHDRAWALS AND ESTIMATED USE, IN MILLION GALLONS PER DAY

$[-$, not applicable $]$

\begin{tabular}{|c|c|c|c|c|c|}
\hline \multirow[b]{2}{*}{ Category } & \multicolumn{3}{|c|}{ Withdrawals } & \multirow[b]{2}{*}{$\begin{array}{l}\text { Total } \\
\text { use }^{1}\end{array}$} & \multirow{2}{*}{$\begin{array}{c}\text { Surface } \\
\text { water } \\
\text { returns }\end{array}$} \\
\hline & $\begin{array}{c}\text { Ground- } \\
\text { water }\end{array}$ & $\begin{array}{c}\text { Surface } \\
\text { water }\end{array}$ & Total & & \\
\hline Public supply & 1.34 & 0.00 & 1.34 & - & \\
\hline Domestic & 0.32 & 0.00 & 0.32 & 1.18 & 0.00 \\
\hline Commercial/public use & 0.00 & 0.00 & 0.00 & 0.27 & 0.00 \\
\hline Industrial & 0.38 & 109.12 & 109.50 & 109.50 & 42.54 \\
\hline Public-supply losses & - & - & - & 0.21 & - \\
\hline Public wastewater treatment & - & - & - & - & 1.16 \\
\hline Mining & 0.00 & 0.00 & 0.00 & 0.00 & 0.00 \\
\hline Irrigation-Crop & 29.44 & 6.71 & 36.15 & 36.15 & 0.00 \\
\hline Irrigation-Golf course & 0.00 & 0.00 & 0.00 & 0.00 & 0.00 \\
\hline Livestock/aquaculture & 0.04 & 0.17 & 0.21 & 0.21 & 0.00 \\
\hline Thermoelectric power & 0.00 & 0.00 & 0.00 & 0.00 & 0.00 \\
\hline TOTAL & 31.52 & 116.00 & 147.52 & 147.52 & 43.70 \\
\hline
\end{tabular}

Withdrawals by Major Industrial Groups
\begin{tabular}{lcc} 
[NAICS, North American Industrial Classification & System code] \\
\hline NAICS & Groundwater & Surface water \\
\hline 322 - Paper, pulp & 0.10 & 109.12 \\
331 - Primary metals & 0.28 & 0.00
\end{tabular}

tal use is total withdrawal plus public supply deliveries and losses.

\section{Withdrawals by Source}

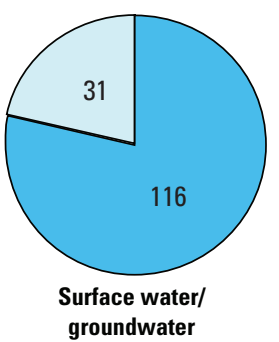

Withdrawals, in million gallons per day

groundwater
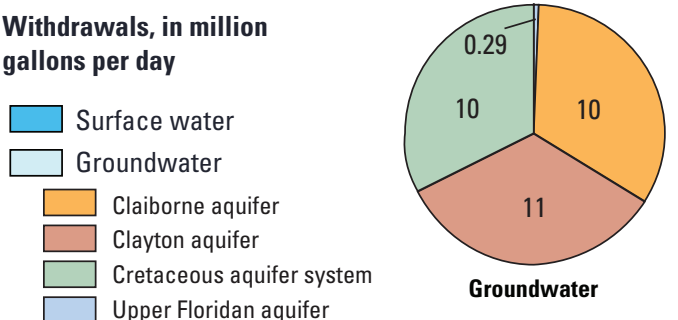

Public-Supply Deliveries by Use Category

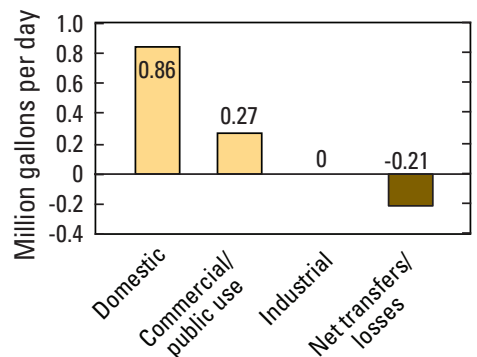

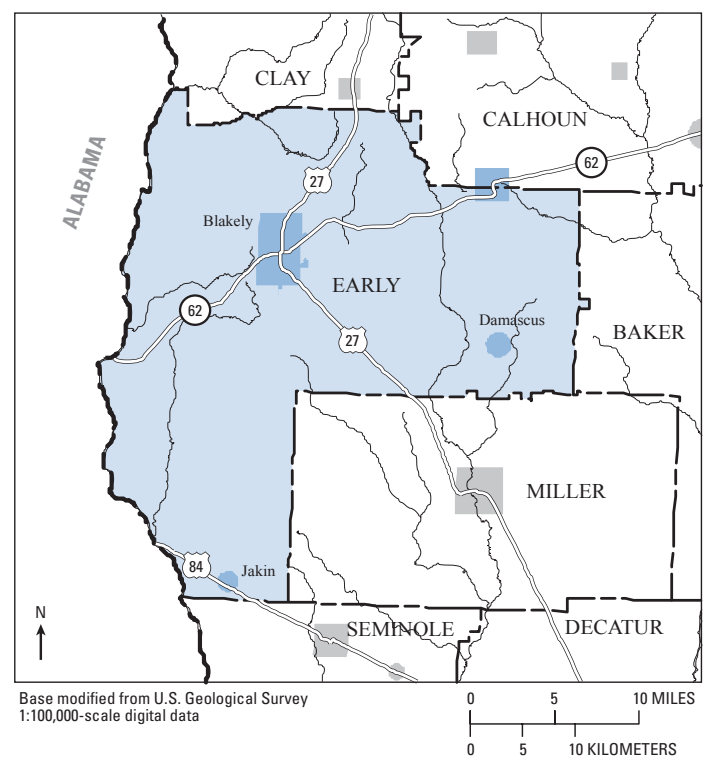

Surface-Water Withdrawals by Year

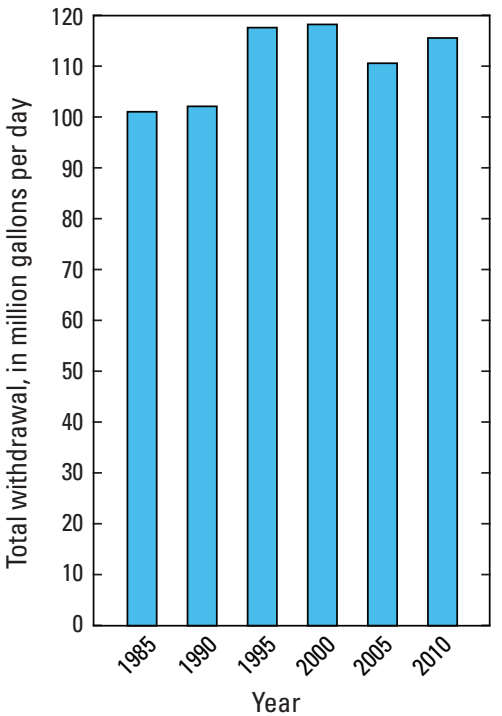

Groundwater Withdrawals by Year

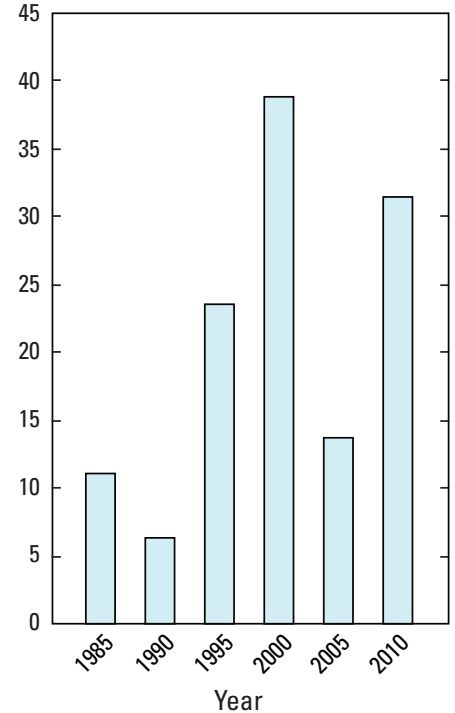




\section{ECHOLS COUNTY}

Population

Population served by public supply-Groundwater

Population served by public supply_-Surface water

Acres irrigated

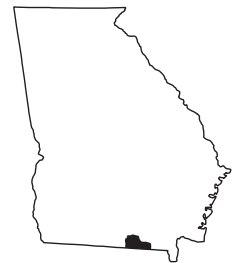

2010 WATER WITHDRAWALS AND ESTIMATED USE, IN MILLION GALLONS PER DAY

$[-$, not applicable; $<$, less than $]$

\begin{tabular}{|c|c|c|c|c|c|}
\hline \multirow[b]{2}{*}{ Category } & \multicolumn{3}{|c|}{ Withdrawals } & \multirow[b]{2}{*}{$\begin{array}{l}\text { Total } \\
\text { use }^{1}\end{array}$} & \multirow{2}{*}{$\begin{array}{c}\text { Surface- } \\
\text { water } \\
\text { returns } \\
\end{array}$} \\
\hline & $\begin{array}{c}\text { Ground- } \\
\text { water }\end{array}$ & $\begin{array}{c}\text { Surface } \\
\text { water }\end{array}$ & Total & & \\
\hline Public supply & 0.05 & 0.00 & 0.05 & - & - \\
\hline Domestic & 0.25 & 0.00 & 0.25 & 0.30 & 0.00 \\
\hline Commercial/public use & 0.00 & 0.00 & 0.00 & 0.00 & 0.00 \\
\hline Industrial & 0.00 & 0.00 & 0.00 & 0.00 & 0.00 \\
\hline Public-supply losses & - & - & - & $<0.01$ & - \\
\hline Public wastewater treatment & - & - & - & - & 0.00 \\
\hline Mining & 0.00 & 0.00 & 0.00 & 0.00 & 0.00 \\
\hline Irrigation-Crop & 0.98 & 0.11 & 1.09 & 1.09 & 0.00 \\
\hline Irrigation-Golf course & 0.00 & 0.00 & 0.00 & 0.00 & 0.00 \\
\hline Livestock/aquaculture & 0.00 & 0.03 & 0.03 & 0.03 & 0.00 \\
\hline Thermoelectric power & 0.00 & 0.00 & 0.00 & 0.00 & 0.00 \\
\hline TOTAL & 1.28 & 0.14 & 1.42 & 1.42 & 0.00 \\
\hline
\end{tabular}

Withdrawals by Major Industrial Groups

[NAICS, North American Industrial Classification System code]

\begin{tabular}{lcc} 
NAICS & Groundwater & Surface water \\
\hline None & - & - \\
\hline
\end{tabular}

Total use is total withdrawal plus public supply deliveries and losses.

\section{Withdrawals by Source}
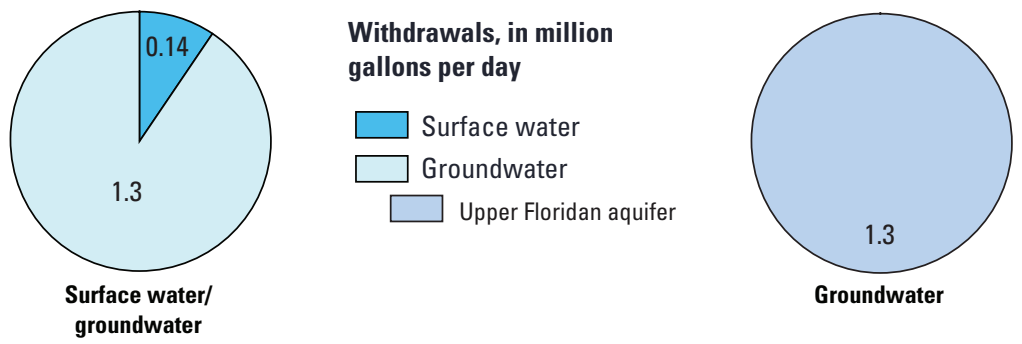

Public-Supply Deliveries by Use Category

Withdrawals by Major Public Suppliers

\begin{tabular}{lcc}
\hline Name & Groundwater & Surface water \\
\hline Echols County & 0.05 & 0.00 \\
Water Authority & & \\
\hline
\end{tabular}

groundwater

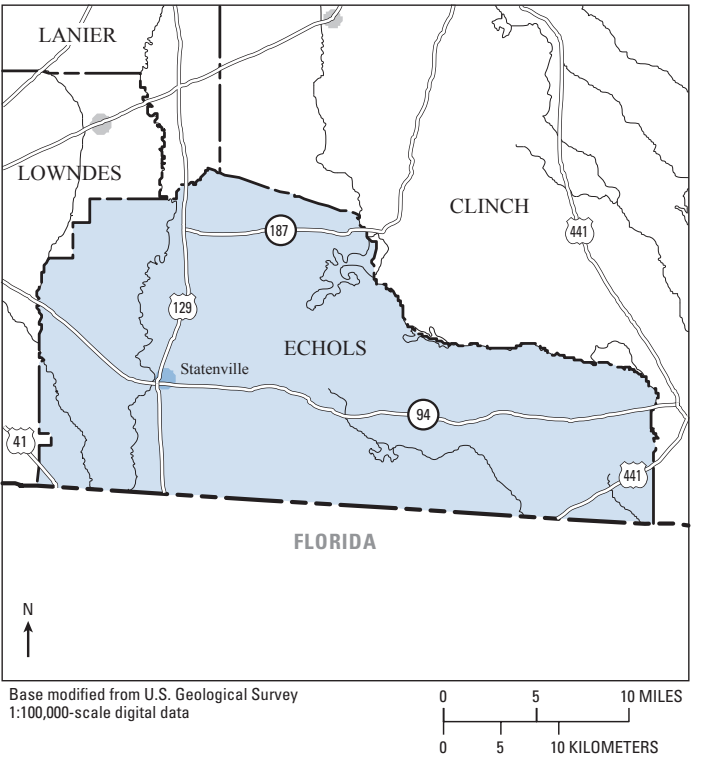

Surface-Water Withdrawals by Year

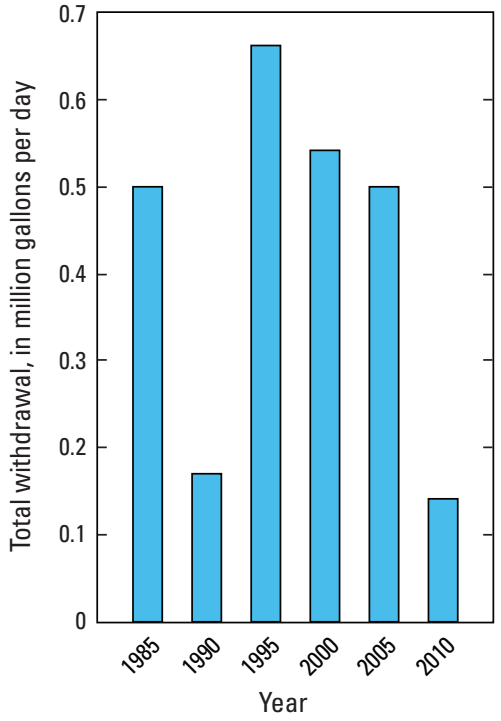

Groundwater Withdrawals by Year

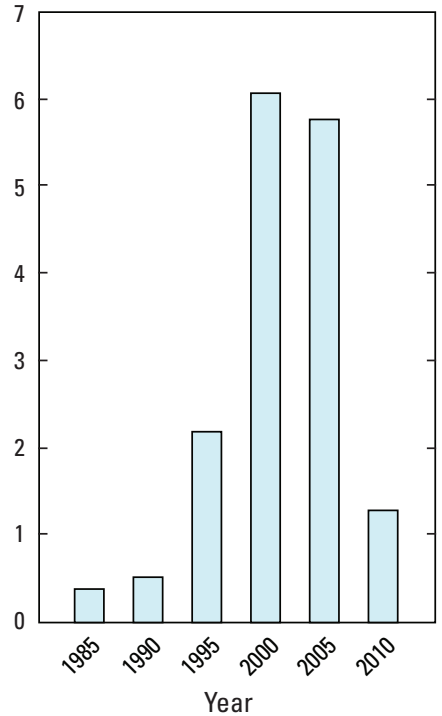




\section{EFFINGHAM COUNTY}

Population

52,250

Population served by public supply-Groundwater $\quad 31,260$

Population served by public supply-Surface water $\quad 1,890$

Acres irrigated

980

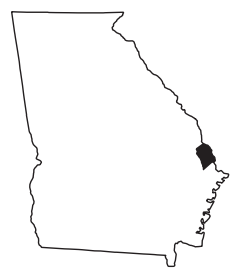

2010 WATER WITHDRAWALS AND ESTIMATED USE, IN MILLION GALLONS PER DAY

[-, not applicable; Mgal/d, million gallons per day $]$

\begin{tabular}{|c|c|c|c|c|c|c|c|c|}
\hline \multirow{3}{*}{ Category } & \multicolumn{3}{|c|}{ Withdrawals } & \multirow{3}{*}{$\begin{array}{l}\text { Total } \\
\text { use }^{1}\end{array}$} & \multirow{3}{*}{$\begin{array}{l}\text { Surface- } \\
\text { water } \\
\text { returns } \\
\end{array}$} & \multirow{2}{*}{\multicolumn{3}{|c|}{$\begin{array}{l}\text { Withdrawals by Major Industrial Groups } \\
\text { [NAICS, North American Industrial Classification System code] }\end{array}$}} \\
\hline & \multirow{2}{*}{$\begin{array}{c}\text { Ground- } \\
\text { water }\end{array}$} & \multirow{2}{*}{$\begin{array}{c}\text { Surface } \\
\text { water }\end{array}$} & \multirow[b]{2}{*}{ Total } & & & & & \\
\hline & & & & & & NAICS & Groundwater & Surface water \\
\hline Public supply & 3.08 & 32.50 & 35.58 & - & - & 322_-Paper, pulp & 0.81 & 14.42 \\
\hline Domestic & 1.43 & 0.00 & 1.43 & 3.94 & 0.00 & 322 -Paper, pulp & 0.81 & 14.42 \\
\hline Commercial/public use & 0.00 & 0.00 & 0.00 & 0.27 & 0.00 & \multicolumn{3}{|c|}{ Withdrawals by Major Public Suppliers } \\
\hline Industrial & 0.81 & 14.42 & 15.23 & 15.56 & 15.38 & Name & Groundwater & Surface water \\
\hline Public-supply losses & - & - & - & 0.47 & - & & & \\
\hline Public wastewater treatment & - & - & - & - & 0.71 & $\begin{array}{l}\text { Savannah Industrial \& } \\
\text { Domestic Water }\end{array}$ & 0.00 & 32.50 \\
\hline Mining & 0.15 & 0.00 & 0.15 & 0.15 & 0.00 & City of Rincon & 0.85 & 0.00 \\
\hline Irrigation-Crop & 0.18 & 0.05 & 0.23 & 0.23 & 0.00 & City of Springfield & 0.71 & 0.00 \\
\hline Irrigation-Golf course & 0.00 & 0.00 & 0.00 & 0.00 & 0.00 & Water Utility Management & 0.39 & 0.00 \\
\hline Livestock/aquaculture & 0.02 & 0.07 & 0.09 & 0.09 & 0.00 & Lakeside Water Company & 0.22 & 0.00 \\
\hline Thermoelectric power & 0.04 & 70.17 & 70.21 & 70.21 & 0.00 & City of Guyton & 0.20 & 0.00 \\
\hline TOTAL & 5.71 & 117.21 & 122.92 & 90.92 & 16.09 & Middle Georgia Water Co. & 0.16 & 0.00 \\
\hline T & & SES. & U. & & unty & Residential subdivision & 0.14 & 0.00 \\
\hline
\end{tabular}

\section{Withdrawals by Source}
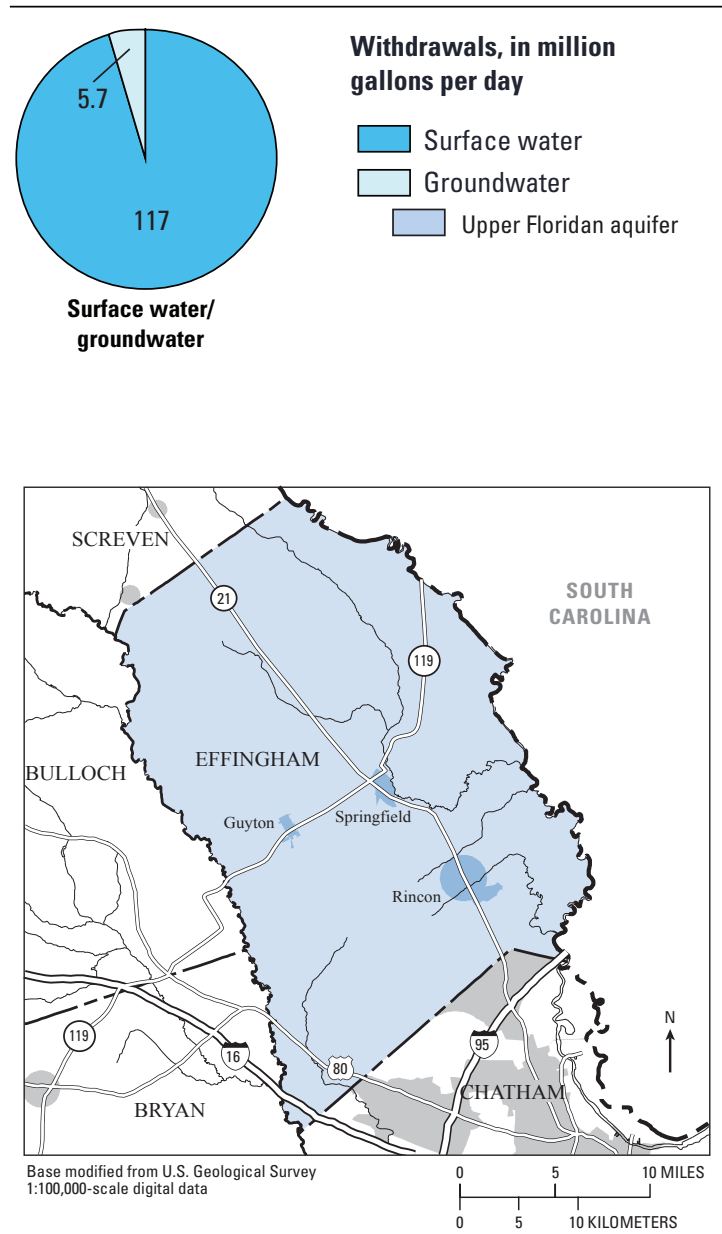

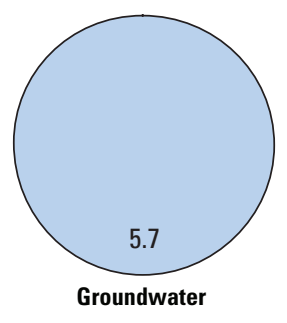

\section{Public-Supply Deliveries ${ }^{1}$ by Use Category}

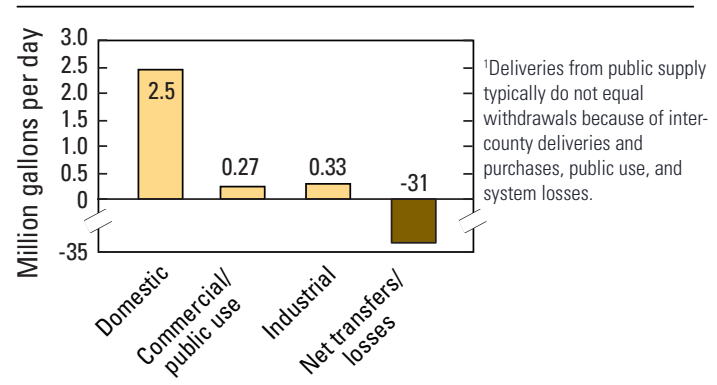

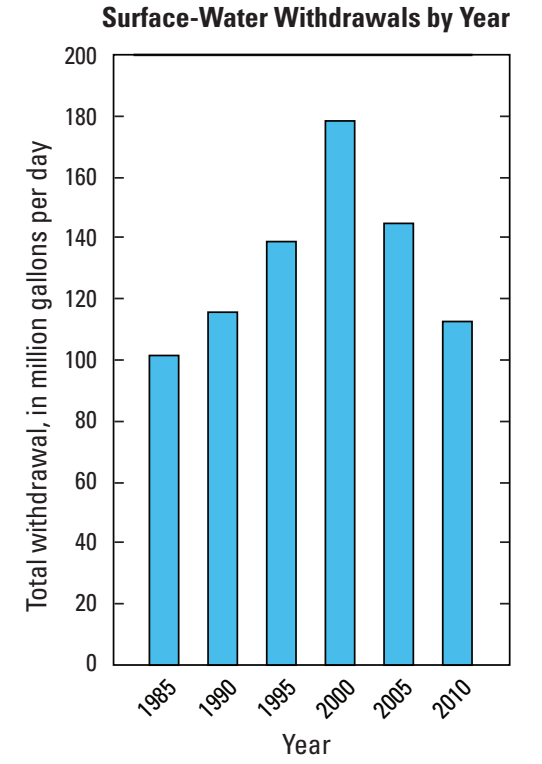

Groundwater Withdrawals by Year

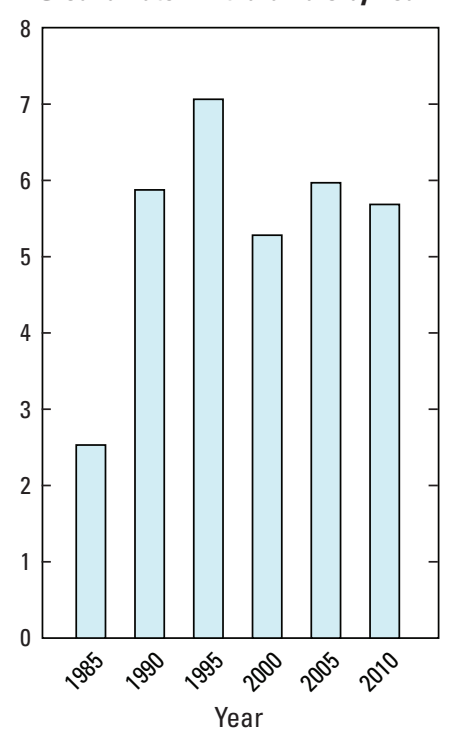




\section{ELBERT COUNTY}

Population

Population served by public supply-Groundwater Population served by public supply-Surface water Acres irrigated
1,100

6,000

575

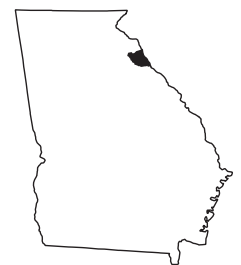

2010 WATER WITHDRAWALS AND ESTIMATED USE, IN MILLION GALLONS PER DAY

[-, not applicable]

\begin{tabular}{lcccccc}
\hline & \multicolumn{3}{c}{ Withdrawals } & & Surface- \\
\cline { 2 - 3 } Category & $\begin{array}{c}\text { Ground- } \\
\text { water }\end{array}$ & $\begin{array}{c}\text { Surface } \\
\text { water }\end{array}$ & Total & & $\begin{array}{c}\text { Total } \\
\text { use }\end{array}$ & $\begin{array}{c}\text { Water } \\
\text { returns }\end{array}$ \\
\hline Public supply & 0.07 & 1.52 & 1.59 & - & - \\
Domestic & 0.98 & 0.00 & 0.98 & 1.66 & 0.00 \\
Commercial/public use & 0.01 & 0.00 & 0.01 & 0.18 & 0.00 \\
Industrial & 0.00 & 0.00 & 0.00 & 0.52 & 0.00 \\
Public-supply losses & - & - & - & 0.22 & - \\
Public wastewater treatment & - & - & - & - & 1.03 \\
Mining & 0.08 & 0.00 & 0.08 & 0.08 & 0.00 \\
Irrigation-Crop & 0.26 & 0.08 & 0.34 & 0.34 & 0.00 \\
Irrigation-Golf course & 0.06 & 0.08 & 0.14 & 0.14 & 0.00 \\
Livestock/aquaculture & 0.02 & 0.30 & 0.32 & 0.32 & 0.00 \\
Thermoelectric power & 0.00 & 0.00 & 0.00 & 0.00 & 0.00 \\
\multicolumn{1}{c}{ TOTAL } & $\mathbf{1 . 4 8}$ & $\mathbf{1 . 9 8}$ & $\mathbf{3 . 4 6}$ & $\mathbf{3 . 4 6}$ & $\mathbf{1 . 0 3}$ \\
\hline
\end{tabular}

Withdrawals by Major Industrial Groups

[NAICS, North American Industrial Classification System code] NAICS

Groundwater

Surface water

None

Withdrawals by Major Public Suppliers

Name Groundwater Surface water

\begin{tabular}{lll}
\hline City of Elberton & 0.00 & 1.52
\end{tabular}

$\begin{array}{lll}\text { City of Bowman } & 0.06 & 0.00\end{array}$

'Total use is total withdrawal plus public supply deliveries and losses.

\section{Withdrawals by Source}
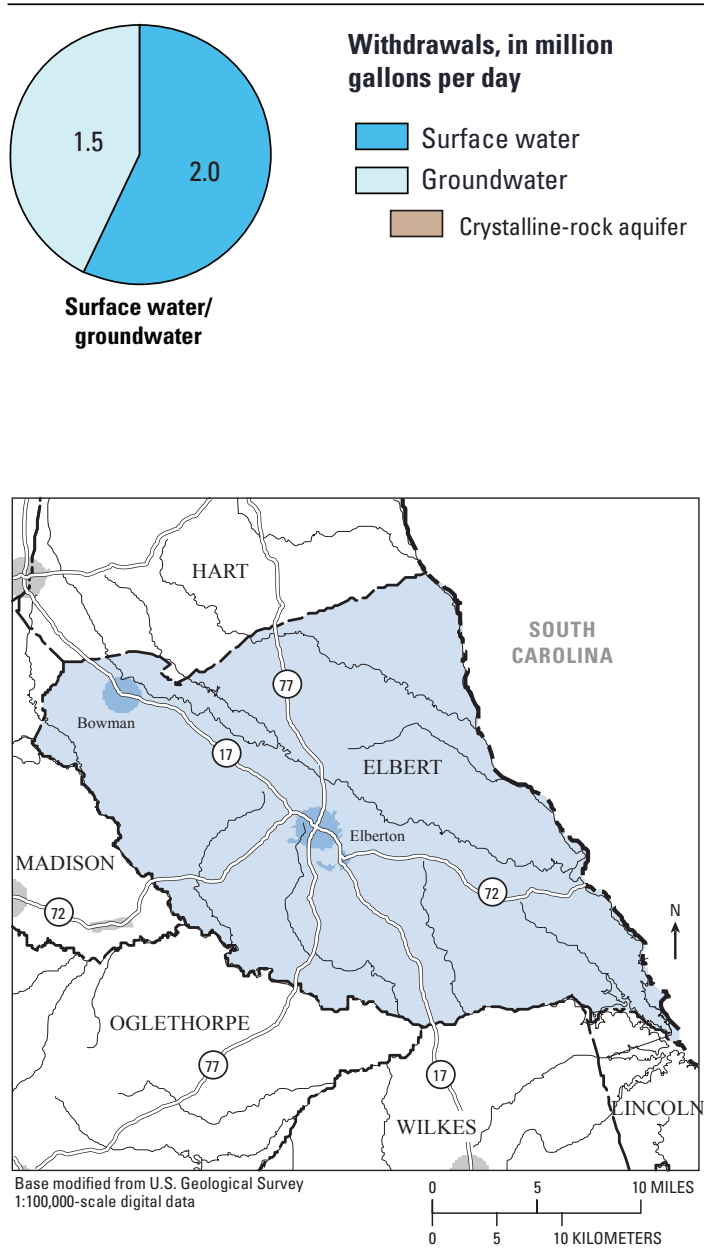

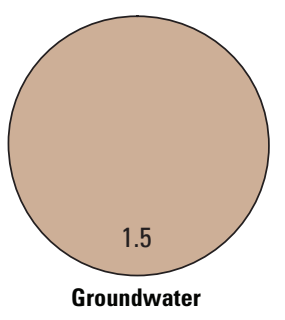

Public-Supply Deliveries by Use Category

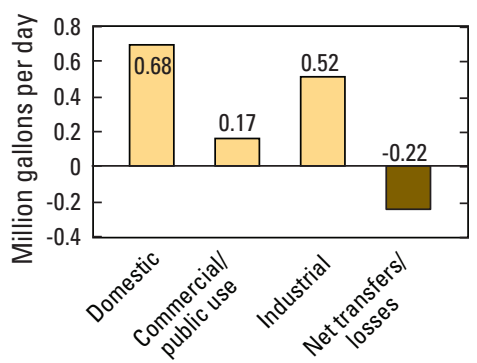

Surface-Water Withdrawals by Year

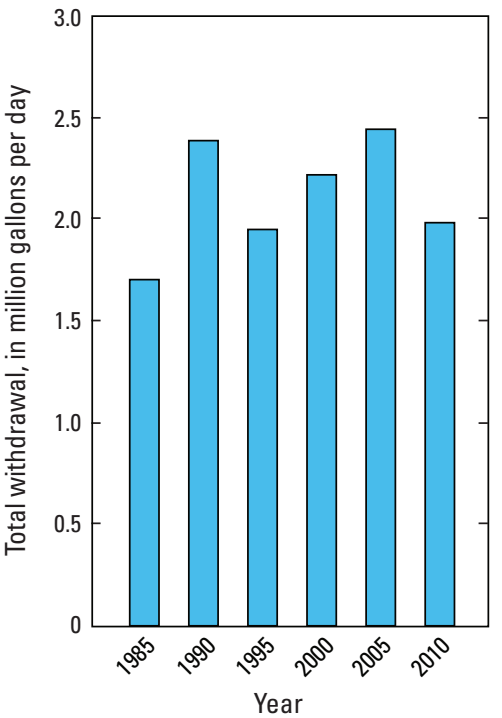

Groundwater Withdrawals by Year

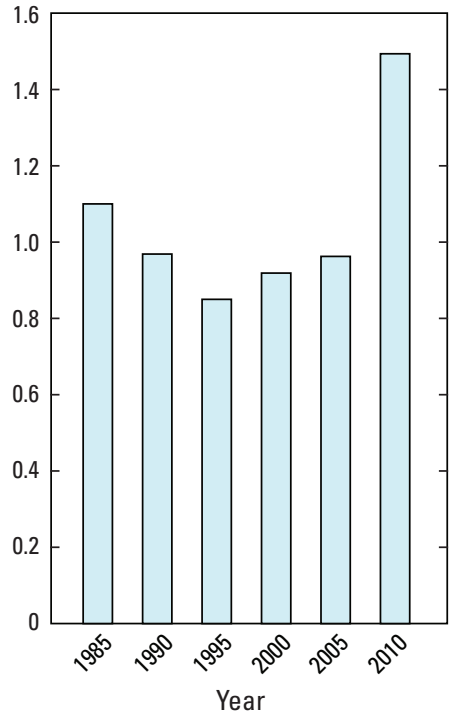




\section{EMANUEL COUNTY}

Population

Population served by public supply-Groundwater $\quad 11,570$

Population served by public supply-Surface water 0

Acres irrigated

3,490

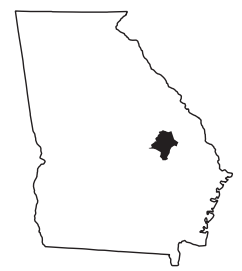

2010 WATER WITHDRAWALS AND ESTIMATED USE, IN MILLION GALLONS PER DAY

$[-$, not applicable $]$

\begin{tabular}{|c|c|c|c|c|c|c|c|c|}
\hline \multirow{3}{*}{ Category } & \multicolumn{3}{|c|}{ Withdrawals } & \multirow{3}{*}{$\begin{array}{l}\text { Total } \\
\text { use }^{1}\end{array}$} & \multirow{3}{*}{$\begin{array}{l}\text { Surface- } \\
\text { water } \\
\text { returns } \\
\end{array}$} & \multirow{2}{*}{\multicolumn{3}{|c|}{$\begin{array}{l}\text { Withdrawals by Major Industrial Groups } \\
\text { [NAICS, North American Industrial Classification System code] }\end{array}$}} \\
\hline & \multirow{2}{*}{$\begin{array}{c}\text { Ground- } \\
\text { water }\end{array}$} & \multirow{2}{*}{$\begin{array}{c}\text { Surface } \\
\text { water }\end{array}$} & \multirow[b]{2}{*}{ Total } & & & & & \\
\hline & & & & & & NAICS & Groundwater & Surface water \\
\hline Public supply & 1.67 & 0.00 & 1.67 & - & - & 311 -Food & 0.97 & 0.00 \\
\hline Domestic & 0.83 & 0.00 & 0.83 & 1.95 & 0.00 & $311-$ Food & 0.97 & 0.00 \\
\hline Commercial/public use & 0.00 & 0.00 & 0.00 & 0.27 & 0.00 & & & \\
\hline Industrial & 0.97 & 0.00 & 0.97 & 1.06 & 0.00 & & & \\
\hline Public-supply losses & - & - & - & 0.19 & - & & & \\
\hline Public wastewater treatment & - & - & - & - & 0.84 & Withdrawals by M & r Public Supp & \\
\hline Mining & 0.00 & 0.00 & 0.00 & 0.00 & 0.00 & Name & Groundwater & Surface water \\
\hline $\begin{array}{l}\text { Irrigation-Crop } \\
\text { Irrigation-Golf course }\end{array}$ & $\begin{array}{l}2.71 \\
0.00\end{array}$ & $\begin{array}{l}0.02 \\
0.00\end{array}$ & $\begin{array}{l}2.73 \\
0.00\end{array}$ & $\begin{array}{l}2.73 \\
0.00\end{array}$ & $\begin{array}{l}0.00 \\
0.00\end{array}$ & City of Swainsboro & 1.16 & 0.00 \\
\hline Livestock/aquaculture & 0.16 & 0.23 & 0.39 & 0.39 & 0.00 & City of Twin City & 0.17 & 0.00 \\
\hline Thermoelectric power & 0.00 & 0.00 & 0.00 & 0.00 & 0.00 & City of Adrian & 0.10 & 0.00 \\
\hline TOTAL & 6.34 & 0.25 & 6.59 & 6.59 & 0.84 & City of Stillmore & 0.09 & 0.00 \\
\hline
\end{tabular}

${ }^{1}$ Total use is total withdrawal plus public supply deliveries and losses.

\section{Withdrawals by Source}

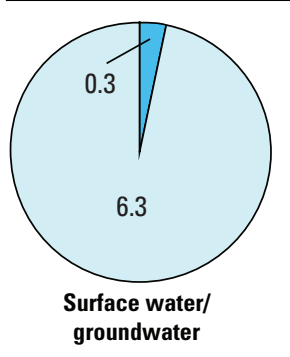

Withdrawals, in million gallons per day

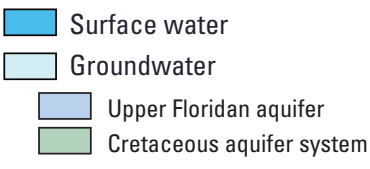

groundwater

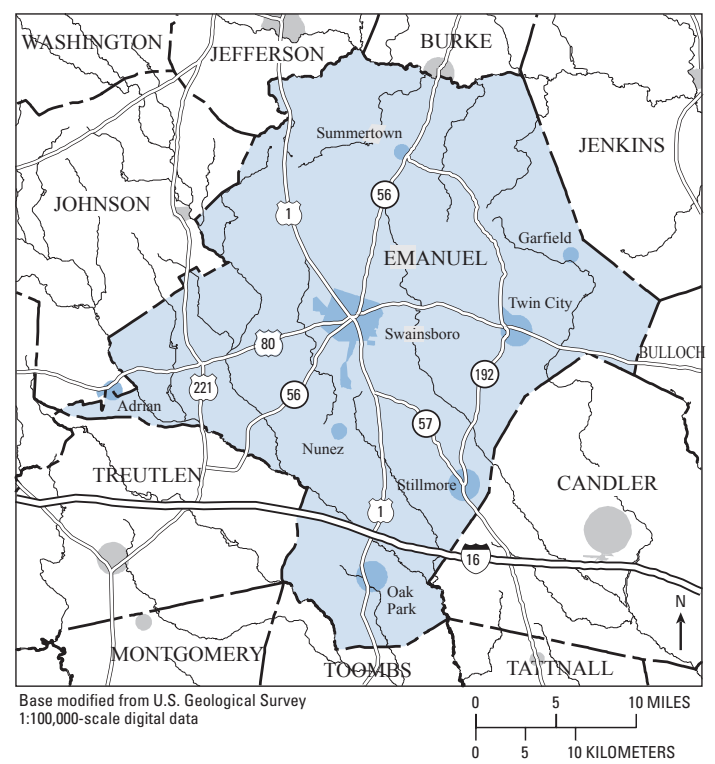

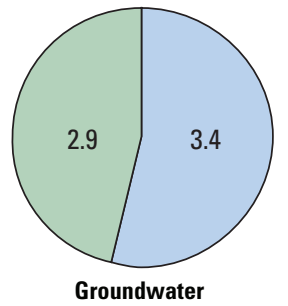

Public-Supply Deliveries by Use Category

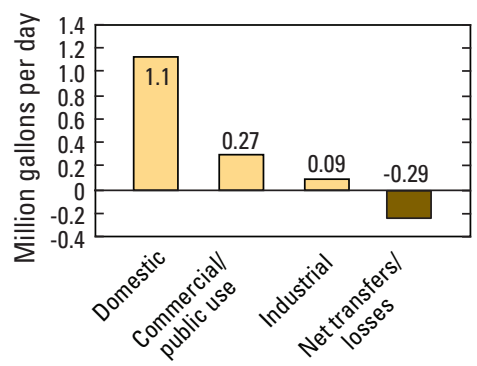

Surface-Water Withdrawals by Year

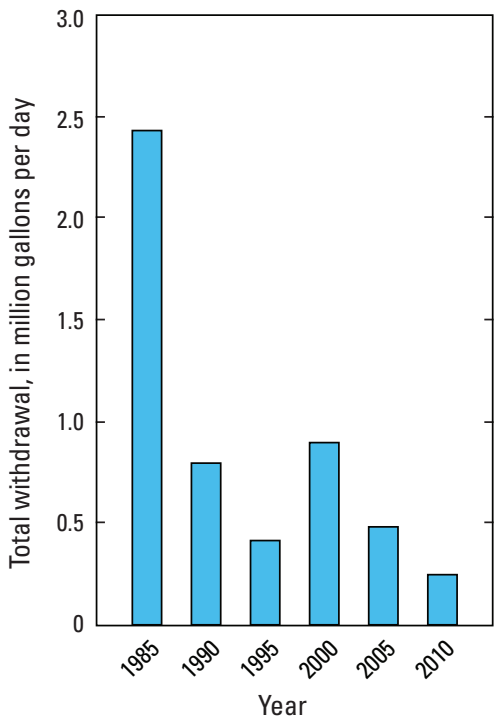

Groundwater Withdrawals by Year

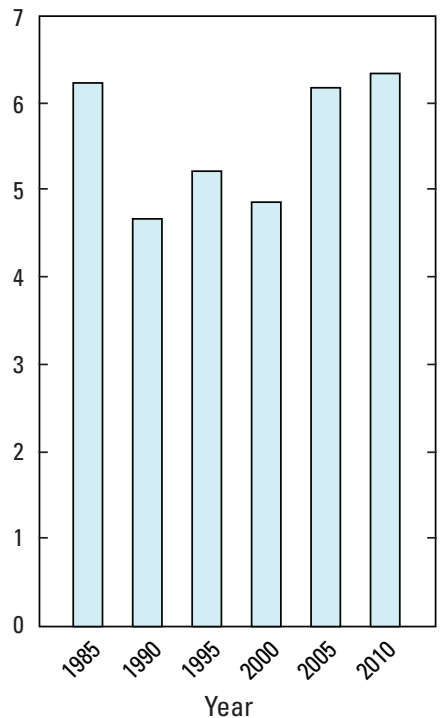


EVANS COUNTY

Population

Population served by public supply-Groundwater

Population served by public supply-Surface water

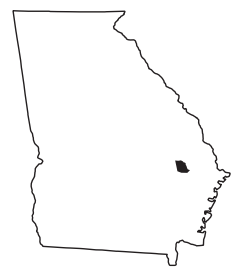

2010 WATER WITHDRAWALS AND ESTIMATED USE, IN MILLION GALLONS PER DAY

$[-$, not applicable $]$

\begin{tabular}{|c|c|c|c|c|c|c|c|c|}
\hline \multirow{3}{*}{ Category } & \multicolumn{3}{|c|}{ Withdrawals } & \multirow{3}{*}{$\begin{array}{l}\text { Total } \\
\text { use }^{1}\end{array}$} & \multirow{3}{*}{$\begin{array}{l}\text { Surface- } \\
\text { water } \\
\text { returns }\end{array}$} & \multirow{2}{*}{\multicolumn{3}{|c|}{$\begin{array}{l}\text { Withdrawals by Major Industrial Groups } \\
\text { [NAICS, North American Industrial Classification System code] }\end{array}$}} \\
\hline & \multirow{2}{*}{$\begin{array}{c}\text { Ground- } \\
\text { water }\end{array}$} & \multirow{2}{*}{$\begin{array}{c}\text { Surface } \\
\text { water }\end{array}$} & \multirow[b]{2}{*}{ Total } & & & & & \\
\hline & & & & & & NAICS & Groundwater & Surface water \\
\hline Public supply & 0.59 & 0.00 & 0.59 & - & - & 311-Food & 1.50 & 0.00 \\
\hline Domestic & 0.52 & 0.00 & 0.52 & 0.92 & 0.00 & & & \\
\hline Commercial/public use & 0.00 & 0.00 & 0.00 & 0.10 & 0.00 & & & \\
\hline Industrial & 1.50 & 0.00 & 1.50 & 1.52 & 0.00 & & & \\
\hline Public-supply losses & - & - & - & 0.07 & - & & & \\
\hline Public wastewater treatment & - & - & - & - & 0.02 & Withdrawals & Public Supp & \\
\hline Mining & 0.03 & 0.00 & 0.03 & 0.03 & 0.00 & Name & Groundwater & Surface water \\
\hline $\begin{array}{l}\text { Irrigation-Crop } \\
\text { Irrigation-Golf course }\end{array}$ & $\begin{array}{l}1.34 \\
0.10\end{array}$ & $\begin{array}{l}0.81 \\
0.00\end{array}$ & $\begin{array}{l}2.15 \\
0.10\end{array}$ & $\begin{array}{l}2.15 \\
0.10\end{array}$ & $\begin{array}{l}0.00 \\
0.00\end{array}$ & City of Claxton & 0.47 & 0.00 \\
\hline Livestock/aquaculture & 0.01 & 0.11 & 0.12 & 0.12 & 0.00 & City of Hagan & 0.08 & 0.00 \\
\hline
\end{tabular}

Thermoelectric power

4.09

0.92

5.01

5.01

0.02

'Total use is total withdrawal plus public supply deliveries and losses.

\section{Withdrawals by Source}

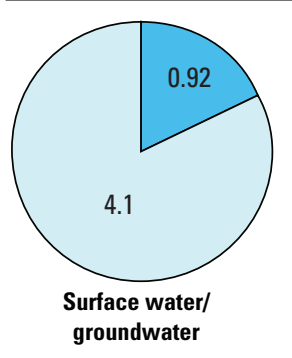

Withdrawals, in million gallons per day
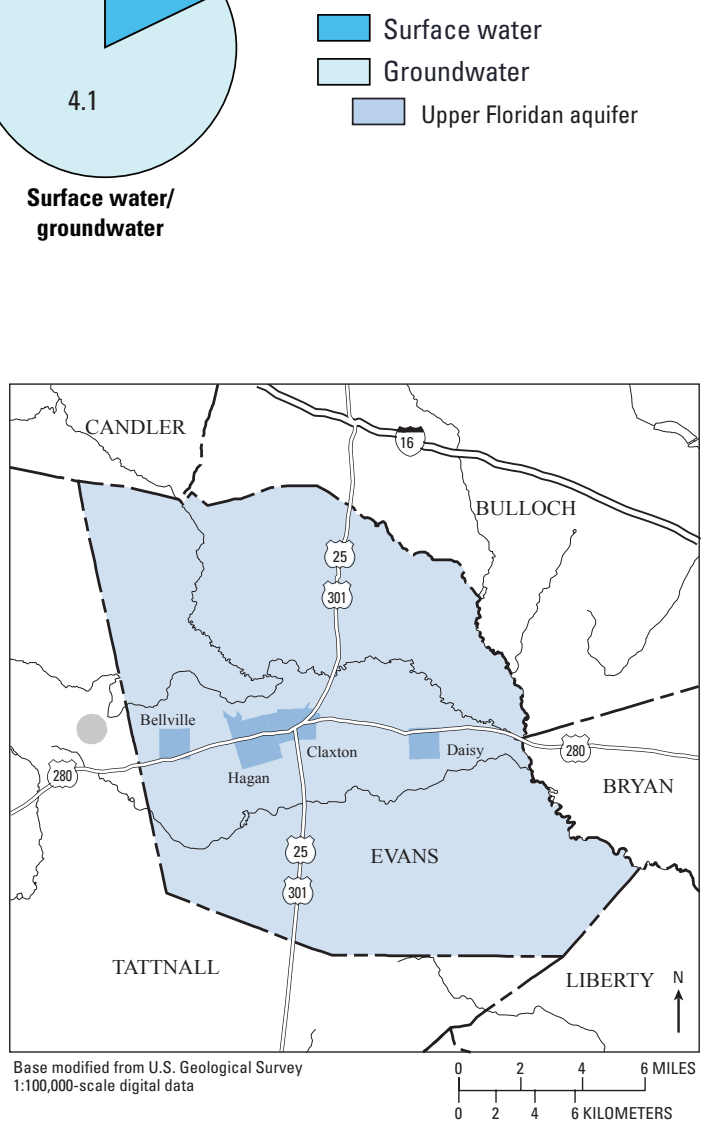

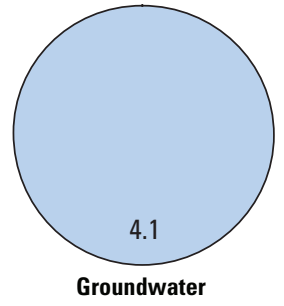

Public-Supply Deliveries by Use Category

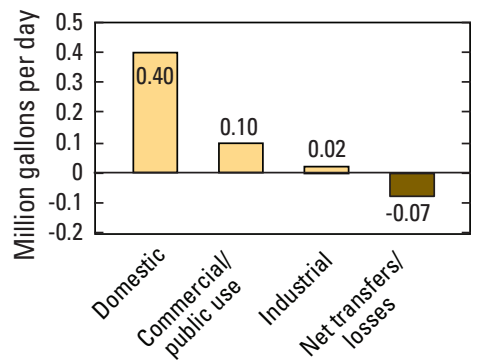

Surface-Water Withdrawals by Year

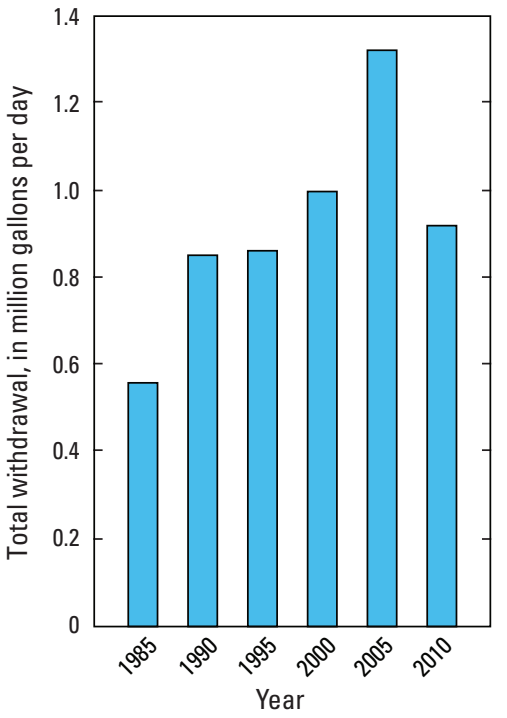

Groundwater Withdrawals by Year

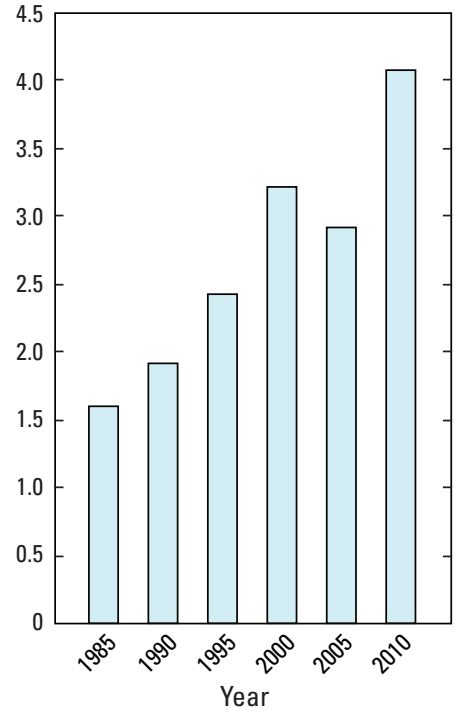




\section{FANNIN COUNTY}

Population

Population served by public supply-Groundwater

Population served by public supply-Surface water

Acres irrigated

10,150

200

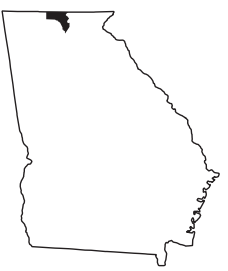

2010 WATER WITHDRAWALS AND ESTIMATED USE, IN MILLION GALLONS PER DAY

$[-$, not applicable; Mgal/d, million gallons per day]

\begin{tabular}{|c|c|c|c|c|c|c|c|c|}
\hline \multirow{3}{*}{ Category } & \multicolumn{3}{|c|}{ Withdrawals } & \multirow{3}{*}{$\begin{array}{l}\text { Total } \\
\text { use }^{1}\end{array}$} & \multirow{3}{*}{$\begin{array}{l}\text { Surface- } \\
\text { water } \\
\text { returns } \\
\end{array}$} & \multirow{2}{*}{\multicolumn{3}{|c|}{$\begin{array}{l}\text { Withdrawals by Major Industrial Groups } \\
\text { [NAICS, North American Industrial Classification System code] }\end{array}$}} \\
\hline & \multirow{2}{*}{$\begin{array}{c}\text { Ground- } \\
\text { water }\end{array}$} & \multirow{2}{*}{$\begin{array}{c}\text { Surface } \\
\text { water }\end{array}$} & \multirow[b]{2}{*}{ Total } & & & & & \\
\hline & & & & & & NAICS & Groundwater & Surface water \\
\hline Public supply & 0.12 & 1.78 & 1.90 & - & - & & & \\
\hline Domestic & 0.89 & 0.00 & 0.89 & 1.98 & 0.00 & None & - & - \\
\hline Commercial/public use & 0.00 & 0.00 & 0.00 & 0.39 & 0.00 & & & \\
\hline Industrial & 0.00 & 0.00 & 0.00 & 0.04 & 0.00 & & & \\
\hline Public-supply losses & - & - & - & 0.27 & - & & & \\
\hline Public wastewater treatment & - & - & - & - & 0.34 & Withdrawals by M & r Public Supp & \\
\hline Mining & 0.00 & 0.00 & 0.00 & 0.00 & 0.00 & Name & Groundwater & Surface water \\
\hline Irrigation-Crop & 0.01 & 0.01 & 0.02 & 0.02 & 0.00 & Naille & & \\
\hline Irrigation-Golf course & 0.00 & 0.00 & 0.00 & 0.00 & 0.00 & City of Blue Ridge & 0.00 & 0.93 \\
\hline Livestock/aquaculture & 0.00 & 6.42 & 6.42 & 6.42 & 1.95 & City of Mccaysville & 0.00 & 0.85 \\
\hline Thermoelectric power & 0.00 & 0.00 & 0.00 & 0.00 & 0.00 & City of Morganton & 0.11 & 0.00 \\
\hline TOTAL & 1.02 & 8.21 & 9.23 & 9.12 & 2.29 & & & \\
\hline
\end{tabular}

'Total use is total withdrawal plus public supply deliveries and losses. An average of $0.12 \mathrm{Mgal} / \mathrm{d}$ was delivered from Fannin County, Georgia

to Polk County, Tennessee in 2010

\section{Withdrawals by Source}

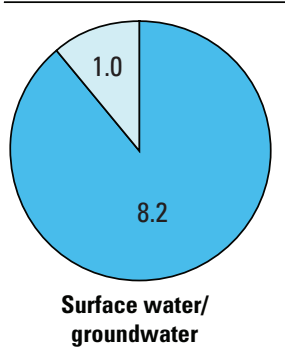

Withdrawals, in million gallons per day

$\square$ Surface water

Groundwater

$\square$ Crystalline-rock aquifer groundwater

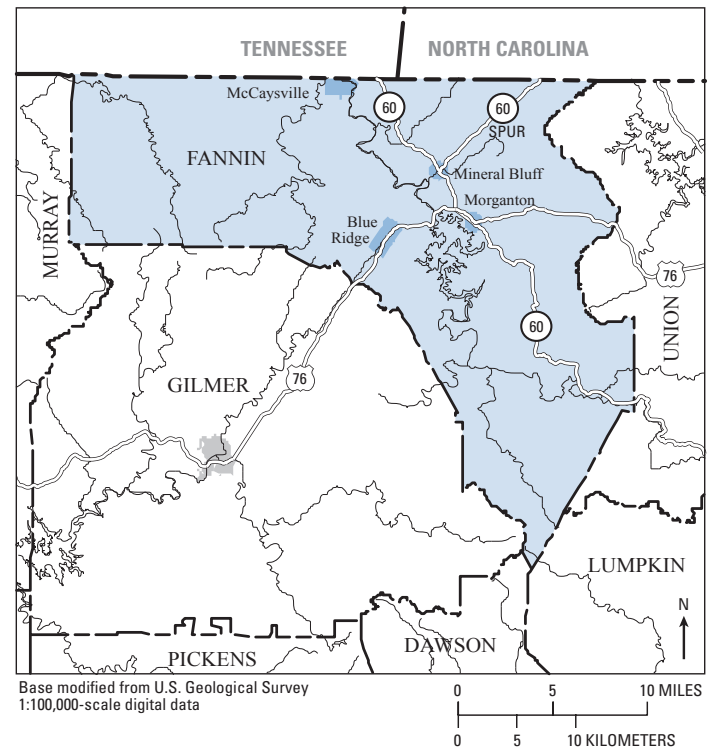

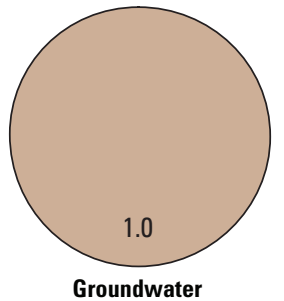

Public-Supply Deliveries ${ }^{1}$ by Use Category

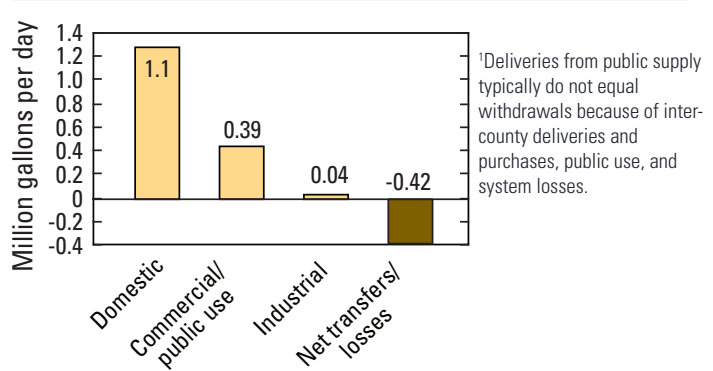

Surface-Water Withdrawals by Year

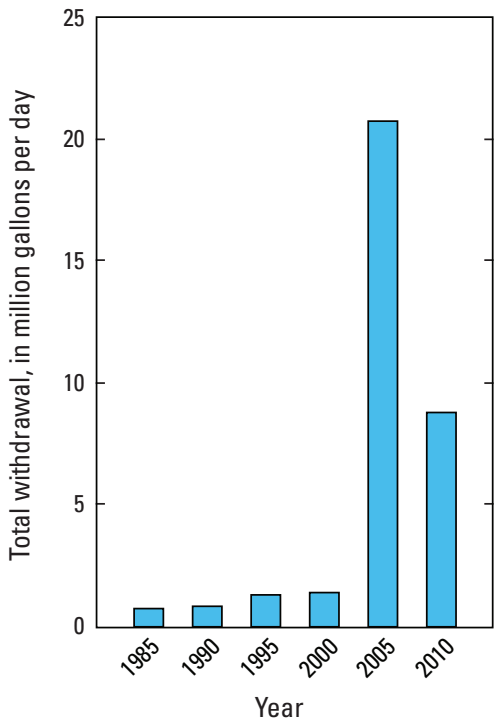

Groundwater Withdrawals by Year

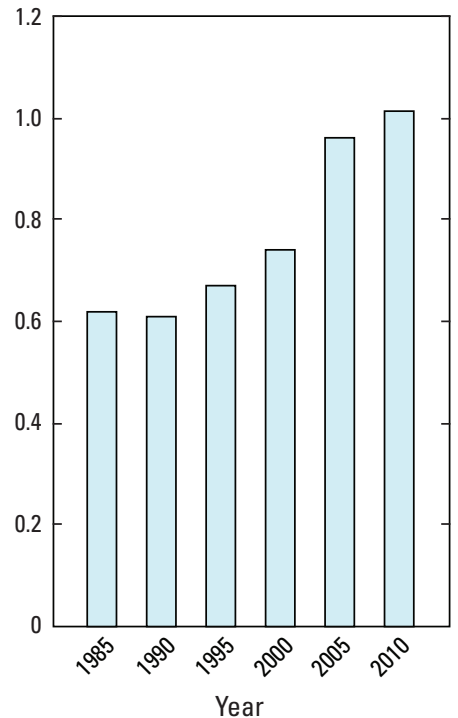




\section{FAYETTE COUNTY}

Population

106,567

Population served by public supply_-Groundwater $\quad 9,300$

Population served by public supply-Surface water $\quad 83,130$

Acres irrigated

645

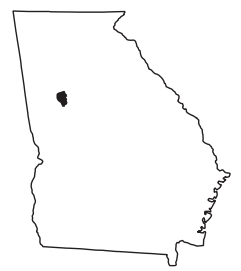

2010 WATER WITHDRAWALS AND ESTIMATED USE, IN MILLION GALLONS PER DAY

$[-$, not applicable; Mgal/d, million gallons per day $]$

\begin{tabular}{|c|c|c|c|c|c|c|c|c|}
\hline \multirow{3}{*}{ Category } & \multicolumn{3}{|c|}{ Withdrawals } & \multirow{3}{*}{$\begin{array}{l}\text { Total } \\
\text { use }^{1}\end{array}$} & \multirow{3}{*}{$\begin{array}{l}\text { Surface- } \\
\text { water } \\
\text { returns } \\
\end{array}$} & \multirow{2}{*}{\multicolumn{3}{|c|}{$\begin{array}{l}\text { Withdrawals by Major Industrial Groups } \\
\text { [NAICS, North American Industrial Classification System code] }\end{array}$}} \\
\hline & \multirow{2}{*}{$\begin{array}{c}\text { Ground- } \\
\text { water }\end{array}$} & \multirow{2}{*}{$\begin{array}{c}\text { Surface } \\
\text { water }\end{array}$} & \multirow[b]{2}{*}{ Total } & & & & & \\
\hline & & & & & & NAICS & Groundwater & Surface water \\
\hline Public supply & 0.93 & 9.73 & 10.66 & - & - & & & \\
\hline Domestic & 1.06 & 0.00 & 1.06 & 8.94 & 0.00 & None & - & - \\
\hline Commercial/public use & 0.00 & 0.00 & 0.00 & 0.92 & 0.00 & & & \\
\hline Industrial & 0.00 & 0.00 & 0.00 & 0.46 & 0.00 & & & \\
\hline Public-supply losses & - & - & - & 1.42 & - & & & \\
\hline Public wastewater treatment & - & - & - & - & 5.26 & Withdrawals by $\mathbf{N}$ & r Public Supp & \\
\hline Mining & 0.18 & 0.00 & 0.18 & 0.18 & 0.17 & Name & Groundwater & Surface water \\
\hline $\begin{array}{l}\text { Irrigation-Crop } \\
\text { Irrigation-Golf course }\end{array}$ & $\begin{array}{l}0.00 \\
0.00\end{array}$ & $\begin{array}{l}0.08 \\
0.79\end{array}$ & $\begin{array}{l}0.08 \\
0.79\end{array}$ & $\begin{array}{l}0.08 \\
0.79\end{array}$ & $\begin{array}{l}0.00 \\
0.00\end{array}$ & Fayette County & 0.12 & 9.02 \\
\hline Livestock/aquaculture & 0.00 & 0.02 & 0.02 & 0.02 & 0.00 & City of Fayetteville & 0.63 & 0.71 \\
\hline
\end{tabular}

Thermoelectric power

0.00

0.00

0.00

0.00

0.00

TOTAL

$2.17 \quad 10.62$

12.79

12.81

5.44

${ }^{1}$ Total use is total withdrawal plus public supply deliveries and losses. The City of Atlanta delivered $0.019 \mathrm{Mgal} / \mathrm{d}$ of water to Fayette County in 2010.

\section{Withdrawals by Source}

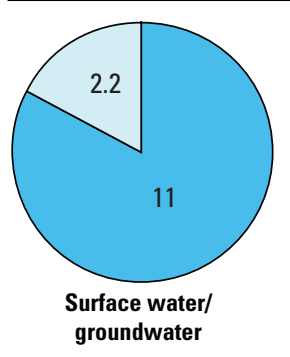

Withdrawals, in million gallons per day

\section{Surface water}

Groundwater

Crystalline-rock aquifer

groundwater

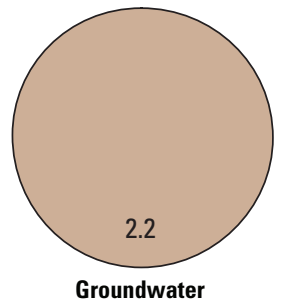

Groundwater
Public-Supply Deliveries ${ }^{1}$ by Use Category

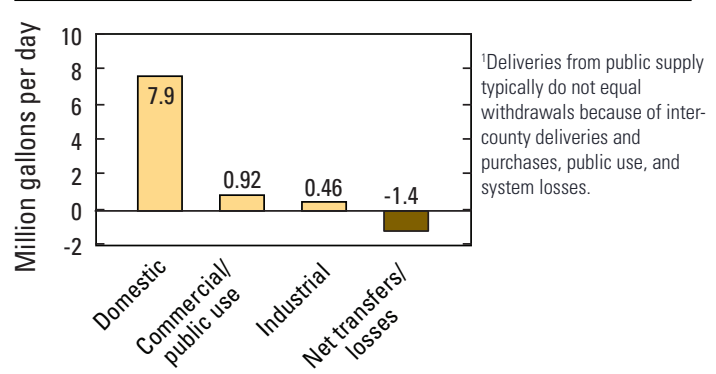

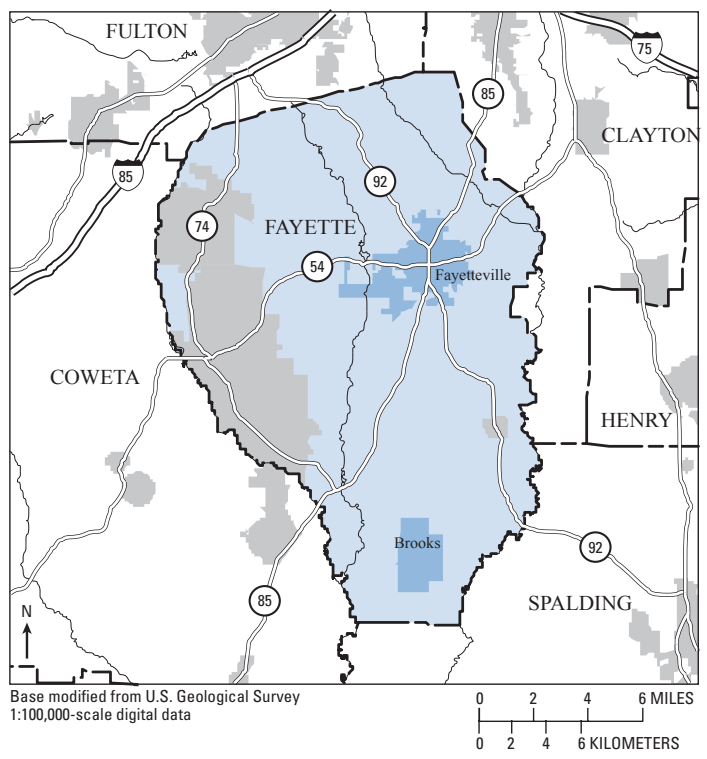

Surface-Water Withdrawals by Year

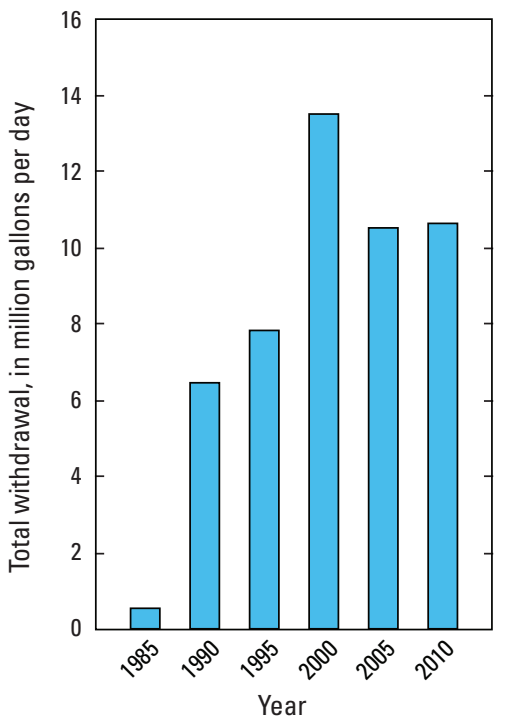

Groundwater Withdrawals by Year

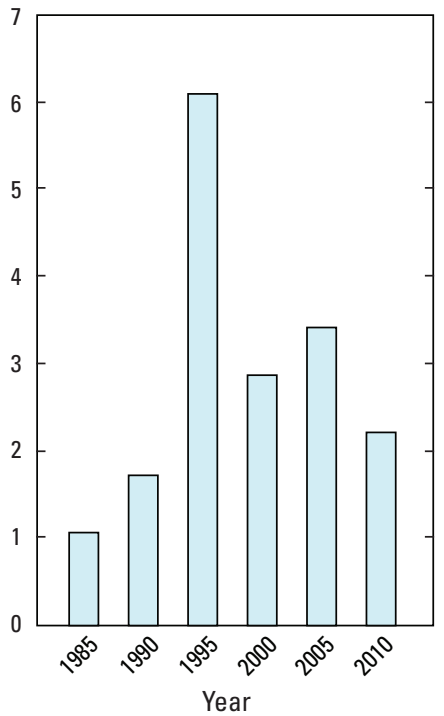




\section{FLOYD COUNTY}

Population

Population served by public supply_Groundwater $\quad 2,820$

Population served by public supply-Surface water $\quad 87,890$

Acres irrigated

2,140

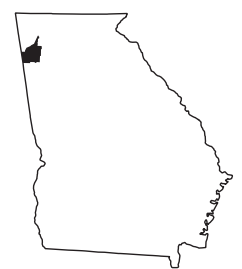

2010 WATER WITHDRAWALS AND ESTIMATED USE, IN MILLION GALLONS PER DAY

[-, not applicabl; Mgal/d, million gallons per daye]

\begin{tabular}{|c|c|c|c|c|c|c|c|c|}
\hline \multirow{3}{*}{ Category } & \multicolumn{3}{|c|}{ Withdrawals } & \multirow{3}{*}{$\begin{array}{l}\text { Total } \\
\text { use }^{1}\end{array}$} & \multirow{3}{*}{$\begin{array}{c}\text { Surface- } \\
\text { water } \\
\text { returns } \\
\end{array}$} & \multirow{2}{*}{\multicolumn{3}{|c|}{$\begin{array}{l}\text { Withdrawals by Major Industrial Groups } \\
\text { [NAICS, North American Industrial Classification System code] }\end{array}$}} \\
\hline & \multirow{2}{*}{$\begin{array}{c}\text { Ground- } \\
\text { water }\end{array}$} & \multirow{2}{*}{$\begin{array}{c}\text { Surface } \\
\text { water }\end{array}$} & \multirow[b]{2}{*}{ Total } & & & & & \\
\hline & & & & & & NAICS & Groundwater & Surface water \\
\hline Public supply & 0.57 & 12.51 & 13.08 & - & & 322-Paper, pulp & 0.00 & 25.71 \\
\hline Domestic & 0.42 & 0.00 & 0.42 & 7.14 & 0.00 & 812-Laundry services & 0.14 & 0.00 \\
\hline $\begin{array}{l}\text { Commercial/public use } \\
\text { Industrial }\end{array}$ & $\begin{array}{l}0.00 \\
0.14\end{array}$ & $\begin{array}{r}0.32 \\
25.71\end{array}$ & $\begin{array}{r}0.32 \\
25.85\end{array}$ & $\begin{array}{r}3.40 \\
27.00\end{array}$ & 0.00 & & & \\
\hline Public-supply losses & - & - & - & 2.27 & - & & & \\
\hline Public wastewater treatment & - & - & - & - & 11.07 & Withdrawals by Majo & r Public Supp & \\
\hline Mining & 0.10 & 0.01 & 0.11 & 0.11 & 0.00 & Name & Groundwater & Surface water \\
\hline $\begin{array}{l}\text { Irrigation-Crop } \\
\text { Irrigation-Golf course }\end{array}$ & $\begin{array}{l}0.05 \\
0.59\end{array}$ & $\begin{array}{l}1.14 \\
0.22\end{array}$ & $\begin{array}{l}1.19 \\
0.81\end{array}$ & $\begin{array}{l}1.19 \\
0.81\end{array}$ & $\begin{array}{l}0.00 \\
0.00\end{array}$ & City of Rome & 0.00 & 8.55 \\
\hline $\begin{array}{l}\text { Irrigation-Golf course } \\
\text { Livestock/aquaculture }\end{array}$ & $\begin{array}{l}0.59 \\
0.03\end{array}$ & $\begin{array}{l}0.22 \\
0.68\end{array}$ & $\begin{array}{l}0.81 \\
0.71\end{array}$ & $\begin{array}{l}0.81 \\
0.71\end{array}$ & 0.00 & Floyd County & 0.28 & 3.08 \\
\hline Thermoelectric power & 0.00 & 432.17 & 432.17 & 432.17 & 432.00 & City of Cave Spring & 0.29 & 0.88 \\
\hline TOTAL & 1.90 & 472.76 & 474.66 & 474.80 & 443.07 & & & \\
\hline
\end{tabular}

${ }^{1}$ Total use is total withdrawal plus public supply deliveries and losses. An average of $0.6 \mathrm{Mgal} / \mathrm{d}$ was delivered from Bartow County, Georgia

to Floyd County, Georgia and 0.13 Mgal/d from Floyd County to DeKalb County, Alabama in 2010.

\section{Withdrawals by Source}
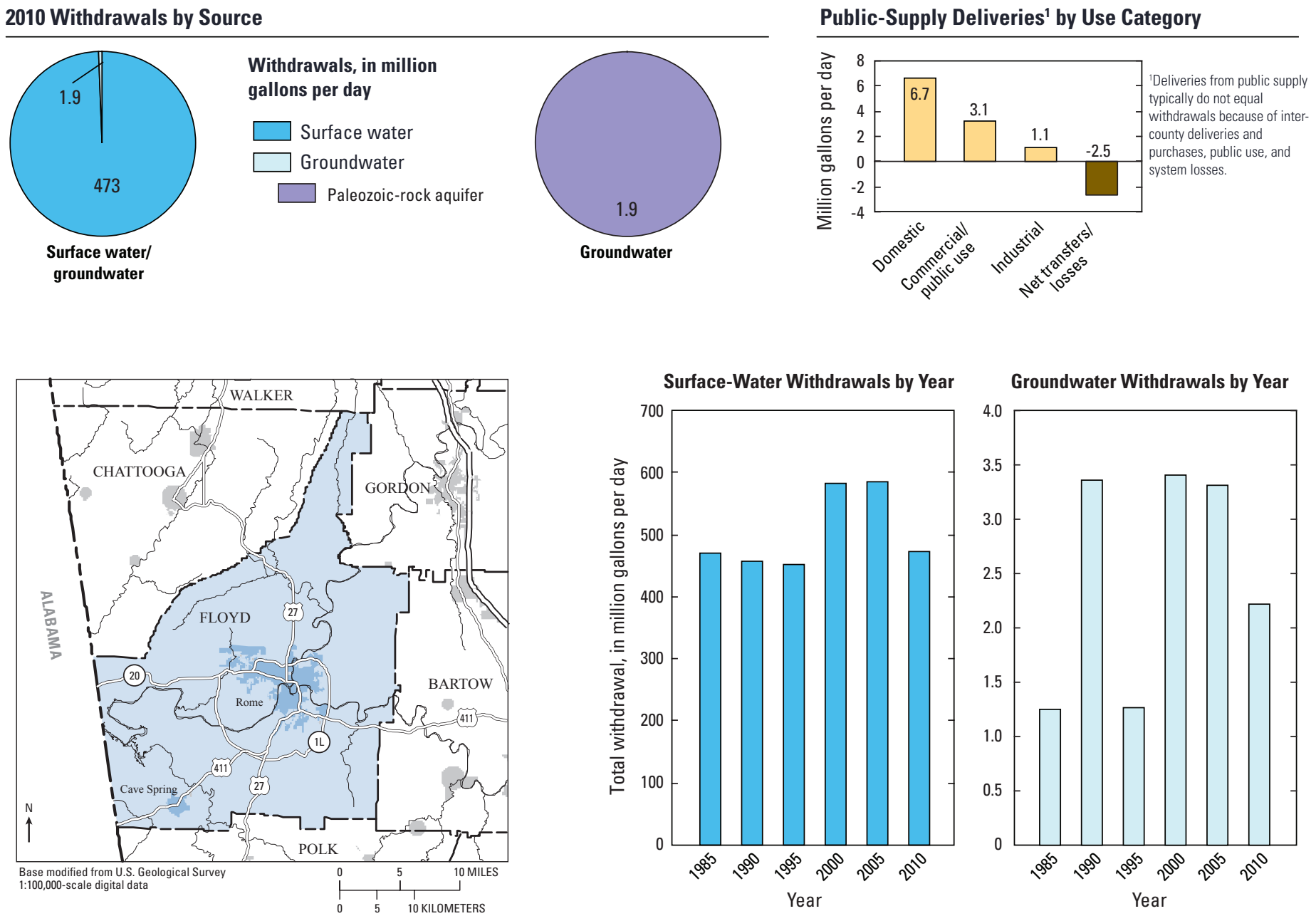

Groundwater Withdrawals by Year

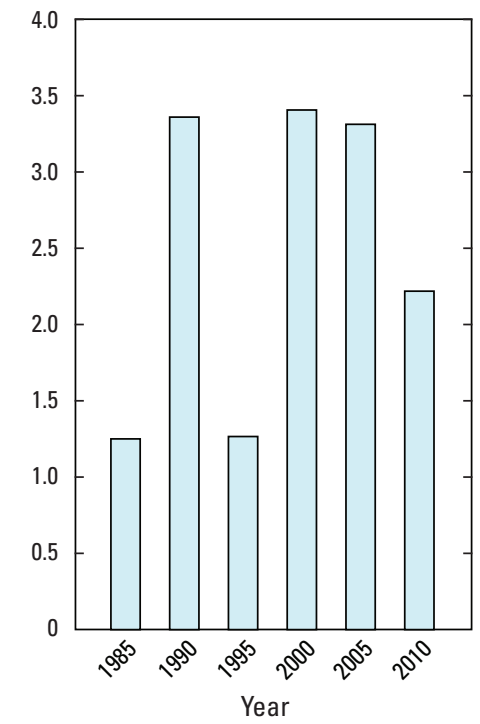




\section{FORSYTH COUNTY}

Population

175,511

Population served by public supply-Groundwater

Population served by public supply_-Surface water 172,890

Acres irrigated

350

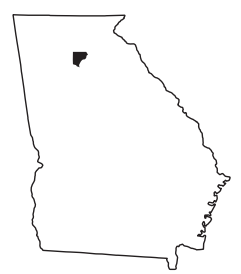

2010 WATER WITHDRAWALS AND ESTIMATED USE, IN MILLION GALLONS PER DAY

$[-$, not applicable; Mgal/d, million gallons per day $]$

\begin{tabular}{|c|c|c|c|c|c|c|c|c|}
\hline \multirow{3}{*}{ Category } & \multicolumn{3}{|c|}{ Withdrawals } & \multirow{3}{*}{$\begin{array}{l}\text { Total } \\
\text { use }^{1}\end{array}$} & \multirow{3}{*}{$\begin{array}{c}\text { Surface- } \\
\text { water } \\
\text { returns } \\
\end{array}$} & \multirow{2}{*}{\multicolumn{3}{|c|}{$\begin{array}{l}\text { Withdrawals by Major Industrial Groups } \\
\text { [NAICS, North American Industrial Classification System code] }\end{array}$}} \\
\hline & \multirow{2}{*}{$\begin{array}{c}\text { Ground- } \\
\text { water }\end{array}$} & \multirow{2}{*}{$\begin{array}{c}\text { Surface } \\
\text { water }\end{array}$} & \multirow[b]{2}{*}{ Total } & & & & & \\
\hline & & & & & & NAICS & Groundwater & Surface water \\
\hline Public supply & 0.04 & 19.18 & 19.22 & - & - & \multirow{2}{*}{ None } & \multirow{2}{*}{-} & \multirow{2}{*}{-} \\
\hline Domestic & 0.17 & 0.00 & 0.17 & 11.67 & 0.00 & & & \\
\hline Commercial/public use & 0.00 & 0.00 & 0.00 & 3.11 & 0.08 & & & \\
\hline Industrial & 0.00 & 0.00 & 0.00 & 2.14 & 0.30 & & & \\
\hline Public-supply losses & - & - & - & 2.42 & - & & & \\
\hline Public wastewater treatment & - & - & - & - & 2.25 & \multicolumn{3}{|c|}{ Withdrawals by Major Public Suppliers } \\
\hline Mining & 0.70 & 0.01 & 0.71 & 0.71 & 0.06 & Name & Groundwater & Surface water \\
\hline $\begin{array}{l}\text { Irrigation-Crop } \\
\text { Irrigation-Golf course }\end{array}$ & $\begin{array}{l}0.23 \\
0.00\end{array}$ & $\begin{array}{l}0.07 \\
0.28\end{array}$ & $\begin{array}{l}0.30 \\
0.28\end{array}$ & $\begin{array}{l}0.30 \\
0.28\end{array}$ & $\begin{array}{l}0.00 \\
0.00\end{array}$ & \multirow{2}{*}{$\begin{array}{l}\text { Forsyth County Water } \\
\& \text { Sewer Authority }\end{array}$} & \multirow{2}{*}{0.00} & \multirow{2}{*}{7.77} \\
\hline Livestock/aquaculture & 0.00 & 6.68 & 6.68 & 6.68 & 0.00 & & & \\
\hline Thermoelectric power & 0.00 & 0.00 & 0.00 & 0.00 & 0.00 & City of Cumming & 0.00 & 11.41 \\
\hline TOTAL & 1.14 & 26.22 & 27.36 & 27.31 & 2.69 & & & \\
\hline
\end{tabular}

Total use is total withdrawal plus public supply deliveries and losses. An average of $0.05 \mathrm{Mgal} / \mathrm{d}$ was delivered from Forsyth County to Dawson County, Georgia in 2010.

\section{Withdrawals by Source}

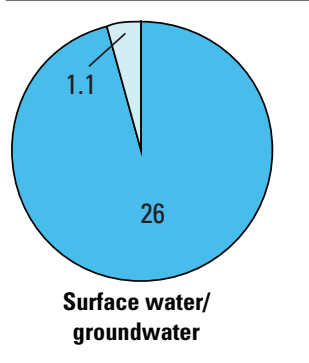

Withdrawals, in million gallons per day

$\square$ Surface water
$\square$ Groundwater
$\square$ Crystalline-rock aquifer

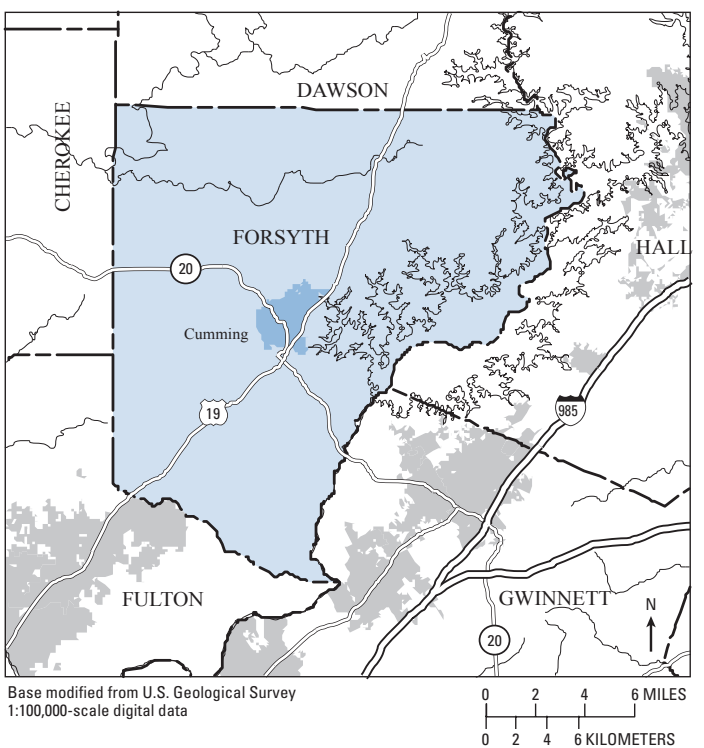

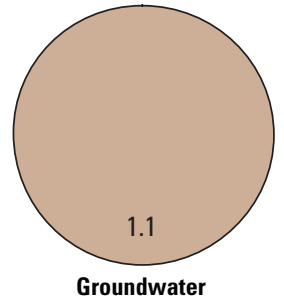

Public-Supply Deliveries ${ }^{1}$ by Use Category

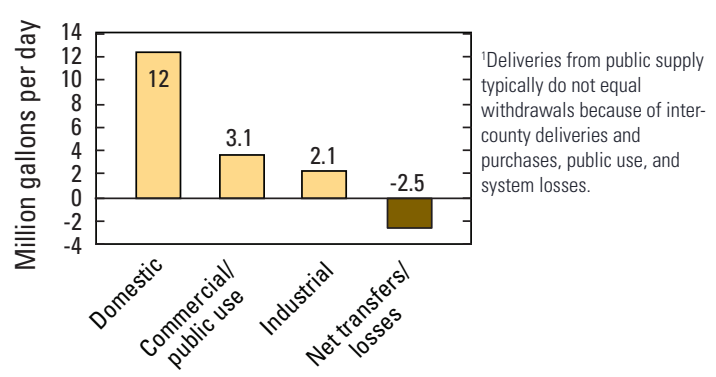

Surface-Water Withdrawals by Year

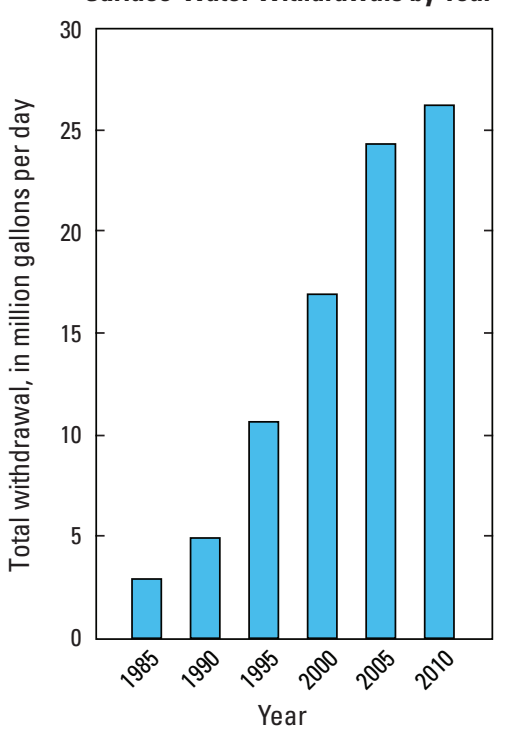

Groundwater Withdrawals by Year

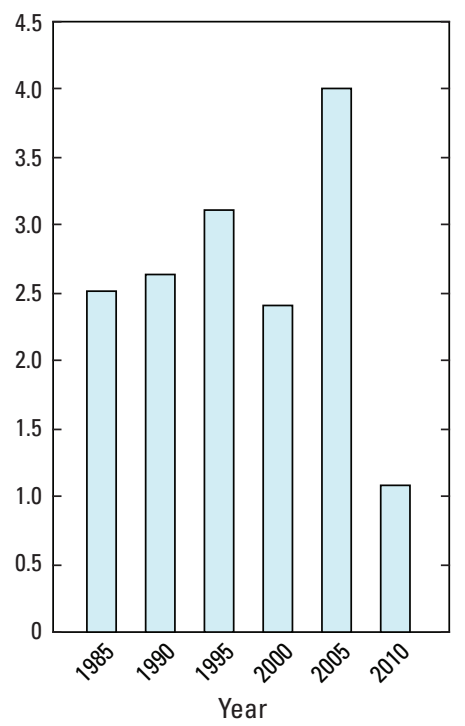




\section{FRANKLIN COUNTY}

Population

Population served by public supply-Groundwater $\quad 4,450$

Population served by public supply-Surface water $\quad 8,270$

Acres irrigated

375

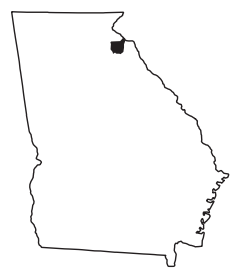

2010 WATER WITHDRAWALS AND ESTIMATED USE, IN MILLION GALLONS PER DAY

[-, not applicable; Mgal/d, million gallons per day]

\begin{tabular}{|c|c|c|c|c|c|c|c|c|}
\hline \multirow{3}{*}{ Category } & \multicolumn{3}{|c|}{ Withdrawals } & \multirow{3}{*}{$\begin{array}{l}\text { Total } \\
\text { use }^{1}\end{array}$} & \multirow{3}{*}{$\begin{array}{l}\text { Surface- } \\
\text { water } \\
\text { returns } \\
\end{array}$} & \multirow{2}{*}{\multicolumn{3}{|c|}{$\begin{array}{l}\text { Withdrawals by Major Industrial Groups } \\
\text { [NAICS, North American Industrial Classification System code] }\end{array}$}} \\
\hline & \multirow{2}{*}{$\begin{array}{c}\text { Ground- } \\
\text { water }\end{array}$} & \multirow{2}{*}{$\begin{array}{c}\text { Surface } \\
\text { water }\end{array}$} & \multirow[b]{2}{*}{ Total } & & & & & \\
\hline & & & & & & NAICS & Groundwater & Surface water \\
\hline Public supply & 0.62 & 1.77 & 2.39 & - & - & None & - & - \\
\hline Domestic & 0.70 & 0.00 & 0.70 & 1.83 & 0.00 & None & - & - \\
\hline Commercial/public use & 0.00 & 0.00 & 0.00 & 0.42 & 0.00 & & & \\
\hline Industrial & 0.00 & 0.00 & 0.00 & 0.40 & 0.00 & & & \\
\hline Public-supply losses & - & - & - & 0.19 & - & & & \\
\hline Public wastewater treatment & - & - & - & - & 0.73 & Withdrawals by Major & Public Supp & \\
\hline Mining & 0.00 & 0.00 & 0.00 & 0.00 & 0.00 & Name & Groundwater & Surface water \\
\hline Irrigation-Crop & 0.00 & 0.08 & 0.08 & 0.08 & 0.00 & City of Lavonia & 0.00 & 1.51 \\
\hline Irrigation-Golf course & 0.19 & 0.15 & 0.34 & 0.34 & 0.00 & City of Royston & 0.00 & 0.26 \\
\hline Livestock/aquaculture & 0.00 & 1.22 & 1.22 & 1.22 & $\begin{array}{l}0.00 \\
0.00\end{array}$ & City of Franklin Springs & 0.52 & 0.00 \\
\hline
\end{tabular}

\section{TOTAL}

$1.51 \quad 3.22$

4.73

4.48

0.73

Total use is total withdrawal plus public supply deliveries and losses. In 2010, an average of $0.08 \mathrm{Mgal} / \mathrm{d}$ was delivered from Stephens

County, Georgia and $0.04 \mathrm{Mgal} / \mathrm{d}$ from Banks County, Georgia to Franklin County; whereas, an average of $0.37 \mathrm{Mgal} / \mathrm{d}$ was delivered to Hart

County, Georgia from Franklin County.

\section{Withdrawals by Source}
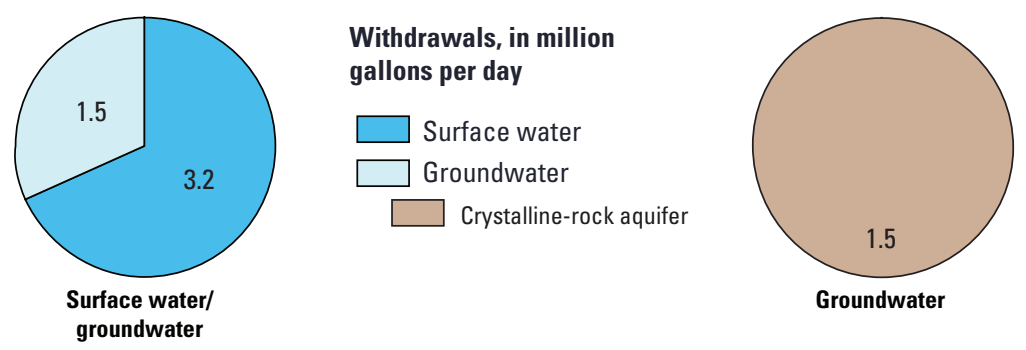

Public-Supply Deliveries ${ }^{1}$ by Use Category
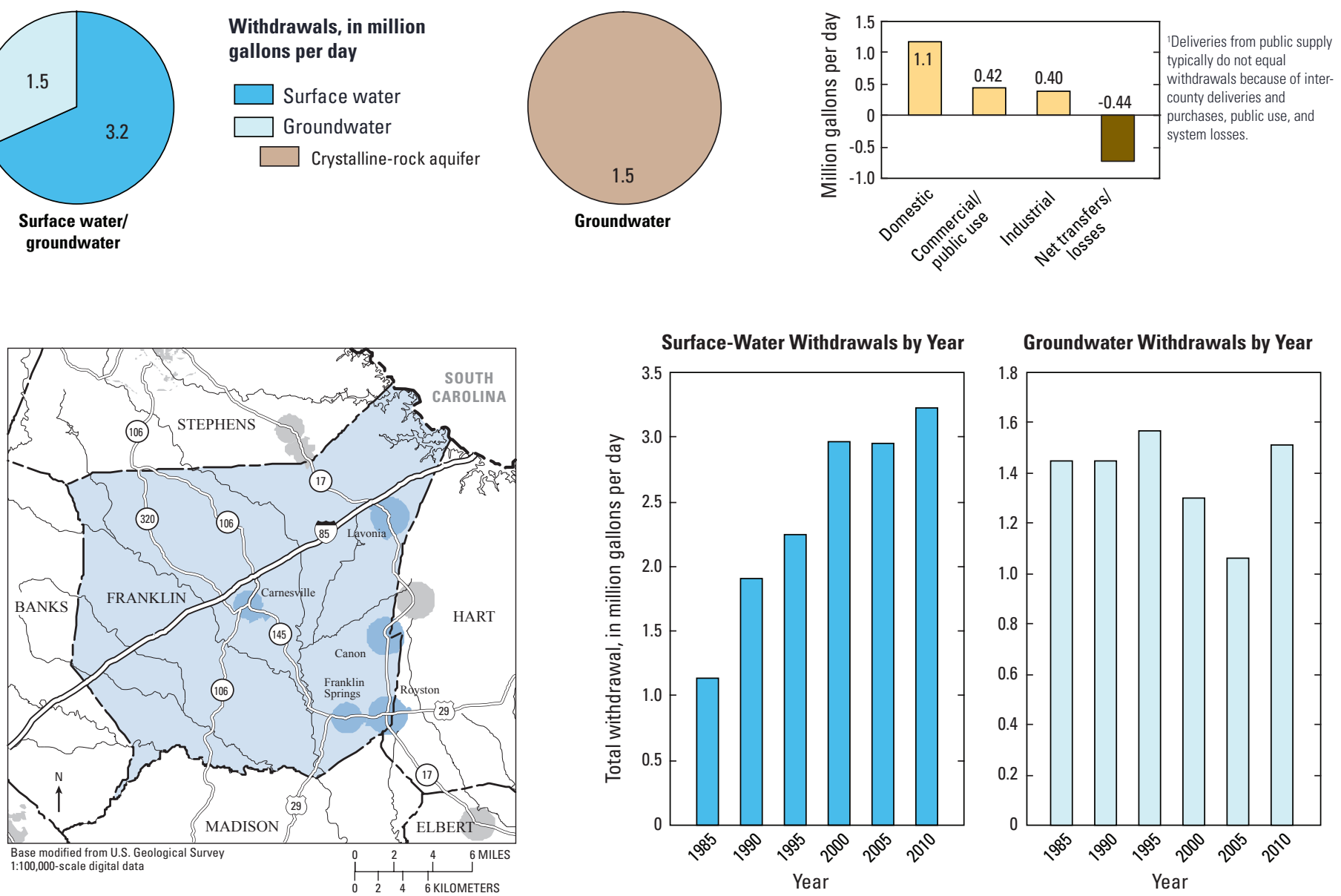

Groundwater Withdrawals by Year

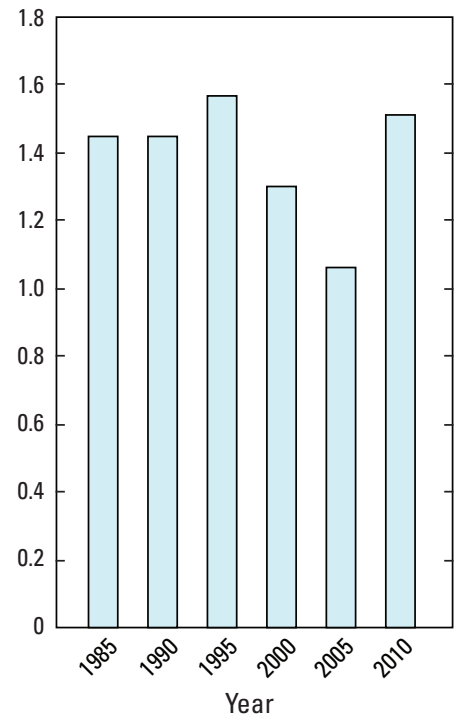




\section{FULTON COUNTY}

Population

920,581

Population served by public supply-Groundwater

Population served by public supply-Surface water 870,810

Acres irrigated

2,180

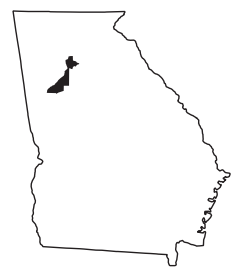

2010 WATER WITHDRAWALS AND ESTIMATED USE, IN MILLION GALLONS PER DAY

[-, not applicable; Mgal/d, million gallons per day]

\begin{tabular}{|c|c|c|c|c|c|c|c|c|}
\hline \multirow{3}{*}{ Category } & \multicolumn{3}{|c|}{ Withdrawals } & \multirow{3}{*}{$\begin{array}{l}\text { Total } \\
\text { use }^{1}\end{array}$} & \multirow{3}{*}{$\begin{array}{c}\text { Surface- } \\
\text { water } \\
\text { returns } \\
\end{array}$} & \multirow{2}{*}{\multicolumn{3}{|c|}{$\begin{array}{l}\text { Withdrawals by Major Industrial Groups } \\
\text { [NAICS, North American Industrial Classification System code] }\end{array}$}} \\
\hline & \multirow{2}{*}{$\begin{array}{c}\text { Ground- } \\
\text { water }\end{array}$} & \multirow{2}{*}{$\begin{array}{c}\text { Surface } \\
\text { water }\end{array}$} & \multirow[b]{2}{*}{ Total } & & & & & \\
\hline & & & & & & NAICS & Groundwater & Surface water \\
\hline Public supply & 0.00 & 200.70 & 200.70 & - & - & & 0.01 & 0.00 \\
\hline Domestic & 3.73 & 0.00 & 3.73 & 87.91 & 0.00 & 334-Electronics & 0.01 & 0.00 \\
\hline Commercial/public use & 0.00 & 0.00 & 0.00 & 9.61 & 0.00 & & & \\
\hline Industrial & 0.01 & 0.00 & 0.01 & 3.53 & 0.01 & Withdrawals by Major & Public Supp & \\
\hline Public-supply losses & - & - & - & 31.80 & - & Name & Groundwater & Surface water \\
\hline Public wastewater treatment & - & - & - & - & 136.16 & City of Atlanta & 0.00 & 84.10 \\
\hline Mining & 0.26 & 0.00 & 0.26 & 0.26 & 1.32 & DeKalb County ${ }^{1}$ & 0.00 & 74.95 \\
\hline Irrigation-Crop & 0.04 & 0.00 & 0.04 & 0.04 & 0.00 & Atlanta-Fulton Co Water & 0.00 & 14.95 \\
\hline Irrigation-Golf course/turf & 0.73 & 2.96 & 3.69 & 6.44 & 0.00 & $\begin{array}{l}\text { Atlanta-Fulton Co. Water } \\
\text { Resources Commission }\end{array}$ & 0.00 & 40.15 \\
\hline Livestock/aquaculture & 0.00 & 0.09 & 0.09 & 0.09 & 0.00 & City of Roswell & 000 & 108 \\
\hline Thermoelectric power & 0.00 & 0.00 & 0.00 & 0.00 & 0.00 & & 0.00 & \\
\hline TOTAL & 4.77 & 203.75 & 208.52 & 139.68 & 137.49 & City of Palmetto & 0.00 & 0.42 \\
\hline
\end{tabular}

${ }^{T}$ Total use is total withdrawal plus public supply deliveries and losses. Does not include water used (4.2 Mgal/d) in the DeKalb County portion of Atlanta's service area. In 2010, an average of $0.05 \mathrm{Mgal} / \mathrm{d}$ was delivered from Cobb County, Georgia and $0.02 \mathrm{Mgal} / \mathrm{d}$ was delivered from

Clayton County, Georgia to Fulton County; whereas, an average of $0.01 \mathrm{Mgal} / \mathrm{d}$ was delivered from Fulton County to Coweta County, Georgia.

\section{Withdrawals by Source}
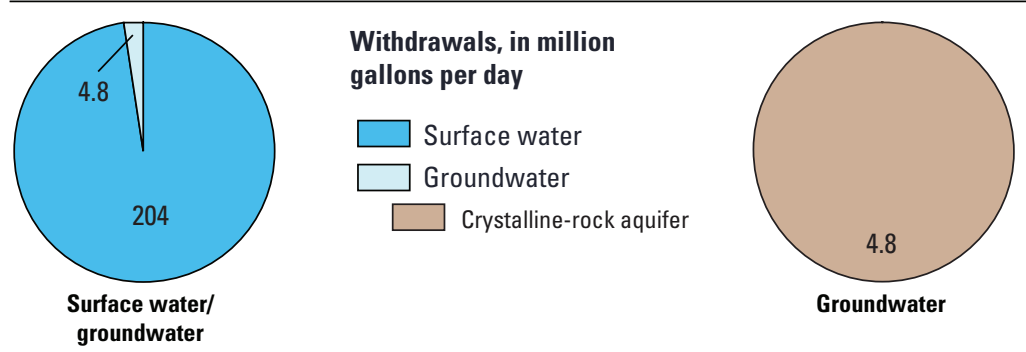

Public-Supply Deliveries' by Use Category
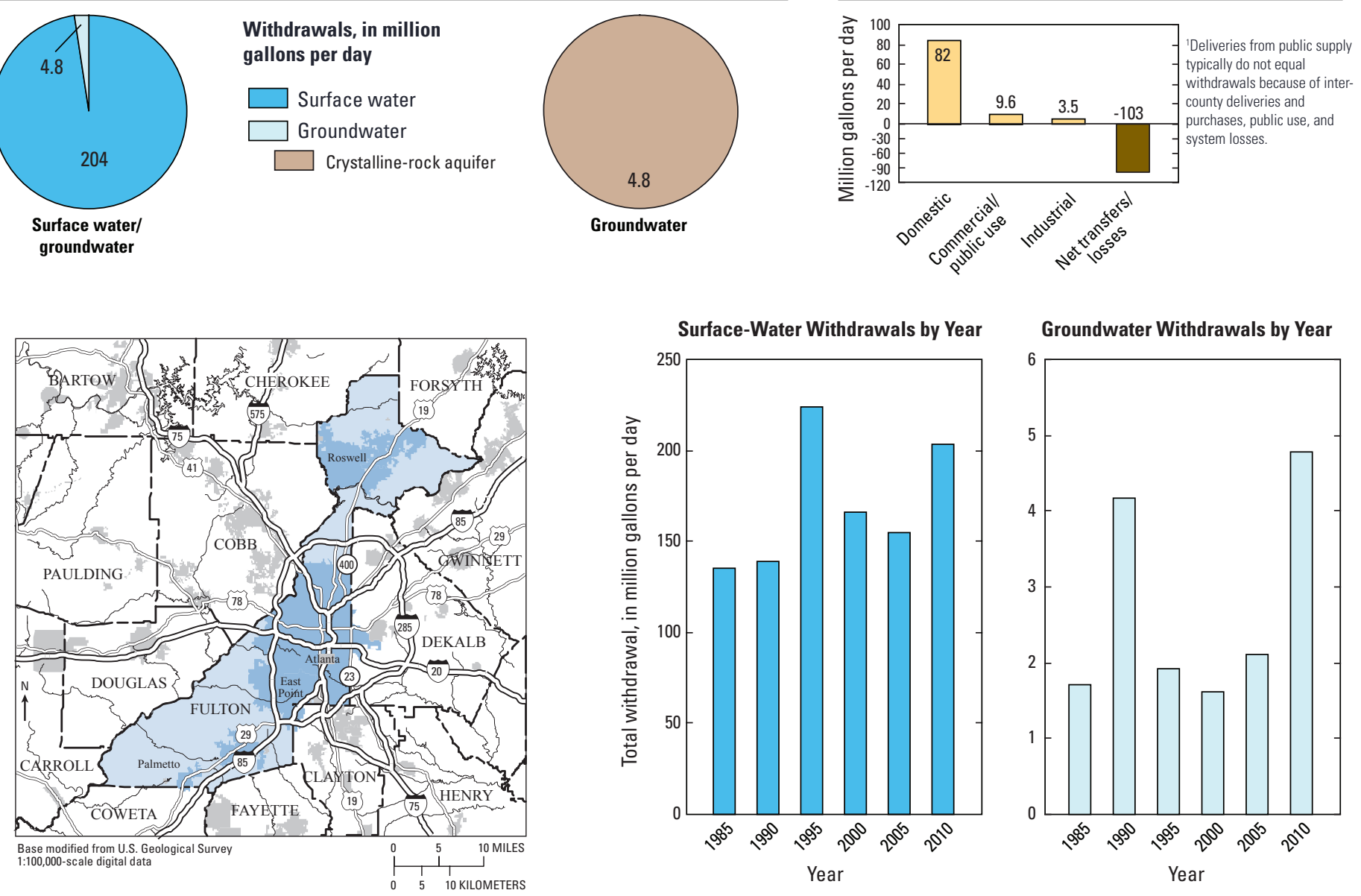


\section{GILMER COUNTY}

Population

Population served by public supply-Groundwater

Population served by public supply-Surface water $\quad 11,280$ Acres irrigated

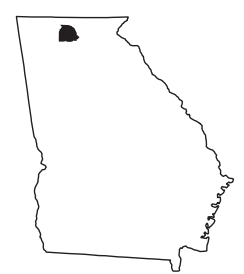

2010 WATER WITHDRAWALS AND ESTIMATED USE, IN MILLION GALLONS PER DAY $[-$, not applicable $]$

\begin{tabular}{|c|c|c|c|c|c|c|c|c|}
\hline \multirow{3}{*}{ Category } & \multicolumn{3}{|c|}{ Withdrawals } & \multirow{3}{*}{$\begin{array}{l}\text { Total } \\
\text { use }^{1}\end{array}$} & \multirow{3}{*}{$\begin{array}{l}\text { Surface- } \\
\text { water } \\
\text { returns } \\
\end{array}$} & \multirow{2}{*}{\multicolumn{3}{|c|}{$\begin{array}{l}\text { Withdrawals by Major Industrial Groups } \\
\text { [NAICS, North American Industrial Classification System code] }\end{array}$}} \\
\hline & \multirow{2}{*}{$\begin{array}{c}\text { Ground- } \\
\text { water }\end{array}$} & \multirow{2}{*}{$\begin{array}{c}\text { Surface } \\
\text { water }\end{array}$} & \multirow[b]{2}{*}{ Total } & & & & & \\
\hline & & & & & & NAICS & Groundwater & Surface water \\
\hline Public supply & 0.06 & 3.05 & 3.11 & - & - & & 1.42 & 0.00 \\
\hline Domestic & 1.21 & 0.00 & 1.21 & 1.86 & 0.00 & 327 -Stone, clay & 1.42 & 0.00 \\
\hline Commercial/public use & 0.00 & 0.00 & 0.00 & 0.46 & 0.00 & & & \\
\hline Industrial & 1.42 & 0.00 & 1.42 & 2.93 & 0.00 & & & \\
\hline Public-supply losses & - & - & - & 0.49 & - & & & \\
\hline Public wastewater treatment & - & - & - & - & 2.09 & Withdrawals by Majo & r Public Supp & \\
\hline Mining & 0.07 & 0.00 & 0.07 & 0.07 & 1.42 & Name & Groundwater & Surface water \\
\hline Irrigation - Crop & 0.00 & 0.00 & 0.00 & 0.00 & 0.00 & & & \\
\hline Irrigation-Golf course & $\begin{array}{l}0.00 \\
0.00\end{array}$ & $\begin{array}{l}0.33 \\
0.70\end{array}$ & $\begin{array}{l}0.33 \\
0.70\end{array}$ & $\begin{array}{l}0.33 \\
0.70\end{array}$ & $\begin{array}{l}0.00 \\
0.00\end{array}$ & $\begin{array}{l}\text { Ellijay-Gilmer County } \\
\text { Water Authority }\end{array}$ & 0.00 & 3.05 \\
\hline $\begin{array}{l}\text { Livestock/aquaculture } \\
\text { Thermoelectric power }\end{array}$ & $\begin{array}{l}0.00 \\
0.00\end{array}$ & $\begin{array}{l}0.70 \\
0.00\end{array}$ & $\begin{array}{l}0.70 \\
0.00\end{array}$ & $\begin{array}{l}0.70 \\
0.00\end{array}$ & $\begin{array}{l}0.00 \\
0.00\end{array}$ & & & \\
\hline TOTAL & 2.76 & 4.08 & 6.84 & 6.84 & 3.51 & & & \\
\hline
\end{tabular}

${ }^{1}$ Total use is total withdrawal plus public supply deliveries and losses.

\section{Withdrawals by Source}
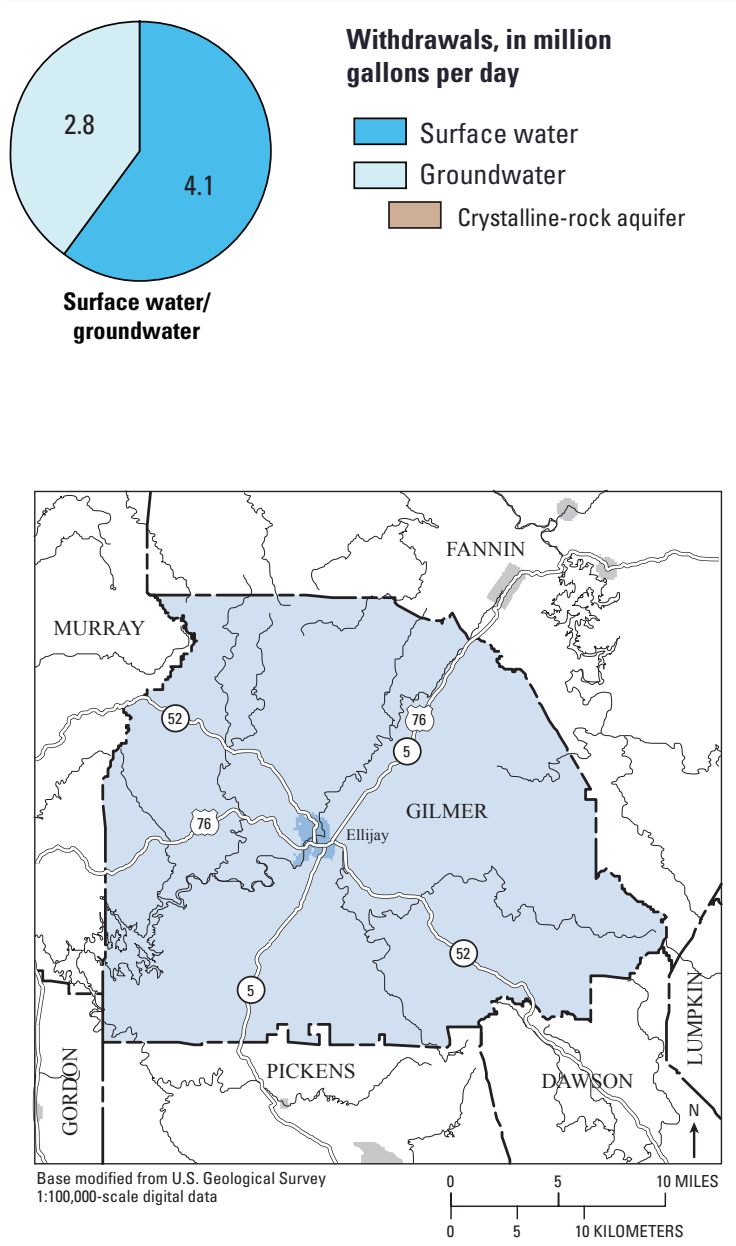

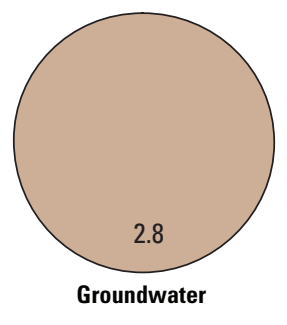

Public-Supply Deliveries by Use Category

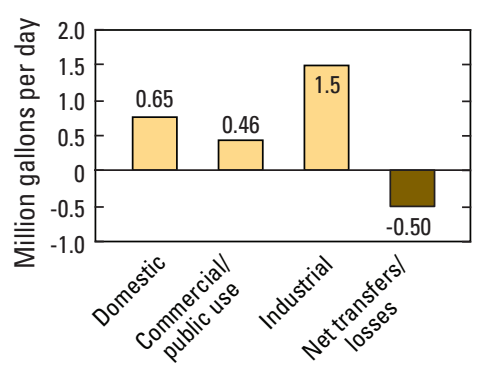

Surface-Water Withdrawals by Year

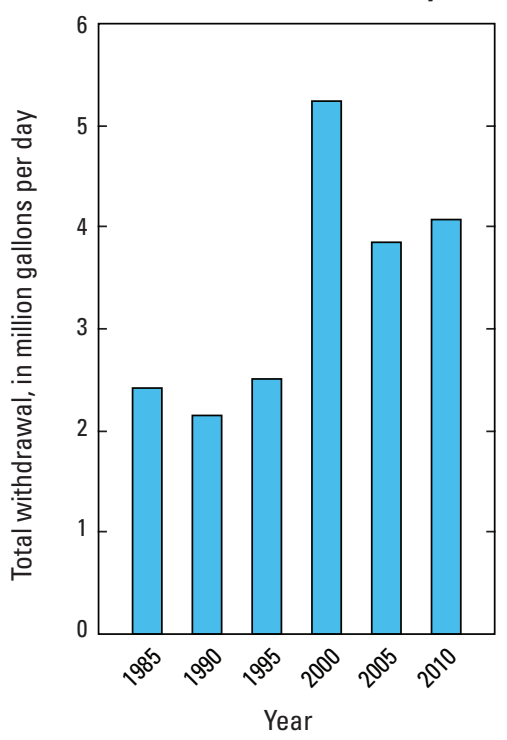

Groundwater Withdrawals by Year

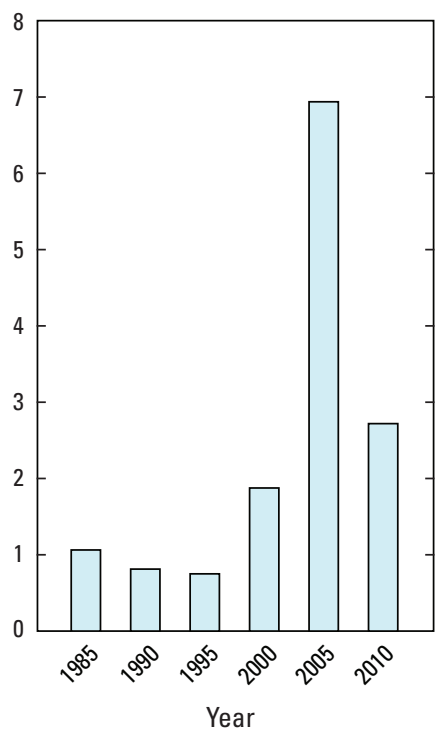




\section{GLASCOCK COUNTY}

Population

3,082

$\begin{array}{lr}\text { Population served by public supply_-Groundwater } & 900 \\ \text { Population served by public supply-Surface water } & 0\end{array}$

Acres irrigated

90

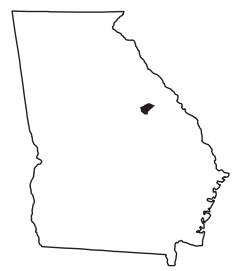

2010 WATER WITHDRAWALS AND ESTIMATED USE, IN MILLION GALLONS PER DAY

$[-$, not applicable; $<$, less than $]$

\begin{tabular}{|c|c|c|c|c|c|c|c|c|}
\hline \multirow{3}{*}{ Category } & \multicolumn{3}{|c|}{ Withdrawals } & \multirow{3}{*}{$\begin{array}{l}\text { Total } \\
\text { use }^{1}\end{array}$} & \multirow{3}{*}{$\begin{array}{l}\text { Surface- } \\
\text { water } \\
\text { returns } \\
\end{array}$} & \multirow{2}{*}{\multicolumn{3}{|c|}{$\begin{array}{l}\text { Withdrawals by Major Industrial Groups } \\
\text { [NAICS, North American Industrial Classification System code] }\end{array}$}} \\
\hline & \multirow{2}{*}{$\begin{array}{c}\text { Ground- } \\
\text { water }\end{array}$} & \multirow{2}{*}{$\begin{array}{c}\text { Surface } \\
\text { water }\end{array}$} & \multirow[b]{2}{*}{ Total } & & & & & \\
\hline & & & & & & NAICS & Groundwater & Surface water \\
\hline Public supply & 0.07 & 0.00 & 0.07 & - & - & & 0.07 & 0.00 \\
\hline Domestic & 0.16 & 0.00 & 0.16 & 0.23 & 0.00 & 327-Stone, clay & 0.07 & 0.00 \\
\hline Commercial/public use & 0.00 & 0.00 & 0.00 & $<0.01$ & 0.00 & & & \\
\hline Industrial & 0.07 & 0.00 & 0.07 & 0.07 & 0.00 & & & \\
\hline Public-supply losses & - & - & - & $<0.01$ & - & & & \\
\hline Public wastewater treatment & - & - & - & - & 0.03 & Withdrawals by & r Public Supp & \\
\hline Mining & 0.00 & 0.00 & 0.00 & 0.00 & 0.56 & Name & Groundwater & Surface water \\
\hline Irrigation-Crop & 0.00 & 0.04 & 0.04 & 0.04 & 0.00 & & & \\
\hline Irrigation-Golf course & 0.00 & 0.00 & 0.00 & 0.00 & 0.00 & Town of Gibson & 0.06 & 0.00 \\
\hline Livestock/aquaculture & 0.02 & 0.04 & 0.06 & 0.06 & 0.00 & Town of Mitchell & 0.01 & 0.00 \\
\hline Thermoelectric power & 0.00 & 0.00 & 0.00 & 0.00 & 0.00 & & & \\
\hline
\end{tabular}

Thermoelectric power

$\begin{array}{lllll}0.00 & 0.00 & 0.00 & 0.00 & 0.00 \\ \mathbf{0 . 3 2} & \mathbf{0 . 0 8} & \mathbf{0 . 4 0} & \mathbf{0 . 4 0} & \mathbf{0 . 5 9}\end{array}$

${ }^{1}$ Total use is total withdrawal plus public supply deliveries and losses.

2010 Withdrawals by Source
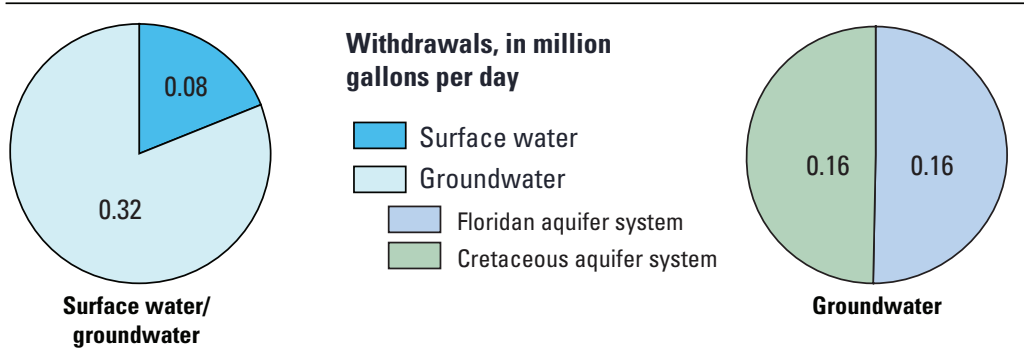

Public-Supply Deliveries by Use Category
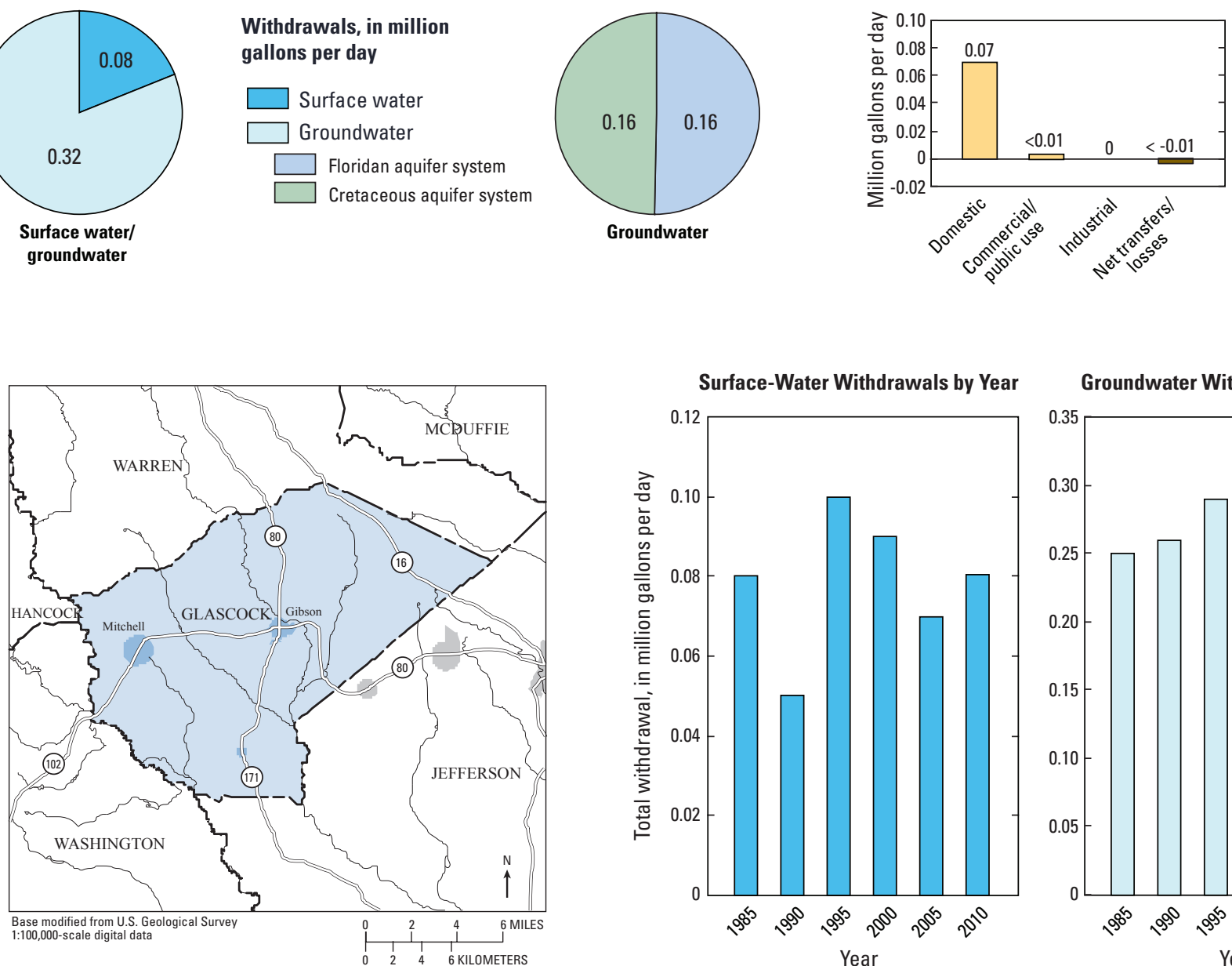

Surface-Water Withdrawals by Year

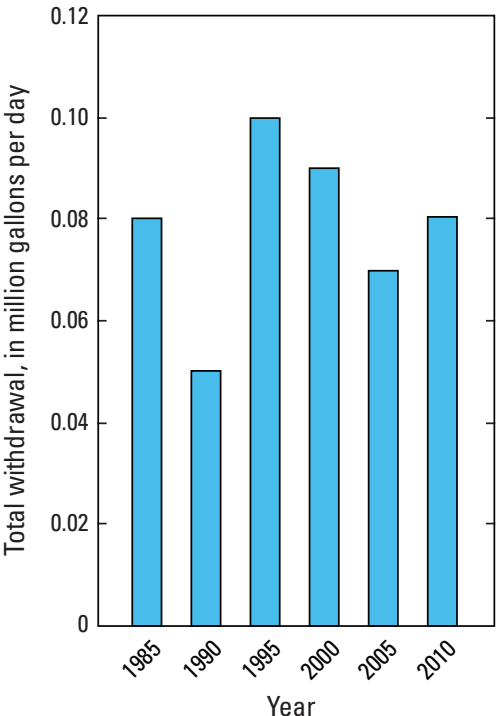

Groundwater Withdrawals by Year

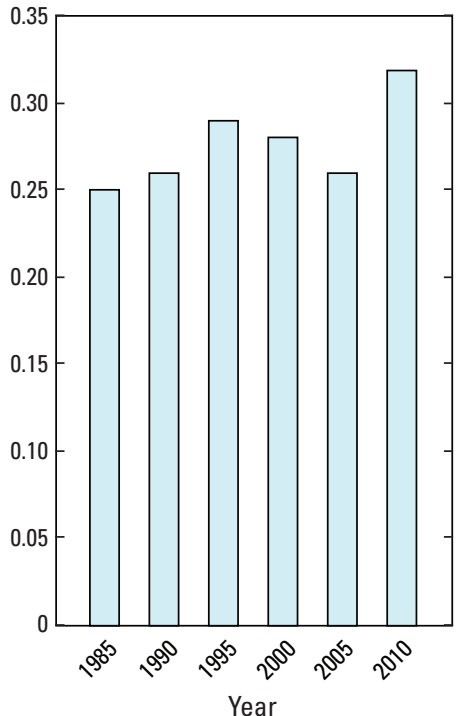




\section{GLYNN COUNTY}

Population

79,626

Population served by public supply-Groundwater $\quad 66,950$

Population served by public supply-Surface water 0

Acres irrigated

2,130

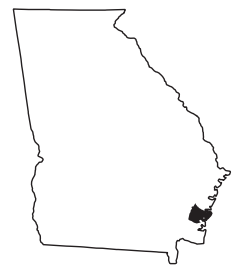

2010 WATER WITHDRAWALS AND ESTIMATED USE, IN MILLION GALLONS PER DAY

$[-$, not applicable $]$

\begin{tabular}{|c|c|c|c|c|c|c|c|c|}
\hline \multirow{3}{*}{ Category } & \multicolumn{3}{|c|}{ Withdrawals } & \multirow{3}{*}{$\begin{array}{l}\text { Total } \\
\text { use }^{1}\end{array}$} & \multirow{3}{*}{$\begin{array}{l}\text { Surface- } \\
\text { water } \\
\text { returns } \\
\end{array}$} & \multirow{2}{*}{\multicolumn{3}{|c|}{$\begin{array}{l}\text { Withdrawals by Major Industrial Groups } \\
\text { [NAICS, North American Industrial Classification System code] }\end{array}$}} \\
\hline & \multirow{2}{*}{$\begin{array}{c}\text { Ground- } \\
\text { water }\end{array}$} & \multirow{2}{*}{$\begin{array}{c}\text { Surface } \\
\text { water }\end{array}$} & \multirow[b]{2}{*}{ Total } & & & & & \\
\hline & & & & & & NAICS & Groundwater & Surface water \\
\hline Public supply & 10.15 & 0.00 & 10.15 & - & - & 322 -Paper, pulp & 32.71 & 0.00 \\
\hline Domestic & 0.95 & 0.00 & 0.95 & 6.72 & 0.00 & 325 -Chemical products & 8.25 & 0.00 \\
\hline Commercial/public use & 0.08 & 0.00 & 0.08 & 2.63 & $\begin{array}{r}0.00 \\
4005\end{array}$ & 311 -Food & 0.26 & 0.00 \\
\hline Industrial & 41.22 & 0.00 & 41.22 & 41.46 & 40.05 & & & \\
\hline Public-supply losses & - & - & - & 1.60 & - & Withdrawals by Major & r Public Supp & \\
\hline $\begin{array}{l}\text { Public wastewater treatment } \\
\text { Mining }\end{array}$ & 0.00 & $\begin{array}{c}- \\
0.00\end{array}$ & 0.00 & $\begin{array}{l}- \\
0.00\end{array}$ & $\begin{array}{l}8.79 \\
0.00\end{array}$ & Name & Groundwater & Surface water \\
\hline Irrigation-Crop & 0.00 & 0.02 & 0.02 & 0.02 & 0.00 & Brunswick-Glynn Co. & 5.13 & 0.00 \\
\hline Irrigation-Golf course & 3.19 & 0.25 & 3.44 & 3.44 & 0.00 & Joint W\&S Authority & & \\
\hline Livestock/aquaculture & 0.00 & 0.67 & 0.67 & 0.67 & 0.00 & St. Simon's Island & 2.59 & 0.00 \\
\hline Thermoelectric power & 0.01 & 8.08 & 8.09 & 8.09 & 8.10 & Sea Island Company & 1.01 & 0.00 \\
\hline TOTAL & 55.60 & 9.01 & 64.62 & 64.62 & 56.94 & Jekyll Island Authority & 0.71 & 0.00 \\
\hline
\end{tabular}

${ }^{1}$ Total use is total withdrawal plus public supply deliveries and losses.

\section{Withdrawals by Source}
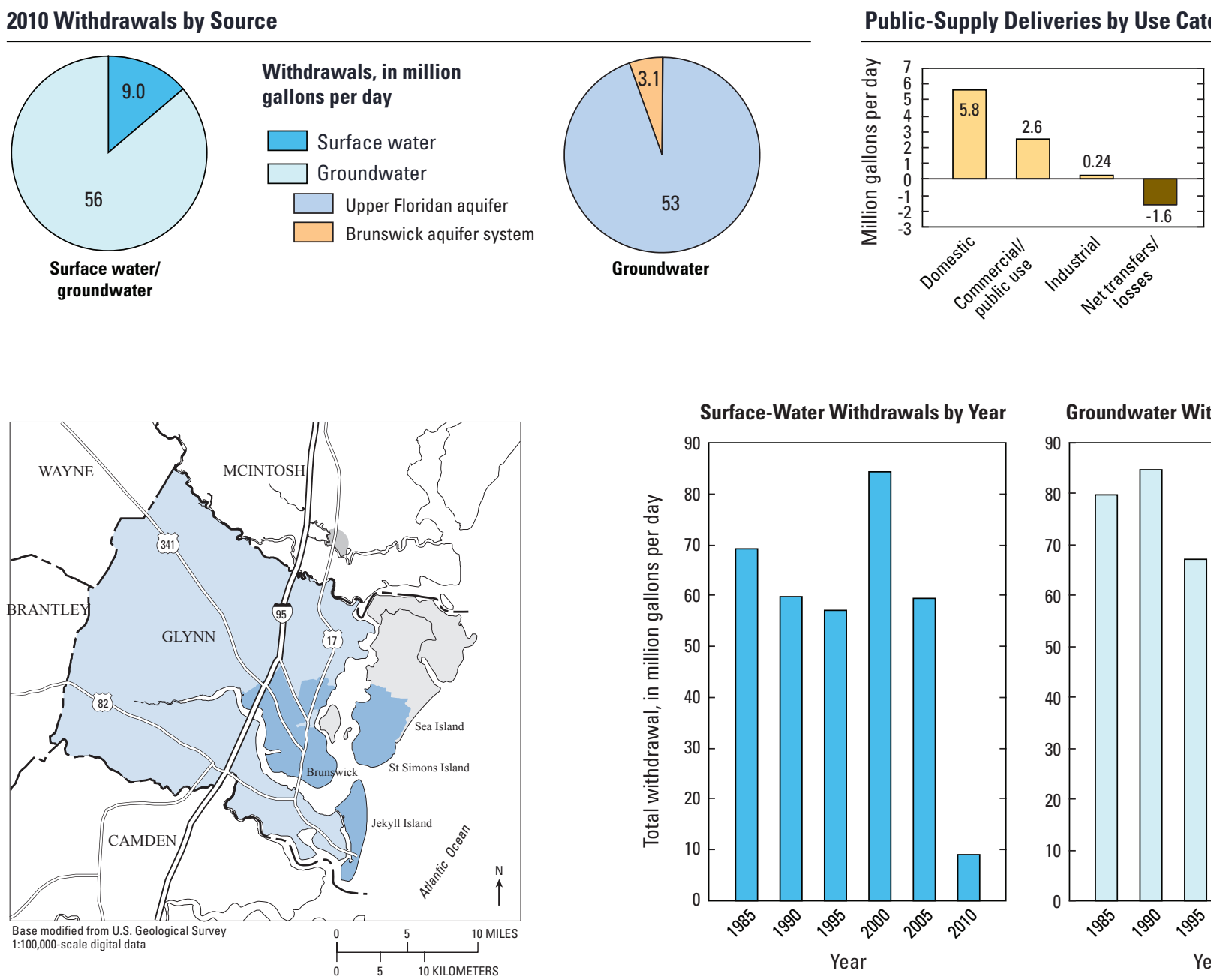

Groundwater Withdrawals by Year

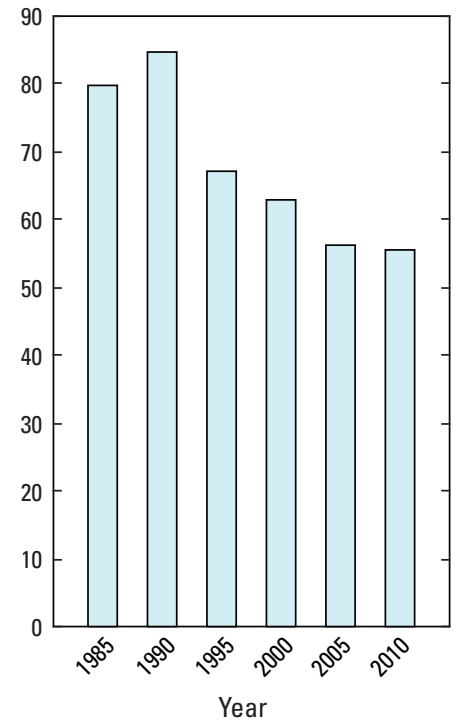


GORDON COUNTY

Population

Population served by public supply-Groundwater $\quad 14,120$

Population served by public supply-Surface water $\quad 32,390$

Acres irrigated

1,970

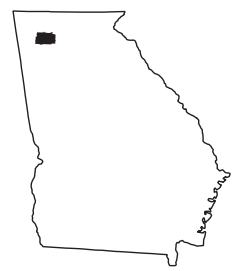

2010 WATER WITHDRAWALS AND ESTIMATED USE, IN MILLION GALLONS PER DAY

$[-$, not applicable; Mgal/d, million gallons per day $]$

\begin{tabular}{|c|c|c|c|c|c|c|c|c|}
\hline \multirow{3}{*}{ Category } & \multicolumn{3}{|c|}{ Withdrawals } & \multirow{3}{*}{$\begin{array}{l}\text { Total } \\
\text { use }^{1}\end{array}$} & \multirow{3}{*}{$\begin{array}{l}\text { Surface- } \\
\text { water } \\
\text { returns } \\
\end{array}$} & \multirow{2}{*}{\multicolumn{3}{|c|}{$\begin{array}{l}\text { Withdrawals by Major Industrial Groups } \\
\text { [NAICS, North American Industrial Classification System code] }\end{array}$}} \\
\hline & \multirow{2}{*}{$\begin{array}{c}\text { Ground- } \\
\text { water }\end{array}$} & \multirow{2}{*}{$\begin{array}{c}\text { Surface } \\
\text { water }\end{array}$} & \multirow[b]{2}{*}{ Total } & & & & & \\
\hline & & & & & & NAICS & Groundwater & Surface water \\
\hline Public supply & 3.19 & 7.33 & 10.52 & - & - & 313-Textiles & 0.06 & 0.00 \\
\hline Domestic & 0.65 & 0.00 & 0.65 & 4.70 & 0.00 & 313 - Iext1les & 0.00 & 0.00 \\
\hline Commercial/public use & 0.00 & 0.00 & 0.00 & 2.42 & 0.01 & & & \\
\hline Industrial & 0.06 & 0.00 & 0.06 & 1.85 & 0.08 & & & \\
\hline Public-supply losses & - & - & - & 1.58 & - & & & \\
\hline Public wastewater treatment & - & - & - & - & 5.37 & Withdrawals b & r Public Supp & \\
\hline Mining & 0.00 & 0.00 & 0.00 & 0.00 & 0.00 & Name & Groundwater & Surface water \\
\hline Irrigation-Crop & 0.01 & 1.30 & 1.31 & 1.31 & 0.00 & Citio & & \\
\hline Irrigation-Golf course & 0.00 & 0.15 & 0.15 & 0.15 & 0.00 & City of Calhoun & 3.18 & 7.33 \\
\hline Livestock/aquaculture & 0.03 & 0.81 & 0.84 & 0.84 & 0.00 & & & \\
\hline Thermoelectric power & 0.00 & 0.00 & 0.00 & 0.00 & 0.00 & & & \\
\hline TOTAL & 3.94 & 9.59 & 13.53 & 12.85 & 5.46 & & & \\
\hline
\end{tabular}

'Total use is total withdrawal plus public supply deliveries and losses. An average of $0.37 \mathrm{Mgal} / \mathrm{d}$ was delivered to Pickens County, Georgia and $0.31 \mathrm{Mgal} / \mathrm{d}$ to Murray County, Georgia in 2010.

2010 Withdrawals by Source
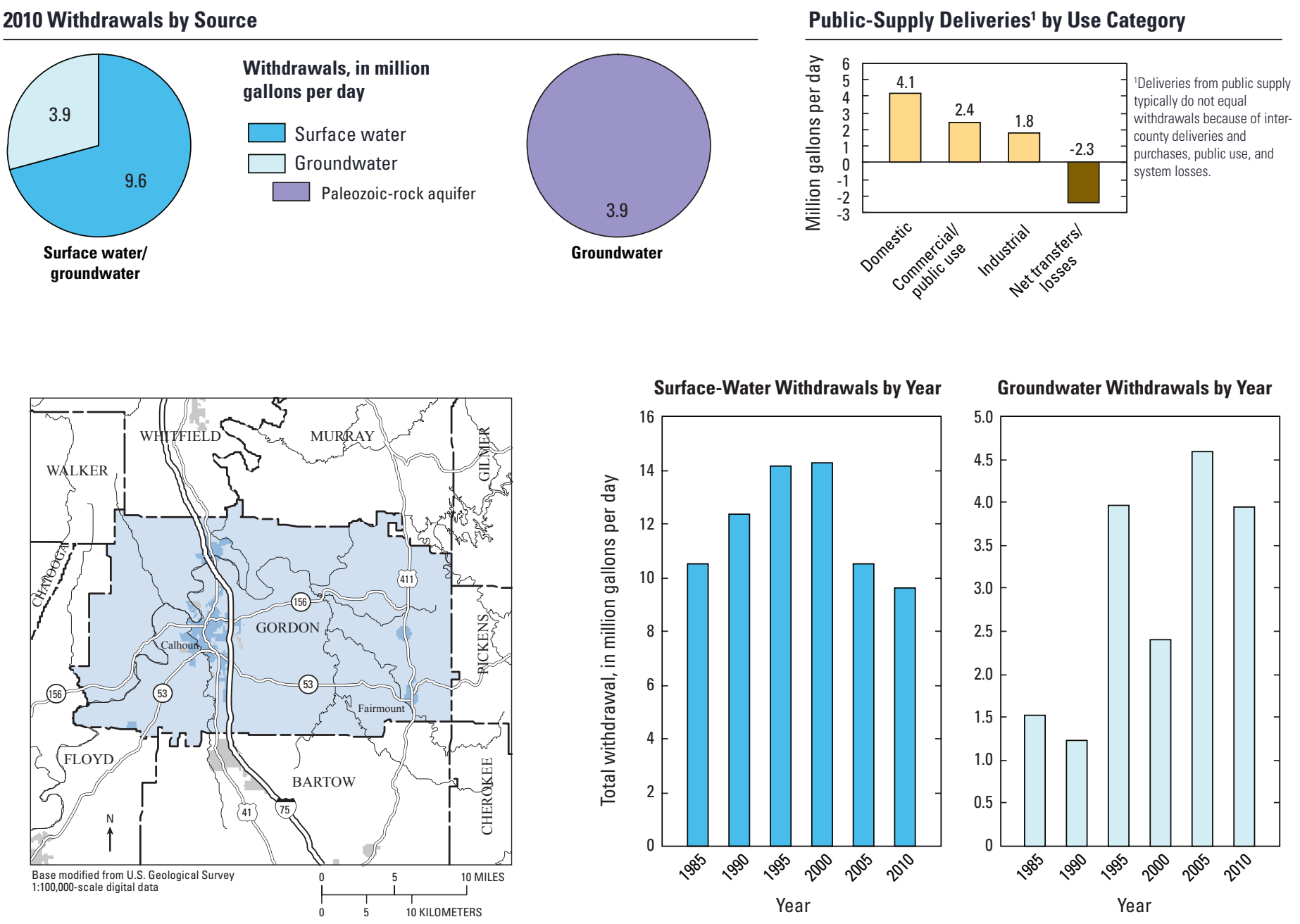

Groundwater Withdrawals by Year

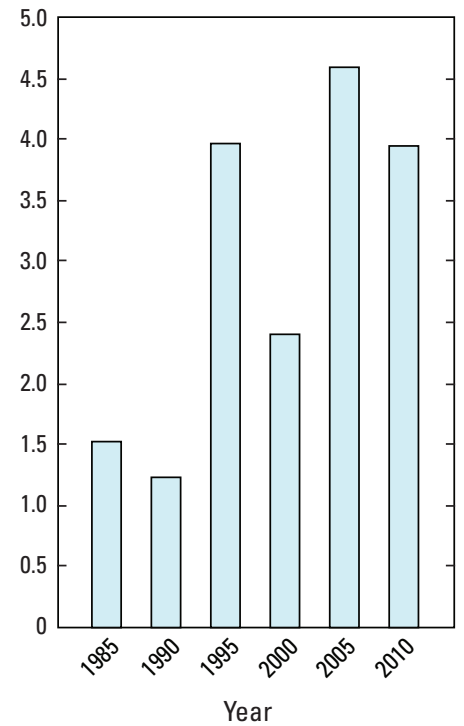




\section{GRADY COUNTY}

Population

Population served by public supply-Groundwater $\quad 11,130$

Population served by public supply-Surface water 0

Acres irrigated

15,950

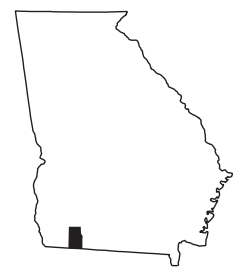

2010 WATER WITHDRAWALS AND ESTIMATED USE, IN MILLION GALLONS PER DAY

$[-$, not applicable]

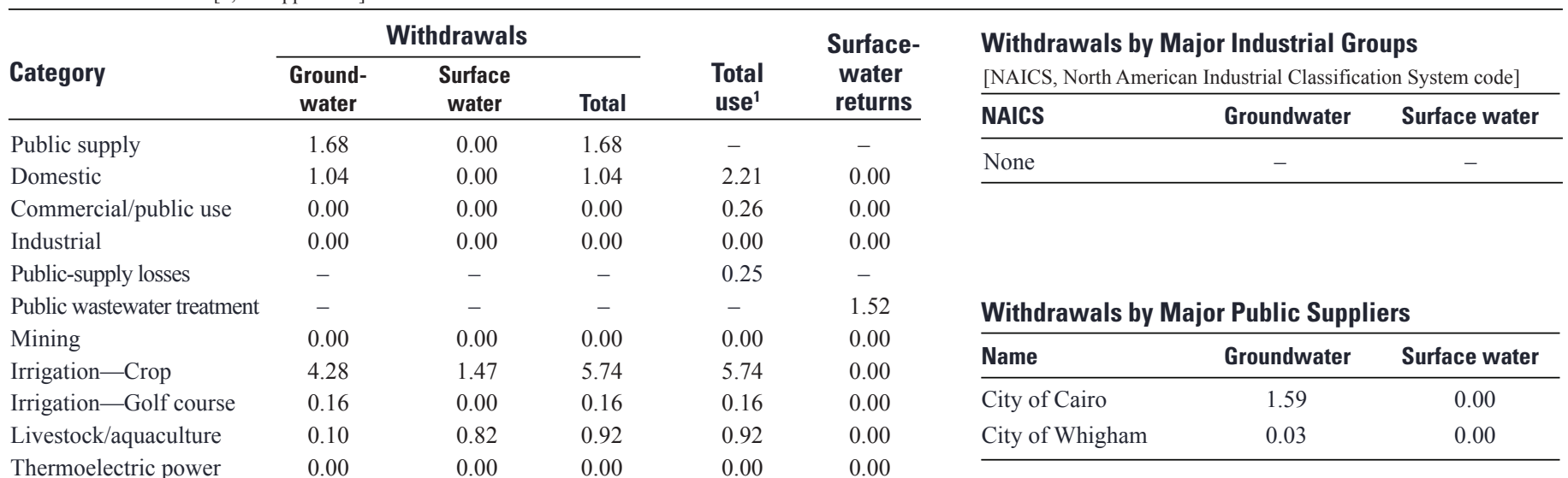

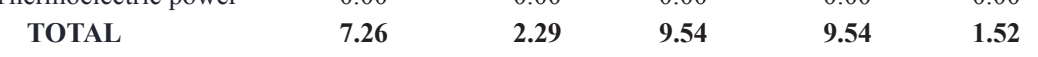

Total use is total withdrawal plus public supply deliveries and losses.

\section{Withdrawals by Source}
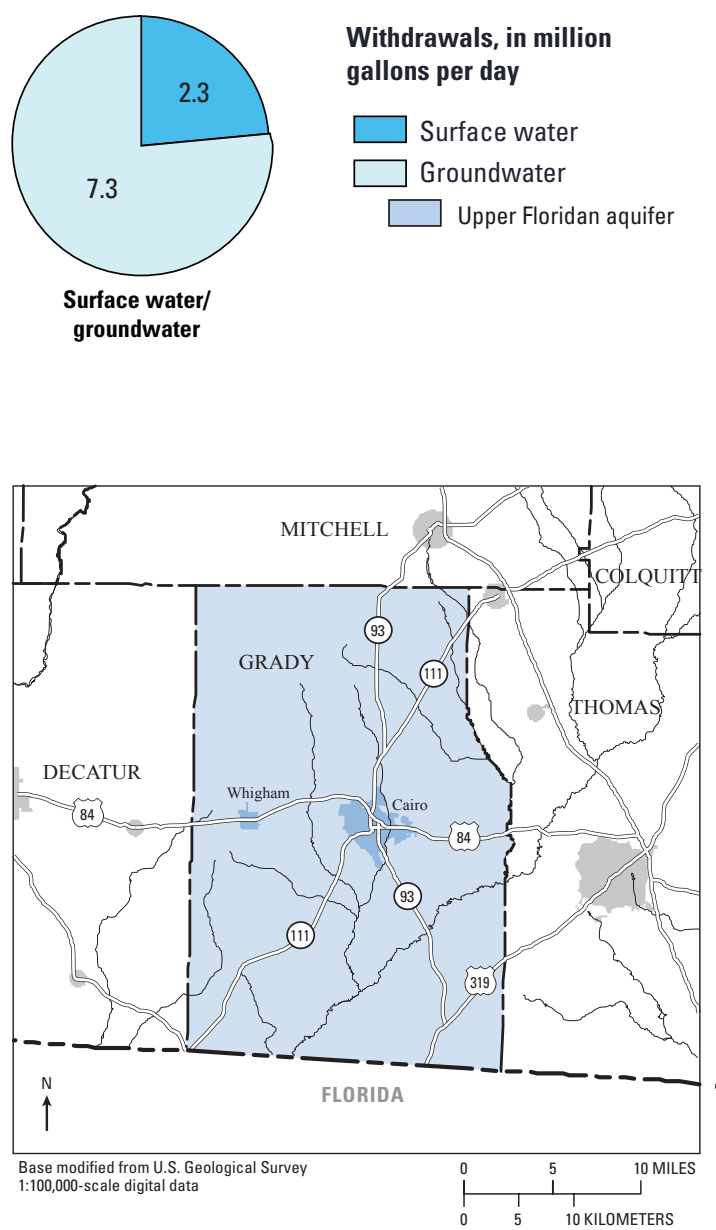

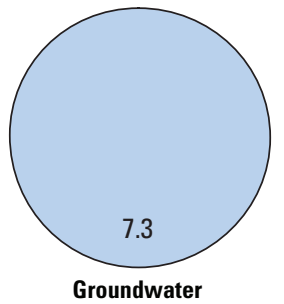

Public-Supply Deliveries by Use Category

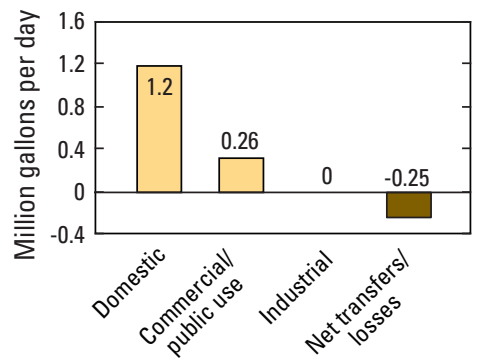

Surface-Water Withdrawals by Year

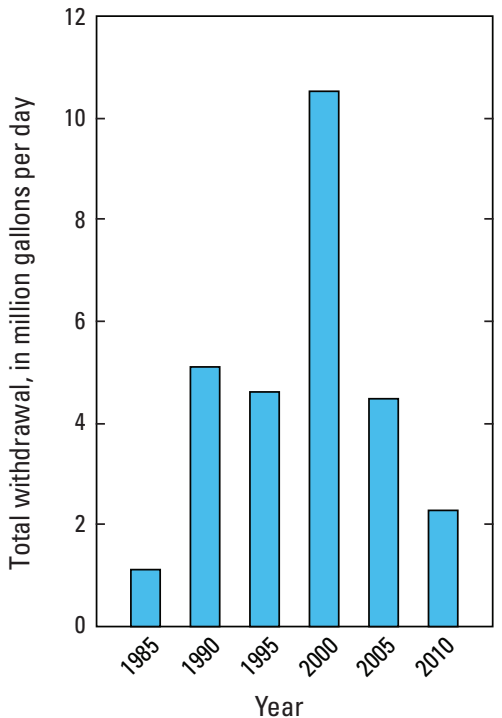

Groundwater Withdrawals by Year

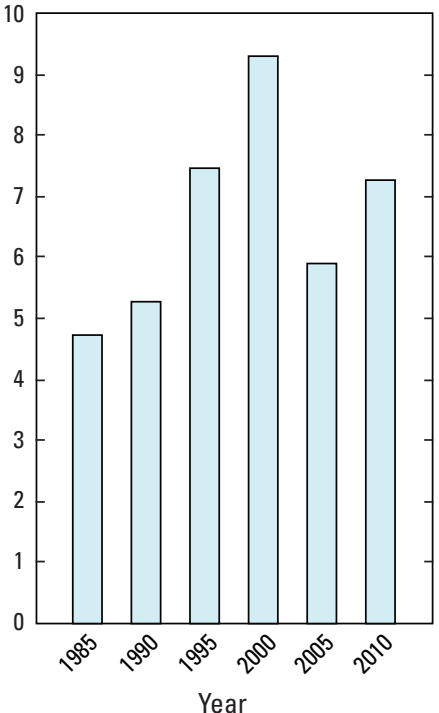




\section{GREENE COUNTY}

Population

Population served by public supply-Groundwater

7,380

Population served by public supply-Surface water $\quad 4,770$

Acres irrigated

890

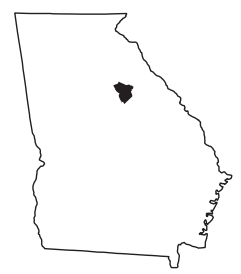

2010 WATER WITHDRAWALS AND ESTIMATED USE, IN MILLION GALLONS PER DAY

$[-$, not applicable]

\begin{tabular}{|c|c|c|c|c|c|c|c|c|}
\hline \multirow{3}{*}{ Category } & \multicolumn{3}{|c|}{ Withdrawals } & \multirow{3}{*}{$\begin{array}{l}\text { Total } \\
\text { use }^{1}\end{array}$} & \multirow{3}{*}{$\begin{array}{c}\text { Surface- } \\
\text { water } \\
\text { returns } \\
\end{array}$} & \multirow{2}{*}{\multicolumn{3}{|c|}{$\begin{array}{l}\text { Withdrawals by Major Industrial Groups } \\
\text { [NAICS, North American Industrial Classification System code] }\end{array}$}} \\
\hline & \multirow{2}{*}{$\begin{array}{l}\text { Ground- } \\
\text { water }\end{array}$} & \multirow{2}{*}{$\begin{array}{c}\text { Surface } \\
\text { water }\end{array}$} & \multirow[b]{2}{*}{ Total } & & & & & \\
\hline & & & & & & NAICS & Groundwater & Surface water \\
\hline Public supply & 1.09 & 0.82 & 1.91 & - & - & None & - & - \\
\hline Domestic & 0.29 & 0.00 & 0.29 & 1.79 & 0.00 & None & & \\
\hline Commercial/public use & 0.00 & 0.00 & 0.00 & 0.21 & 0.00 & & & \\
\hline Industrial & 0.00 & 0.00 & 0.00 & 0.00 & 0.02 & & & \\
\hline Public-supply losses & - & - & - & 0.20 & - & & & \\
\hline Public wastewater treatment & - & - & - & - & 0.19 & Withdrawals by Major & Public Supp & \\
\hline Mining & 0.08 & 0.00 & 0.08 & 0.08 & 0.00 & Name G & Groundwater & Surface water \\
\hline Irrigation-Crop & 0.28 & 0.00 & 0.28 & 0.28 & 0.00 & & & \\
\hline Irrigation-Golf course & 0.00 & 1.63 & 1.63 & 1.63 & 0.00 & Piedmont Water Company & 0.95 & 0.00 \\
\hline Livestock/aquaculture & 0.00 & 0.30 & 0.30 & 0.30 & 0.00 & City of Greensboro & 0.00 & 0.62 \\
\hline Thermoelectric power & 0.00 & 0.00 & 0.00 & 0.00 & 0.00 & City of Union Point & 0.00 & 0.20 \\
\hline TOTAL & 1.74 & 2.75 & 4.49 & 4.49 & 0.21 & & & \\
\hline
\end{tabular}

'Total use is total withdrawal plus public supply deliveries and losses.

\section{Withdrawals by Source}

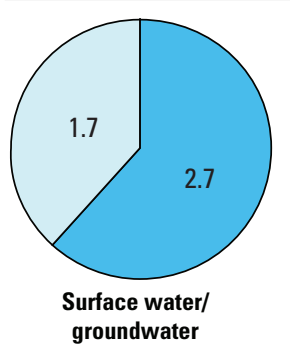

Withdrawals, in million gallons per day
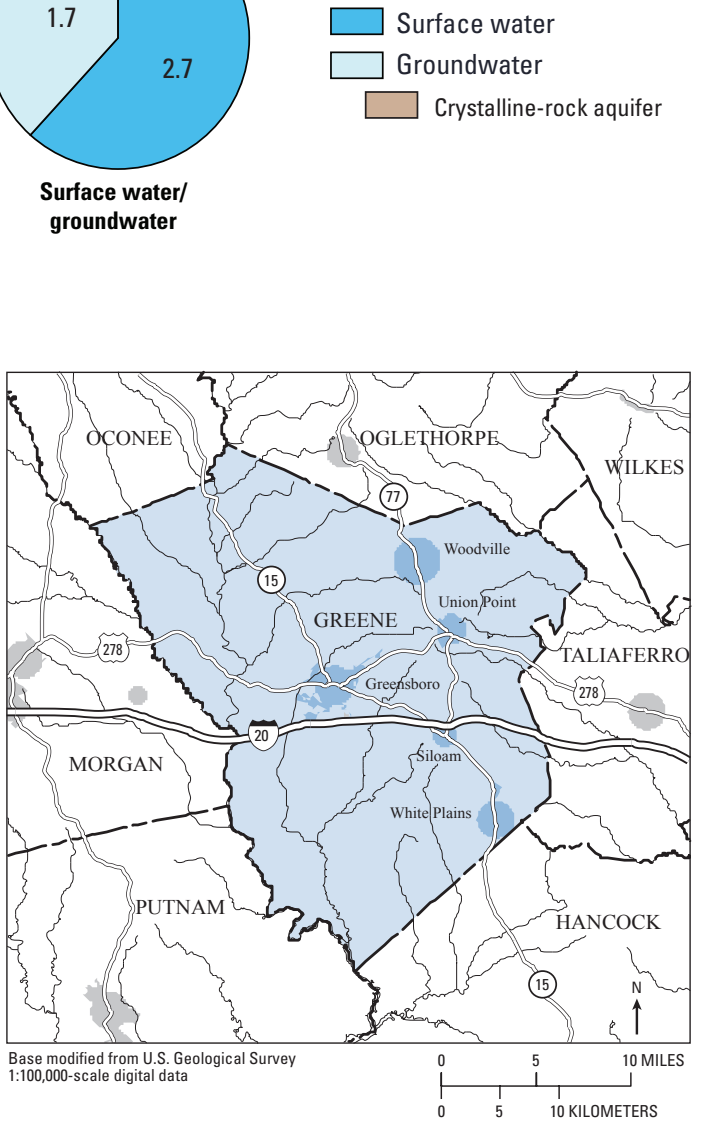

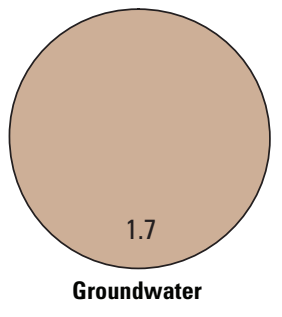

Public-Supply Deliveries by Use Category

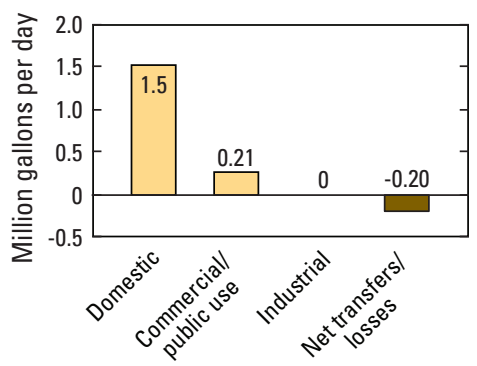

Surface-Water Withdrawals by Year

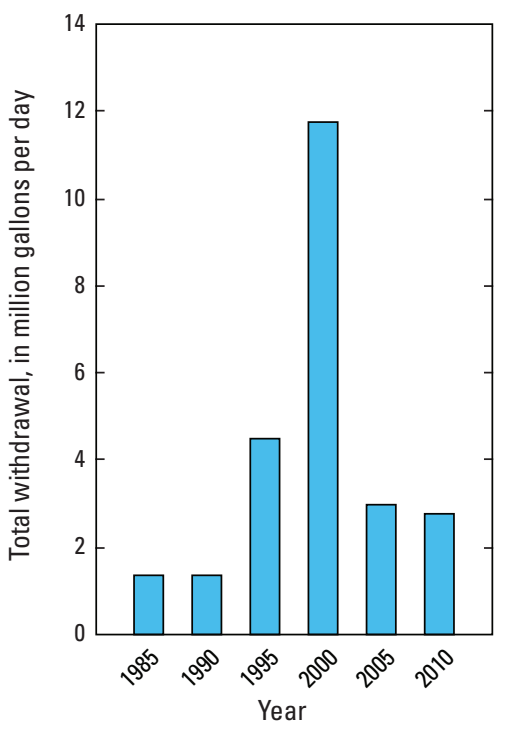

Groundwater Withdrawals by Year

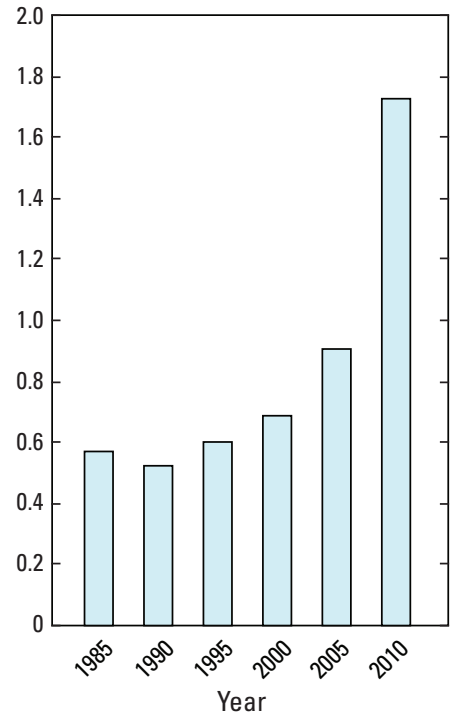




\section{GWINNETT COUNTY}

Population

805,321

Population served by public supply-Groundwater

Population served by public supply_-Surface water 801,131

Acres irrigated

1,000

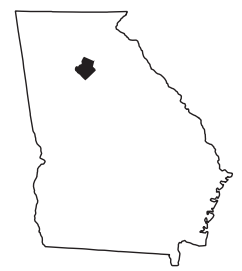

2010 WATER WITHDRAWALS AND ESTIMATED USE, IN MILLION GALLONS PER DAY

$[-$, not applicable; Mgal/d, million gallons per day $]$

\begin{tabular}{|c|c|c|c|c|c|c|c|c|}
\hline \multirow{3}{*}{ Category } & \multicolumn{3}{|c|}{ Withdrawals } & \multirow{3}{*}{$\begin{array}{l}\text { Total } \\
\text { use }^{1}\end{array}$} & \multirow{3}{*}{$\begin{array}{l}\text { Surface- } \\
\text { water } \\
\text { returns } \\
\end{array}$} & \multirow{2}{*}{\multicolumn{3}{|c|}{$\begin{array}{l}\text { Withdrawals by Major Industrial Groups } \\
\text { [NAICS, North American Industrial Classification System code] }\end{array}$}} \\
\hline & \multirow{2}{*}{$\begin{array}{c}\text { Ground- } \\
\text { water }\end{array}$} & \multirow{2}{*}{$\begin{array}{c}\text { Surface } \\
\text { water }\end{array}$} & \multirow[b]{2}{*}{ Total } & & & & & \\
\hline & & & & & & NAICS & Groundwater & Surface water \\
\hline Public supply & 0.41 & 0.00 & 0.41 & - & - & None & - & - \\
\hline Domestic & 0.00 & 0.00 & 0.00 & 51.83 & 0.00 & None & - & - \\
\hline Commercial/public use & 0.00 & 0.00 & 0.00 & 21.47 & 0.00 & & & \\
\hline Industrial & 0.00 & 0.00 & 0.00 & 0.50 & 0.82 & & & \\
\hline Public-supply losses & - & - & - & 4.55 & - & & & \\
\hline Public wastewater treatment & - & - & - & - & 38.03 & Withdrawals by Maj & r Public Supp & \\
\hline Mining & 0.39 & 0.01 & 0.40 & 0.40 & 1.17 & Name & Groundwater & Surface water \\
\hline Irrigation-Crop & 0.02 & 0.00 & 0.02 & 0.02 & $\begin{array}{l}0.00 \\
0.00\end{array}$ & City of Lawrenceville & 0.25 & 0.00 \\
\hline Irrigation-Golf course & 0.07 & 1.78 & 1.85 & 1.85 & 0.00 & City of Suwanee & 0.06 & 0.00 \\
\hline Livestock/aquaculture & 0.01 & 0.01 & 0.02 & 0.02 & 0.00 & & & \\
\hline Thermoelectric power & 0.00 & 0.00 & 0.00 & 0.00 & 0.00 & Gwinnett County Dept. of & Vater Resources w & Araws water \\
\hline TOTAL & 0.90 & 1.80 & 2.70 & 80.64 & 40.02 & from Lake Sidney Lanier & an intake location & Hall County \\
\hline
\end{tabular}

Total use is total withdrawal plus public supply deliveries and losses. An average of $76.73 \mathrm{Mgal} / \mathrm{d}$ was withdrawn from Hall County, Georgia, but delivered to and used in Gwinnett County in 2010. About 29 percent of the Braselton service area is in Gwinnett County.

\section{Withdrawals by Source}
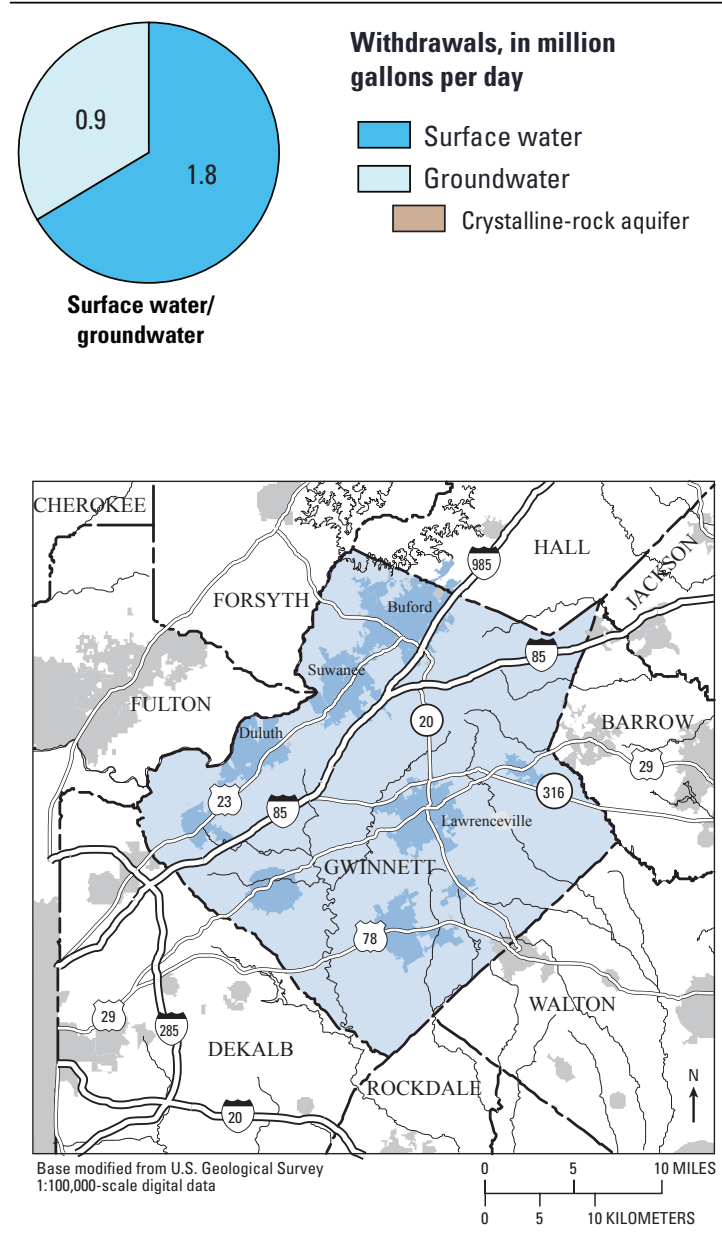

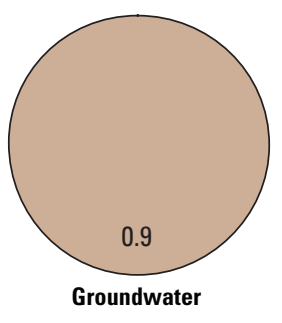

Public-Supply Deliveries' by Use Category

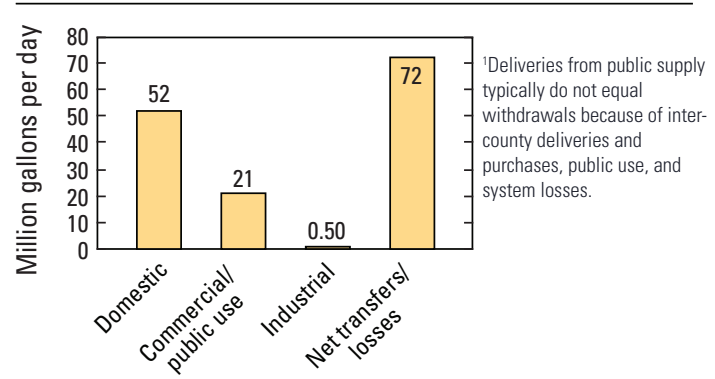

Surface-Water Withdrawals by Year

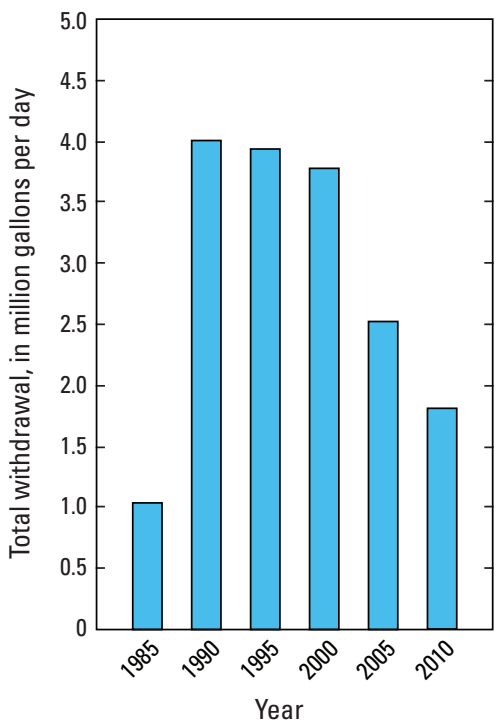

Groundwater Withdrawals by Year

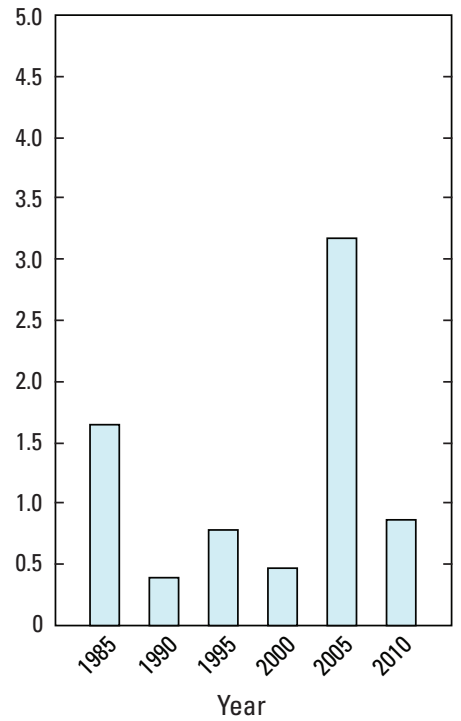


HABERSHAM COUNTY

Population

43,041

Population served by public supply-Groundwater

12,740

Population served by public supply-Surface water Acres irrigated
14,930

470

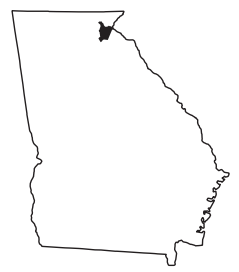

2010 WATER WITHDRAWALS AND ESTIMATED USE, IN MILLION GALLONS PER DAY

[-, not applicable; Mgal/d, million gallons per day]

\begin{tabular}{|c|c|c|c|c|c|c|c|c|}
\hline \multirow{3}{*}{ Category } & \multicolumn{3}{|c|}{ Withdrawals } & \multirow{3}{*}{$\begin{array}{l}\text { Total } \\
\text { use }^{1}\end{array}$} & \multirow{3}{*}{$\begin{array}{l}\text { Surface- } \\
\text { water } \\
\text { returns } \\
\end{array}$} & \multirow{2}{*}{\multicolumn{3}{|c|}{$\begin{array}{l}\text { Withdrawals by Major Industrial Groups } \\
\text { [NAICS, North American Industrial Classification System code] }\end{array}$}} \\
\hline & \multirow{2}{*}{$\begin{array}{c}\text { Ground- } \\
\text { water }\end{array}$} & \multirow{2}{*}{$\begin{array}{c}\text { Surface } \\
\text { water }\end{array}$} & \multirow{2}{*}{ Total } & & & & & \\
\hline & & & & & & NAICS & Groundwater & Surface water \\
\hline Public supply & 1.07 & 4.21 & 5.28 & - & - & & & \\
\hline Domestic & 1.15 & 0.00 & 1.15 & 3.82 & 0.00 & None & - & - \\
\hline Commercial/public use & 0.00 & 0.00 & 0.00 & 0.54 & 0.00 & & & \\
\hline Industrial & 0.00 & 0.00 & 0.00 & 1.41 & 0.19 & & & \\
\hline Public-supply losses & - & - & - & 0.78 & - & Withdrawals by M & r Public Supp & \\
\hline Public wastewater treatment & - & - & - & - & 2.90 & Name & Groundwater & Surface water \\
\hline $\begin{array}{l}\text { Mining } \\
\text { Irrigation-Crop }\end{array}$ & $\begin{array}{l}0.06 \\
0.02\end{array}$ & $\begin{array}{l}0.00 \\
0.26\end{array}$ & $\begin{array}{l}0.06 \\
0.28\end{array}$ & $\begin{array}{l}0.06 \\
0.28\end{array}$ & $\begin{array}{l}0.00 \\
0.00\end{array}$ & City of Cornelia & 0.00 & 1.89 \\
\hline Irrigation-Golf course & 0.00 & 0.30 & 0.30 & 0.30 & 0.00 & City of Baldwin & 0.00 & 1.63 \\
\hline Livestock/aquaculture & 0.01 & 5.07 & 5.08 & 5.08 & 0.00 & City of Clarkesville & 0.00 & 0.69 \\
\hline Thermoelectric power & 0.00 & 0.00 & 0.00 & 0.00 & 0.00 & City of Alto & 0.45 & 0.00 \\
\hline TOTAL & 2.31 & 9.84 & 12.15 & 12.27 & 3.19 & City of Demorest & 0.45 & 0.00 \\
\hline
\end{tabular}

'Total use is total withdrawal plus public supply deliveries and losses. An average of $0.12 \mathrm{Mgal} / \mathrm{d}$ was delivered from Stephens County,

Georgia to Habersham County in 2010.

\section{Withdrawals by Source}
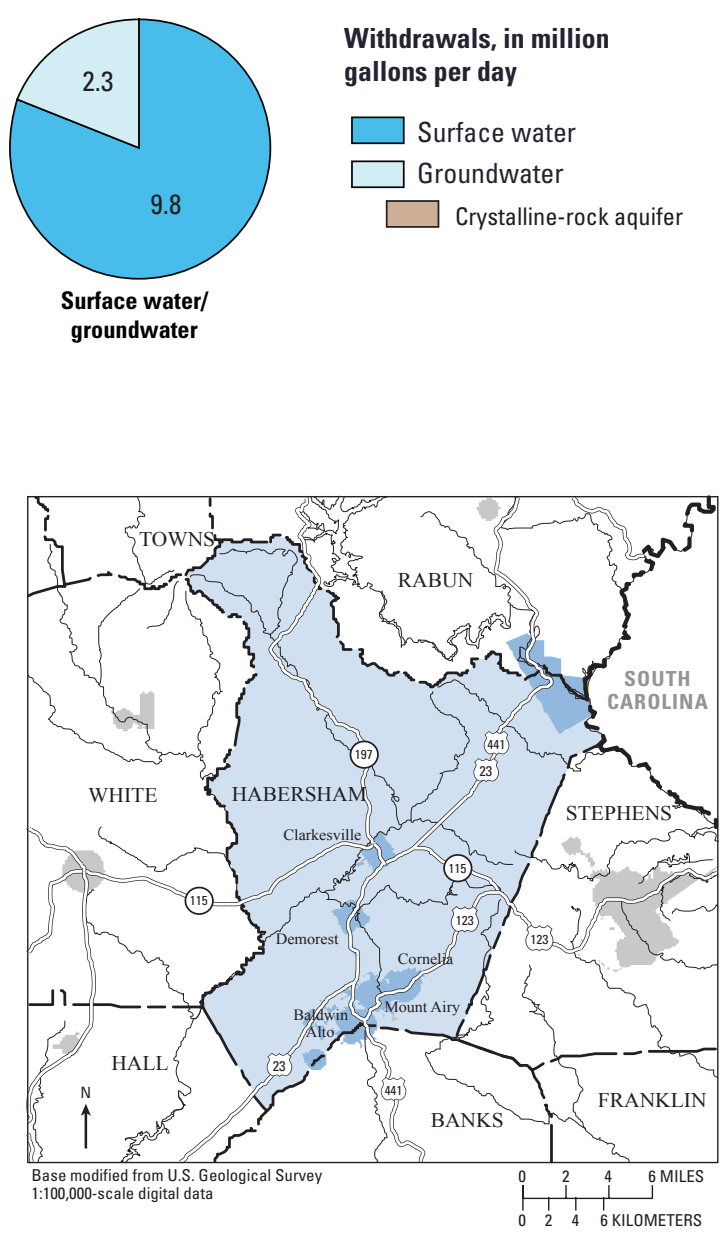

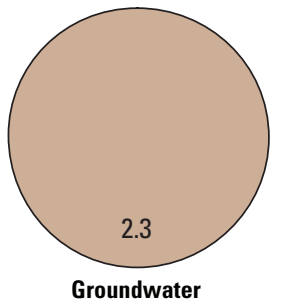

Public-Supply Deliveries ${ }^{1}$ by Use Category

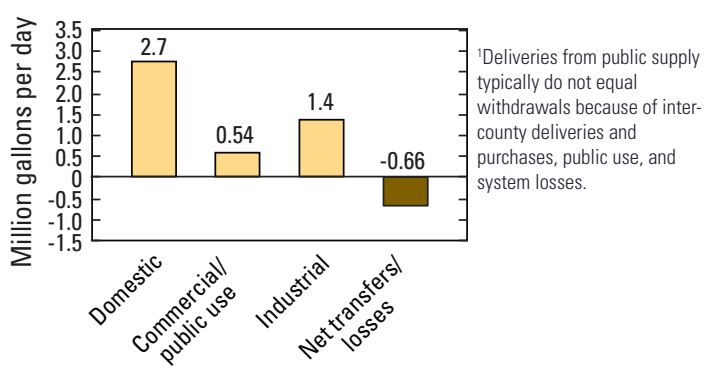

Surface-Water Withdrawals by Year

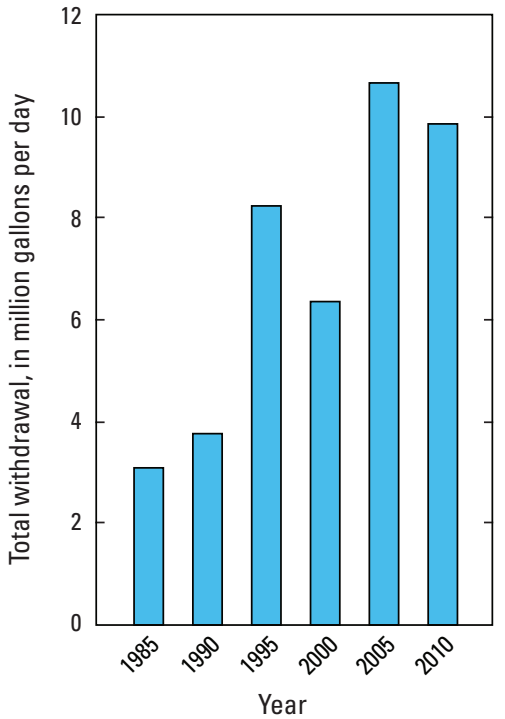

Groundwater Withdrawals by Year

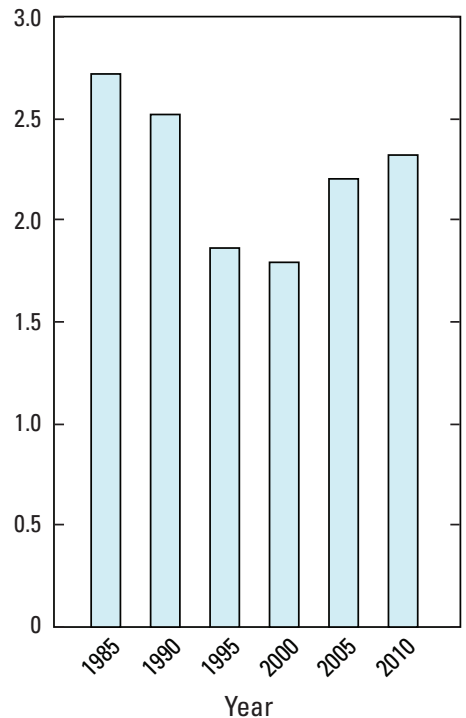




\section{HALL COUNTY}

Population

179,684

Population served by public supply-Groundwater

7,150

Population served by public supply-Surface water 144,670

Acres irrigated

830

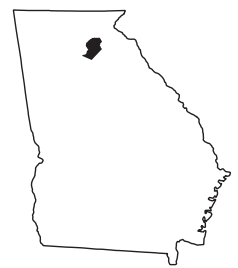

2010 WATER WITHDRAWALS AND ESTIMATED USE, IN MILLION GALLONS PER DAY

[-, not applicable; Mgal/d, million gallons per day]

\begin{tabular}{lcccccc}
\hline \multirow{2}{*}{ Category } & \multicolumn{3}{c}{ Withdrawals } & & Surface- \\
\cline { 2 - 4 } & $\begin{array}{c}\text { Ground- } \\
\text { water }\end{array}$ & $\begin{array}{c}\text { Surface } \\
\text { water }\end{array}$ & Total & $\begin{array}{c}\text { Total } \\
\text { use }\end{array}$ & $\begin{array}{c}\text { Water } \\
\text { returns }\end{array}$ \\
\hline Public supply & 0.55 & 94.24 & 94.79 & - & - \\
Domestic & 2.09 & 0.00 & 2.09 & 10.52 & 0.00 \\
Commercial/public use & 0.00 & 0.00 & 0.00 & 2.16 & 0.00 \\
Industrial & 0.21 & 0.00 & 0.21 & 5.06 & 0.00 \\
Public-supply losses & - & - & - & 2.69 & - \\
Public wastewater treatment & - & - & - & - & 9.03 \\
Mining & 0.20 & 0.00 & 0.20 & 0.20 & 0.00 \\
Irrigation-Crop & 0.07 & 0.04 & 0.11 & 0.11 & 0.00 \\
Irrigation-Golf course & 0.41 & 0.85 & 1.26 & 21.51 & 0.00 \\
Livestock/aquaculture & 0.09 & 1.48 & 1.57 & 1.57 & 0.00 \\
Thermoelectric power & 0.00 & 0.00 & 0.00 & 0.00 & 0.00 \\
$\quad$ TOTAL & $\mathbf{3 . 6 2}$ & $\mathbf{9 6 . 6 1}$ & $\mathbf{1 0 0 . 2 3}$ & $\mathbf{2 3 . 8 2}$ & $\mathbf{9 . 0 3}$ \\
\hline
\end{tabular}

${ }^{1}$ Total use is total withdrawal plus public supply deliveries and losses. An average of $76.72 \mathrm{Mgal} / \mathrm{d}$ was withdrawn in Hall County and

delivered to and used in Gwinnett County, Georgia in 2010. ${ }^{2}$ Includes $0.25 \mathrm{Mgal} / \mathrm{d}$ of water delivered for irrigation.

\section{Withdrawals by Source}
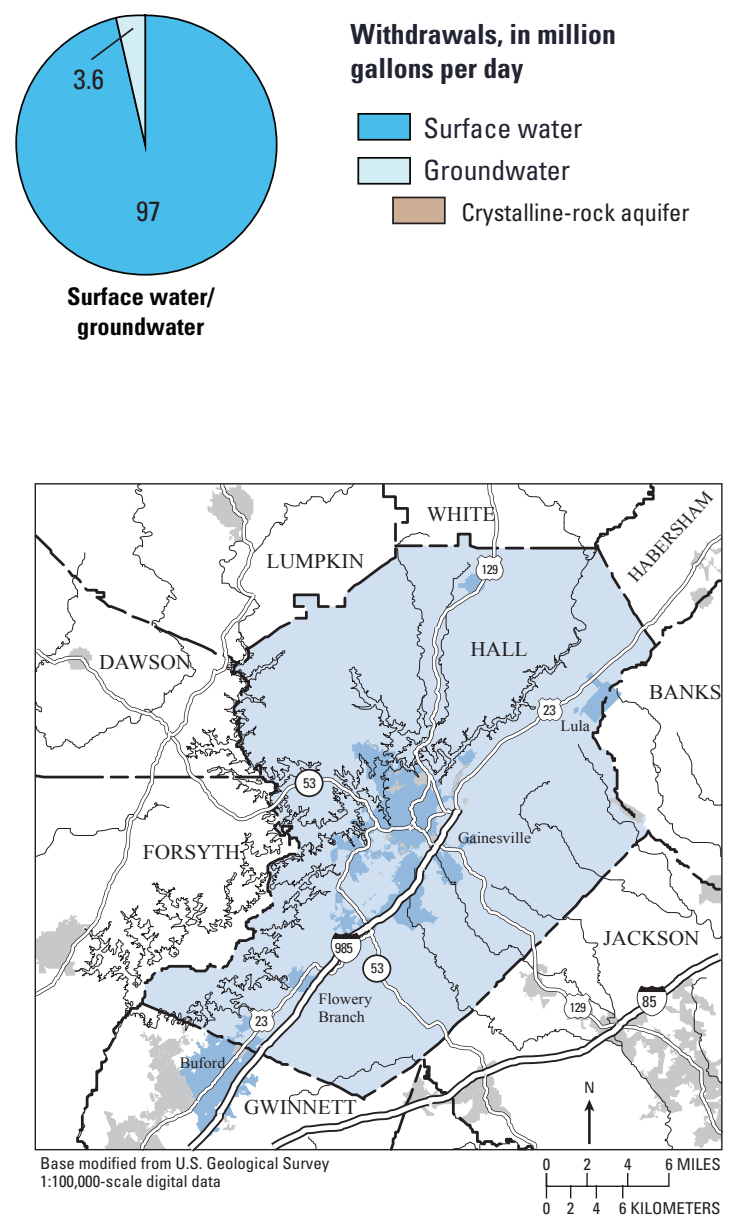
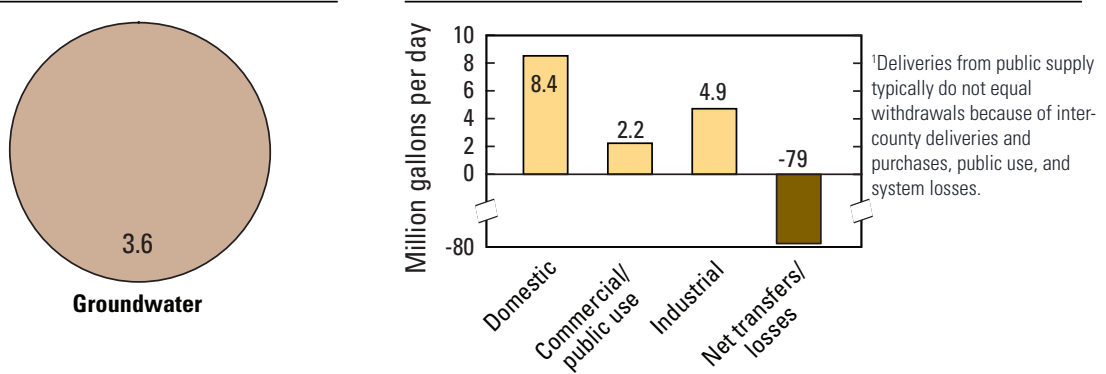

\section{Public-Supply Deliveries ${ }^{1}$ by Use Category}

Withdrawals by Major Industrial Groups

[NAICS, North American Industrial Classification System code]

\begin{tabular}{lcc}
\hline NAICS & Groundwater & Surface water \\
\hline 311 -Food products & 0.21 & 0.00 \\
\hline
\end{tabular}

Withdrawals by Major Public Suppliers

\begin{tabular}{lcc}
\hline Name & Groundwater & Surface water \\
\hline Gwinnett Co. Dept. of & 0.00 & 175.46 \\
Water Resources & & 17.52 \\
City of Gainesville & 0.00 & ${ }^{1} 1.26$ \\
City of Buford & 0.00 & 0.00 \\
City of Flowery Branch & 0.23 & \\
\hline
\end{tabular}

${ }^{1}$ Water withdrawn is used in Gwinnett County, Georgia. 


\section{HANCOCK COUNTY}

Population

Population served by public supply-Groundwater

Population served by public supply-Surface water Acres irrigated
3,150

3,650

310

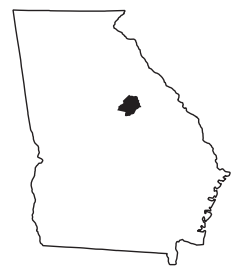

2010 WATER WITHDRAWALS AND ESTIMATED USE, IN MILLION GALLONS PER DAY

$[-$, not applicable $]$

\begin{tabular}{|c|c|c|c|c|c|c|c|c|}
\hline \multirow{3}{*}{ Category } & \multicolumn{3}{|c|}{ Withdrawals } & \multirow{3}{*}{$\begin{array}{l}\text { Total } \\
\text { use }^{1}\end{array}$} & \multirow{3}{*}{$\begin{array}{l}\text { Surface- } \\
\text { water } \\
\text { returns } \\
\end{array}$} & \multirow{2}{*}{\multicolumn{3}{|c|}{$\begin{array}{l}\text { Withdrawals by Major Industrial Groups } \\
\text { [NAICS, North American Industrial Classification System code] }\end{array}$}} \\
\hline & \multirow{2}{*}{$\begin{array}{c}\text { Ground- } \\
\text { water }\end{array}$} & \multirow{2}{*}{$\begin{array}{c}\text { Surface } \\
\text { water }\end{array}$} & \multirow[b]{2}{*}{ Total } & & & & & \\
\hline & & & & & & NAICS & Groundwater & Surface water \\
\hline \multirow{2}{*}{$\begin{array}{l}\text { Public supply } \\
\text { Domestic }\end{array}$} & 0.23 & 1.12 & 1.35 & - & \multirow{2}{*}{-} & \multirow{2}{*}{ None } & \multirow[b]{2}{*}{-} & \multirow{2}{*}{-} \\
\hline & 0.20 & 0.00 & 0.20 & 0.71 & & & & \\
\hline Commercial/public use & 0.00 & 0.00 & 0.00 & 0.62 & 0.00 & & & \\
\hline Industrial & 0.00 & 0.00 & 0.00 & 0.00 & 0.00 & & & \\
\hline Public-supply losses & - & - & - & 0.22 & - & & & \\
\hline Public wastewater treatment & - & - & - & - & 0.02 & \multicolumn{3}{|c|}{ Withdrawals by Major Public Suppliers } \\
\hline \multirow{3}{*}{$\begin{array}{l}\text { Mining } \\
\text { Irrigation-Crop } \\
\text { Irrigation-Golf course }\end{array}$} & 0.06 & 0.00 & 0.06 & 0.06 & 0.00 & \multirow{2}{*}{ Name } & Groundwater & \multirow[t]{2}{*}{ Surface water } \\
\hline & 0.52 & 0.13 & 0.65 & 0.65 & 0.00 & & & \\
\hline & 0.00 & 0.00 & 0.00 & 0.00 & 0.00 & City of Sparta & 0.00 & 1.12 \\
\hline Livestock/aquaculture & 0.00 & 0.05 & 0.05 & 0.05 & 0.00 & Piedmont Water Company & 0.17 & 0.00 \\
\hline Thermoelectric power & 0.00 & 0.00 & 0.00 & 0.00 & 0.00 & & & \\
\hline
\end{tabular}

Thermoelectric power

1.01

$\mathbf{1 . 3 0}$

2.31

2.31

0.02

'Total use is total withdrawal plus public supply deliveries and losses.

\section{Withdrawals by Source}
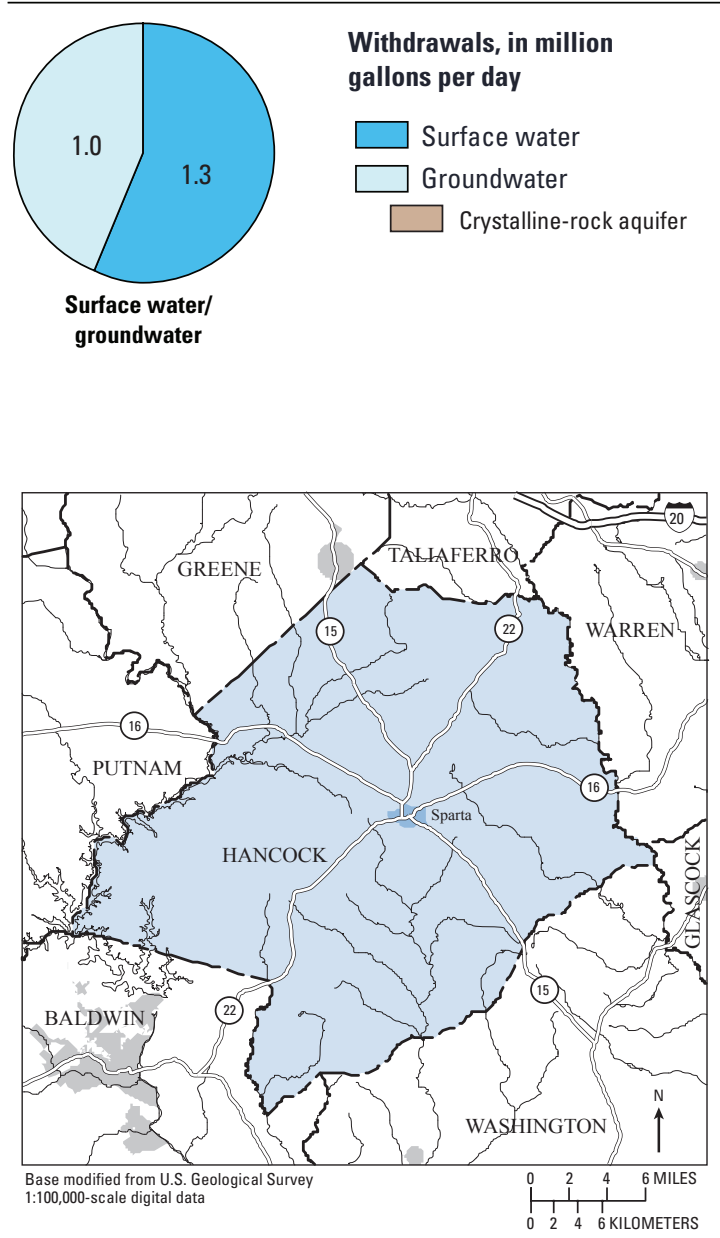

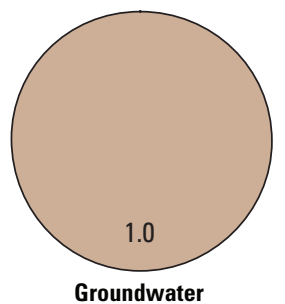

Public-Supply Deliveries by Use Category

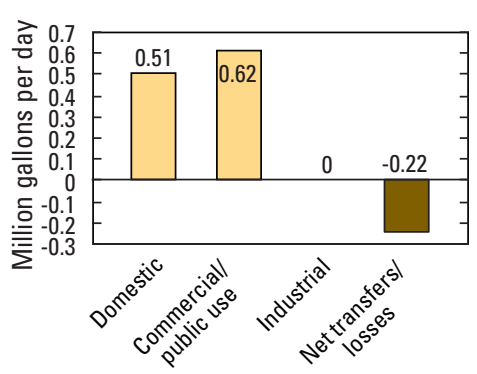

Surface-Water Withdrawals by Year

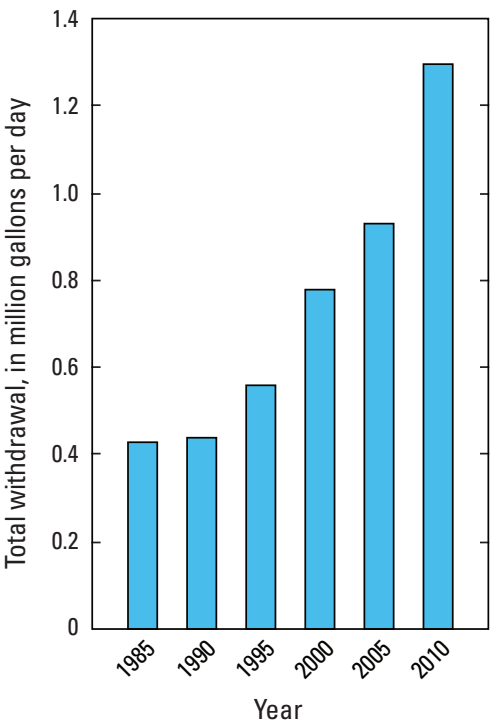

Groundwater Withdrawals by Year

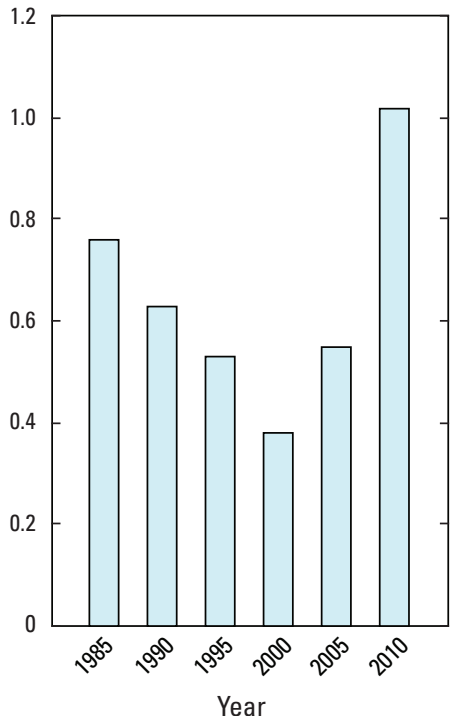


HARALSON COUNTY

Population

Population served by public supply-Groundwater

Population served by public supply-Surface water 22,430

Acres irrigated

30

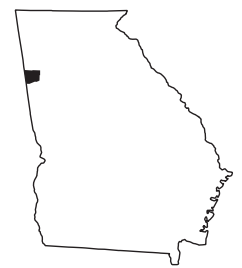

2010 WATER WITHDRAWALS AND ESTIMATED USE, IN MILLION GALLONS PER DAY

[-, not applicable; Mgal/d, million gallons per day]

\begin{tabular}{|c|c|c|c|c|c|c|c|c|}
\hline \multirow{3}{*}{ Category } & \multicolumn{3}{|c|}{ Withdrawals } & \multirow{3}{*}{$\begin{array}{l}\text { Total } \\
\text { use }^{1}\end{array}$} & \multirow{3}{*}{$\begin{array}{l}\text { Surface- } \\
\text { water } \\
\text { returns }\end{array}$} & \multirow{2}{*}{\multicolumn{3}{|c|}{$\begin{array}{l}\text { Withdrawals by Major Industrial Groups } \\
\text { [NAICS, North American Industrial Classification System code] }\end{array}$}} \\
\hline & \multirow{2}{*}{$\begin{array}{c}\text { Ground- } \\
\text { water }\end{array}$} & \multirow{2}{*}{$\begin{array}{c}\text { Surface } \\
\text { water }\end{array}$} & \multirow[b]{2}{*}{ Total } & & & & & \\
\hline & & & & & & NAICS & Groundwater & Surface water \\
\hline Public supply & 0.09 & 2.53 & 2.62 & - & - & None & & \\
\hline Domestic & 0.45 & 0.00 & 0.45 & 2.10 & 0.00 & None & - & - \\
\hline Commercial/public use & 0.00 & 0.00 & 0.00 & 0.47 & 0.00 & & & \\
\hline Industrial & 0.00 & 0.00 & 0.00 & 0.05 & 0.00 & & & \\
\hline Public-supply losses & - & - & - & 0.65 & - & & & \\
\hline Public wastewater treatment & - & - & - & - & 0.81 & Withdrawals by Majo & r Public Supp & \\
\hline Mining & 0.00 & 0.00 & 0.00 & 0.00 & 0.00 & Name & Groundwater & Surface water \\
\hline Irrigation-Crop & 0.00 & 0.00 & 0.00 & 0.00 & 0.00 & Naille & & \\
\hline Irrigation-Golf course & 0.00 & 0.22 & 0.22 & 0.22 & 0.00 & Haralson County Water & 0.09 & 2.34 \\
\hline Livestock/aquaculture & 0.05 & 0.57 & 0.62 & 0.62 & 0.00 & Authority & & \\
\hline Thermoelectric power & 0.00 & 0.00 & 0.00 & 0.00 & 0.00 & City of Bremen & 0.00 & 0.19 \\
\hline TOTAL & 0.59 & 3.32 & 3.91 & 4.11 & 0.81 & & & \\
\hline
\end{tabular}

'Total use is total withdrawal plus public supply deliveries and losses. An average of $0.2 \mathrm{Mgal} / \mathrm{d}$ was delivered from Cleburne County, Alabama to Haralson County, Georgia in 2010

\section{Withdrawals by Source}

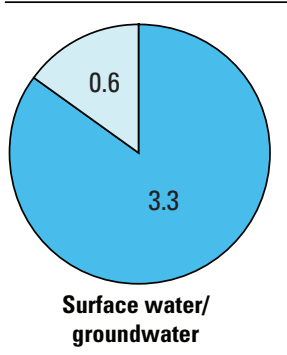

Withdrawals, in million gallons per day

$\square$ Surface water

$\square$ Groundwater

$\square$ Crystalline-rock aquifer groundwater

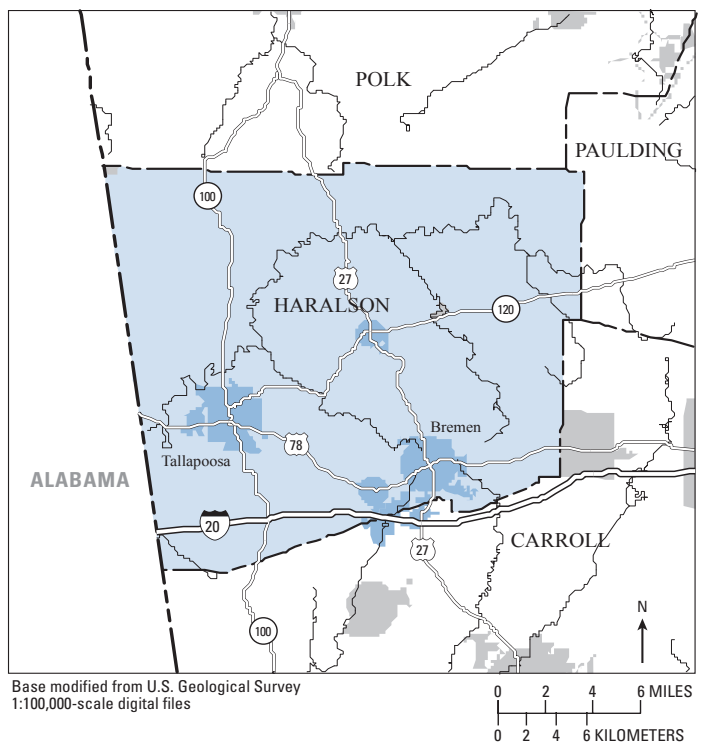

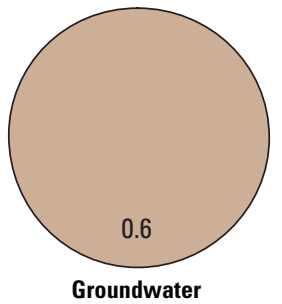

Public-Supply Deliveries ${ }^{1}$ by Use Category

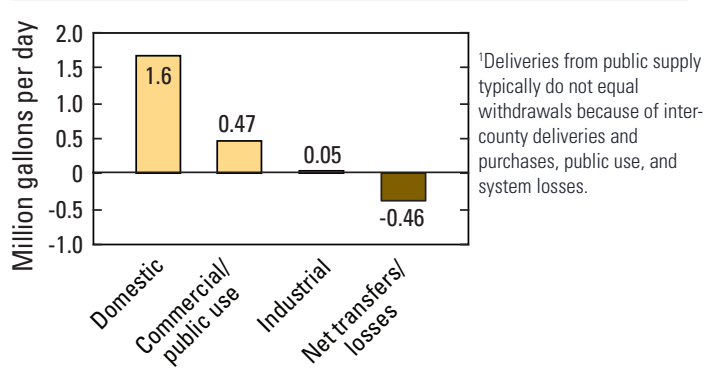

Surface-Water Withdrawals by Year

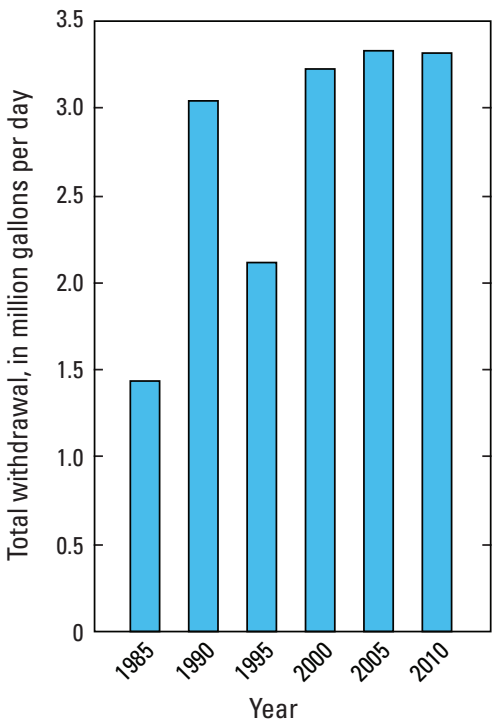

Groundwater Withdrawals by Year

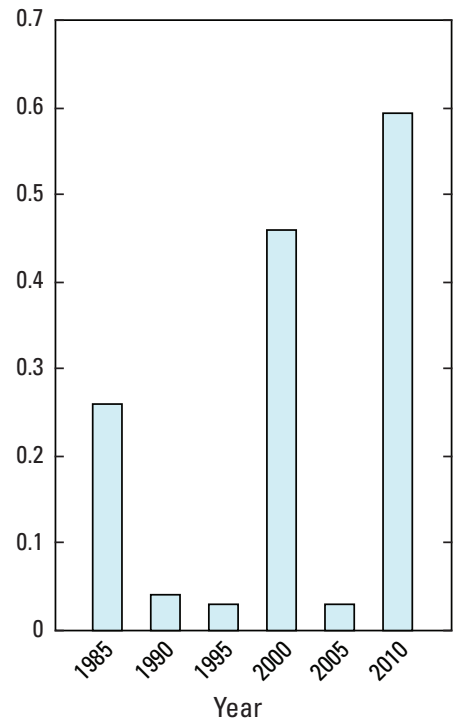


HARRIS COUNTY

Population

32,024

Population served by public supply_-Groundwater

Population served by public supply-Surface water Acres irrigated
5,250

20,730

220

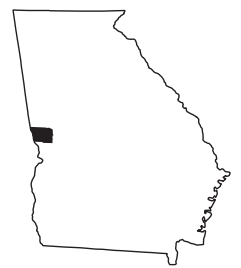

2010 WATER WITHDRAWALS AND ESTIMATED USE, IN MILLION GALLONS PER DAY

$[-$, not applicable; Mgal/d, million gallons per day $]$

\begin{tabular}{|c|c|c|c|c|c|}
\hline \multirow[b]{2}{*}{ Category } & \multicolumn{3}{|c|}{ Withdrawals } & \multirow[b]{2}{*}{$\begin{array}{l}\text { Total } \\
\text { use }^{1}\end{array}$} & \multirow{2}{*}{$\begin{array}{c}\text { Surface- } \\
\text { water } \\
\text { returns }\end{array}$} \\
\hline & $\begin{array}{c}\text { Ground- } \\
\text { water }\end{array}$ & $\begin{array}{c}\text { Surface } \\
\text { water }\end{array}$ & Total & & \\
\hline Public supply & 0.41 & 5.86 & 6.27 & - & - \\
\hline Domestic & 0.45 & 0.00 & 0.45 & 2.62 & 0.00 \\
\hline Commercial/public use & 0.00 & 0.00 & 0.00 & 0.12 & 0.13 \\
\hline Industrial & 0.00 & 0.00 & 0.00 & 0.94 & 0.00 \\
\hline Public-supply losses & - & - & - & 0.21 & - \\
\hline Public wastewater treatment & - & - & - & - & 1.39 \\
\hline Mining & 0.13 & 0.00 & 0.13 & 0.13 & 0.00 \\
\hline Irrigation-Crop & 0.15 & 0.00 & 0.15 & 0.15 & 0.00 \\
\hline Irrigation-Golf course & 0.00 & 0.05 & 0.05 & 0.05 & 0.00 \\
\hline Livestock/aquaculture & 0.00 & 0.05 & 0.05 & 0.05 & 0.00 \\
\hline Thermoelectric power & 0.00 & 0.00 & 0.00 & 0.00 & 0.00 \\
\hline TOTAL & 1.14 & 5.96 & 7.10 & 4.27 & 1.52 \\
\hline
\end{tabular}

${ }^{1}$ Total use is total withdrawal plus public supply deliveries and losses. An average of $0.82 \mathrm{Mgal} / \mathrm{d}$ was delivered from Muscogee County,

Georgia and $0.08 \mathrm{Mgal} / \mathrm{d}$ from Talbot County, Georgia to Harris County in 2010. An average of $3.73 \mathrm{Mgal} / \mathrm{d}$ was withdrawn in Harris County,

Georgia but delivered to and used in Chambers County, Alabama in 2010.

\section{Withdrawals by Source}
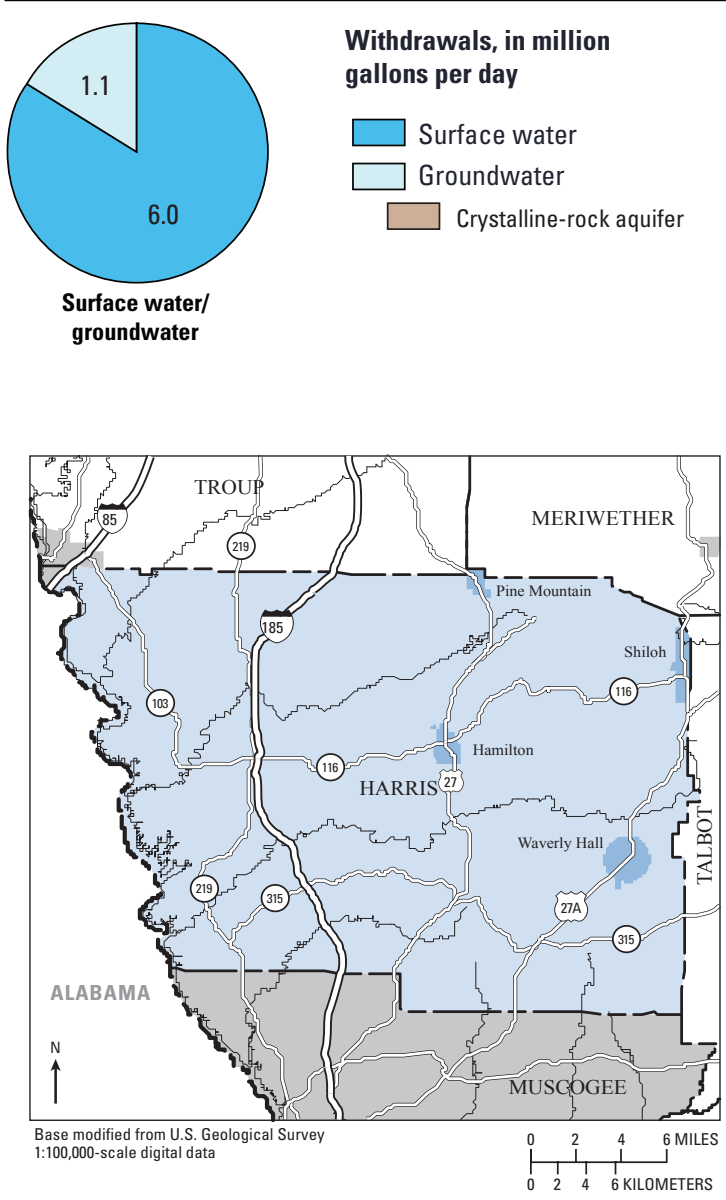

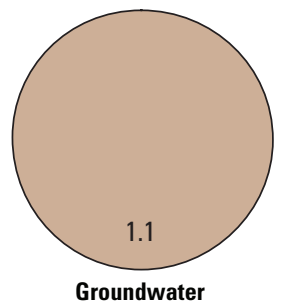

Public-Supply Deliveries' by Use Category

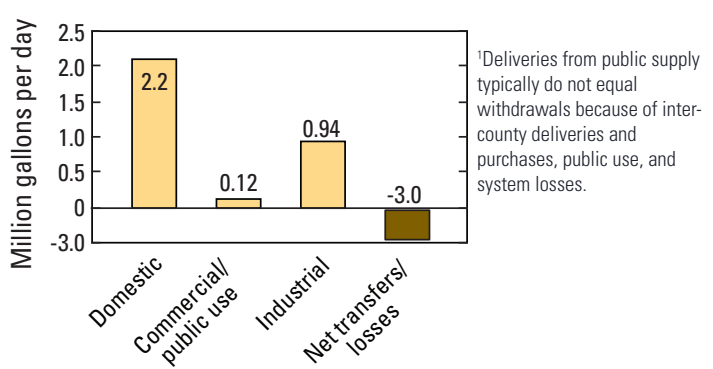

Surface-Water Withdrawals by Year

Groundwater Withdrawals by Year
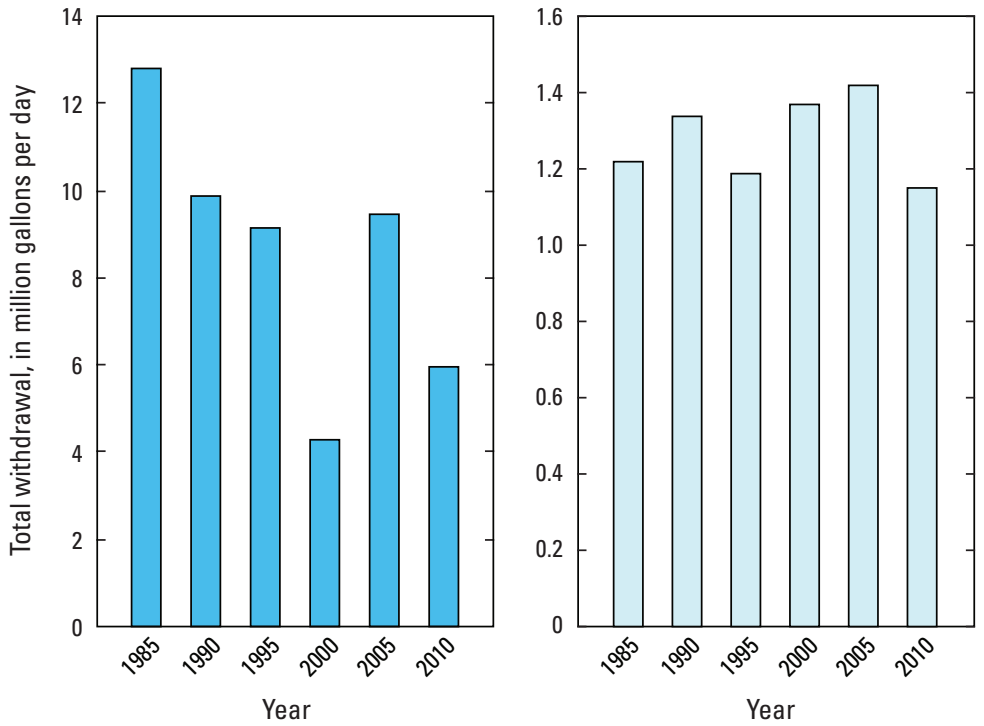


\section{HART COUNTY}

Population

Population served by public supply-Groundwater

Population served by public supply-Surface water $\quad 7,900$ Acres irrigated

990

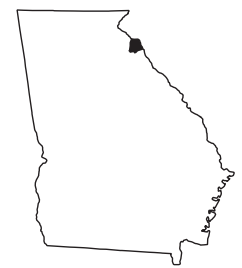

2010 WATER WITHDRAWALS AND ESTIMATED USE, IN MILLION GALLONS PER DAY

$[-$, not applicable; Mgal/d, million gallons per day $]$

\begin{tabular}{|c|c|c|c|c|c|c|c|c|}
\hline \multirow{3}{*}{ Category } & \multicolumn{3}{|c|}{ Withdrawals } & \multirow{3}{*}{$\begin{array}{l}\text { Total } \\
\text { use }^{1}\end{array}$} & \multirow{3}{*}{$\begin{array}{c}\text { Surface- } \\
\text { water } \\
\text { returns } \\
\end{array}$} & \multirow{2}{*}{\multicolumn{3}{|c|}{$\begin{array}{l}\text { Withdrawals by Major Industrial Groups } \\
\text { [NAICS, North American Industrial Classification System code] }\end{array}$}} \\
\hline & \multirow{2}{*}{$\begin{array}{l}\text { Ground- } \\
\text { water }\end{array}$} & \multirow{2}{*}{$\begin{array}{c}\text { Surface } \\
\text { water }\end{array}$} & \multirow[b]{2}{*}{ Total } & & & & & \\
\hline & & & & & & NAICS & Groundwater & Surface water \\
\hline Public supply & 0.13 & 1.02 & 1.15 & - & - & & - & - \\
\hline Domestic & 1.13 & 0.00 & 1.13 & 2.11 & 0.00 & None & - & - \\
\hline Commercial/public use & 0.00 & 0.00 & 0.00 & 0.35 & 0.00 & & & \\
\hline Industrial & 0.00 & 0.00 & 0.00 & 0.04 & 0.00 & & & \\
\hline Public-supply losses & - & - & - & 0.24 & - & & & \\
\hline Public wastewater treatment & - & - & - & - & 0.00 & Withdrawals b & r Public Supp & \\
\hline Mining & 0.00 & 0.00 & 0.00 & 0.00 & 0.00 & Name & Groundwater & Surface water \\
\hline $\begin{array}{l}\text { Irrigation-Crop } \\
\text { Irrigation-Golf course }\end{array}$ & $\begin{array}{l}0.25 \\
0.00\end{array}$ & $\begin{array}{l}0.61 \\
0.26\end{array}$ & $\begin{array}{l}0.86 \\
0.26\end{array}$ & $\begin{array}{l}0.86 \\
0.26\end{array}$ & $\begin{array}{l}0.00 \\
0.00\end{array}$ & City of Hartwell & 0.00 & 1.02 \\
\hline Livestock/aquaculture & 0.00 & 0.80 & 0.80 & 0.80 & 0.00 & & & \\
\hline Thermoelectric power & 0.00 & 0.00 & 0.00 & 0.00 & 0.00 & & & \\
\hline TOTAL & 1.51 & 2.69 & 4.20 & 4.66 & 0.00 & & & \\
\hline
\end{tabular}

${ }^{1}$ Total use is total withdrawal plus public supply deliveries and losses. An average of $0.08 \mathrm{Mgal} / \mathrm{d}$ was delivered to Franklin County, Georgia in 2010. Parts of the cities of Royston and Canon lie in Hart County.

\section{Withdrawals by Source}
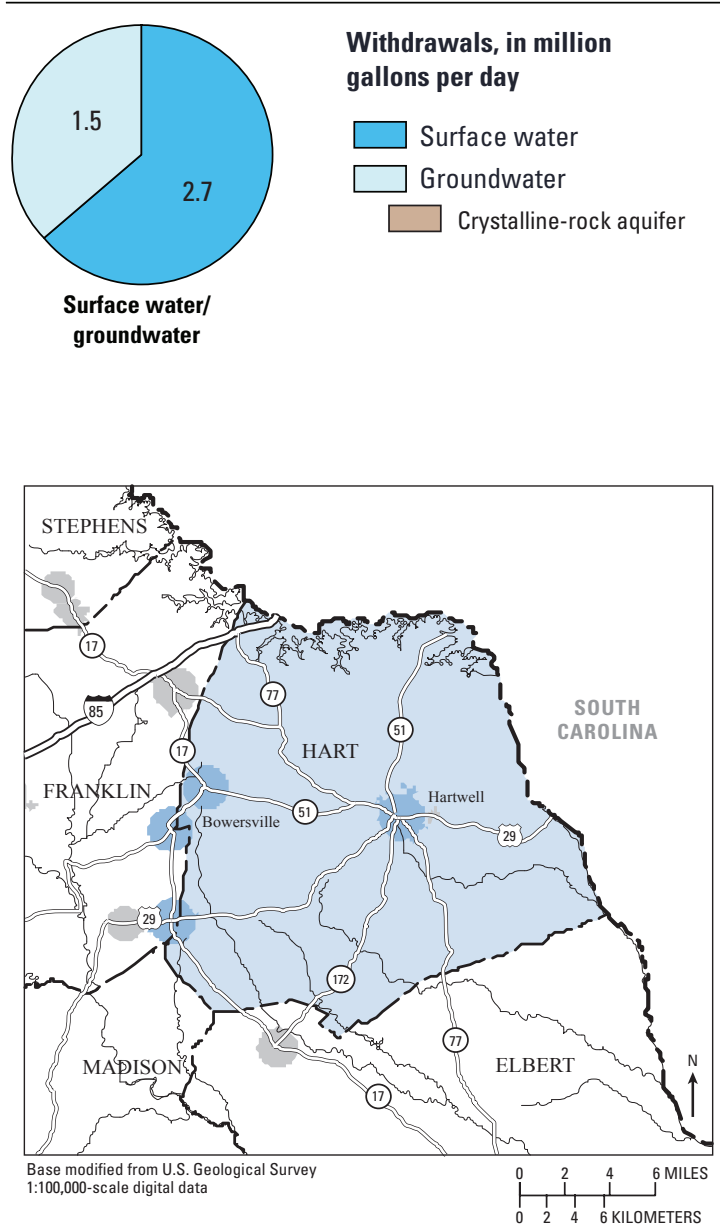

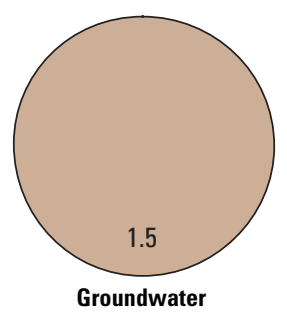

Public-Supply Deliveries' by Use Category

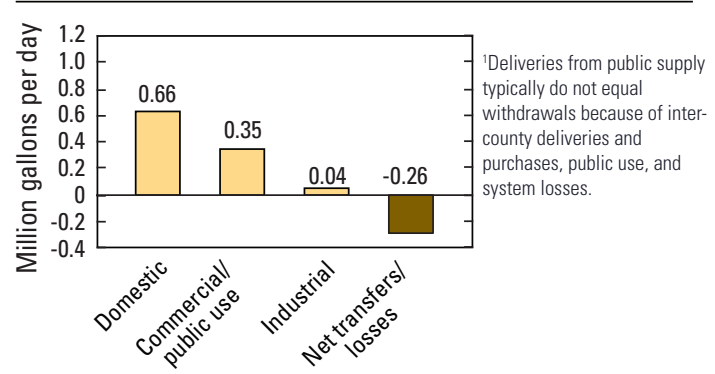

Surface-Water Withdrawals by Year

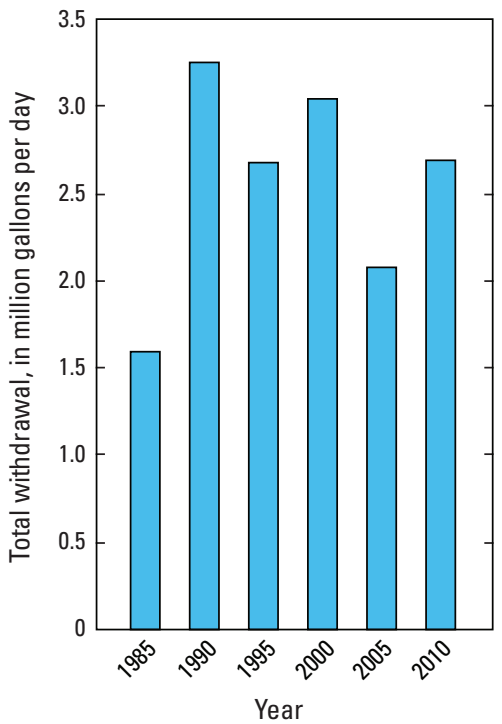

Groundwater Withdrawals by Year

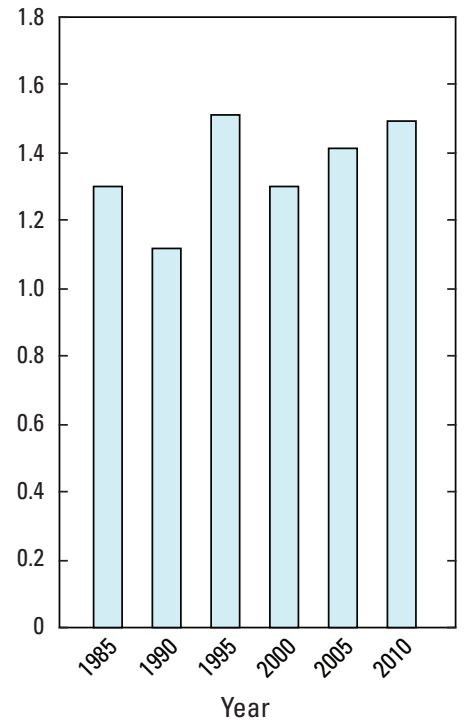




\section{HEARD COUNTY}

Population

Population served by public supply-Groundwater

Population served by public supply-Surface water $\quad 7,530$

Acres irrigated

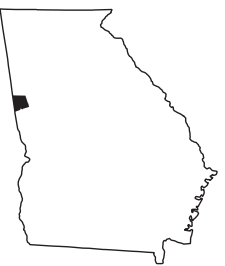

2010 WATER WITHDRAWALS AND ESTIMATED USE, IN MILLION GALLONS PER DAY

$[-$, not applicable; $<$, less than $]$

\begin{tabular}{|c|c|c|c|c|c|c|c|c|}
\hline \multirow{3}{*}{ Category } & \multicolumn{3}{|c|}{ Withdrawals } & \multirow{3}{*}{$\begin{array}{l}\text { Total } \\
\text { use }^{1}\end{array}$} & \multirow{3}{*}{$\begin{array}{l}\text { Surface- } \\
\text { water } \\
\text { returns } \\
\end{array}$} & \multirow{2}{*}{\multicolumn{3}{|c|}{$\begin{array}{l}\text { Withdrawals by Major Industrial Groups } \\
\text { [NAICS, North American Industrial Classification System code] }\end{array}$}} \\
\hline & \multirow{2}{*}{$\begin{array}{c}\text { Ground- } \\
\text { water }\end{array}$} & \multirow{2}{*}{$\begin{array}{c}\text { Surface } \\
\text { water }\end{array}$} & \multirow[b]{2}{*}{ Total } & & & & & \\
\hline & & & & & & NAICS & Groundwater & Surface water \\
\hline Public supply & 0.06 & 1.40 & 1.46 & - & - & & & - \\
\hline Domestic & 0.26 & 0.00 & 0.26 & 0.86 & 0.00 & None & - & - \\
\hline Commercial/public use & 0.00 & 0.00 & 0.00 & 0.55 & 0.00 & & & \\
\hline Industrial & 0.00 & 0.00 & 0.00 & $<0.01$ & 0.00 & & & \\
\hline Public-supply losses & - & - & - & 0.31 & - & & & \\
\hline Public wastewater treatment & - & - & - & - & 0.00 & Withdrawals by $\mathbf{M}$ & r Public Supp & \\
\hline Mining & 0.00 & 0.00 & 0.00 & 0.00 & 0.00 & Name & Groundwater & Surface water \\
\hline Irrigation-Crop & 0.00 & 0.00 & 0.00 & 0.00 & 0.00 & & & \\
\hline Irrigation-Golf course & 0.00 & 0.00 & 0.00 & 0.00 & 0.00 & Heard County Water & 0.00 & 1.40 \\
\hline Livestock/aquaculture & 0.00 & 0.16 & 0.16 & 0.16 & 0.00 & Authority & & \\
\hline Thermoelectric power & 0.00 & 48.61 & 48.61 & 48.61 & 0.00 & City of Ephesus & 0.06 & 0.00 \\
\hline
\end{tabular}

'Total use is total withdrawal plus public supply deliveries and losses.

\section{Withdrawals by Source}
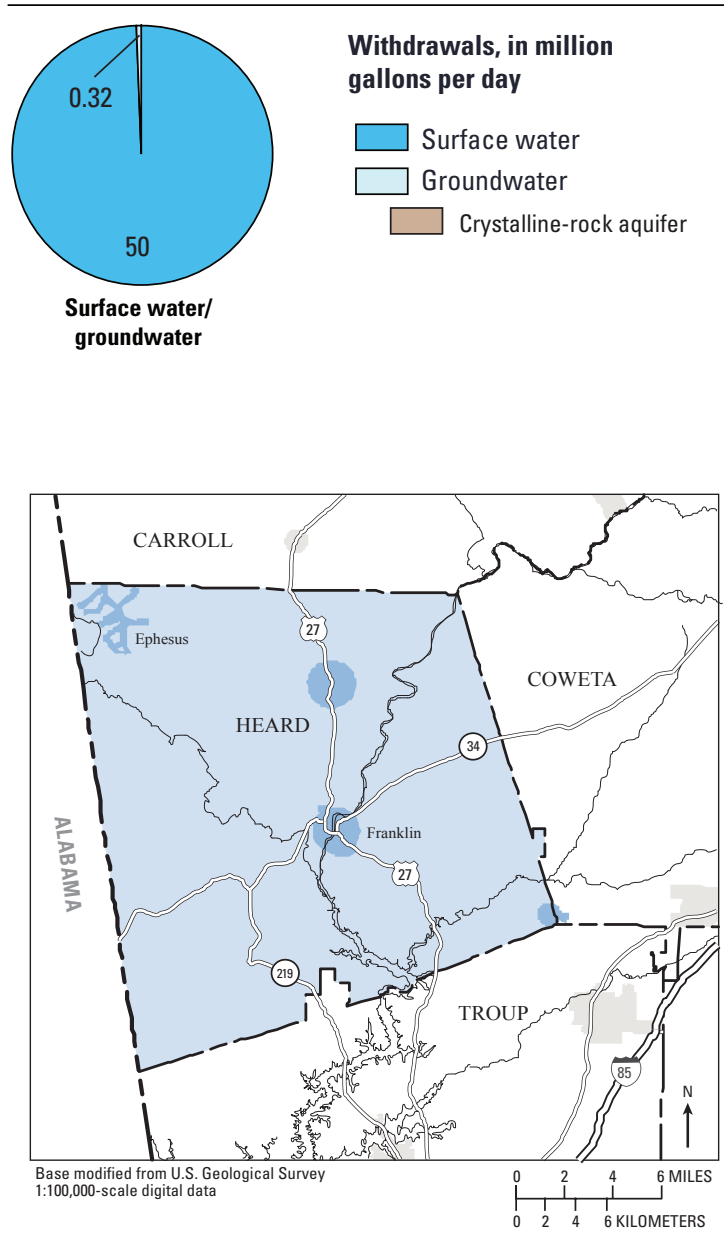

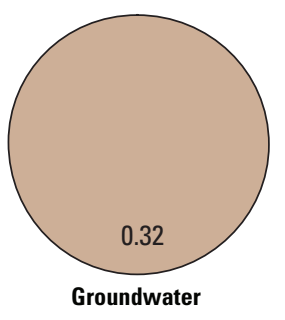

Public-Supply Deliveries by Use Category

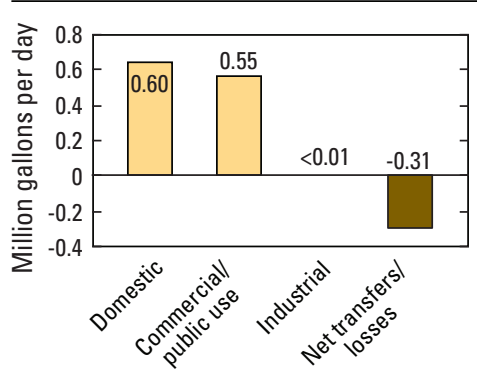

Surface-Water Withdrawals by Year

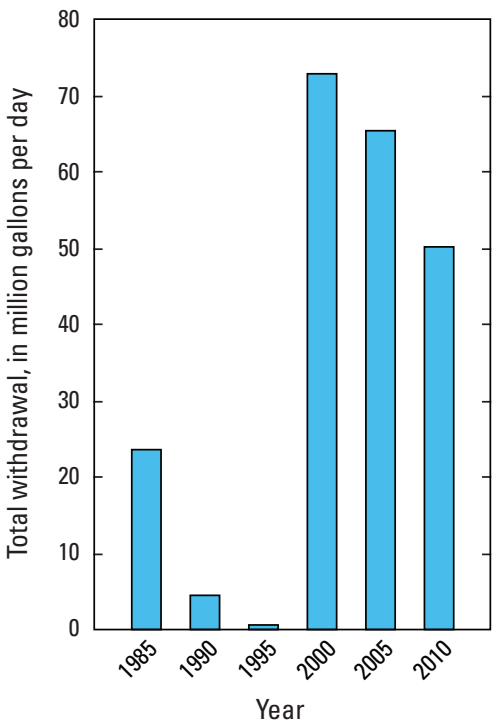

Groundwater Withdrawals by Year

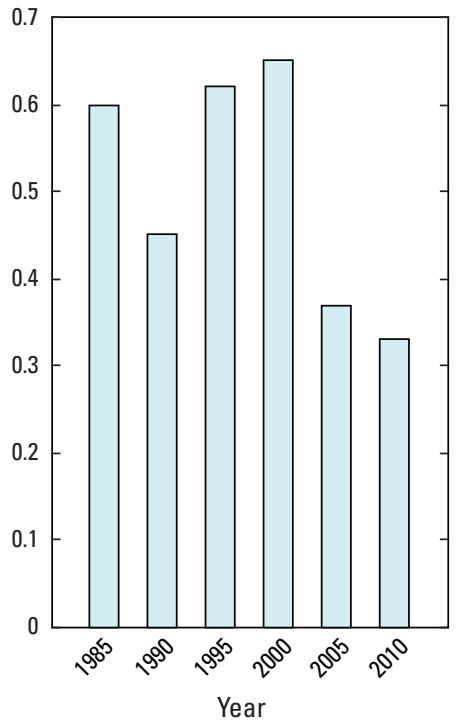




\section{HENRY COUNTY}

Population

203,922

Population served by public supply-Groundwater $\quad 5,080$

Population served by public supply-Surface water 172,000

Acres irrigated

880

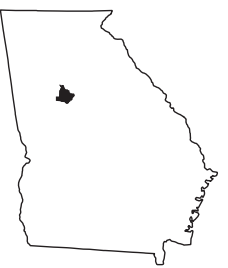

2010 WATER WITHDRAWALS AND ESTIMATED USE, IN MILLION GALLONS PER DAY

$[-$, not applicable; Mgal/d, million gallons per day]

\begin{tabular}{lccccc}
\hline \multirow{2}{*}{ Category } & \multicolumn{3}{c}{ Withdrawals } & & Surface- \\
\cline { 2 - 4 } & $\begin{array}{c}\text { Ground- } \\
\text { water }\end{array}$ & $\begin{array}{c}\text { Surface } \\
\text { water }\end{array}$ & Total & $\begin{array}{c}\text { Total } \\
\text { use }^{1}\end{array}$ & $\begin{array}{c}\text { water } \\
\text { returns }\end{array}$ \\
\hline Public supply & 0.58 & 36.90 & 37.48 & - & - \\
Domestic & 2.01 & 0.00 & 2.01 & 15.88 & 0.00 \\
Commercial/public use & 0.00 & 0.00 & 0.00 & 2.10 & 0.00 \\
Industrial & 0.00 & 0.00 & 0.00 & 0.49 & 0.00 \\
Public-supply losses & - & - & - & 2.80 & - \\
Public wastewater treatment & - & - & - & - & 22.65 \\
Mining & 0.19 & 0.00 & 0.19 & 0.19 & 0.44 \\
Irrigation-Crop & 0.00 & 0.08 & 0.08 & 0.08 & 0.00 \\
Irrigation-Golf course & 1.52 & 0.81 & 2.33 & 2.33 & 0.00 \\
Livestock/aquaculture & 0.06 & 0.80 & 0.86 & 0.86 & 0.00 \\
Thermoelectric power & 0.00 & 0.00 & 0.00 & 0.00 & 0.00 \\
\multicolumn{1}{c}{ TOTAL } & $\mathbf{4 . 3 6}$ & $\mathbf{3 8 . 5 9}$ & $\mathbf{4 2 . 9 5}$ & $\mathbf{2 4 . 7 3}$ & $\mathbf{2 3 . 0 9}$ \\
\hline
\end{tabular}

'Total use is total withdrawal plus public supply deliveries and losses. An average of $0.13 \mathrm{Mgal} / \mathrm{d}$ was delivered from DeKalb County, Georgia to Henry County, Georgia in 2010.

\section{Withdrawals by Source}

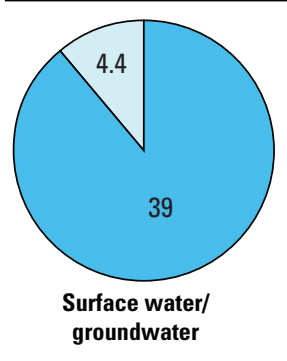

Withdrawals, in million gallons per day

Surface water
Groundwater
$\square$ Crystalline-rock aquifer

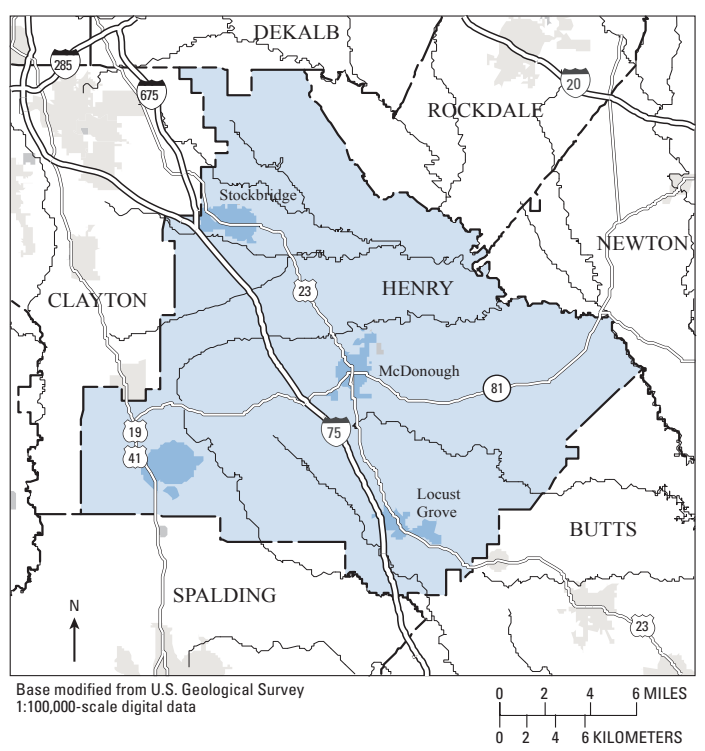

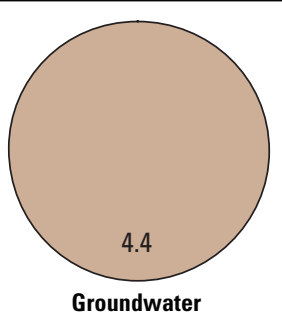

Groundwater
Withdrawals by Major Industrial Groups

[NAICS, North American Industrial Classification System code]

\begin{tabular}{lcc}
\hline NAICS & Groundwater & Surface water \\
\hline None & - & - \\
\hline
\end{tabular}

Withdrawals by Major Public Suppliers

\begin{tabular}{lcc}
\hline Name & Groundwater & Surface water \\
\hline $\begin{array}{l}\text { Henry County Water } \\
\text { Authority }\end{array}$ & 0.00 & 17.33 \\
Clayton County Water & 0.00 & ${ }^{1} 15.23$ \\
Authority & 0.00 & 1.15 \\
City of McDonough & 0.00 & 0.29 \\
City of Locust Grove & 0.28 & 0.00 \\
City of Stockbridge & & \\
\hline
\end{tabular}

'Withdrawn from Little Cotton Indian Creek in Henry County, Georgia and delivered to Harbin Reservoir (Edwin Blalock) in Henry County, Georgia. Withdrawals from Harbin

Reservoir are delivered and used in Clayton County, Georgia.

Public-Supply Deliveries ${ }^{1}$ by Use Category

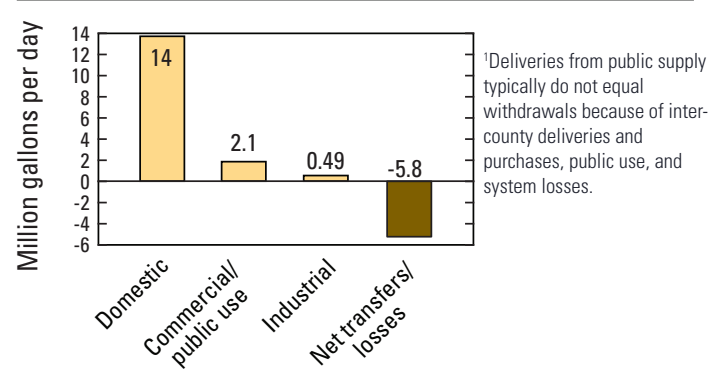

Surface-Water Withdrawals by Year

Groundwater Withdrawals by Year
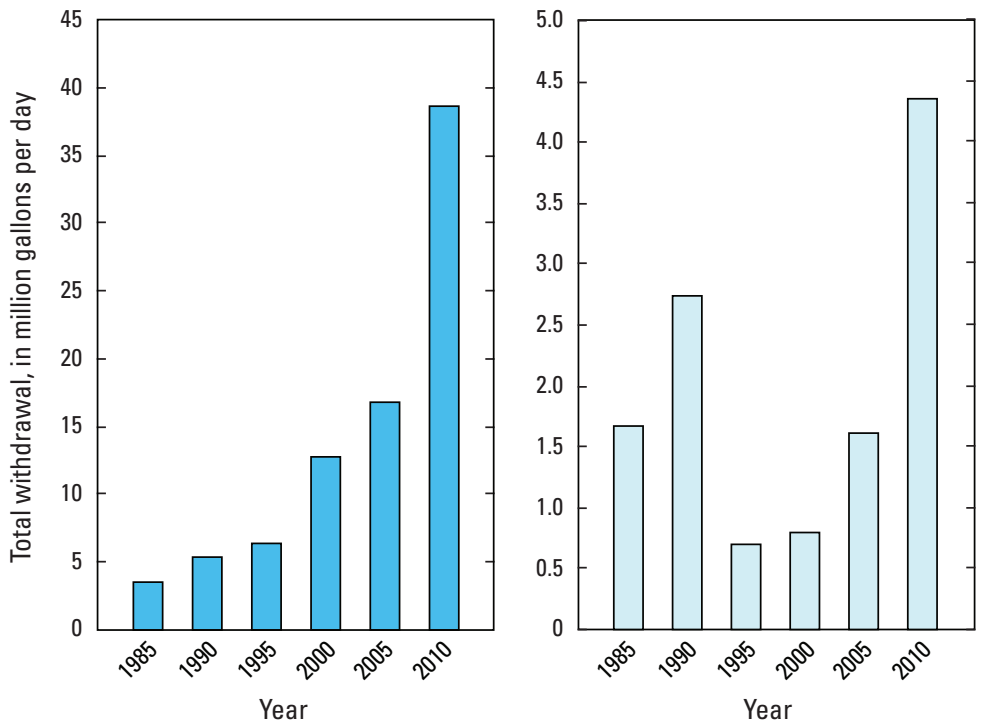


\section{HOUSTON COUNTY}

Population

139,900

Population served by public supply-Groundwater $\quad 137,570$

Population served by public supply-Surface water 0

Acres irrigated

8,910

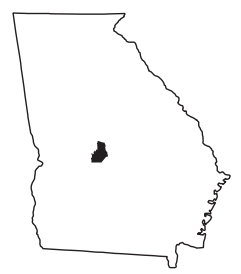

2010 WATER WITHDRAWALS AND ESTIMATED USE, IN MILLION GALLONS PER DAY

[-, not applicable; Mgal/d, million gallons per day]

\begin{tabular}{|c|c|c|c|c|c|c|c|c|}
\hline \multirow{3}{*}{ Category } & \multicolumn{3}{|c|}{ Withdrawals } & \multirow{3}{*}{$\begin{array}{l}\text { Total } \\
\text { use }^{1}\end{array}$} & \multirow{3}{*}{$\begin{array}{l}\text { Surface- } \\
\text { water } \\
\text { returns } \\
\end{array}$} & \multirow{2}{*}{\multicolumn{3}{|c|}{$\begin{array}{l}\text { Withdrawals by Major Industrial Groups } \\
\text { [NAICS, North American Industrial Classification System code] }\end{array}$}} \\
\hline & \multirow{2}{*}{$\begin{array}{c}\text { Ground- } \\
\text { water }\end{array}$} & \multirow{2}{*}{$\begin{array}{c}\text { Surface } \\
\text { water }\end{array}$} & \multirow[b]{2}{*}{ Total } & & & & & \\
\hline & & & & & & NAICS & Groundwater & Surface water \\
\hline Public supply & 23.76 & 0.00 & 23.76 & - & - & 311-Food & 2.64 & 0.00 \\
\hline Domestic & 0.17 & 0.00 & 0.17 & 14.64 & 0.00 & 327 -Stone, clay & 0.24 & 0.00 \\
\hline $\begin{array}{l}\text { Commercial/public use } \\
\text { Industrial }\end{array}$ & $\begin{array}{l}0.00 \\
2.96\end{array}$ & $\begin{array}{l}0.00 \\
0.00\end{array}$ & $\begin{array}{l}0.00 \\
2.96\end{array}$ & $\begin{array}{l}2.56 \\
4.11\end{array}$ & $\begin{array}{l}0.00 \\
1.16\end{array}$ & & & \\
\hline Public-supply losses & - & - & - & 3.65 & - & Withdrawals by Majo & r Public Supp & \\
\hline Public wastewater treatment & - & - & - & - & $\begin{array}{r}10.32 \\
0.00\end{array}$ & Name & Groundwater & Surface water \\
\hline $\begin{array}{l}\text { Mining } \\
\text { Irrigation-Crop }\end{array}$ & $\begin{array}{l}0.01 \\
4.87\end{array}$ & $\begin{array}{l}0.01 \\
0.19\end{array}$ & $\begin{array}{l}0.02 \\
5.06\end{array}$ & $\begin{array}{l}0.02 \\
5.06\end{array}$ & 0.00 & Houston County & 10.62 & 0.00 \\
\hline Irrigation-Golf course & 0.55 & 0.34 & 0.89 & 0.89 & 0.00 & City of Warner Robins & 8.01 & 0.00 \\
\hline Livestock/aquaculture & 0.01 & 0.04 & 0.05 & 0.05 & 0.00 & City of Perry & 2.33 & 0.00 \\
\hline Thermoelectric power & 0.00 & 0.00 & 0.00 & 0.00 & 0.00 & USAF Robins Airbase & 1.55 & 0.00 \\
\hline TOTAL & 32.33 & 0.58 & 32.91 & 30.98 & 11.48 & City of Centerville & 1.11 & 0.00 \\
\hline
\end{tabular}

${ }^{1} T o t a l$ use is total withdrawal plus public supply deliveries and losses. An average of $0.88 \mathrm{Mgal} / \mathrm{d}$ included in the industrial category was water withdrawn for groundwater remediation in 2010. Domestic and commercial customers in Peach County, Georgia were served by

public water suppliers in Houston County, Georgia in 2010.

\section{Withdrawals by Source}
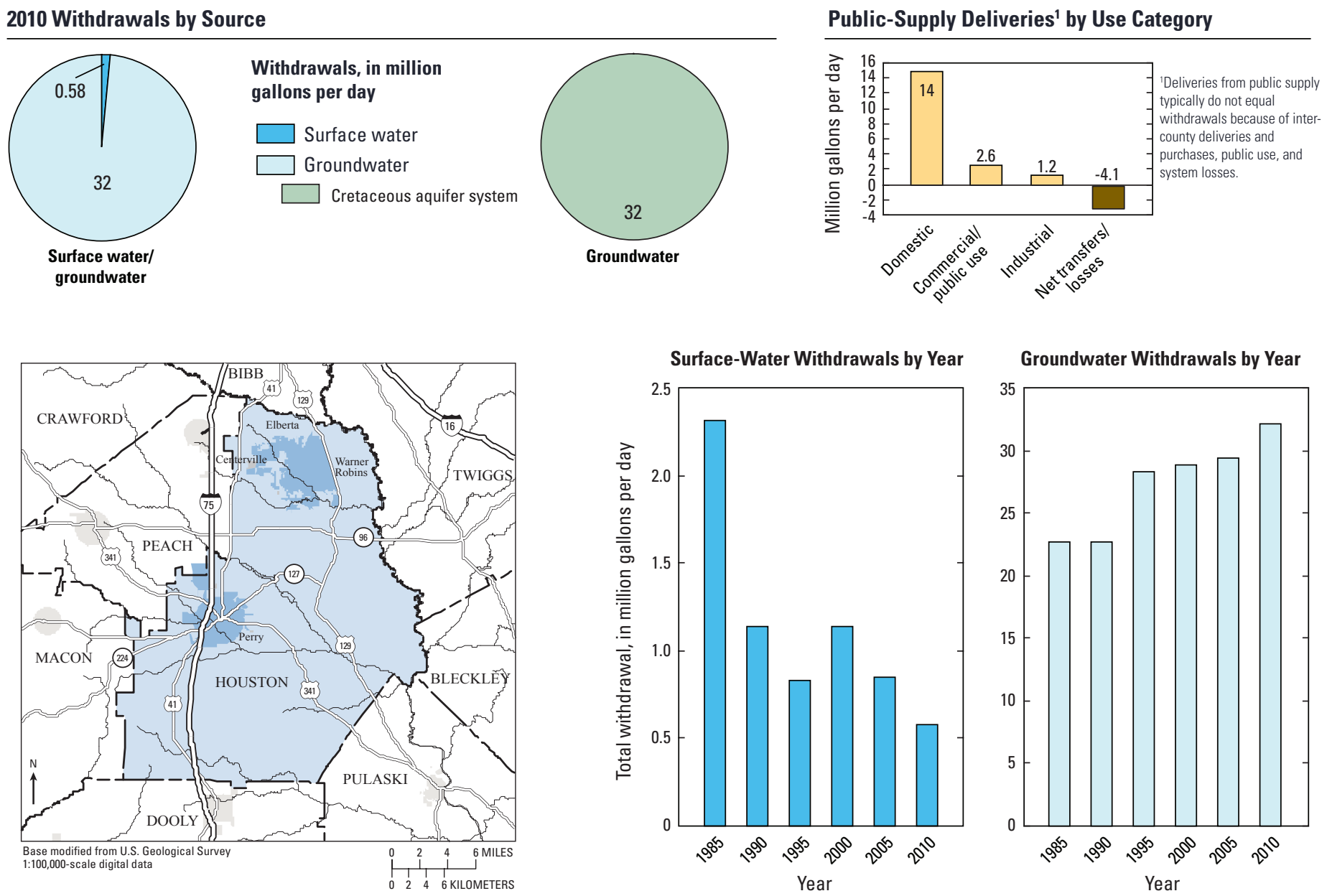
IRWIN COUNTY

Population

Population served by public supply_Groundwater $\quad 4,030$

Population served by public supply-Surface water 0

Acres irrigated

31,050

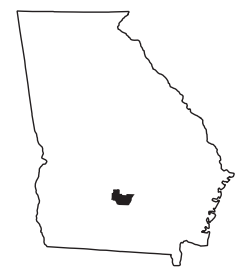

2010 WATER WITHDRAWALS AND ESTIMATED USE, IN MILLION GALLONS PER DAY

$[-$, not applicable $]$

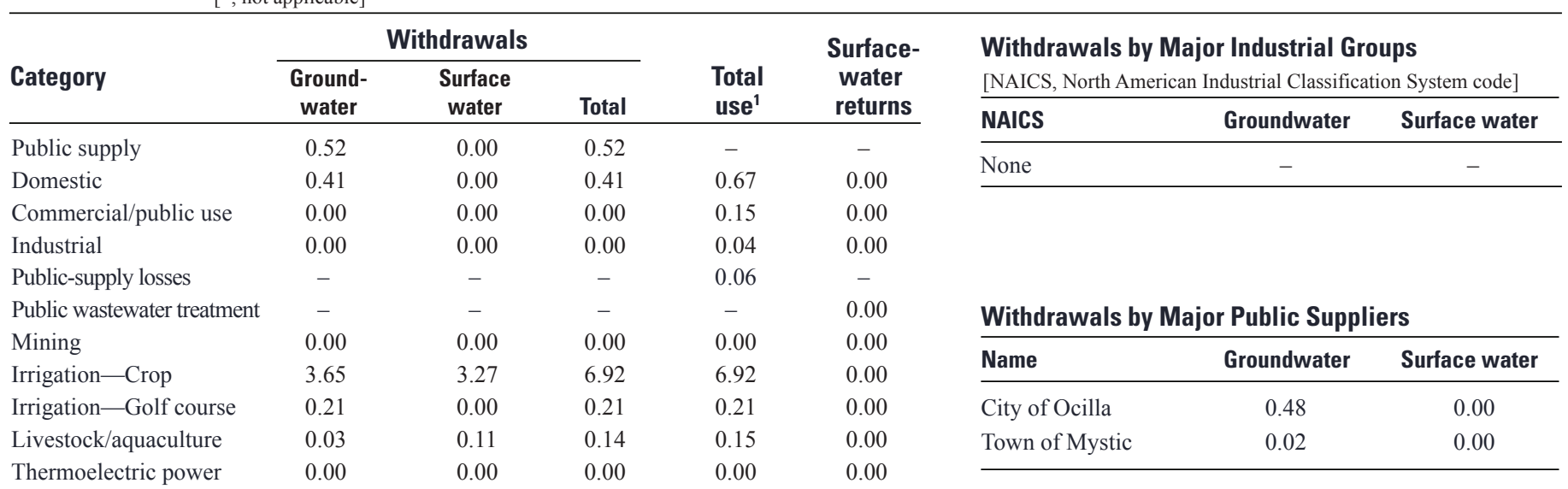

TOTAL

4.82

3.38

8.20

8.20

$\mathbf{0 . 0 0}$

'Total use is total withdrawal plus public supply deliveries and losses.

\section{Withdrawals by Source}
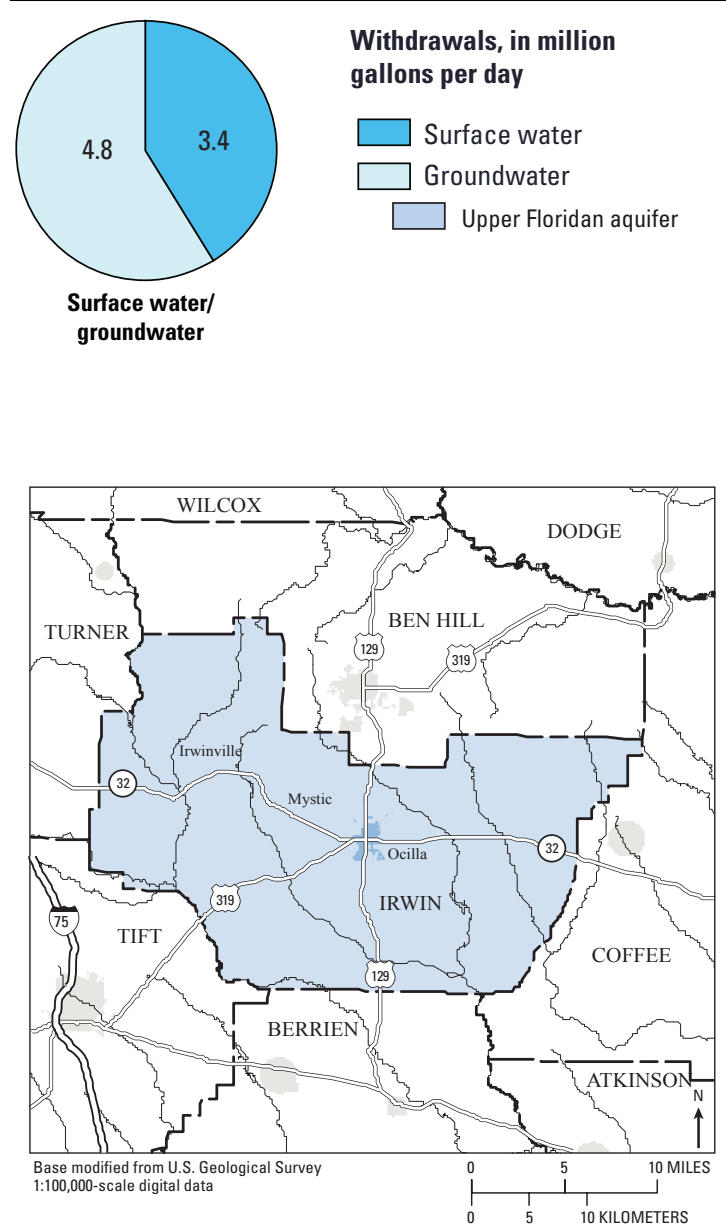

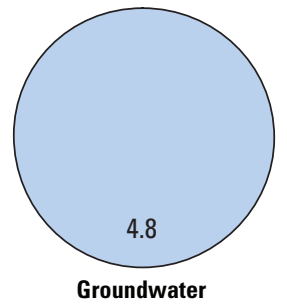

Public-Supply Deliveries by Use Category

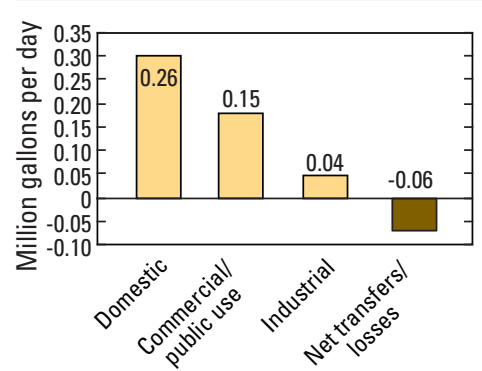

Surface-Water Withdrawals by Year

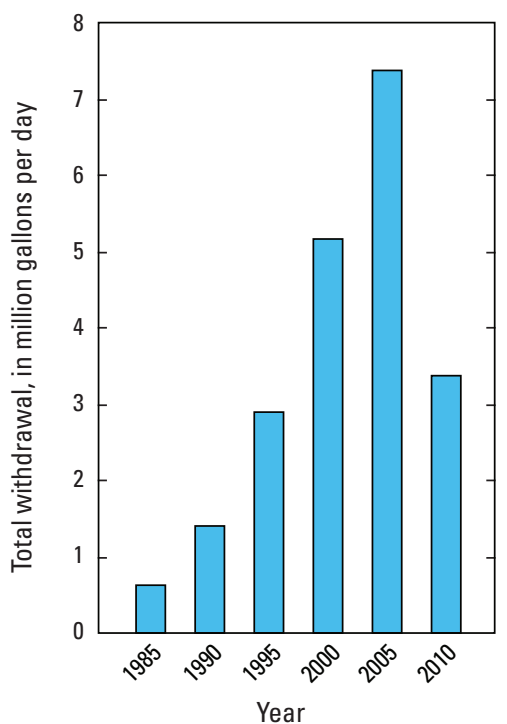

Groundwater Withdrawals by Year

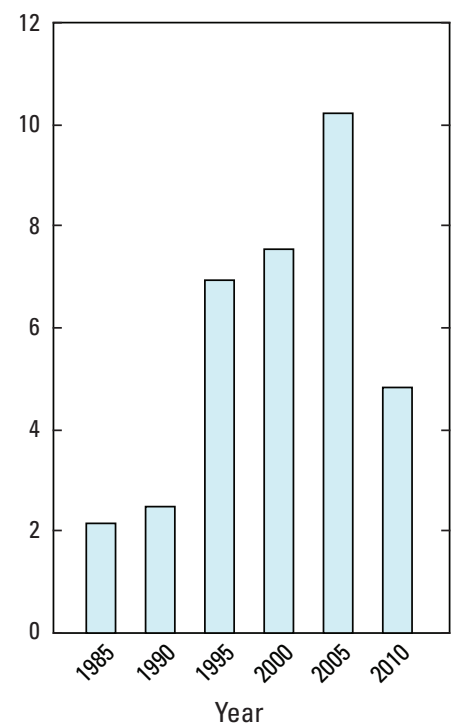


JACKSON COUNTY

Population

60,485

Population served by public supply-Groundwater $\quad 3,180$

Population served by public supply-Surface water $\quad 42,160$

Acres irrigated

310

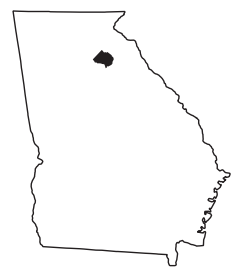

2010 WATER WITHDRAWALS AND ESTIMATED USE, IN MILLION GALLONS PER DAY

[-, not applicable; Mgal/d, million gallons per day]

\begin{tabular}{|c|c|c|c|c|c|c|c|c|}
\hline \multirow{3}{*}{ Category } & \multicolumn{3}{|c|}{ Withdrawals } & \multirow{3}{*}{$\begin{array}{l}\text { Total } \\
\text { use }^{1}\end{array}$} & \multirow{3}{*}{$\begin{array}{l}\text { Surface- } \\
\text { water } \\
\text { returns } \\
\end{array}$} & \multirow{2}{*}{\multicolumn{3}{|c|}{$\begin{array}{l}\text { Withdrawals by Major Industrial Groups } \\
\text { [NAICS, North American Industrial Classification System code] }\end{array}$}} \\
\hline & \multirow{2}{*}{$\begin{array}{c}\text { Ground- } \\
\text { water }\end{array}$} & \multirow{2}{*}{$\begin{array}{c}\text { Surface } \\
\text { water }\end{array}$} & \multirow[b]{2}{*}{ Total } & & & & & \\
\hline & & & & & & NAICS & Groundwater & Surface water \\
\hline Public supply & 0.31 & 10.52 & 10.83 & & - & 311 -Food products & 0.48 & 0.00 \\
\hline Domestic & 1.14 & 0.00 & 1.14 & 4.75 & 0.00 & & 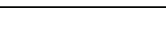 & \\
\hline Commercial/public use & 0.00 & 0.00 & 0.00 & 1.21 & 0.00 & & & \\
\hline Industrial & 0.48 & 0.00 & 0.48 & 0.93 & 0.00 & & & \\
\hline Public-supply losses & - & - & - & 1.01 & - & & & \\
\hline Public wastewater treatment & - & - & - & - & 1.93 & Withdrawals by Ma & Public Supp & \\
\hline Mining & 0.09 & 0.00 & 0.09 & 0.09 & 0.00 & Name & Groundwater & Surface water \\
\hline Irrigation-Crop & 0.09 & 0.03 & 0.12 & 0.12 & 0.00 & Upper Oconee Basin & & \\
\hline Irrigation-Golf course & 0.00 & 0.31 & 0.31 & 0.31 & 0.00 & Water Authority & 0.00 & 9.40 \\
\hline Livestock/aquaculture & 0.25 & 3.38 & 3.63 & 3.63 & 0.00 & City of Jefferson & 0.00 & 1.12 \\
\hline Thermoelectric power & 0.00 & 0.00 & 0.00 & 0.00 & 0.00 & City of Braselton & 0.13 & 0.00 \\
\hline TOTAL & 2.36 & 14.24 & 16.60 & 12.05 & 1.93 & & & \\
\hline
\end{tabular}

Total use is total withdrawal plus public supply deliveries and losses. An average of $1.0 \mathrm{Mgal} / \mathrm{d}$ was delivered to Clarke County, Georgia,

$1.65 \mathrm{Mgal} / \mathrm{d}$ to Barrow County, Georgia, $2.1 \mathrm{Mgal} / \mathrm{d}$ to Oconee County, Georgia, and $0.06 \mathrm{Mgal} / \mathrm{d}$ was delivered to Banks County, Georgia

in 2010. An average of $0.02 \mathrm{Mgal} / \mathrm{d}$ was delivered from Gwinnett County, Georgia to Jackson County in 2010.

\section{Withdrawals by Source}

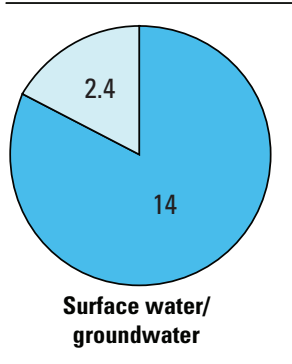

Withdrawals, in million gallons per day

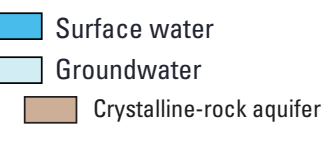

Surface water/

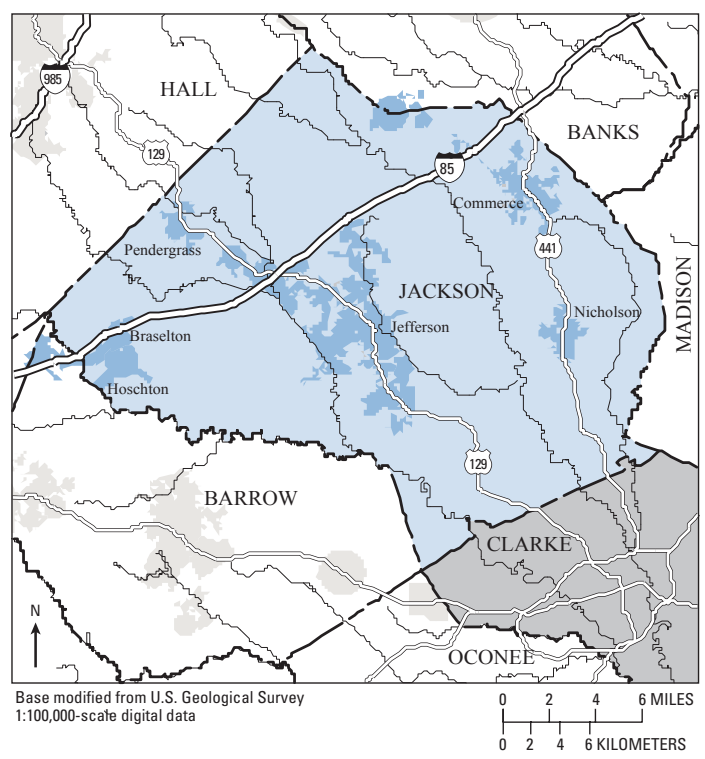

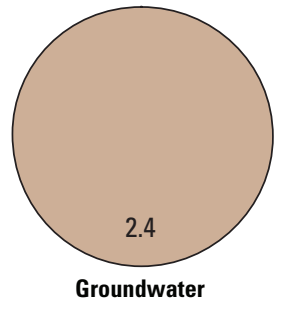

Public-Supply Deliveries' by Use Category

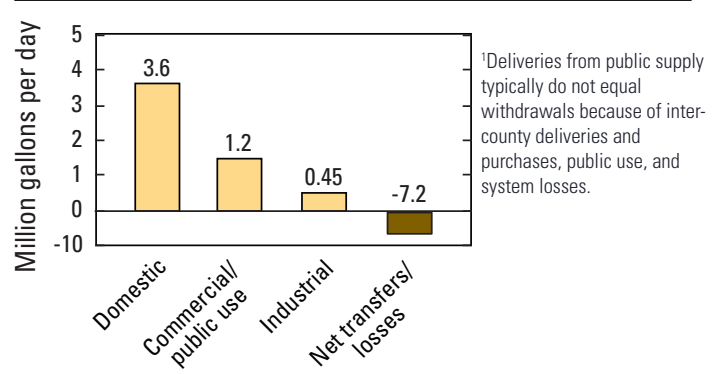

Surface-Water Withdrawals by Year

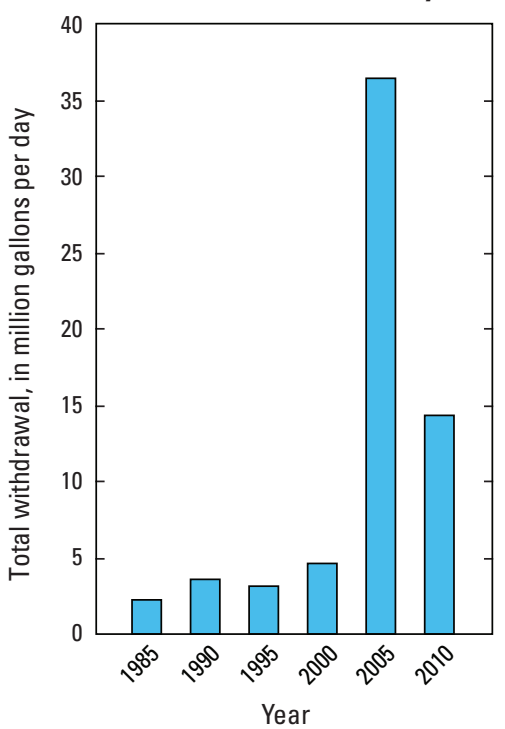

Groundwater Withdrawals by Year

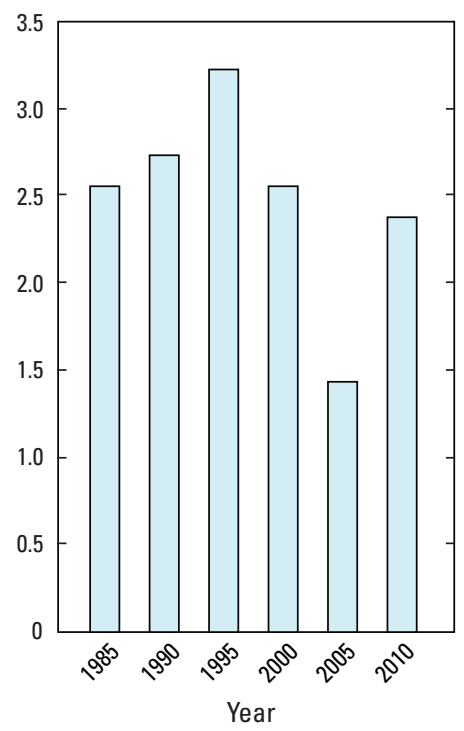




\section{JASPER COUNTY}

Population

13,900

Population served by public supply-Groundwater $\quad 3,770$

Population served by public supply-Surface water $\quad 2,930$

Acres irrigated

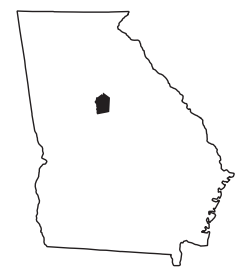

2010 WATER WITHDRAWALS AND ESTIMATED USE, IN MILLION GALLONS PER DAY

$[-$, not applicable; Mgal/d, million gallons per day $]$

\begin{tabular}{|c|c|c|c|c|c|c|c|c|}
\hline \multirow{3}{*}{ Category } & \multicolumn{3}{|c|}{ Withdrawals } & \multirow{3}{*}{$\begin{array}{l}\text { Total } \\
\text { use }^{1}\end{array}$} & \multirow{3}{*}{$\begin{array}{l}\text { Surface- } \\
\text { water } \\
\text { returns } \\
\end{array}$} & \multirow{2}{*}{\multicolumn{3}{|c|}{$\begin{array}{l}\text { Withdrawals by Major Industrial Groups } \\
\text { [NAICS, North American Industrial Classification System code] }\end{array}$}} \\
\hline & \multirow{2}{*}{$\begin{array}{c}\text { Ground- } \\
\text { water }\end{array}$} & \multirow{2}{*}{$\begin{array}{c}\text { Surface } \\
\text { water }\end{array}$} & \multirow[b]{2}{*}{ Total } & & & & & \\
\hline & & & & & & NAICS & Groundwater & Surface water \\
\hline Public supply & 0.42 & 0.46 & 0.88 & - & - & None & & - \\
\hline Domestic & 0.54 & 0.00 & 0.54 & 1.14 & 0.00 & None & - & - \\
\hline Commercial/public use & 0.00 & 0.00 & 0.00 & 0.20 & 0.00 & & & \\
\hline Industrial & 0.00 & 0.80 & 0.80 & 0.84 & 0.00 & & & \\
\hline Public-supply losses & - & - & - & 0.11 & - & & & \\
\hline Public wastewater treatment & - & - & - & - & 0.23 & Withdrawals by Ma & r Public Supp & \\
\hline Mining & 0.04 & 0.00 & 0.04 & 0.04 & 0.00 & Name & Groundwater & Surface water \\
\hline Irrigation-Crop & 0.40 & 0.06 & 0.46 & 0.46 & 0.00 & & & \\
\hline Irrigation-Golf course & 0.00 & 0.00 & 0.00 & 0.00 & 0.00 & City of Monticello & 0.19 & 0.46 \\
\hline Livestock/aquaculture & 0.00 & 0.18 & 0.18 & 0.18 & 0.00 & Turtle Cove Property & 0.18 & 0.00 \\
\hline Thermoelectric power & 0.00 & 0.00 & 0.00 & 0.00 & 0.00 & Owners Association & 0.10 & 0.00 \\
\hline TOTAL & 1.40 & 1.50 & 2.90 & 2.97 & 0.23 & & & \\
\hline
\end{tabular}

'Total use is total withdrawal plus public supply deliveries and losses. An average of $0.07 \mathrm{Mgal} / \mathrm{d}$ was delivered from Newton County,

Georgia to Jasper County in 2010.

\section{Withdrawals by Source}
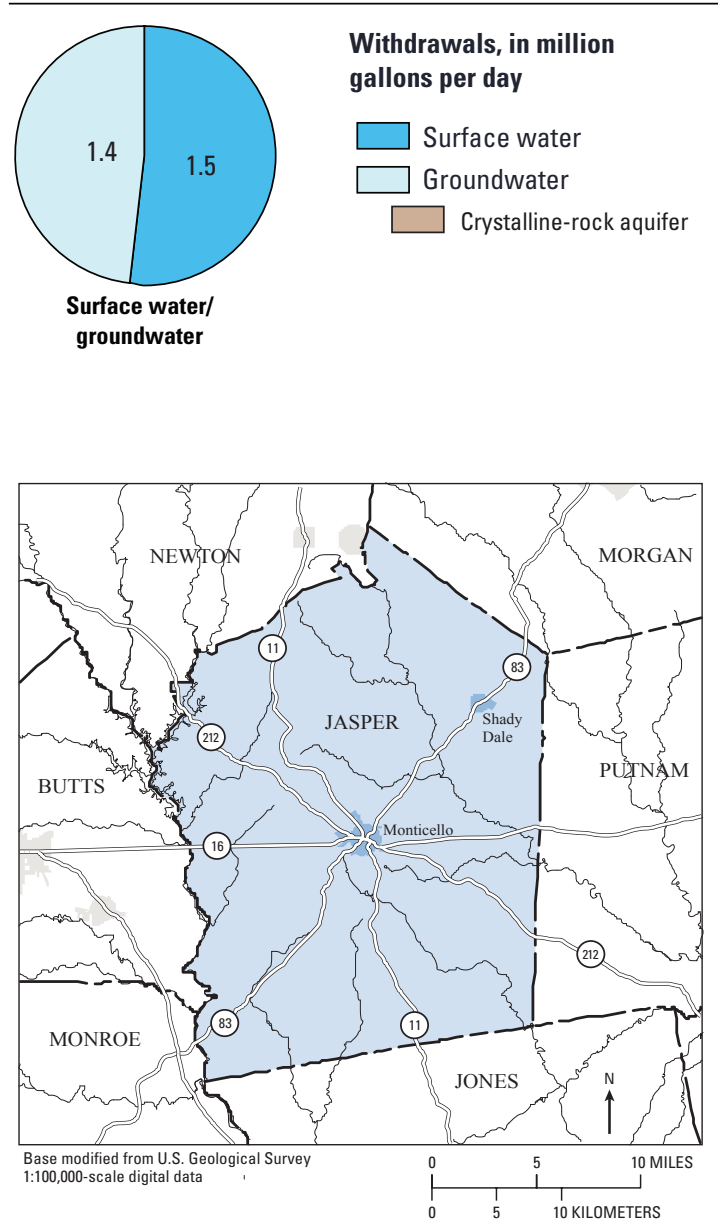

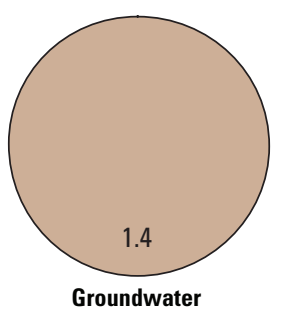

Public-Supply Deliveries ${ }^{1}$ by Use Category

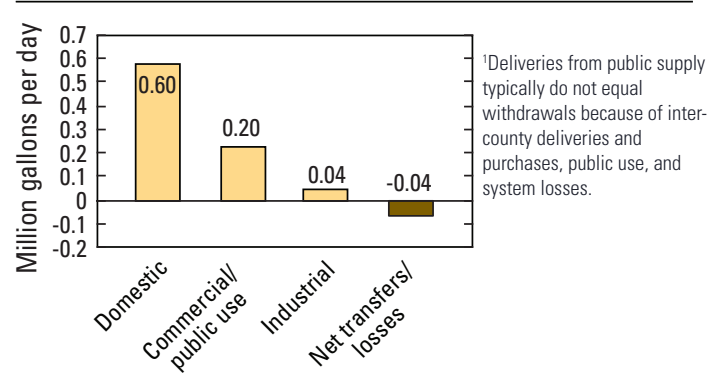

Surface-Water Withdrawals by Year

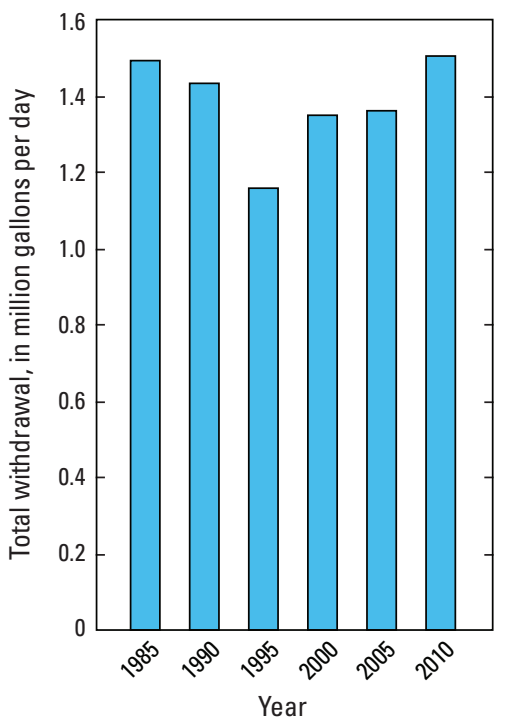

Groundwater Withdrawals by Year

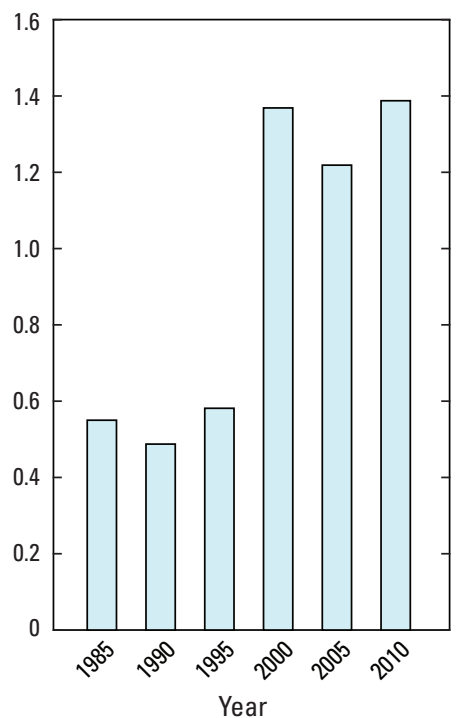




\section{JEFF DAVIS COUNTY}

Population

15,068

6,960

Population served by public supply-Groundwater

Population served by public supply-Surface water Acres irrigated
0
9,838

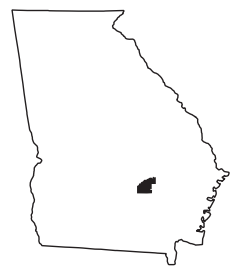

2010 WATER WITHDRAWALS AND ESTIMATED USE, IN MILLION GALLONS PER DAY

$[-$, not applicable $]$

\begin{tabular}{|c|c|c|c|c|c|c|c|c|}
\hline \multirow{3}{*}{ Category } & \multicolumn{3}{|c|}{ Withdrawals } & \multirow{3}{*}{$\begin{array}{l}\text { Total } \\
\text { use }^{1}\end{array}$} & \multirow{3}{*}{$\begin{array}{l}\text { Surface- } \\
\text { water } \\
\text { returns } \\
\end{array}$} & \multirow{2}{*}{\multicolumn{3}{|c|}{$\begin{array}{l}\text { Withdrawals by Major Industrial Groups } \\
\text { [NAICS, North American Industrial Classification System code] }\end{array}$}} \\
\hline & \multirow{2}{*}{$\begin{array}{c}\text { Ground- } \\
\text { water }\end{array}$} & \multirow{2}{*}{$\begin{array}{c}\text { Surface } \\
\text { water }\end{array}$} & \multirow[b]{2}{*}{ Total } & & & & & \\
\hline & & & & & & NAICS & Groundwater & Surface water \\
\hline Public supply & 0.84 & 0.00 & 0.84 & \multirow{2}{*}{$\begin{array}{l}- \\
1.13\end{array}$} & \multirow{2}{*}{$\begin{array}{c}- \\
0.00\end{array}$} & \multirow{2}{*}{313 -Textiles } & \multirow{2}{*}{0.05} & \multirow{2}{*}{0.00} \\
\hline Domestic & 0.61 & 0.00 & 0.61 & & & & & \\
\hline Commercial/public use & 0.00 & 0.00 & 0.00 & 0.07 & 0.00 & \multirow{4}{*}{\multicolumn{3}{|c|}{ Withdrawals by Major Public Suppliers }} \\
\hline Industrial & 0.05 & 0.00 & 0.05 & 0.10 & 0.00 & & & \\
\hline Public-supply losses & - & - & - & 0.20 & - & & & \\
\hline Public wastewater treatment & - & - & - & - & 0.71 & & & \\
\hline $\begin{array}{l}\text { Mining } \\
\text { Irrigation-Crop }\end{array}$ & 0.00 & 0.00 & 0.00 & 0.00 & 0.00 & Name & Groundwater & Surface water \\
\hline Irrigation-Golf course & 0.05 & 0.00 & 0.05 & 0.05 & 0.00 & City of Hazelhurst & 0.80 & 0.00 \\
\hline Livestock/aquaculture & 0.27 & 0.36 & 0.63 & 0.63 & 0.00 & & & \\
\hline Thermoelectric power & 0.00 & 0.00 & 0.00 & 0.00 & 0.00 & & & \\
\hline TOTAL & 3.41 & 0.98 & 4.39 & 4.39 & 0.71 & & & \\
\hline
\end{tabular}

\section{Withdrawals by Source}
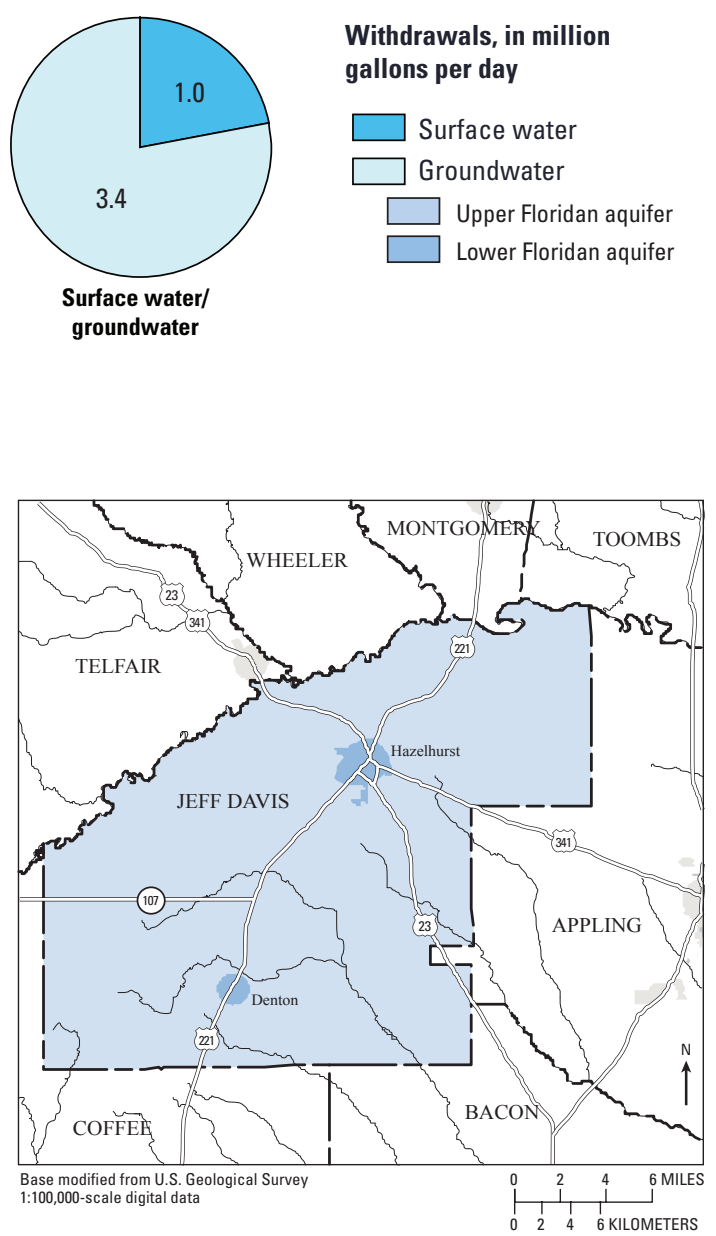

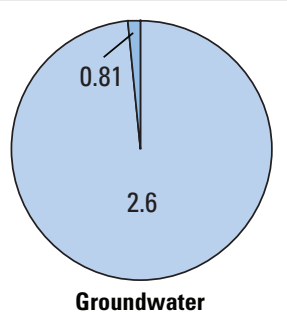

Public-Supply Deliveries by Use Category

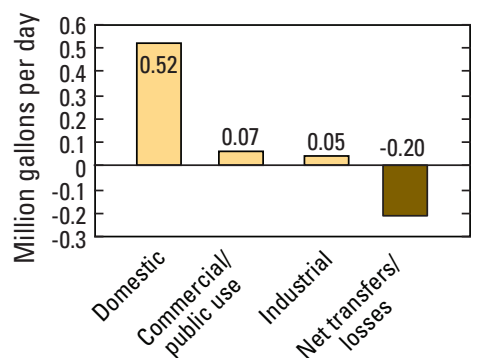

Surface-Water Withdrawals by Year

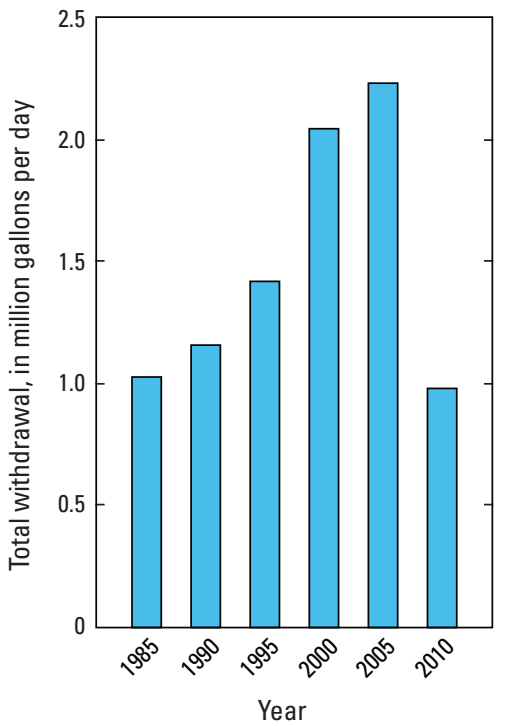

Groundwater Withdrawals by Year

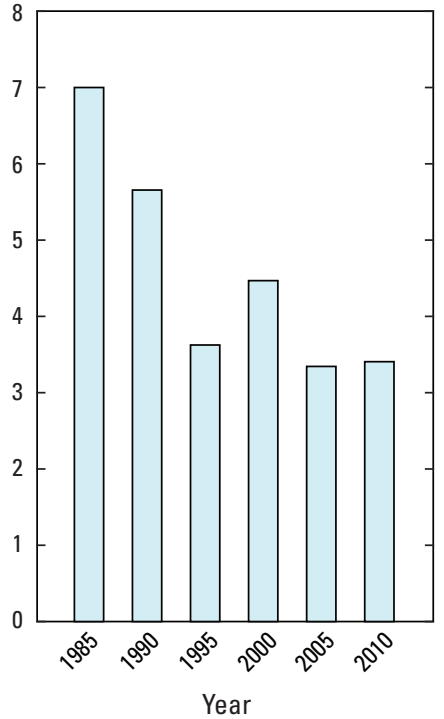




\section{JEFFERSON COUNTY}

Population

16,930

Population served by public supply_-Groundwater $\quad 9,060$

Population served by public supply-Surface water 0

Acres irrigated

16,015

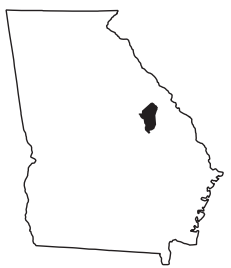

2010 WATER WITHDRAWALS AND ESTIMATED USE, IN MILLION GALLONS PER DAY

$[-$, not applicable $]$

\begin{tabular}{|c|c|c|c|c|c|c|c|c|}
\hline \multirow{3}{*}{ Category } & \multicolumn{3}{|c|}{ Withdrawals } & \multirow{3}{*}{$\begin{array}{l}\text { Total } \\
\text { use }^{1}\end{array}$} & \multirow{3}{*}{$\begin{array}{l}\text { Surface- } \\
\text { water } \\
\text { returns } \\
\end{array}$} & \multirow{2}{*}{\multicolumn{3}{|c|}{$\begin{array}{l}\text { Withdrawals by Major Industrial Groups } \\
\text { [NAICS, North American Industrial Classification System code] }\end{array}$}} \\
\hline & \multirow{2}{*}{$\begin{array}{c}\text { Ground- } \\
\text { water }\end{array}$} & \multirow{2}{*}{$\begin{array}{c}\text { Surface } \\
\text { water }\end{array}$} & \multirow[b]{2}{*}{ Total } & & & & & \\
\hline & & & & & & NAICS G & Groundwater & Surface water \\
\hline Public supply & 1.35 & 0.00 & 1.35 & - & - & 212-Kaolin and ball clay & $y \quad 4.14$ & 3.17 \\
\hline Domestic & 0.59 & 0.00 & 0.59 & 1.50 & 0.00 & & & \\
\hline Commercial/public use & 0.00 & 0.00 & 0.00 & 0.27 & 0.00 & & & \\
\hline Industrial & 4.14 & 3.17 & 7.31 & 7.34 & 0.00 & & & \\
\hline Public-supply losses & - & - & - & 0.14 & - & & & \\
\hline Public wastewater treatment & - & - & - & - & 1.01 & & & \\
\hline Mining & 0.00 & 0.00 & 0.00 & 0.00 & 2.07 & Withdrawals by Major & Public Supp & \\
\hline Irrigation-Crop & 7.01 & 0.00 & 7.01 & 7.01 & 0.00 & Name & Groundwater & Surface water \\
\hline Irrigation-Golf course & 0.04 & 0.00 & 0.04 & 0.04 & 0.00 & & & \\
\hline Livestock/aquaculture & 0.53 & 0.21 & 0.74 & 0.74 & 0.00 & City of Louisville & 0.71 & 0.00 \\
\hline Thermoelectric power & 0.00 & 0.00 & 0.00 & 0.00 & 0.00 & City of Wadley & 0.37 & 0.00 \\
\hline TOTAL & 13.66 & 3.38 & 17.04 & 17.04 & 3.08 & City of Wrens & 0.17 & 0.00 \\
\hline
\end{tabular}

'Total use is total withdrawal plus public supply deliveries and losses.

\section{Withdrawals by Source}

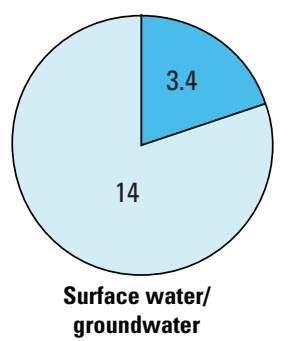

\section{Withdrawals, in million} gallons per day

$\square$ Surface water
$\square$ Groundwater
$\square$ Upper Floridan aquifer
$\square$ Cretaceous aquifer system

groundwater

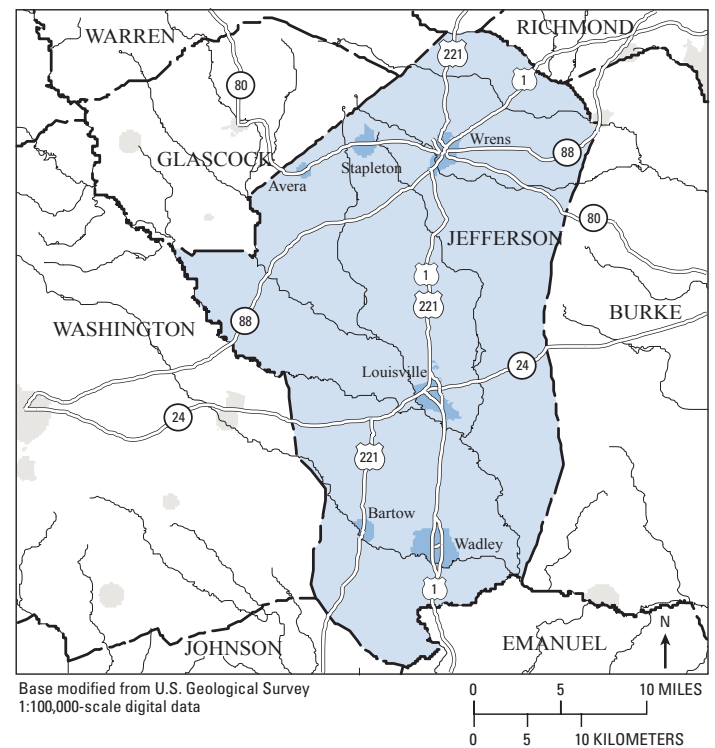

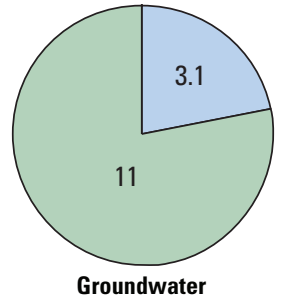

Public-Supply Deliveries by Use Category

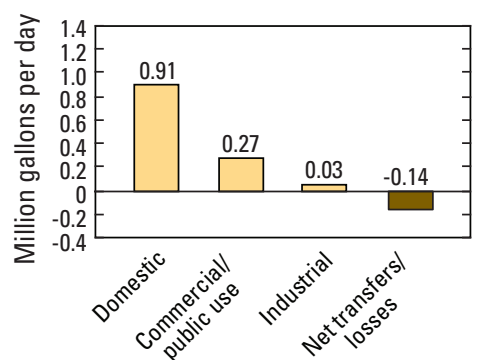

Surface-Water Withdrawals by Year

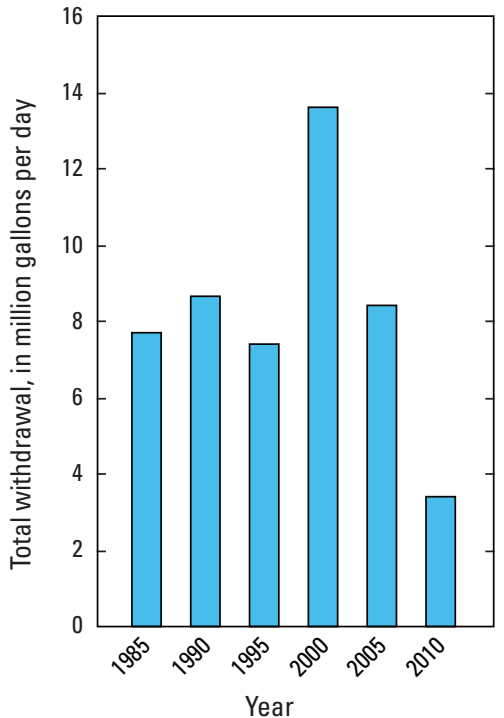

Groundwater Withdrawals by Year

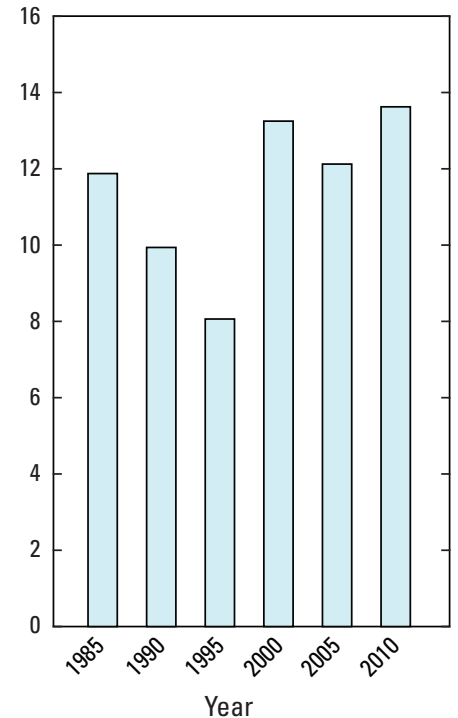




\section{JENKINS COUNTY}

Population

8,340

Population served by public supply-Groundwater

Population served by public supply-Surface water Acres irrigated
3,340

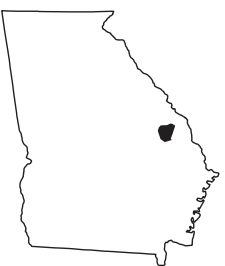

2010 WATER WITHDRAWALS AND ESTIMATED USE, IN MILLION GALLONS PER DAY

$[-$, not applicable $]$

\begin{tabular}{|c|c|c|c|c|c|c|c|c|}
\hline \multirow{3}{*}{ Category } & \multicolumn{3}{|c|}{ Withdrawals } & \multirow{3}{*}{$\begin{array}{l}\text { Total } \\
\text { use }^{1}\end{array}$} & \multirow{3}{*}{$\begin{array}{c}\text { Surface- } \\
\text { water } \\
\text { returns } \\
\end{array}$} & \multirow{2}{*}{\multicolumn{3}{|c|}{$\begin{array}{l}\text { Withdrawals by Major Industrial Groups } \\
\text { [NAICS, North American Industrial Classification System code] }\end{array}$}} \\
\hline & \multirow{2}{*}{$\begin{array}{c}\text { Ground- } \\
\text { water }\end{array}$} & \multirow{2}{*}{$\begin{array}{c}\text { Surface } \\
\text { water }\end{array}$} & \multirow[b]{2}{*}{ Total } & & & & & \\
\hline & & & & & & NAICS & Groundwater & Surface water \\
\hline Public supply & 0.35 & 0.00 & 0.35 & - & - & None & - & - \\
\hline Domestic & 0.38 & 0.00 & 0.38 & 0.60 & 0.00 & & & \\
\hline Commercial/public use & 0.00 & 0.00 & 0.00 & 0.06 & 0.00 & & & \\
\hline Industrial & 0.00 & 0.00 & 0.00 & 0.00 & 0.00 & & & \\
\hline Public-supply losses & - & - & - & 0.07 & - & & & \\
\hline Public wastewater treatment & - & - & - & - & 0.72 & Withdrawals & Public Supp & \\
\hline $\begin{array}{l}\text { Mining } \\
\text { Irrigation-Crop }\end{array}$ & $\begin{array}{l}0.00 \\
1.30\end{array}$ & $\begin{array}{l}0.00 \\
0.73\end{array}$ & $\begin{array}{l}0.00 \\
2.03\end{array}$ & $\begin{array}{l}0.00 \\
2.03\end{array}$ & $\begin{array}{l}0.00 \\
0.00\end{array}$ & Name & Groundwater & Surface water \\
\hline Irrigation-Golf course & 0.00 & 0.00 & 0.00 & 0.00 & 0.00 & City of Millen & 0.34 & 0.00 \\
\hline Livestock/aquaculture & 0.12 & 0.04 & 0.16 & 0.16 & 0.00 & & & \\
\hline Thermoelectric power & 0.00 & 0.00 & 0.00 & 0.00 & 0.00 & & & \\
\hline TOTAL & 2.15 & 0.77 & 2.92 & 2.92 & 0.72 & & & \\
\hline
\end{tabular}

'Total use is total withdrawal plus public supply deliveries and losses.

\section{Withdrawals by Source}

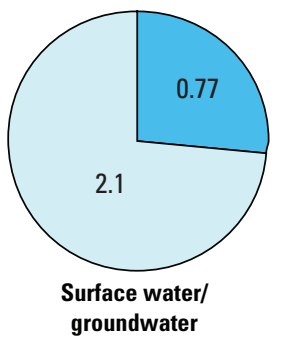

Withdrawals, in million gallons per day

$\square$ Surface water
$\square$ Groundwater
$\square$ Upper Floridan aquifer
$\square$ Cretaceous aquifer system

groundwater

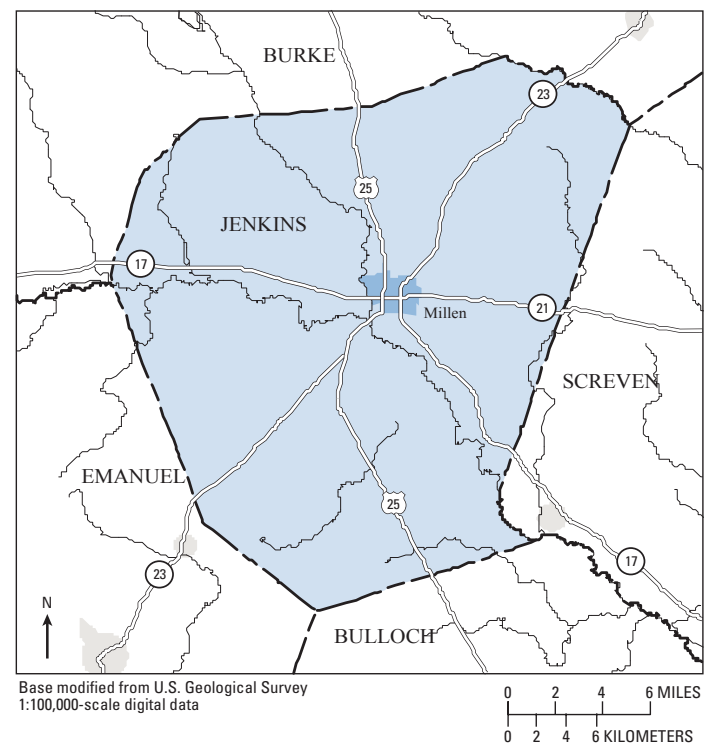

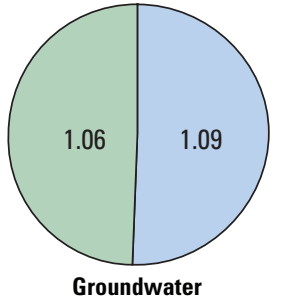

Public-Supply Deliveries by Use Category

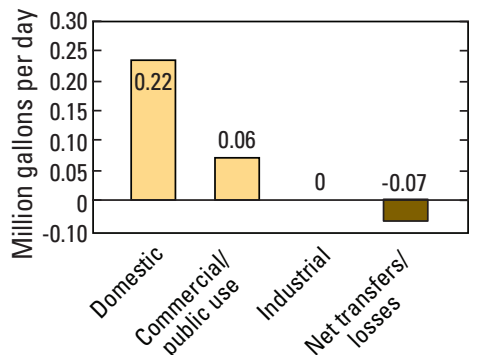

Surface-Water Withdrawals by Year

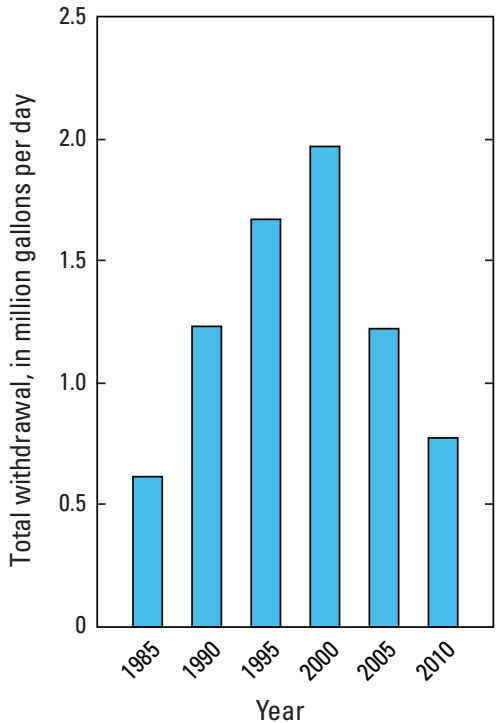

Groundwater Withdrawals by Year

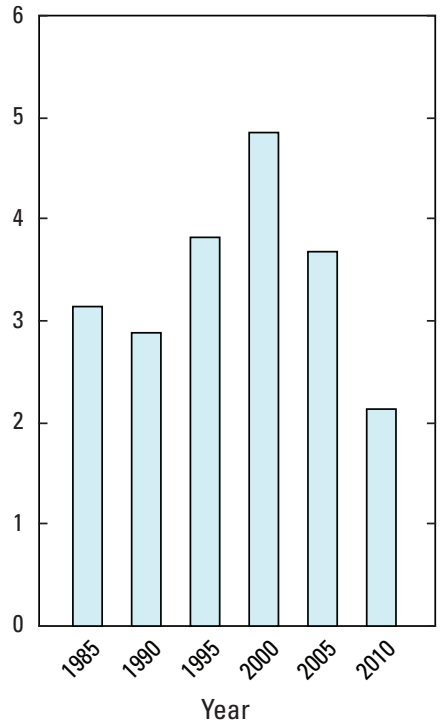


JOHNSON COUNTY

Population

Population served by public supply-Groundwater $\quad 3,060$

Population served by public supply-Surface water 0

Acres irrigated

1,640

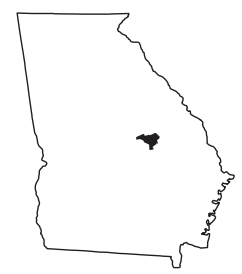

2010 WATER WITHDRAWALS AND ESTIMATED USE, IN MILLION GALLONS PER DAY

$[-$, not applicable $]$

\begin{tabular}{|c|c|c|c|c|c|c|c|c|}
\hline \multirow{3}{*}{ Category } & \multicolumn{3}{|c|}{ Withdrawals } & \multirow{3}{*}{$\begin{array}{l}\text { Total } \\
\text { use }^{1}\end{array}$} & \multirow{3}{*}{$\begin{array}{c}\text { Surface- } \\
\text { water } \\
\text { returns } \\
\end{array}$} & \multirow{2}{*}{\multicolumn{3}{|c|}{$\begin{array}{l}\text { Withdrawals by Major Industrial Groups } \\
\text { [NAICS, North American Industrial Classification System code] }\end{array}$}} \\
\hline & \multirow{2}{*}{$\begin{array}{c}\text { Ground- } \\
\text { water }\end{array}$} & \multirow{2}{*}{$\begin{array}{c}\text { Surface } \\
\text { water }\end{array}$} & \multirow[b]{2}{*}{ Total } & & & & & \\
\hline & & & & & & NAICS & Groundwater & Surface water \\
\hline Public supply & 0.56 & 0.00 & 0.56 & - & - & None & - & - \\
\hline Domestic & 0.52 & 0.00 & 0.52 & 0.72 & 0.00 & None & - & \\
\hline Commercial/public use & 0.00 & 0.00 & 0.00 & 0.28 & 0.00 & & & \\
\hline Industrial & 0.00 & 0.00 & 0.00 & 0.00 & 0.00 & & & \\
\hline Public-supply losses & - & - & - & 0.08 & - & & & \\
\hline Public wastewater treatment & - & - & - & - & 0.70 & Withdrawals by M & r Public Supp & \\
\hline Mining & 0.00 & 0.00 & 0.00 & 0.00 & 0.00 & Name & Groundwater & Surface water \\
\hline Irrigation-Crop & 0.71 & 0.07 & 0.78 & 0.78 & 0.00 & & & \\
\hline Irrigation-Golf course & 0.00 & 0.00 & 0.00 & 0.00 & 0.00 & City of Wrightsville & 0.51 & 0.00 \\
\hline Livestock/aquaculture & 0.02 & 0.06 & 0.08 & 0.08 & 0.00 & Town of Kite & 0.04 & 0.00 \\
\hline Thermoelectric power & 0.00 & 0.00 & 0.00 & 0.00 & 0.00 & & & \\
\hline
\end{tabular}

Thermoelectric power

$1.81 \quad 0.13$

1.94

$\mathbf{1 . 9 4}$

0.00

'Total use is total withdrawal plus public supply deliveries and losses.

\section{Withdrawals by Source}

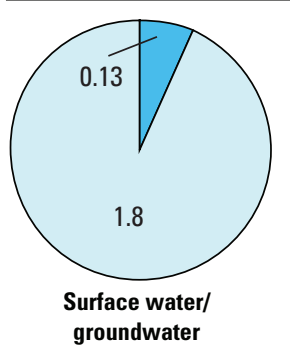

Withdrawals, in million gallons per day
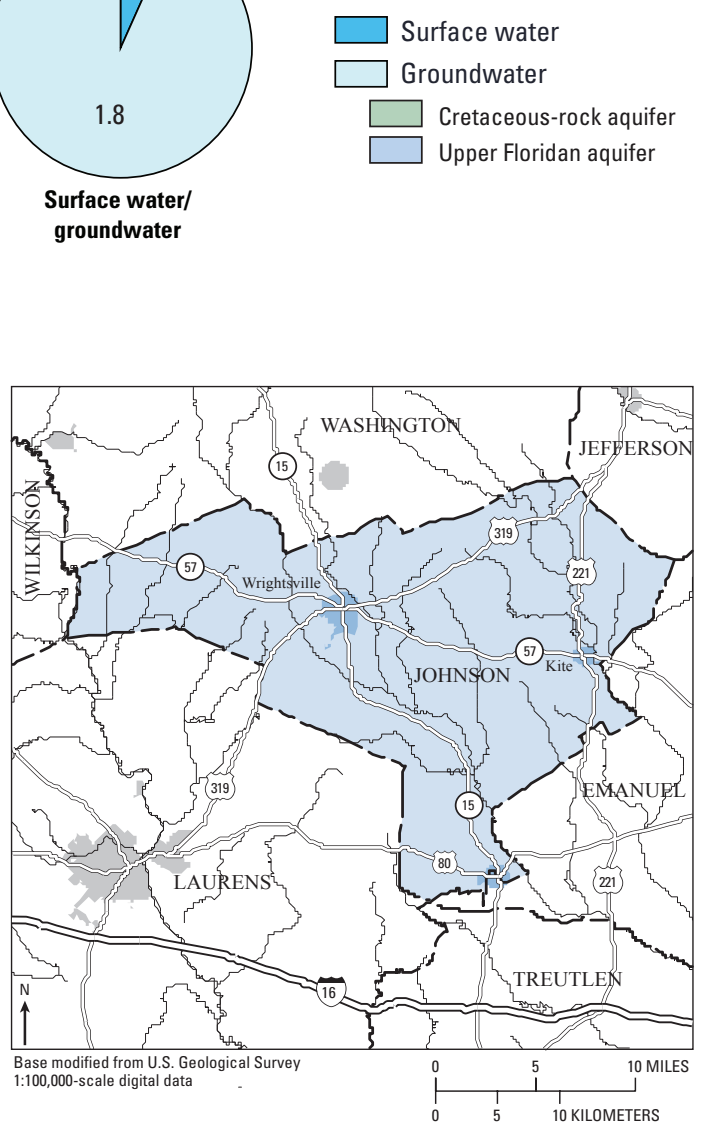

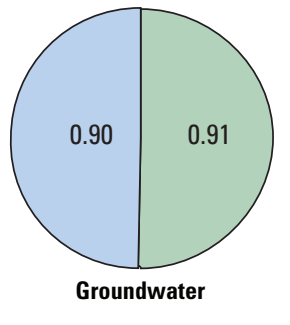

Public-Supply Deliveries by Use Category

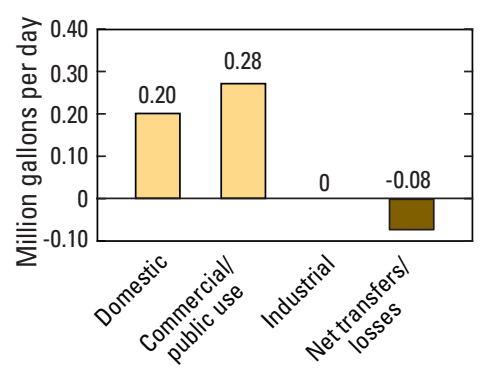

Surface-Water Withdrawals by Year

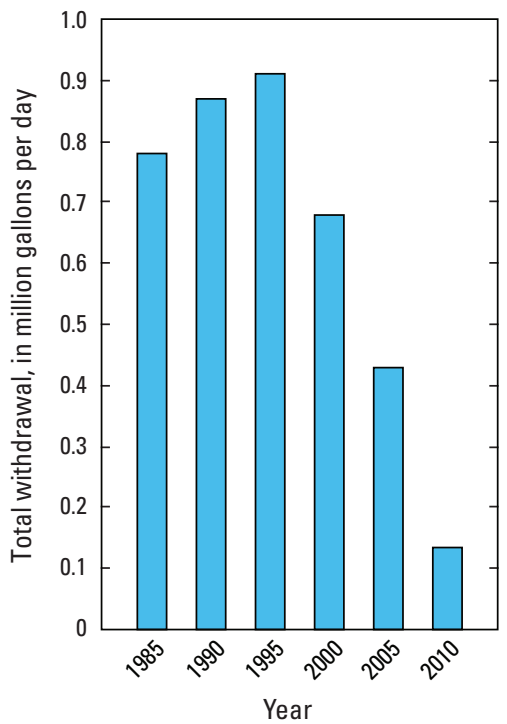

Groundwater Withdrawals by Year

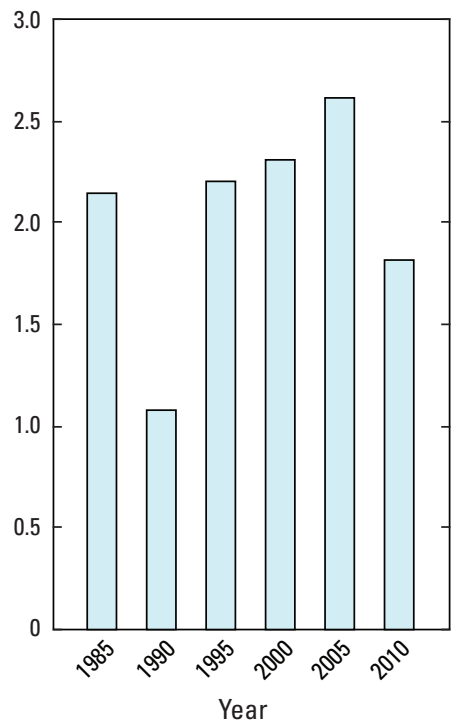




\section{JONES COUNTY}

Population

28,669

Population served by public supply-Groundwater

Population served by public supply-Surface water

Acres irrigated
13,940

610

230

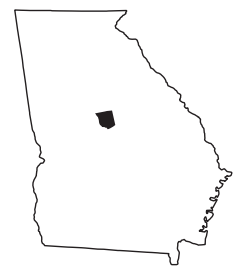

2010 WATER WITHDRAWALS AND ESTIMATED USE, IN MILLION GALLONS PER DAY

$[-$, not applicable; Mgal/d, million gallons per day]

\begin{tabular}{|c|c|c|c|c|c|}
\hline \multirow[b]{2}{*}{ Category } & \multicolumn{3}{|c|}{ Withdrawals } & \multirow[b]{2}{*}{$\begin{array}{l}\text { Total } \\
\text { use }^{1}\end{array}$} & \multirow{2}{*}{$\begin{array}{c}\text { Surface- } \\
\text { water } \\
\text { returns }\end{array}$} \\
\hline & $\begin{array}{c}\text { Ground- } \\
\text { water }\end{array}$ & $\begin{array}{c}\text { Surface } \\
\text { water }\end{array}$ & Total & & \\
\hline Public supply & 1.67 & 2.78 & 4.45 & - & - \\
\hline Domestic & 1.06 & 0.00 & 1.06 & 2.35 & 0.00 \\
\hline Commercial/public use & 0.00 & 0.00 & 0.00 & 0.14 & 0.00 \\
\hline Industrial & 0.00 & 0.00 & 0.00 & 0.00 & 0.00 \\
\hline Public-supply losses & - & - & - & 0.26 & - \\
\hline Public wastewater treatment & - & - & - & - & 0.25 \\
\hline Mining & 0.94 & 0.01 & 0.95 & 0.95 & 1.26 \\
\hline Irrigation-Crop & 0.00 & 0.02 & 0.02 & 0.02 & 0.00 \\
\hline Irrigation-Golf course & 0.00 & 0.25 & 0.25 & 0.25 & 0.00 \\
\hline Livestock/aquaculture & 0.00 & 0.10 & 0.10 & 0.10 & 0.00 \\
\hline Thermoelectric power & 0.00 & 0.00 & 0.00 & 0.00 & 0.00 \\
\hline TOTAL & 3.67 & 3.16 & 6.83 & 4.07 & 1.51 \\
\hline
\end{tabular}

Total use is total withdrawal plus public supply deliveries and losses. An average of $2.78 \mathrm{Mgal} / \mathrm{d}$ was delivered from Jones County to Bibb County, Georgia in 2010 .

\section{Withdrawals by Source}

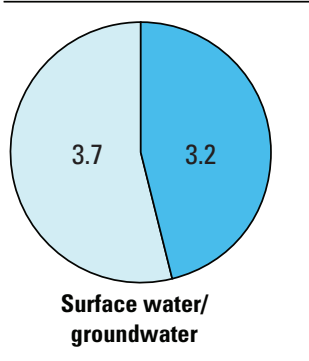

Withdrawals, in million gallons per day
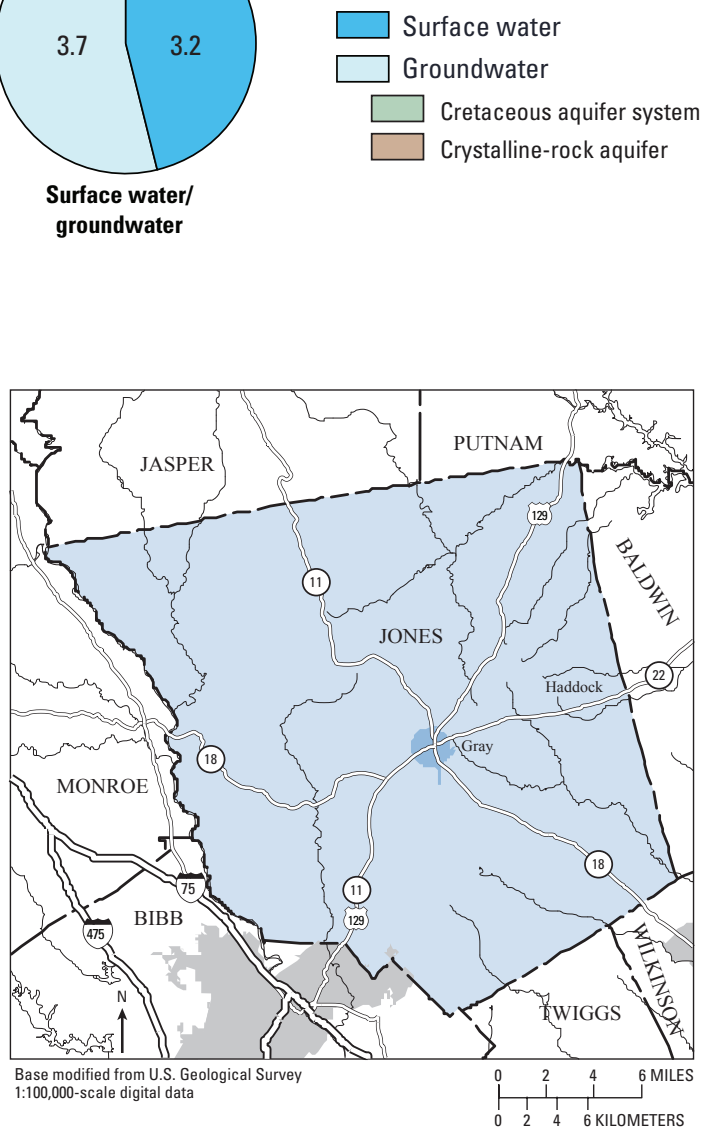

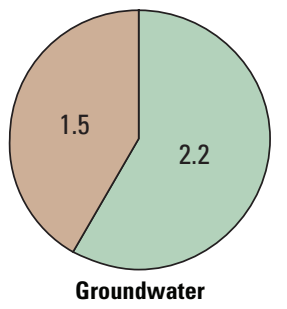

Public-Supply Deliveries' by Use Category

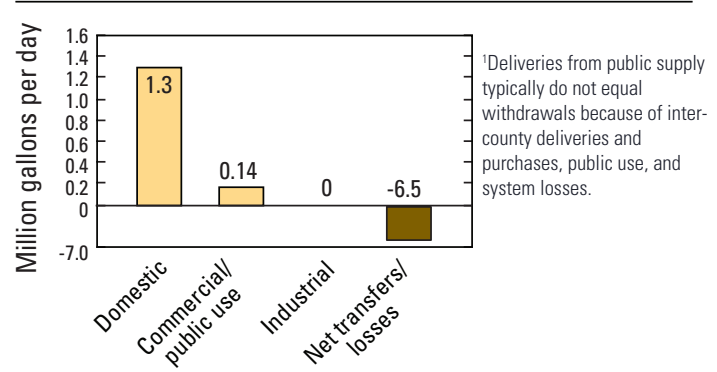

Withdrawals by Major Industrial Groups

[NAICS, North American Industrial Classification System code]

NAICS Groundwater Surface water

None

Withdrawals by Major Public Suppliers

\begin{tabular}{|c|c|c|}
\hline Name & undwater & Surface water \\
\hline Macon Water Authority ${ }^{1}$ & 0.00 & 2.78 \\
\hline Jones County Water Comm. & 1.12 & 0.00 \\
\hline City of Gray & 0.46 & 0.00 \\
\hline
\end{tabular}




\section{LAMAR COUNTY}

Population

18,317

Population served by public supply-Groundwater

Population served by public supply-Surface water $\quad 8,210$

Acres irrigated

670

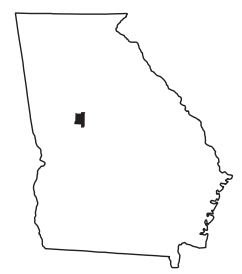

2010 WATER WITHDRAWALS AND ESTIMATED USE, IN MILLION GALLONS PER DAY

$[-$, not applicable $]$

\begin{tabular}{|c|c|c|c|c|c|c|c|c|}
\hline \multirow{3}{*}{ Category } & \multicolumn{3}{|c|}{ Withdrawals } & \multirow{3}{*}{$\begin{array}{l}\text { Total } \\
\text { use }^{1}\end{array}$} & \multirow{3}{*}{$\begin{array}{l}\text { Surface- } \\
\text { water } \\
\text { returns } \\
\end{array}$} & \multirow{2}{*}{\multicolumn{3}{|c|}{$\begin{array}{l}\text { Withdrawals by Major Industrial Groups } \\
\text { [NAICS, North American Industrial Classification System code] }\end{array}$}} \\
\hline & \multirow{2}{*}{$\begin{array}{c}\text { Ground- } \\
\text { water }\end{array}$} & \multirow{2}{*}{$\begin{array}{c}\text { Surface } \\
\text { water }\end{array}$} & \multirow[b]{2}{*}{ Total } & & & & & \\
\hline & & & & & & NAICS & Groundwater & Surface water \\
\hline Public supply & 0.01 & 1.53 & 1.54 & - & - & None & - & - \\
\hline Domestic & 0.75 & 0.00 & 0.75 & 1.87 & 0.00 & None & - & - \\
\hline Commercial/public use & 0.00 & 0.00 & 0.00 & 0.18 & 0.00 & & & \\
\hline Industrial & 0.00 & 0.00 & 0.00 & 0.01 & 0.00 & & & \\
\hline Public-supply losses & - & - & - & 0.23 & - & & & \\
\hline Public wastewater treatment & - & - & - & - & 2.33 & Withdrawals by $\mathbf{N}$ & r Public Supp & \\
\hline Mining & 0.00 & 0.00 & 0.00 & 0.00 & 0.00 & & & \\
\hline Irrigation-Crop & 1.22 & 0.00 & 1.22 & 1.22 & 0.00 & Name & Groundwater & Surface water \\
\hline Irrigation-Golf course & 0.00 & 0.13 & 0.13 & 0.13 & 0.00 & City of Barnesville & 0.00 & 1.53 \\
\hline Livestock/aquaculture & 0.00 & 0.19 & 0.19 & 0.19 & 0.00 & & & \\
\hline Thermoelectric power & 0.00 & 0.00 & 0.00 & 0.00 & 0.00 & & & \\
\hline TOTAL & 1.98 & 1.85 & 3.83 & 3.83 & 2.33 & & & \\
\hline
\end{tabular}

'Total use is total withdrawal plus public supply deliveries and losses.

\section{Withdrawals by Source}

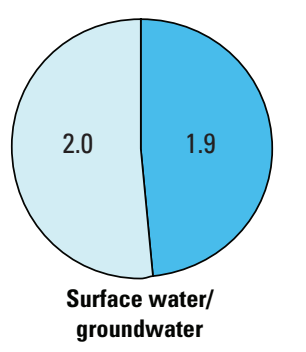

Withdrawals, in million gallons per day

$\square$ Surface water
$\square$ Groundwater
$\square$ Crystalline-rock aquifer

groundwater

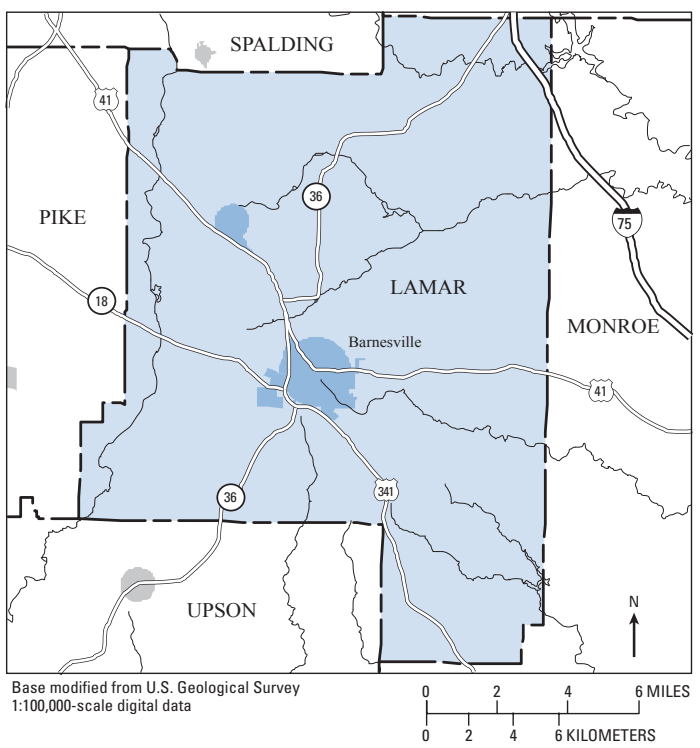

Public-Supply Deliveries by Use Category
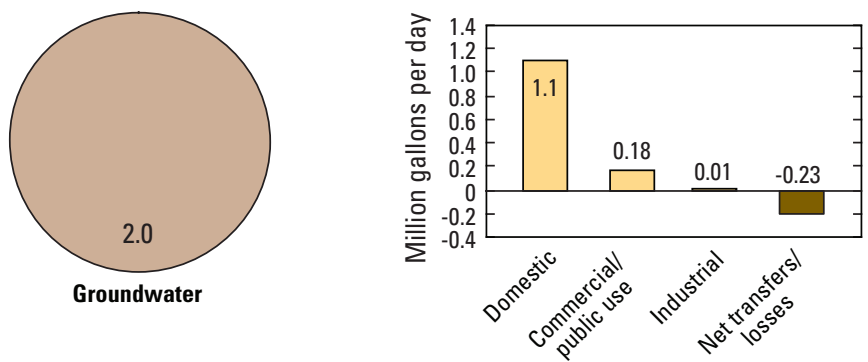

Surface-Water Withdrawals by Year

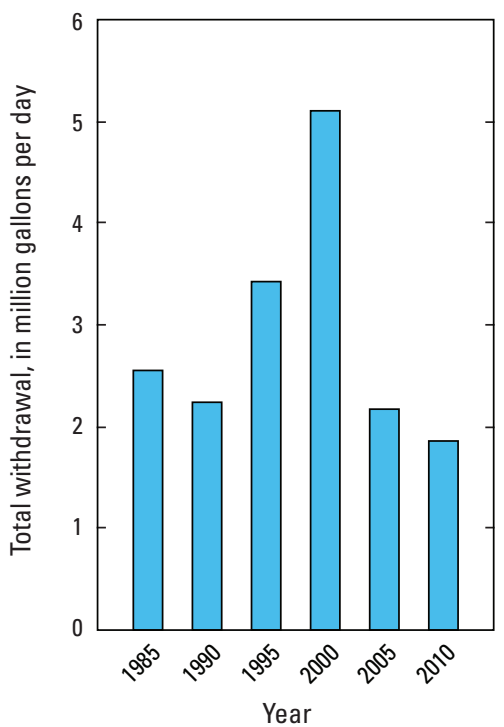

Groundwater Withdrawals by Year

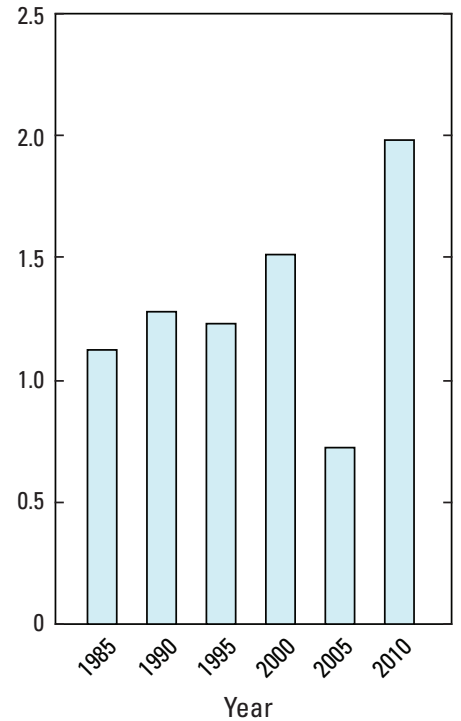


LANIER COUNTY

Population

10,078

Population served by public supply-Groundwater $\quad 4,360$

Population served by public supply-Surface water 0

Acres irrigated

5,600

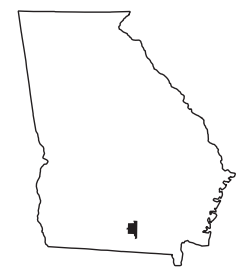

2010 WATER WITHDRAWALS AND ESTIMATED USE, IN MILLION GALLONS PER DAY

\begin{tabular}{|c|c|c|c|c|c|}
\hline \multirow[b]{2}{*}{ Category } & \multicolumn{3}{|c|}{ Withdrawals } & \multirow[b]{2}{*}{$\begin{array}{l}\text { Total } \\
\text { use }^{1}\end{array}$} & \multirow{2}{*}{$\begin{array}{c}\text { Surface- } \\
\text { water } \\
\text { returns } \\
\end{array}$} \\
\hline & $\begin{array}{c}\text { Ground- } \\
\text { water }\end{array}$ & $\begin{array}{c}\text { Surface } \\
\text { water }\end{array}$ & Total & & \\
\hline Public supply & 0.52 & 0.00 & 0.52 & - & - \\
\hline Domestic & 0.43 & 0.00 & 0.43 & 0.76 & 0.00 \\
\hline Commercial/public use & 0.00 & 0.00 & 0.00 & 0.12 & 0.00 \\
\hline Industrial & 0.00 & 0.00 & 0.00 & 0.00 & 0.00 \\
\hline Public-supply losses & - & - & - & 0.07 & - \\
\hline Public wastewater treatment & - & - & - & - & 0.09 \\
\hline Mining & 0.09 & 0.00 & 0.09 & 0.09 & 0.00 \\
\hline Irrigation-Crop & 0.79 & 0.08 & 0.87 & 0.87 & 0.00 \\
\hline Irrigation-Golf course & 0.00 & 0.00 & 0.00 & 0.00 & 0.00 \\
\hline Livestock/aquaculture & 0.02 & 0.02 & 0.04 & 0.04 & 0.00 \\
\hline Thermoelectric power & 0.00 & 0.00 & 0.00 & 0.00 & 0.00 \\
\hline TOTAL & 1.85 & 0.10 & 1.95 & 1.95 & 0.09 \\
\hline
\end{tabular}

Withdrawals by Major Industrial Groups

[NAICS, North American Industrial Classification System code]

\begin{tabular}{lcc}
\hline NAICS & Groundwater & Surface water \\
\hline None & - & - \\
\hline
\end{tabular}

Total use is total withdrawal plus public supply deliveries and losses.

\section{Withdrawals by Source}

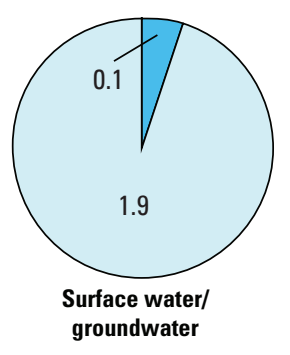

Withdrawals, in million gallons per day

Surface water

Groundwater

$\square$ Upper Floridan aquifer

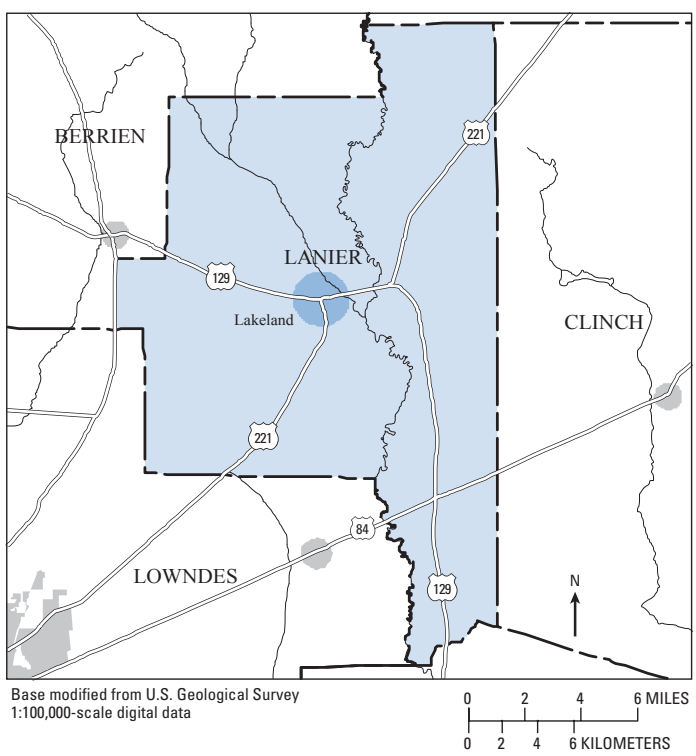

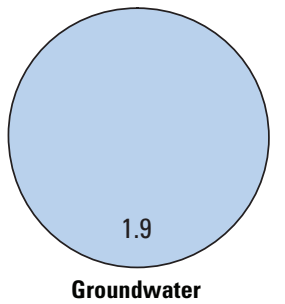

Public-Supply Deliveries by Use Category

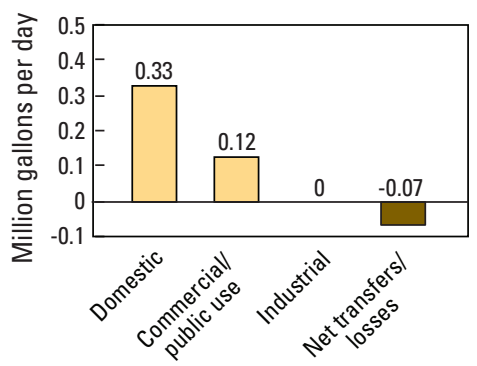

Withdrawals by Major Public Suppliers

\begin{tabular}{lcc}
\hline Name & Groundwater & Surface water \\
\hline City of Lakeland & 0.47 & 0.00 \\
\hline
\end{tabular}




\section{LAURENS COUNTY}

Population

Population served by public supply-Groundwater $\quad 13,300$

Population served by public supply_-Surface water $\quad 8,690$

Acres irrigated

7,455

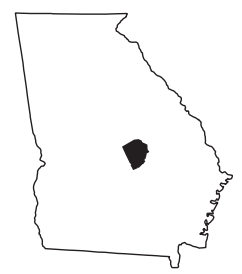

2010 WATER WITHDRAWALS AND ESTIMATED USE, IN MILLION GALLONS PER DAY

$[-$, not applicable $]$

\begin{tabular}{|c|c|c|c|c|c|c|c|c|}
\hline \multirow[b]{3}{*}{ Category } & \multicolumn{3}{|c|}{ Withdrawals } & \multirow{3}{*}{$\begin{array}{l}\text { Total } \\
\text { use }^{1}\end{array}$} & \multirow{3}{*}{$\begin{array}{l}\text { Surface- } \\
\text { water } \\
\text { returns } \\
\end{array}$} & \multirow{2}{*}{\multicolumn{3}{|c|}{$\begin{array}{l}\text { Withdrawals by Major Industrial Groups } \\
\text { [NAICS, North American Industrial Classification System code] }\end{array}$}} \\
\hline & \multirow{2}{*}{$\begin{array}{l}\text { Ground- } \\
\text { water }\end{array}$} & \multirow{2}{*}{$\begin{array}{c}\text { Surface } \\
\text { water }\end{array}$} & \multirow[b]{2}{*}{ Total } & & & & & \\
\hline & & & & & & NAICS & Groundwater & Surface water \\
\hline Public supply & 2.14 & 1.78 & 3.92 & - & - & & & 14.11 \\
\hline Domestic & 1.98 & 0.00 & 1.98 & 4.15 & 0.00 & 322-Paper, pulp & 1.60 & 14.11 \\
\hline Commercial/public use & 0.00 & 0.00 & 0.00 & 0.51 & 0.00 & 314-Textiles & 0.39 & 0.00 \\
\hline Industrial & 1.99 & 14.11 & 16.10 & 16.49 & 15.91 & & & \\
\hline Public-supply losses & - & - & - & 0.85 & - & & & \\
\hline Public wastewater treatment & - & - & - & - & 2.17 & Withdrawals by Major & Public Supp & \\
\hline Mining & 0.00 & 0.00 & 0.00 & 0.00 & 0.00 & Name & Groundwater & Surface water \\
\hline Irrigation - Crop & 3.55 & 1.28 & 4.83 & 4.83 & 0.00 & City of Dublin & 130 & \\
\hline Irrigation-Golf course & 0.16 & 0.08 & 0.24 & 0.24 & 0.00 & City of Dublin & $\begin{array}{l}1.30 \\
0.42\end{array}$ & $\begin{array}{l}1.78 \\
0.00\end{array}$ \\
\hline Livestock/aquaculture & 0.03 & 0.10 & 0.13 & 0.13 & 0.00 & City of East Dublin & 0.42 & 0.00 \\
\hline Thermoelectric power & 0.00 & 0.00 & 0.00 & 0.00 & 0.00 & Laurens Co. Water System & 0.11 & 0.00 \\
\hline TOTAL & 9.85 & 17.35 & 27.20 & 27.20 & 18.08 & City of Dudley & 0.09 & 0.00 \\
\hline
\end{tabular}

Total use is total withdrawal plus public supply deliveries and losses.

\section{Withdrawals by Source}

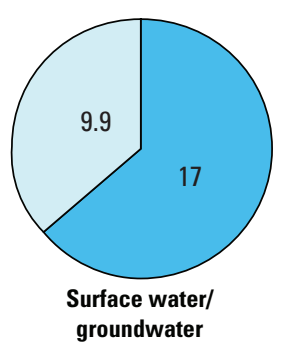

Withdrawals, in million gallons per day

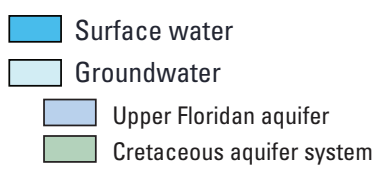

groundwater

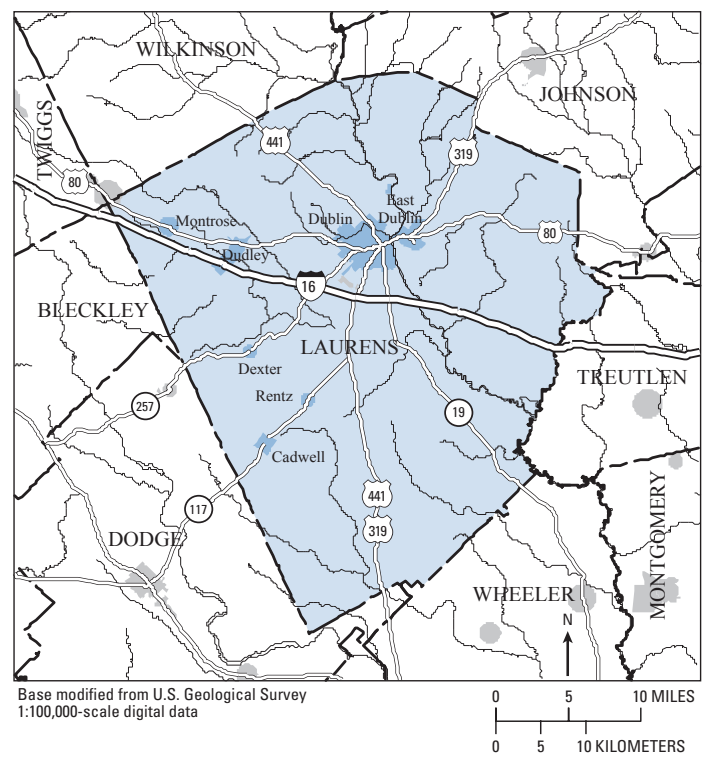

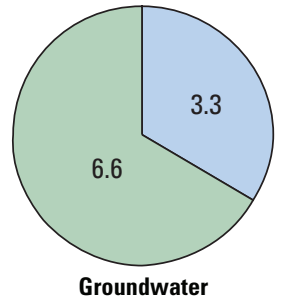

Public-Supply Deliveries by Use Category

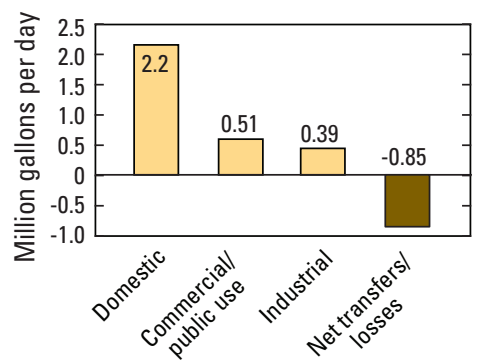

Surface-Water Withdrawals by Year

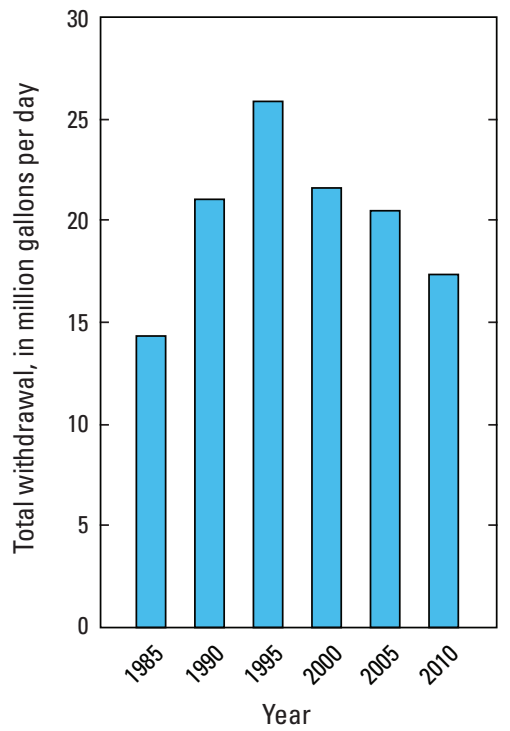

Groundwater Withdrawals by Year

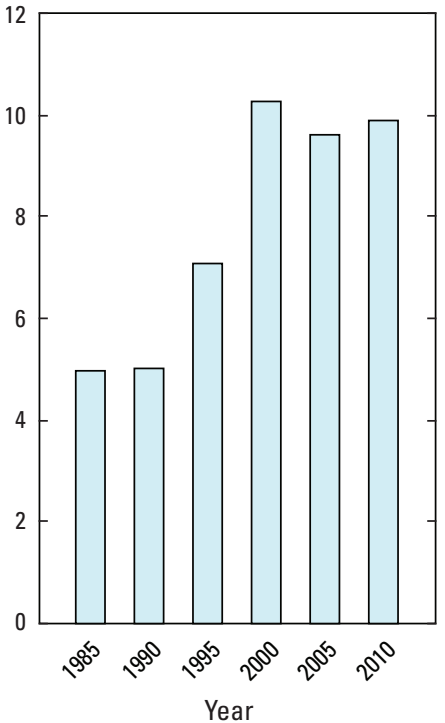




\section{LEE COUNTY}

Population

28,298

Population served by public supply-Groundwater

Population served by public supply-Surface water Acres irrigated
17,920

45,170

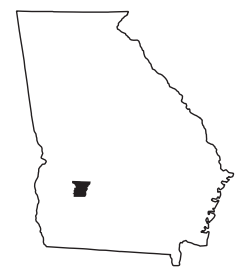

2010 WATER WITHDRAWALS AND ESTIMATED USE, IN MILLION GALLONS PER DAY

$[-$, not applicable $]$

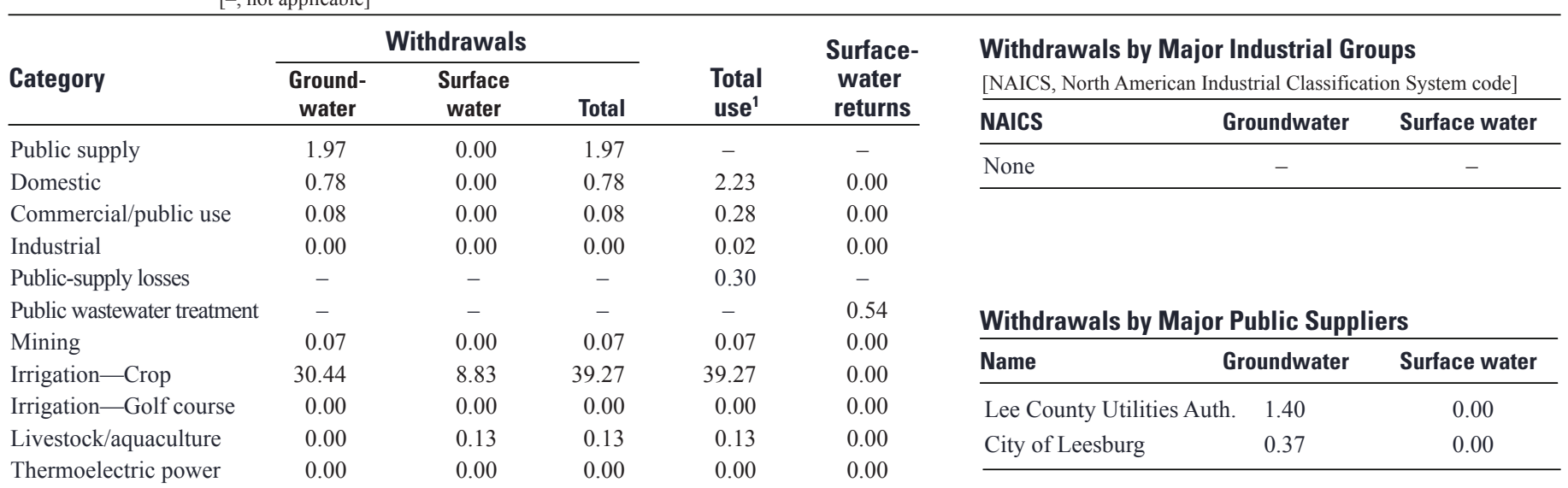

\begin{tabular}{lrrrrr} 
TOTAL & 33.34 & 8.96 & 42.30 & 42.30 & 0.54 \\
\hline
\end{tabular}

TTotal use is total withdrawal plus public supply deliveries and losses.

2010 Withdrawals by Source

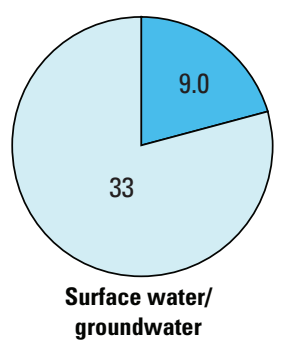

\begin{tabular}{|c|c|}
\hline \multicolumn{2}{|c|}{$\begin{array}{l}\text { Withdrawals, in million } \\
\text { gallons per day }\end{array}$} \\
\hline & Surface water \\
\hline & Groundwater \\
\hline & Upper Floridan aquifer \\
\hline & Claiborne aquifer \\
\hline & Clayton aquifer \\
\hline & Cretaceous aquifer system \\
\hline
\end{tabular}

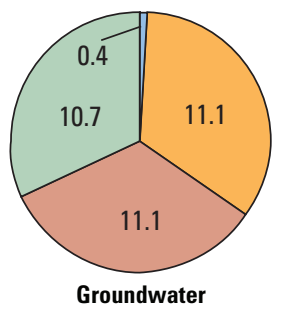

Groundwater
Public-Supply Deliveries by Use Category

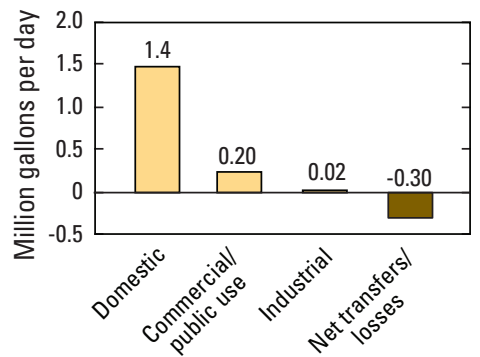

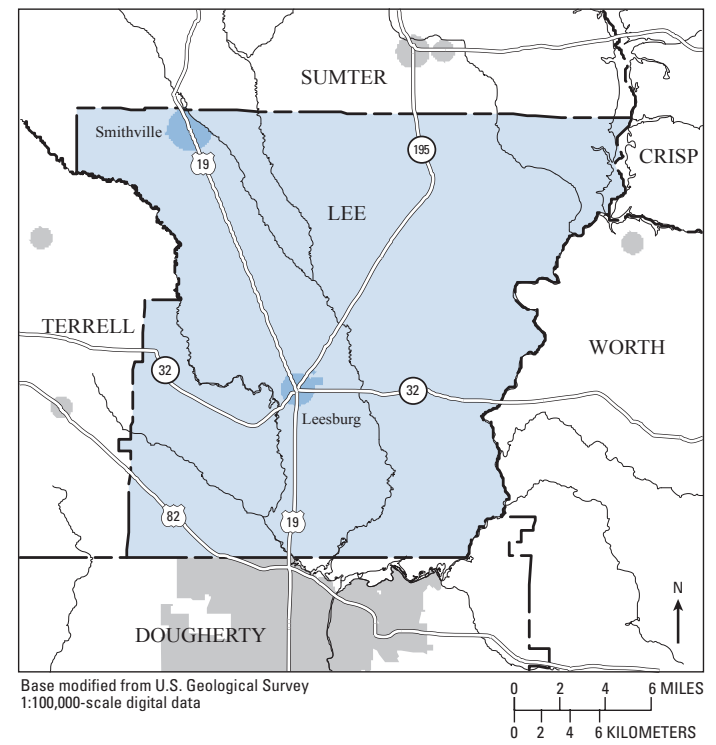

Surface-Water Withdrawals by Year

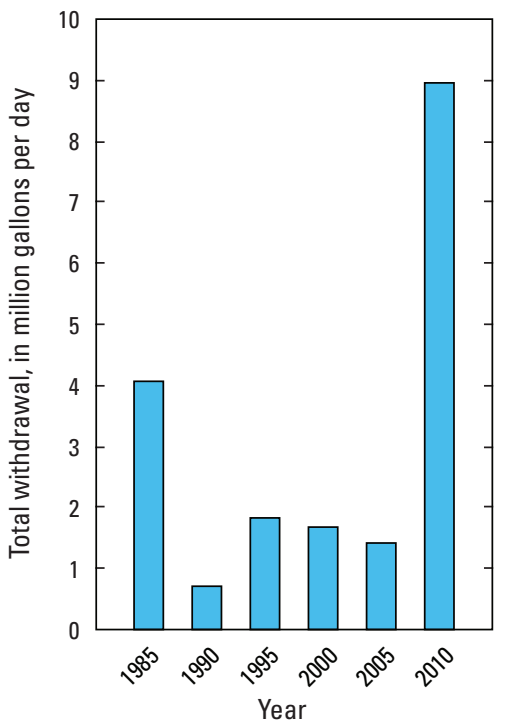

Groundwater Withdrawals by Year

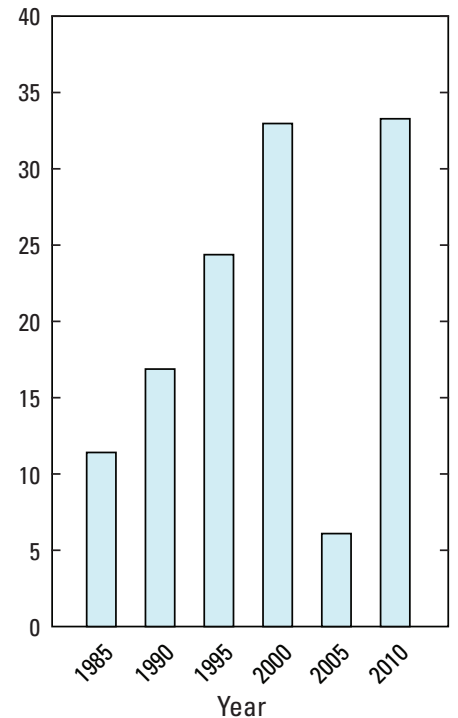




\section{LIBERTY COUNTY}

Population

Population served by public supply-Groundwater $\quad 54,200$

Population served by public supply-Surface water 0

Acres irrigated

30

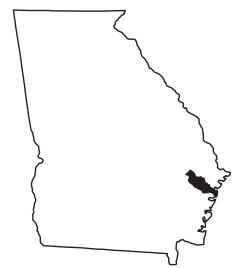

2010 WATER WITHDRAWALS AND ESTIMATED USE, IN MILLION GALLONS PER DAY

$[-$, not applicable $]$

\begin{tabular}{|c|c|c|c|c|c|c|c|c|}
\hline \multirow{3}{*}{ Category } & \multicolumn{3}{|c|}{ Withdrawals } & \multirow{3}{*}{$\begin{array}{l}\text { Total } \\
\text { use }^{1}\end{array}$} & \multirow{3}{*}{$\begin{array}{l}\text { Surface- } \\
\text { water } \\
\text { returns } \\
\end{array}$} & \multirow{2}{*}{\multicolumn{3}{|c|}{$\begin{array}{l}\text { Withdrawals by Major Industrial Groups } \\
\text { [NAICS, North American Industrial Classification System code] }\end{array}$}} \\
\hline & \multirow{2}{*}{$\begin{array}{c}\text { Ground- } \\
\text { water }\end{array}$} & \multirow{2}{*}{$\begin{array}{c}\text { Surface } \\
\text { water }\end{array}$} & \multirow[b]{2}{*}{ Total } & & & & & \\
\hline & & & & & & NAICS & Groundwater & Surface water \\
\hline Public supply & 6.75 & 0.00 & 6.75 & - & - & 322-Paper, pulp & 8.96 & 000 \\
\hline Domestic & 0.69 & 0.00 & 0.69 & 4.03 & 0.00 & $322-$ - & 8.96 & 0.00 \\
\hline Commercial/public use & 0.05 & 0.00 & 0.05 & 0.73 & 0.00 & & & \\
\hline Industrial & 8.96 & 0.00 & 8.96 & 10.75 & 6.94 & & & \\
\hline Public-supply losses & - & - & - & 0.94 & - & Withdrawals by Major & Public Supp & \\
\hline Public wastewater treatment & - & - & - & - & $\begin{array}{l}4.87 \\
0.00\end{array}$ & Name G & Groundwater & Surface water \\
\hline $\begin{array}{l}\text { Mining } \\
\text { Irrigation-Crop }\end{array}$ & $\begin{array}{l}0.00 \\
0.00\end{array}$ & $\begin{array}{l}0.09 \\
0.02\end{array}$ & $\begin{array}{l}0.09 \\
0.02\end{array}$ & $\begin{array}{l}0.09 \\
0.02\end{array}$ & $\begin{array}{l}0.00 \\
0.00\end{array}$ & City of Hinesville & 2.97 & 0.00 \\
\hline Irrigation-Golf course & 0.00 & 0.00 & 0.00 & 0.00 & 0.00 & U.S. Army-Fort Stewart & 1.90 & 0.00 \\
\hline Livestock/aquaculture & 0.00 & 0.01 & 0.01 & 0.01 & 0.00 & City of Riceboro & 0.82 & 0.00 \\
\hline Thermoelectric power & 0.00 & 0.00 & 0.00 & 0.00 & 0.00 & City of Walthourville & 0.38 & 0.00 \\
\hline TOTAL & 16.45 & 0.12 & 16.57 & 16.57 & 11.81 & Water Utility Management & $\mathrm{t} \quad 0.23$ & 0.00 \\
\hline
\end{tabular}

2010 Withdrawals by Source
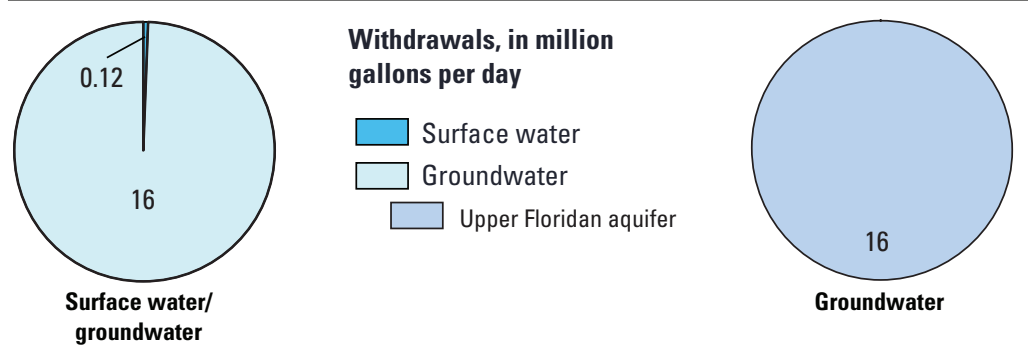

Public-Supply Deliveries by Use Category
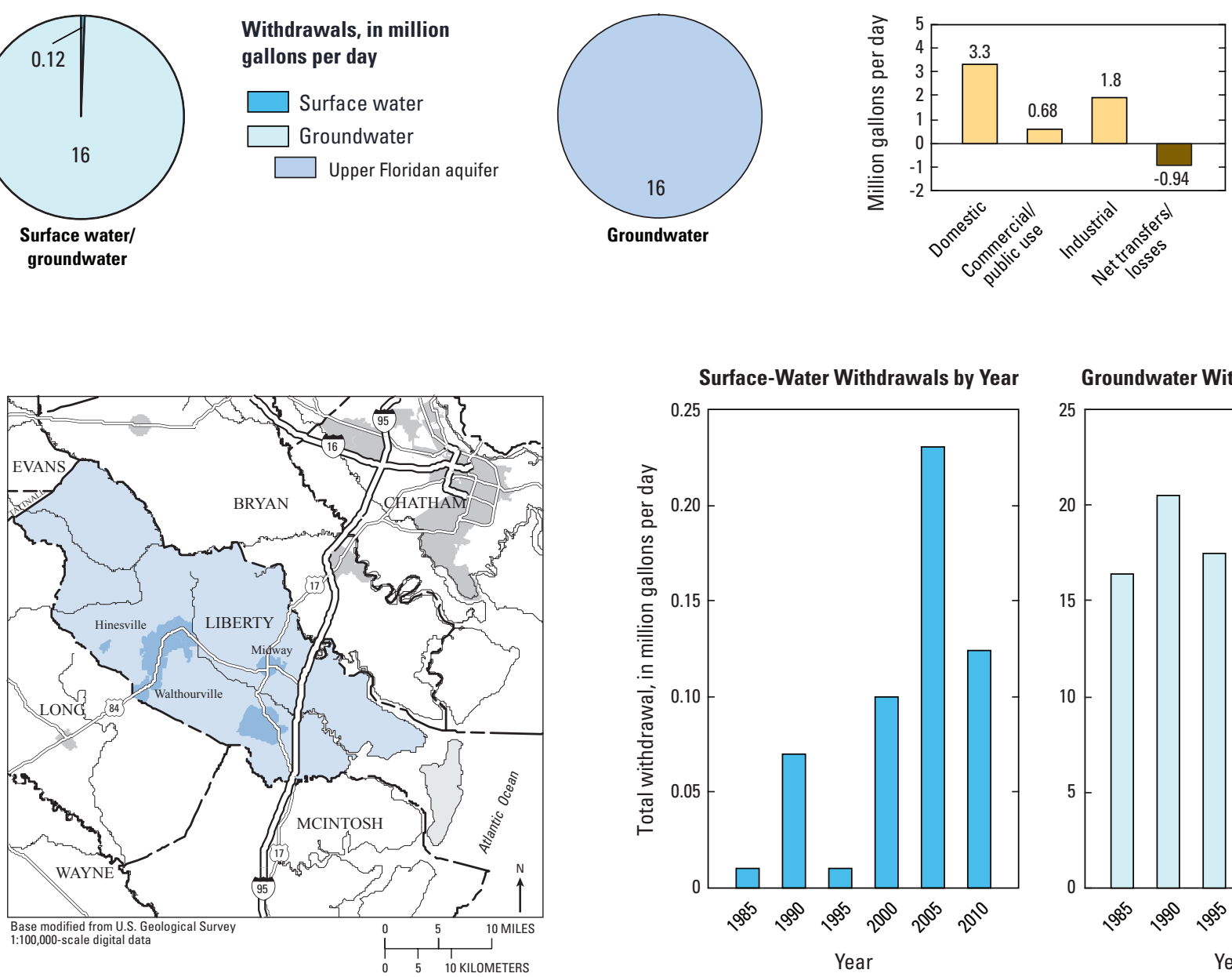

Groundwater Withdrawals by Year

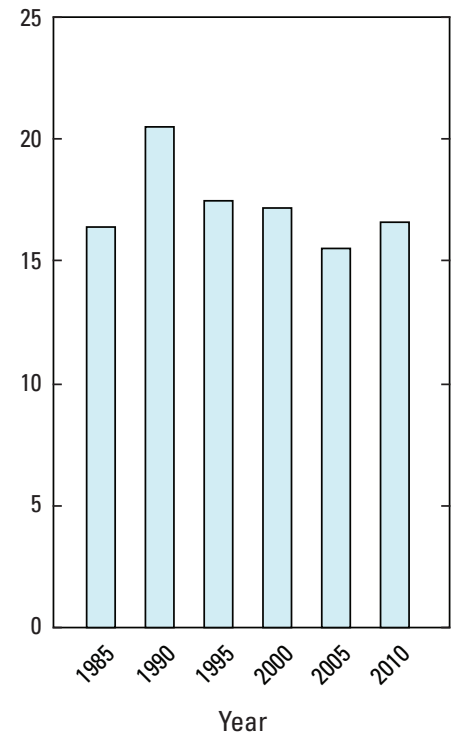




\section{LINCOLN COUNTY}

Population

Population served by public supply-Groundwater

Population served by public supply-Surface water $\quad 4,620$ Acres irrigated

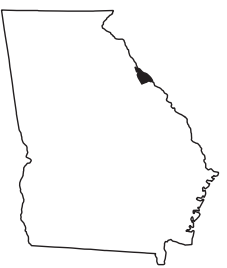

2010 WATER WITHDRAWALS AND ESTIMATED USE, IN MILLION GALLONS PER DAY

$[-$, not applicable $]$

\begin{tabular}{|c|c|c|c|c|c|c|c|c|}
\hline \multirow{3}{*}{ Category } & \multicolumn{3}{|c|}{ Withdrawals } & \multirow{3}{*}{$\begin{array}{l}\text { Total } \\
\text { use }^{1}\end{array}$} & \multirow{3}{*}{$\begin{array}{l}\text { Surface- } \\
\text { water } \\
\text { returns } \\
\end{array}$} & \multirow{2}{*}{\multicolumn{3}{|c|}{$\begin{array}{l}\text { Withdrawals by Major Industrial Groups } \\
\text { [NAICS, North American Industrial Classification System code] }\end{array}$}} \\
\hline & \multirow{2}{*}{$\begin{array}{c}\text { Ground- } \\
\text { water }\end{array}$} & \multirow{2}{*}{$\begin{array}{c}\text { Surface } \\
\text { water }\end{array}$} & \multirow[b]{2}{*}{ Total } & & & & & \\
\hline & & & & & & NAICS & Groundwater & Surface water \\
\hline Public supply & 0.05 & 0.46 & 0.51 & - & - & \multirow{2}{*}{311 -Food products } & \multirow{2}{*}{1.01} & \multirow{2}{*}{0.00} \\
\hline Domestic & 0.20 & 0.00 & 0.20 & 0.58 & 0.00 & & & \\
\hline Commercial/public use & 0.00 & 0.00 & 0.00 & 0.06 & 0.00 & & & \\
\hline Industrial & 1.01 & 0.00 & 1.01 & 1.02 & 0.00 & & & \\
\hline Public-supply losses & - & - & - & 0.06 & - & & & \\
\hline Public wastewater treatment & - & - & - & - & 0.25 & \multicolumn{3}{|c|}{ Withdrawals by Major Public Suppliers } \\
\hline Mining & 0.00 & 0.00 & 0.00 & 0.00 & 0.00 & \multirow{2}{*}{ Name } & \multirow[t]{2}{*}{ Groundwater } & \multirow[t]{2}{*}{ Surface water } \\
\hline Irrigation-Crop & 0.00 & 0.00 & 0.00 & 0.00 & 0.00 & & & \\
\hline Irrigation-Golf course & 0.00 & 0.00 & 0.00 & 0.00 & 0.00 & City of Lincolnton & 0.00 & 0.46 \\
\hline Livestock/aquaculture & 0.00 & 0.11 & 0.11 & 0.11 & 0.00 & Lincoln Co. Water System & 0.04 & 0.00 \\
\hline Thermoelectric power & 0.00 & 0.00 & 0.00 & 0.00 & 0.00 & & & \\
\hline
\end{tabular}

0.57

1.83

1.83

0.25

'Total use is total withdrawal plus public supply deliveries and losses.

\section{Withdrawals by Source}
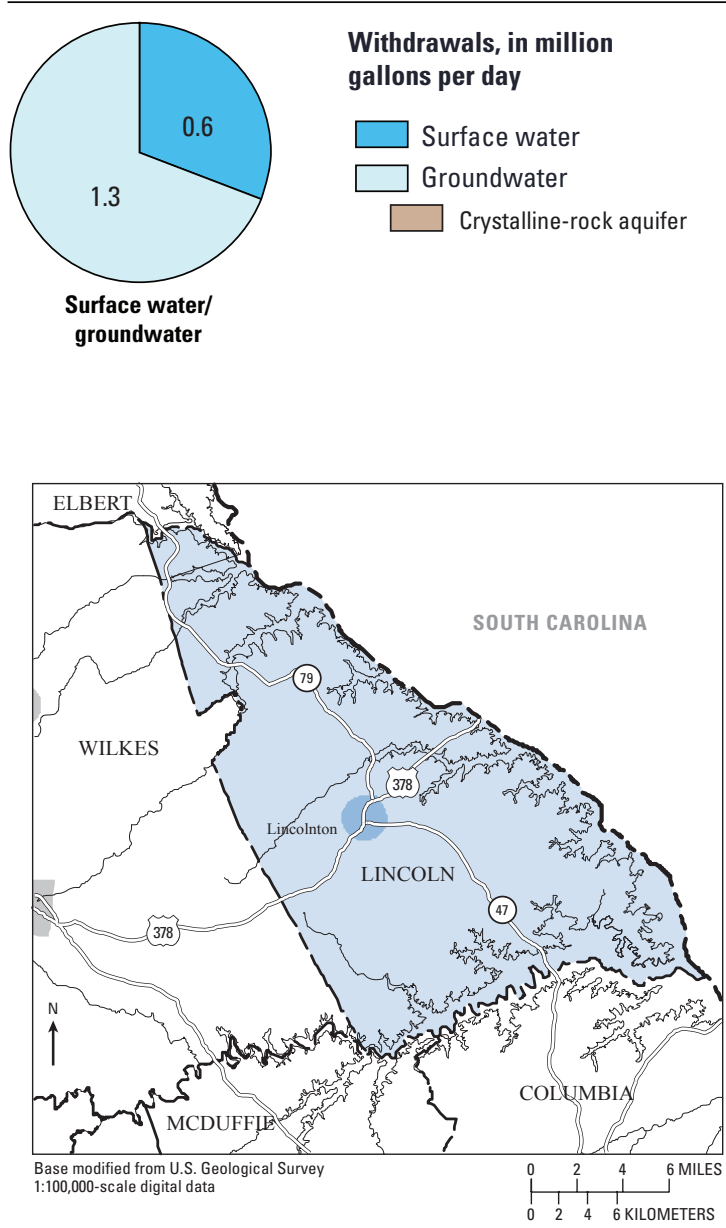

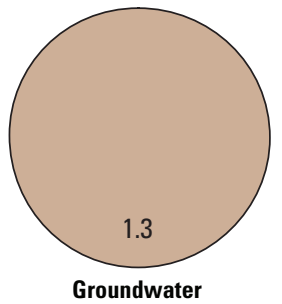

Public-Supply Deliveries by Use Category

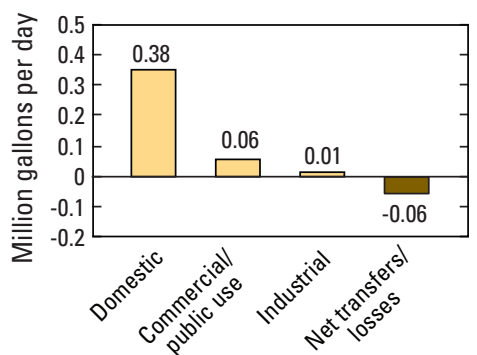

Surface-Water Withdrawals by Year

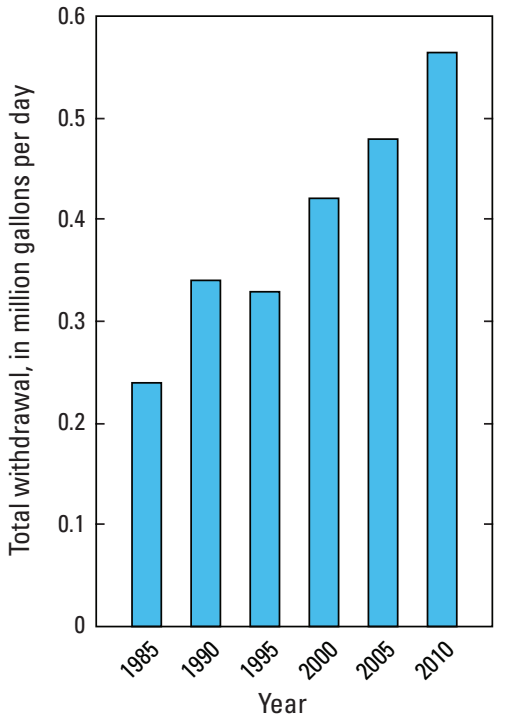

Groundwater Withdrawals by Year

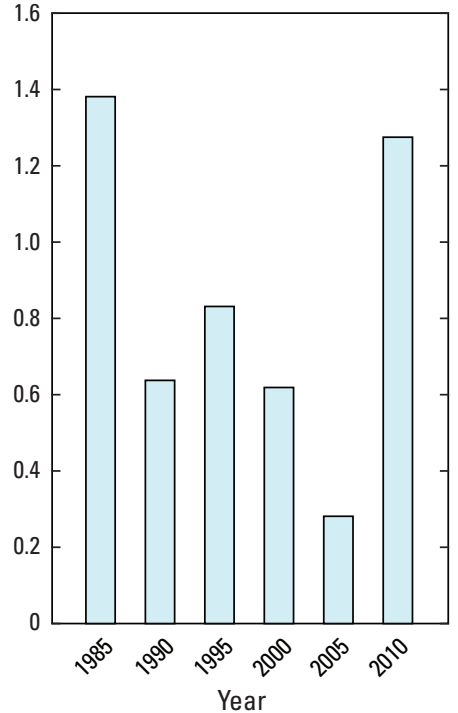




\section{LONG COUNTY}

Population

Population served by public supply-Groundwater $\quad 4,560$

Population served by public supply-Surface water 0

Acres irrigated

960

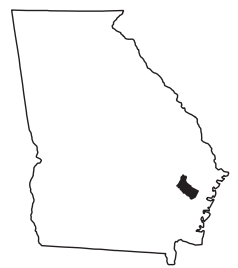

2010 WATER WITHDRAWALS AND ESTIMATED USE, IN MILLION GALLONS PER DAY

$[-$, not applicable $]$

\begin{tabular}{|c|c|c|c|c|c|c|c|c|}
\hline \multirow{3}{*}{ Category } & \multicolumn{3}{|c|}{ Withdrawals } & \multirow{3}{*}{$\begin{array}{l}\text { Total } \\
\text { use }^{1}\end{array}$} & \multirow{3}{*}{$\begin{array}{l}\text { Surface- } \\
\text { water } \\
\text { returns } \\
\end{array}$} & \multirow{2}{*}{\multicolumn{3}{|c|}{$\begin{array}{l}\text { Withdrawals by Major Industrial Groups } \\
\text { [NAICS, North American Industrial Classification System code] }\end{array}$}} \\
\hline & \multirow{2}{*}{$\begin{array}{c}\text { Ground- } \\
\text { water }\end{array}$} & \multirow{2}{*}{$\begin{array}{c}\text { Surface } \\
\text { water }\end{array}$} & \multirow[b]{2}{*}{ Total } & & & & & \\
\hline & & & & & & NAICS & Groundwater & Surface water \\
\hline Public supply & 0.54 & 0.00 & 0.54 & - & - & None & & - \\
\hline Domestic & 0.74 & 0.00 & 0.74 & 1.06 & 0.00 & None & - & - \\
\hline Commercial/public use & 0.01 & 0.00 & 0.01 & 0.15 & 0.00 & & & \\
\hline Industrial & 0.00 & 0.00 & 0.00 & 0.00 & 0.00 & & & \\
\hline Public-supply losses & - & - & - & 0.08 & - & & & \\
\hline Public wastewater treatment & - & - & - & - & 0.02 & Withdrawals by & r Public Supp| & \\
\hline Mining & 0.30 & 0.01 & 0.31 & 0.31 & 0.00 & Name & Groundwater & Surface water \\
\hline $\begin{array}{l}\text { Irrigation-Crop } \\
\text { Irrigation-Golf course }\end{array}$ & $\begin{array}{l}0.05 \\
0.00\end{array}$ & $\begin{array}{l}0.41 \\
0.00\end{array}$ & $\begin{array}{l}0.46 \\
0.00\end{array}$ & $\begin{array}{l}0.46 \\
0.00\end{array}$ & $\begin{array}{l}0.00 \\
0.00\end{array}$ & City of Ludowici & 0.39 & 0.00 \\
\hline Livestock/aquaculture & 0.00 & 0.05 & 0.05 & 0.05 & 0.00 & & & \\
\hline Thermoelectric power & 0.00 & 0.00 & 0.00 & 0.00 & 0.00 & & & \\
\hline TOTAL & 1.64 & 0.47 & 2.11 & 2.11 & 0.02 & & & \\
\hline
\end{tabular}

\section{Withdrawals by Source}
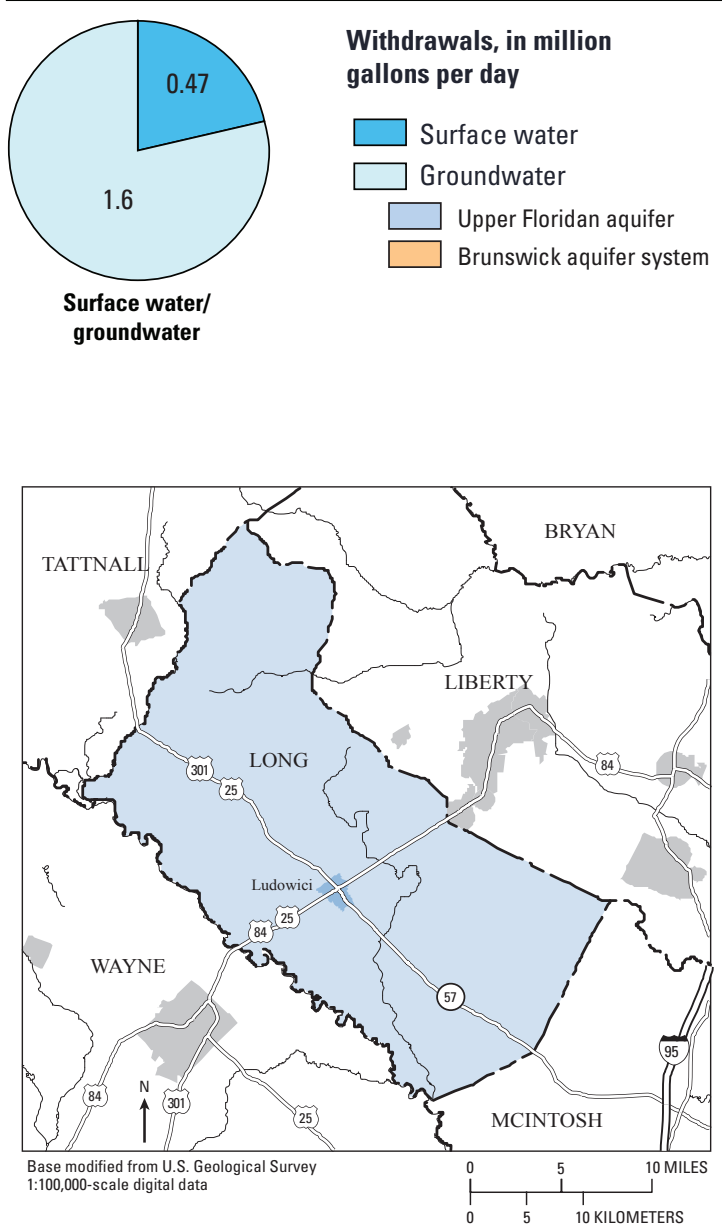

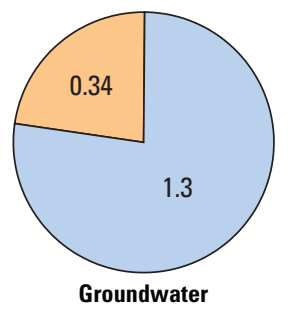

Public-Supply Deliveries by Use Category

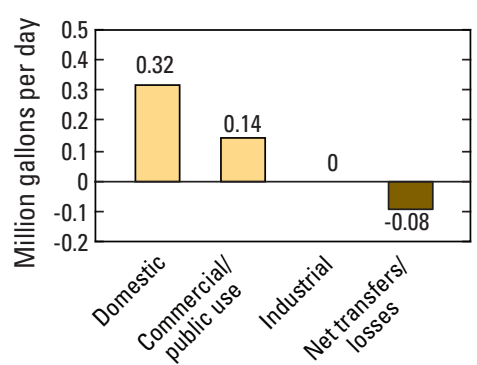

Surface-Water Withdrawals by Year

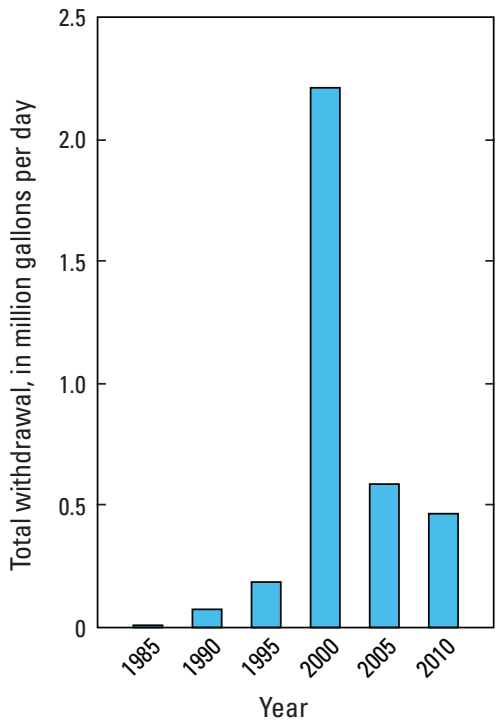

Groundwater Withdrawals by Year

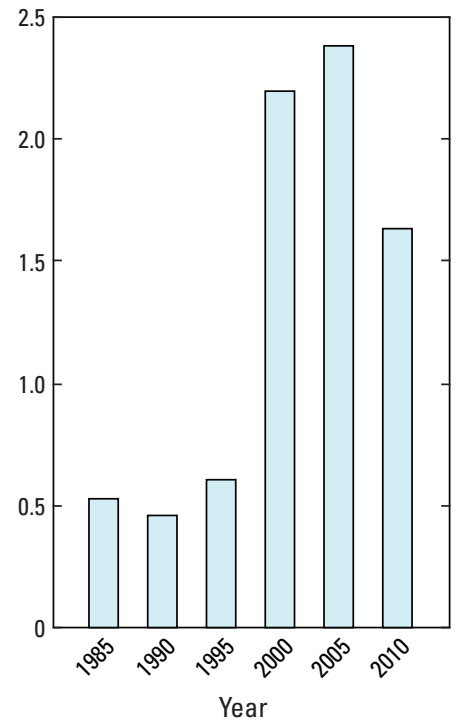




\section{LOWNDES COUNTY}

Population

109,233

Population served by public supply-Groundwater Population served by public supply-Surface water Acres irrigated
92,400

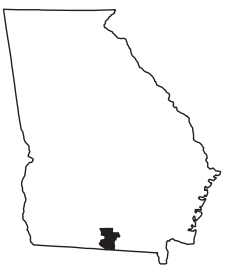

2010 WATER WITHDRAWALS AND ESTIMATED USE, IN MILLION GALLONS PER DAY

$[-$, not applicable $]$

\begin{tabular}{|c|c|c|c|c|c|}
\hline \multirow[b]{2}{*}{ Category } & \multicolumn{3}{|c|}{ Withdrawals } & \multirow[b]{2}{*}{$\begin{array}{c}\text { Total } \\
\text { use }^{1}\end{array}$} & \multirow{2}{*}{$\begin{array}{c}\text { Surface } \\
\text { water } \\
\text { returns }\end{array}$} \\
\hline & $\begin{array}{c}\text { Ground- } \\
\text { water }\end{array}$ & $\begin{array}{c}\text { Surface } \\
\text { water }\end{array}$ & Total & & \\
\hline Public supply & 14.01 & 0.00 & 14.01 & - & - \\
\hline Domestic & 1.26 & 0.00 & 1.26 & 8.92 & 0.00 \\
\hline Commercial/public use & 0.00 & 0.00 & 0.00 & 3.08 & 0.00 \\
\hline Industrial & 8.99 & 0.00 & 8.99 & 10.13 & 0.00 \\
\hline Public-supply losses & - & - & - & 2.13 & - \\
\hline Public wastewater treatment & - & - & - & - & 9.70 \\
\hline Mining & 0.00 & 0.00 & 0.00 & 0.00 & 0.00 \\
\hline Irrigation-Crop & 1.04 & 0.14 & 1.18 & 1.18 & 0.00 \\
\hline Irrigation-Golf course & 0.26 & 0.00 & 0.26 & 0.26 & 0.00 \\
\hline Livestock/aquaculture & 0.03 & 0.06 & 0.09 & 0.09 & 0.00 \\
\hline Thermoelectric power & 0.00 & 0.00 & 0.00 & 0.00 & 0.00 \\
\hline TOTAL & 25.59 & 0.20 & 25.79 & 25.79 & 9.70 \\
\hline
\end{tabular}

'Total use is total withdrawal plus public supply deliveries and losses.

\section{Withdrawals by Source}

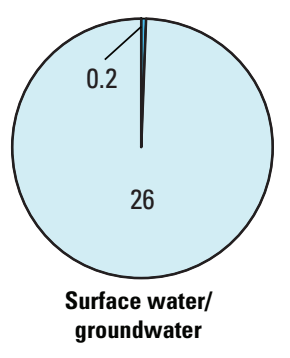

Withdrawals, in million gallons per day

Surface water

Groundwater

$\square$ Upper Floridan aquifer groundwater

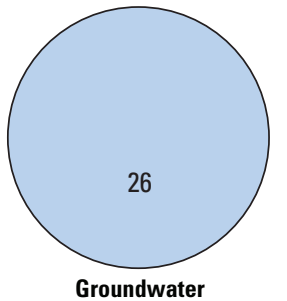

Groundwater
Withdrawals by Major Industrial Groups

[NAICS, North American Industrial Classification System code]

\begin{tabular}{lcc}
\hline NAICS & Groundwater & Surface water \\
\hline 322-Paper, pulp & 8.28 & 0.00 \\
325-Chemical products & 0.66 & 0.00 \\
335-Misc. manufacturing & 0.05 & 0.00
\end{tabular}

Withdrawals by Major Public Suppliers

Name Groundwater Surface water

$\begin{array}{lll}\text { City of Valdosta } & 10.79 & 0.00\end{array}$

$\begin{array}{lll}\text { Lowndes County } & 1.58 & 0.00\end{array}$

Water System

$\begin{array}{lll}\text { USAF Moody Airbase } & 0.36 & 0.00\end{array}$

$\begin{array}{lll}\text { City of Hahira } & 0.14 & 0.00\end{array}$

$\begin{array}{lll}\text { City of Lake Park } & 0.10 & 0.00\end{array}$

City of Remerton $\quad 0.08 \quad 0.00$

Public-Supply Deliveries by Use Category

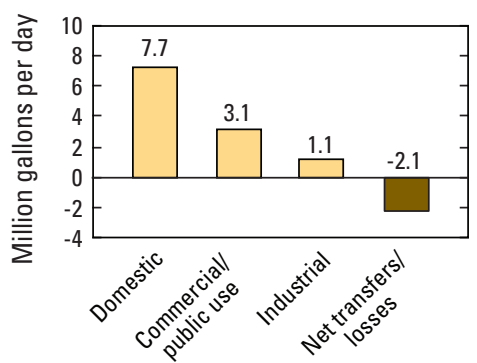

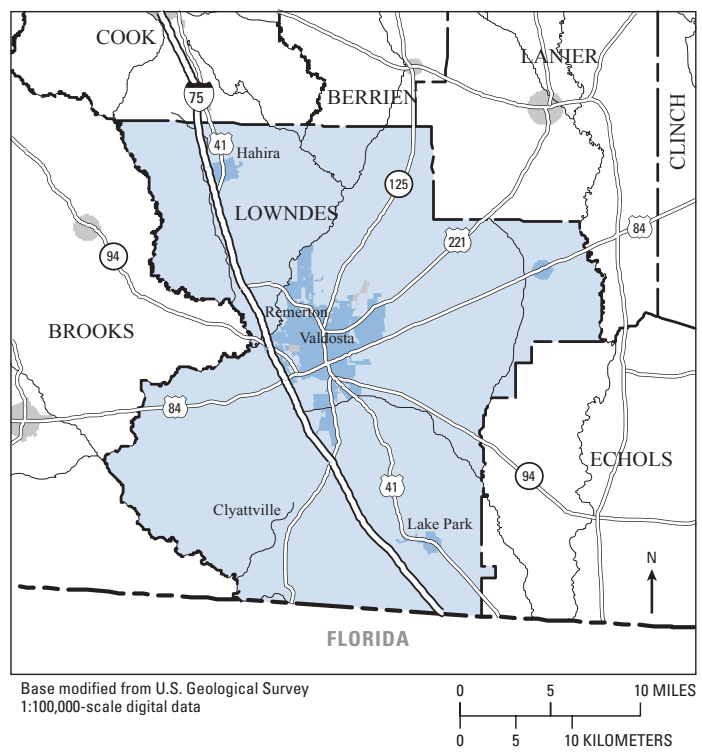

Surface-Water Withdrawals by Year

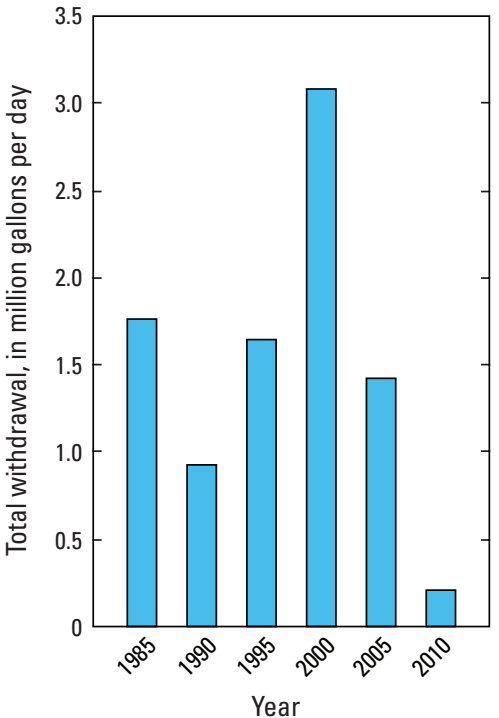

Groundwater Withdrawals by Year

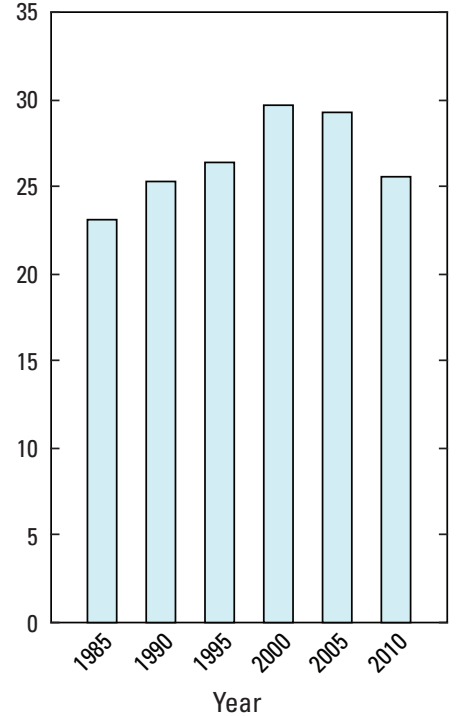




\section{LUMPKIN COUNTY}

Population

Population served by public supply-Groundwater $\quad 4,320$

Population served by public supply-Surface water $\quad 6,000$

Acres irrigated

230

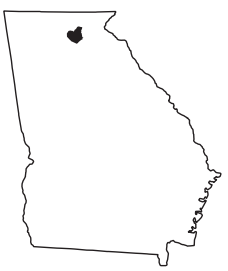

2010 WATER WITHDRAWALS AND ESTIMATED USE, IN MILLION GALLONS PER DAY

$[-$, not applicable $]$

\begin{tabular}{|c|c|c|c|c|c|c|c|c|}
\hline \multirow{3}{*}{ Category } & \multicolumn{3}{|c|}{ Withdrawals } & \multirow{3}{*}{$\begin{array}{l}\text { Total } \\
\text { use }^{1}\end{array}$} & \multirow{3}{*}{$\begin{array}{l}\text { Surface- } \\
\text { water } \\
\text { returns }\end{array}$} & \multirow{2}{*}{\multicolumn{3}{|c|}{$\begin{array}{l}\text { Withdrawals by Major Industrial Groups } \\
\text { [NAICS, North American Industrial Classification System code] }\end{array}$}} \\
\hline & \multirow{2}{*}{$\begin{array}{c}\text { Ground- } \\
\text { water }\end{array}$} & \multirow{2}{*}{$\begin{array}{c}\text { Surface } \\
\text { water }\end{array}$} & \multirow[b]{2}{*}{ Total } & & & & & \\
\hline & & & & & & NAICS & Groundwater & Surface water \\
\hline Public supply & 0.34 & 0.98 & 1.32 & - & - & & & \\
\hline Domestic & 1.47 & 0.00 & 1.47 & 2.19 & 0.00 & None & - & - \\
\hline Commercial/public use & 0.00 & 0.00 & 0.00 & 0.28 & 0.00 & & & \\
\hline Industrial & 0.00 & 0.00 & 0.00 & 0.14 & 0.00 & & & \\
\hline Public-supply losses & - & - & - & 0.18 & - & & & \\
\hline Public wastewater treatment & - & - & - & - & 0.62 & Withdrawals by 1 & r Public Supp & \\
\hline Mining & 0.00 & 0.04 & 0.04 & 0.04 & 0.00 & Name & Groundwater & Surface water \\
\hline Irrigation - Crop & 0.10 & 0.02 & 0.12 & 0.12 & 0.00 & & & \\
\hline Irrigation-Golf course & 0.16 & 0.00 & 0.16 & 0.16 & 0.00 & City of Dahlonega & 0.10 & 0.98 \\
\hline Livestock/aquaculture & 0.00 & 0.15 & 0.15 & 0.15 & 0.00 & Lumpkin County & & \\
\hline Thermoelectric power & 0.00 & 0.00 & 0.00 & 0.00 & 0.00 & Water and Sewer & 0.14 & 0.00 \\
\hline TOTAL & 2.07 & 1.19 & 3.26 & 3.26 & 0.62 & Authority & & \\
\hline
\end{tabular}

Total use is total withdrawal plus public supply deliveries and losses.

\section{Withdrawals by Source}
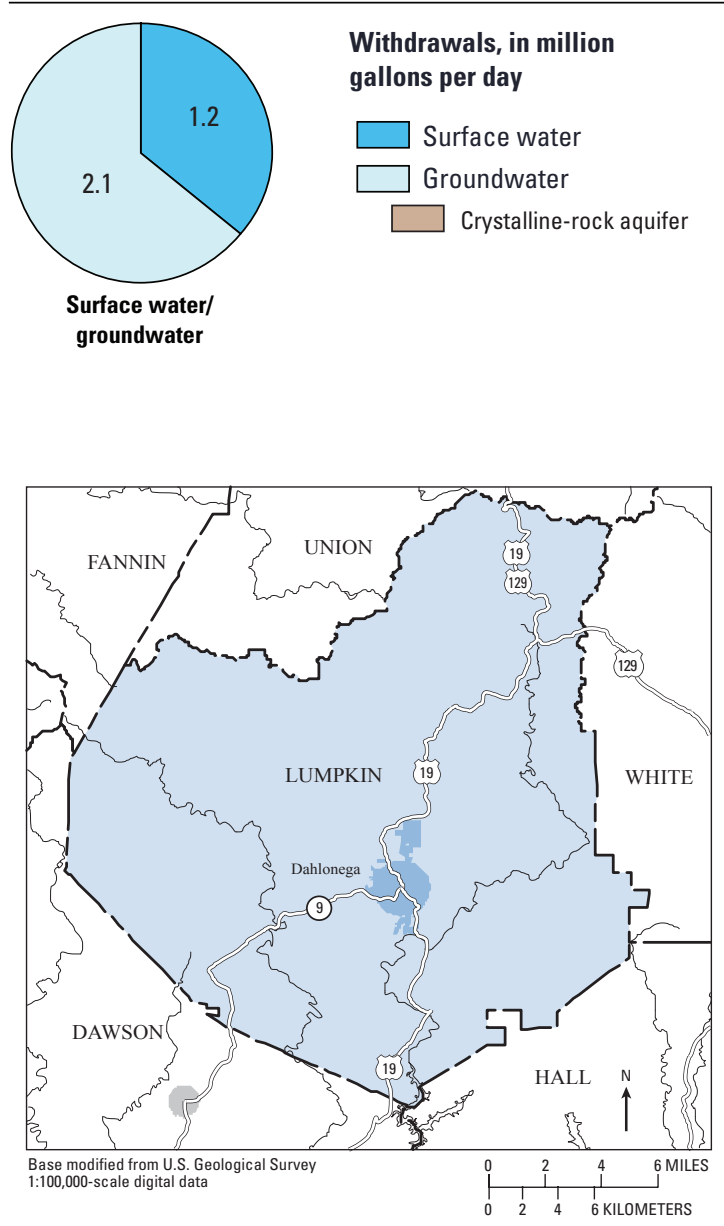

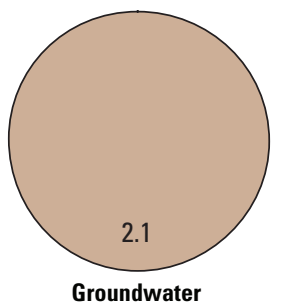

Public-Supply Deliveries by Use Category

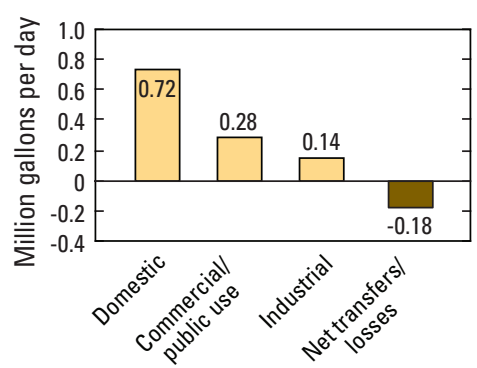

Surface-Water Withdrawals by Year

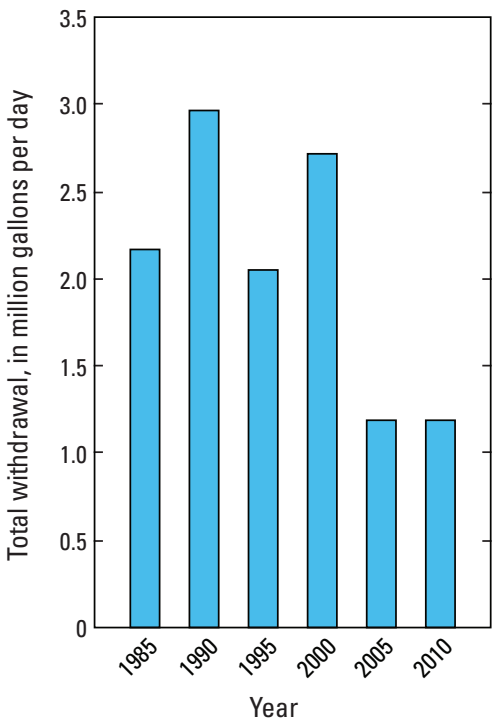

Groundwater Withdrawals by Year

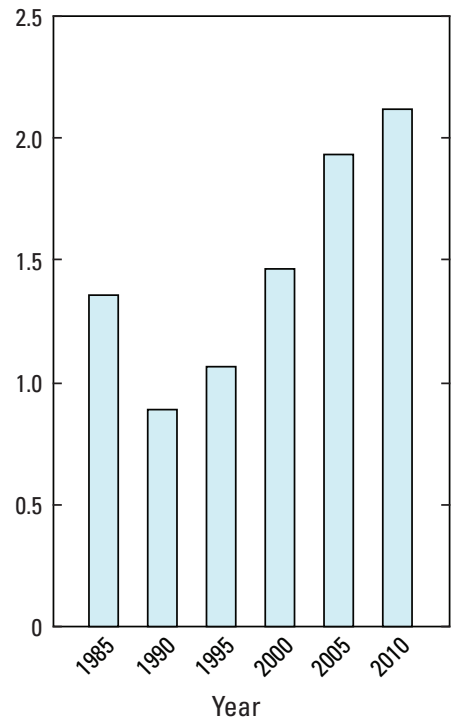


MACON COUNTY

Population

14,740

$\begin{array}{lr}\text { Population served by public supply_-Groundwater } & 6,570 \\ \text { Population served by public supply_-Surface water } & 0\end{array}$

Acres irrigated

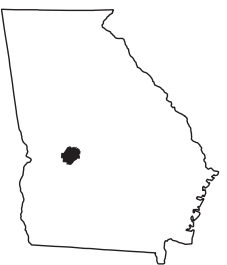

2010 WATER WITHDRAWALS AND ESTIMATED USE, IN MILLION GALLONS PER DAY

$[-$, not applicable $]$

\begin{tabular}{|c|c|c|c|c|c|c|c|c|}
\hline \multirow{3}{*}{ Category } & \multicolumn{3}{|c|}{ Withdrawals } & \multirow{3}{*}{$\begin{array}{c}\text { Total } \\
\text { use }^{1}\end{array}$} & \multirow{3}{*}{$\begin{array}{l}\text { Surface- } \\
\text { water } \\
\text { returns } \\
\end{array}$} & \multirow{2}{*}{\multicolumn{3}{|c|}{$\begin{array}{l}\text { Withdrawals by Major Industrial Groups } \\
\text { [NAICS, North American Industrial Classification System code] }\end{array}$}} \\
\hline & \multirow{2}{*}{$\begin{array}{c}\text { Ground- } \\
\text { water }\end{array}$} & \multirow{2}{*}{$\begin{array}{c}\begin{array}{c}\text { Surface } \\
\text { water }\end{array} \\
\end{array}$} & \multirow[b]{2}{*}{ Total } & & & & & \\
\hline & & & & & & NAICS & Groundwater & Surface water \\
\hline Public supply & 0.88 & 0.00 & 0.88 & - & - & 325 -Paper, pulp & 0.57 & 9.24 \\
\hline $\begin{array}{l}\text { Domestic } \\
\text { Commercial/public use }\end{array}$ & $\begin{array}{l}0.61 \\
0.01\end{array}$ & $\begin{array}{l}0.00 \\
0.00\end{array}$ & $\begin{array}{l}0.61 \\
0.01\end{array}$ & $\begin{array}{l}1.09 \\
0.09\end{array}$ & $\begin{array}{l}0.00 \\
0.00\end{array}$ & 311 -Food products & 0.19 & 0.00 \\
\hline Industrial & 0.81 & 9.24 & $\begin{array}{r}0.01 \\
10.05\end{array}$ & 10.20 & 0.02 & 212-Kaolin and ball clay & 0.05 & 0.00 \\
\hline
\end{tabular}

Public-supply losses

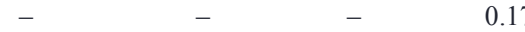

Public wastewater treatment

Mining

$-$

Irrigation-Crop

0.00

$-$

10.54

0.00

Irrigation-Golf course

0.00

4.20

0.00

Livestock/aquaculture

0.13

0.00

14.74

$-$

1.31

Thermoelectric power

0.00

0.53

0.00

0.00

0.00

0.00

14.74

0.00

TOTAL

12.98

0.00

0.66

0.66

0.00

13.97

26.95

0.00

0.00

1.33

'Total use is total withdrawal plus public supply deliveries and losses

\section{Withdrawals by Source}

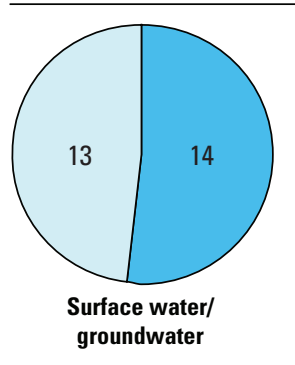

Withdrawals, in million gallons per day
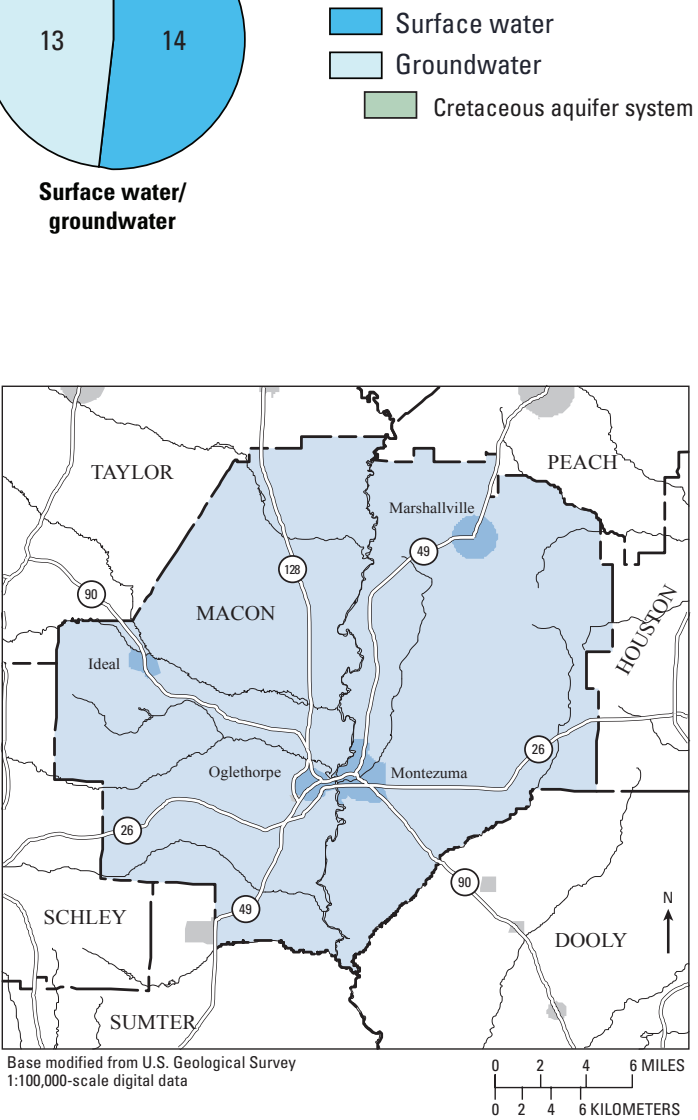

Surface-Water Withdrawals by Year

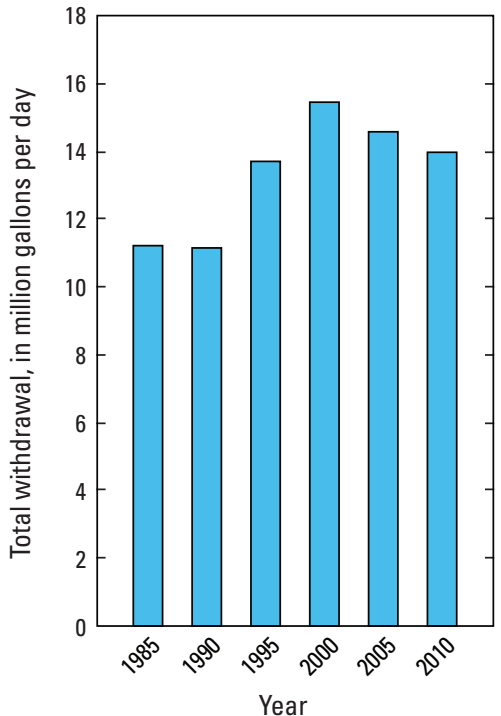

Groundwater Withdrawals by Year

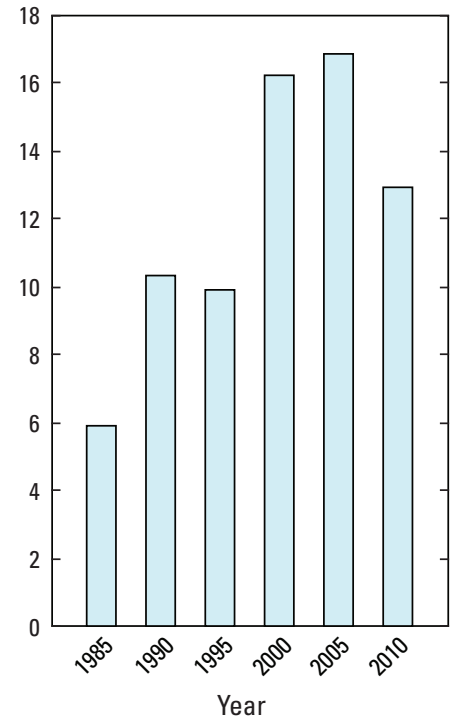

Withdrawals by Major Public Suppliers

\begin{tabular}{lcc}
\hline Name & Groundwater & Surface water \\
\hline City of Montezuma & 0.30 & 0.00 \\
City of Oglethorpe & 0.44 & 0.00 \\
City of Marshallville & 0.14 & 0.00 \\
\hline
\end{tabular}

Public-Supply Deliveries by Use Category

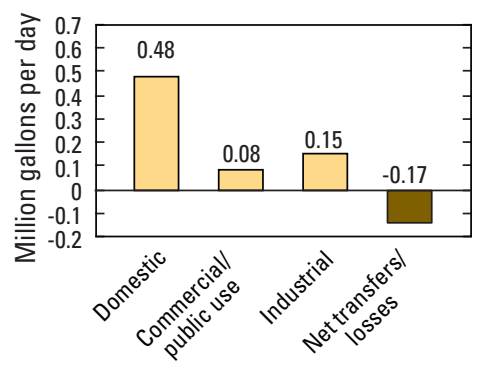


MADISON COUNTY

Population

Population served by public supply-Groundwater $\quad 7,840$

Population served by public supply-Surface water 0

Acres irrigated

140

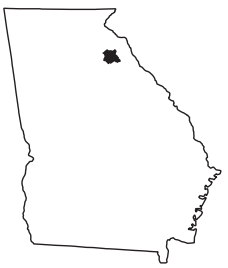

2010 WATER WITHDRAWALS AND ESTIMATED USE, IN MILLION GALLONS PER DAY

[-, not applicable; Mgal/d, million gallons per day]

\begin{tabular}{|c|c|c|c|c|c|c|c|c|}
\hline \multirow{3}{*}{ Category } & \multicolumn{3}{|c|}{ Withdrawals } & \multirow{3}{*}{$\begin{array}{l}\text { Total } \\
\text { use }^{1}\end{array}$} & \multirow{3}{*}{$\begin{array}{l}\text { Surface- } \\
\text { water } \\
\text { returns } \\
\end{array}$} & \multirow{2}{*}{\multicolumn{3}{|c|}{$\begin{array}{l}\text { Withdrawals by Major Industrial Groups } \\
\text { [NAICS, North American Industrial Classification System code] }\end{array}$}} \\
\hline & \multirow{2}{*}{$\begin{array}{c}\text { Ground- } \\
\text { water }\end{array}$} & \multirow{2}{*}{$\begin{array}{c}\text { Surface } \\
\text { water }\end{array}$} & \multirow[b]{2}{*}{ Total } & & & & & \\
\hline & & & & & & NAICS & Groundwater & Surface water \\
\hline Public supply & 0.50 & 0.00 & 0.50 & - & - & 327 -Stone, clay & 0.00 & 0.10 \\
\hline Domestic & 1.52 & 0.00 & 1.52 & 1.94 & 0.00 & 321 -stone, cray & 0.00 & 0.10 \\
\hline Commercial/public use & 0.00 & 0.00 & 0.00 & 0.05 & 0.00 & & & \\
\hline Industrial & 0.00 & 0.10 & 0.10 & 0.11 & 0.00 & & & \\
\hline Public-supply losses & - & - & - & 0.15 & - & & & \\
\hline Public wastewater treatment & - & - & - & - & 0.05 & Withdrawals by Ma & r Public Supp & \\
\hline Mining & 0.02 & 0.00 & 0.02 & 0.02 & 0.00 & Name & Groundwater & Surface water \\
\hline Irrigation-Crop & 0.00 & 0.00 & 0.00 & 0.00 & 0.00 & & & \\
\hline Irrigation-Golf course & 0.00 & 0.30 & 0.30 & 0.30 & 0.00 & City of Danielsville & 0.12 & 0.00 \\
\hline Livestock/aquaculture & 0.00 & 0.80 & 0.80 & 0.80 & 0.00 & City of Comer & 0.12 & 0.00 \\
\hline Thermoelectric power & 0.00 & 0.00 & 0.00 & 0.00 & 0.00 & Piedmont Water Co. & 0.08 & 0.00 \\
\hline TOTAL & 2.04 & 1.20 & 3.24 & 3.37 & 0.05 & City of Colbert & 0.05 & 0.00 \\
\hline
\end{tabular}

'Total use is total withdrawal plus public supply deliveries and losses. An average of $0.13 \mathrm{Mgal} / \mathrm{d}$ was delivered from Franklin County,

Georgia to Madison County in 2010.

\section{Withdrawals by Source}
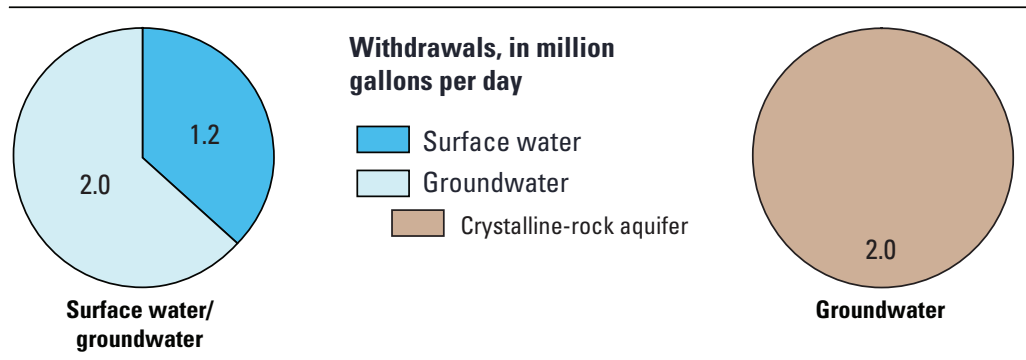

Public-Supply Deliveries ${ }^{1}$ by Use Category
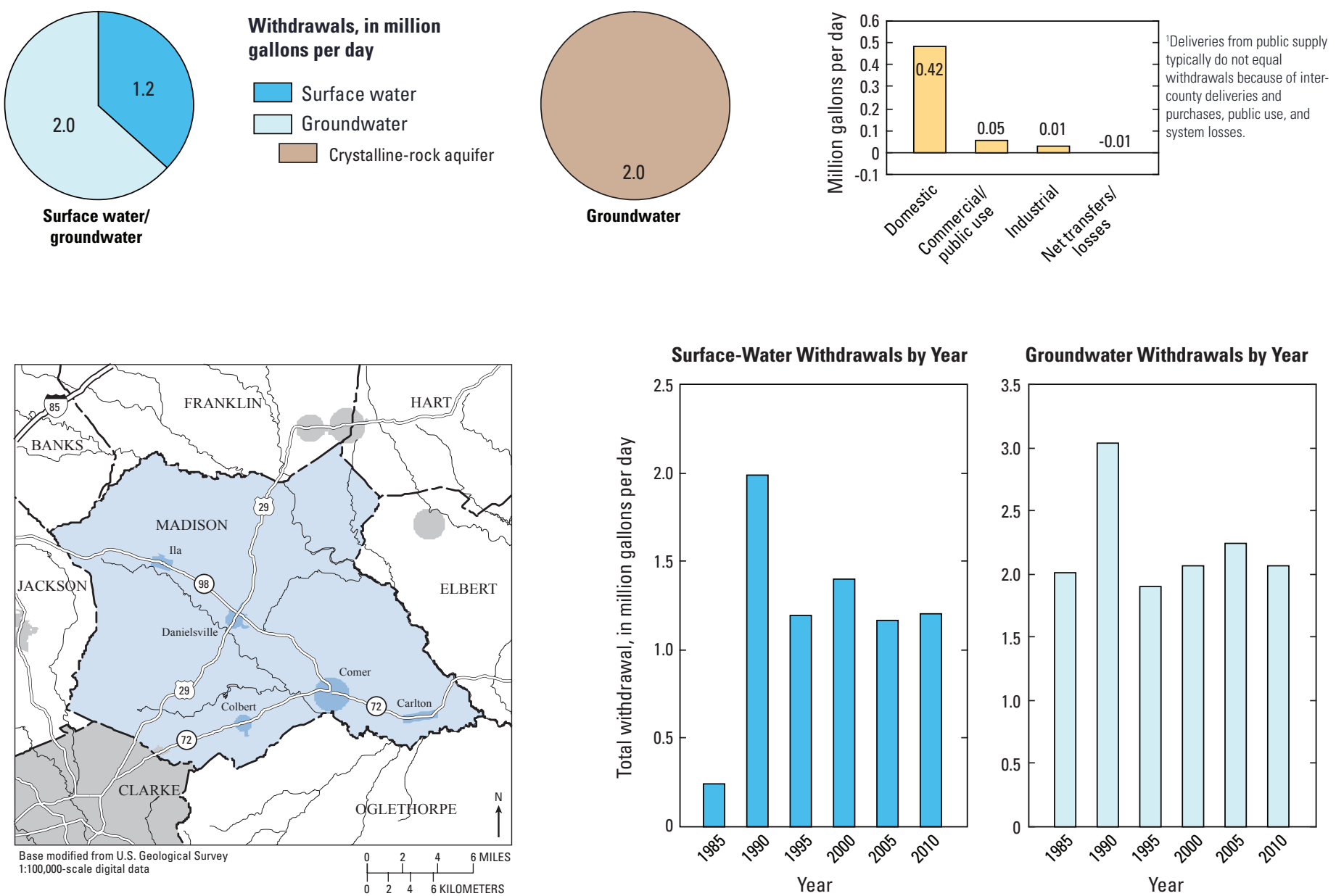

Groundwater Withdrawals by Year

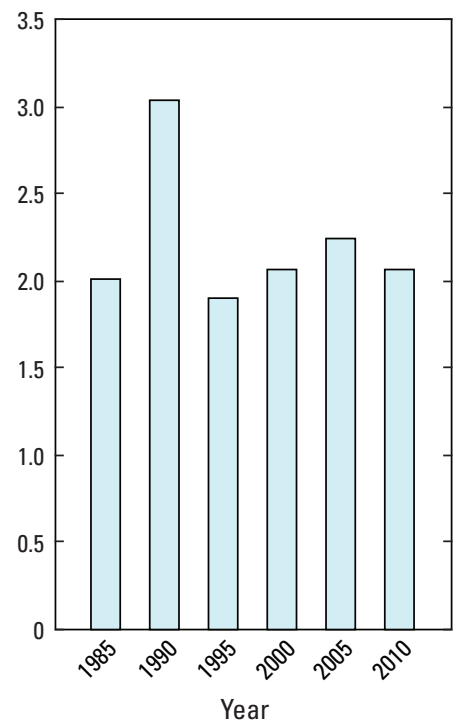


MARION COUNTY

Population

Population served by public supply-Groundwater

Population served by public supply-Surface water

Acres irrigated
8,742

6,290

5,770

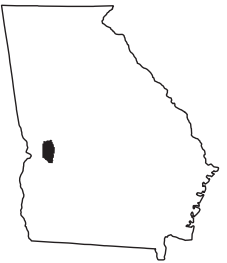

2010 WATER WITHDRAWALS AND ESTIMATED USE, IN MILLION GALLONS PER DAY

$[-$, not applicable $]$

\begin{tabular}{|c|c|c|c|c|c|}
\hline \multirow[b]{2}{*}{ Category } & \multicolumn{3}{|c|}{ Withdrawals } & \multirow[b]{2}{*}{$\begin{array}{l}\text { Total } \\
\text { use }^{1}\end{array}$} & \multirow{2}{*}{$\begin{array}{c}\text { Surface- } \\
\text { water } \\
\text { returns }\end{array}$} \\
\hline & $\begin{array}{c}\text { Ground- } \\
\text { water }\end{array}$ & $\begin{array}{c}\text { Surface } \\
\text { water }\end{array}$ & Total & & \\
\hline Public supply & 0.72 & 0.00 & 0.72 & - & - \\
\hline Domestic & 0.18 & 0.00 & 0.18 & 0.65 & 0.00 \\
\hline Commercial/public use & 0.00 & 0.00 & 0.00 & 0.06 & 0.00 \\
\hline Industrial & 0.00 & 0.27 & 0.27 & 0.32 & 0.28 \\
\hline Public-supply losses & - & - & - & 0.14 & - \\
\hline Public wastewater treatment & - & - & - & - & 0.20 \\
\hline Mining & 0.24 & 0.00 & 0.24 & 0.24 & 0.00 \\
\hline Irrigation-Crop & 0.08 & 1.23 & 1.31 & 1.31 & 0.00 \\
\hline Irrigation-Golf course & 0.00 & 0.00 & 0.00 & 0.00 & 0.00 \\
\hline Livestock/aquaculture & 0.02 & 0.12 & 0.14 & 0.14 & 0.00 \\
\hline Thermoelectric power & 0.00 & 0.00 & 0.00 & 0.00 & 0.00 \\
\hline TOTAL & 1.24 & 1.62 & 2.86 & 2.86 & 0.48 \\
\hline
\end{tabular}

Withdrawals by Major Industrial Groups

[NAICS, North American Industrial Classification System code]

NAICS Groundwater Surface water

212 -Kaolin and ball clay $\quad 0.00 \quad 0.27$

Name Groundwater Surface water

Buena Vista-Marion

County Water System

0.72

0.00

'Total use is total withdrawal plus public supply deliveries and losses.

\section{Withdrawals by Source}
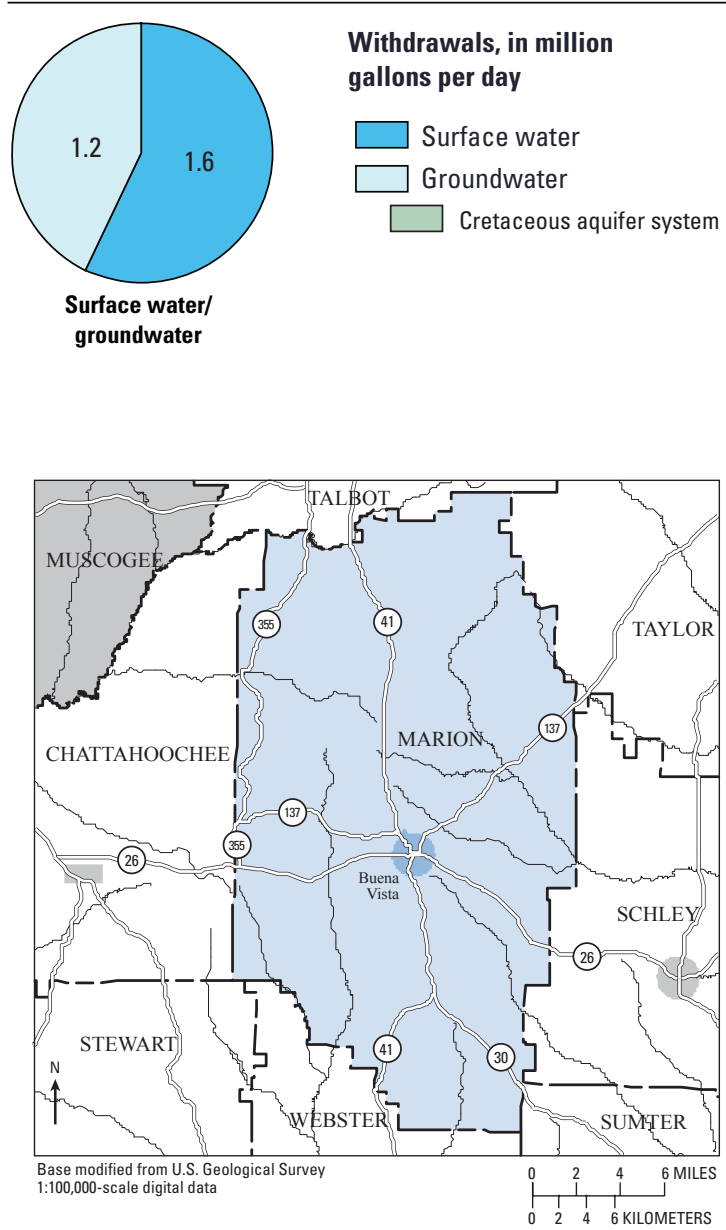

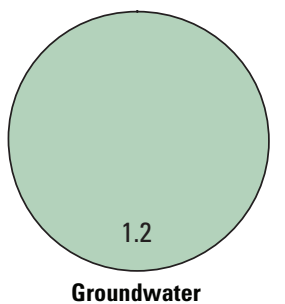

Public-Supply Deliveries by Use Category

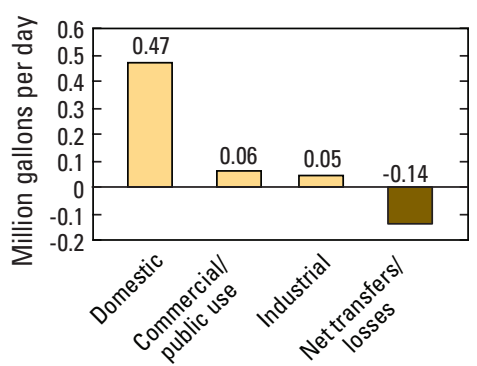

Surface-Water Withdrawals by Year

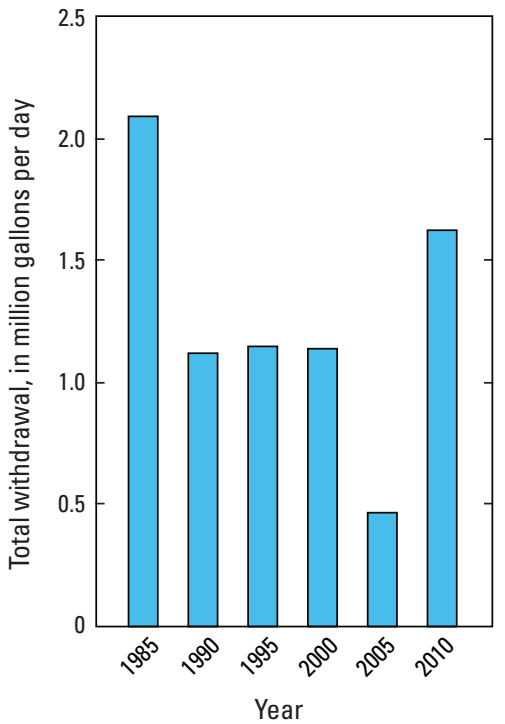

Groundwater Withdrawals by Year

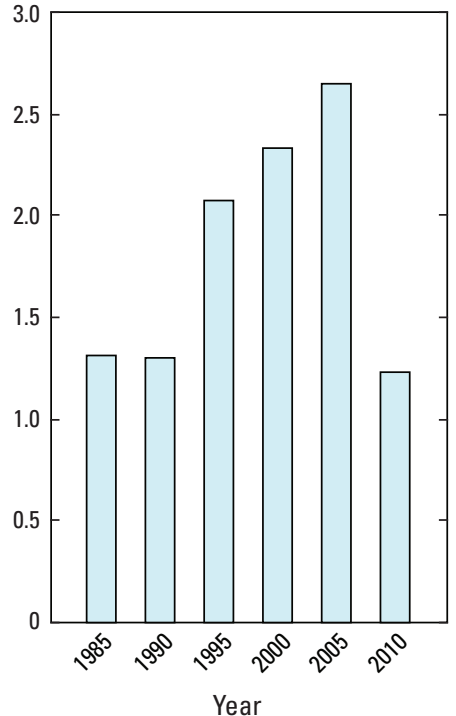


MCDUFFIE COUNTY

Population

21,875

Population served by public supply-Groundwater

Population served by public supply-Surface water $\quad 14,780$

Acres irrigated

900

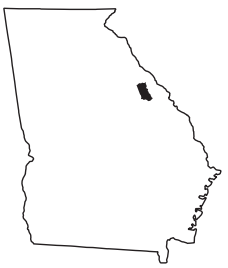

2010 WATER WITHDRAWALS AND ESTIMATED USE, IN MILLION GALLONS PER DAY

$[-$, not applicable $]$

\begin{tabular}{|c|c|c|c|c|c|c|c|c|}
\hline \multirow{3}{*}{ Category } & \multicolumn{3}{|c|}{ Withdrawals } & \multirow{3}{*}{$\begin{array}{l}\text { Total } \\
\text { use }^{1}\end{array}$} & \multirow{3}{*}{$\begin{array}{l}\text { Surface- } \\
\text { water } \\
\text { returns } \\
\end{array}$} & \multirow{2}{*}{\multicolumn{3}{|c|}{$\begin{array}{l}\text { Withdrawals by Major Industrial Groups } \\
\text { [NAICS, North American Industrial Classification System code] }\end{array}$}} \\
\hline & \multirow{2}{*}{$\begin{array}{c}\text { Ground- } \\
\text { water }\end{array}$} & \multirow{2}{*}{$\begin{array}{c}\text { Surface } \\
\text { water }\end{array}$} & \multirow[b]{2}{*}{ Total } & & & & & \\
\hline & & & & & & NAICS & Groundwater & Surface water \\
\hline \multirow{2}{*}{ Public supply } & 0.00 & 2.20 & 2.20 & \multirow{2}{*}{$\begin{array}{l}- \\
1.50\end{array}$} & \multirow{2}{*}{$\begin{array}{c}- \\
0.00\end{array}$} & \multirow{2}{*}{ None } & \multirow{2}{*}{-} & \multirow{2}{*}{-} \\
\hline & 0.53 & 0.00 & 0.53 & & & & & \\
\hline Commercial/public use & 0.00 & 0.00 & 0.00 & 0.52 & 0.00 & \multirow{4}{*}{\multicolumn{3}{|c|}{ Withdrawals by Major Public Suppliers }} \\
\hline Industrial & 0.00 & 0.00 & 0.00 & 0.35 & 0.00 & & & \\
\hline Public-supply losses & - & - & - & 0.36 & - & & & \\
\hline \multirow{2}{*}{$\begin{array}{l}\text { Public wastewater treatment } \\
\text { Mining }\end{array}$} & - & \multirow{2}{*}{0.00} & \multirow{2}{*}{0.00} & \multirow{2}{*}{0.00} & \multirow{2}{*}{$\begin{array}{l}1.21 \\
0.00\end{array}$} & & & \\
\hline & 0.00 & & & & & Name & Groundwater & Surface water \\
\hline Irrigation-Golf course & 0.00 & 0.39 & 0.39 & 0.39 & 0.00 & \multirow{3}{*}{$\begin{array}{l}\text { Thomson-McDuffie } \\
\text { County Water and } \\
\text { Sewer Commission }\end{array}$} & & \\
\hline Livestock/aquaculture & 0.00 & 0.14 & 0.14 & 0.14 & 0.00 & & 0.00 & 2.20 \\
\hline Thermoelectric power & 0.00 & 0.00 & 0.00 & 0.00 & 0.00 & & & \\
\hline TOTAL & 0.67 & 2.78 & 3.45 & 3.45 & 1.21 & & & \\
\hline
\end{tabular}

${ }^{1}$ Total use is total withdrawal plus public supply deliveries and losses.

\section{Withdrawals by Source}

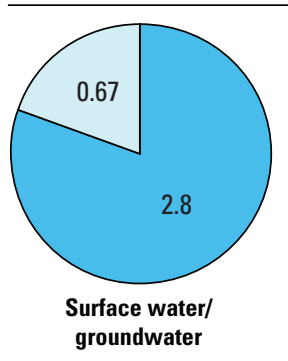

Withdrawals, in million gallons per day

$\square$ Surface water

$\square$ Groundwater

$\square$ Crystalline-rock aquifer groundwater

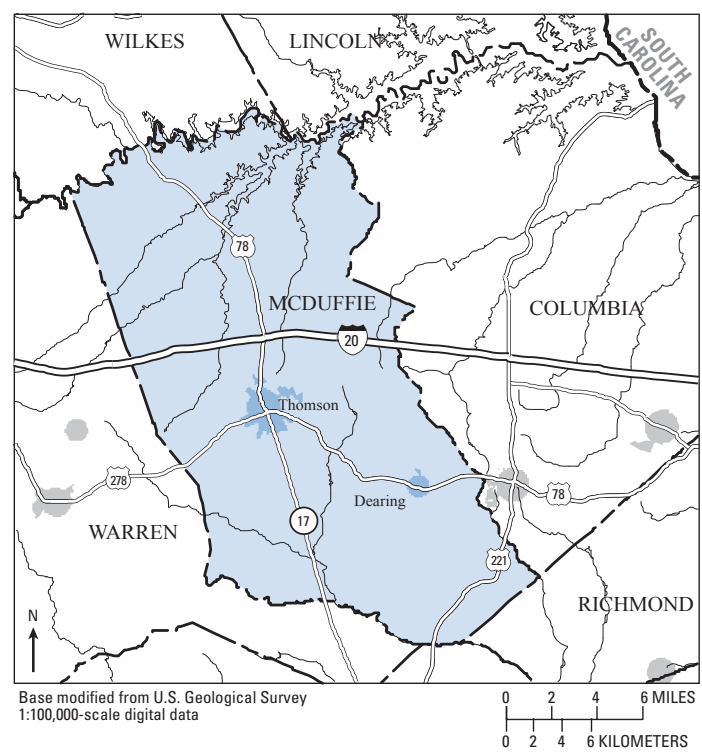

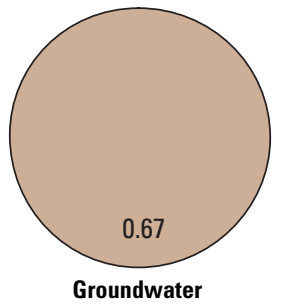

Public-Supply Deliveries by Use Category

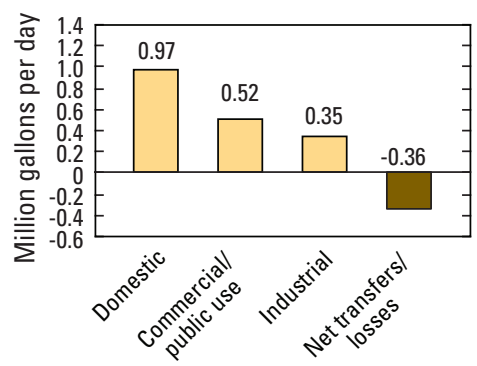

Surface-Water Withdrawals by Year

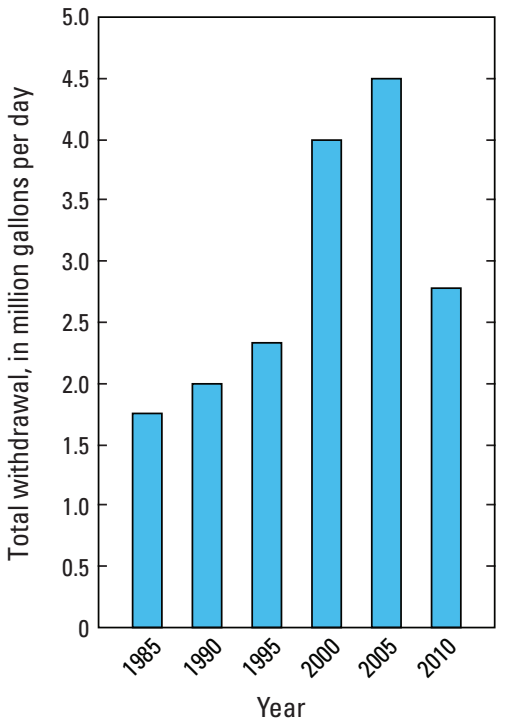

Groundwater Withdrawals by Year

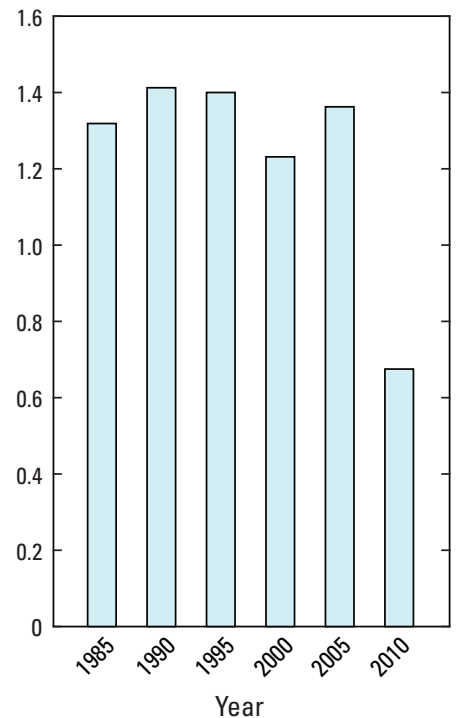




\section{MCINTOSH COUNTY}

Population

14,333

Population served by public supply-Groundwater

10,870

Population served by public supply-Surface water Acres irrigated

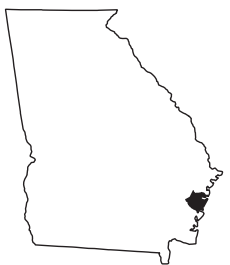

2010 WATER WITHDRAWALS AND ESTIMATED USE, IN MILLION GALLONS PER DAY

$[-$, not applicable; $<$, less than $]$

\begin{tabular}{|c|c|c|c|c|c|c|c|c|}
\hline \multirow{3}{*}{ Category } & \multicolumn{3}{|c|}{ Withdrawals } & \multirow{3}{*}{$\begin{array}{l}\text { Total } \\
\text { use }^{1}\end{array}$} & \multirow{3}{*}{$\begin{array}{l}\text { Surface- } \\
\text { water } \\
\text { returns } \\
\end{array}$} & \multirow{2}{*}{\multicolumn{3}{|c|}{$\begin{array}{l}\text { Withdrawals by Major Industrial Groups } \\
\text { [NAICS, North American Industrial Classification System code] }\end{array}$}} \\
\hline & \multirow{2}{*}{$\begin{array}{c}\text { Ground- } \\
\text { water }\end{array}$} & \multirow{2}{*}{$\begin{array}{c}\text { Surface } \\
\text { water }\end{array}$} & \multirow[b]{2}{*}{ Total } & & & & & \\
\hline & & & & & & NAICS & Groundwater & Surface water \\
\hline Public supply & 1.05 & 0.00 & 1.05 & - & - & & & \\
\hline Domestic & 0.26 & 0.00 & 0.26 & 1.21 & 0.00 & None & - & - \\
\hline Commercial/public use & 0.00 & 0.00 & 0.00 & 0.04 & 0.00 & & & \\
\hline Industrial & 0.00 & 0.00 & 0.00 & $<0.01$ & 0.00 & & & \\
\hline Public-supply losses & - & - & - & 0.06 & - & Withdrawals by Major & Public Supp & \\
\hline Public wastewater treatment & - & - & - & - & 0.26 & Name & Groundwater & Surface water \\
\hline Mining & 0.00 & 0.00 & 0.00 & 0.00 & 0.00 & & & \\
\hline Irrigation-Crop & 0.00 & 0.00 & 0.00 & 0.00 & 0.00 & City of Darien & 0.40 & 0.00 \\
\hline Irrigation-Golf course & 0.25 & 0.00 & 0.25 & 0.25 & 0.00 & W and D Utilities & 0.20 & 0.00 \\
\hline Livestock/aquaculture & 0.00 & 0.00 & 0.00 & 0.00 & 0.00 & McIntosh County Water & 0.17 & 0.00 \\
\hline Thermoelectric power & 0.00 & 0.00 & 0.00 & 0.00 & 0.00 & System & 0.17 & 0.00 \\
\hline TOTAL & 1.56 & 0.00 & 1.56 & 1.56 & 0.26 & Water Utility Management & 0.11 & 0.00 \\
\hline
\end{tabular}

Total use is total withdrawal plus public supply deliveries and losses.

\section{Withdrawals by Source}
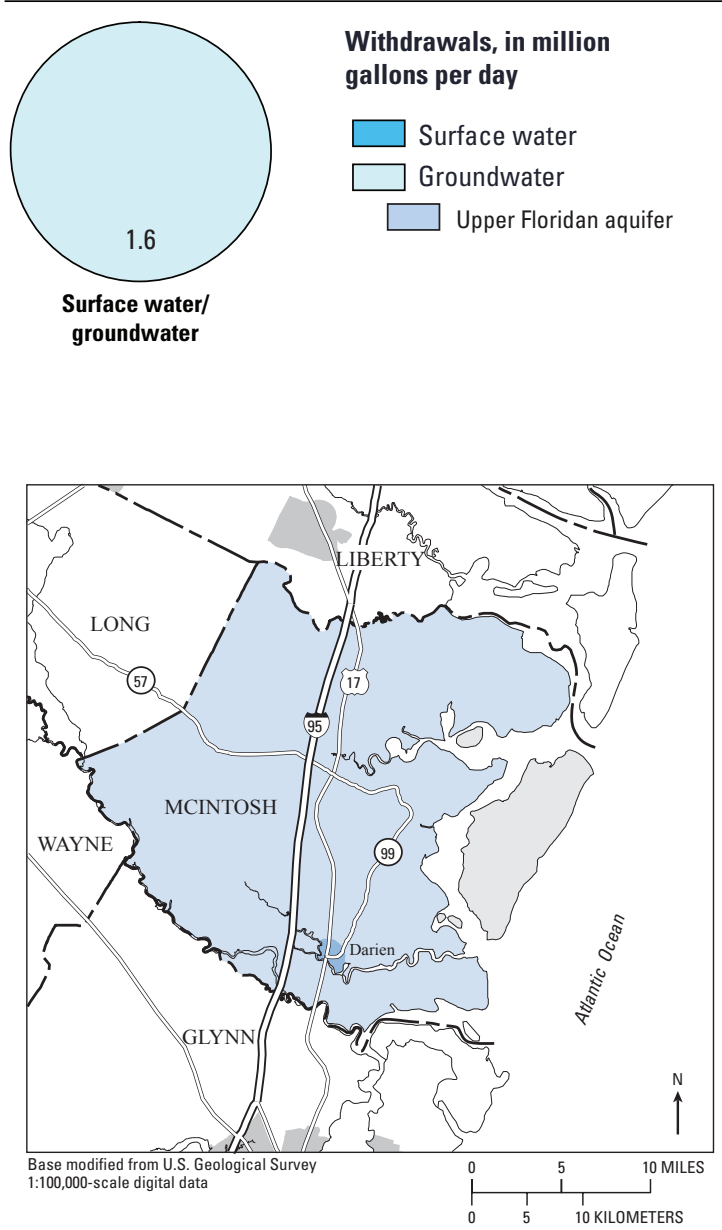

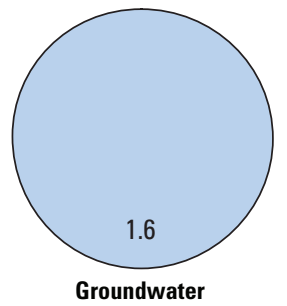

Public-Supply Deliveries by Use Category

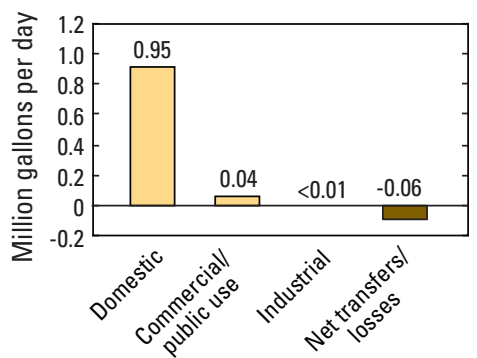

Surface-Water Withdrawals by Year

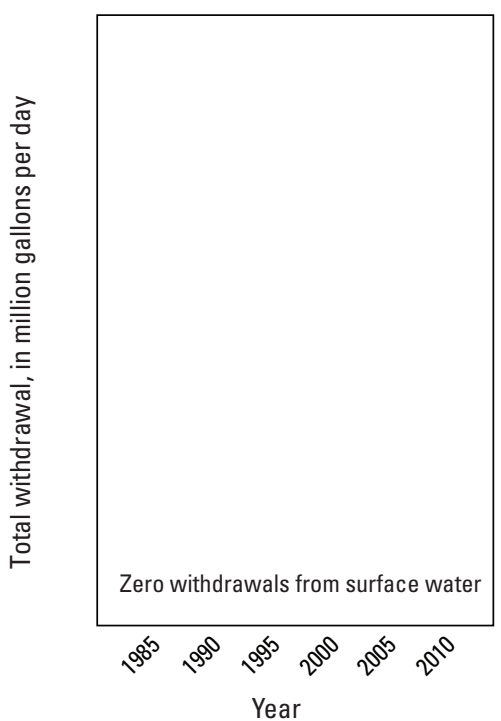

Groundwater Withdrawals by Year

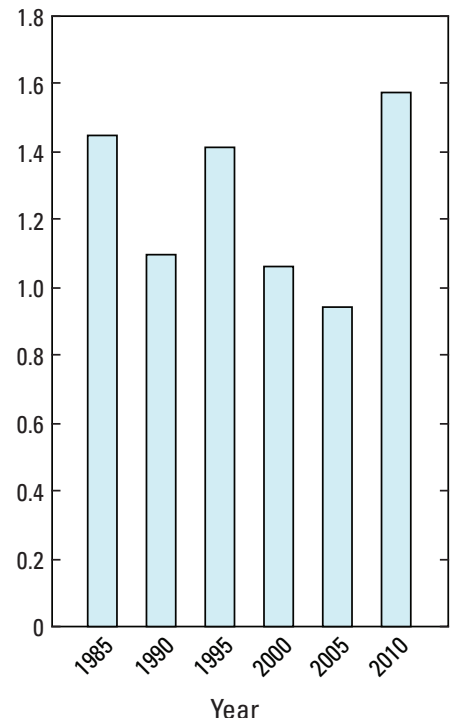


MERIWETHER COUNTY

Population

Population served by public supply-Groundwater

Population served by public supply-Surface water

8,440

Acres irrigated

320

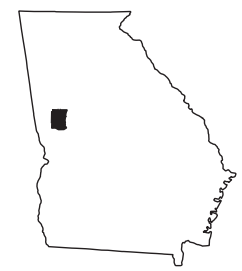

2010 WATER WITHDRAWALS AND ESTIMATED USE, IN MILLION GALLONS PER DAY

$[-$, not applicable; Mgal/d, million gallons per day $]$

\begin{tabular}{|c|c|c|c|c|c|c|c|c|}
\hline \multirow{3}{*}{ Category } & \multicolumn{3}{|c|}{ Withdrawals } & \multirow{3}{*}{$\begin{array}{l}\text { Total } \\
\text { use }^{1}\end{array}$} & \multirow{3}{*}{$\begin{array}{l}\text { Surface- } \\
\text { water } \\
\text { returns } \\
\end{array}$} & \multirow{2}{*}{\multicolumn{3}{|c|}{$\begin{array}{l}\text { Withdrawals by Major Industrial Groups } \\
\text { [NAICS, North American Industrial Classification System code] }\end{array}$}} \\
\hline & \multirow{2}{*}{$\begin{array}{c}\text { Ground- } \\
\text { water }\end{array}$} & \multirow{2}{*}{$\begin{array}{c}\text { Surface } \\
\text { water }\end{array}$} & \multirow[b]{2}{*}{ Total } & & & & & \\
\hline & & & & & & NAICS & Groundwater & Surface water \\
\hline Public supply & 0.11 & 0.12 & 0.23 & - & - & \multirow{2}{*}{321 -Wood } & \multirow{2}{*}{0.11} & \multirow{2}{*}{0.00} \\
\hline Domestic & 0.97 & 0.00 & 0.97 & 1.69 & 0.00 & & & \\
\hline Commercial/public use & 0.07 & 0.14 & 0.21 & 0.45 & 0.00 & & & \\
\hline Industrial & 0.11 & 0.00 & 0.11 & 0.12 & 0.00 & & & \\
\hline Public-supply losses & - & - & - & 0.24 & - & & & \\
\hline Public wastewater treatment & - & - & - & - & 0.35 & \multicolumn{3}{|c|}{ Withdrawals by Major Public Suppliers } \\
\hline Mining & 0.00 & 0.00 & 0.00 & 0.00 & 0.00 & \multirow{2}{*}{ Name } & \multirow[t]{2}{*}{ Groundwater } & \multirow{2}{*}{ Surface water } \\
\hline Irrigation-Crop & 0.09 & 0.05 & 0.14 & 0.14 & 0.00 & & & \\
\hline Irrigation-Golf course & 0.00 & 0.07 & 0.07 & 0.07 & 0.00 & City of Woodbury & 0.00 & 0.12 \\
\hline Livestock/aquaculture & 0.00 & 0.26 & 0.26 & 0.26 & 0.00 & City of Warm Springs & 0.05 & 0.00 \\
\hline Thermoelectric power & 0.00 & 0.00 & 0.00 & 0.00 & 0.00 & City of Luthersville & 0.04 & 0.00 \\
\hline TOTAL & 1.35 & 0.64 & 1.99 & 2.97 & 0.35 & & & \\
\hline
\end{tabular}

Total use is total withdrawal plus public supply deliveries and losses. An average of $0.69 \mathrm{Mgal} / \mathrm{d}$ was delivered from Talbot County, Georgia

and $0.24 \mathrm{Mgal} / \mathrm{d}$ from Troup County, Georgia to Meriwether County in 2010.

\section{Withdrawals by Source}
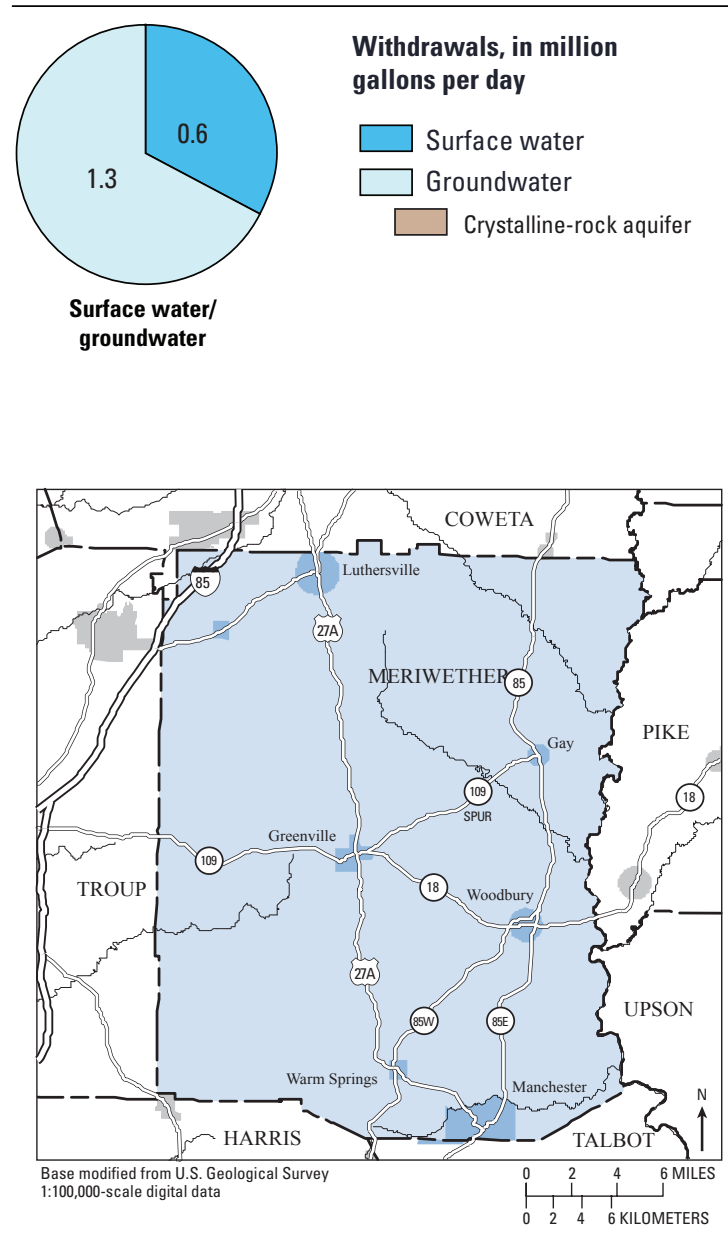

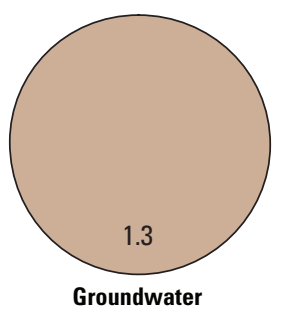

Public-Supply Deliveries ${ }^{1}$ by Use Category

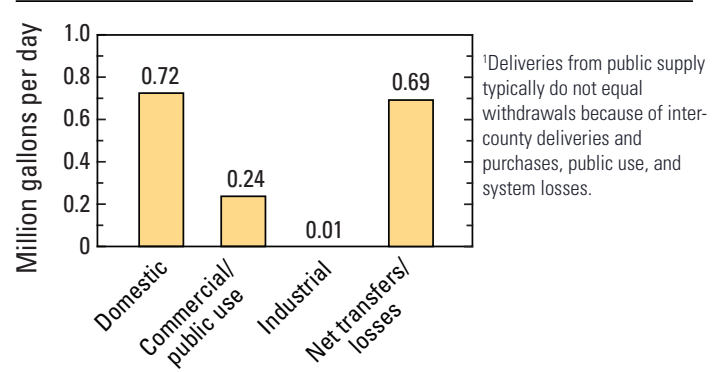

Surface-Water Withdrawals by Year

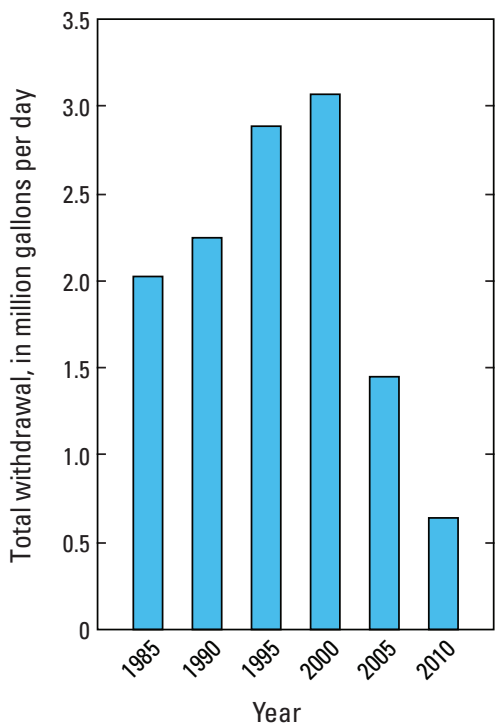

Groundwater Withdrawals by Year

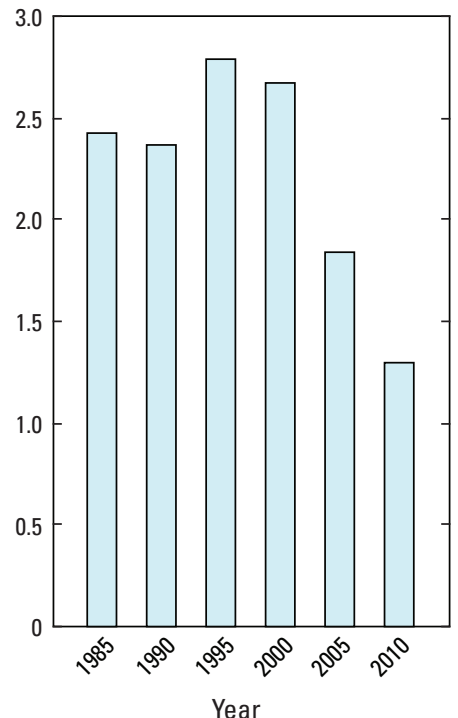




\section{MILLER COUNTY}

Population

Population served by public supply-Groundwater

Population served by public supply-Surface water Acres irrigated
6,125

1,970

0

59,290

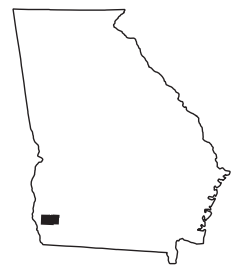

2010 WATER WITHDRAWALS AND ESTIMATED USE, IN MILLION GALLONS PER DAY

$[-$, not applicable $]$

\begin{tabular}{|c|c|c|c|c|c|c|c|c|}
\hline \multirow{3}{*}{ Category } & \multicolumn{3}{|c|}{ Withdrawals } & \multirow{3}{*}{$\begin{array}{l}\text { Total } \\
\text { use }^{1}\end{array}$} & \multirow{3}{*}{$\begin{array}{l}\text { Surface- } \\
\text { water } \\
\text { returns } \\
\end{array}$} & \multirow{2}{*}{\multicolumn{3}{|c|}{$\begin{array}{l}\text { Withdrawals by Major Industrial Groups } \\
\text { [NAICS, North American Industrial Classification System code] }\end{array}$}} \\
\hline & \multirow{2}{*}{$\begin{array}{c}\text { Ground- } \\
\text { water }\end{array}$} & \multirow{2}{*}{$\begin{array}{c}\text { Surface } \\
\text { water }\end{array}$} & \multirow[b]{2}{*}{ Total } & & & & & \\
\hline & & & & & & NAICS & Groundwater & Surface water \\
\hline Public supply & 0.27 & 0.00 & 0.27 & - & - & None & - & - \\
\hline Domestic & 0.31 & 0.00 & 0.31 & 0.46 & 0.00 & None & - & - \\
\hline Commercial/public use & 0.00 & 0.00 & 0.00 & 0.07 & 0.00 & & & \\
\hline Industrial & 0.00 & 0.00 & 0.00 & 0.00 & 0.00 & & & \\
\hline Public-supply losses & - & - & - & 0.05 & - & & & \\
\hline Public wastewater treatment & - & - & - & - & 0.27 & Withdrawals b & Public Supp & \\
\hline Mining & $\begin{array}{r}0.00 \\
45.13\end{array}$ & $\begin{array}{l}0.00 \\
0.15\end{array}$ & $\begin{array}{r}0.00 \\
45.28\end{array}$ & $\begin{array}{r}0.00 \\
45.28\end{array}$ & $\begin{array}{l}0.00 \\
0.00\end{array}$ & Name & Groundwater & Surface water \\
\hline $\begin{array}{l}\text { Irrigation-Crop } \\
\text { Irrigation-Golf course }\end{array}$ & $\begin{array}{r}45.13 \\
0.00\end{array}$ & $\begin{array}{l}0.15 \\
0.00\end{array}$ & $\begin{array}{r}45.20 \\
0.00\end{array}$ & $\begin{array}{r}45.20 \\
0.00\end{array}$ & 0.00 & & & $\cap \cap 0$ \\
\hline Livestock/aquaculture & 0.00 & 0.22 & 0.22 & 0.22 & 0.00 & City of Colquitt & 0.21 & 0.00 \\
\hline
\end{tabular}

Thermoelectric power

0.00

0.00

0.00

0.00

0.00

TOTAL

45.71

0.37

46.08

46.08

0.27

'Total use is total withdrawal plus public supply deliveries and losses.

\section{Withdrawals by Source}

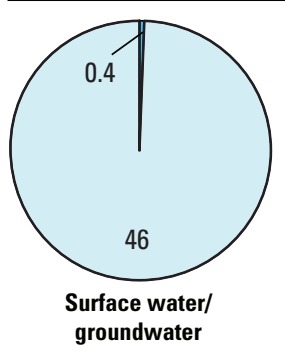

Withdrawals, in million gallons per day

Surface water

Groundwater

$\square$ Upper Floridan aquifer

Upper Floridan aquifer

groundwater

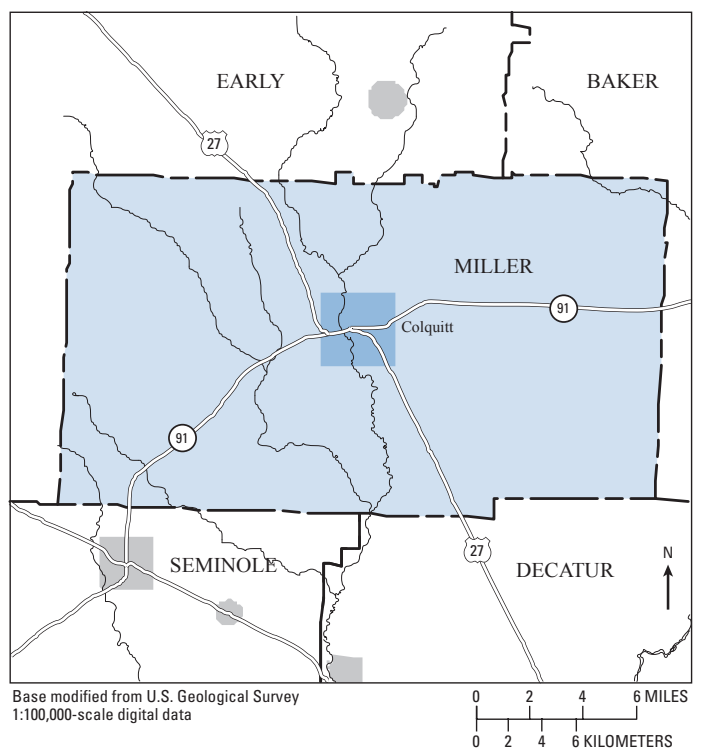

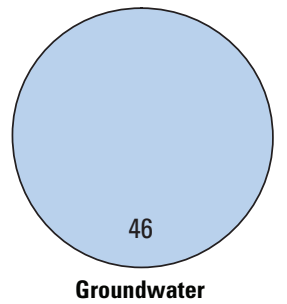

Public-Supply Deliveries by Use Category

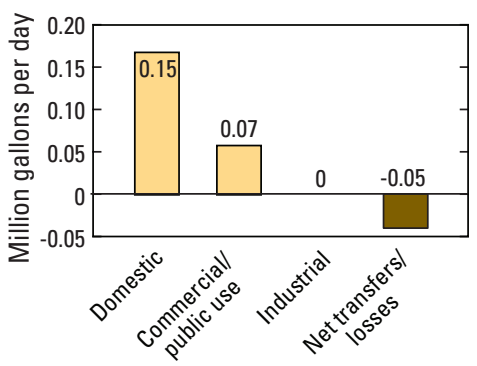

Surface-Water Withdrawals by Year

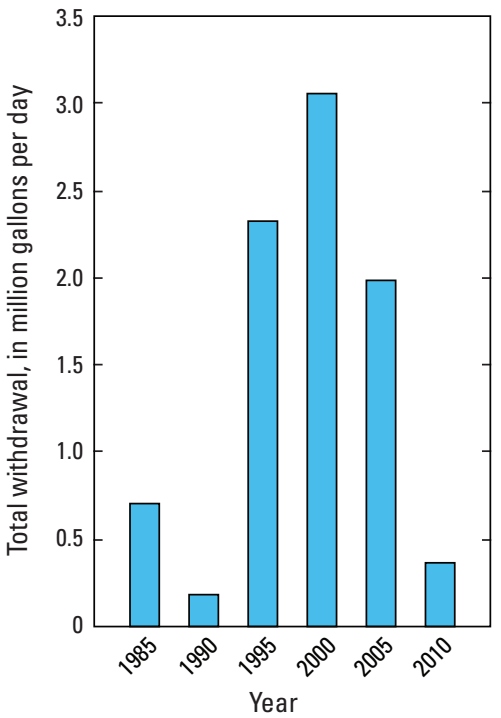

Groundwater Withdrawals by Year

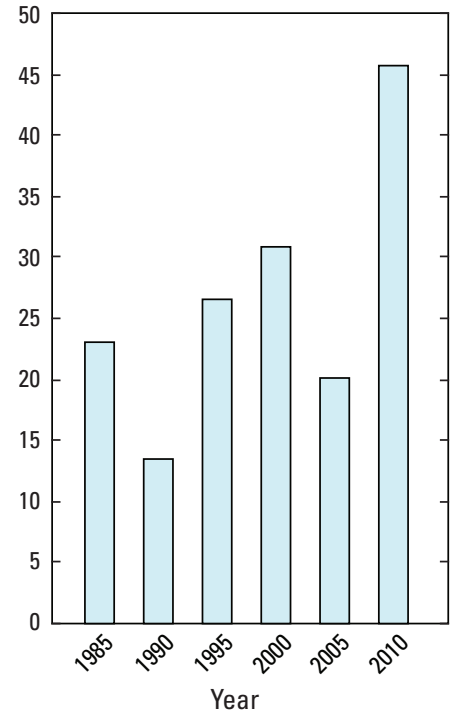


MITCHELL COUNTY

Population

Population served by public supply-Groundwater $\quad 11,790$

Population served by public supply-Surface water 0

Acres irrigated

81,325

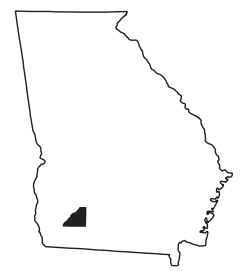

2010 WATER WITHDRAWALS AND ESTIMATED USE, IN MILLION GALLONS PER DAY

$[-$, not applicable $]$

\begin{tabular}{|c|c|c|c|c|c|c|c|c|}
\hline \multirow{3}{*}{ Category } & \multicolumn{3}{|c|}{ Withdrawals } & \multirow{3}{*}{$\begin{array}{l}\text { Total } \\
\text { use }^{1}\end{array}$} & \multirow{3}{*}{$\begin{array}{l}\text { Surface- } \\
\text { water } \\
\text { returns }\end{array}$} & \multirow{2}{*}{\multicolumn{3}{|c|}{$\begin{array}{l}\text { Withdrawals by Major Industrial Groups } \\
\text { [NAICS, North American Industrial Classification System code] }\end{array}$}} \\
\hline & \multirow{2}{*}{$\begin{array}{c}\text { Ground- } \\
\text { water }\end{array}$} & \multirow{2}{*}{$\begin{array}{c}\text { Surface } \\
\text { water }\end{array}$} & \multirow[b]{2}{*}{ Total } & & & & & \\
\hline & & & & & & NAICS & Groundwater & Surface water \\
\hline Public supply & 3.86 & 0.00 & 3.86 & - & - & 325-Chemical products & 0.84 & 0.00 \\
\hline Domestic & 0.88 & 0.00 & 0.88 & 1.89 & 0.00 & & & \\
\hline Commercial/public use & 0.24 & 0.00 & 0.24 & 0.92 & 0.00 & & & \\
\hline Industrial & 0.84 & 0.00 & 0.84 & 2.61 & 0.00 & & & \\
\hline Public-supply losses & - & - & - & 0.40 & - & & & \\
\hline Public wastewater treatment & - & - & - & - & 0.08 & & & \\
\hline Mining & 0.00 & 0.00 & 0.00 & 0.00 & 0.00 & & & \\
\hline Irrigation-Crop & 57.67 & 1.18 & 58.85 & 58.85 & 0.00 & Withdrawals by Major & Public Supp & \\
\hline Irrigation-Golf course & 0.06 & 0.00 & 0.06 & 0.06 & 0.00 & Name & Groundwater & Surface water \\
\hline Livestock/aquaculture & 0.01 & 0.68 & 0.69 & 0.69 & 0.00 & & & \\
\hline Thermoelectric power & 0.14 & 0.00 & 0.14 & 0.14 & 0.00 & City of Camilla & 3.06 & 0.00 \\
\hline TOTAL & 63.70 & 1.86 & 65.56 & 65.56 & 0.08 & City of Pelham & 0.68 & 0.00 \\
\hline
\end{tabular}

${ }^{1}$ Total use is total withdrawal plus public supply deliveries and losses.

\section{Withdrawals by Source}

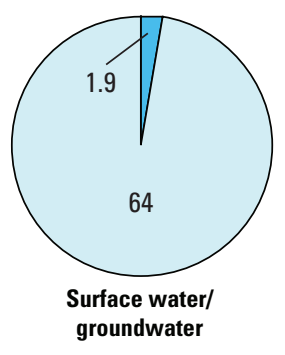

Withdrawals, in million gallons per day

Surface water

Groundwater

$\square$ Upper Floridan aquifer

groundwater

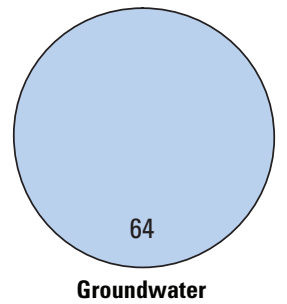

Public-Supply Deliveries by Use Category

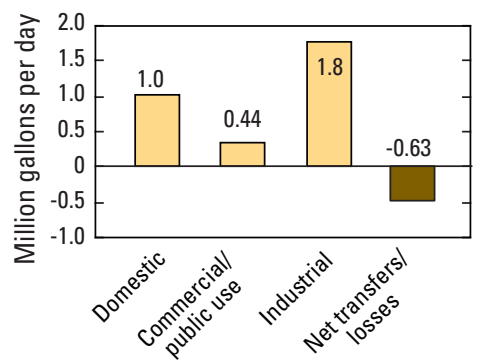

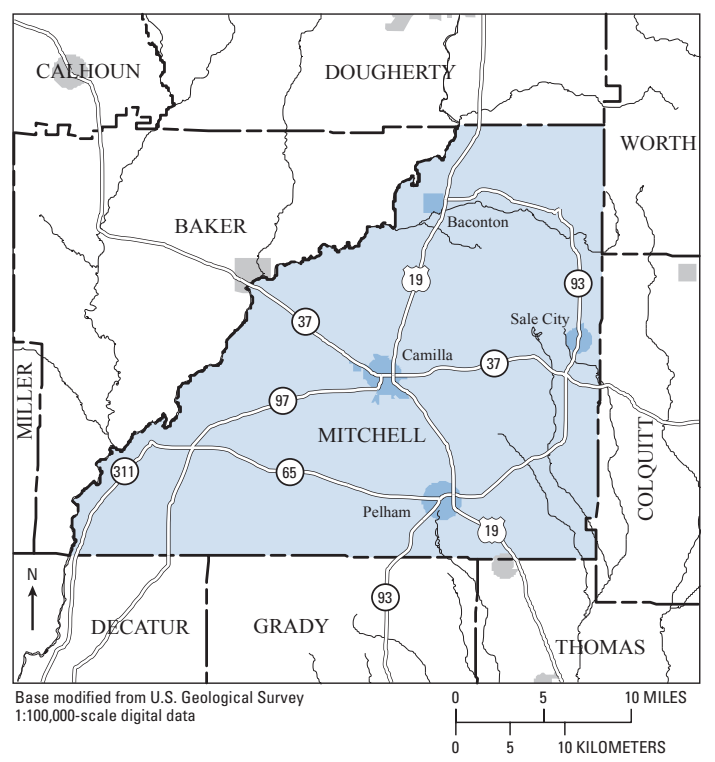

Surface-Water Withdrawals by Year

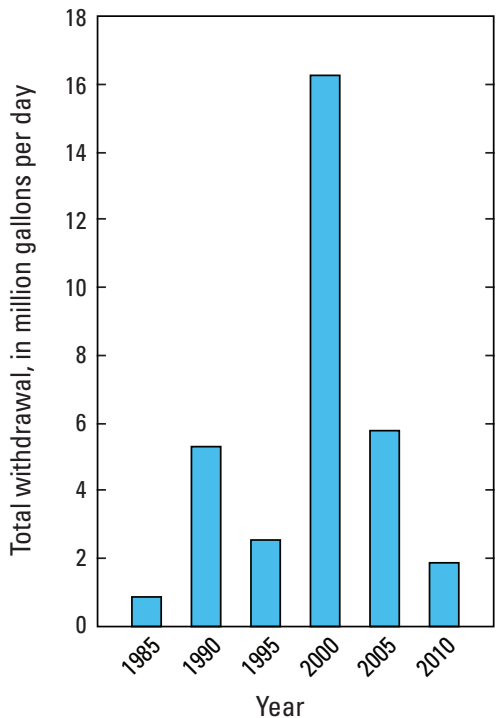

Groundwater Withdrawals by Year

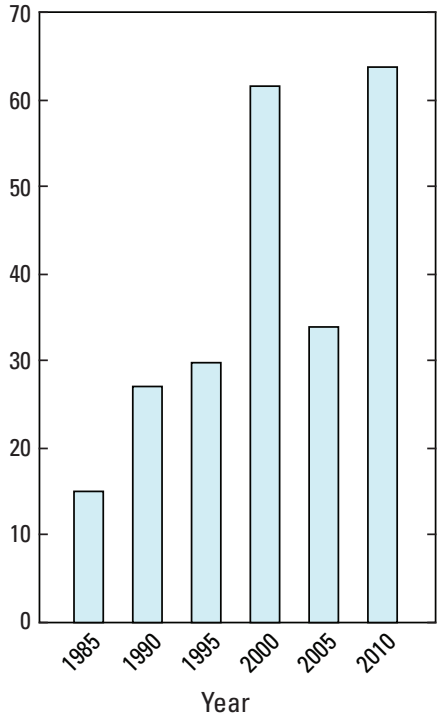


MONROE COUNTY

Population

26,424

Population served by public supply-Groundwater

Population served by public supply-Surface water Acres irrigated
10,370

420

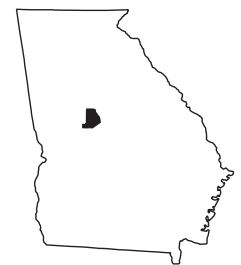

2010 WATER WITHDRAWALS AND ESTIMATED USE, IN MILLION GALLONS PER DAY

[-, not applicable; Mgal/d, million gallons per day]

\begin{tabular}{|c|c|c|c|c|c|c|c|c|}
\hline \multirow{3}{*}{ Category } & \multicolumn{3}{|c|}{ Withdrawals } & \multirow{3}{*}{$\begin{array}{l}\text { Total } \\
\text { use }^{1}\end{array}$} & \multirow{3}{*}{$\begin{array}{l}\text { Surface- } \\
\text { water } \\
\text { returns } \\
\end{array}$} & \multirow{2}{*}{\multicolumn{3}{|c|}{$\begin{array}{l}\text { Withdrawals by Major Industrial Groups } \\
\text { [NAICS, North American Industrial Classification System code] }\end{array}$}} \\
\hline & \multirow{2}{*}{$\begin{array}{c}\text { Ground- } \\
\text { water }\end{array}$} & \multirow{2}{*}{$\begin{array}{c}\text { Surface } \\
\text { water }\end{array}$} & \multirow[b]{2}{*}{ Total } & & & & & \\
\hline & & & & & & NAICS & Groundwater & Surface water \\
\hline \multirow{2}{*}{$\begin{array}{l}\text { Public supply } \\
\text { Domestic }\end{array}$} & 0.01 & 1.50 & 1.51 & \multirow{2}{*}{-} & \multirow{2}{*}{$\begin{array}{c}- \\
0.00\end{array}$} & \multirow{2}{*}{ None } & \multirow[t]{2}{*}{-} & \multirow[t]{2}{*}{-} \\
\hline & 1.19 & 0.00 & 1.19 & & & & & \\
\hline Commercial/public use & 0.01 & 0.00 & 0.01 & 0.23 & 0.00 & \multirow{4}{*}{\multicolumn{3}{|c|}{ Withdrawals by Major Public Suppliers }} \\
\hline Industrial & 0.00 & 0.00 & 0.00 & 0.02 & 0.00 & & & \\
\hline Public-supply losses & - & - & - & 0.28 & - & & & \\
\hline Public wastewater treatment & - & - & - & - & 0.70 & & & \\
\hline $\begin{array}{l}\text { Mining } \\
\text { Irrigation-Crop }\end{array}$ & 0.45 & 0.01 & 0.46 & 0.46 & 0.22 & Name & Groundwater & Surface water \\
\hline Irrigation-Golf course & 0.25 & 0.26 & 0.51 & 0.51 & 0.00 & City of Forsyth & 0.00 & 1.50 \\
\hline Livestock/aquaculture & 0.00 & 0.17 & 0.17 & 0.17 & 0.00 & & & \\
\hline Thermoelectric power & 0.06 & 55.17 & 55.23 & 55.23 & 0.00 & & & \\
\hline TOTAL & 2.09 & 57.12 & 59.21 & 59.55 & 0.92 & & & \\
\hline
\end{tabular}

'Total use is total withdrawal plus public supply deliveries and losses. An average of $0.23 \mathrm{Mgal} / \mathrm{d}$ was delivered from Bibb County, Georgia and $0.13 \mathrm{Mgal} / \mathrm{d}$ from Butts County, Georgia to Monroe County in 2010.

\section{Withdrawals by Source}

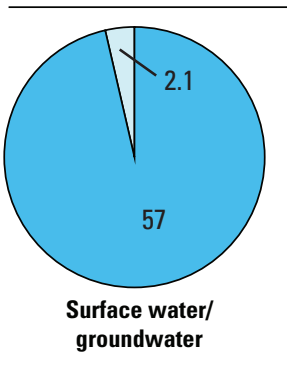

Withdrawals, in million gallons per day

$\square$ Surface water

Groundwater

Crystalline-rock aquifer groundwater

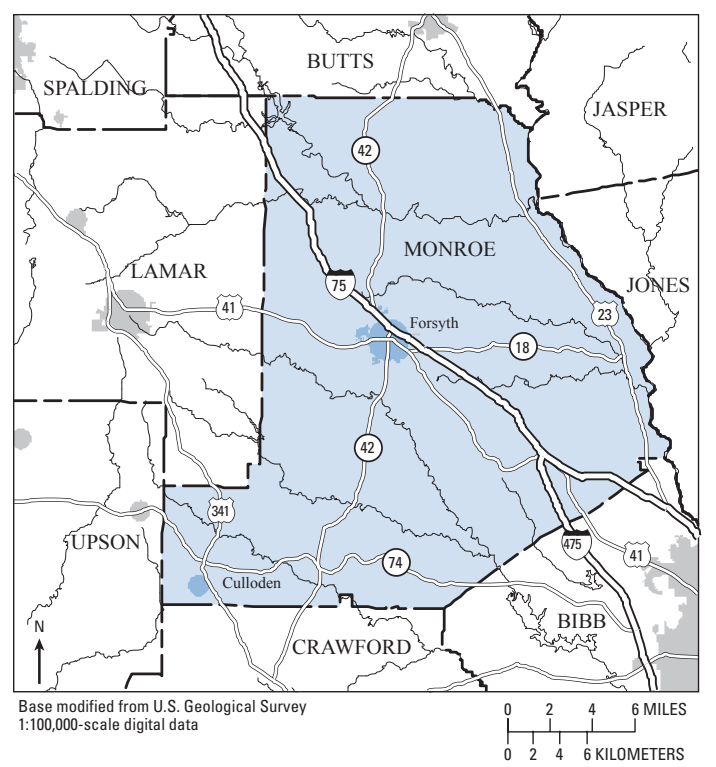

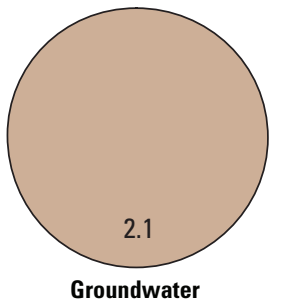

Public-Supply Deliveries ${ }^{1}$ by Use Category

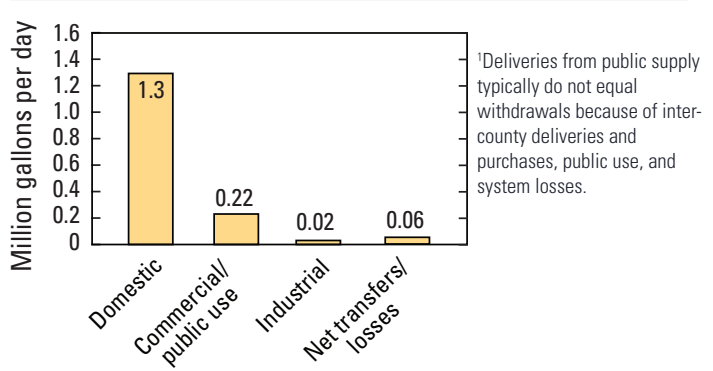

Surface-Water Withdrawals by Year

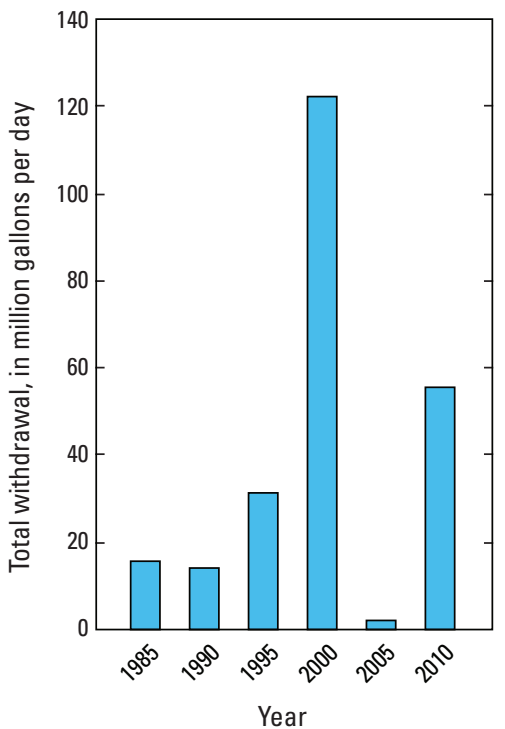

Groundwater Withdrawals by Year

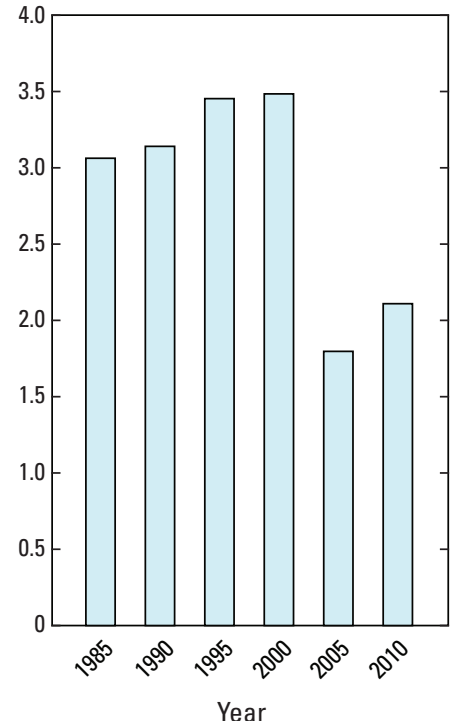




\section{MONTGOMERY COUNTY}

Population

9,123

Population served by public supply-Groundwater $\quad 3,730$

Population served by public supply-Surface water 0

Acres irrigated

2,033

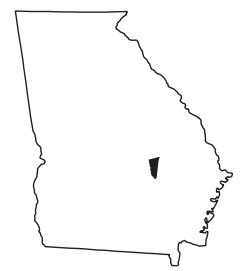

2010 WATER WITHDRAWALS AND ESTIMATED USE, IN MILLION GALLONS PER DAY

$[-$, not applicable; $<$, less than $]$

\begin{tabular}{|c|c|c|c|c|c|c|c|c|}
\hline \multirow{3}{*}{ Category } & \multicolumn{3}{|c|}{ Withdrawals } & \multirow{3}{*}{$\begin{array}{l}\text { Total } \\
\text { use }^{1}\end{array}$} & \multirow{3}{*}{$\begin{array}{l}\text { Surface- } \\
\text { water } \\
\text { returns } \\
\end{array}$} & \multirow{2}{*}{\multicolumn{3}{|c|}{$\begin{array}{l}\text { Withdrawals by Major Industrial Groups } \\
\text { [NAICS, North American Industrial Classification System code] }\end{array}$}} \\
\hline & \multirow{2}{*}{$\begin{array}{c}\text { Ground- } \\
\text { water }\end{array}$} & \multirow{2}{*}{$\begin{array}{c}\text { Surface } \\
\text { water }\end{array}$} & \multirow[b]{2}{*}{ Total } & & & & & \\
\hline & & & & & & NAICS & Groundwater & Surface water \\
\hline Public supply & 0.45 & 0.00 & 0.45 & - & - & & & \\
\hline Domestic & 0.40 & 0.00 & 0.40 & 0.77 & 0.00 & None & - & - \\
\hline Commercial/public use & 0.00 & 0.00 & 0.00 & 0.07 & 0.00 & & & \\
\hline Industrial & 0.00 & 0.00 & 0.00 & 0.00 & 0.00 & & & \\
\hline Public-supply losses & - & - & - & 0.01 & - & & & \\
\hline Public wastewater treatment & - & - & - & - & 0.31 & Withdrawals by Maj & r Public Supp & \\
\hline $\begin{array}{l}\text { Mining } \\
\text { Irrigation-Crop }\end{array}$ & $\begin{array}{l}0.06 \\
0.47\end{array}$ & $\begin{array}{l}0.00 \\
0.09\end{array}$ & $\begin{array}{l}0.06 \\
0.56\end{array}$ & $\begin{array}{l}0.06 \\
0.56\end{array}$ & $\begin{array}{l}0.00 \\
0.00\end{array}$ & Name & Groundwater & Surface water \\
\hline Irrigation-Golf course & 0.00 & 0.25 & 0.25 & 0.25 & 0.00 & City of Mount Vernon & 0.33 & 0.00 \\
\hline Livestock/aquaculture & 0.07 & 0.72 & 0.79 & 0.79 & 0.00 & Town of Uvalda & 0.04 & 0.00 \\
\hline Thermoelectric power & 0.00 & 0.00 & 0.00 & 0.00 & 0.00 & Town of Ailey & 0.03 & 0.00 \\
\hline
\end{tabular}

'Total use is total withdrawal plus public supply deliveries and losses.

\section{Withdrawals by Source}
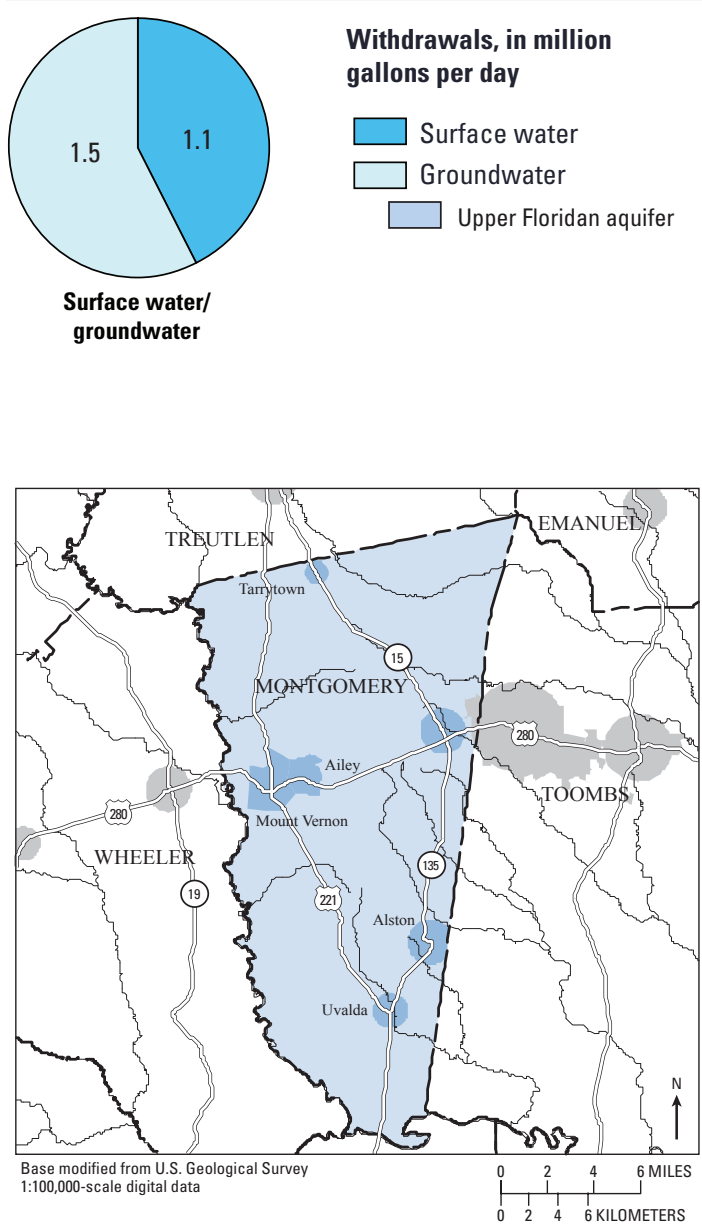

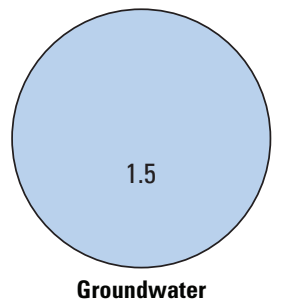

Public-Supply Deliveries by Use Category

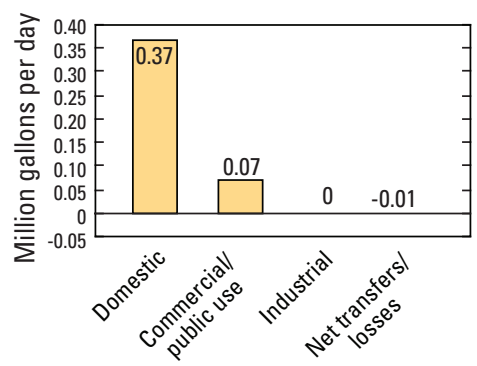

Surface-Water Withdrawals by Year

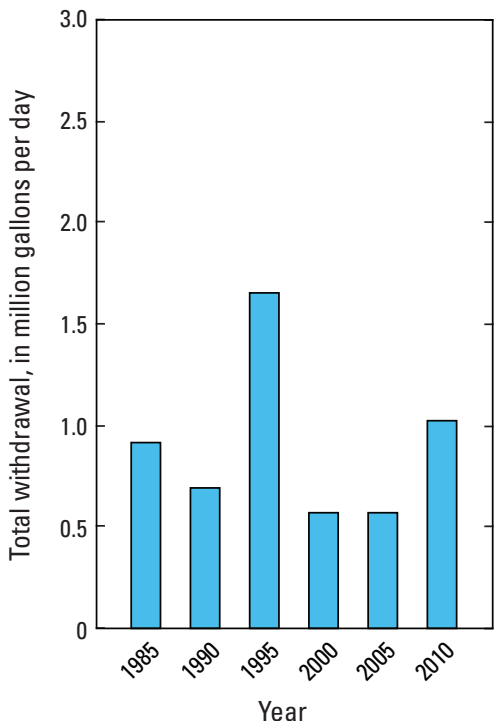

Groundwater Withdrawals by Year

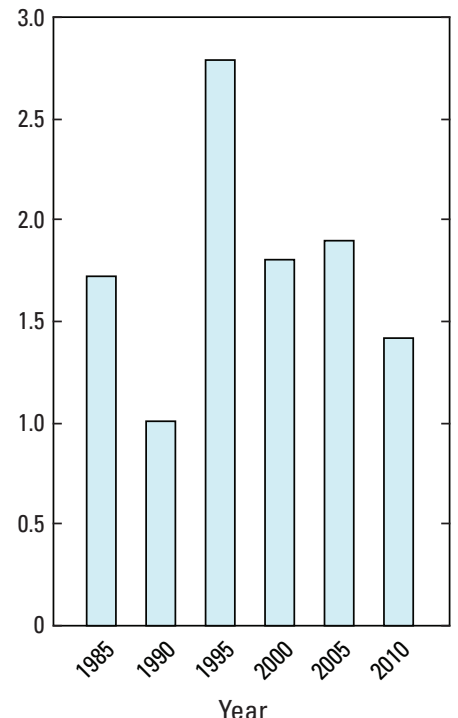




\section{MORGAN COUNTY}

Population

17,868

Population served by public supply-Groundwater

Population served by public supply-Surface water Acres irrigated
1,570

9,370

1,400

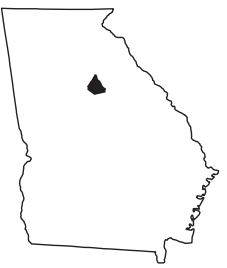

2010 WATER WITHDRAWALS AND ESTIMATED USE, IN MILLION GALLONS PER DAY

$[-$, not applicable $]$

\begin{tabular}{|c|c|c|c|c|c|c|c|c|}
\hline \multirow{3}{*}{ Category } & \multicolumn{3}{|c|}{ Withdrawals } & \multirow{3}{*}{$\begin{array}{l}\text { Total } \\
\text { use }^{1}\end{array}$} & \multirow{3}{*}{$\begin{array}{l}\text { Surface- } \\
\text { water } \\
\text { returns }\end{array}$} & \multirow{2}{*}{\multicolumn{3}{|c|}{$\begin{array}{l}\text { Withdrawals by Major Industrial Groups } \\
\text { [NAICS, North American Industrial Classification System code] }\end{array}$}} \\
\hline & \multirow{2}{*}{$\begin{array}{c}\text { Ground- } \\
\text { water }\end{array}$} & \multirow{2}{*}{$\begin{array}{c}\text { Surface } \\
\text { water }\end{array}$} & \multirow[b]{2}{*}{ Total } & & & & & \\
\hline & & & & & & NAICS G & Groundwater & Surface water \\
\hline Public supply & 0.19 & 1.31 & 1.50 & - & - & & & 000 \\
\hline Domestic & 0.52 & 0.00 & 0.52 & 1.34 & 0.00 & 322-Paper, pulp & 0.05 & 0.00 \\
\hline Commercial/public use & 0.01 & 0.00 & 0.01 & 0.47 & 0.00 & & & \\
\hline Industrial & 0.05 & 0.00 & 0.05 & 0.28 & 0.05 & & & \\
\hline Public-supply losses & - & - & - & 0.18 & - & & & \\
\hline Public wastewater treatment & - & - & - & - & 0.66 & Withdrawals by Major F & Public Sup & \\
\hline Mining & 0.00 & 0.00 & 0.00 & 0.00 & 0.00 & Name & Groundwater & Surface water \\
\hline Irrigation-Crop & 0.03 & 1.30 & 1.33 & 1.33 & 0.00 & & & \\
\hline Irrigation-Golf course & 0.00 & 0.66 & 0.66 & 0.66 & 0.00 & City of Madison & 0.00 & 1.31 \\
\hline Livestock/aquaculture & 0.06 & 1.22 & 1.28 & 1.28 & 0.00 & City of Rutledge & 0.07 & 0.00 \\
\hline Thermoelectric power & 0.00 & 0.00 & 0.00 & 0.00 & 0.00 & City of Bostwick & 0.06 & 0.00 \\
\hline TOTAL & 0.86 & 4.49 & 5.35 & 5.35 & 0.71 & Piedmont Water Company & 0.05 & 0.00 \\
\hline
\end{tabular}

Total use is total withdrawal plus public supply deliveries and losses.

2010 Withdrawals by Source

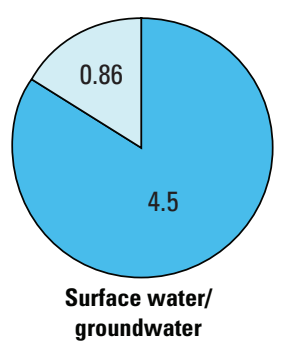

Withdrawals, in million gallons per day

$\square$ Surface water

$\square$ Groundwater

$\square$ Crystalline-rock aquifer

groundwater

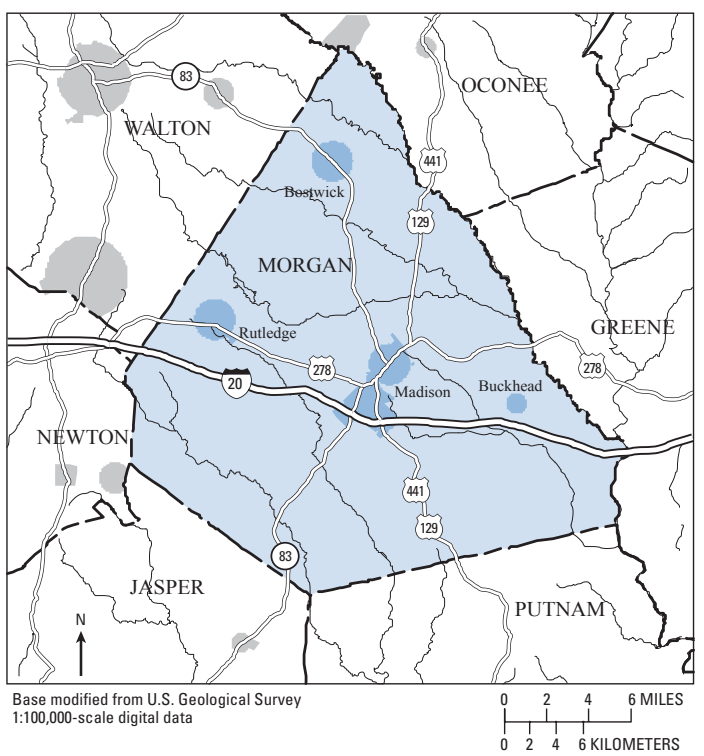

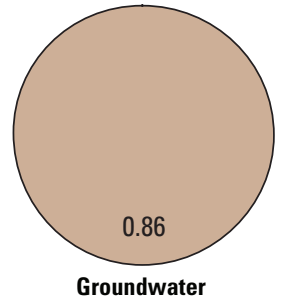

Public-Supply Deliveries by Use Category

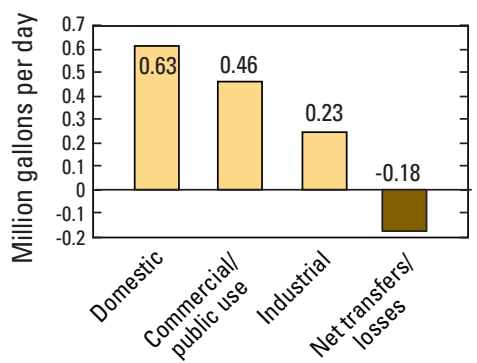

Surface-Water Withdrawals by Year

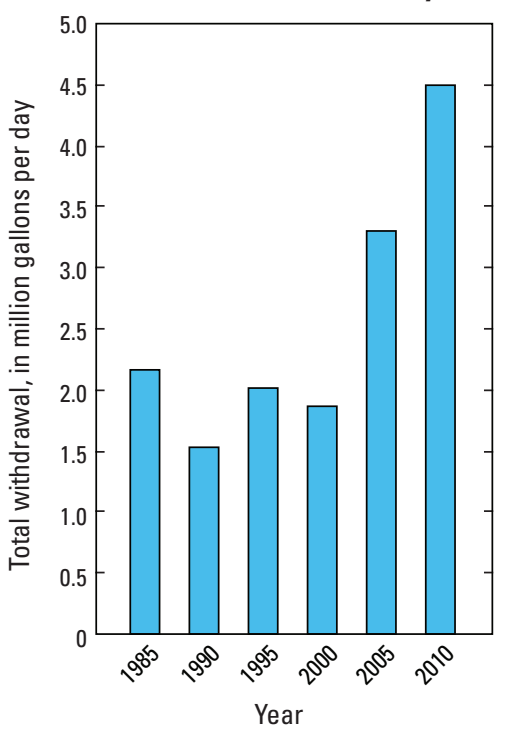

Groundwater Withdrawals by Year

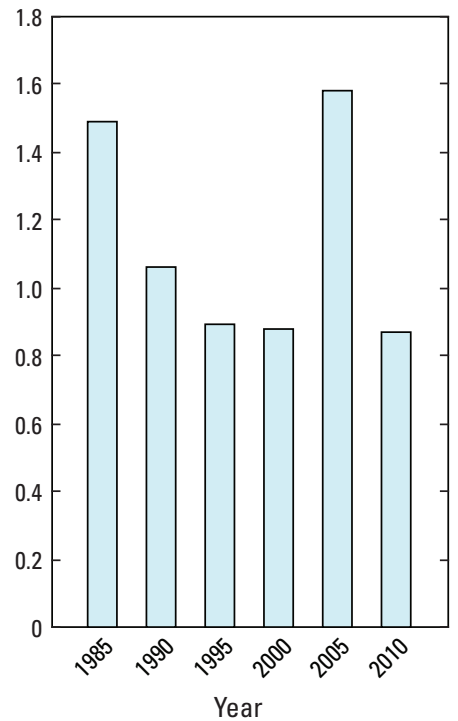




\section{MURRAY COUNTY}

Population

39,628

Population served by public supply-Groundwater $\quad 12,740$

Population served by public supply-Surface water $\quad 22,180$

Acres irrigated

260

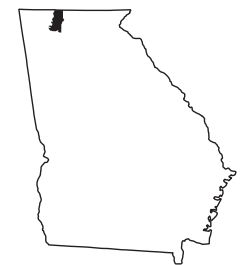

2010 WATER WITHDRAWALS AND ESTIMATED USE, IN MILLION GALLONS PER DAY

$[-$, not applicable; Mgal/d, million gallons per day $]$

\begin{tabular}{|c|c|c|c|c|c|c|c|c|}
\hline \multirow{3}{*}{ Category } & \multicolumn{3}{|c|}{ Withdrawals } & \multirow{3}{*}{$\begin{array}{l}\text { Total } \\
\text { use }^{1}\end{array}$} & \multirow{3}{*}{$\begin{array}{l}\text { Surface- } \\
\text { water } \\
\text { returns } \\
\end{array}$} & \multirow{2}{*}{\multicolumn{3}{|c|}{$\begin{array}{l}\text { Withdrawals by Major Industrial Groups } \\
\text { [NAICS, North American Industrial Classification System code] }\end{array}$}} \\
\hline & \multirow{2}{*}{$\begin{array}{c}\text { Ground- } \\
\text { water }\end{array}$} & \multirow{2}{*}{$\begin{array}{c}\text { Surface } \\
\text { water }\end{array}$} & \multirow[b]{2}{*}{ Total } & & & & & \\
\hline & & & & & & NAICS & Groundwater & Surface water \\
\hline Public supply & 0.76 & 1.89 & 2.65 & \multirow{2}{*}{$\begin{array}{l}- \\
2.74\end{array}$} & \multirow{2}{*}{0.00} & \multirow{2}{*}{ None } & \multirow{2}{*}{-} & \multirow{2}{*}{-} \\
\hline Domestic & 0.35 & 0.00 & 0.35 & & & & & \\
\hline Commercial/public use & 0.00 & 0.00 & 0.00 & 0.35 & 0.00 & \multirow{4}{*}{\multicolumn{3}{|c|}{ Withdrawals by Major Public Suppliers }} \\
\hline Industrial & 0.00 & 0.00 & 0.00 & 0.48 & 0.00 & & & \\
\hline Public-supply losses & - & - & - & 0.41 & - & & & \\
\hline Public wastewater treatment & - & - & - & - & 1.27 & & & \\
\hline \multirow{3}{*}{$\begin{array}{l}\text { Mining } \\
\text { Irrigation-Crop } \\
\text { Irrigation-Golf course }\end{array}$} & 0.02 & 0.00 & 0.02 & 0.02 & 0.00 & Name & \multirow{2}{*}{ Groundwater } & \multirow{2}{*}{ Surface water } \\
\hline & 0.00 & 0.23 & 0.23 & 0.23 & 0.00 & Name & & \\
\hline & 0.00 & 0.00 & 0.00 & 0.00 & 0.00 & \multirow{2}{*}{$\begin{array}{l}\text { Chatsworth Water Works } \\
\text { Commission }\end{array}$} & \multirow{2}{*}{0.75} & \multirow{2}{*}{1.89} \\
\hline Livestock/aquaculture & 0.06 & 0.55 & 0.61 & 0.61 & 0.00 & & & \\
\hline Thermoelectric power & 0.00 & 0.00 & 0.00 & 0.00 & 0.00 & & & \\
\hline
\end{tabular}

$\begin{array}{lllll}1.19 & 2.67 & 3.86 & 4.84 & 1.27\end{array}$

'Total use is total withdrawal plus public supply deliveries and losses. An average of $0.34 \mathrm{Mgal} / \mathrm{d}$ was delivered from Gordon County, Georgia to Murray County, $0.14 \mathrm{Mgal} / \mathrm{d}$ from Polk County, Tennessee, and 0.5 Mgal/d from Whitfield County, Georgia to Murray County in 2010.

\section{Withdrawals by Source}
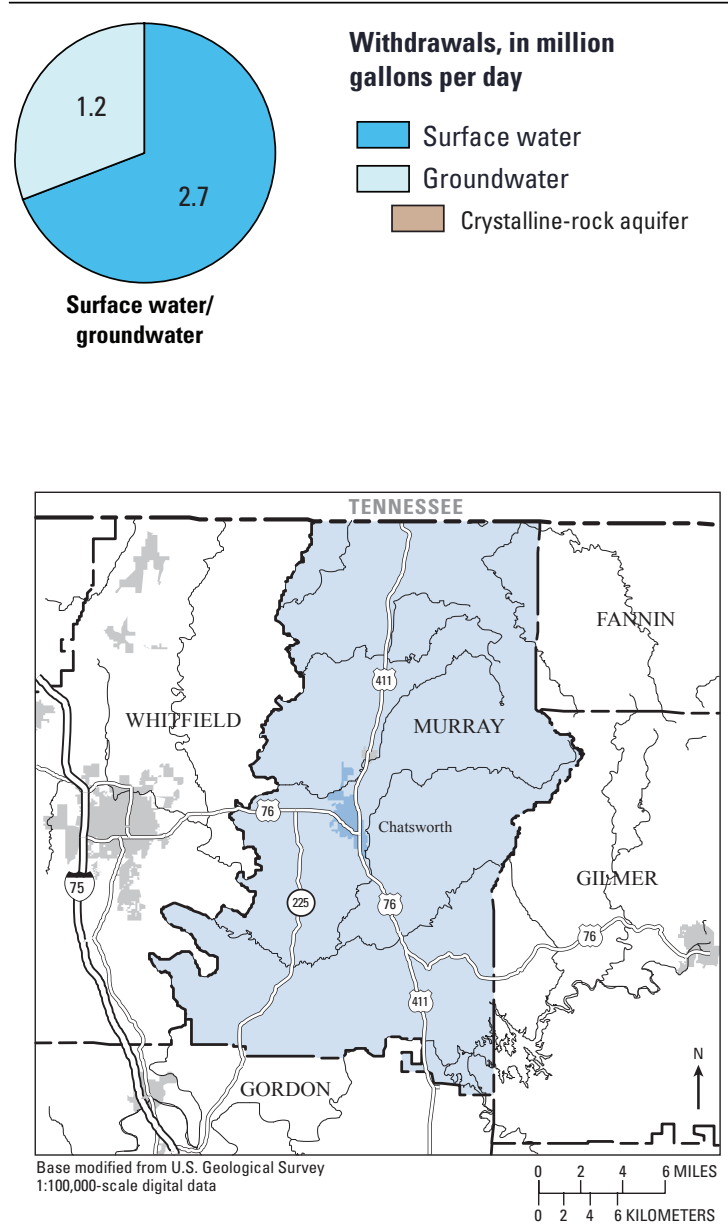

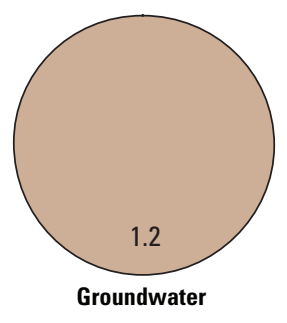

Public-Supply Deliveries' by Use Category

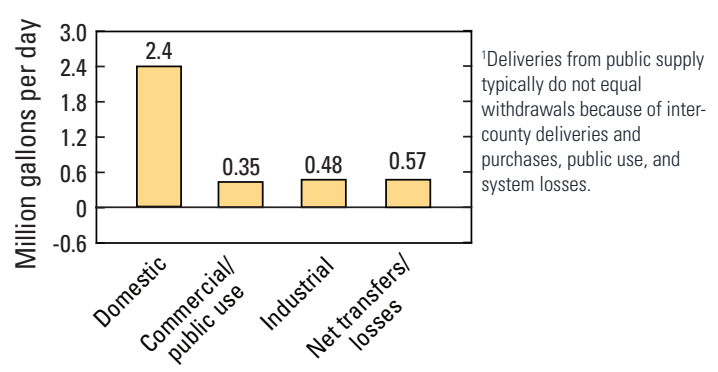

Surface-Water Withdrawals by Year

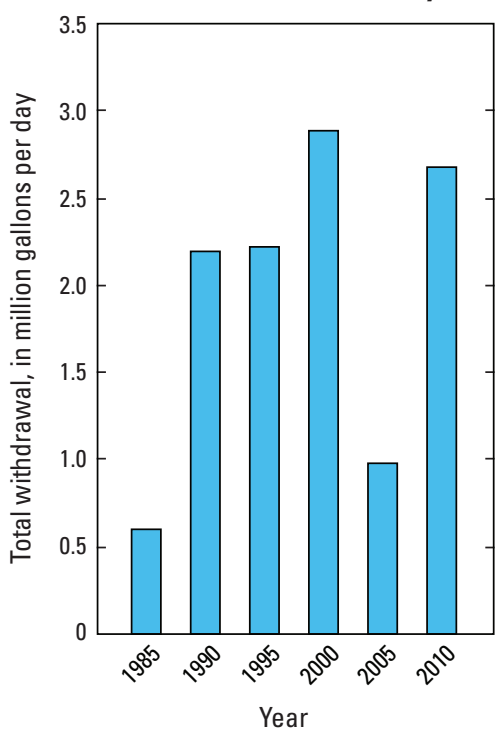

Groundwater Withdrawals by Year

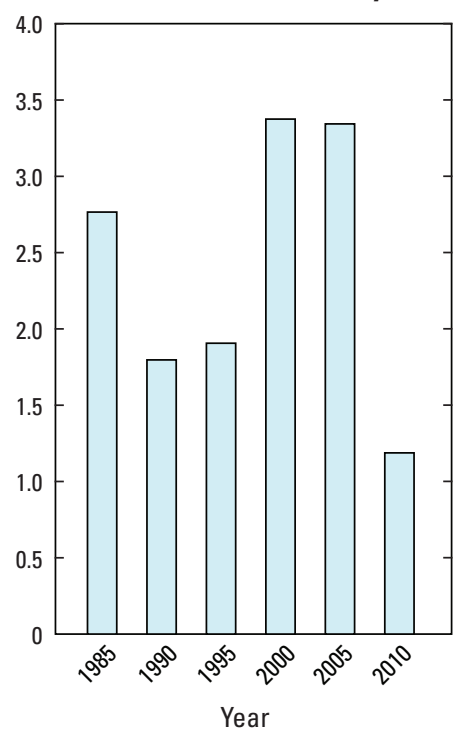




\section{MUSCOGEE COUNTY}

Population

189,890

Population served by public supply-Groundwater

Population served by public supply-Surface water 189,880

Acres irrigated

420

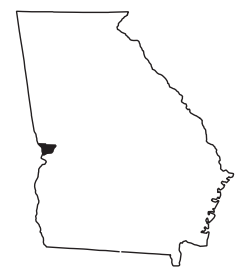

2010 WATER WITHDRAWALS AND ESTIMATED USE, IN MILLION GALLONS PER DAY

[-, not applicable; Mgal/d, million gallons per day]

\begin{tabular}{|c|c|c|c|c|c|c|c|c|}
\hline \multirow{3}{*}{ Category } & \multicolumn{3}{|c|}{ Withdrawals } & \multirow{3}{*}{$\begin{array}{l}\text { Total } \\
\text { use }^{1}\end{array}$} & \multirow{3}{*}{$\begin{array}{l}\text { Surface- } \\
\text { water } \\
\text { returns } \\
\end{array}$} & \multirow{2}{*}{\multicolumn{3}{|c|}{$\begin{array}{l}\text { Withdrawals by Major Industrial Groups } \\
\text { [NAICS, North American Industrial Classification System code] }\end{array}$}} \\
\hline & \multirow{2}{*}{$\begin{array}{c}\text { Ground- } \\
\text { water }\end{array}$} & \multirow{2}{*}{$\begin{array}{c}\text { Surface } \\
\text { water }\end{array}$} & \multirow[b]{2}{*}{ Total } & & & & & \\
\hline & & & & & & NAICS & Groundwater & Surface water \\
\hline Public supply & 0.00 & 37.30 & 37.30 & - & - & 325-Chemical products & 0.01 & 0.00 \\
\hline Domestic & 0.00 & 0.00 & 0.00 & 17.33 & 0.00 & & & \\
\hline Commercial/public use & 0.00 & 0.00 & 0.00 & 6.46 & 0.00 & & & \\
\hline Industrial & 0.01 & 0.00 & 0.01 & 1.76 & 0.00 & & & \\
\hline Public-supply losses & - & - & - & 5.24 & - & & & \\
\hline Public wastewater treatment & - & - & - & - & 30.39 & \multicolumn{3}{|c|}{ Withdrawals by Major Public Suppliers } \\
\hline $\begin{array}{l}\text { Mining } \\
\text { Irrigation-Crop }\end{array}$ & $\begin{array}{l}0.55 \\
0.05\end{array}$ & $\begin{array}{l}0.01 \\
0.00\end{array}$ & $\begin{array}{l}0.56 \\
0.05\end{array}$ & $\begin{array}{l}0.56 \\
0.05\end{array}$ & $\begin{array}{l}2.58 \\
0.00\end{array}$ & Name & Groundwater & Surface water \\
\hline Irrigation-Golf course & 0.16 & 0.66 & 0.82 & 0.82 & 0.00 & City of Columbus & 0.00 & 34.94 \\
\hline Livestock/aquaculture & 0.00 & 0.00 & 0.00 & 0.00 & 0.00 & Smith Water Authority ${ }^{1}$ & 0.00 & 2.36 \\
\hline Thermoelectric power & 0.00 & 4.59 & 4.59 & 4.59 & 0.00 & & & \\
\hline TOTAL & 0.77 & 42.56 & 43.33 & 36.81 & 32.97 & & & \\
\hline
\end{tabular}

'Total use is total withdrawal plus public supply deliveries and losses. An average of $2.36 \mathrm{Mgal} / \mathrm{d}$ was withdrawn in Muscogee County

and delivered to Lee County, Alabama in 2010. An average of $3.27 \mathrm{Mgal} / \mathrm{d}$ was delivered to Chattahoochee County, Georgia, $0.82 \mathrm{Mgal} / \mathrm{d}$

to Harris County, Georgia and 0.07 to Talbot County, Georgia from Muscogee County in 2010.

2010 Withdrawals by Source
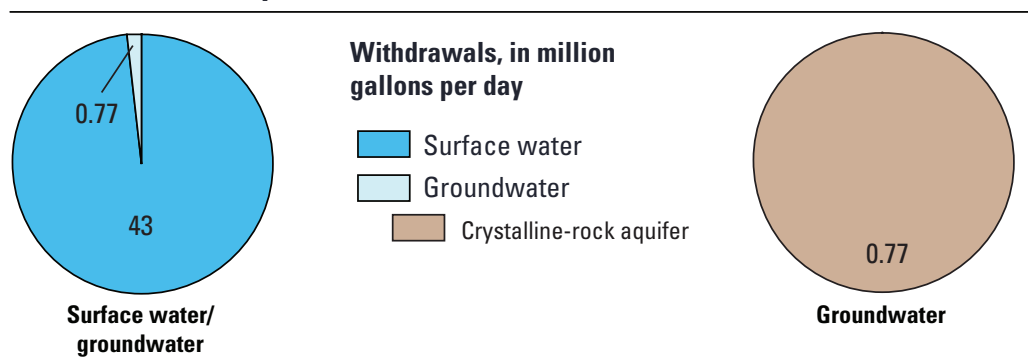

Public-Supply Deliveries ${ }^{1}$ by Use Category
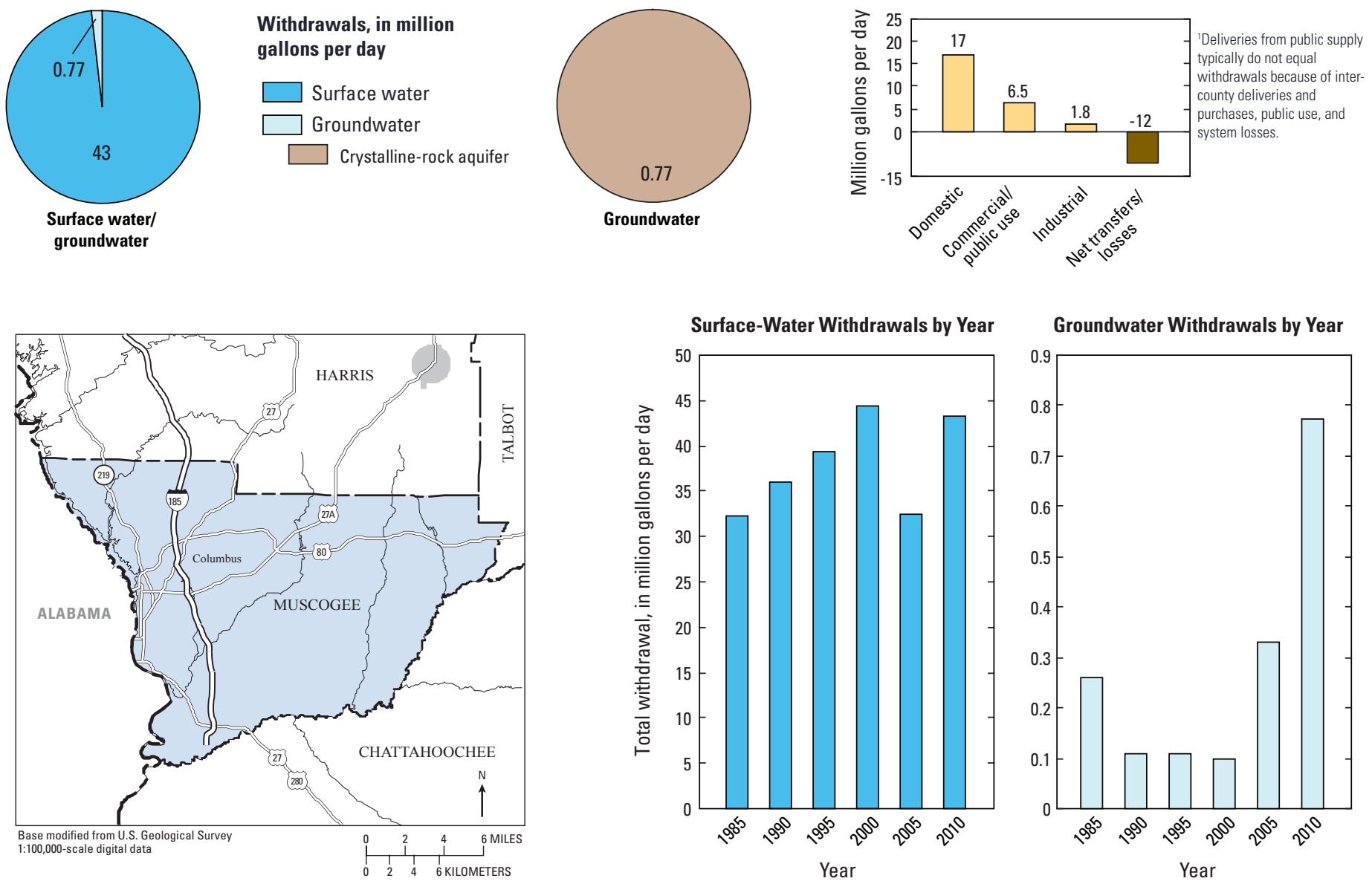

Groundwater Withdrawals by Year

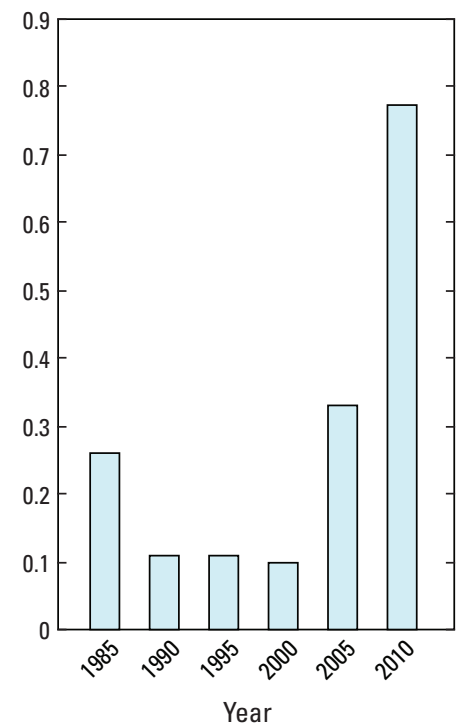




\section{NEWTON COUNTY}

Population

Population served by public supply-Groundwater $\quad 1,360$

Population served by public supply-Surface water $\quad 95,790$

Acres irrigated

500

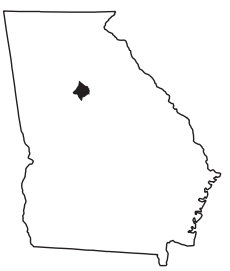

2010 WATER WITHDRAWALS AND ESTIMATED USE, IN MILLION GALLONS PER DAY (Mgal/d)

$[-$, not applicable; Mgal/d, million gallons per day $]$

\begin{tabular}{|c|c|c|c|c|c|c|c|c|}
\hline \multirow{3}{*}{ Category } & \multicolumn{3}{|c|}{ Withdrawals } & \multirow{3}{*}{$\begin{array}{l}\text { Total } \\
\text { use }^{1}\end{array}$} & \multirow{3}{*}{$\begin{array}{c}\text { Surface- } \\
\text { water } \\
\text { returns } \\
\end{array}$} & \multirow{2}{*}{\multicolumn{3}{|c|}{$\begin{array}{l}\text { Withdrawals by Major Industrial Groups } \\
\text { [NAICS, North American Industrial Classification System code] }\end{array}$}} \\
\hline & \multirow{2}{*}{$\begin{array}{c}\text { Ground- } \\
\text { water }\end{array}$} & \multirow{2}{*}{$\begin{array}{c}\text { Surface } \\
\text { water }\end{array}$} & \multirow[b]{2}{*}{ Total } & & & & & \\
\hline & & & & & & NAICS & Groundwater & Surface water \\
\hline Public supply & 0.13 & 12.70 & 12.83 & - & - & None & - & - \\
\hline Domestic & 0.21 & 0.00 & 0.21 & 5.95 & 0.00 & None & - & - \\
\hline Commercial/public use & 0.00 & 0.00 & 0.00 & 1.00 & 0.00 & & & \\
\hline Industrial & 0.00 & 0.00 & 0.00 & 0.43 & 0.00 & & & \\
\hline Public-supply losses & - & - & - & 1.28 & - & & & \\
\hline Public wastewater treatment & - & - & - & - & 0.03 & Withdrawals by Majo & Public Supp & \\
\hline Mining & 0.10 & 0.00 & 0.10 & 0.10 & 0.00 & Name & Groundwater & Surface water \\
\hline $\begin{array}{l}\text { Irrigation-Crop } \\
\text { Irrigation-Colf course }\end{array}$ & 0.01 & 0.02 & 0.03 & 0.03 & $\begin{array}{l}0.00 \\
0.00\end{array}$ & & & \\
\hline $\begin{array}{l}\text { Irrigation-Golf course } \\
\text { Livestock/aquaculture }\end{array}$ & 0.33 & 0.46 & 0.79 & 0.79 & $\begin{array}{l}0.00 \\
0.00\end{array}$ & $\begin{array}{l}\text { Newton County Water- } \\
\text { Sewerage Authority }\end{array}$ & 0.00 & 12.84 \\
\hline Livestock/aquaculture & 0.02 & 0.12 & 0.14 & 0.14 & 0.00 & & & \\
\hline Thermoelectric power & 0.00 & 0.00 & 0.00 & 0.00 & 0.00 & & & \\
\hline
\end{tabular}

$\mathbf{0 . 8 0}$

13.30

14.10

9.72

$\mathbf{0 . 0 3}$

${ }^{1}$ Total use is total withdrawal plus public supply deliveries and losses. An average of $4.33 \mathrm{Mgal} / \mathrm{d}$ was delivered to Walton County, Georgia and $0.05 \mathrm{Mgal} / \mathrm{d}$ to Jasper County, Georgia from Newton County in 2010.

\section{Withdrawals by Source}

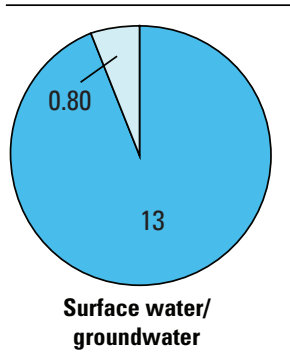

Withdrawals, in million gallons per day

$\square$ Surface water

$\square$ Groundwater

$\square$ Crystalline-rock aquifer groundwater

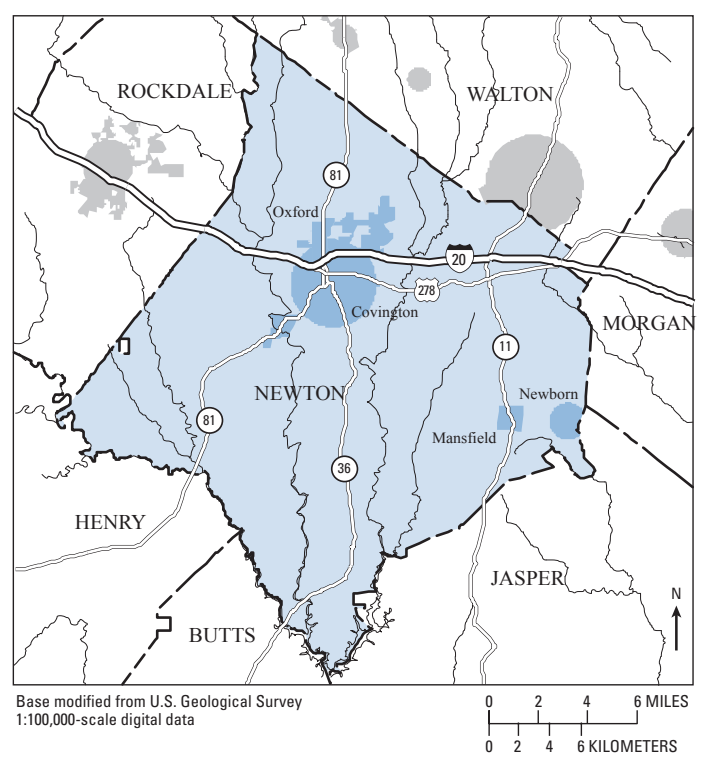

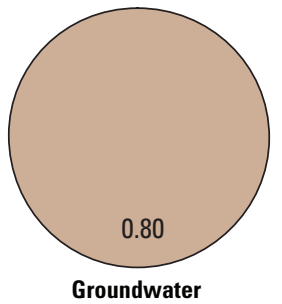

Public-Supply Deliveries ${ }^{1}$ by Use Category

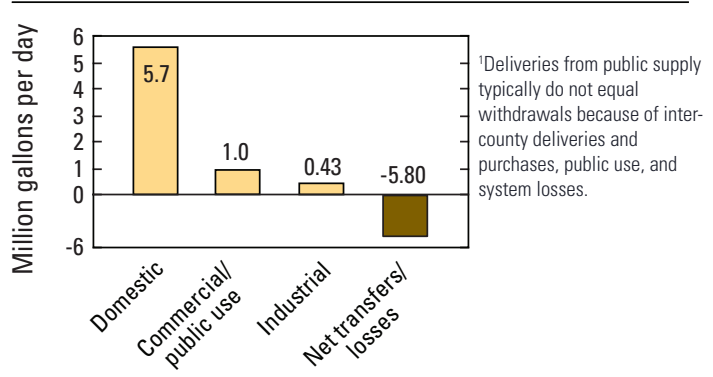

Surface-Water Withdrawals by Year

Groundwater Withdrawals by Year
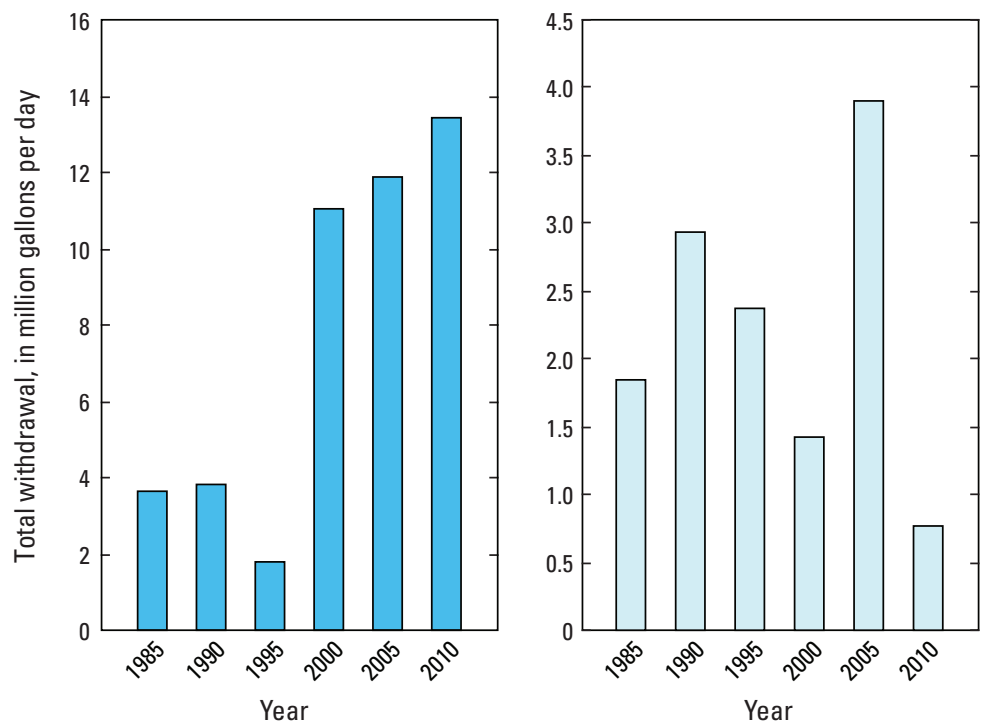


\section{OCONEE COUNTY}

Population

32,808

Population served by public supply-Groundwater

Population served by public supply_-Surface water Acres irrigated
4,720

19,200

1,505

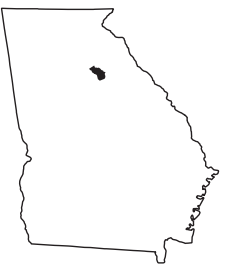

2010 WATER WITHDRAWALS AND ESTIMATED USE, IN MILLION GALLONS PER DAY

[-, not applicable; Mgal/d, million gallons per day]

\begin{tabular}{|c|c|c|c|c|c|c|c|c|}
\hline \multirow{3}{*}{ Category } & \multicolumn{3}{|c|}{ Withdrawals } & \multirow{3}{*}{$\begin{array}{l}\text { Total } \\
\text { use }^{1}\end{array}$} & \multirow{3}{*}{$\begin{array}{c}\text { Surface- } \\
\text { water } \\
\text { returns } \\
\end{array}$} & \multirow{2}{*}{\multicolumn{3}{|c|}{$\begin{array}{l}\text { Withdrawals by Major Industrial Groups } \\
\text { [NAICS, North American Industrial Classification System code] }\end{array}$}} \\
\hline & \multirow{2}{*}{$\begin{array}{c}\text { Ground- } \\
\text { water }\end{array}$} & \multirow{2}{*}{$\begin{array}{c}\text { Surface } \\
\text { water }\end{array}$} & \multirow[b]{2}{*}{ Total } & & & & & \\
\hline & & & & & & NAICS G & Groundwater & Surface water \\
\hline Public supply & 0.37 & 0.00 & 0.37 & - & - & None & - & - \\
\hline Domestic & 0.67 & 0.00 & 0.67 & 2.30 & 0.00 & None & - & - \\
\hline Commercial/public use & 0.00 & 0.00 & 0.00 & 0.86 & 0.00 & & & \\
\hline Industrial & 0.00 & 0.00 & 0.00 & 0.04 & 0.00 & & & \\
\hline Public-supply losses & - & - & - & 0.30 & - & & & \\
\hline Public wastewater treatment & - & - & - & - & 0.36 & Withdrawals by Major & Public Supp & \\
\hline Mining & 0.00 & 0.00 & 0.00 & $\begin{array}{l}0.00 \\
373\end{array}$ & $\begin{array}{l}0.00 \\
0.00\end{array}$ & Name G & Groundwater & Surface water \\
\hline $\begin{array}{l}\text { Irrigation-Crop } \\
\text { Irrigation-Golf course }\end{array}$ & $\begin{array}{l}3.73 \\
0.00\end{array}$ & $\begin{array}{l}0.00 \\
0.29\end{array}$ & $\begin{array}{l}3.73 \\
0.29\end{array}$ & $\begin{array}{l}3.73 \\
0.29\end{array}$ & $\begin{array}{l}0.00 \\
0.00\end{array}$ & & & \\
\hline Livestock/aquaculture & $\begin{array}{l}0.00 \\
0.16\end{array}$ & $\begin{array}{l}0.29 \\
2.07\end{array}$ & 2.23 & 2.23 & 0.00 & Oconee County & 0.18 & 0.00 \\
\hline Thermoelectric power & 0.00 & 0.00 & 0.00 & 0.00 & 0.00 & Piedmont Water Company & 0.13 & 0.00 \\
\hline
\end{tabular}

TOTAL

4.93

2.36

7.29

9.75

0.36

'Total use is total withdrawal plus public supply deliveries and losses. An average of $2.5 \mathrm{Mgal} / \mathrm{d}$ was delivered from Jackson County, Georgia and $0.10 \mathrm{Mgal} / \mathrm{d}$ from Barrow County, Georgia to Oconee County; whereas, $0.13 \mathrm{Mgal} / \mathrm{d}$ was delivered from Oconee County to Walton County, Georgia in 2010.

\section{Withdrawals by Source}
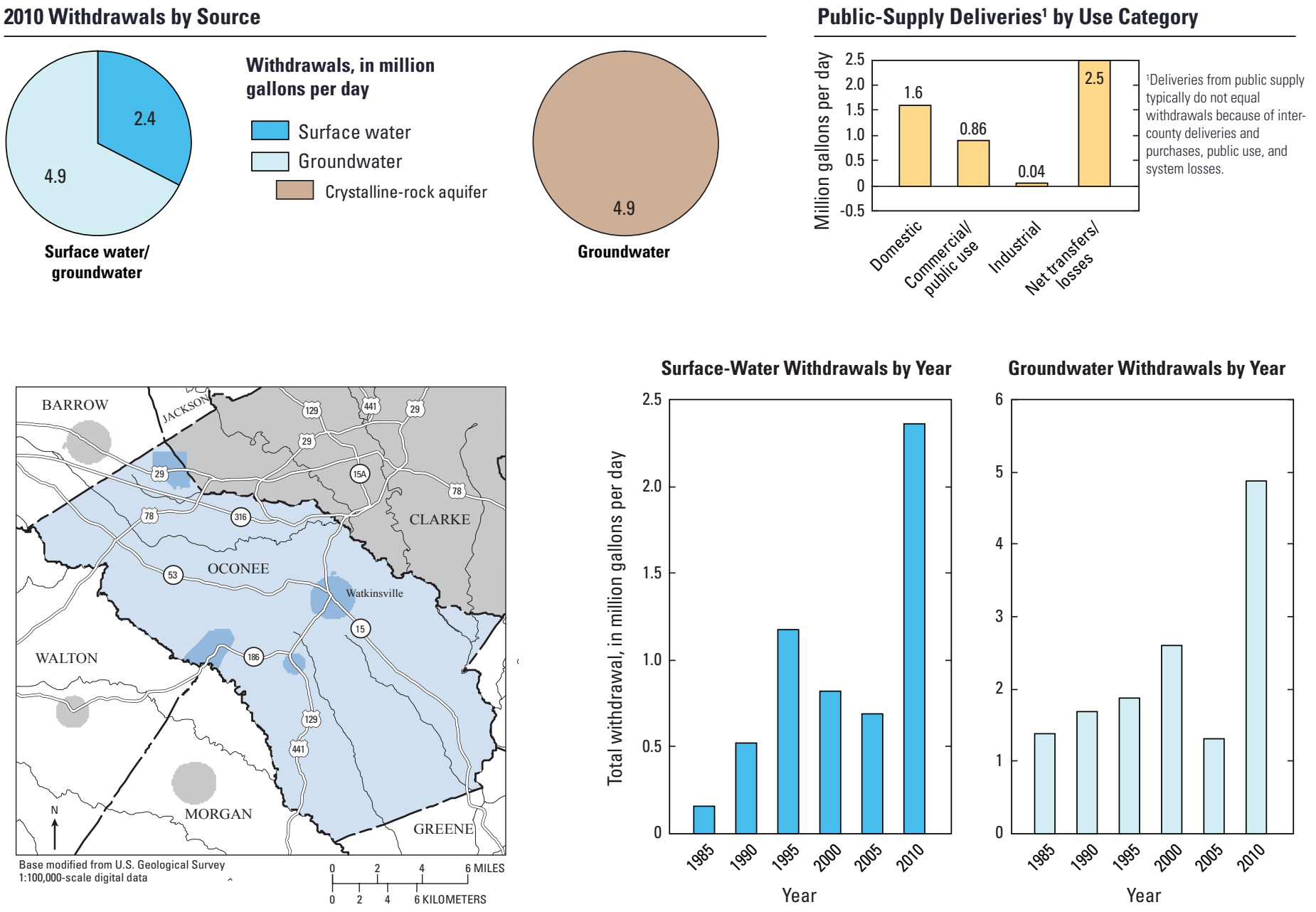


\section{OGLETHORPE COUNTY}

Population

Population served by public supply-Groundwater $\quad 2,910$

Population served by public supply-Surface water $\quad 980$

Acres irrigated

390

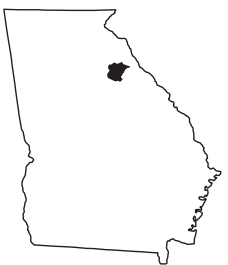

2010 WATER WITHDRAWALS AND ESTIMATED USE, IN MILLION GALLONS PER DAY

$[-$, not applicable $]$

\begin{tabular}{|c|c|c|c|c|c|c|c|c|}
\hline \multirow{3}{*}{ Category } & \multicolumn{3}{|c|}{ Withdrawals } & \multirow{3}{*}{$\begin{array}{l}\text { Total } \\
\text { use }^{1}\end{array}$} & \multirow{3}{*}{$\begin{array}{l}\text { Surface- } \\
\text { water } \\
\text { returns }\end{array}$} & \multirow{2}{*}{\multicolumn{3}{|c|}{$\begin{array}{l}\text { Withdrawals by Major Industrial Groups } \\
\text { [NAICS, North American Industrial Classification System code] }\end{array}$}} \\
\hline & \multirow{2}{*}{$\begin{array}{c}\text { Ground- } \\
\text { water }\end{array}$} & \multirow{2}{*}{$\begin{array}{c}\text { Surface } \\
\text { water }\end{array}$} & \multirow[b]{2}{*}{ Total } & & & & & \\
\hline & & & & & & NAICS & Groundwater & Surface water \\
\hline \multirow{2}{*}{$\begin{array}{l}\text { Public supply } \\
\text { Domestic }\end{array}$} & 0.20 & 0.10 & 0.30 & \multirow{2}{*}{$\begin{array}{l}- \\
1.11\end{array}$} & \multirow{2}{*}{$\begin{array}{c}- \\
0.00\end{array}$} & \multirow{2}{*}{ None } & \multirow{2}{*}{-} & \multirow{2}{*}{-} \\
\hline & 0.83 & 0.00 & 0.83 & & & & & \\
\hline Commercial/public use & 0.00 & 0.00 & 0.00 & 0.00 & 0.00 & \multirow{4}{*}{\multicolumn{3}{|c|}{ Withdrawals by Major Public Suppliers }} \\
\hline Industrial & 0.00 & 0.00 & 0.00 & 0.00 & 0.00 & & & \\
\hline Public-supply losses & - & - & - & 0.02 & - & & & \\
\hline Public wastewater treatment & - & - & - & - & 0.05 & & & \\
\hline $\begin{array}{l}\text { Mining } \\
\text { Irrigation-Crop }\end{array}$ & 0.00 & 0.00 & 0.00 & $\begin{array}{l}0.00 \\
0.31\end{array}$ & $\begin{array}{l}0.00 \\
0.00\end{array}$ & Name & Groundwater & Surface water \\
\hline Irrigation-Golf course & 0.00 & 0.00 & 0.00 & 0.00 & 0.00 & City of Crawford & 0.00 & 0.10 \\
\hline Livestock/aquaculture & 0.00 & 0.76 & 0.76 & 0.76 & 0.00 & City of Lexington & 0.06 & 0.00 \\
\hline Thermoelectric power & 0.00 & 0.00 & 0.00 & 0.00 & 0.00 & City of Arnoldsville & 0.03 & 0.00 \\
\hline TOTAL & 1.28 & 0.92 & 2.20 & 2.20 & 0.05 & & & \\
\hline
\end{tabular}

${ }^{1}$ Total use is total withdrawal plus public supply deliveries and losses.

\section{Withdrawals by Source}
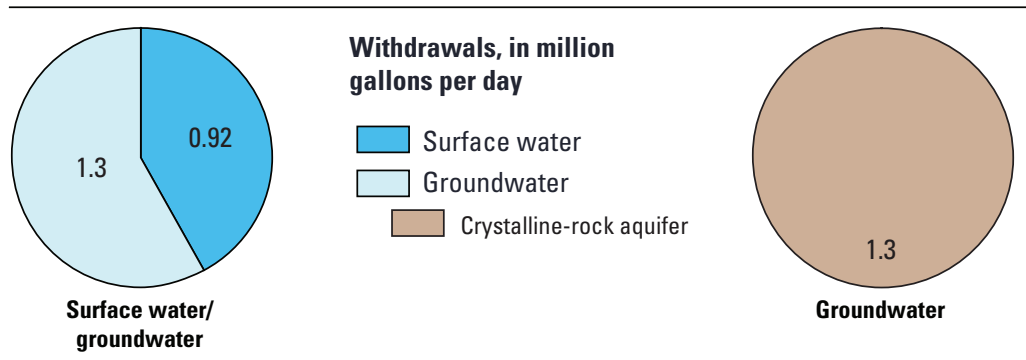

Public-Supply Deliveries by Use Category

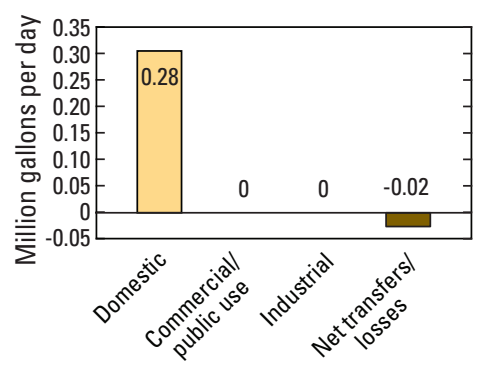

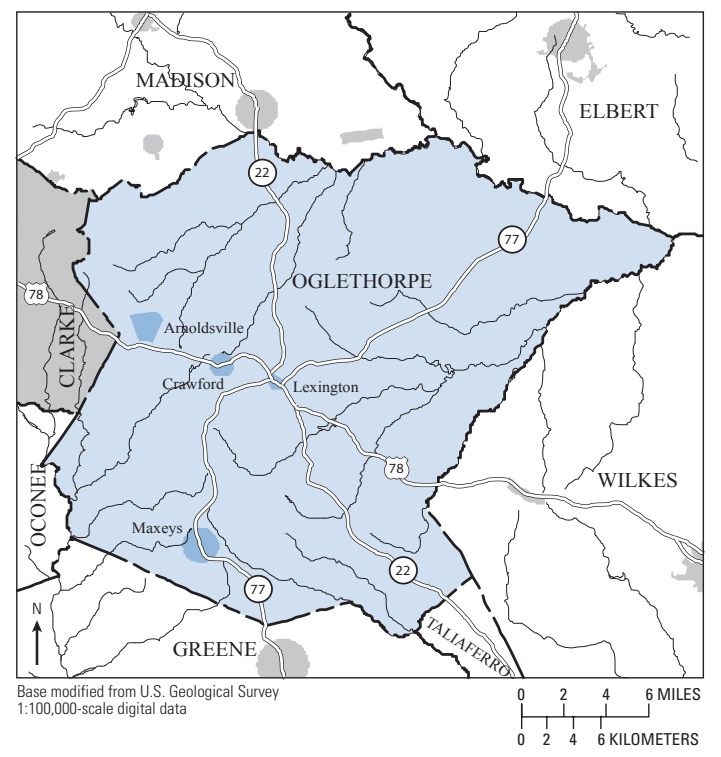

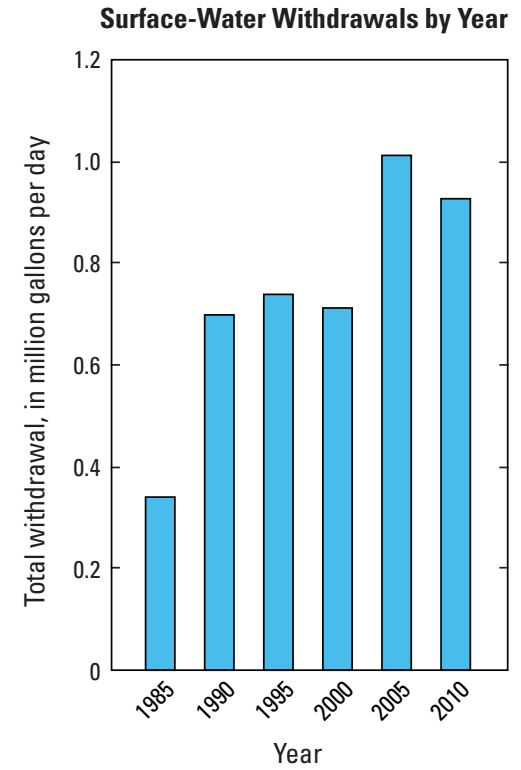

Groundwater Withdrawals by Year

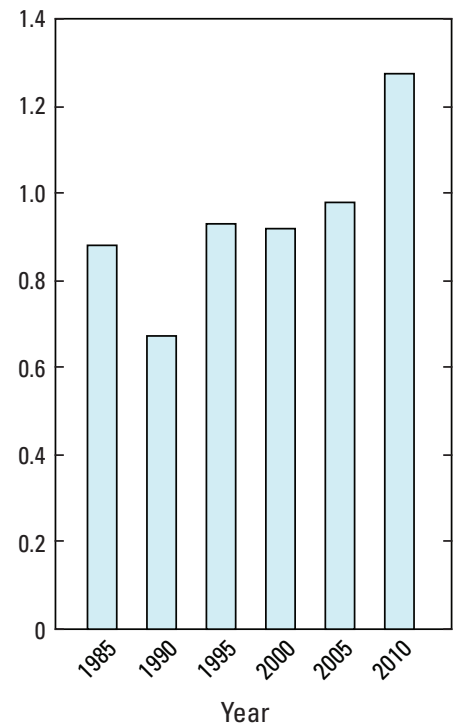




\section{PAULDING COUNTY}

Population

142,324

Population served by public supply-Groundwater

Population served by public supply-Surface water 130,110

Acres irrigated
1,670

447

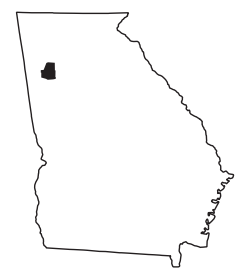

2010 WATER WITHDRAWALS AND ESTIMATED USE, IN MILLION GALLONS PER DAY

$[-$, not applicable; Mgal/d, million gallons per day $]$

\begin{tabular}{|c|c|c|c|c|c|c|c|c|}
\hline \multirow{3}{*}{ Category } & \multicolumn{3}{|c|}{ Withdrawals } & \multirow{3}{*}{$\begin{array}{l}\text { Total } \\
\text { use }^{1}\end{array}$} & \multirow{3}{*}{$\begin{array}{l}\text { Surface- } \\
\text { water } \\
\text { returns } \\
\end{array}$} & \multirow{2}{*}{\multicolumn{3}{|c|}{$\begin{array}{l}\text { Withdrawals by Major Industrial Groups } \\
\text { [NAICS, North American Industrial Classification System code] }\end{array}$}} \\
\hline & \multirow{2}{*}{$\begin{array}{c}\text { Ground- } \\
\text { water }\end{array}$} & \multirow{2}{*}{$\begin{array}{c}\text { Surface } \\
\text { water }\end{array}$} & \multirow[b]{2}{*}{ Total } & & & & & \\
\hline & & & & & & NAICS & Groundwater & Surface water \\
\hline Public supply & 0.13 & 0.00 & 0.13 & - & \multirow{2}{*}{$\begin{array}{c}- \\
0.00\end{array}$} & \multirow{2}{*}{ None } & \multirow{2}{*}{-} & \multirow{2}{*}{-} \\
\hline Domestic & 0.79 & 0.00 & 0.79 & 8.88 & & & & \\
\hline Commercial/public use & 0.00 & 0.00 & 0.00 & 0.67 & 0.00 & & & \\
\hline Industrial & 0.00 & 0.00 & 0.00 & 0.16 & 0.00 & & & \\
\hline Public-supply losses & - & - & - & 1.74 & - & & & \\
\hline \multirow{2}{*}{$\begin{array}{l}\text { Public wastewater treatment } \\
\text { Mining }\end{array}$} & \multirow{2}{*}{$\begin{array}{c}- \\
0.20\end{array}$} & \multirow{2}{*}{$\begin{array}{c}- \\
0.00\end{array}$} & \multirow{2}{*}{$\begin{array}{c}- \\
0.20\end{array}$} & \multirow{2}{*}{$\begin{array}{l}- \\
0.20\end{array}$} & 0.71 & \multicolumn{3}{|c|}{ Withdrawals by Major Public Suppliers } \\
\hline & & & & & $\begin{array}{l}0.00 \\
0.00\end{array}$ & Name & Groundwater & Surface water \\
\hline $\begin{array}{l}\text { Irrigation-Crop } \\
\text { Irrigation-Golf course }\end{array}$ & $\begin{array}{l}0.13 \\
0.00\end{array}$ & $\begin{array}{l}0.00 \\
0.72\end{array}$ & $\begin{array}{l}0.13 \\
0.72\end{array}$ & $\begin{array}{l}0.13 \\
0.72\end{array}$ & $\begin{array}{l}0.00 \\
0.00\end{array}$ & City of $\mathrm{H}$ & 007 & 000 \\
\hline Livestock/aquaculture & 0.00 & 0.11 & 0.11 & 0.11 & 0.00 & City of Hiram & 0.07 & 0.00 \\
\hline Thermoelectric power & 0.00 & 0.00 & 0.00 & 0.00 & 0.00 & City or Danas & 0.00 & 0.00 \\
\hline
\end{tabular}

1.25

0.83

2.08

12.61

0.71

'Total use is total withdrawal plus public supply deliveries and losses. An average of $10.53 \mathrm{Mgal} / \mathrm{d}$ was delivered from Cobb County, Georgia to Paulding County in 2010.

\section{Withdrawals by Source}
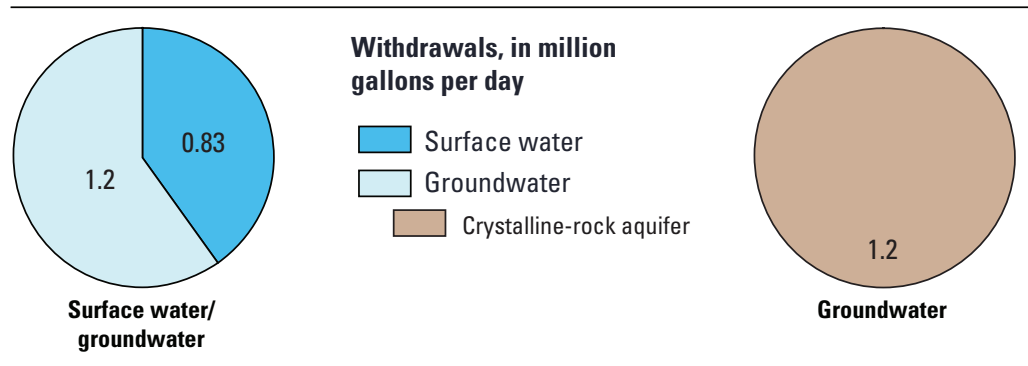

Public-Supply Deliveries ${ }^{1}$ by Use Category
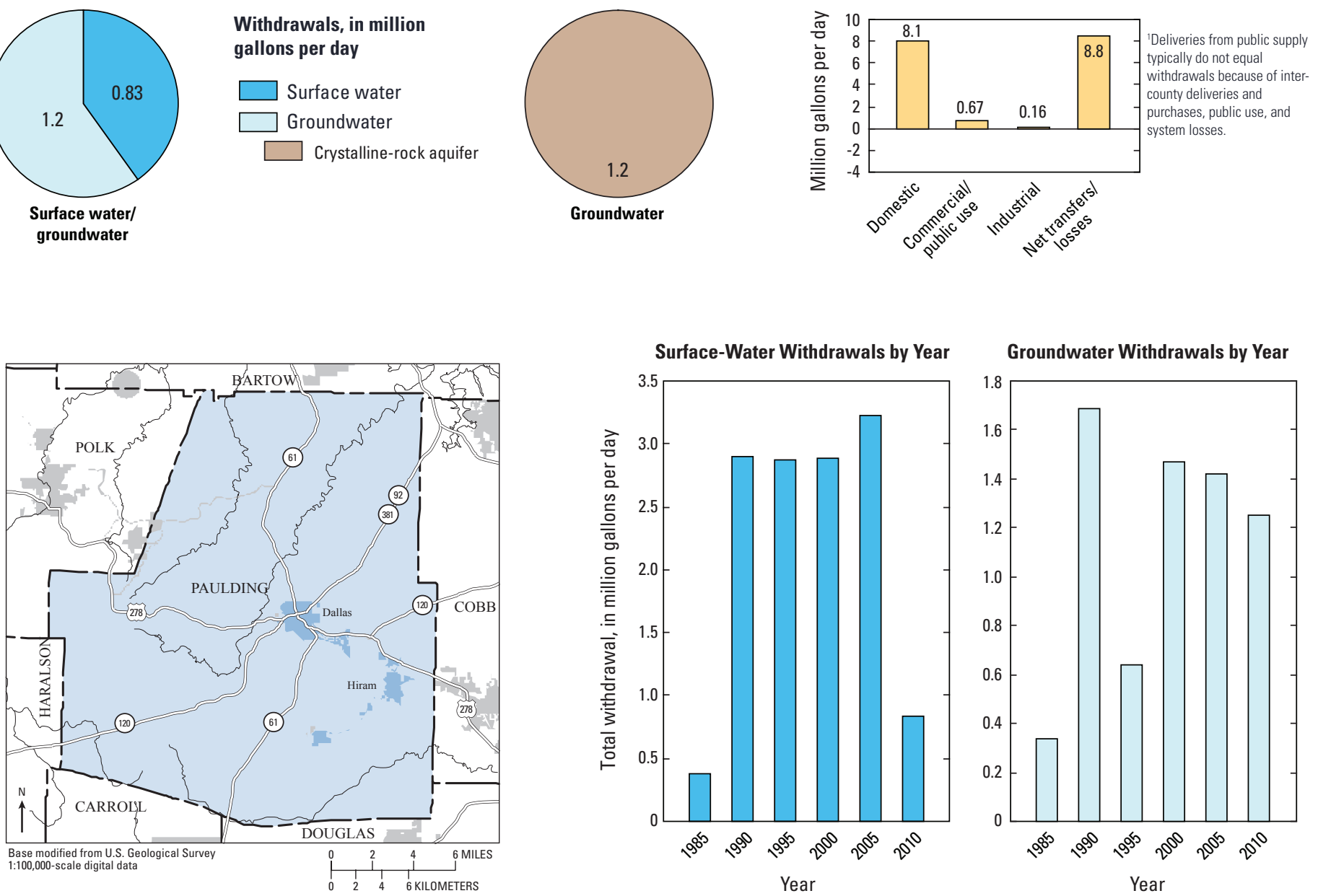

Surface-Water Withdrawals by Year

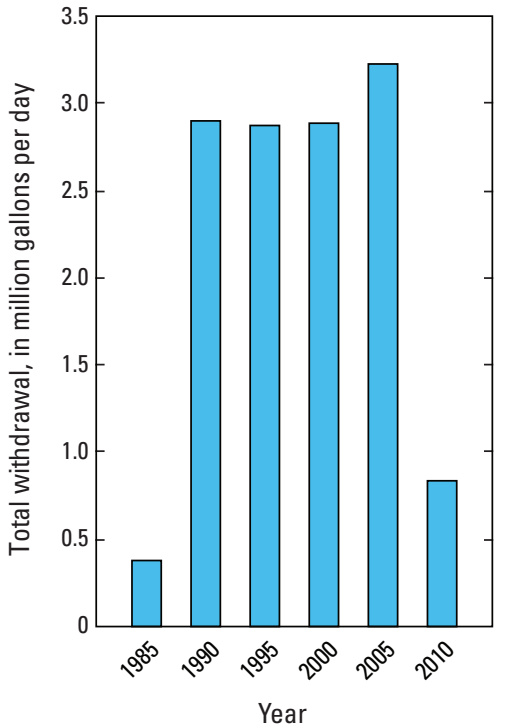

Groundwater Withdrawals by Year

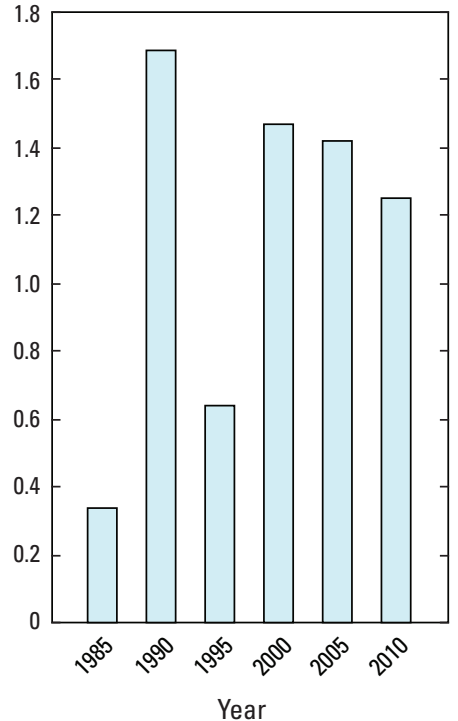




\section{PEACH COUNTY}

Population

Population served by public supply-Groundwater

Population served by public supply-Surface water Acres irrigated

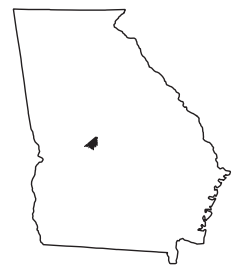

2010 WATER WITHDRAWALS AND ESTIMATED USE, IN MILLION GALLONS PER DAY

$[-$, not applicable $]$

\begin{tabular}{|c|c|c|c|c|c|c|c|c|}
\hline \multirow{3}{*}{ Category } & \multicolumn{3}{|c|}{ Withdrawals } & \multirow{3}{*}{$\begin{array}{l}\text { Total } \\
\text { use }^{1}\end{array}$} & \multirow{3}{*}{$\begin{array}{c}\text { Surface- } \\
\text { water } \\
\text { returns } \\
\end{array}$} & \multirow{2}{*}{\multicolumn{3}{|c|}{$\begin{array}{l}\text { Withdrawals by Major Industrial Groups } \\
\text { [NAICS, North American Industrial Classification System code] }\end{array}$}} \\
\hline & \multirow{2}{*}{$\begin{array}{c}\text { Ground- } \\
\text { water }\end{array}$} & \multirow{2}{*}{$\begin{array}{c}\text { Surface } \\
\text { water }\end{array}$} & \multirow[b]{2}{*}{ Total } & & & & & \\
\hline & & & & & & NAICS & Groundwater & Surface water \\
\hline Commercial/public use & 0.00 & 0.00 & 0.00 & 0.52 & 0.00 & & & \\
\hline Industrial & 0.00 & 0.00 & 0.00 & 0.09 & 0.00 & & & \\
\hline $\begin{array}{l}\text { Mining } \\
\text { Irrigation__Cron }\end{array}$ & $\begin{array}{l}0.00 \\
5.14\end{array}$ & $\begin{array}{l}0.00 \\
0.08\end{array}$ & $\begin{array}{l}0.00 \\
5.22\end{array}$ & $\begin{array}{l}0.00 \\
5.22\end{array}$ & $\begin{array}{l}0.00 \\
0.00\end{array}$ & Name & Groundwater & Surface water \\
\hline Irrigation-Golf course & 0.00 & 0.00 & 0.00 & 0.00 & 0.00 & Fort Valley Utility & & \\
\hline Livestock/aquaculture & 0.01 & 0.03 & 0.04 & 0.04 & 0.00 & Commission & 2.15 & 0.00 \\
\hline Thermoelectric power & 0.00 & 0.00 & 0.00 & 0.00 & 0.00 & City of Byron & 0.46 & 0.00 \\
\hline
\end{tabular}

TOTAL $8.55 \quad 0.11$

\section{Withdrawals by Source}
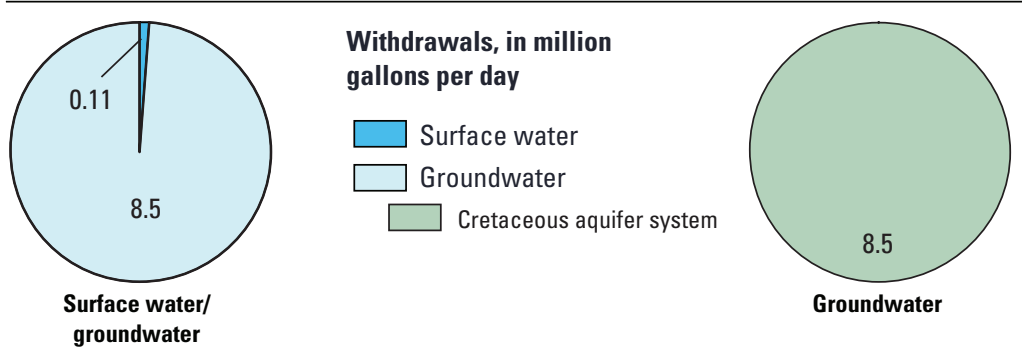

Public-Supply Deliveries by Use Category

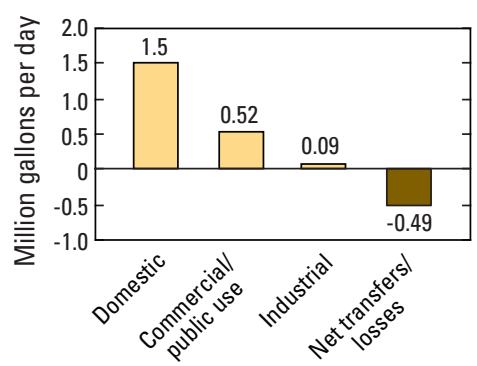

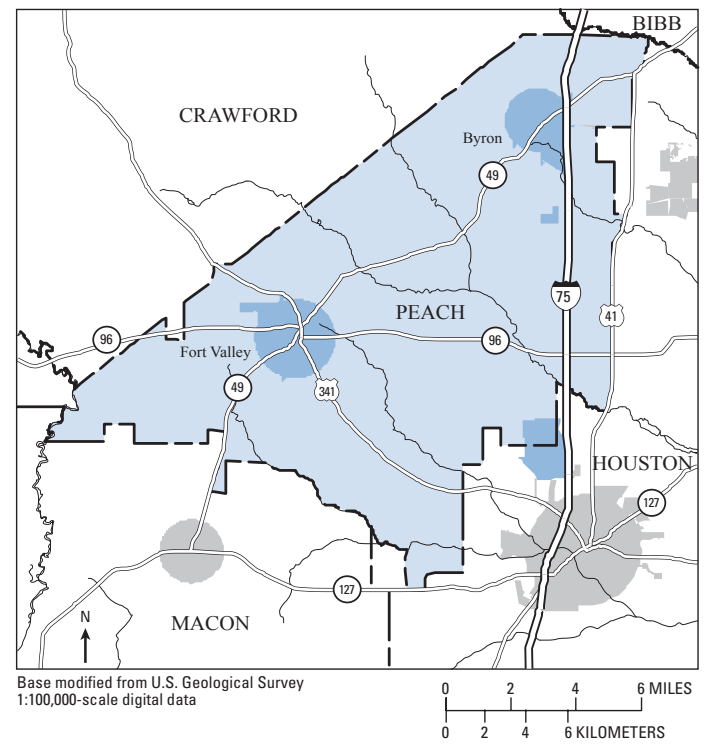

Surface-Water Withdrawals by Year

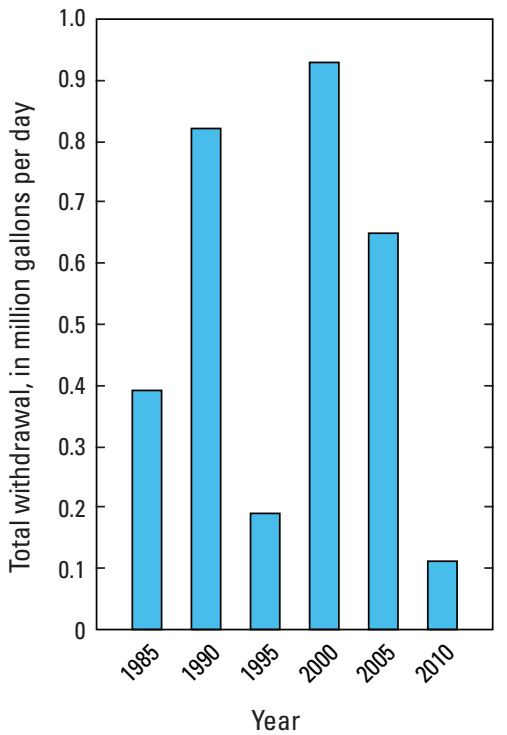

Groundwater Withdrawals by Year

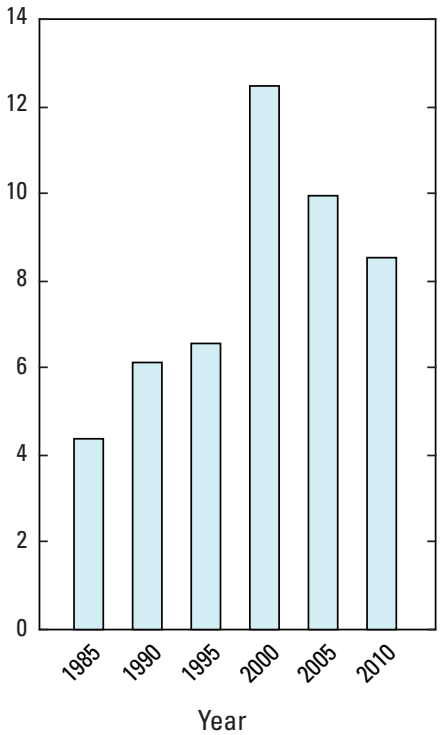


PICKENS COUNTY

Population

Population served by public supply-Groundwater $\quad 9,090$

Population served by public supply-Surface water $\quad 13,170$

Acres irrigated

60

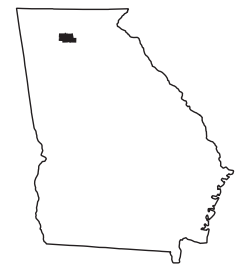

2010 WATER WITHDRAWALS AND ESTIMATED USE, IN MILLION GALLONS PER DAY

$[-$, not applicable; Mgal/d, million gallons per day $]$

\begin{tabular}{|c|c|c|c|c|c|c|c|c|}
\hline \multirow{3}{*}{ Category } & \multicolumn{3}{|c|}{ Withdrawals } & \multirow{3}{*}{$\begin{array}{l}\text { Total } \\
\text { use }^{1}\end{array}$} & \multirow{3}{*}{$\begin{array}{l}\text { Surface- } \\
\text { water } \\
\text { returns } \\
\end{array}$} & \multirow{2}{*}{\multicolumn{3}{|c|}{$\begin{array}{l}\text { Withdrawals by Major Industrial Groups } \\
\text { [NAICS, North American Industrial Classification System code] }\end{array}$}} \\
\hline & \multirow{2}{*}{$\begin{array}{c}\text { Ground- } \\
\text { water }\end{array}$} & \multirow{2}{*}{$\begin{array}{c}\text { Surface } \\
\text { water }\end{array}$} & \multirow[b]{2}{*}{ Total } & & & & & \\
\hline & & & & & & NAICS & Groundwater & Surface water \\
\hline \multirow{2}{*}{$\begin{array}{l}\text { Public supply } \\
\text { Domestic }\end{array}$} & 0.73 & 2.21 & 2.94 & \multirow{2}{*}{$\begin{array}{l}- \\
3.07\end{array}$} & \multirow{2}{*}{$\begin{array}{c}- \\
0.00\end{array}$} & \multirow{2}{*}{ None } & \multirow{2}{*}{-} & \multirow{2}{*}{-} \\
\hline & 0.54 & 0.00 & 0.54 & & & & & \\
\hline Commercial/public use & 0.00 & 0.00 & 0.00 & 0.36 & 0.00 & \multirow{4}{*}{\multicolumn{3}{|c|}{ Withdrawals by Major Public Suppliers }} \\
\hline Industrial & 0.00 & 0.00 & 0.00 & 0.10 & 0.24 & & & \\
\hline Public-supply losses & - & - & - & 0.55 & - & & & \\
\hline Public wastewater treatment & - & - & - & - & 0.56 & & & \\
\hline \multirow{2}{*}{$\begin{array}{l}\text { Mining } \\
\text { Irrigation-Crop }\end{array}$} & 0.22 & 0.00 & 0.22 & 0.22 & 0.03 & \multirow[t]{2}{*}{ Name } & \multirow[t]{2}{*}{ Groundwater } & \multirow{2}{*}{ Surface water } \\
\hline & 0.01 & 0.00 & 0.01 & 0.01 & 0.00 & & & \\
\hline Irrigation-Golf course & 0.00 & 0.10 & 0.10 & 0.10 & 0.00 & City of Jasper & 0.73 & 1.00 \\
\hline Livestock/aquaculture & 0.00 & 0.26 & 0.26 & 0.26 & 0.00 & Big Canoe Utilities & 0.00 & 0.98 \\
\hline Thermoelectric power & 0.00 & 0.00 & 0.00 & 0.00 & 0.00 & Bent Tree Community & 0.00 & 0.23 \\
\hline TOTAL & 1.50 & 2.57 & 4.07 & 4.67 & 0.83 & & & \\
\hline
\end{tabular}

Total use is total withdrawal plus public supply deliveries and losses. An average of $0.37 \mathrm{Mgal} / \mathrm{d}$ was delivered from Gordon County,

Georgia, $0.18 \mathrm{Mgal} / \mathrm{d}$ from Cherokee County, Georgia, and 0.05 from Newton County, Georgia to Pickens County in 2010.

\section{Withdrawals by Source}

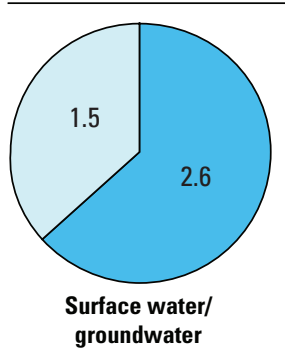

Withdrawals, in million gallons per day

$\square$ Surface water
$\square$ Groundwater
$\square$ Crystalline-rock aquifer
groundwater

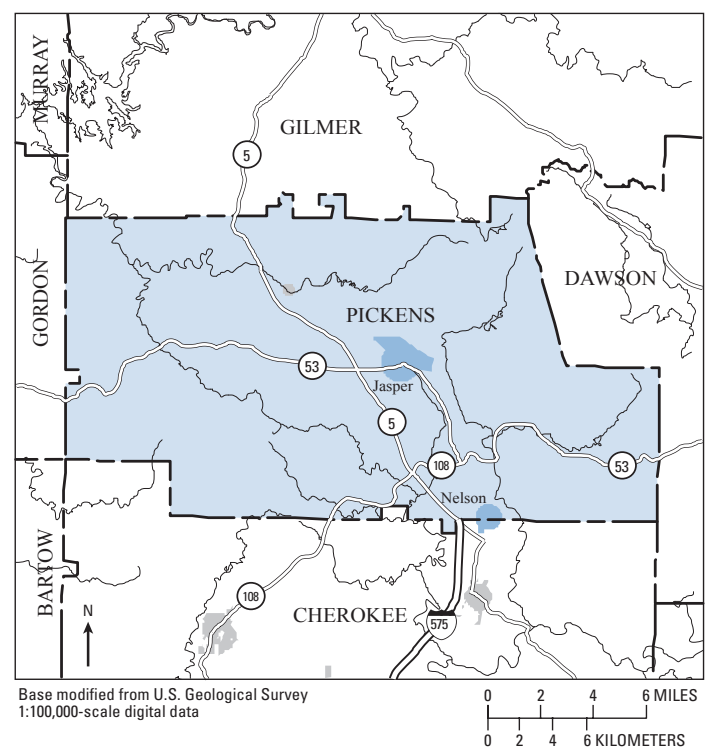

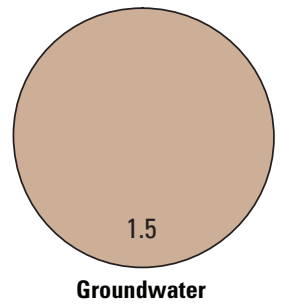

Public-Supply Deliveries ${ }^{1}$ by Use Category

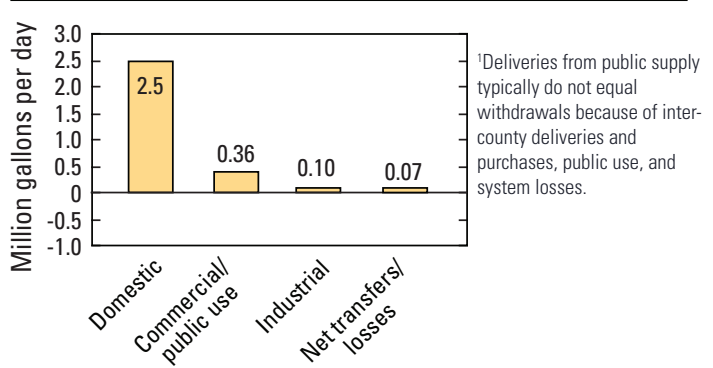

Surface-Water Withdrawals by Year

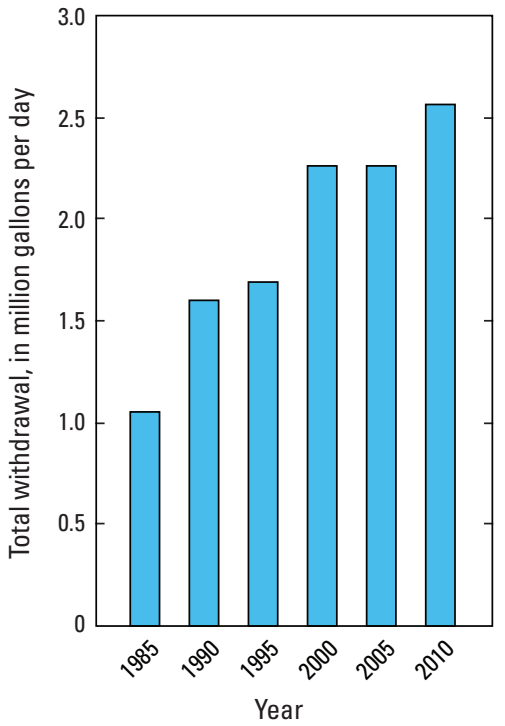

Groundwater Withdrawals by Year

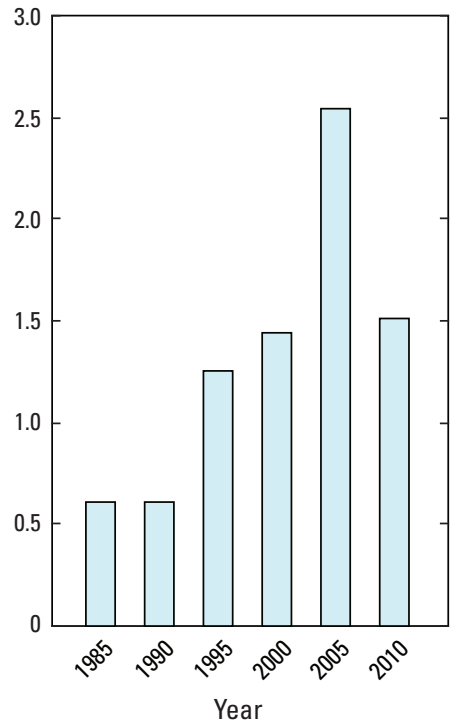




\section{PIERCE COUNTY}

Population

18,758

Population served by public supply-Groundwater $\quad 6,760$

Population served by public supply-Surface water 0

Acres irrigated

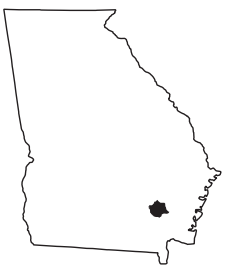

2010 WATER WITHDRAWALS AND ESTIMATED USE, IN MILLION GALLONS PER DAY

$[-$, not applicable]

\begin{tabular}{|c|c|c|c|c|c|c|c|c|}
\hline \multirow{3}{*}{ Category } & \multicolumn{3}{|c|}{ Withdrawals } & \multirow{3}{*}{$\begin{array}{l}\text { Total } \\
\text { use }^{1}\end{array}$} & \multirow{3}{*}{$\begin{array}{l}\text { Surface- } \\
\text { water } \\
\text { returns }\end{array}$} & \multirow{2}{*}{\multicolumn{3}{|c|}{$\begin{array}{l}\text { Withdrawals by Major Industrial Groups } \\
\text { [NAICS, North American Industrial Classification System code] }\end{array}$}} \\
\hline & \multirow{2}{*}{$\begin{array}{c}\text { Ground- } \\
\text { water }\end{array}$} & \multirow{2}{*}{$\begin{array}{c}\text { Surface } \\
\text { water }\end{array}$} & \multirow[b]{2}{*}{ Total } & & & & & \\
\hline & & & & & & NAICS & Groundwater & Surface water \\
\hline Public supply & 0.74 & 0.00 & 0.74 & - & - & 322-Paper, pulp & 0.08 & 0.00 \\
\hline Domestic & 0.90 & 0.00 & 0.90 & 1.44 & 0.00 & & & \\
\hline Commercial/public use & 0.00 & 0.00 & 0.00 & 0.07 & 0.00 & & & \\
\hline Industrial & 0.08 & 0.00 & 0.08 & 0.13 & 0.00 & & & \\
\hline Public-supply losses & - & - & - & 0.08 & - & & & \\
\hline Public wastewater treatment & - & - & - & - & 0.00 & Withdrawals by I & r Public Supp & \\
\hline $\begin{array}{l}\text { Mining } \\
\text { Irrigation-Crop }\end{array}$ & $\begin{array}{l}0.00 \\
1.87\end{array}$ & $\begin{array}{l}0.00 \\
0.33\end{array}$ & $\begin{array}{l}0.00 \\
2.20\end{array}$ & $\begin{array}{l}0.00 \\
2.20\end{array}$ & $\begin{array}{l}0.00 \\
0.00\end{array}$ & Name & Groundwater & Surface water \\
\hline Irrigation-Golf course & 0.11 & 0.00 & 0.11 & 0.11 & 0.00 & City of Blackshear & 0.48 & 0.00 \\
\hline Livestock/aquaculture & 0.02 & 0.08 & 0.10 & 0.10 & 0.00 & W and D Utilities & 0.12 & 0.00 \\
\hline Thermoelectric power & 0.00 & 0.00 & 0.00 & 0.00 & 0.00 & City of Patterson & 0.06 & 0.00 \\
\hline
\end{tabular}

'Total use is total withdrawal plus public supply deliveries and losses.

\section{Withdrawals by Source}
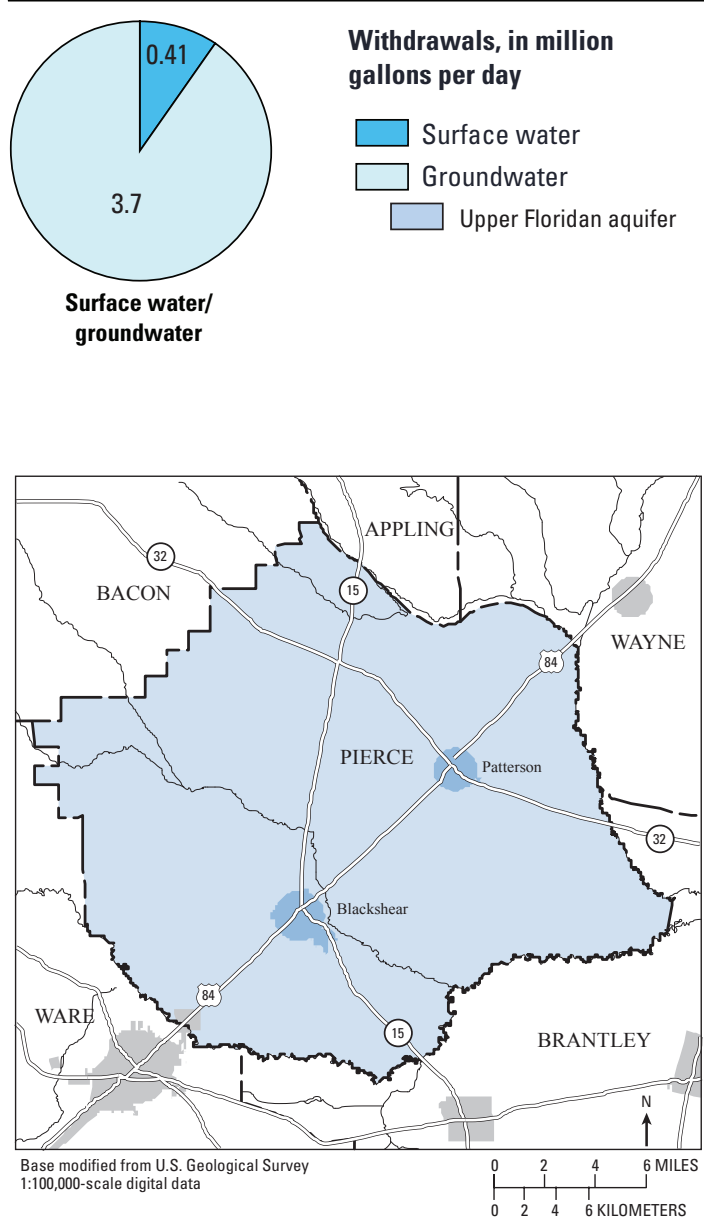

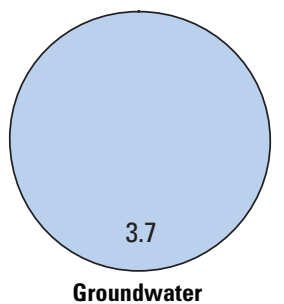

Public-Supply Deliveries by Use Category

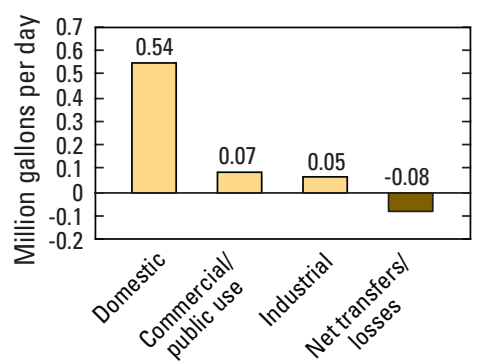

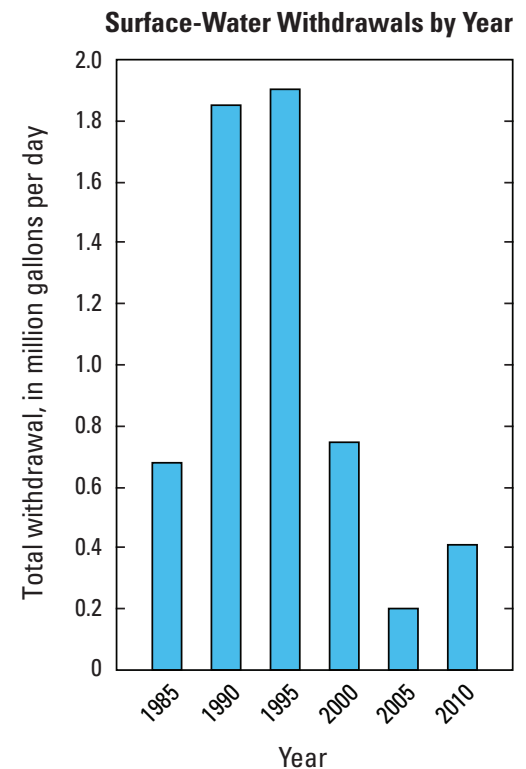

Groundwater Withdrawals by Year

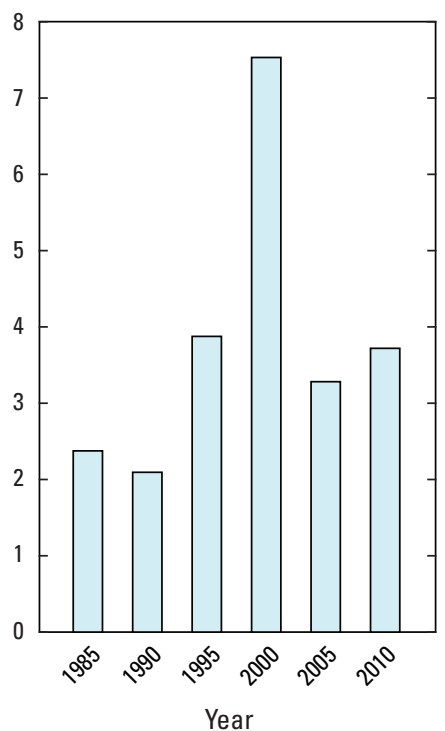




\section{PIKE COUNTY}

Population

Population served by public supply-Groundwater Population served by public supply-Surface water

1,250

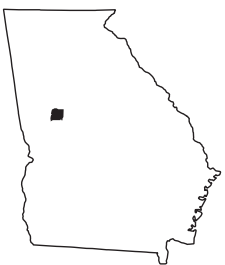

2010 WATER WITHDRAWALS AND ESTIMATED USE, IN MILLION GALLONS PER DAY

$[-$, not applicable; Mgal/d, million gallons per day $]$

\begin{tabular}{|c|c|c|c|c|c|}
\hline \multirow[b]{2}{*}{ Category } & \multicolumn{3}{|c|}{ Withdrawals } & \multirow[b]{2}{*}{$\begin{array}{l}\text { Total } \\
\text { use }^{1}\end{array}$} & \multirow{2}{*}{$\begin{array}{c}\text { Surface- } \\
\text { water } \\
\text { returns }\end{array}$} \\
\hline & $\begin{array}{c}\text { Ground- } \\
\text { water }\end{array}$ & $\begin{array}{c}\text { Surface } \\
\text { water }\end{array}$ & Total & & \\
\hline Public supply & 0.29 & 4.15 & 4.44 & - & - \\
\hline Domestic & 0.93 & 0.00 & 0.93 & 1.37 & 0.00 \\
\hline Commercial/public use & 0.00 & 0.00 & 0.00 & 0.09 & 0.01 \\
\hline Industrial & 0.00 & 0.00 & 0.00 & 0.00 & 0.00 \\
\hline Public-supply losses & - & - & - & 0.06 & - \\
\hline Public wastewater treatment & - & - & - & - & 0.14 \\
\hline Mining & 0.00 & 0.00 & 0.00 & 0.00 & 0.00 \\
\hline Irrigation-Crop & 0.00 & 1.00 & 1.00 & 1.00 & 0.00 \\
\hline Irrigation-Golf course & 0.00 & 0.39 & 0.39 & 0.39 & 0.00 \\
\hline Livestock/aquaculture & 0.02 & 0.14 & 0.16 & 0.16 & 0.00 \\
\hline Thermoelectric power & 0.00 & 0.00 & 0.00 & 0.00 & 0.00 \\
\hline TOTAL & 1.24 & 5.68 & 6.92 & 3.07 & 0.15 \\
\hline
\end{tabular}

'Total use is total withdrawal plus public supply deliveries and losses. An average of $3.88 \mathrm{Mgal} / \mathrm{d}$ was delivered to Spalding County, Georgia from Pike County in 2010.

\section{Withdrawals by Source}

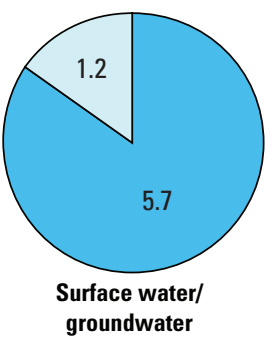

Withdrawals, in million gallons per day

groundwater
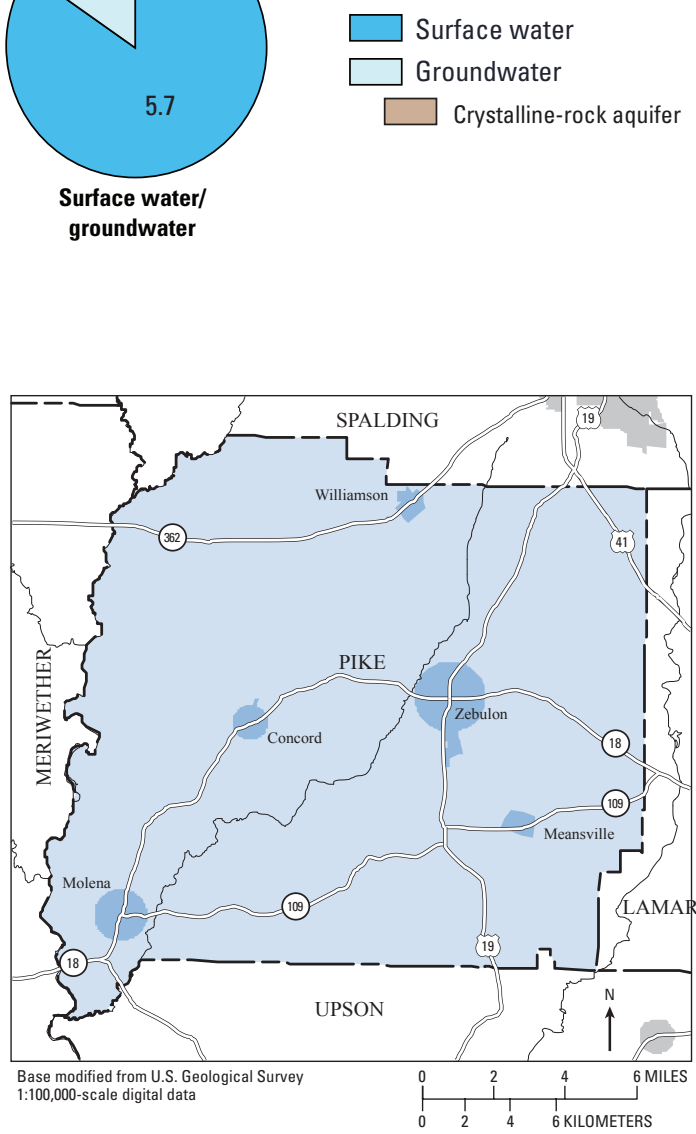

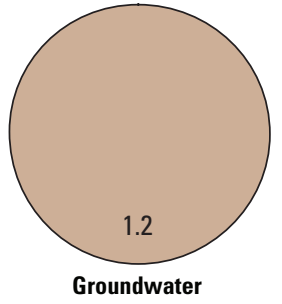

Public-Supply Deliveries ${ }^{1}$ by Use Category

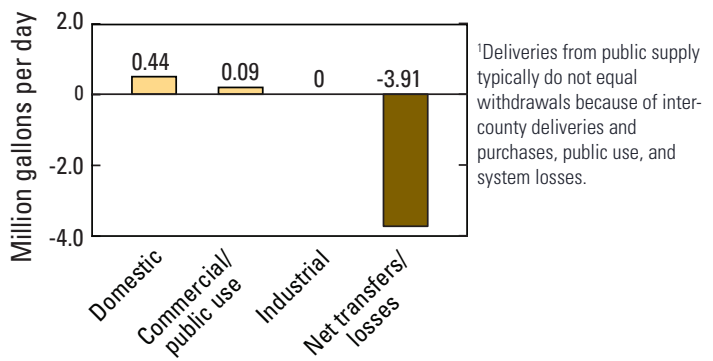

Withdrawals by Major Public Suppliers

\begin{tabular}{lcc}
\hline Name & Groundwater & Surface water \\
\hline City of Griffin & 0.00 & 14.15 \\
Pike County Rural & 0.21 & 0.00 \\
Development & 0.03 & 0.00 \\
City of Concord & 0.03 & 0.00 \\
City of Molena &
\end{tabular}

'This water was delivered to Spalding County, Georgia.

[NAICS, North American Industrial Classification System code]

NAICS

Groundwater

Surface water

None 


\section{POLK COUNTY}

Population

Population served by public supply-Groundwater

Population served by public supply-Surface water $\quad 33,840$ Acres irrigated

150

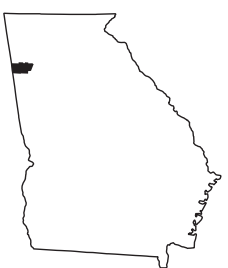

2010 WATER WITHDRAWALS AND ESTIMATED USE, IN MILLION GALLONS PER DAY

[-, not applicable; Mgal/d, million gallons per day]

\begin{tabular}{|c|c|c|c|c|c|c|c|c|}
\hline \multirow{3}{*}{ Category } & \multicolumn{3}{|c|}{ Withdrawals } & \multirow{3}{*}{$\begin{array}{l}\text { Total } \\
\text { use }^{1}\end{array}$} & \multirow{3}{*}{$\begin{array}{l}\text { Surface- } \\
\text { water } \\
\text { returns } \\
\end{array}$} & \multirow{2}{*}{\multicolumn{3}{|c|}{$\begin{array}{l}\text { Withdrawals by Major Industrial Groups } \\
\text { [NAICS, North American Industrial Classification System code] }\end{array}$}} \\
\hline & \multirow{2}{*}{$\begin{array}{c}\text { Ground- } \\
\text { water }\end{array}$} & \multirow{2}{*}{$\begin{array}{c}\text { Surface } \\
\text { water }\end{array}$} & \multirow[b]{2}{*}{ Total } & & & & & \\
\hline & & & & & & NAICS & Groundwater & Surface water \\
\hline Public supply & 1.35 & 4.15 & 5.50 & - & - & \multirow{2}{*}{ 325-Chemical products } & \multirow{2}{*}{1.29} & \multirow{2}{*}{0.00} \\
\hline Domestic & 0.07 & 0.00 & 0.07 & 3.27 & 0.00 & & & \\
\hline Commercial/public use & 0.00 & 0.00 & 0.00 & 0.51 & 0.00 & & & \\
\hline Industrial & 1.29 & 0.00 & 1.29 & 1.74 & 0.24 & & & \\
\hline Public-supply losses & - & - & - & 0.87 & - & & & \\
\hline Public wastewater treatment & - & - & - & - & 3.46 & \multicolumn{3}{|c|}{ Withdrawals by Major Public Suppliers } \\
\hline $\begin{array}{l}\text { Mining } \\
\text { Irrigation-Crop }\end{array}$ & $\begin{array}{l}0.08 \\
0.01\end{array}$ & $\begin{array}{l}0.00 \\
0.00\end{array}$ & $\begin{array}{l}0.08 \\
0.01\end{array}$ & $\begin{array}{l}0.08 \\
0.01\end{array}$ & $\begin{array}{l}0.00 \\
0.00\end{array}$ & Name & Groundwater & Surface water \\
\hline Irrigation-Golf course & 0.25 & 0.00 & 0.25 & 0.25 & 0.00 & \multirow{2}{*}{$\begin{array}{l}\text { Polk County Water } \\
\text { Authority }\end{array}$} & \multirow{2}{*}{0.00} & \multirow{2}{*}{2.37} \\
\hline Livestock/aquaculture & 0.04 & 0.52 & 0.56 & 0.56 & 0.00 & & & \\
\hline Thermoelectric power & 0.00 & 0.00 & 0.00 & 0.00 & 0.00 & City of Cedartown & 0.00 & 1.78 \\
\hline TOTAL & 3.09 & 4.67 & 7.76 & 7.29 & 3.70 & City of Rockmart & 1.35 & 0.00 \\
\hline
\end{tabular}

${ }^{1}$ Total use is total withdrawal plus public supply deliveries and losses. An average of $0.47 \mathrm{Mgal} / \mathrm{d}$ was delivered to Bartow County, Georgia from Polk County in 2010.

\section{Withdrawals by Source}

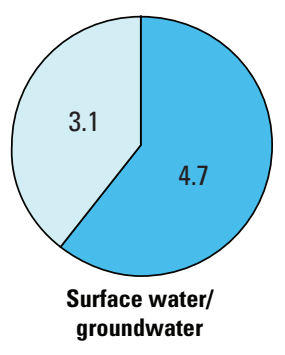

Withdrawals, in million gallons per day

$\square$ Surface water
$\square$ Groundwater
$\square$ Paleozoic-rock aquifer

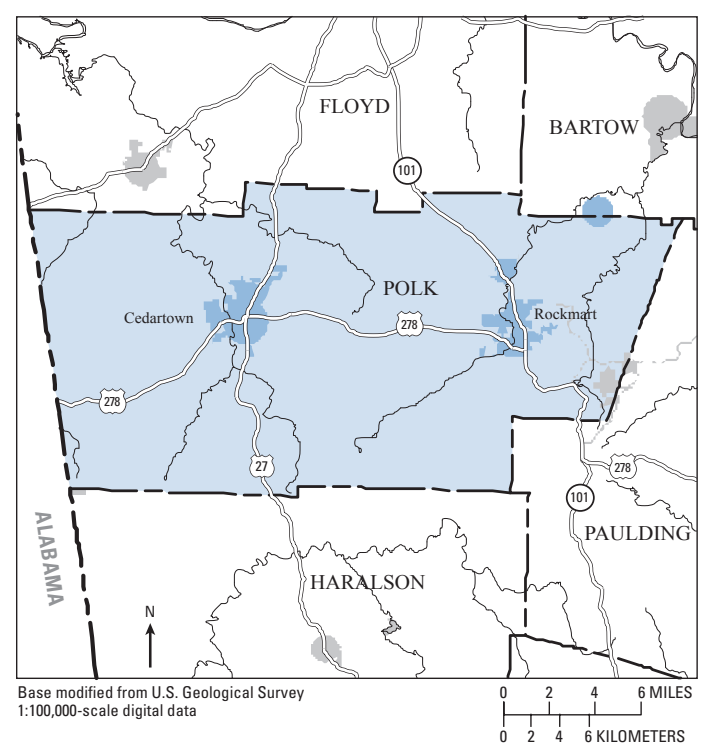

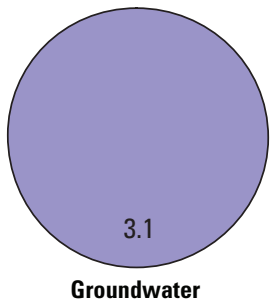

Public-Supply Deliveries ${ }^{1}$ by Use Category

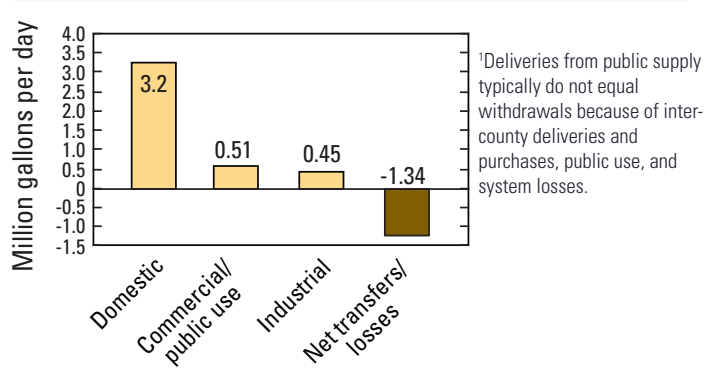

Surface-Water Withdrawals by Year

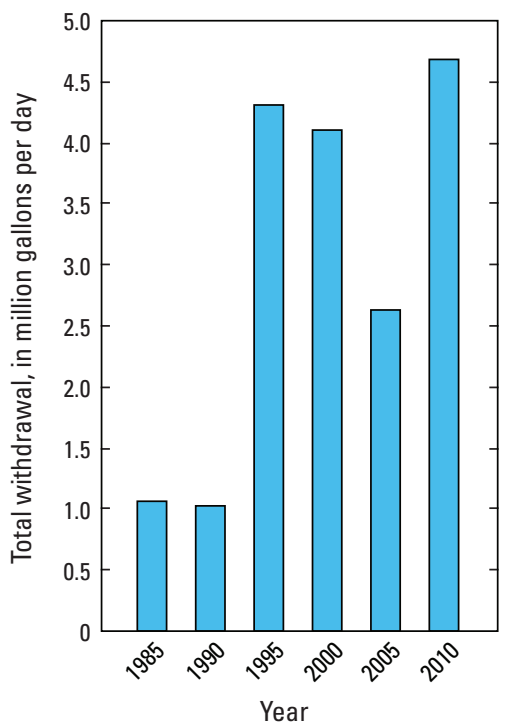

Groundwater Withdrawals by Year

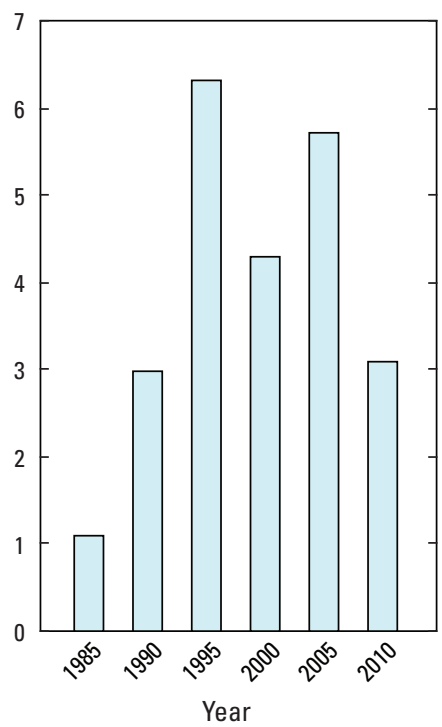




\section{PULASKI COUNTY}

Population

$\begin{array}{lr}\text { Population served by public supply-Groundwater } & 5,080 \\ \text { Population served by public supply-Surface water } & 0\end{array}$

Acres irrigated

17,310

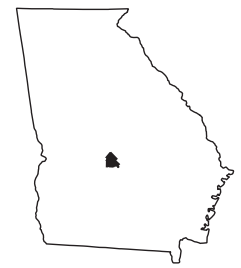

2010 WATER WITHDRAWALS AND ESTIMATED USE, IN MILLION GALLONS PER DAY

$[-$, not applicable]

\begin{tabular}{|c|c|c|c|c|c|c|c|c|}
\hline \multirow{3}{*}{ Category } & \multicolumn{3}{|c|}{ Withdrawals } & \multirow{3}{*}{$\begin{array}{l}\text { Total } \\
\text { use }^{1}\end{array}$} & \multirow{3}{*}{$\begin{array}{l}\text { Surface- } \\
\text { water } \\
\text { returns } \\
\end{array}$} & \multirow{2}{*}{\multicolumn{3}{|c|}{$\begin{array}{l}\text { Withdrawals by Major Industrial Groups } \\
\text { [NAICS, North American Industrial Classification System code] }\end{array}$}} \\
\hline & \multirow{2}{*}{$\begin{array}{c}\text { Ground- } \\
\text { water }\end{array}$} & \multirow{2}{*}{$\begin{array}{c}\text { Surface } \\
\text { water }\end{array}$} & \multirow[b]{2}{*}{ Total } & & & & & \\
\hline & & & & & & NAICS & Groundwater & Surface water \\
\hline Public supply & 1.00 & 0.00 & 1.00 & - & - & 322-Paper, pulp & 0.54 & 0.00 \\
\hline Domestic & 0.52 & 0.00 & 0.52 & 1.11 & 0.00 & & 0.34 & 0.00 \\
\hline Commercial/public use & 0.00 & 0.00 & 0.00 & 0.17 & 0.00 & & & \\
\hline Industrial & 0.54 & 0.00 & 0.54 & 0.55 & 0.45 & & & \\
\hline Public-supply losses & - & - & - & 0.23 & - & & & \\
\hline Public wastewater treatment & - & - & - & - & 0.83 & Withdrawals by Major & Public Supp & \\
\hline Mining & $\begin{array}{r}0.00 \\
1013\end{array}$ & 0.00 & 0.00 & $\begin{array}{r}0.00 \\
13.31\end{array}$ & $\begin{array}{l}0.00 \\
0.00\end{array}$ & Name & Groundwater & Surface water \\
\hline $\begin{array}{l}\text { Irrigation-Crop } \\
\text { Irrigation-Golf course }\end{array}$ & $\begin{array}{r}10.13 \\
0.00\end{array}$ & $\begin{array}{l}3.18 \\
0.00\end{array}$ & $\begin{array}{r}13.31 \\
0.00\end{array}$ & $\begin{array}{r}13.31 \\
0.00\end{array}$ & $\begin{array}{l}0.00 \\
0.00\end{array}$ & City of Hawkinsville & 0.88 & 0.00 \\
\hline Livestock/aquaculture & 0.00 & 0.02 & 0.02 & 0.02 & 0.00 & Hartford Water Authority & 0.12 & 0.00 \\
\hline Thermoelectric power & 0.00 & 0.00 & 0.00 & 0.00 & 0.00 & & & \\
\hline
\end{tabular}

TOTAL

12.19

3.20

15.39

15.39

1.28

'Total use is total withdrawal plus public supply deliveries and losses.

2010 Withdrawals by Source
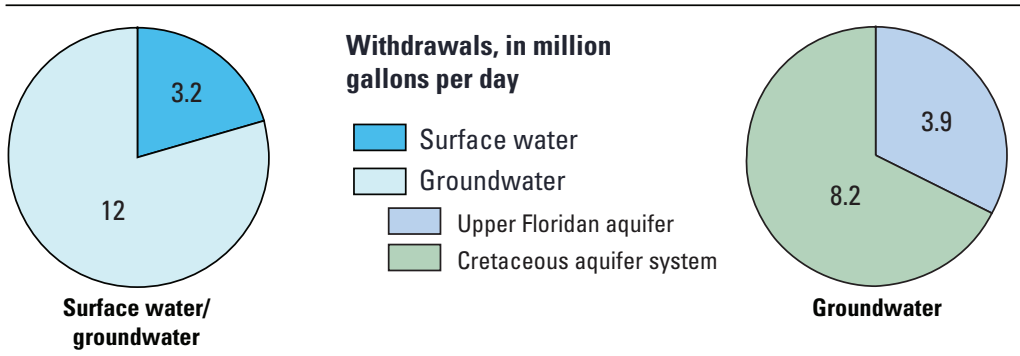

Public-Supply Deliveries by Use Category
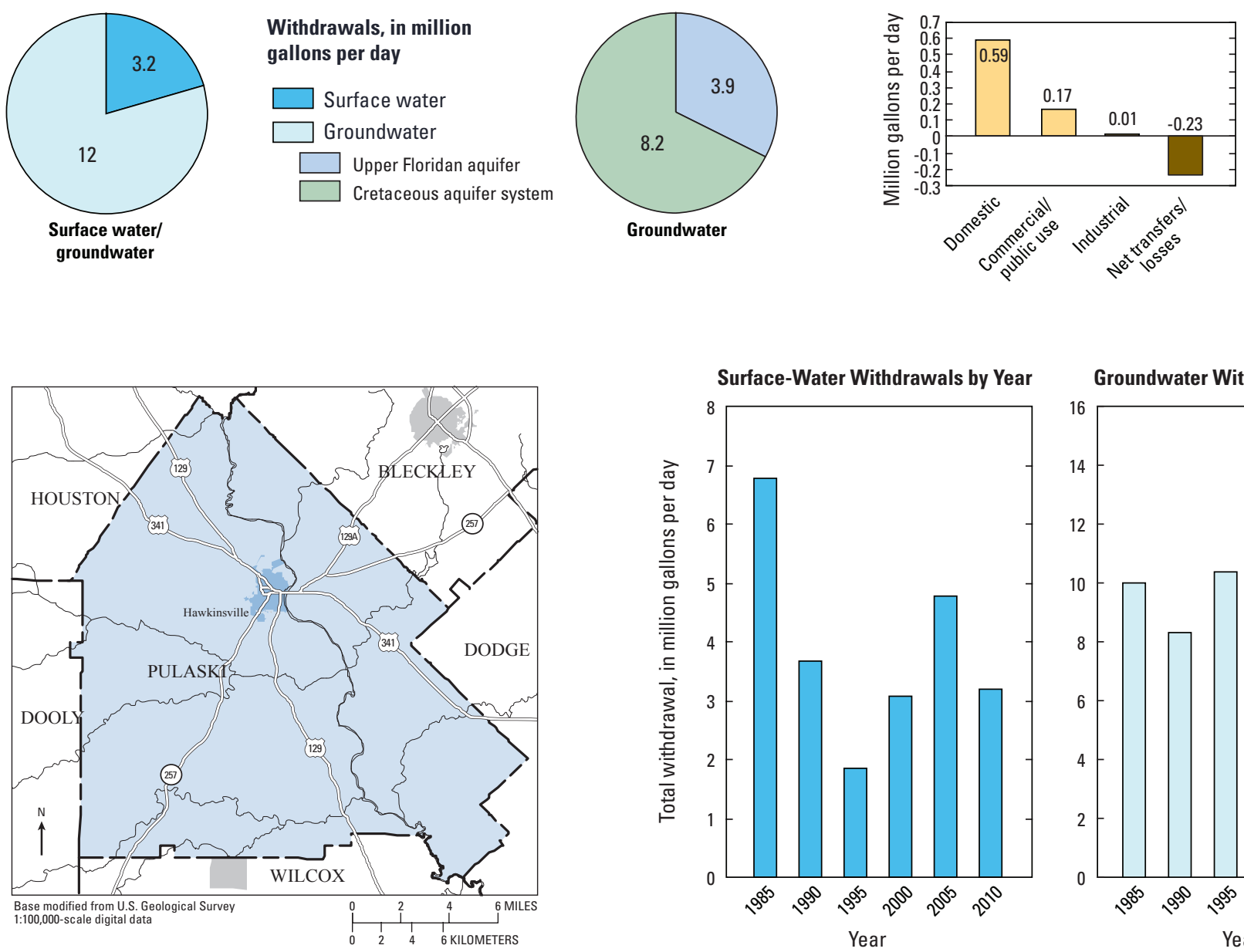

Groundwater Withdrawals by Year

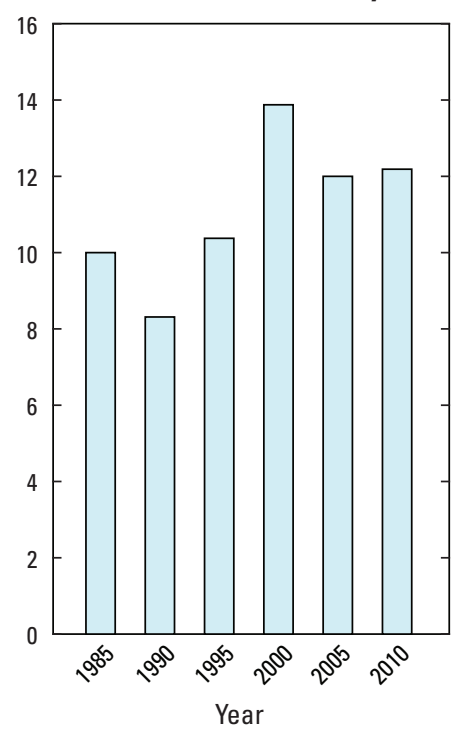




\section{PUTNAM COUNTY}

Population

Population served by public supply-Groundwater

Population served by public supply-Surface water $\quad 12,270$ Acres irrigated

540

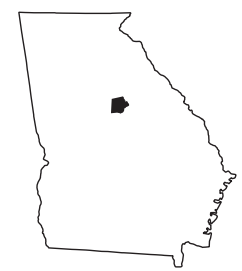

2010 WATER WITHDRAWALS AND ESTIMATED USE, IN MILLION GALLONS PER DAY

$[-$, not applicable; Mgal/d, million gallons per day $]$

\begin{tabular}{|c|c|c|c|c|c|c|c|c|}
\hline \multirow{3}{*}{ Category } & \multicolumn{3}{|c|}{ Withdrawals } & \multirow{3}{*}{$\begin{array}{l}\text { Total } \\
\text { use }^{1}\end{array}$} & \multirow{3}{*}{$\begin{array}{l}\text { Surface- } \\
\text { water } \\
\text { returns } \\
\end{array}$} & \multirow{2}{*}{\multicolumn{3}{|c|}{$\begin{array}{l}\text { Withdrawals by Major Industrial Groups } \\
\text { [NAICS, North American Industrial Classification System code] }\end{array}$}} \\
\hline & \multirow{2}{*}{$\begin{array}{l}\text { Ground- } \\
\text { water }\end{array}$} & \multirow{2}{*}{$\begin{array}{c}\text { Surface } \\
\text { water }\end{array}$} & \multirow[b]{2}{*}{ Total } & & & & & \\
\hline & & & & & & NAICS & Groundwater & Surface water \\
\hline Public supply & 0.53 & 3.66 & 4.19 & - & - & \multirow{2}{*}{ 322-Paper, pulp } & \multirow{2}{*}{0.04} & \multirow{2}{*}{0.00} \\
\hline Domestic & 0.19 & 0.00 & 0.19 & 1.12 & 0.00 & & & \\
\hline Commercial/public use & 0.00 & 0.00 & 0.00 & 0.33 & 0.00 & & & \\
\hline Industrial & 0.04 & 0.00 & 0.04 & 0.09 & 0.00 & & & \\
\hline Public-supply losses & - & - & - & 0.78 & - & & & \\
\hline Public wastewater treatment & - & - & - & - & 0.51 & \multicolumn{3}{|c|}{ Withdrawals by Major Public Suppliers } \\
\hline Mining & 0.00 & 0.00 & 0.00 & 0.00 & 0.00 & Name & Groundwater & Surface water \\
\hline $\begin{array}{l}\text { Irrigation-Crop } \\
\text { Irrigation-Golf course }\end{array}$ & $\begin{array}{l}0.25 \\
0.00\end{array}$ & $\begin{array}{l}0.06 \\
0.16\end{array}$ & $\begin{array}{l}0.31 \\
0.16\end{array}$ & $\begin{array}{l}0.31 \\
0.16\end{array}$ & $\begin{array}{l}0.00 \\
0.00\end{array}$ & Sinclair Water Authority & 0.00 & 3.66 \\
\hline Livestock/aquaculture & 0.00 & 0.24 & 0.24 & 0.24 & 0.00 & Piedmont Water Company & 0.35 & 0.00 \\
\hline Thermoelectric power & 0.00 & 952.42 & 952.42 & 952.42 & 368.73 & & & \\
\hline
\end{tabular}

1.01

956.54

957.55

955.45

369.24

'Total use is total withdrawal plus public supply deliveries and losses. An average of $2.1 \mathrm{Mgal} / \mathrm{d}$ was delivered to Baldwin County, Georgia from Putnam County in 2010.

\section{Withdrawals by Source}
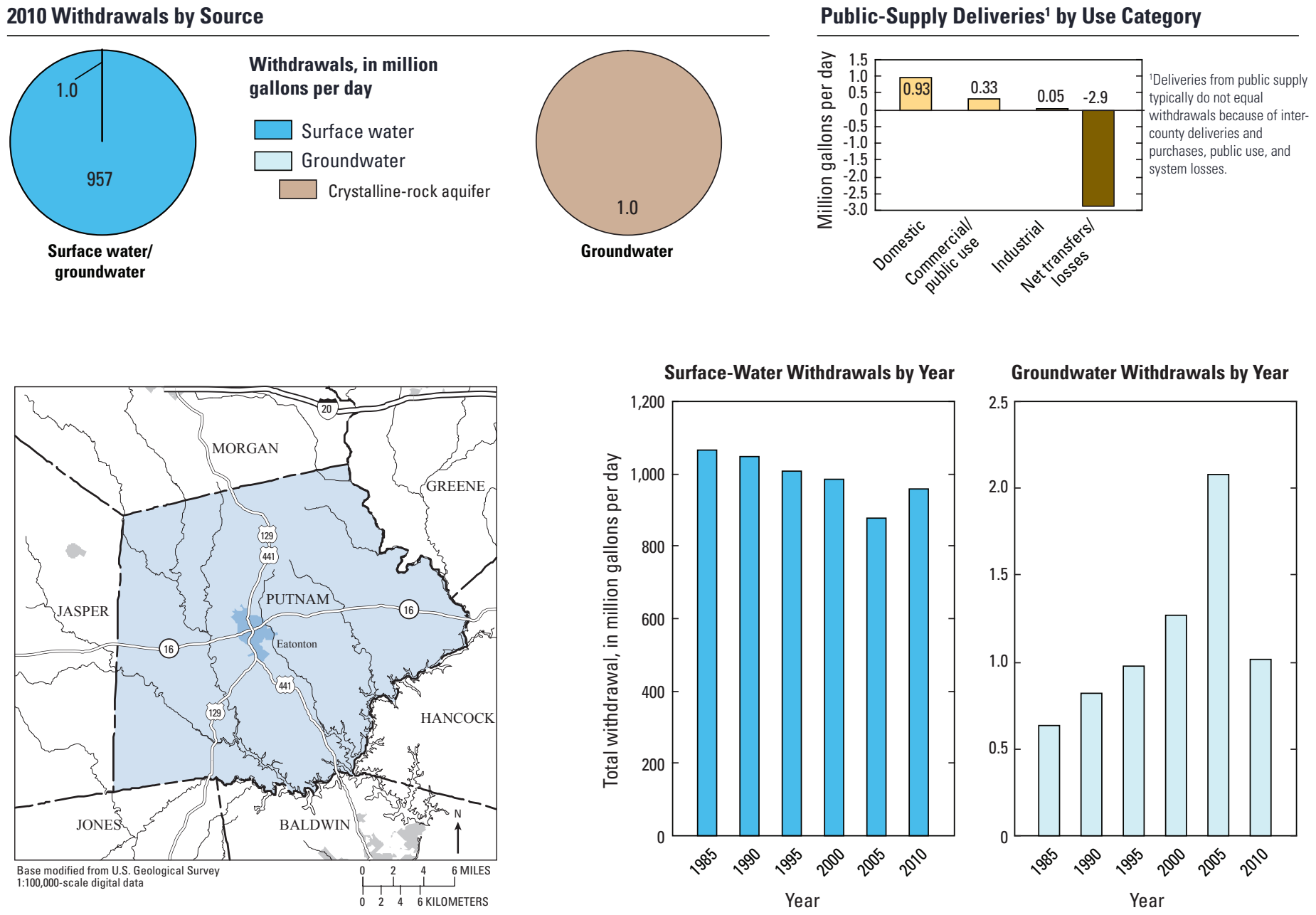


\section{OUITMAN COUNTY}

Population

2,513

Population served by public supply-Groundwater

Population served by public supply-Surface water Acres irrigated
1,860

0

500

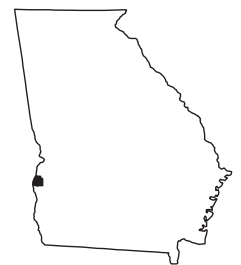

2010 WATER WITHDRAWALS AND ESTIMATED USE, IN MILLION GALLONS PER DAY

$[-$, not applicable; $<$, less than]

\begin{tabular}{|c|c|c|c|c|c|c|c|c|}
\hline \multirow[b]{3}{*}{ Category } & \multicolumn{3}{|c|}{ Withdrawals } & \multirow{3}{*}{$\begin{array}{l}\text { Total } \\
\text { use }^{1}\end{array}$} & \multirow{3}{*}{$\begin{array}{l}\text { Surface- } \\
\text { water } \\
\text { returns } \\
\end{array}$} & \multirow{2}{*}{\multicolumn{3}{|c|}{$\begin{array}{l}\text { Withdrawals by Major Industrial Groups } \\
\text { [NAICS, North American Industrial Classification System code] }\end{array}$}} \\
\hline & \multirow{2}{*}{$\begin{array}{c}\text { Ground- } \\
\text { water }\end{array}$} & \multirow{2}{*}{$\begin{array}{c}\text { Surface } \\
\text { water }\end{array}$} & \multirow[b]{2}{*}{ Total } & & & & & \\
\hline & & & & & & NAICS & Groundwater & Surface water \\
\hline Public supply & 0.14 & 0.00 & 0.14 & \multirow{2}{*}{$\begin{array}{l}- \\
0.19\end{array}$} & \multirow{2}{*}{$\begin{array}{c}- \\
0.00\end{array}$} & \multirow{2}{*}{ None } & \multirow{2}{*}{-} & \multirow{2}{*}{-} \\
\hline Domestic & 0.05 & 0.00 & 0.05 & & & & & \\
\hline Commercial/public use & 0.00 & 0.00 & 0.00 & 0.00 & 0.00 & \multirow{4}{*}{\multicolumn{3}{|c|}{ Withdrawals by Major Public Suppliers }} \\
\hline Industrial & 0.00 & 0.00 & 0.00 & 0.00 & 0.00 & & & \\
\hline Public-supply losses & - & - & - & $<0.01$ & - & & & \\
\hline \multirow{2}{*}{$\begin{array}{l}\text { Public wastewater treatment } \\
\text { Mining }\end{array}$} & - & - & \multirow{2}{*}{0.00} & \multirow{2}{*}{0.00} & \multirow{2}{*}{$\begin{array}{l}0.00 \\
0.00\end{array}$} & & & \\
\hline & 0.00 & 0.00 & & & & \multirow[t]{2}{*}{ Name } & \multirow{2}{*}{ Groundwater } & \multirow{2}{*}{ Surface water } \\
\hline Irrigation-Crop & 0.00 & 0.25 & 0.25 & 0.25 & 0.00 & & & \\
\hline Irrigation-Golf course & 0.00 & 0.00 & 0.00 & 0.00 & 0.00 & \multirow{2}{*}{$\begin{array}{l}\text { Georgetown-Quitman } \\
\text { County Commission }\end{array}$} & \multirow[b]{2}{*}{0.14} & \multirow[b]{2}{*}{0.00} \\
\hline Livestock/aquaculture & 0.00 & 0.02 & 0.02 & 0.02 & 0.00 & & & \\
\hline Thermoelectric power & 0.00 & 0.00 & 0.00 & 0.00 & 0.00 & & & \\
\hline
\end{tabular}

$\begin{array}{lllll}0.19 & 0.27 & 0.46 & 0.46 & 0.00\end{array}$

'Total use is total withdrawal plus public supply deliveries and losses.

\section{Withdrawals by Source}
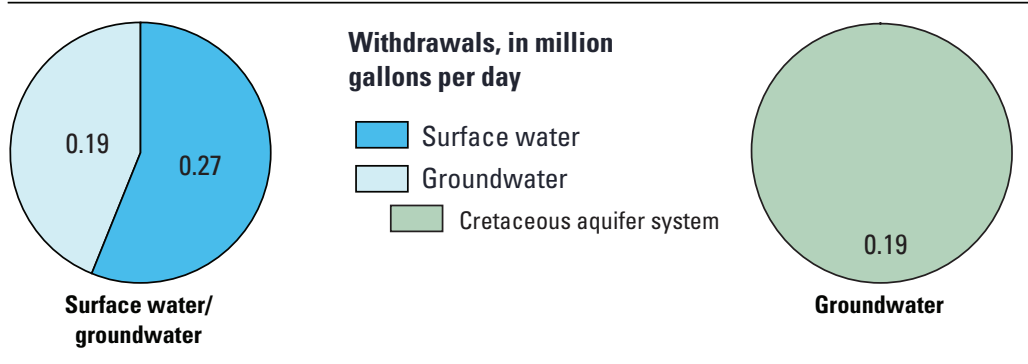

Public-Supply Deliveries by Use Category
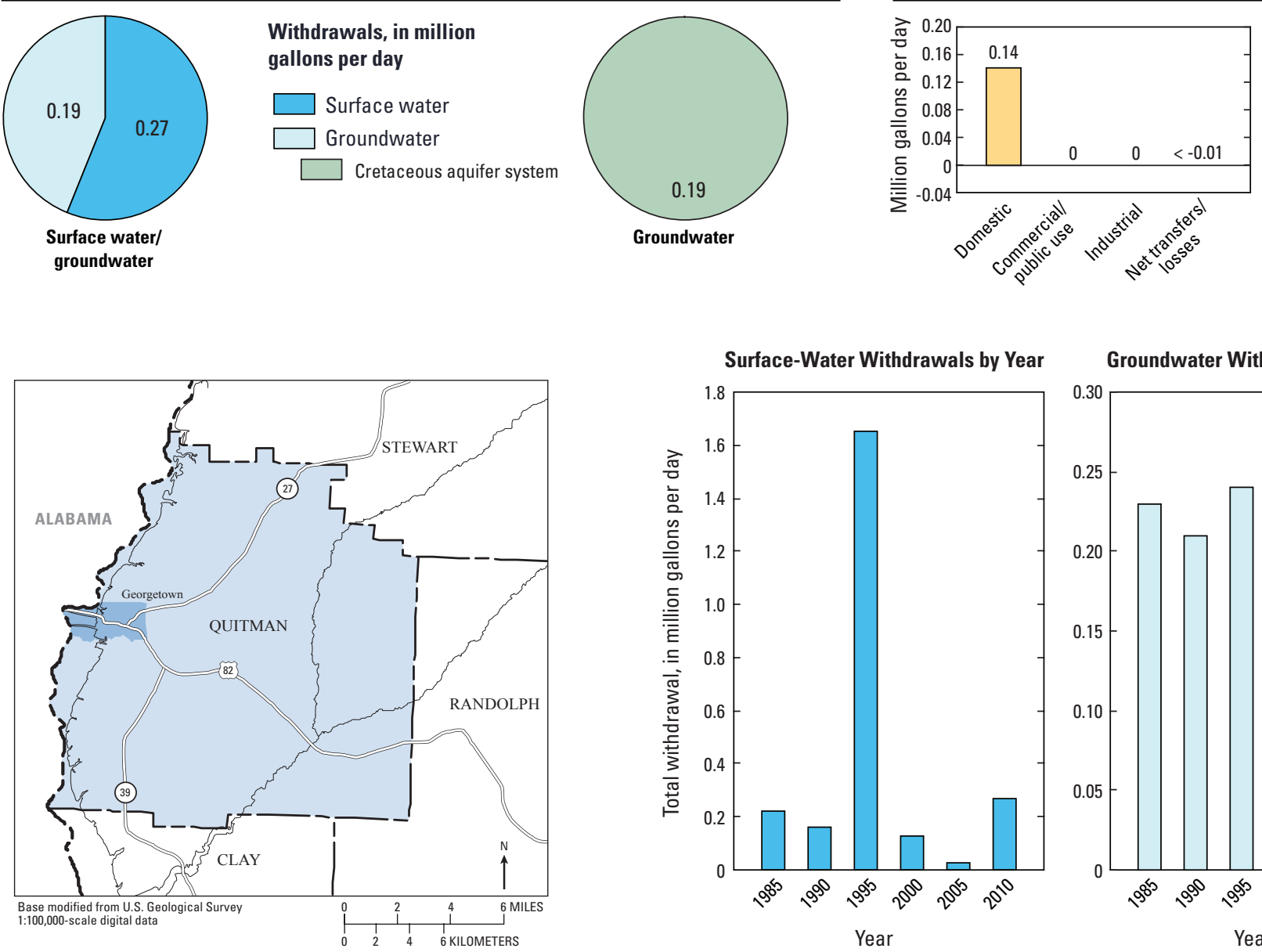

Groundwater Withdrawals by Year

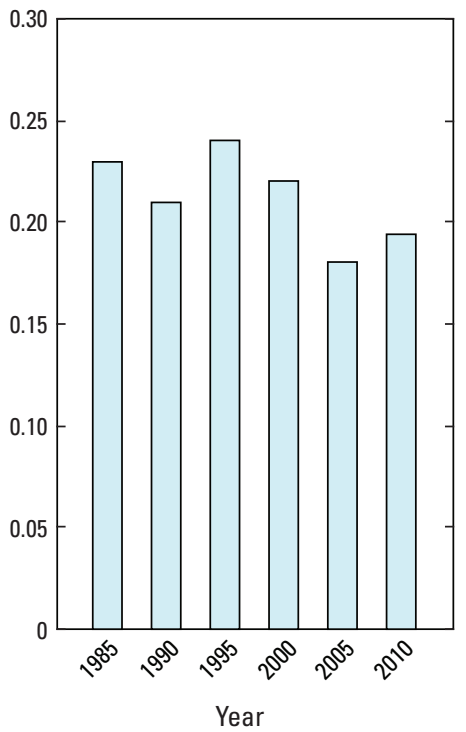




\section{RABUN COUNTY}

Population

16,276

Population served by public supply-Groundwater $\quad 920$

Population served by public supply-Surface water $\quad 10,760$

Acres irrigated

280

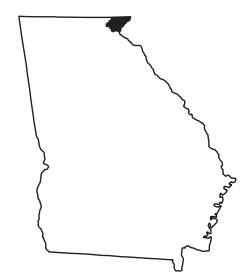

2010 WATER WITHDRAWALS AND ESTIMATED USE, IN MILLION GALLONS PER DAY

$[-$, not applicable $]$

\begin{tabular}{|c|c|c|c|c|c|c|c|c|}
\hline \multirow{3}{*}{ Category } & \multicolumn{3}{|c|}{ Withdrawals } & \multirow{3}{*}{$\begin{array}{l}\text { Total } \\
\text { use }^{1}\end{array}$} & \multirow{3}{*}{$\begin{array}{c}\text { Surface- } \\
\text { water } \\
\text { returns } \\
\end{array}$} & \multirow{2}{*}{\multicolumn{3}{|c|}{$\begin{array}{l}\text { Withdrawals by Major Industrial Groups } \\
\text { [NAICS, North American Industrial Classification System code] }\end{array}$}} \\
\hline & \multirow{2}{*}{$\begin{array}{l}\text { Ground- } \\
\text { water }\end{array}$} & \multirow{2}{*}{$\begin{array}{c}\text { Surface } \\
\text { water }\end{array}$} & \multirow[b]{2}{*}{ Total } & & & & & \\
\hline & & & & & & NAICS & Groundwater & Surface water \\
\hline Public supply & 0.13 & 1.37 & 1.50 & - & - & & & \\
\hline Domestic & 0.34 & 0.00 & 0.34 & 1.24 & 0.00 & 313-Textiles & 0.10 & 0.00 \\
\hline Commercial/public use & 0.00 & 0.00 & 0.00 & 0.04 & 0.00 & & & \\
\hline Industrial & 0.10 & 0.00 & 0.10 & 0.10 & 0.90 & & & \\
\hline Public-supply losses & - & - & - & 0.56 & - & & & \\
\hline Public wastewater treatment & - & - & - & - & 0.50 & Withdrawals by Majo & r Public Supp & \\
\hline Mining & 0.06 & 0.00 & 0.06 & 0.06 & 0.00 & Name & Groundwater & Surface water \\
\hline Irrigation-Crop & 0.02 & 0.00 & 0.02 & 0.02 & 0.00 & Name & Grouinuwater & \\
\hline Irrigation-Golf course & 0.00 & 0.40 & 0.40 & 0.40 & 0.00 & Clayton-Rabun County & & \\
\hline Livestock/aquaculture & 0.03 & 0.39 & 0.42 & 0.42 & 0.00 & $\begin{array}{l}\text { Water and Sewerage } \\
\text { Authority }\end{array}$ & 0.00 & 1.37 \\
\hline Thermoelectric power & 0.00 & 0.29 & 0.29 & 0.29 & 0.00 & & & \\
\hline TOTAL & 0.68 & 2.45 & 3.13 & 3.13 & 1.40 & City of Sky Valley & 0.08 & 0.00 \\
\hline
\end{tabular}

${ }^{1}$ Total use is total withdrawal plus public supply deliveries and losses.

\section{Withdrawals by Source}
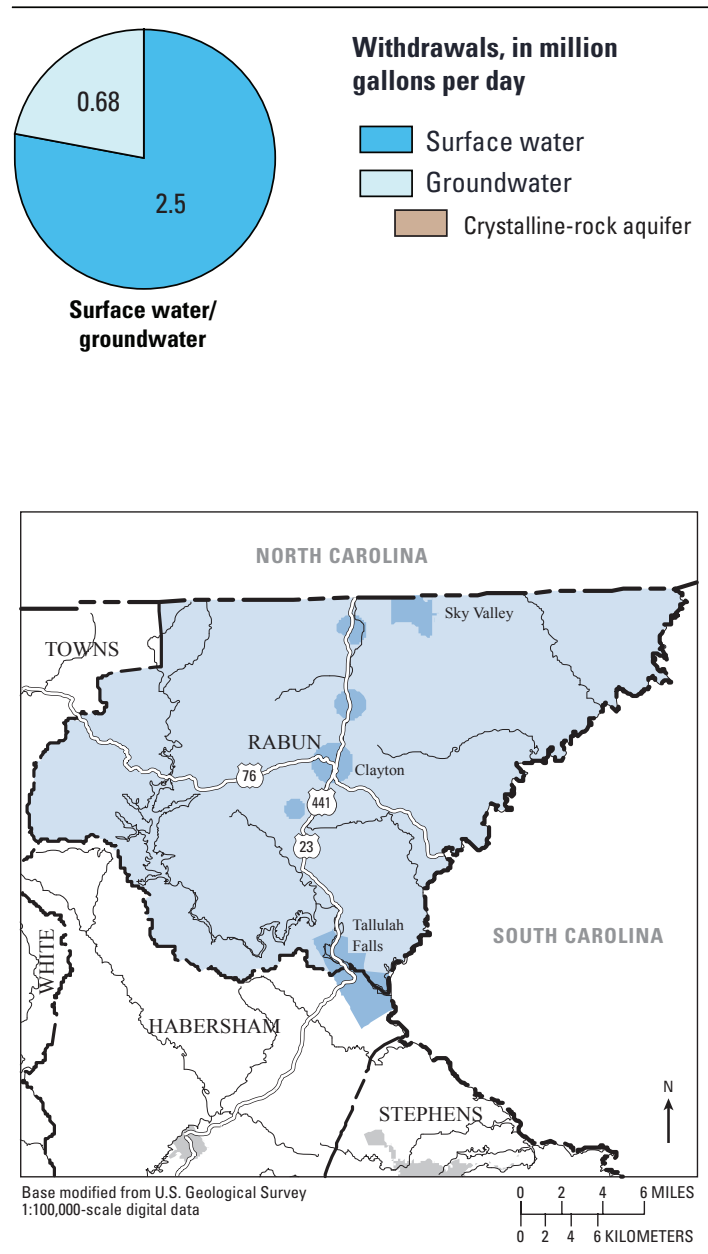

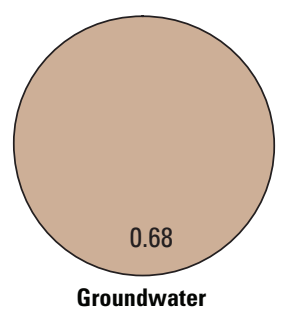

Public-Supply Deliveries by Use Category

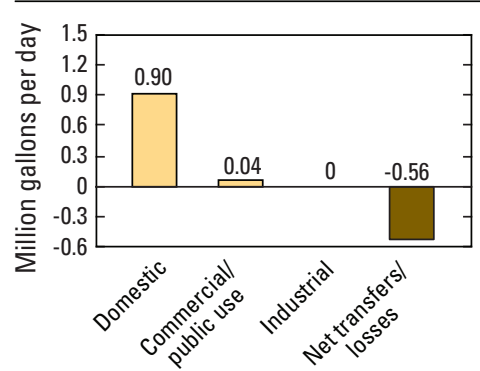

Surface-Water Withdrawals by Year

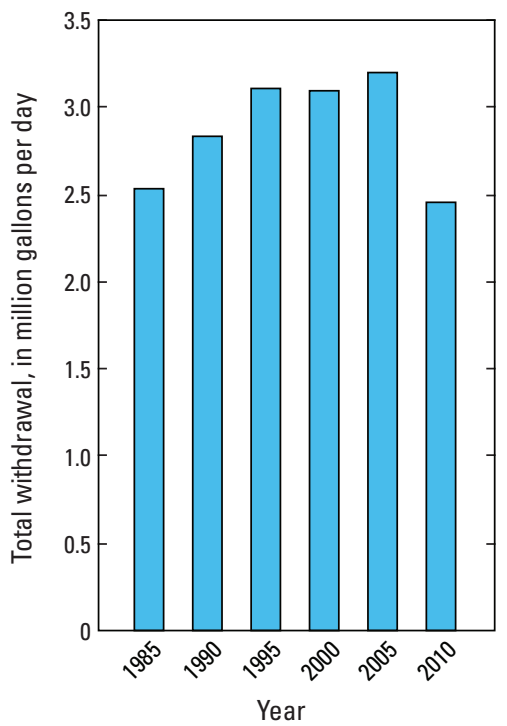

Groundwater Withdrawals by Year

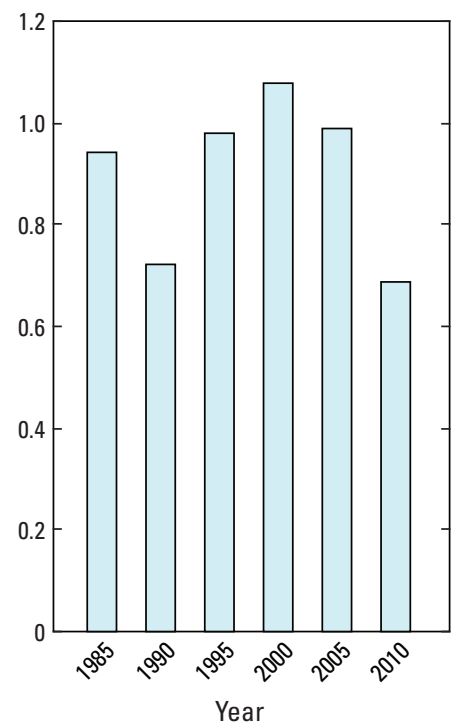




\section{RANDOLPH COUNTY}

Population

Population served by public supply-Groundwater Population served by public supply-Surface water Acres irrigated
5,090

0

26,004

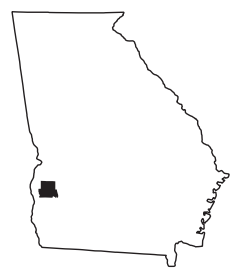

2010 WATER WITHDRAWALS AND ESTIMATED USE, IN MILLION GALLONS PER DAY

$[-$, not applicable $]$

\begin{tabular}{|c|c|c|c|c|c|c|c|c|}
\hline \multirow{3}{*}{ Category } & \multicolumn{3}{|c|}{ Withdrawals } & \multirow{3}{*}{$\begin{array}{l}\text { Total } \\
\text { use }^{1}\end{array}$} & \multirow{3}{*}{$\begin{array}{c}\text { Surface- } \\
\text { water } \\
\text { returns } \\
\end{array}$} & \multirow{2}{*}{\multicolumn{3}{|c|}{$\begin{array}{l}\text { Withdrawals by Major Industrial Groups } \\
\text { [NAICS, North American Industrial Classification System code] }\end{array}$}} \\
\hline & \multirow{2}{*}{$\begin{array}{c}\text { Ground- } \\
\text { water }\end{array}$} & \multirow{2}{*}{$\begin{array}{c}\text { Surface } \\
\text { water }\end{array}$} & \multirow[b]{2}{*}{ Total } & & & & & \\
\hline & & & & & & NAICS & Groundwater & Surface water \\
\hline Public supply & 0.57 & 0.00 & 0.57 & - & - & & 0.18 & 0.00 \\
\hline Domestic & 0.20 & 0.00 & 0.20 & 0.59 & 0.00 & $311-\mathrm{Food}$ & 0.18 & 0.00 \\
\hline Commercial/public use & 0.00 & 0.00 & 0.00 & 0.08 & 0.00 & & & \\
\hline Industrial & 0.18 & 0.00 & 0.18 & 0.18 & 0.00 & & & \\
\hline Public-supply losses & - & - & - & 0.09 & - & & & \\
\hline Public wastewater treatment & - & - & - & - & 0.39 & Withdrawals by & r Public Supp & \\
\hline Mining & 0.00 & $\begin{array}{r}0.00 \\
11.59\end{array}$ & $\begin{array}{r}0.00 \\
2128\end{array}$ & $\begin{array}{r}0.00 \\
2128\end{array}$ & 0.00 & Name & Groundwater & Surface water \\
\hline $\begin{array}{l}\text { Irrigation-Crop } \\
\text { Irrigation-Golf course }\end{array}$ & $\begin{array}{l}9.69 \\
0.00\end{array}$ & $\begin{array}{r}11.59 \\
0.06\end{array}$ & $\begin{array}{r}21.28 \\
0.06\end{array}$ & $\begin{array}{r}21.28 \\
0.06\end{array}$ & $\begin{array}{l}0.00 \\
0.00\end{array}$ & City of Cuthbert & 0.33 & 0.00 \\
\hline Livestock/aquaculture & 0.01 & 0.05 & 0.06 & 0.07 & 0.00 & City of Shellman & 0.21 & 0.00 \\
\hline Thermoelectric power & 0.00 & 0.00 & 0.00 & 0.00 & 0.00 & & & \\
\hline
\end{tabular}

10.65

11.70

22.35

22.35

0.39

${ }^{1}$ Total use is total withdrawal plus public supply deliveries and losses.

\section{Withdrawals by Source}
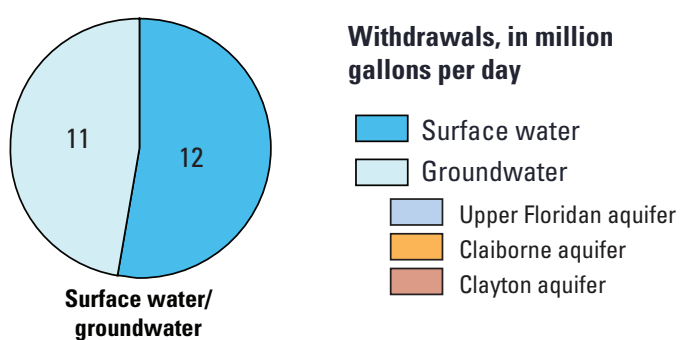

groundwater

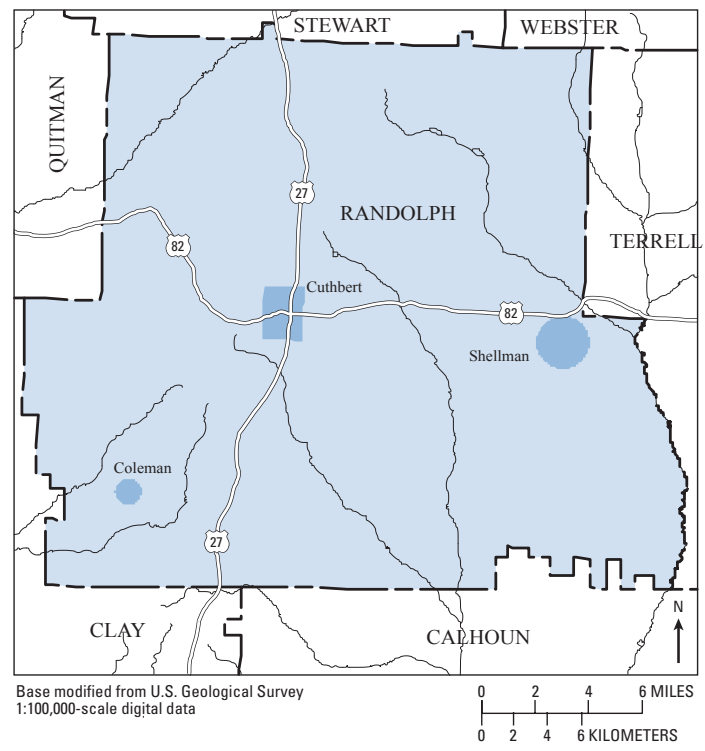

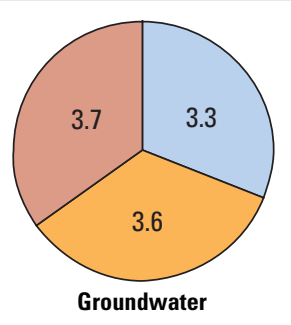

Public-Supply Deliveries by Use Category

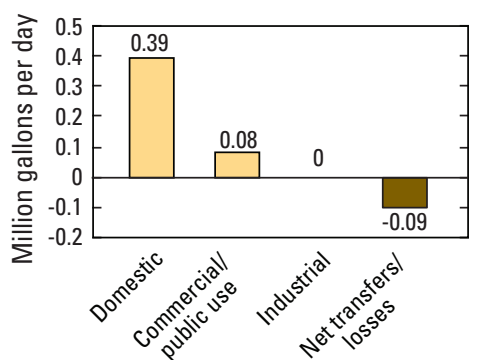

Surface-Water Withdrawals by Year

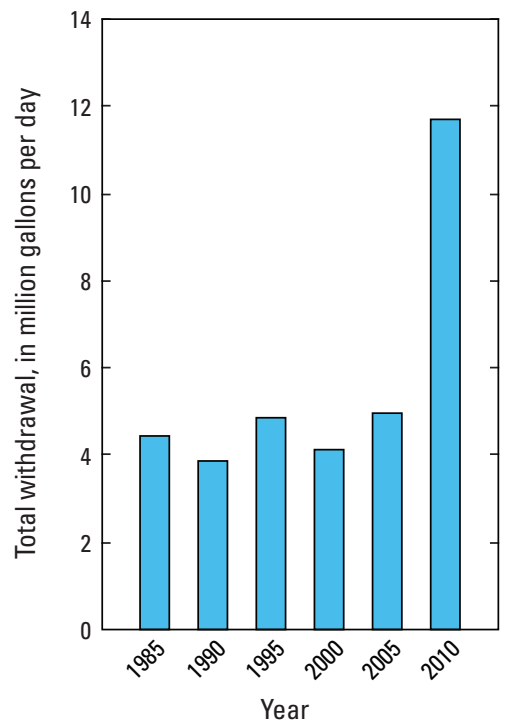

Groundwater Withdrawals by Year

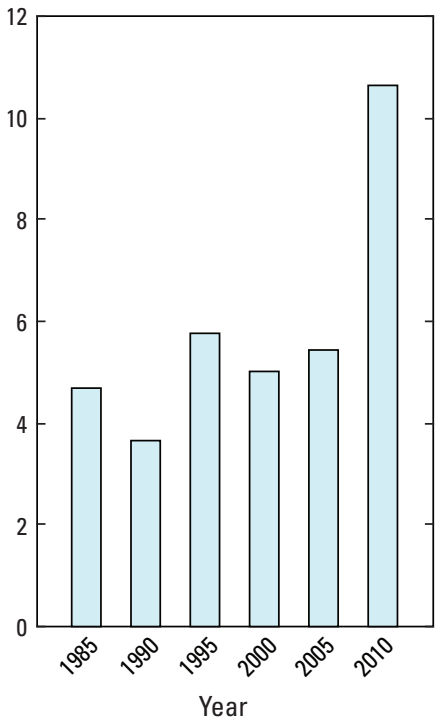


RICHMOND COUNTY

Population

200,549

Population served by public supply-Groundwater $\quad 35,960$

Population served by public supply-Surface water 159,920

Acres irrigated

500

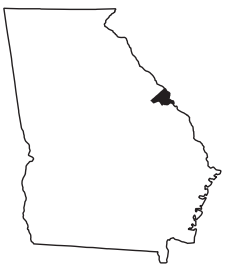

2010 WATER WITHDRAWALS AND ESTIMATED USE, IN MILLION GALLONS PER DAY

$[-$, not applicable $]$

\begin{tabular}{|c|c|c|c|c|c|c|c|c|}
\hline \multirow{3}{*}{ Category } & \multicolumn{3}{|c|}{ Withdrawals } & \multirow{3}{*}{$\begin{array}{l}\text { Total } \\
\text { use }^{1}\end{array}$} & \multirow{3}{*}{$\begin{array}{l}\text { Surface- } \\
\text { water } \\
\text { returns }\end{array}$} & \multirow{2}{*}{\multicolumn{3}{|c|}{$\begin{array}{l}\text { Withdrawals by Major Industrial Groups } \\
\text { [NAICS, North American Industrial Classification System code] }\end{array}$}} \\
\hline & \multirow{2}{*}{$\begin{array}{c}\text { Ground- } \\
\text { water }\end{array}$} & \multirow{2}{*}{$\begin{array}{c}\text { Surface } \\
\text { water }\end{array}$} & \multirow[b]{2}{*}{ Total } & & & & & \\
\hline & & & & & & NAICS & Groundwater & Surface water \\
\hline Public supply & 7.33 & 36.66 & 43.99 & - & - & & & \\
\hline Domestic & 0.35 & 0.00 & 0.35 & 15.20 & 0.00 & 322_-Paper, pulp & 0.00 & 55.46 \\
\hline Commercial/public use & 0.19 & 0.00 & 0.19 & 14.92 & 0.00 & 325 - Chemical products & 1.39 & 10.18 \\
\hline Industrial & 2.13 & 65.64 & 67.77 & 73.41 & 47.85 & 327 -Stone, clay & 0.48 & 0.00 \\
\hline Public-supply losses & - & - & - & 8.77 & - & $321-$ Wood & 0.26 & 0.00 \\
\hline Public wastewater treatment & - & - & - & - & 35.32 & \multirow{2}{*}{\multicolumn{3}{|c|}{ Withdrawals by Major Public Suppliers }} \\
\hline Mining & 1.53 & 0.00 & 1.53 & 1.53 & 0.00 & & & \\
\hline Irrigation-Crop & 0.14 & 0.00 & 0.14 & 0.14 & 0.00 & Name & Groundwater & Surface water \\
\hline Irrigation-Golf course & 0.24 & 0.52 & 0.76 & 0.76 & 0.00 & & & \\
\hline Livestock/aquaculture & 0.02 & 0.02 & 0.04 & 0.04 & 0.00 & $\begin{array}{l}\text { Augusta-Richmond } \\
\text { County Utilities }\end{array}$ & 6.77 & 36.63 \\
\hline Thermoelectric power & 0.00 & 0.00 & 0.00 & 0.00 & 0.00 & City of Hephzibah & 0.37 & 0.00 \\
\hline TOTAL & 11.93 & 102.84 & 114.77 & 114.77 & 83.17 & City of Blythe & 0.08 & 0.00 \\
\hline
\end{tabular}

\section{Withdrawals by Source}
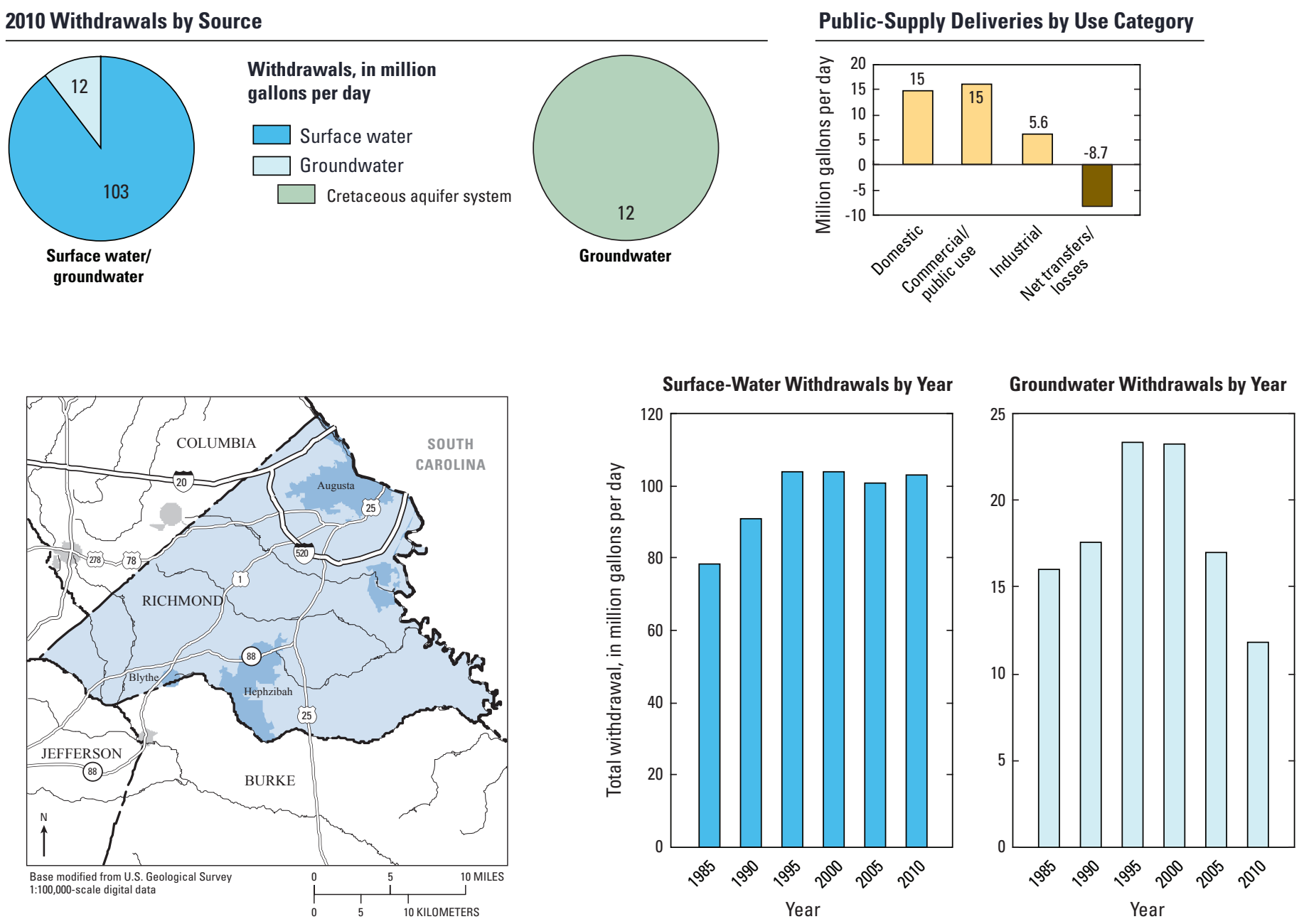


\section{ROCKDALE COUNTY}

Population

85,215

Population served by public supply-Groundwater

Population served by public supply-Surface water Acres irrigated
2,510

68,410

434

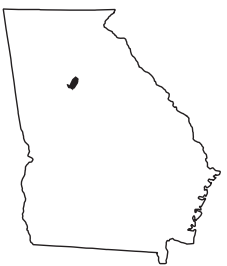

2010 WATER WITHDRAWALS AND ESTIMATED USE, IN MILLION GALLONS PER DAY

$[-$, not applicable $]$

\begin{tabular}{|c|c|c|c|c|c|c|c|c|}
\hline \multirow{3}{*}{ Category } & \multicolumn{3}{|c|}{ Withdrawals } & \multirow{3}{*}{$\begin{array}{l}\text { Total } \\
\text { use }^{1}\end{array}$} & \multirow{3}{*}{$\begin{array}{l}\text { Surface- } \\
\text { water } \\
\text { returns } \\
\end{array}$} & \multirow{2}{*}{\multicolumn{3}{|c|}{$\begin{array}{l}\text { Withdrawals by Major Industrial Groups } \\
\text { [NAICS, North American Industrial Classification System code] }\end{array}$}} \\
\hline & \multirow{2}{*}{$\begin{array}{c}\text { Ground- } \\
\text { water }\end{array}$} & \multirow{2}{*}{$\begin{array}{c}\text { Surface } \\
\text { water }\end{array}$} & \multirow[b]{2}{*}{ Total } & & & & & \\
\hline & & & & & & NAICS Gro & oundwater & Surface water \\
\hline Public supply & 0.24 & 11.57 & 11.81 & - & - & & & \\
\hline Domestic & 1.07 & 0.00 & 1.07 & 6.52 & 0.00 & None & - & - \\
\hline Commercial/public use & 0.01 & 0.00 & 0.01 & 3.02 & 0.00 & & & \\
\hline Industrial & 0.00 & 0.00 & 0.00 & 1.27 & 0.00 & & & \\
\hline Public-supply losses & - & - & - & 2.08 & - & & & \\
\hline Public wastewater treatment & - & - & - & - & 5.12 & Withdrawals by Major Pu & ublic Supp & \\
\hline Mining & 0.00 & 0.00 & 0.00 & 0.00 & 0.00 & Name & oundwater & Surface water \\
\hline Irrigation-Crop & 0.00 & 0.00 & 0.00 & 0.00 & 0.00 & & & \\
\hline Irrigation-Golf course & 0.10 & 0.61 & 0.71 & 0.71 & 0.00 & Rockdale County Water & 0.00 & 11.57 \\
\hline Livestock/aquaculture & 0.00 & 0.03 & 0.03 & 0.03 & 0.00 & Resources & & \\
\hline Thermoelectric power & 0.00 & 0.00 & 0.00 & 0.00 & 0.00 & Lakeview Utilities & 0.14 & 0.00 \\
\hline TOTAL & 1.42 & 12.21 & 13.63 & 13.63 & 5.12 & Poyntsource Solutions, Inc. & 0.10 & 0.00 \\
\hline
\end{tabular}

${ }^{1} T o t a l$ use is total withdrawal plus public supply deliveries and losses.

\section{Withdrawals by Source}
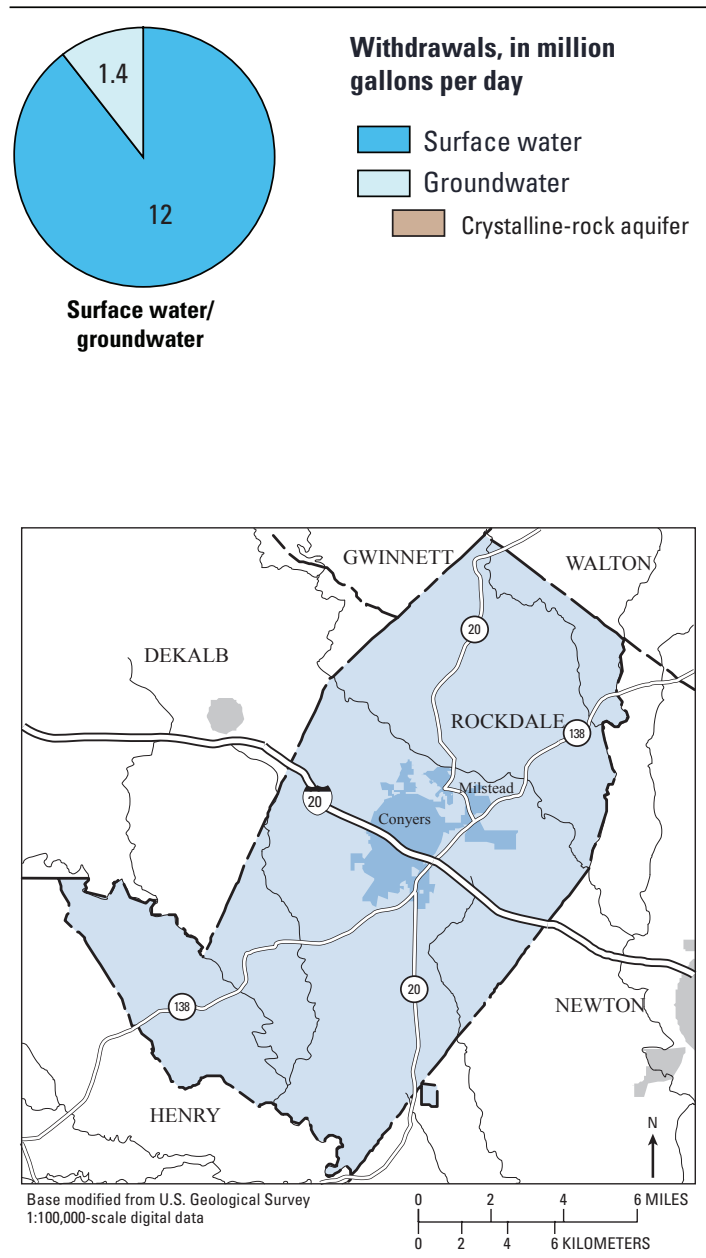

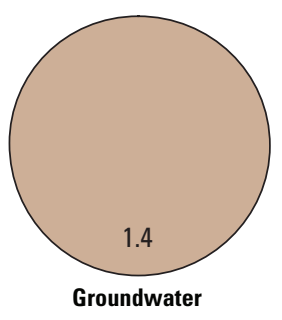

Public-Supply Deliveries by Use Category

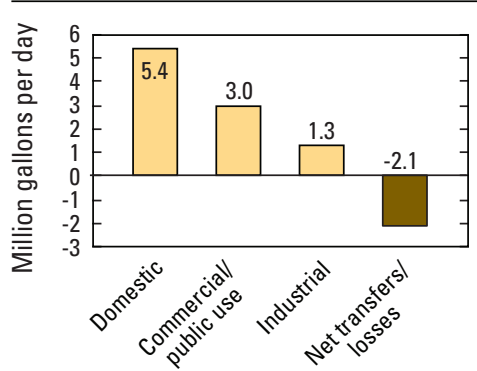

Surface-Water Withdrawals by Year

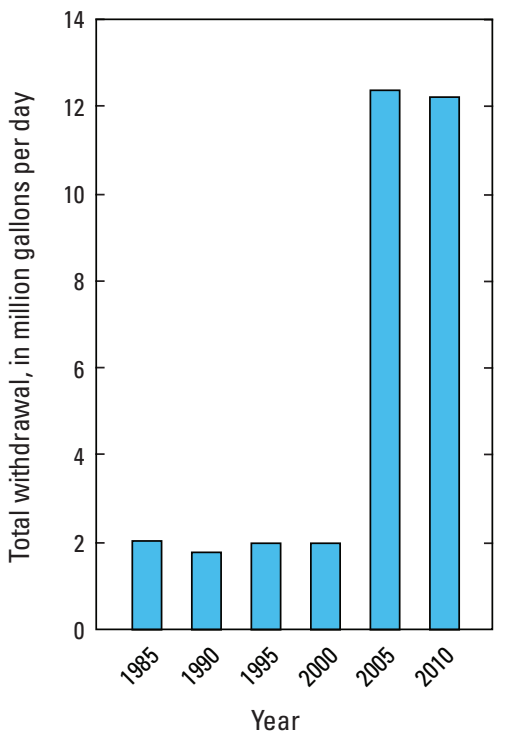

Groundwater Withdrawals by Year

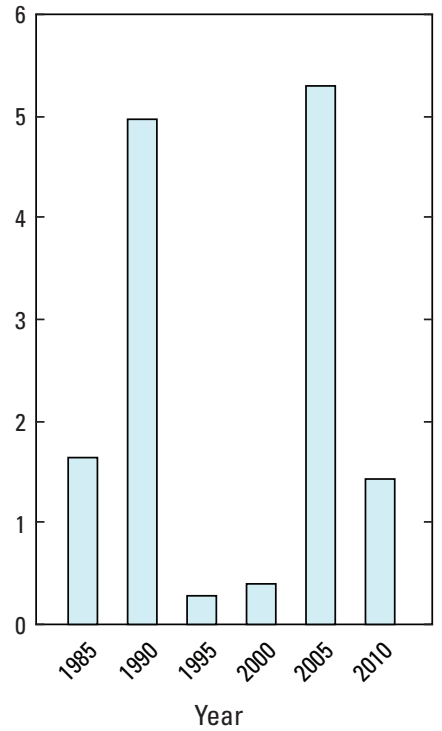




\section{SCHLEY COUNTY}

Population

Population served by public supply-Groundwater

Population served by public supply-Surface water

4,530

Acres irrigated

1,790

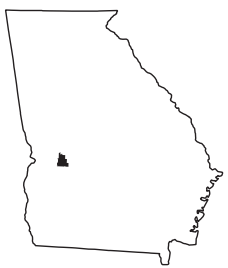

2010 WATER WITHDRAWALS AND ESTIMATED USE, IN MILLION GALLONS PER DAY

$[-$, not applicable $]$

\begin{tabular}{|c|c|c|c|c|c|c|c|c|}
\hline \multirow{3}{*}{ Category } & \multicolumn{3}{|c|}{ Withdrawals } & \multirow{3}{*}{$\begin{array}{c}\text { Total } \\
\text { use }^{1}\end{array}$} & \multirow{3}{*}{$\begin{array}{c}\text { Surface- } \\
\text { water } \\
\text { returns } \\
\end{array}$} & \multirow{2}{*}{\multicolumn{3}{|c|}{$\begin{array}{l}\text { Withdrawals by Major Industrial Groups } \\
\text { [NAICS, North American Industrial Classification System code] }\end{array}$}} \\
\hline & \multirow{2}{*}{$\begin{array}{l}\text { Ground- } \\
\text { water }\end{array}$} & \multirow{2}{*}{$\begin{array}{c}\text { Surface } \\
\text { water }\end{array}$} & \multirow[b]{2}{*}{ Total } & & & & & \\
\hline & & & & & & NAICS & Groundwater & Surface water \\
\hline \multirow{2}{*}{$\begin{array}{l}\text { Public supply } \\
\text { Domestic }\end{array}$} & 0.43 & 0.00 & 0.43 & \multirow{2}{*}{$\begin{array}{l}- \\
0.36\end{array}$} & \multirow{2}{*}{-} & \multirow{2}{*}{ None } & \multirow{2}{*}{-} & \multirow{2}{*}{-} \\
\hline & 0.04 & 0.00 & 0.04 & & & & & \\
\hline Commercial/public use & 0.00 & 0.00 & 0.00 & 0.03 & 0.00 & \multirow{4}{*}{\multicolumn{3}{|c|}{ Withdrawals by Major Public Suppliers }} \\
\hline Industrial & 0.00 & 0.00 & 0.00 & 0.01 & 0.00 & & & \\
\hline Public-supply losses & - & - & - & 0.07 & - & & & \\
\hline Public wastewater treatment & - & - & - & - & 0.13 & & & \\
\hline \multirow{2}{*}{$\begin{array}{l}\text { Mining } \\
\text { Irrigation-Crop }\end{array}$} & 0.00 & 0.00 & 0.00 & 0.00 & 0.00 & Name & Groundwater & Surface water \\
\hline & $\begin{array}{l}0.08 \\
0.00\end{array}$ & $\begin{array}{l}0.32 \\
0.07\end{array}$ & $\begin{array}{l}0.40 \\
0.07\end{array}$ & $\begin{array}{l}0.40 \\
0.07\end{array}$ & $\begin{array}{l}0.00 \\
0.00\end{array}$ & \multirow{2}{*}{$\begin{array}{l}\text { Schley County } \\
\text { Water System }\end{array}$} & \multirow{2}{*}{0.20} & \multirow{2}{*}{0.00} \\
\hline Livestock/aquaculture & 0.02 & 0.12 & 0.14 & 0.14 & 0.00 & & & \\
\hline \multirow{2}{*}{$\begin{array}{l}\text { Thermoelectric power } \\
\text { TOTAL }\end{array}$} & 0.00 & 0.00 & 0.00 & 0.00 & 0.00 & \multirow[t]{2}{*}{ City of Ellaville } & \multirow[t]{2}{*}{0.23} & \multirow[t]{2}{*}{0.00} \\
\hline & 0.57 & 0.51 & 1.08 & 1.08 & 0.13 & & & \\
\hline
\end{tabular}

${ }^{1}$ Total use is total withdrawal plus public supply deliveries and losses.

\section{Withdrawals by Source}
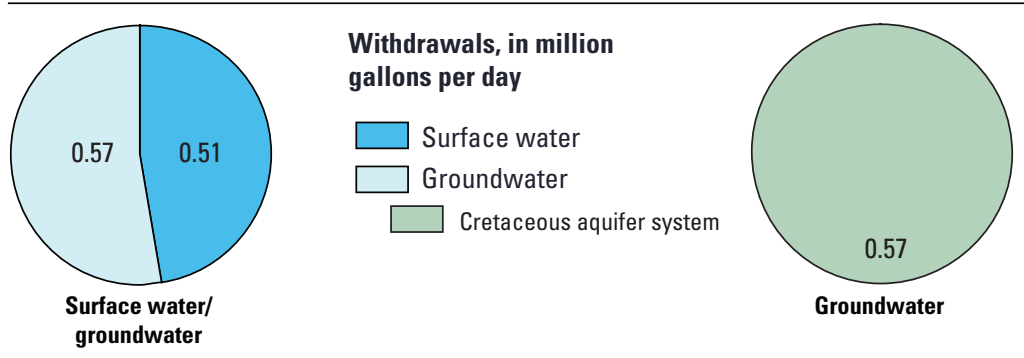

Public-Supply Deliveries by Use Category

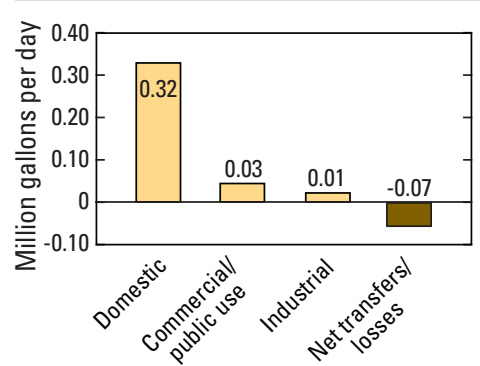

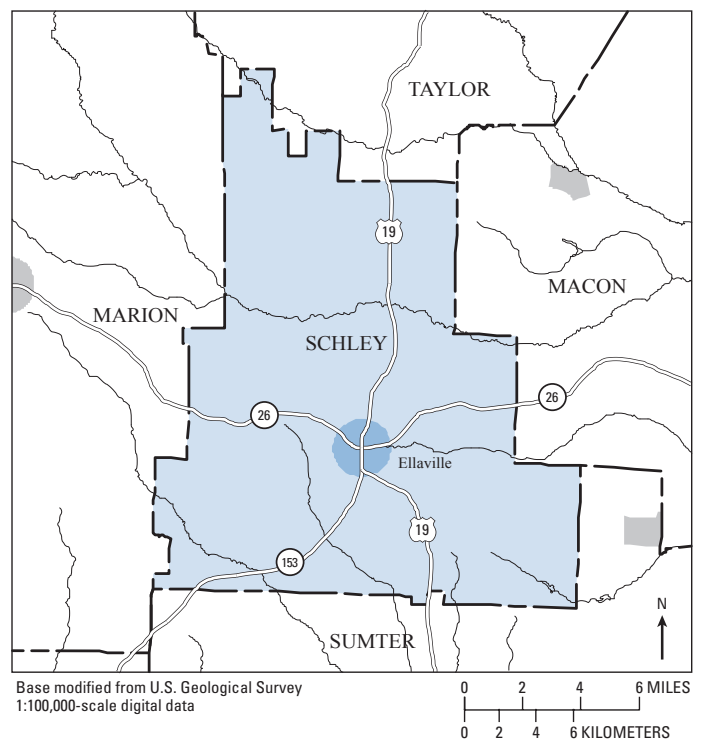

Surface-Water Withdrawals by Year

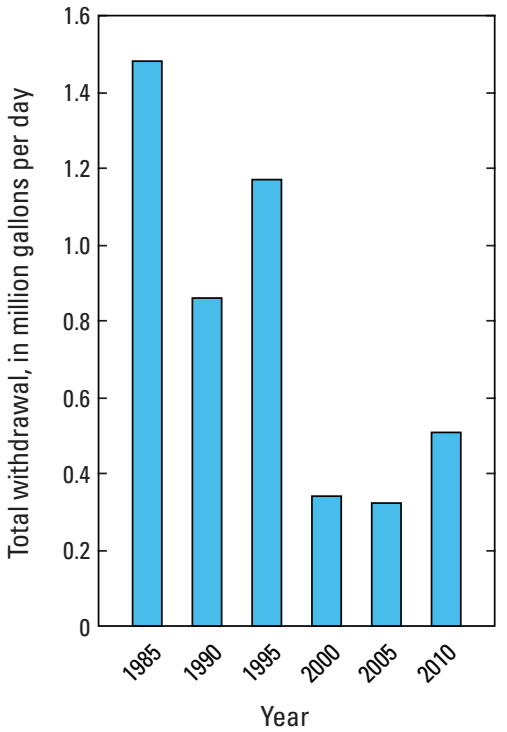

Groundwater Withdrawals by Year

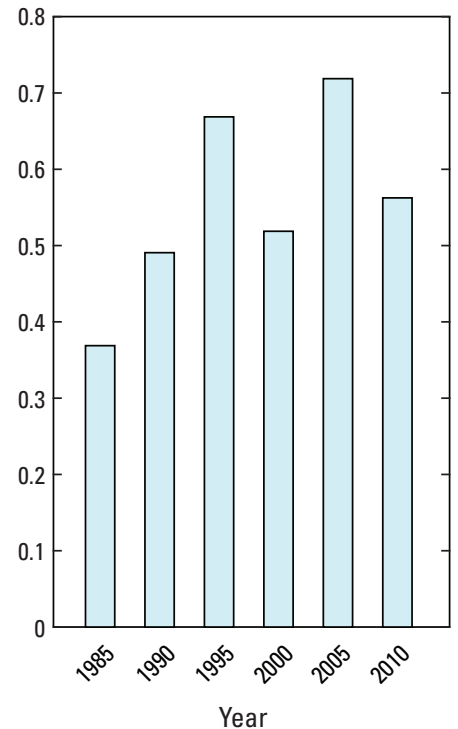




\section{SCREVEN COUNTY}

Population

Population served by public supply-Groundwater $\quad 4,870$

Population served by public supply-Surface water 0

Acres irrigated

21,540

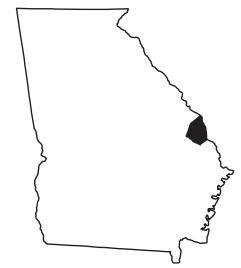

2010 WATER WITHDRAWALS AND ESTIMATED USE, IN MILLION GALLONS PER DAY

$[-$, not applicable $]$

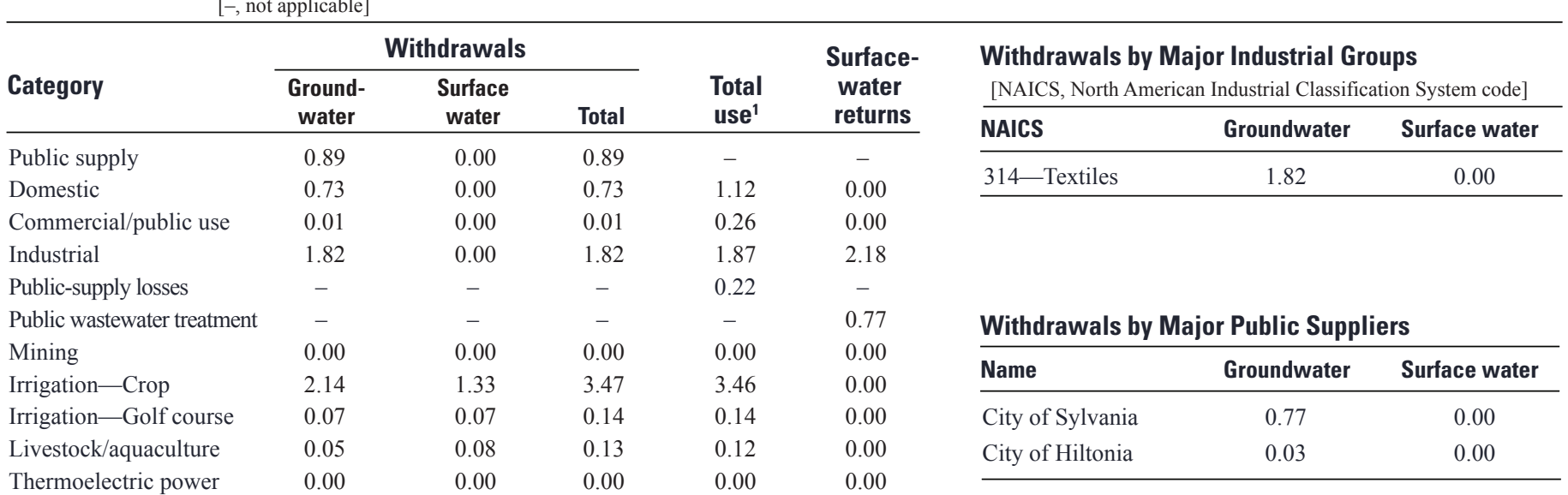

TOTAL

$5.71 \quad 1.48$

7.19

7.19

2.95

${ }^{1}$ Total use is total withdrawal plus public supply deliveries and losses.

2010 Withdrawals by Source
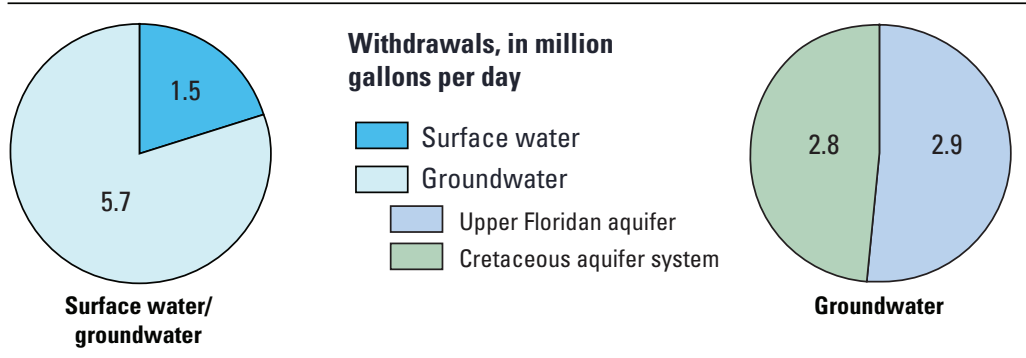

Public-Supply Deliveries by Use Category
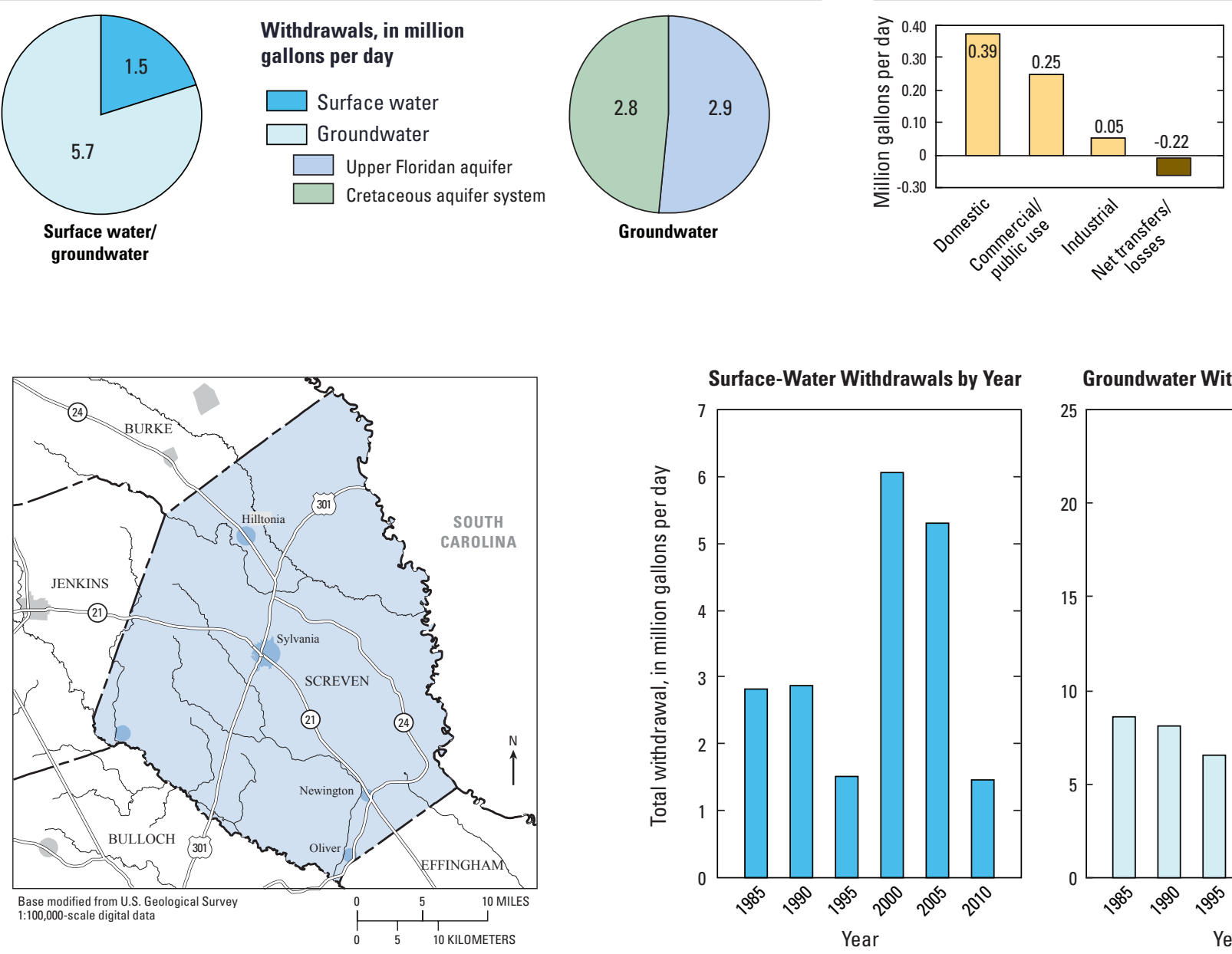

Groundwater Withdrawals by Year

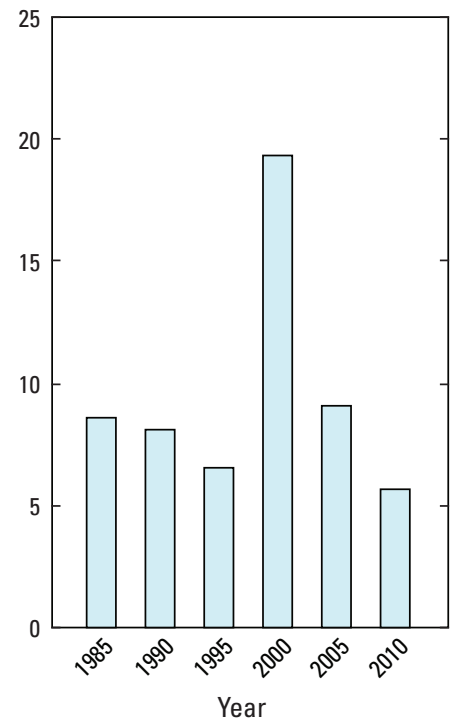




\section{SEMINOLE COUNTY}

Population

Population served by public supply-Groundwater

Population served by public supply-Surface water Acres irrigated

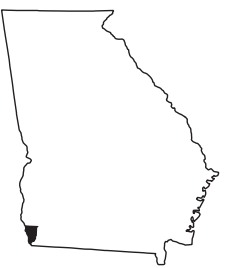

2010 WATER WITHDRAWALS AND ESTIMATED USE, IN MILLION GALLONS PER DAY

$[-$, not applicable $]$

\begin{tabular}{|c|c|c|c|c|c|c|c|c|}
\hline \multirow{3}{*}{ Category } & \multicolumn{3}{|c|}{ Withdrawals } & \multirow{3}{*}{$\begin{array}{l}\text { Total } \\
\text { use }^{1}\end{array}$} & \multirow{3}{*}{$\begin{array}{c}\text { Surface- } \\
\text { water } \\
\text { returns } \\
\end{array}$} & \multirow{2}{*}{\multicolumn{3}{|c|}{$\begin{array}{l}\text { Withdrawals by Major Industrial Groups } \\
\text { [NAICS, North American Industrial Classification System code] }\end{array}$}} \\
\hline & \multirow{2}{*}{$\begin{array}{c}\text { Ground- } \\
\text { water }\end{array}$} & \multirow{2}{*}{$\begin{array}{c}\text { Surface } \\
\text { water }\end{array}$} & \multirow[b]{2}{*}{ Total } & & & & & \\
\hline & & & & & & NAICS & Groundwater & Surface water \\
\hline Public supply & 0.58 & 0.00 & 0.58 & - & - & & & \\
\hline Domestic & 0.37 & 0.00 & 0.37 & 0.78 & 0.00 & None & - & - \\
\hline Commercial/public use & 0.00 & 0.00 & 0.00 & 0.08 & 0.00 & & & \\
\hline Industrial & 0.00 & 0.00 & 0.00 & 0.01 & 0.00 & & & \\
\hline Public-supply losses & - & - & - & 0.08 & - & & & \\
\hline Public wastewater treatment & - & - & - & - & 0.43 & Withdrawals by Maj & r Public Supp & \\
\hline Mining & 0.00 & 0.00 & 0.00 & 0.00 & 0.00 & Name & Groundwater & Surface water \\
\hline $\begin{array}{l}\text { Irrigation-Crop } \\
\text { Irrigation-Golf course }\end{array}$ & $\begin{array}{r}43.46 \\
0.26\end{array}$ & $\begin{array}{l}0.50 \\
0.00\end{array}$ & $\begin{array}{r}43.96 \\
0.26\end{array}$ & $\begin{array}{r}43.96 \\
0.26\end{array}$ & $\begin{array}{l}0.00 \\
0.00\end{array}$ & City of Donalsonville & 0.52 & 0.00 \\
\hline Livestock/aquaculture & 0.06 & 0.01 & 0.07 & 0.07 & 0.00 & & & \\
\hline Thermoelectric power & 0.00 & 0.00 & 0.00 & 0.00 & 0.00 & & & \\
\hline TOTAL & 44.73 & 0.51 & 45.24 & 45.24 & 0.43 & & & \\
\hline
\end{tabular}

\section{Withdrawals by Source}
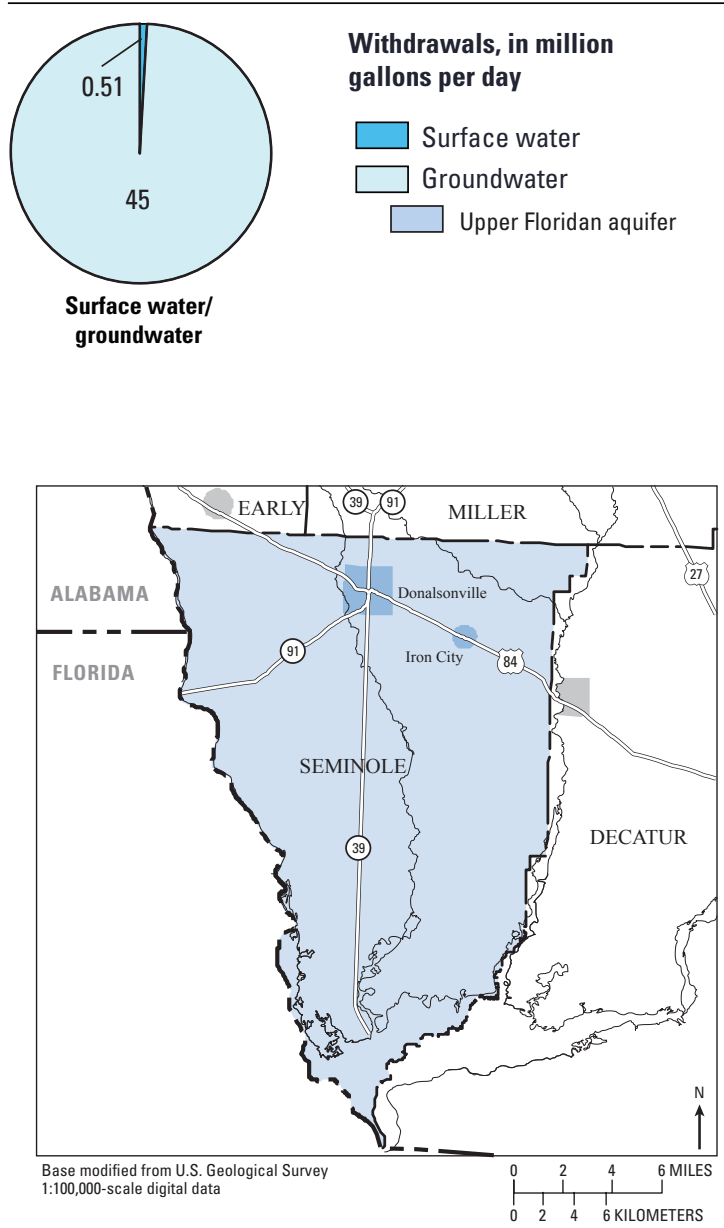

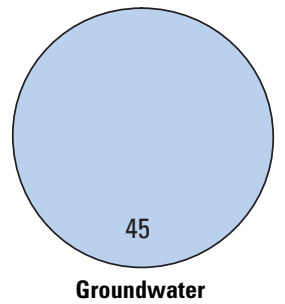

Public-Supply Deliveries by Use Category

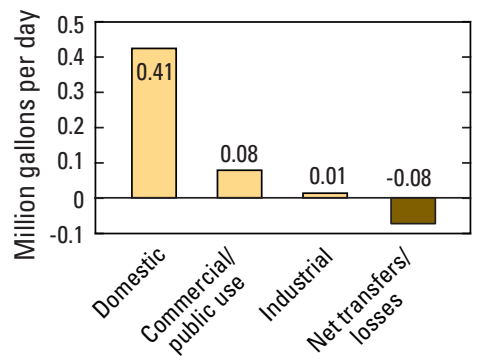

Surface-Water Withdrawals by Year

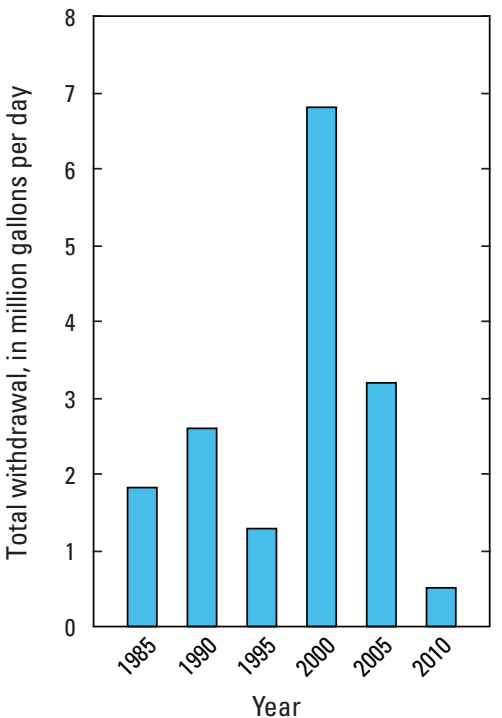

Groundwater Withdrawals by Year

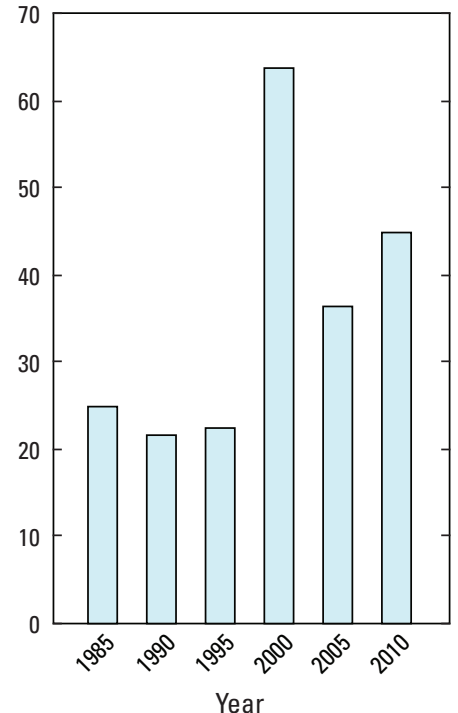




\section{SPALDING COUNTY}

Population

64,073

Population served by public supply-Groundwater $\quad 1,110$

Population served by public supply-Surface water $\quad 55,760$

Acres irrigated

250

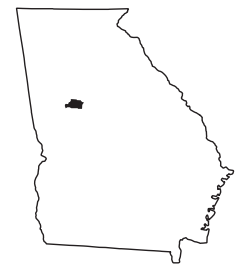

2010 WATER WITHDRAWALS AND ESTIMATED USE, IN MILLION GALLONS PER DAY

[-, not applicable; Mgal/d, million gallons per day $]$

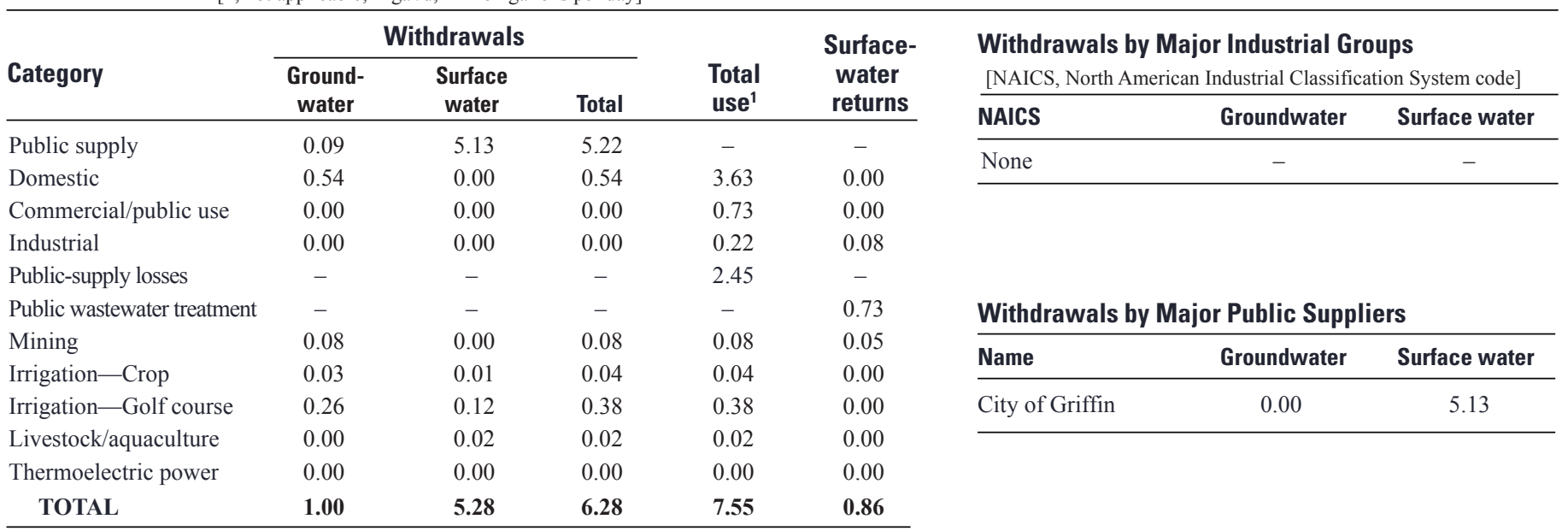

'Total use is total withdrawal plus public supply deliveries and losses. An average of $3.7 \mathrm{Mgal} / \mathrm{d}$ was delivered from Pike County, Georgia

to Spalding County and 2.43 Mgal/d was delivered from Spalding County to Coweta County, Georgia in 2010.

\section{Withdrawals by Source}
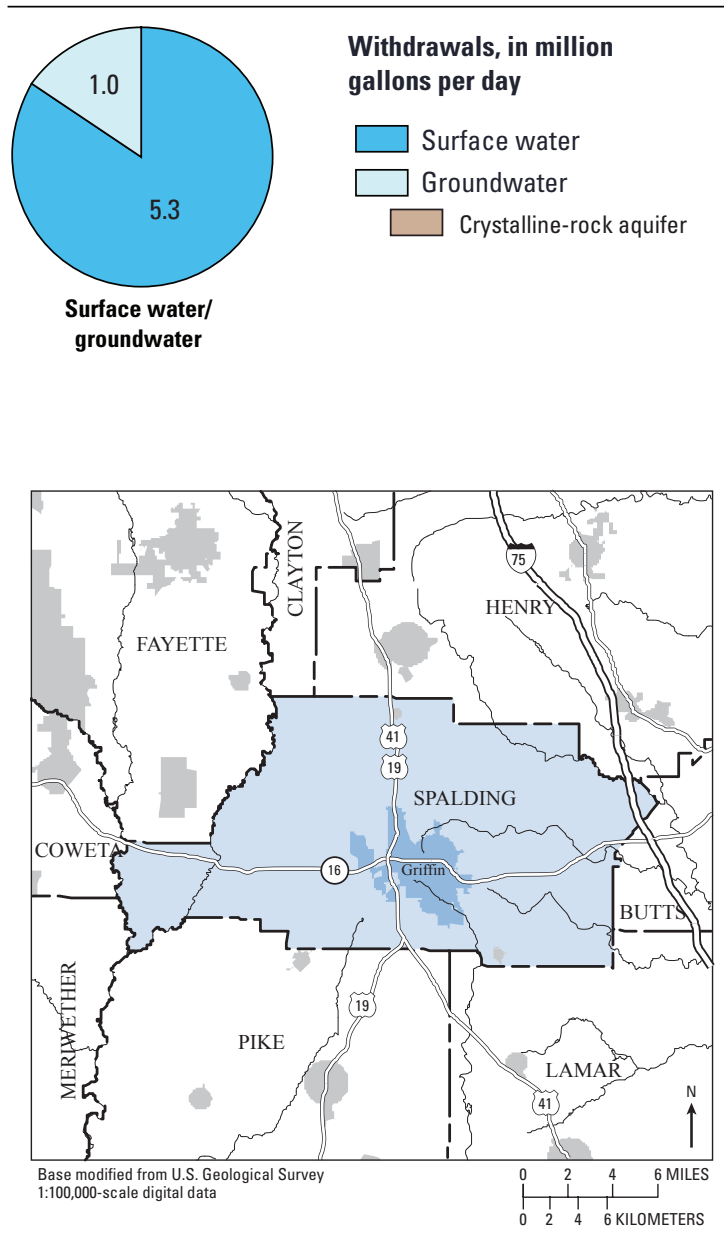

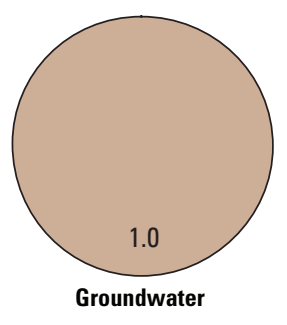

Public-Supply Deliveries ${ }^{1}$ by Use Category

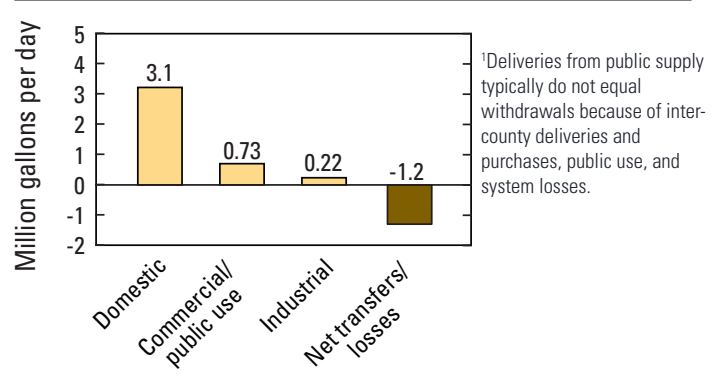

Surface-Water Withdrawals by Year

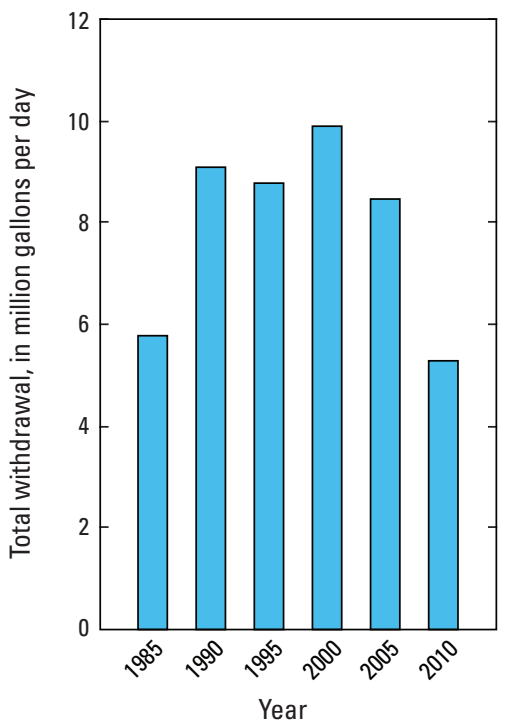

Groundwater Withdrawals by Year

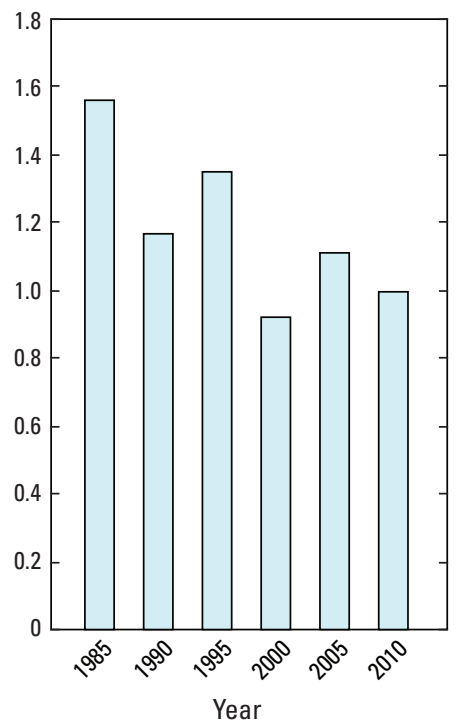




\section{STEPHENS COUNTY}

Population

26,175

Population served by public supply-Groundwater

Population served by public supply_-Surface water $\quad 23,500$

Acres irrigated

500

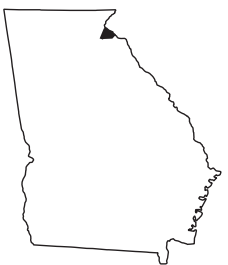

2010 WATER WITHDRAWALS AND ESTIMATED USE, IN MILLION GALLONS PER DAY

[-, not applicable; Mgal/d, million gallons per day]

\begin{tabular}{|c|c|c|c|c|c|c|c|c|}
\hline \multirow{3}{*}{ Category } & \multicolumn{3}{|c|}{ Withdrawals } & \multirow{3}{*}{$\begin{array}{l}\text { Total } \\
\text { use }^{1}\end{array}$} & \multirow{3}{*}{$\begin{array}{l}\text { Surface- } \\
\text { water } \\
\text { returns } \\
\end{array}$} & \multirow{2}{*}{\multicolumn{3}{|c|}{$\begin{array}{l}\text { Withdrawals by Major Industrial Groups } \\
\text { [NAICS, North American Industrial Classification System code] }\end{array}$}} \\
\hline & \multirow{2}{*}{$\begin{array}{c}\text { Ground- } \\
\text { water }\end{array}$} & \multirow{2}{*}{$\begin{array}{c}\text { Surface } \\
\text { water }\end{array}$} & \multirow[b]{2}{*}{ Total } & & & & & \\
\hline & & & & & & NAICS & Groundwater & Surface water \\
\hline Public supply & 0.05 & 3.08 & 3.13 & - & - & & - & - \\
\hline Domestic & 0.16 & 0.00 & 0.16 & 2.00 & 0.00 & None & - & - \\
\hline Commercial/public use & 0.08 & 0.00 & 0.08 & 0.51 & 0.03 & & & \\
\hline Industrial & 0.00 & 0.00 & 0.00 & 0.31 & 0.00 & & & \\
\hline Public-supply losses & - & - & - & 0.43 & - & & & \\
\hline Public wastewater treatment & - & - & - & - & 1.03 & Withdrawals & r Public Supp & \\
\hline Mining & 0.12 & 0.00 & 0.12 & 0.12 & 0.00 & Name & Groundwater & Surface water \\
\hline $\begin{array}{l}\text { Irrigation-Crop } \\
\text { Irrigation-Golf course }\end{array}$ & $\begin{array}{l}0.00 \\
2.29\end{array}$ & $\begin{array}{l}0.00 \\
0.00\end{array}$ & $\begin{array}{l}0.00 \\
2.29\end{array}$ & $\begin{array}{l}0.00 \\
2.29\end{array}$ & $\begin{array}{l}0.00 \\
0.00\end{array}$ & City of Toccoa & 0.00 & 3.08 \\
\hline Livestock/aquaculture & 0.00 & 0.18 & 0.18 & 0.18 & 0.00 & & & \\
\hline Thermoelectric power & 0.00 & 0.00 & 0.00 & 0.00 & 0.00 & & & \\
\hline TOTAL & 2.70 & 3.26 & 5.96 & 5.84 & 1.06 & & & \\
\hline
\end{tabular}

'Total use is total withdrawal plus public supply deliveries and losses. An average of $0.12 \mathrm{Mgal} / \mathrm{d}$ was delivered to Habersham County,

Georgia from Stephens County in 2010.

\section{Withdrawals by Source}
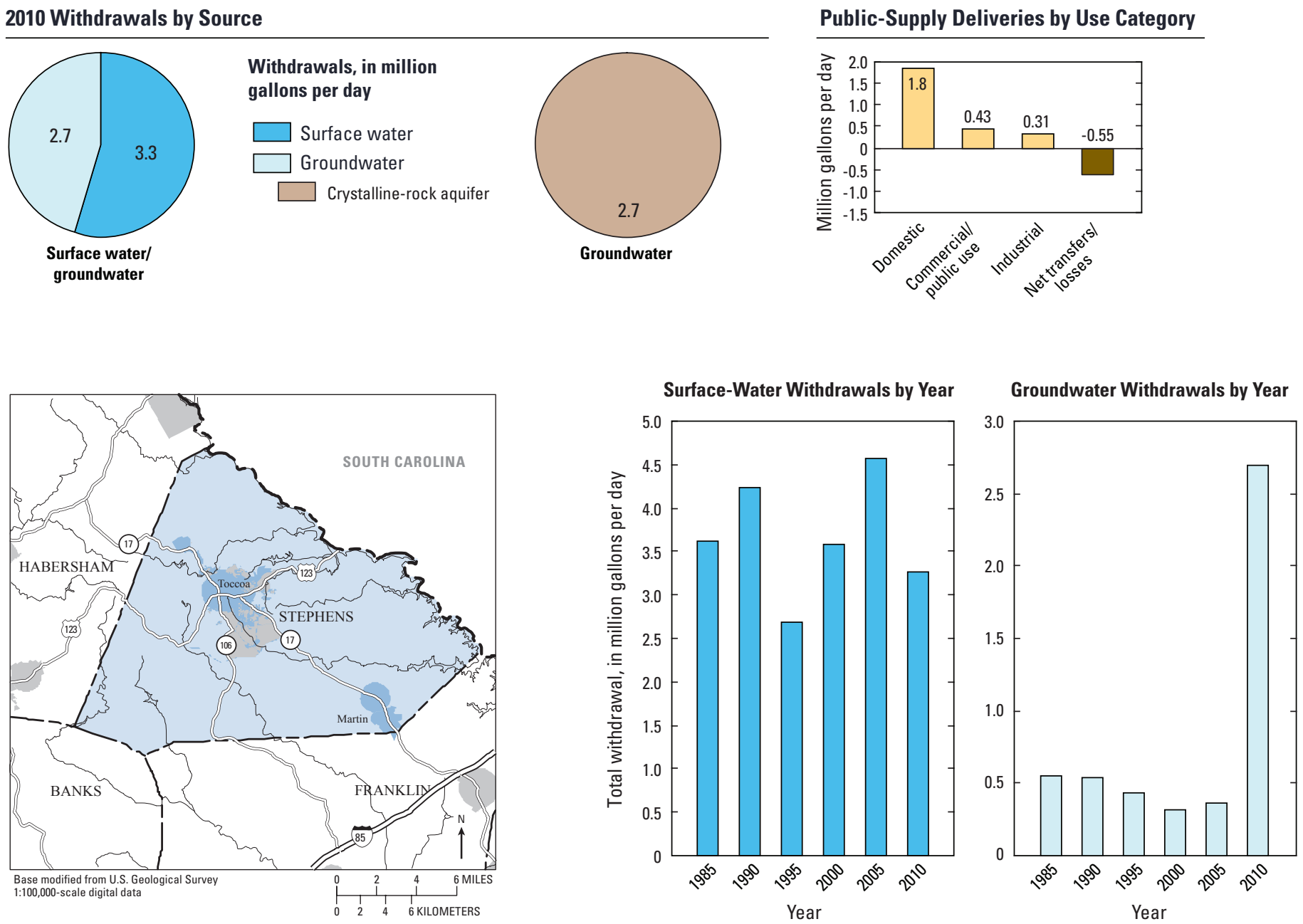


\section{STEWART COUNTY}

Population

6,058

Population served by public supply-Groundwater

Population served by public supply-Surface water Acres irrigated
4,260

3,520

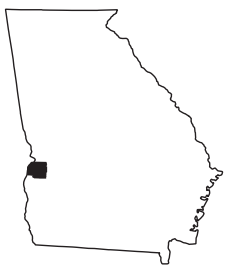

2010 WATER WITHDRAWALS AND ESTIMATED USE, IN MILLION GALLONS PER DAY

$[-$, not applicable $]$

\begin{tabular}{|c|c|c|c|c|c|c|c|c|}
\hline \multirow{3}{*}{ Category } & \multicolumn{3}{|c|}{ Withdrawals } & \multirow{3}{*}{$\begin{array}{l}\text { Total } \\
\text { use }^{1}\end{array}$} & \multirow{3}{*}{$\begin{array}{l}\text { Surface- } \\
\text { water } \\
\text { returns } \\
\end{array}$} & \multirow{2}{*}{\multicolumn{3}{|c|}{$\begin{array}{l}\text { Withdrawals by Major Industrial Groups } \\
\text { [NAICS, North American Industrial Classification System code] }\end{array}$}} \\
\hline & \multirow{2}{*}{$\begin{array}{c}\text { Ground- } \\
\text { water }\end{array}$} & \multirow{2}{*}{$\begin{array}{c}\text { Surface } \\
\text { water }\end{array}$} & \multirow[b]{2}{*}{ Total } & & & & & \\
\hline & & & & & & NAICS & Groundwater & Surface water \\
\hline Public supply & 0.40 & 0.00 & 0.40 & \multirow{2}{*}{$\begin{array}{c}- \\
0.45\end{array}$} & \multirow{2}{*}{$\begin{array}{c}- \\
0.00\end{array}$} & \multirow{2}{*}{ None } & \multirow[t]{2}{*}{-} & \multirow{2}{*}{-} \\
\hline Domestic & 0.13 & 0.00 & 0.13 & & & & & \\
\hline Commercial/public use & 0.00 & 0.00 & 0.00 & 0.04 & 0.00 & \multirow{4}{*}{\multicolumn{3}{|c|}{ Withdrawals by Major Public Suppliers }} \\
\hline Industrial & 0.00 & 0.00 & 0.00 & 0.00 & 0.00 & & & \\
\hline Public-supply losses & - & - & - & 0.04 & - & & & \\
\hline Public wastewater treatment & - & - & - & - & 0.36 & & & \\
\hline \multirow{3}{*}{$\begin{array}{l}\text { Mining } \\
\text { Irrigation-Crop } \\
\text { Irrigation-Golf course } \\
\text { Livestock/aquaculture }\end{array}$} & $\begin{array}{l}0.00 \\
0.04\end{array}$ & $\begin{array}{l}0.00 \\
0.92\end{array}$ & $\begin{array}{l}0.00 \\
0.96\end{array}$ & $\begin{array}{l}0.00 \\
0.96\end{array}$ & $\begin{array}{l}0.00 \\
0.00\end{array}$ & Name & Groundwater & Surface water \\
\hline & 0.00 & 0.00 & 0.00 & 0.00 & 0.00 & \multirow{2}{*}{$\begin{array}{l}\text { Stewart County } \\
\text { Water System }\end{array}$} & \multirow{2}{*}{0.16} & \multirow{2}{*}{0.00} \\
\hline & 0.00 & 0.02 & 0.02 & 0.02 & 0.00 & & & \\
\hline \multirow{2}{*}{$\begin{array}{l}\text { Thermoelectric power } \\
\text { TOTAL }\end{array}$} & 0.00 & 0.00 & 0.00 & 0.00 & 0.00 & \multirow{2}{*}{$\begin{array}{l}\text { City of Lumpkin } \\
\text { City of Richland }\end{array}$} & 0.13 & 0.00 \\
\hline & 0.57 & 0.94 & 1.51 & 1.51 & 0.36 & & 0.10 & 0.00 \\
\hline
\end{tabular}

Total use is total withdrawal plus public supply deliveries and losses.

\section{Withdrawals by Source}
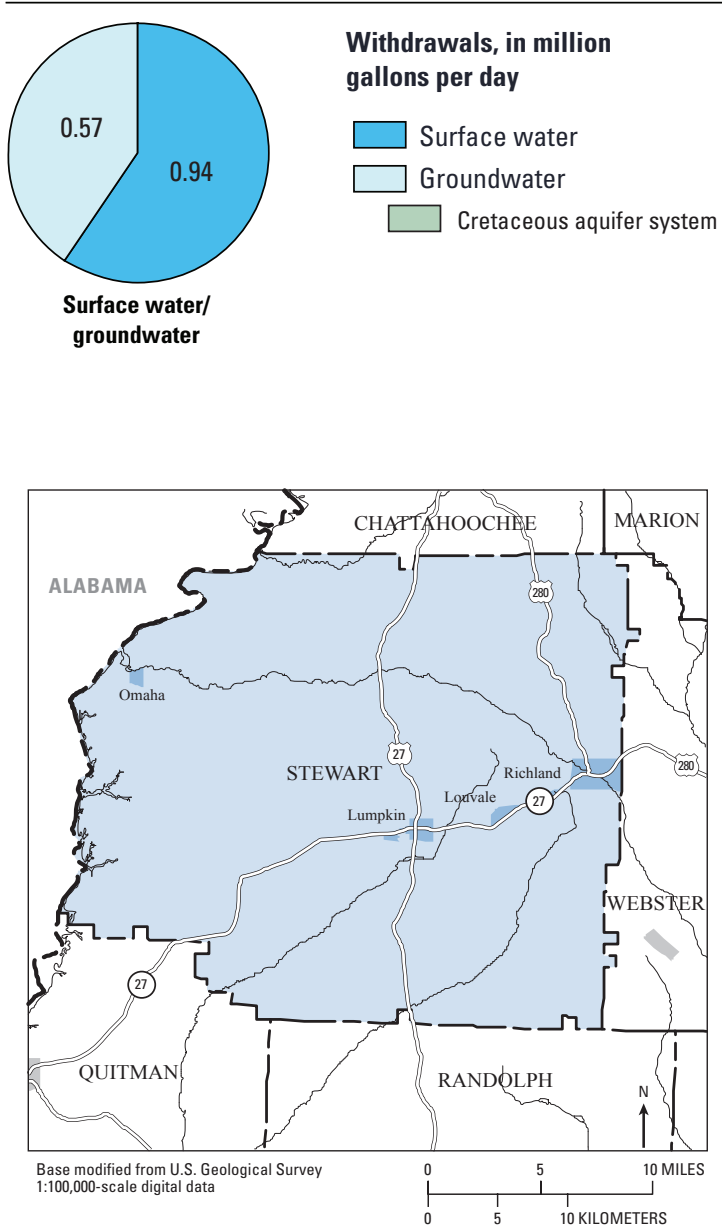

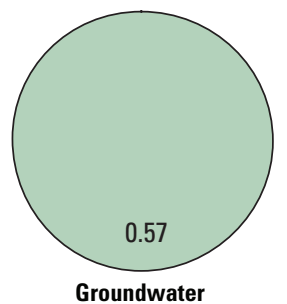

Public-Supply Deliveries by Use Category

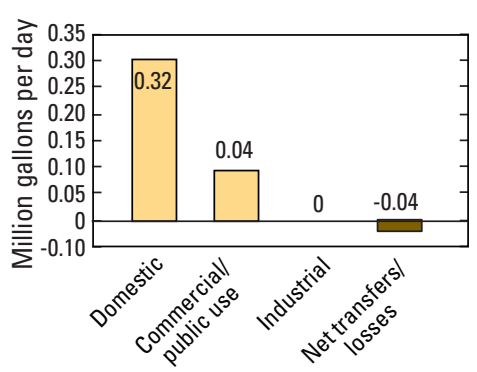

Surface-Water Withdrawals by Year

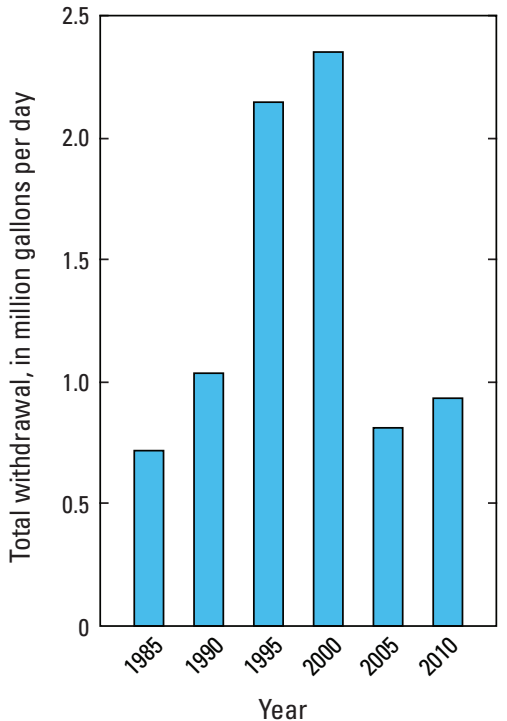

Groundwater Withdrawals by Year

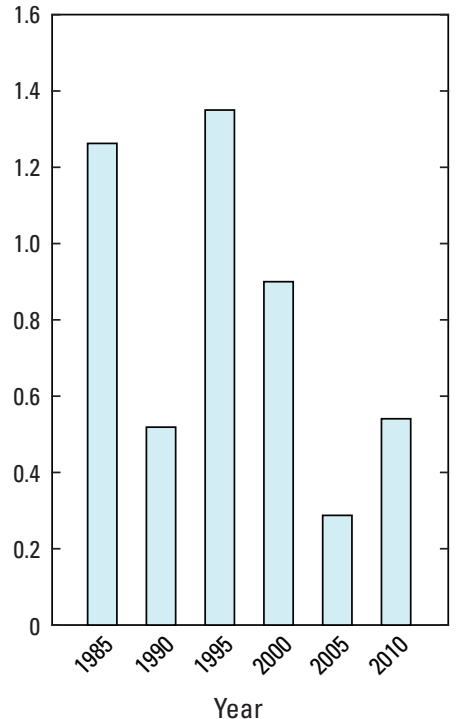




\section{SUMTER COUNTY}

Population

Population served by public supply-Groundwater

Population served by public supply-Surface water Acres irrigated
20,470

48,124

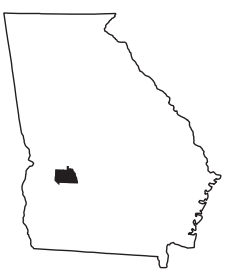

2010 WATER WITHDRAWALS AND ESTIMATED USE, IN MILLION GALLONS PER DAY

$[-$, not applicable $]$

\begin{tabular}{|c|c|c|c|c|c|c|c|c|}
\hline \multirow{3}{*}{ Category } & \multicolumn{3}{|c|}{ Withdrawals } & \multirow{3}{*}{$\begin{array}{c}\text { Total } \\
\text { use }^{1}\end{array}$} & \multirow{3}{*}{$\begin{array}{l}\text { Surface- } \\
\text { water } \\
\text { returns }\end{array}$} & \multirow{2}{*}{\multicolumn{3}{|c|}{$\begin{array}{l}\text { Withdrawals by Major Industrial Groups } \\
\text { [NAICS, North American Industrial Classification System code] }\end{array}$}} \\
\hline & \multirow{2}{*}{$\begin{array}{c}\text { Ground- } \\
\text { water }\end{array}$} & \multirow{2}{*}{$\begin{array}{c}\text { Surface } \\
\text { water }\end{array}$} & \multirow[b]{2}{*}{ Total } & & & & & \\
\hline & & & & & & NAICS & Groundwater & Surface water \\
\hline Public supply & 2.78 & 0.00 & 2.78 & - & - & $212-$ Kaolin and ball $\mathrm{c}$ & ay 0.26 & 0.00 \\
\hline Domestic & 0.93 & 0.00 & 0.93 & 2.61 & 0.00 & & & \\
\hline Commercial/public use & 0.02 & 0.00 & 0.02 & 0.48 & 0.00 & & & \\
\hline Industrial & 0.26 & 0.00 & 0.26 & 0.29 & 0.00 & & & \\
\hline Public-supply losses & - & - & - & 0.61 & - & & & \\
\hline Public wastewater treatment & - & - & - & - & 2.63 & Withdrawals by Majo & r Public Supp & \\
\hline Mining & 1.60 & 0.00 & 1.60 & 1.60 & 0.00 & Name & Groundwater & Surface water \\
\hline Irrigation-Crop & 22.88 & 12.42 & 35.30 & 35.30 & 0.00 & & & \\
\hline Irrigation-Golf course & 0.10 & 0.00 & 0.10 & 0.10 & 0.00 & City of Americus & 2.55 & 0.00 \\
\hline Livestock/aquaculture & 0.07 & 0.47 & 0.54 & 0.54 & 0.00 & Town of Leslie & 0.04 & 0.00 \\
\hline Thermoelectric power & 0.00 & 0.00 & 0.00 & 0.00 & 0.00 & Town of Desoto & 0.03 & 0.00 \\
\hline TOTAL & 28.64 & 12.89 & 41.53 & 41.53 & 2.63 & Town of Andersonville & 0.03 & 0.00 \\
\hline
\end{tabular}

2010 Withdrawals by Source
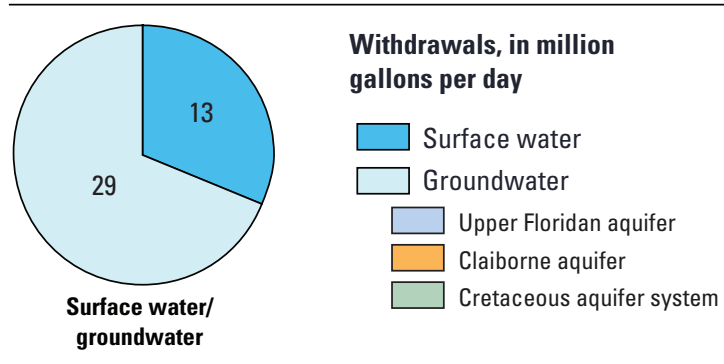

groundwater

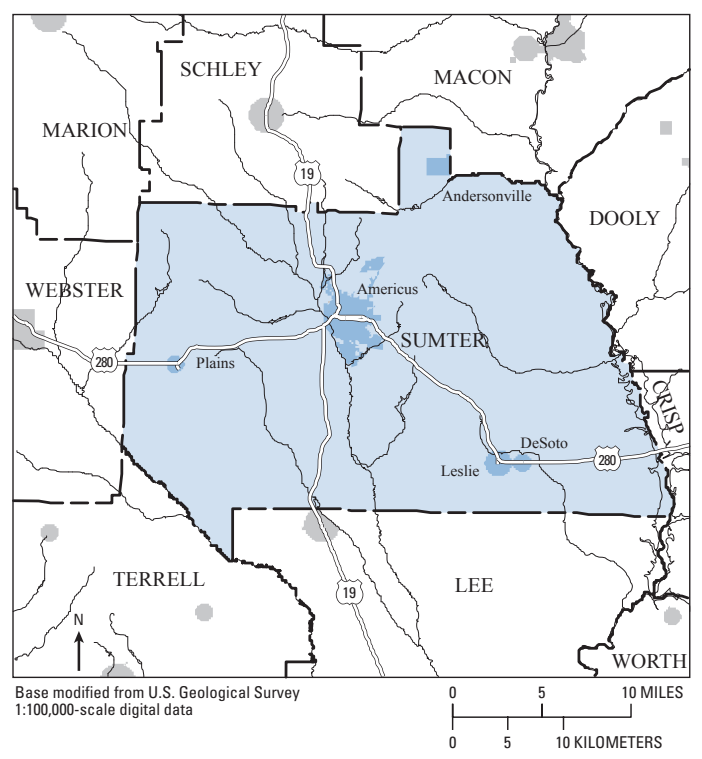

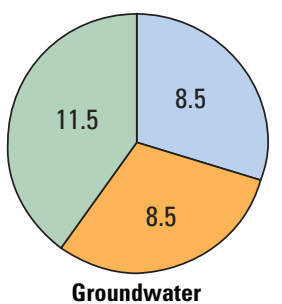

Public-Supply Deliveries by Use Category

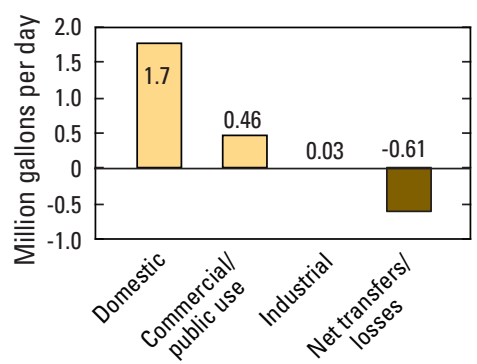

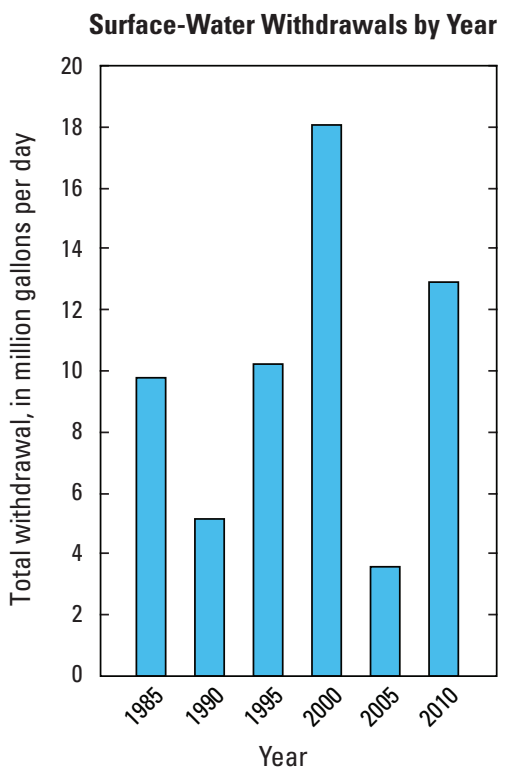

Groundwater Withdrawals by Year

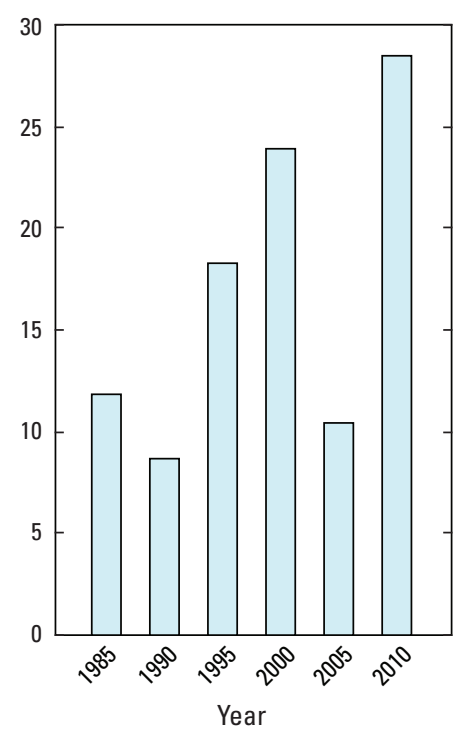




\section{TALBOT COUNTY}

Population

6,865

Population served by public supply-Groundwater $\quad 1,570$

Population served by public supply-Surface water $\quad 2,940$

Acres irrigated

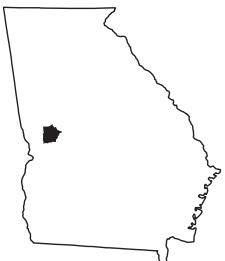

2010 WATER WITHDRAWALS AND ESTIMATED USE, IN MILLION GALLONS PER DAY

$[-$, not applicable; Mgal/d, million gallons per day]

\begin{tabular}{|c|c|c|c|c|c|c|c|c|}
\hline \multirow{3}{*}{ Category } & \multicolumn{3}{|c|}{ Withdrawals } & \multirow{3}{*}{$\begin{array}{l}\text { Total } \\
\text { use }^{1}\end{array}$} & \multirow{3}{*}{$\begin{array}{c}\text { Surface- } \\
\text { water } \\
\text { returns } \\
\end{array}$} & \multirow{2}{*}{\multicolumn{3}{|c|}{$\begin{array}{l}\text { Withdrawals by Major Industrial Groups } \\
\text { [NAICS, North American Industrial Classification System code] }\end{array}$}} \\
\hline & \multirow{2}{*}{$\begin{array}{c}\text { Ground- } \\
\text { water }\end{array}$} & \multirow{2}{*}{$\begin{array}{c}\text { Surface } \\
\text { water }\end{array}$} & \multirow[b]{2}{*}{ Total } & & & & & \\
\hline & & & & & & NAICS & Groundwater & Surface water \\
\hline Public supply & 0.18 & 1.04 & 1.22 & - & - & None & & \\
\hline Domestic & 0.18 & 0.00 & 0.18 & 0.56 & 0.00 & None & - & - \\
\hline Commercial/public use & 0.00 & 0.00 & 0.00 & 0.09 & 0.00 & & & \\
\hline Industrial & 0.00 & 0.00 & 0.00 & 0.00 & 0.00 & & & \\
\hline Public-supply losses & - & - & - & 0.09 & - & & & \\
\hline Public wastewater treatment & - & - & - & - & 0.05 & Withdrawals by $\mathbf{M}$ & Public Supp & \\
\hline Mining & 0.80 & 0.01 & 0.81 & 0.81 & 0.00 & Name & Groundwater & Surface water \\
\hline Irrigation-Crop & 0.01 & 0.00 & 0.01 & 0.01 & 0.00 & & & \\
\hline Irrigation-Golf course & 0.00 & 0.00 & 0.00 & 0.00 & 0.00 & City of Manchester ${ }^{1}$ & 0.00 & 1.04 \\
\hline Livestock/aquaculture & 0.00 & 0.30 & 0.30 & 0.30 & 0.00 & City of Talbotton & 0.12 & 0.00 \\
\hline Thermoelectric power & 0.00 & 0.00 & 0.00 & 0.00 & 0.00 & City of Woodland & 0.03 & 0.00 \\
\hline TOTAL & 1.17 & 1.35 & 2.52 & 1.86 & 0.15 & s in Meriwe & nty, Georgia & \\
\hline
\end{tabular}

'Total use is total withdrawal plus public supply deliveries and losses. An average of $1.04 \mathrm{Mgal} / \mathrm{d}$ was delivered to Meriwether County,

Georgia and $0.07 \mathrm{Mgal} / \mathrm{d}$ was delivered from Muscogee County, Georgia to Talbot County in 2010.

\section{Withdrawals by Source}

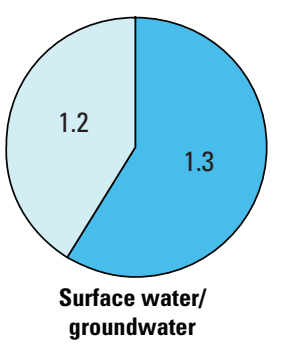

Withdrawals, in million gallons per day

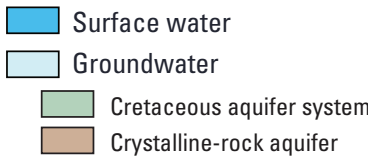
groundwater

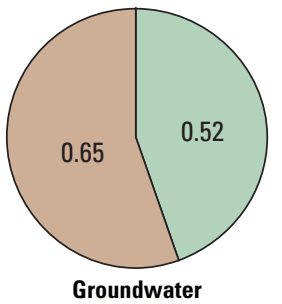

Groundwater
Public-Supply Deliveries ${ }^{1}$ by Use Category

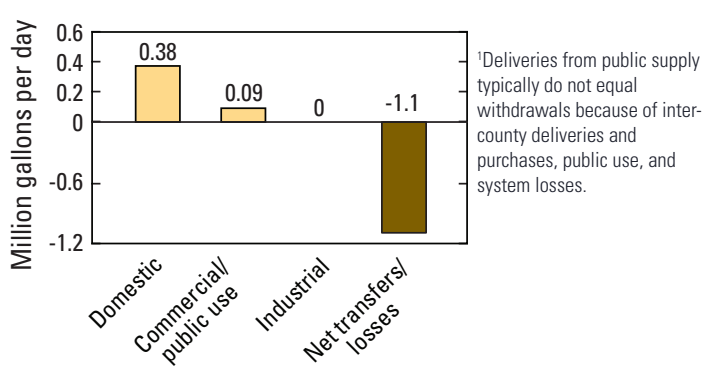

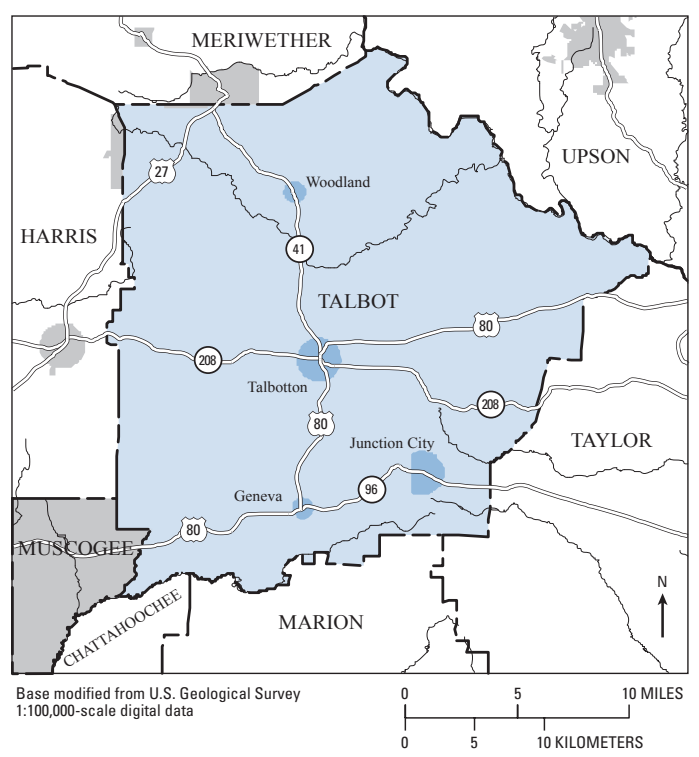

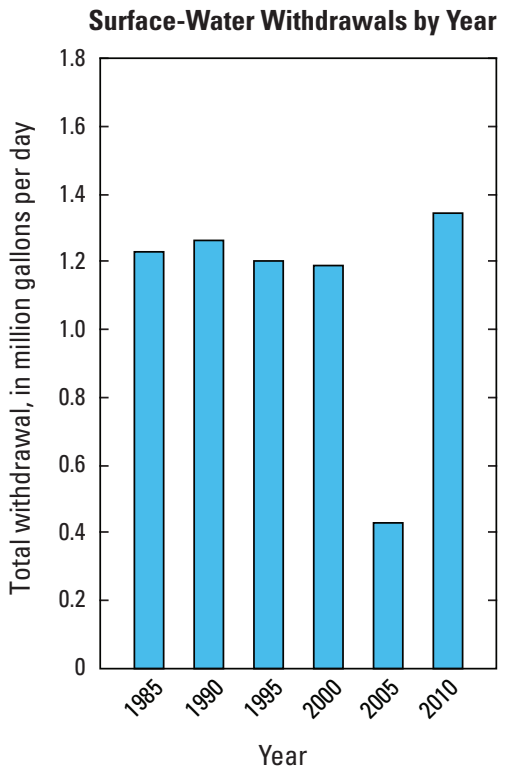

Groundwater Withdrawals by Year

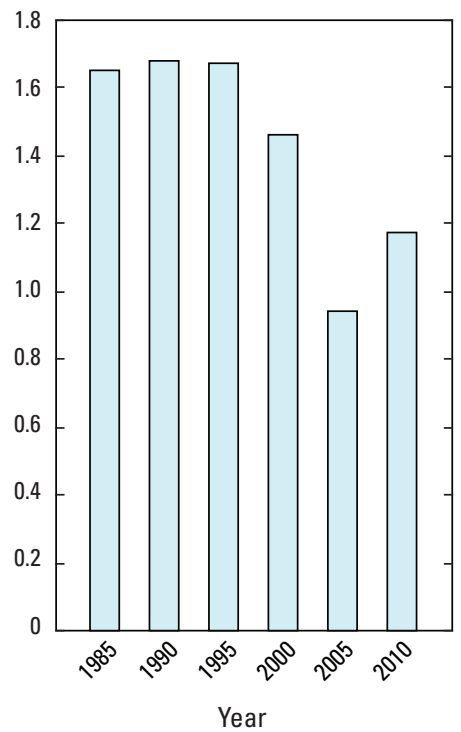




\section{TALIAFERRO COUNTY}

Population

Population served by public supply-Groundwater

Population served by public supply-Surface water

Acres irrigated

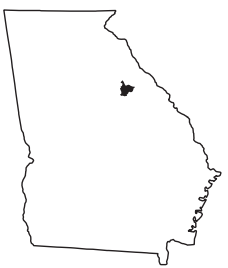

2010 WATER WITHDRAWALS AND ESTIMATED USE, IN MILLION GALLONS PER DAY

$[-$, not applicable; $<$, less than $]$

\begin{tabular}{|c|c|c|c|c|c|c|c|c|}
\hline \multirow{3}{*}{ Category } & \multicolumn{3}{|c|}{ Withdrawals } & \multirow{3}{*}{$\begin{array}{l}\text { Total } \\
\text { use }^{1}\end{array}$} & \multirow{3}{*}{$\begin{array}{l}\text { Surface- } \\
\text { water } \\
\text { returns } \\
\end{array}$} & \multirow{2}{*}{\multicolumn{3}{|c|}{$\begin{array}{l}\text { Withdrawals by Major Industrial Groups } \\
\text { [NAICS, North American Industrial Classification System code] }\end{array}$}} \\
\hline & \multirow{2}{*}{$\begin{array}{c}\text { Ground- } \\
\text { water }\end{array}$} & \multirow{2}{*}{$\begin{array}{c}\text { Surface } \\
\text { water }\end{array}$} & \multirow[b]{2}{*}{ Total } & & & & & \\
\hline & & & & & & NAICS & Groundwater & Surface water \\
\hline Public supply & 0.05 & 0.00 & 0.05 & - & - & & & \\
\hline Domestic & 0.08 & 0.00 & 0.08 & 0.13 & 0.00 & None & - & - \\
\hline Commercial/public use & 0.00 & 0.00 & 0.00 & 0.00 & 0.00 & & & \\
\hline Industrial & 0.00 & 0.00 & 0.00 & 0.00 & 0.00 & & & \\
\hline Public-supply losses & - & - & - & $<0.01$ & - & & & \\
\hline Public wastewater treatment & - & - & - & - & 0.05 & Withdrawals by Maj & r Public Supp & \\
\hline Mining & 0.00 & 0.00 & 0.00 & 0.00 & 0.00 & Name & Groundwater & Surface water \\
\hline Irrigation-Crop & 0.00 & 0.00 & 0.00 & 0.00 & 0.00 & & & \\
\hline Irrigation-Golf course & 0.00 & 0.00 & 0.00 & 0.00 & 0.00 & City of Crawfordville & 0.05 & 0.00 \\
\hline Livestock/aquaculture & 0.00 & 0.00 & 0.00 & 0.00 & 0.00 & & & \\
\hline Thermoelectric power & 0.00 & 0.00 & 0.00 & 0.00 & 0.00 & & & \\
\hline TOTAL & 0.13 & 0.00 & 0.13 & 0.13 & 0.05 & & & \\
\hline
\end{tabular}

'Total use is total withdrawal plus public supply deliveries and losses.

\section{Withdrawals by Source}
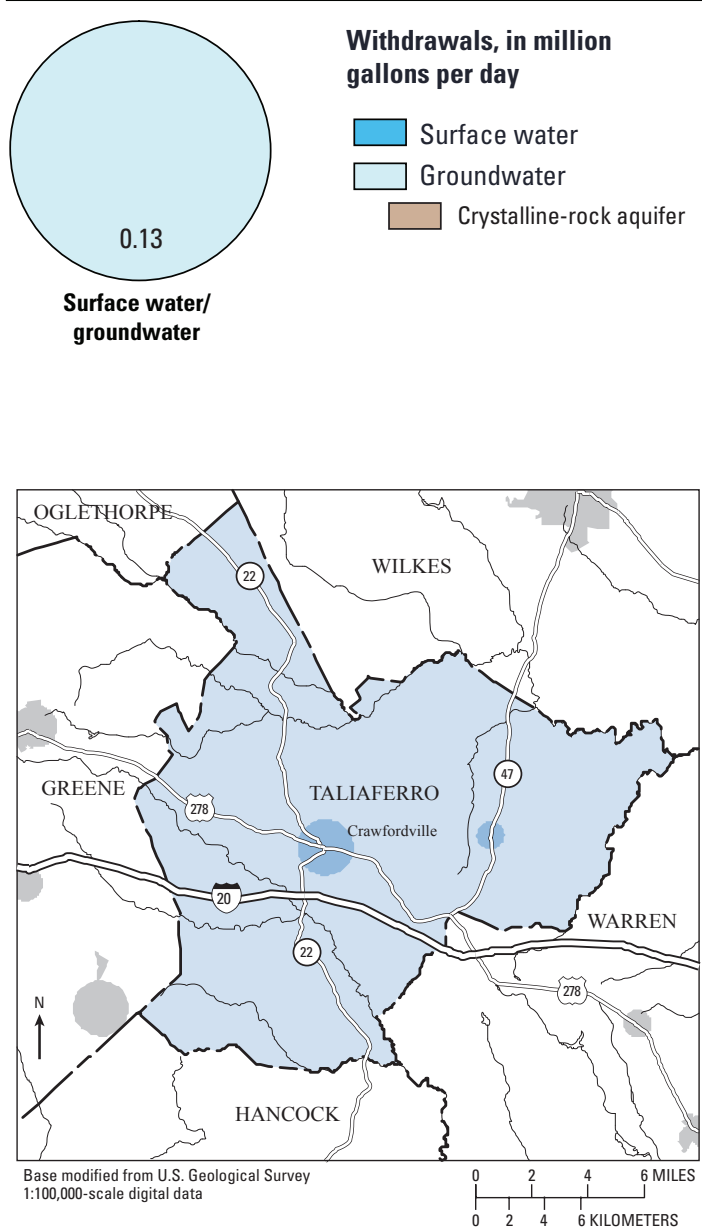

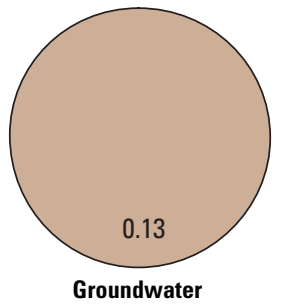

Public-Supply Deliveries by Use Category

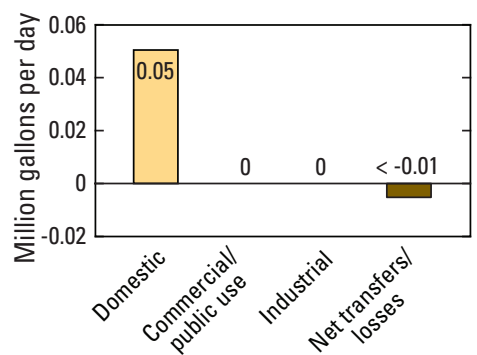

Surface-Water Withdrawals by Year

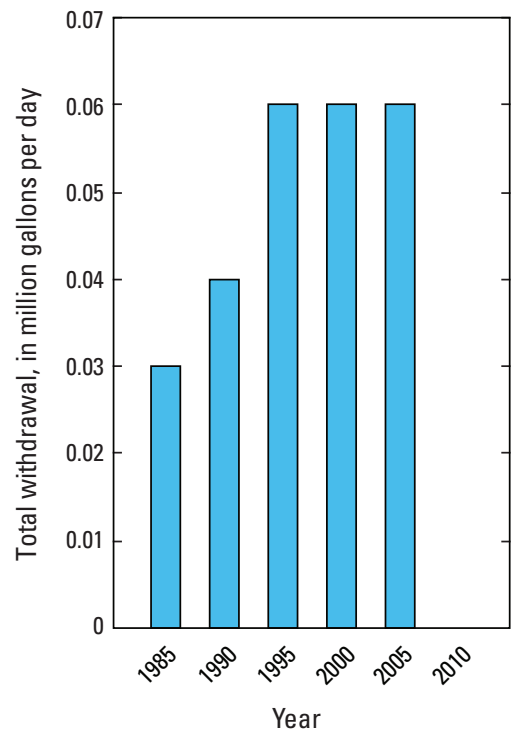

Groundwater Withdrawals by Year

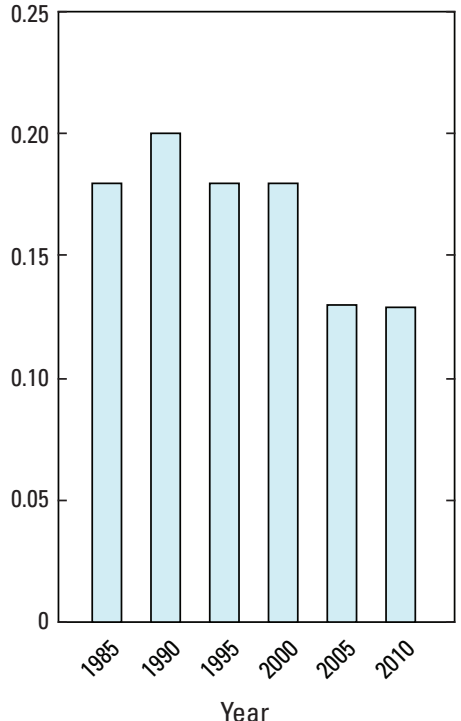




\section{TATTNALL COUNTY}

Population

25,520

Population served by public supply-Groundwater $\quad 7,900$

Population served by public supply-Surface water 0

Acres irrigated

16,540

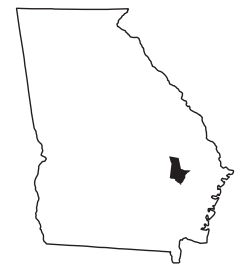

2010 WATER WITHDRAWALS AND ESTIMATED USE, IN MILLION GALLONS PER DAY

$[-$, not applicable $]$

\begin{tabular}{|c|c|c|c|c|c|c|c|c|}
\hline \multirow{3}{*}{ Category } & \multicolumn{3}{|c|}{ Withdrawals } & \multirow{3}{*}{$\begin{array}{l}\text { Total } \\
\text { use }^{1}\end{array}$} & \multirow{3}{*}{$\begin{array}{l}\text { Surface- } \\
\text { water } \\
\text { returns } \\
\end{array}$} & \multirow{2}{*}{\multicolumn{3}{|c|}{$\begin{array}{l}\text { Withdrawals by Major Industrial Groups } \\
\text { [NAICS, North American Industrial Classification System code] }\end{array}$}} \\
\hline & \multirow{2}{*}{$\begin{array}{c}\text { Ground- } \\
\text { water }\end{array}$} & \multirow{2}{*}{$\begin{array}{c}\text { Surface } \\
\text { water }\end{array}$} & \multirow[b]{2}{*}{ Total } & & & & & \\
\hline & & & & & & NAICS & Groundwater & Surface water \\
\hline Public supply & 1.11 & 0.00 & 1.11 & - & - & & & 0.00 \\
\hline Domestic & 1.32 & 0.00 & 1.32 & 1.89 & 0.00 & 322-Paper, pulp & 0.03 & 0.00 \\
\hline Commercial/public use & 0.22 & 0.00 & 0.22 & 0.38 & 0.75 & & & \\
\hline Industrial & 0.03 & 0.00 & 0.03 & 0.23 & 0.00 & & & \\
\hline Public-supply losses & - & - & - & 0.18 & - & & & \\
\hline Public wastewater treatment & - & - & - & - & 1.13 & Withdrawals by & r Public Supp & \\
\hline Mining & $\begin{array}{l}0.01 \\
185\end{array}$ & 0.00 & $\begin{array}{l}0.01 \\
487\end{array}$ & $\begin{array}{l}0.01 \\
487\end{array}$ & 0.00 & Name & Groundwater & Surface water \\
\hline Irrigation-Crop & 1.85 & $\begin{array}{l}3.02 \\
0.00\end{array}$ & $\begin{array}{l}4.87 \\
0.16\end{array}$ & $\begin{array}{l}4.87 \\
0.16\end{array}$ & $\begin{array}{l}0.00 \\
0.00\end{array}$ & & & \\
\hline & 0.16 & 0.00 & 0.16 & 0.16 & $\begin{array}{l}0.00 \\
000\end{array}$ & City of Glennville & 0.73 & 0.00 \\
\hline Livestock/aquaculture & 0.15 & 1.51 & 1.66 & 1.66 & 0.00 & City of Reidsville & 0.28 & 0.00 \\
\hline Thermoelectric power & 0.00 & 0.00 & 0.00 & 0.00 & 0.00 & City of Collins & 0.04 & 0.00 \\
\hline
\end{tabular}

'Total use is total withdrawal plus public supply deliveries and losses.

2010 Withdrawals by Source
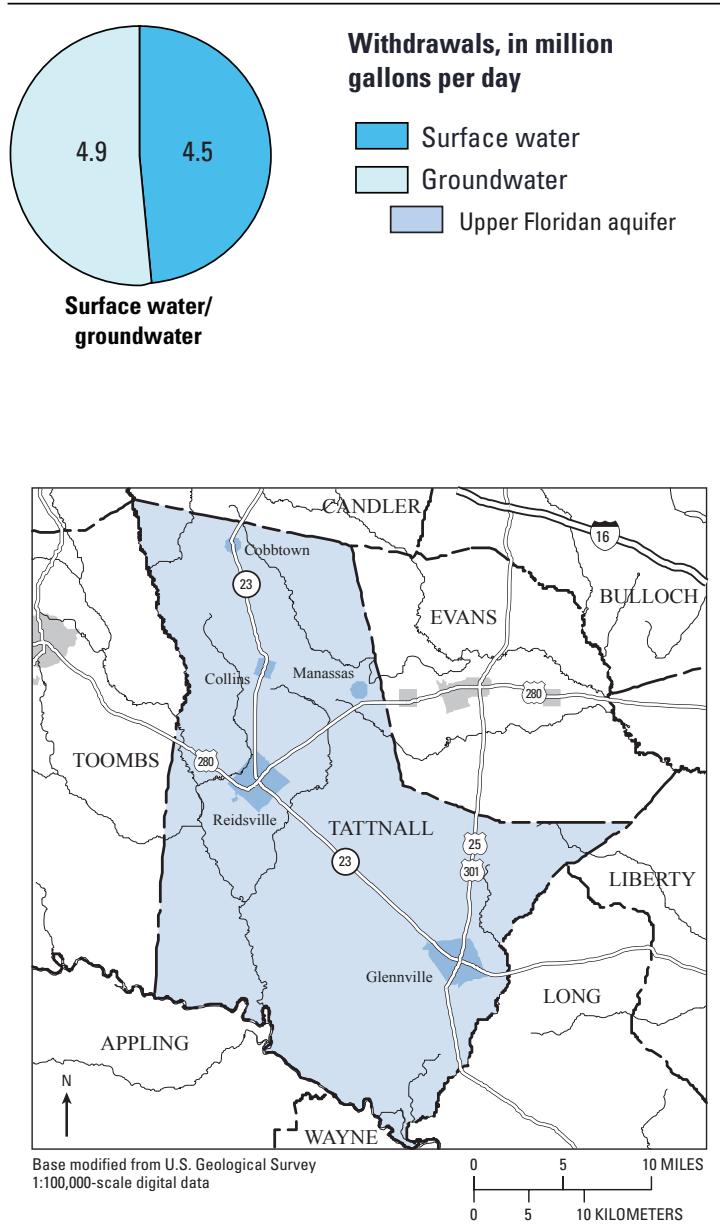

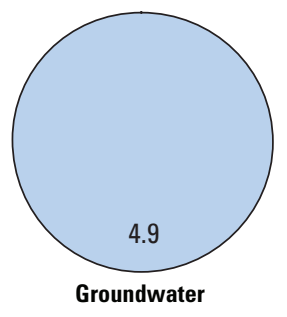

Public-Supply Deliveries by Use Category

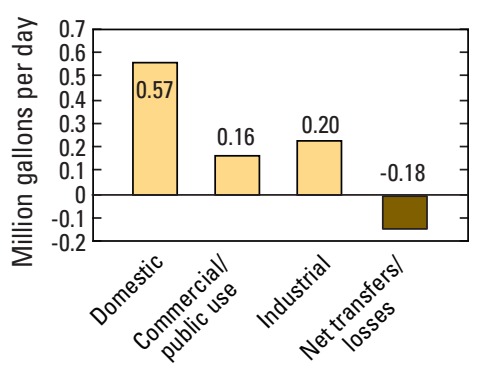

Surface-Water Withdrawals by Year

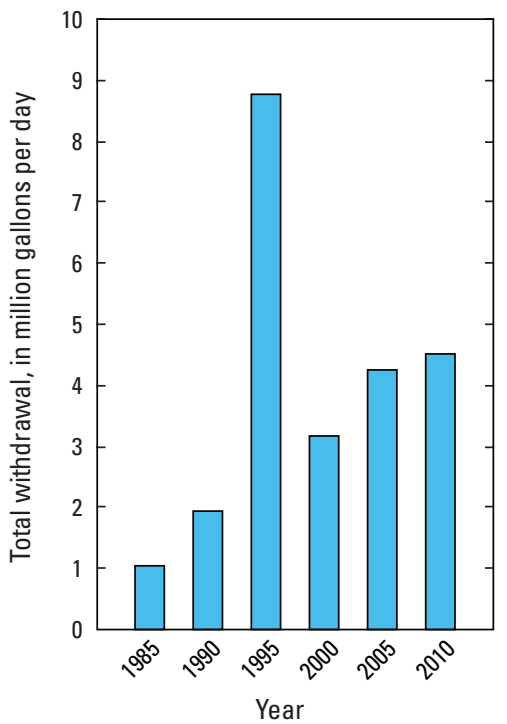

Groundwater Withdrawals by Year

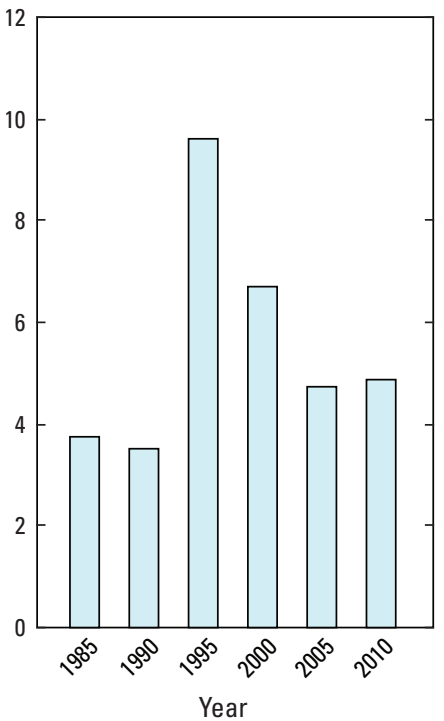




\section{TAYLOR COUNTY}

Population

8,906

Population served by public supply-Groundwater $\quad 3,760$

Population served by public supply_-Surface water 0

Acres irrigated

3,890

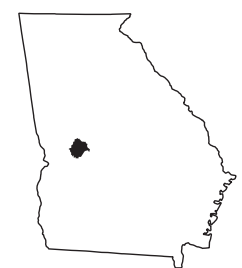

2010 WATER WITHDRAWALS AND ESTIMATED USE, IN MILLION GALLONS PER DAY

$[-$, not applicable $]$

\begin{tabular}{|c|c|c|c|c|c|c|c|c|}
\hline \multirow{3}{*}{ Category } & \multicolumn{3}{|c|}{ Withdrawals } & \multirow{3}{*}{$\begin{array}{l}\text { Total } \\
\text { use }^{1}\end{array}$} & \multirow{3}{*}{$\begin{array}{l}\text { Surface- } \\
\text { water } \\
\text { returns }\end{array}$} & \multirow{2}{*}{\multicolumn{3}{|c|}{$\begin{array}{l}\text { Withdrawals by Major Industrial Groups } \\
\text { [NAICS, North American Industrial Classification System code] }\end{array}$}} \\
\hline & \multirow{2}{*}{$\begin{array}{c}\text { Ground- } \\
\text { water }\end{array}$} & \multirow{2}{*}{$\begin{array}{c}\text { Surface } \\
\text { water }\end{array}$} & \multirow[b]{2}{*}{ Total } & & & & & \\
\hline & & & & & & NAICS & Groundwater & Surface water \\
\hline Public supply & 0.76 & 0.00 & 0.76 & - & - & & & \\
\hline Domestic & 0.39 & 0.00 & 0.39 & 0.95 & 0.00 & 423-Stone, clay & 0.00 & 1.72 \\
\hline Commercial/public use & 0.01 & 0.00 & 0.01 & 0.09 & 0.00 & & & \\
\hline Industrial & 0.00 & 1.72 & 1.72 & 1.72 & 0.00 & & & \\
\hline Public-supply losses & - & - & - & 0.12 & - & & & \\
\hline Public wastewater treatment & - & - & - & - & 0.07 & Withdrawals by & r Public Supp & \\
\hline Mining & 0.09 & 0.00 & 0.09 & 0.09 & 0.00 & Name & Groundwater & Surface water \\
\hline $\begin{array}{l}\text { Irrigation-Crop } \\
\text { Irrigation-Golf course }\end{array}$ & $\begin{array}{l}1.25 \\
0.00\end{array}$ & $\begin{array}{l}0.95 \\
0.00\end{array}$ & $\begin{array}{l}2.20 \\
0.00\end{array}$ & $\begin{array}{l}2.20 \\
0.00\end{array}$ & $\begin{array}{l}0.00 \\
0.00\end{array}$ & City of Butler & 0.59 & 0.00 \\
\hline Livestock/aquaculture & 0.07 & 0.75 & 0.82 & 0.82 & 0.00 & City of Reynolds & 0.16 & 0.00 \\
\hline Thermoelectric power & 0.00 & 0.00 & 0.00 & 0.00 & 0.00 & & & \\
\hline
\end{tabular}

TOTAL

$2.57 \quad 3.42$

5.99

5.99

0.07

'Total use is total withdrawal plus public supply deliveries and losses.

\section{Withdrawals by Source}
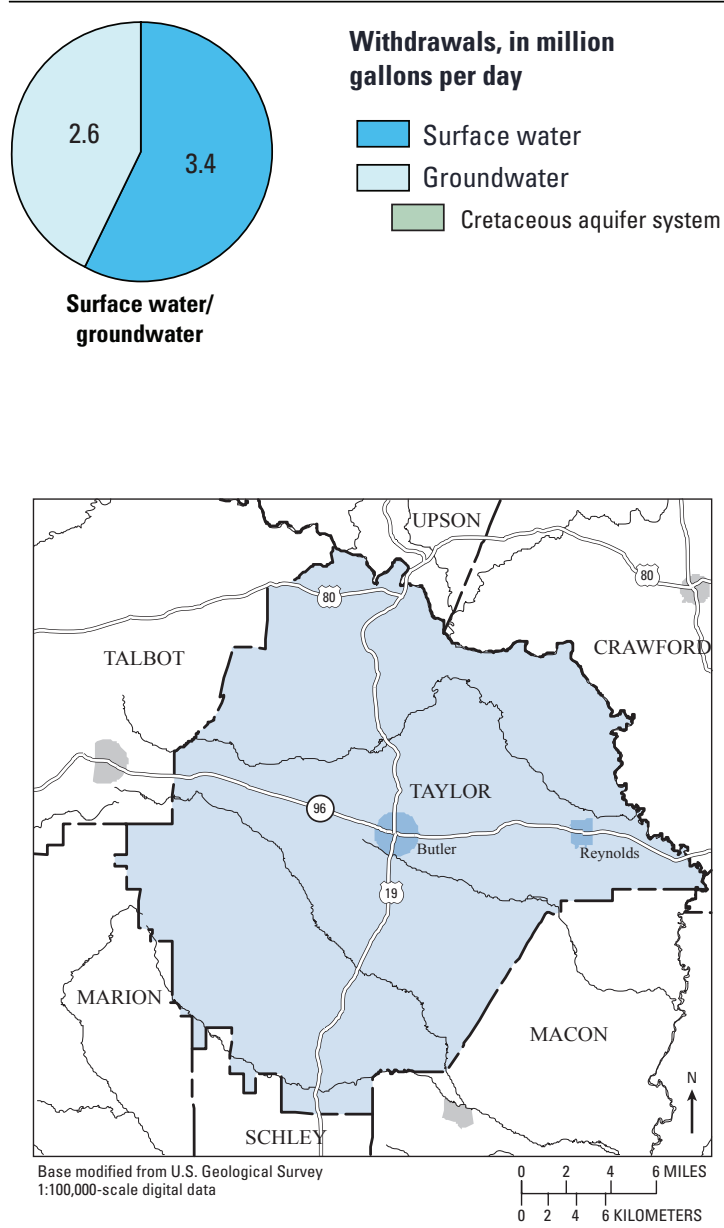

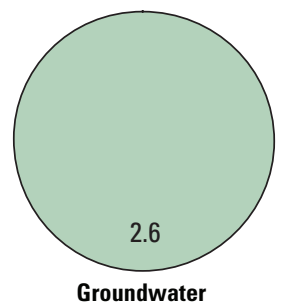

Public-Supply Deliveries by Use Category

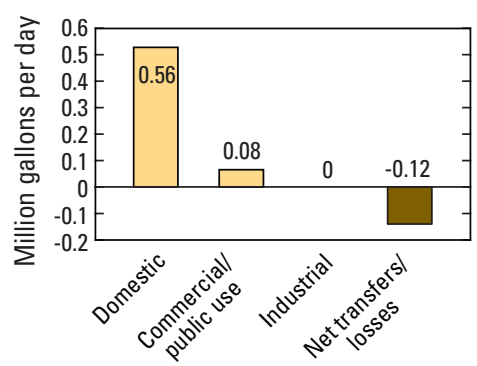

Surface-Water Withdrawals by Year

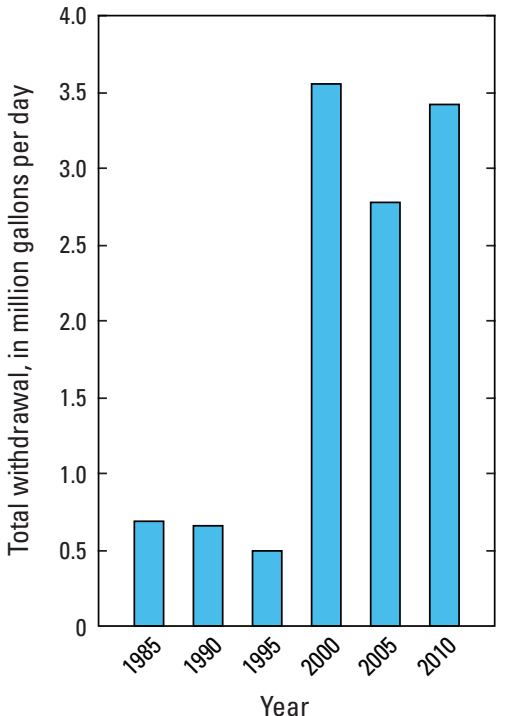

Groundwater Withdrawals by Year

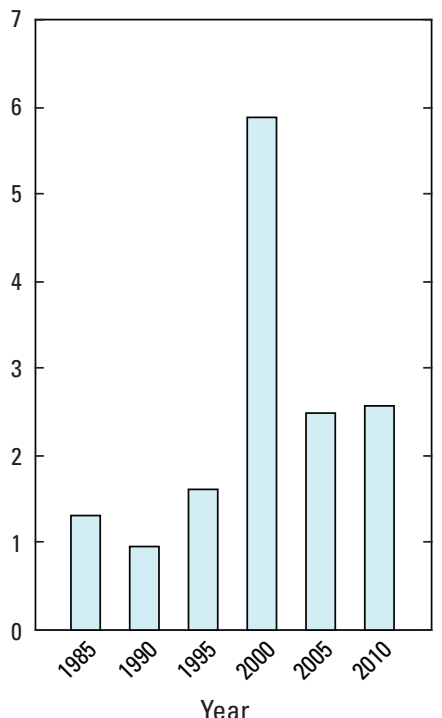




\section{TELFAIR COUNTY}

Population

16,500

Population served by public supply-Groundwater

Population served by public supply-Surface water Acres irrigated
7,830

6,180

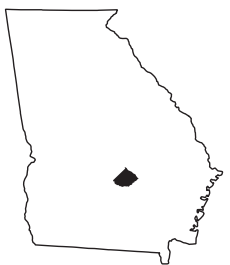

2010 WATER WITHDRAWALS AND ESTIMATED USE, IN MILLION GALLONS PER DAY

$[-$, not applicable $]$

\begin{tabular}{|c|c|c|c|c|c|c|c|c|}
\hline \multirow{3}{*}{ Category } & \multicolumn{3}{|c|}{ Withdrawals } & \multirow{3}{*}{$\begin{array}{l}\text { Total } \\
\text { use }^{1}\end{array}$} & \multirow{3}{*}{$\begin{array}{c}\text { Surface- } \\
\text { water } \\
\text { returns } \\
\end{array}$} & \multirow{2}{*}{\multicolumn{3}{|c|}{$\begin{array}{l}\text { Withdrawals by Major Industrial Groups } \\
\text { [NAICS, North American Industrial Classification System code] }\end{array}$}} \\
\hline & \multirow{2}{*}{$\begin{array}{l}\text { Ground- } \\
\text { water }\end{array}$} & \multirow{2}{*}{$\begin{array}{c}\text { Surface } \\
\text { water }\end{array}$} & \multirow[b]{2}{*}{ Total } & & & & & \\
\hline & & & & & & NAICS & Groundwater & Surface water \\
\hline Public supply & 1.43 & 0.00 & 1.43 & - & - & None & - & - \\
\hline Domestic & 0.65 & 0.00 & 0.65 & 1.38 & 0.00 & None & - & - \\
\hline Commercial/public use & 0.00 & 0.00 & 0.00 & 0.37 & 0.00 & & & \\
\hline Industrial & 0.00 & 0.00 & 0.00 & 0.06 & 0.00 & & & \\
\hline Public-supply losses & - & - & - & 0.27 & - & & & \\
\hline Public wastewater treatment & - & - & - & - & 0.74 & Withdrawals by M & Public Supp & \\
\hline Mining & 0.00 & 0.00 & 0.00 & 0.00 & 0.00 & Name & Groundwater & Surface water \\
\hline Irrigation-Crop & 2.13 & 1.02 & 3.15 & 3.15 & 0.00 & & & \\
\hline Irrigation-Golf course & 0.00 & 0.00 & 0.00 & 0.00 & 0.00 & City of McRae & 0.65 & 0.00 \\
\hline Livestock/aquaculture & 0.01 & 0.05 & 0.06 & 0.06 & 0.00 & City of Helena & 0.43 & 0.00 \\
\hline Thermoelectric power & 0.00 & 0.00 & 0.00 & 0.00 & 0.00 & City of Lumber City & 0.19 & 0.00 \\
\hline TOTAL & 4.22 & 1.07 & 5.29 & 5.29 & 0.74 & City of Milan & 0.14 & 0.00 \\
\hline
\end{tabular}

'Total use is total withdrawal plus public supply deliveries and losses.

2010 Withdrawals by Source
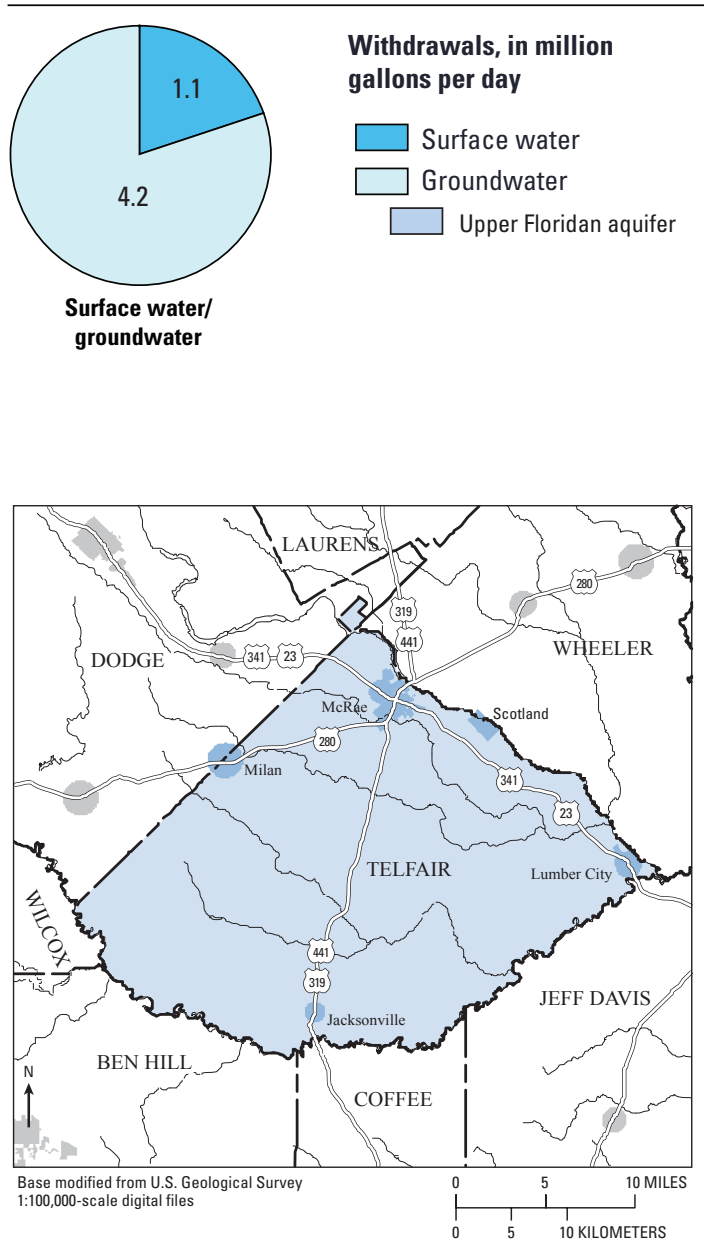

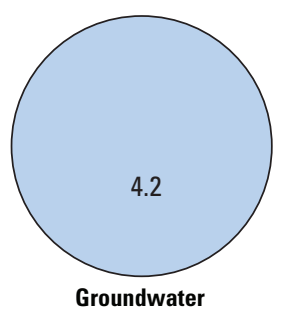

Public-Supply Deliveries by Use Category

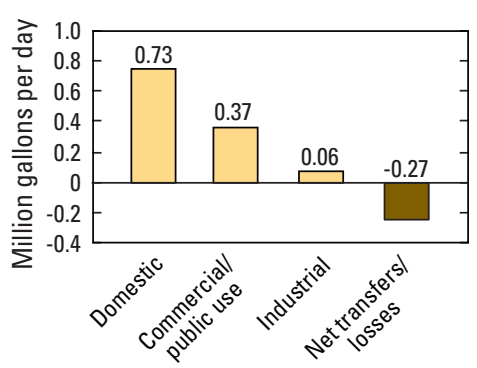

Surface-Water Withdrawals by Year

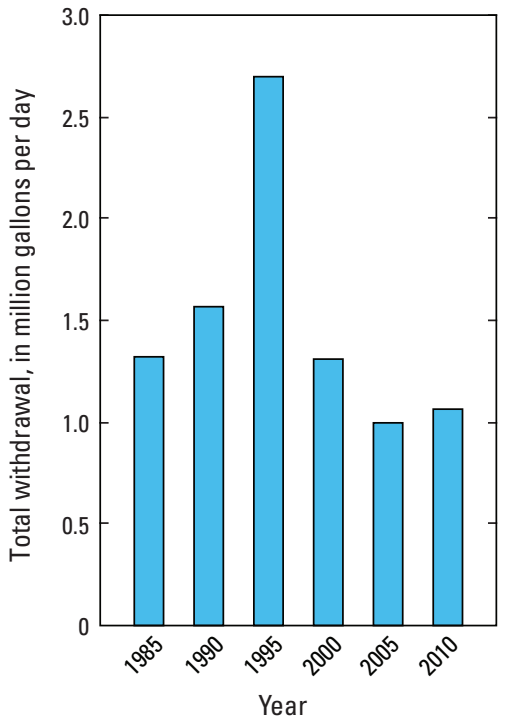

Groundwater Withdrawals by Year

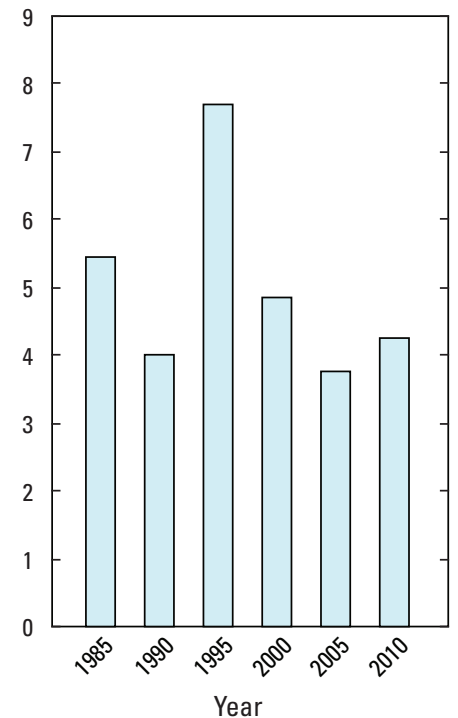




\section{TERRELL COUNTY}

Population

9,315

$\begin{array}{lr}\text { Population served by public supply-Groundwater } & 6,700 \\ \text { Population served by public supply-Surface water } & 0\end{array}$

Acres irrigated

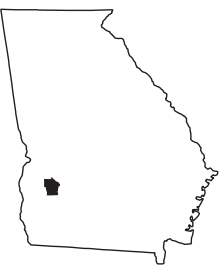

2010 WATER WITHDRAWALS AND ESTIMATED USE, IN MILLION GALLONS PER DAY

$[-$, not applicable $]$

\begin{tabular}{|c|c|c|c|c|c|c|c|c|}
\hline \multirow{3}{*}{ Category } & \multicolumn{3}{|c|}{ Withdrawals } & \multirow{3}{*}{$\begin{array}{l}\text { Total } \\
\text { use }^{1}\end{array}$} & \multirow{3}{*}{$\begin{array}{l}\text { Surface- } \\
\text { water } \\
\text { returns } \\
\end{array}$} & \multirow{2}{*}{\multicolumn{3}{|c|}{$\begin{array}{l}\text { Withdrawals by Major Industrial Groups } \\
\text { [NAICS, North American Industrial Classification System code] }\end{array}$}} \\
\hline & \multirow{2}{*}{$\begin{array}{c}\text { Ground- } \\
\text { water }\end{array}$} & \multirow{2}{*}{$\begin{array}{c}\text { Surface } \\
\text { water }\end{array}$} & \multirow[b]{2}{*}{ Total } & & & & & \\
\hline & & & & & & NAICS & Groundwater & Surface water \\
\hline Public supply & 1.59 & 0.00 & 1.59 & - & - & None & - & - \\
\hline Domestic & 0.20 & 0.00 & 0.20 & 0.74 & 0.00 & & & \\
\hline Commercial/public use & 0.01 & 0.00 & 0.01 & 0.18 & 0.00 & & & \\
\hline Industrial & 0.00 & 0.00 & 0.00 & 0.28 & 0.00 & & & \\
\hline Public-supply losses & - & - & - & 0.60 & - & & & \\
\hline Public wastewater treatment & - & - & - & - & 1.53 & Withdrawals b & r Public Supp & \\
\hline Mining & 0.00 & 0.00 & 0.00 & 0.00 & 0.00 & Name & Groundwater & Surface water \\
\hline Irrigation-Crop & 11.88 & 10.90 & 22.78 & 22.78 & 0.00 & & & \\
\hline Irrigation-Golf course & 0.00 & 0.04 & 0.04 & 0.04 & 0.00 & City of Dawson & 1.54 & 0.00 \\
\hline Livestock/aquaculture & 0.11 & 0.05 & 0.16 & 0.16 & 0.00 & & & \\
\hline Thermoelectric power & 0.00 & 0.00 & 0.00 & 0.00 & 0.00 & & & \\
\hline TOTAL & 13.79 & 10.99 & 24.78 & 24.78 & 1.53 & & & \\
\hline
\end{tabular}

${ }^{1}$ Total use is total withdrawal plus public supply deliveries and losses.

\section{Withdrawals by Source}
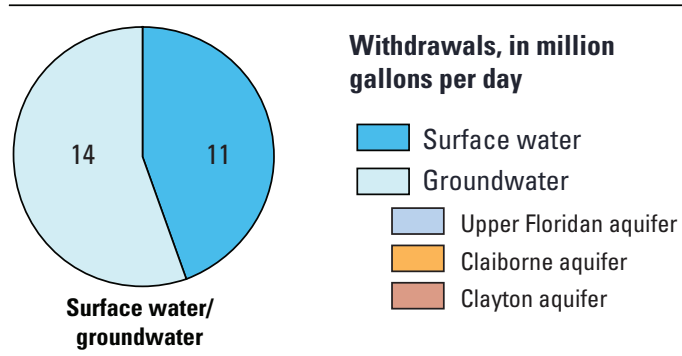

groundwater

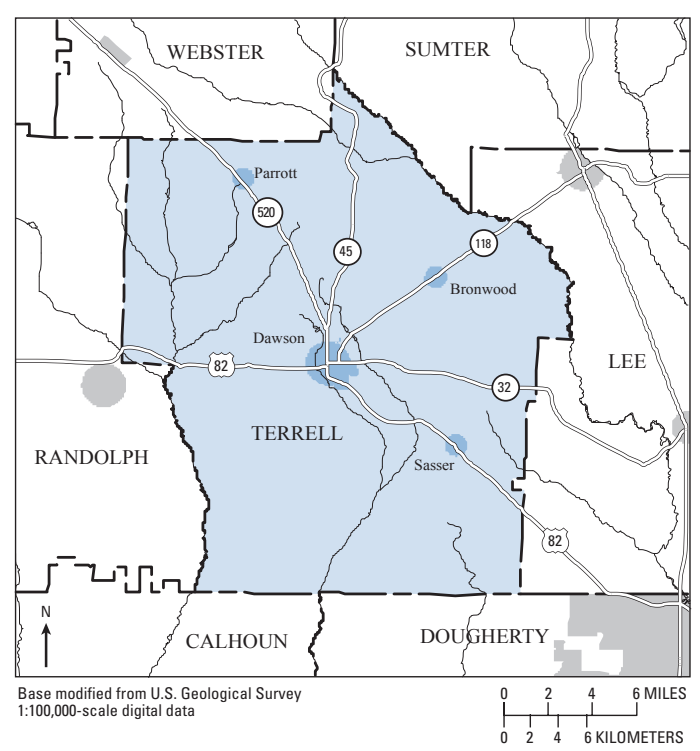

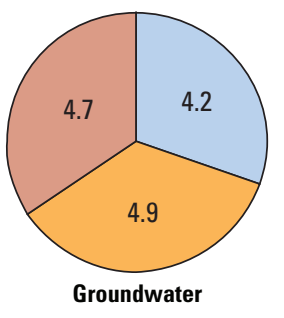

Public-Supply Deliveries by Use Category

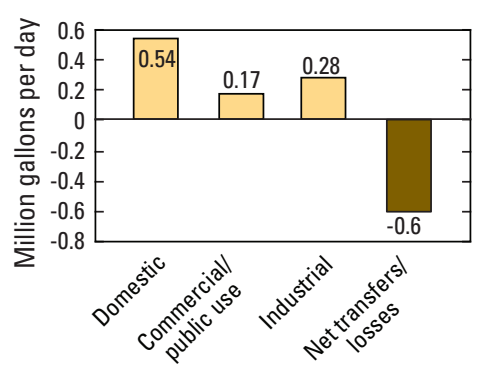

Surface-Water Withdrawals by Year

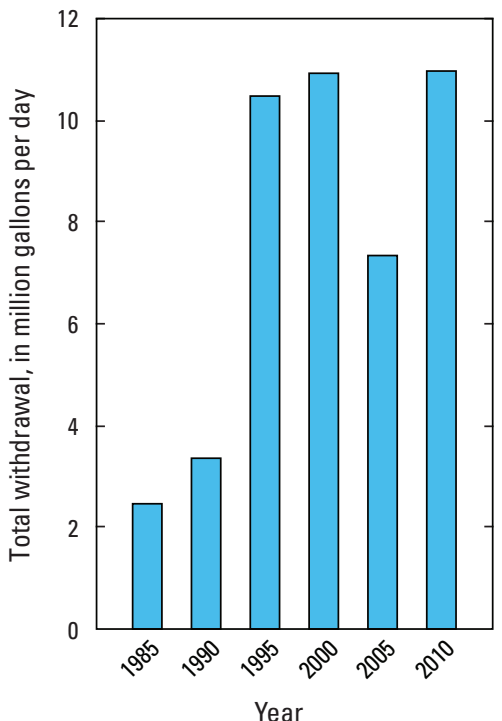

Groundwater Withdrawals by Year

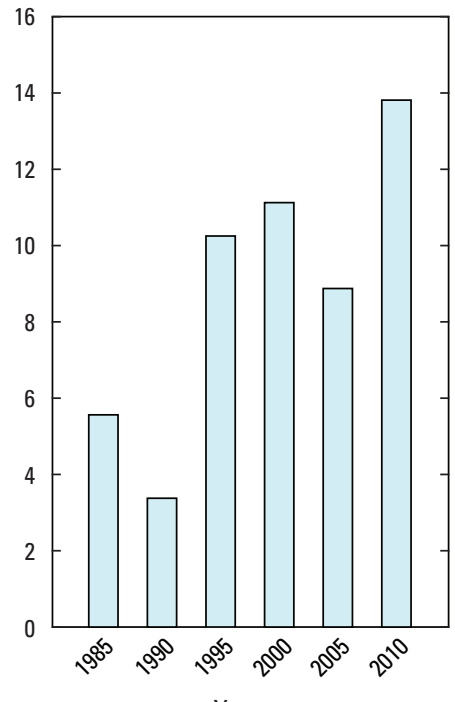




\section{THOMAS COUNTY}

Population

Population served by public supply-Groundwater

Population served by public supply-Surface water Acres irrigated
44,720

30,940

$$
0
$$

12,410

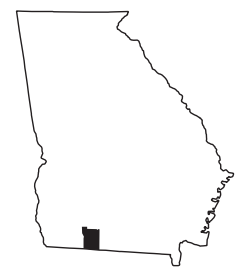

2010 WATER WITHDRAWALS AND ESTIMATED USE, IN MILLION GALLONS PER DAY [-, not applicable]

\begin{tabular}{|c|c|c|c|c|c|c|c|c|}
\hline \multirow{3}{*}{ Category } & \multicolumn{3}{|c|}{ Withdrawals } & \multirow{3}{*}{$\begin{array}{l}\text { Total } \\
\text { use }^{1}\end{array}$} & \multirow{3}{*}{$\begin{array}{l}\text { Surface- } \\
\text { water } \\
\text { returns } \\
\end{array}$} & \multirow{2}{*}{\multicolumn{3}{|c|}{$\begin{array}{l}\text { Withdrawals by Major Industrial Groups } \\
\text { [NAICS, North American Industrial Classification System code] }\end{array}$}} \\
\hline & \multirow{2}{*}{$\begin{array}{c}\text { Ground- } \\
\text { water }\end{array}$} & \multirow{2}{*}{$\begin{array}{c}\text { Surface } \\
\text { water }\end{array}$} & \multirow[b]{2}{*}{ Total } & & & & & \\
\hline & & & & & & NAICS & Groundwater & Surface water \\
\hline Public supply & 5.49 & 0.00 & 5.49 & - & - & & 0.14 & 0.00 \\
\hline $\begin{array}{l}\text { Domestic } \\
\text { Commercial/public use }\end{array}$ & $\begin{array}{l}1.03 \\
0.03\end{array}$ & $\begin{array}{l}0.00 \\
0.00\end{array}$ & $\begin{array}{l}1.03 \\
0.03\end{array}$ & $\begin{array}{l}3.49 \\
1.24\end{array}$ & $\begin{array}{l}0.00 \\
0.00\end{array}$ & $\begin{array}{l}327 \text {-Stone, clay } \\
311 \text {-Food }\end{array}$ & 0.05 & 0.00 \\
\hline Industrial & 0.19 & 0.00 & 0.19 & 0.55 & 0.00 & Withdrawals by M & r Public Supp & \\
\hline $\begin{array}{l}\text { Public-supply losses } \\
\text { Public wastewater treatment }\end{array}$ & - & - & - & $\begin{array}{l}1.46 \\
-\end{array}$ & $\begin{array}{c}- \\
4.03\end{array}$ & Name & Groundwater & Surface water \\
\hline Mining & 1.02 & 0.01 & 1.03 & 1.03 & 0.03 & City of Thomasville & 4.75 & 0.00 \\
\hline Irrigation-Crop & 2.66 & 0.10 & 2.76 & 2.76 & 0.00 & City of Boston & 0.24 & 0.00 \\
\hline Irrigation-Golf course & 0.25 & 0.00 & 0.25 & 0.25 & 0.00 & City of Meigs & 0.15 & 0.00 \\
\hline Livestock/aquaculture & 0.16 & 0.13 & 0.29 & 0.29 & 0.00 & City of Coolidge & 0.05 & 0.00 \\
\hline Thermoelectric power & 0.00 & 0.00 & 0.00 & 0.00 & 0.00 & $\begin{array}{l}\text { City of Coolidge } \\
\text { City of Ochlocknee }\end{array}$ & 0.05 & 0.00 \\
\hline TOTAL & 10.83 & 0.24 & 11.07 & 11.07 & 4.06 & $\begin{array}{l}\text { City of Ochlocknee } \\
\text { City of Pavo }\end{array}$ & $\begin{array}{l}0.05 \\
0.05\end{array}$ & $\begin{array}{l}0.00 \\
0.00\end{array}$ \\
\hline
\end{tabular}

\section{Withdrawals by Source}

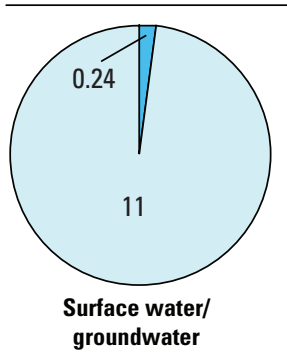

Withdrawals, in million gallons per day

Surface water

Groundwater

$\square$ Upper Floridan aquifer groundwater

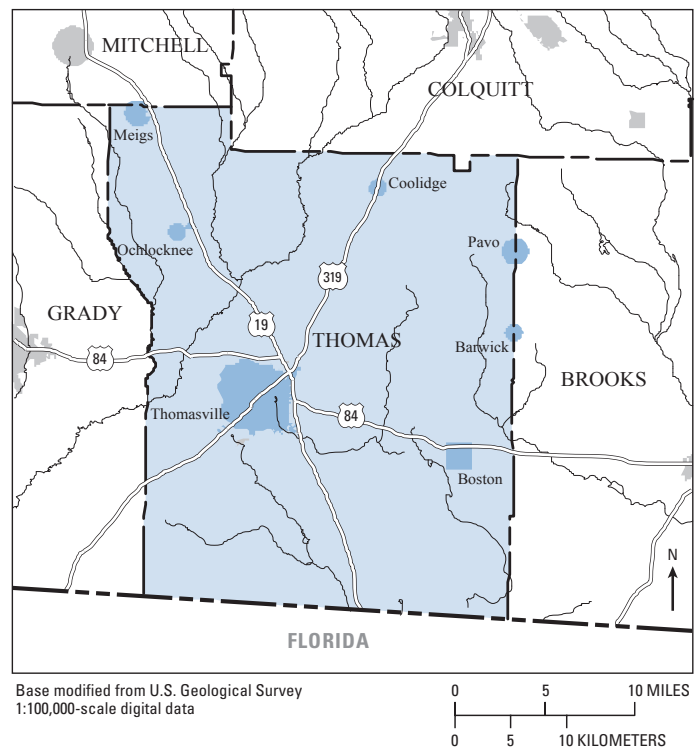

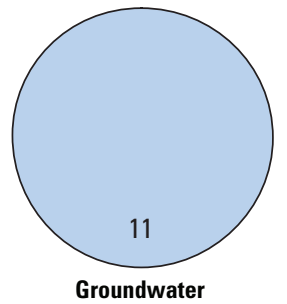

Public-Supply Deliveries by Use Category

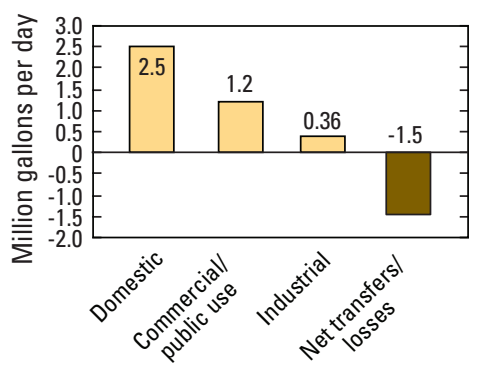

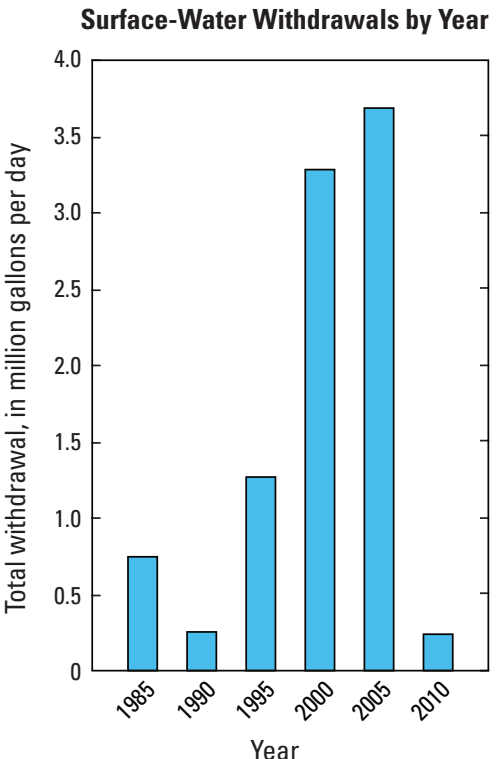

Groundwater Withdrawals by Year

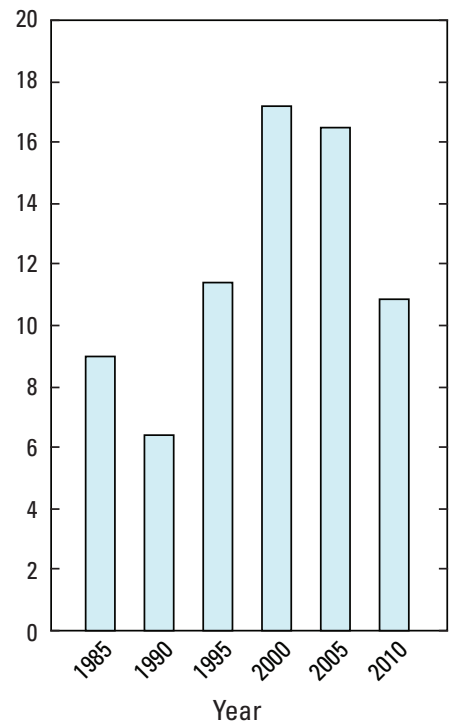




\section{TIFT COUNTY}

Population

Population served by public supply-Groundwater

Population served by public supply-Surface water Acres irrigated
40,118

28,610

20,950

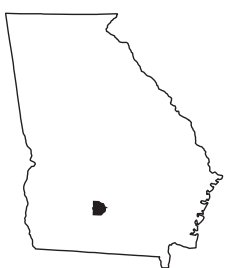

2010 WATER WITHDRAWALS AND ESTIMATED USE, IN MILLION GALLONS PER DAY

$[-$, not applicable $]$

\begin{tabular}{|c|c|c|c|c|c|c|c|c|}
\hline \multirow{3}{*}{ Category } & \multicolumn{3}{|c|}{ Withdrawals } & \multirow{3}{*}{$\begin{array}{l}\text { Total } \\
\text { use }^{1}\end{array}$} & \multirow{3}{*}{$\begin{array}{l}\text { Surface- } \\
\text { water } \\
\text { returns } \\
\end{array}$} & \multirow{2}{*}{\multicolumn{3}{|c|}{$\begin{array}{l}\text { Withdrawals by Major Industrial Groups } \\
\text { [NAICS, North American Industrial Classification System code] }\end{array}$}} \\
\hline & \multirow{2}{*}{$\begin{array}{l}\text { Ground- } \\
\text { water }\end{array}$} & \multirow{2}{*}{$\begin{array}{c}\text { Surface } \\
\text { water }\end{array}$} & \multirow[b]{2}{*}{ Total } & & & & & \\
\hline & & & & & & NAICS & Groundwater & Surface water \\
\hline Public supply & 5.62 & 0.00 & 5.62 & \multirow{2}{*}{$\begin{array}{c}- \\
4.63\end{array}$} & \multirow{2}{*}{0.00} & \multirow{2}{*}{ None } & \multirow{2}{*}{-} & \multirow{2}{*}{-} \\
\hline Domestic & 0.86 & 0.00 & 0.86 & & & & & \\
\hline Commercial/public use & 0.17 & 0.00 & 0.17 & 1.18 & 0.00 & \multirow{4}{*}{\multicolumn{3}{|c|}{ Withdrawals by Major Public Suppliers }} \\
\hline Industrial & 0.00 & 0.00 & 0.00 & 0.05 & 0.00 & & & \\
\hline Public-supply losses & - & - & - & 0.79 & - & & & \\
\hline Public wastewater treatment & - & \multirow{2}{*}{0.00} & - & - & \multirow{2}{*}{$\begin{array}{l}4.80 \\
0.00\end{array}$} & & & \\
\hline \multirow{2}{*}{$\begin{array}{l}\text { Mining } \\
\text { Irrigation-Crop }\end{array}$} & 0.00 & & 0.00 & 0.00 & & \multirow{2}{*}{ Name } & \multirow{2}{*}{ Groundwater } & \multirow{2}{*}{ Surface water } \\
\hline & 5.03 & 3.49 & 8.52 & 8.52 & 0.00 & & & \\
\hline Irrigation-Golf course & 0.52 & 0.00 & 0.52 & 0.52 & 0.00 & \multirow{2}{*}{$\begin{array}{l}\text { Tifton-Tift County } \\
\text { Water System }\end{array}$} & \multirow{2}{*}{5.38} & \multirow{2}{*}{0.00} \\
\hline Livestock/aquaculture & 0.03 & 0.09 & 0.12 & 0.12 & 0.00 & & & \\
\hline Thermoelectric power & 0.00 & 0.00 & 0.00 & 0.00 & 0.00 & City of Omega & 0.13 & 0.00 \\
\hline TOTAL & 12.23 & 3.58 & 15.81 & 15.81 & 4.80 & & & \\
\hline
\end{tabular}

${ }^{1}$ Total use is total withdrawal plus public supply deliveries and losses.

\section{Withdrawals by Source}
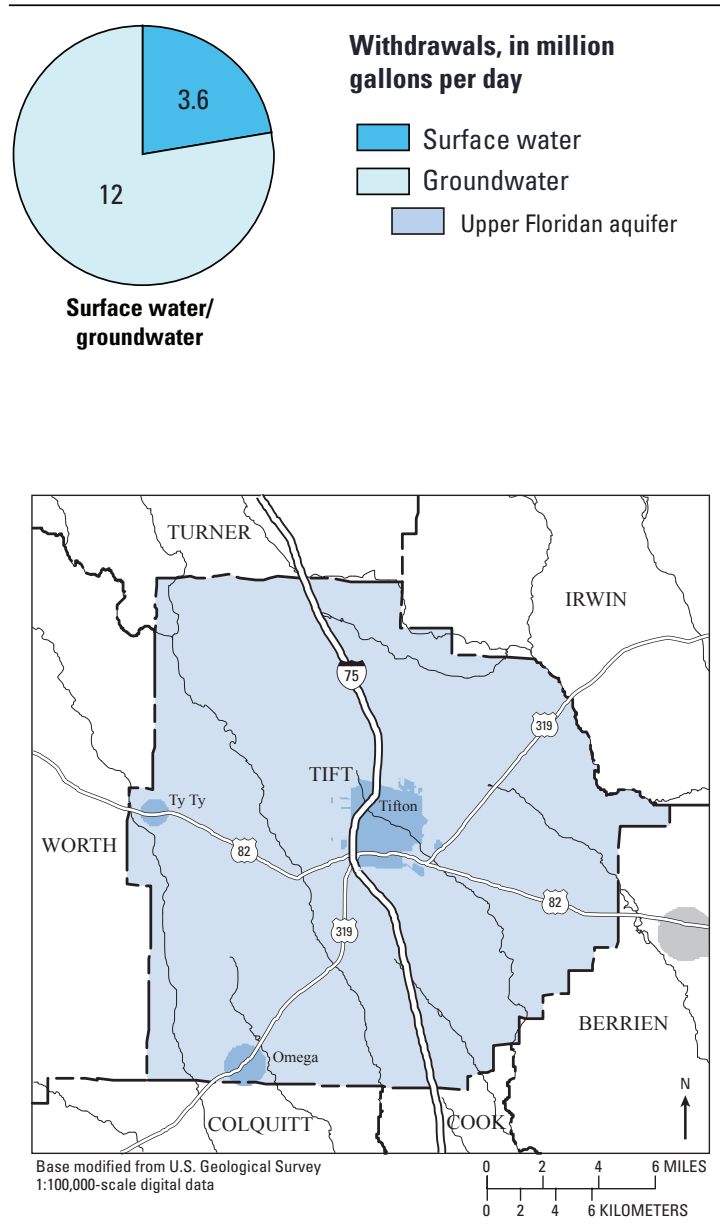

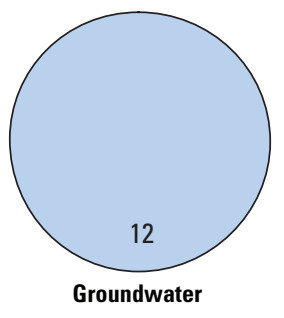

Public-Supply Deliveries by Use Category

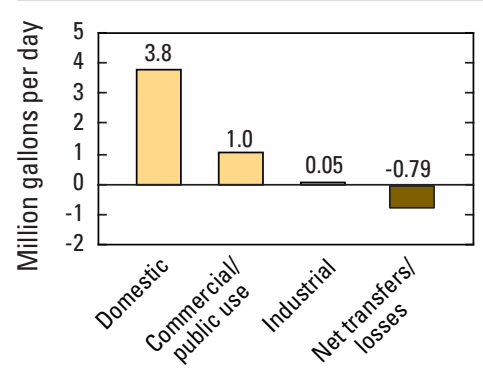

Surface-Water Withdrawals by Year

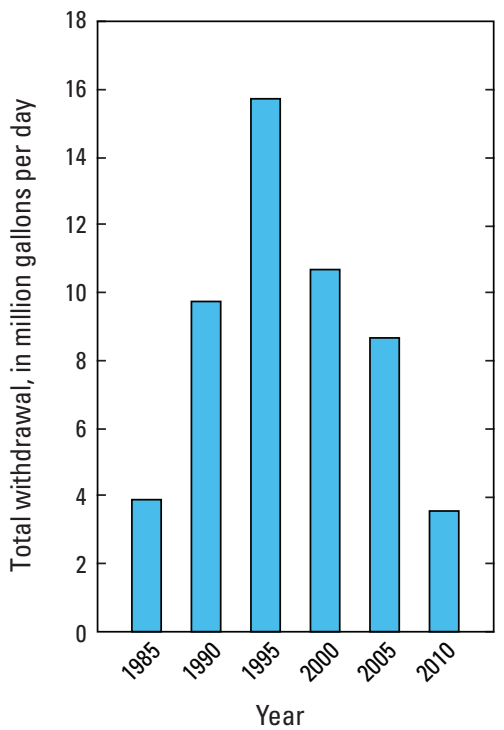

Groundwater Withdrawals by Year

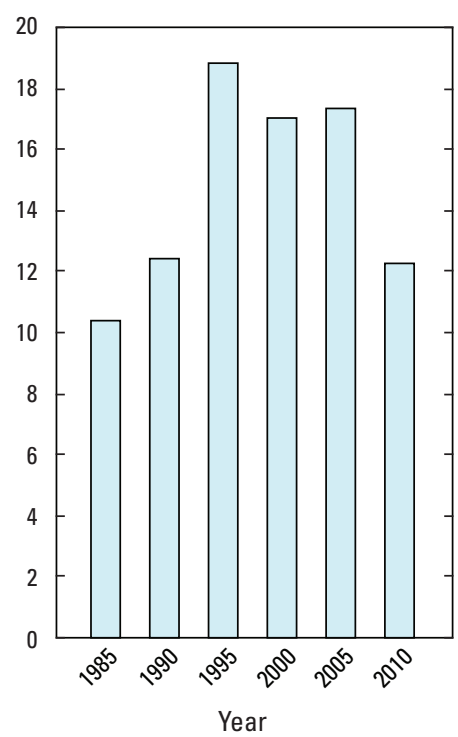




\section{TOOMBS COUNTY}

Population

Population served by public supply-Groundwater

Population served by public supply-Surface water Acres irrigated
27,223

18,720

0

11,440

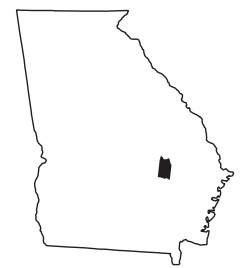

2010 WATER WITHDRAWALS AND ESTIMATED USE, IN MILLION GALLONS PER DAY

$[-$, not applicable $]$

\begin{tabular}{|c|c|c|c|c|c|c|c|c|}
\hline \multirow{3}{*}{ Category } & \multicolumn{3}{|c|}{ Withdrawals } & \multirow{3}{*}{$\begin{array}{l}\text { Total } \\
\text { use }^{1}\end{array}$} & \multirow{3}{*}{$\begin{array}{l}\text { Surface- } \\
\text { water } \\
\text { returns } \\
\end{array}$} & \multirow{2}{*}{\multicolumn{3}{|c|}{$\begin{array}{l}\text { Withdrawals by Major Industrial Groups } \\
\text { [NAICS, North American Industrial Classification System code] }\end{array}$}} \\
\hline & \multirow{2}{*}{$\begin{array}{c}\text { Ground- } \\
\text { water }\end{array}$} & \multirow{2}{*}{$\begin{array}{c}\text { Surface } \\
\text { water }\end{array}$} & \multirow[b]{2}{*}{ Total } & & & & & \\
\hline & & & & & & NAICS & Groundwater & Surface water \\
\hline \multirow{2}{*}{$\begin{array}{l}\text { Public supply } \\
\text { Domestic }\end{array}$} & 2.92 & 0.00 & 2.92 & \multirow{2}{*}{$\begin{array}{l}- \\
2.09\end{array}$} & \multirow{2}{*}{-} & \multirow{2}{*}{ None } & \multirow{2}{*}{-} & \multirow[t]{2}{*}{-} \\
\hline & 0.64 & 0.00 & 0.64 & & & & & \\
\hline Commercial/public use & 0.00 & 0.00 & 0.00 & 0.52 & 0.00 & \multirow{4}{*}{\multicolumn{3}{|c|}{ Withdrawals by Major Public Suppliers }} \\
\hline Industrial & 0.00 & 0.00 & 0.00 & 0.44 & 0.00 & & & \\
\hline Public-supply losses & - & - & - & 0.51 & - & & & \\
\hline \multirow{2}{*}{$\begin{array}{l}\text { Public wastewater treatment } \\
\text { Mining }\end{array}$} & - & \multirow{2}{*}{0.00} & \multirow{2}{*}{$\begin{array}{c}- \\
0.06\end{array}$} & \multirow{2}{*}{$\begin{array}{c}- \\
0.06\end{array}$} & \multirow{2}{*}{$\begin{array}{l}1.40 \\
0.00\end{array}$} & & & \\
\hline & 0.06 & & & & & Name & Groundwater & Surface water \\
\hline Irrigation-Golf course & 0.05 & 0.09 & 0.14 & 0.14 & 0.00 & City of Vidalia & 1.75 & 0.00 \\
\hline Livestock/aquaculture & 0.02 & 0.12 & 0.14 & 0.14 & 0.00 & City of Lyons & 1.08 & 0.00 \\
\hline Thermoelectric power & 0.00 & 0.00 & 0.00 & 0.00 & 0.00 & & & \\
\hline
\end{tabular}

$6.73 \quad 3.97$

10.70

10.70

1.40

'Total use is total withdrawal plus public supply deliveries and losses.

\section{Withdrawals by Source}
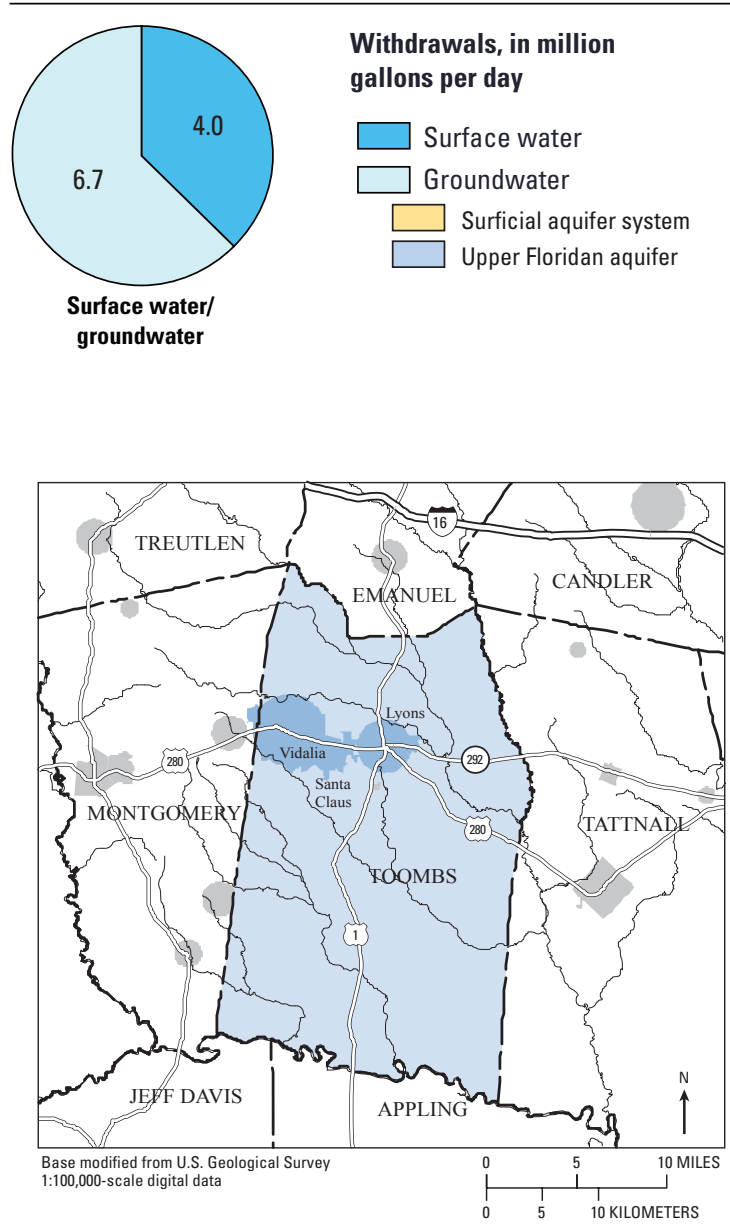

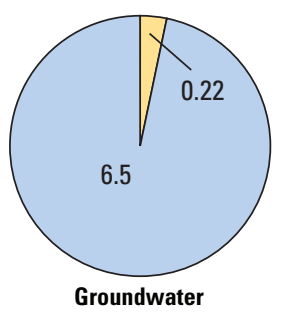

Public-Supply Deliveries by Use Category

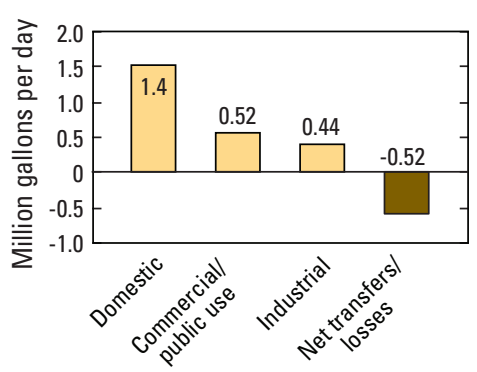

Surface-Water Withdrawals by Year

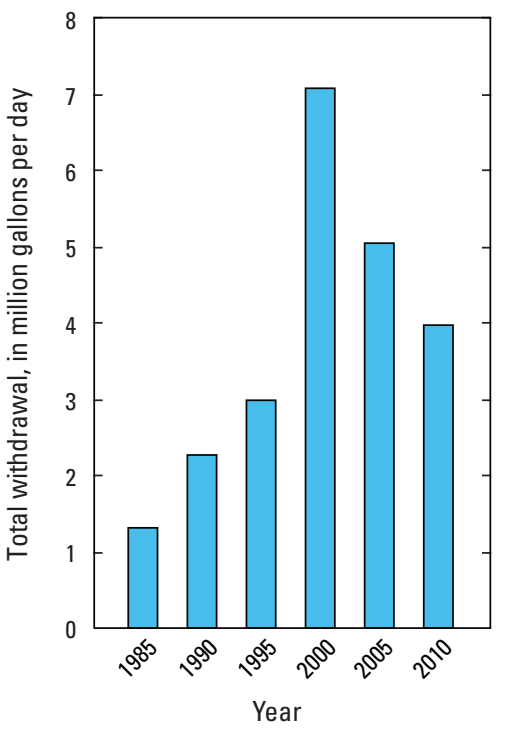

Groundwater Withdrawals by Year

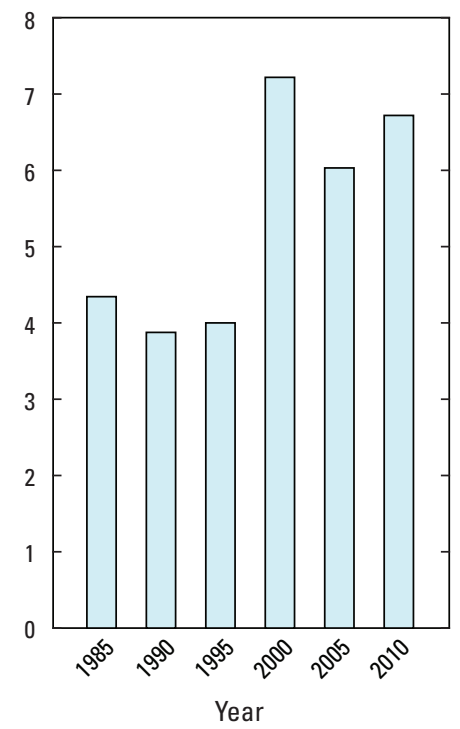




\section{TOWNS COUNTY}

Population

10,471

Population served by public supply-Groundwater

Population served by public supply-Surface water Acres irrigated
2,310

7,440

100

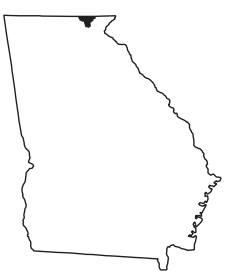

2010 WATER WITHDRAWALS AND ESTIMATED USE, IN MILLION GALLONS PER DAY

$[-$, not applicable $]$

\begin{tabular}{|c|c|c|c|c|c|c|c|c|}
\hline \multirow{3}{*}{ Category } & \multicolumn{3}{|c|}{ Withdrawals } & \multirow{3}{*}{$\begin{array}{l}\text { Total } \\
\text { use }\end{array}$} & \multirow{3}{*}{$\begin{array}{l}\text { Surface- } \\
\text { water } \\
\text { returns }\end{array}$} & \multirow{2}{*}{\multicolumn{3}{|c|}{$\begin{array}{l}\text { Withdrawals by Major Industrial Groups } \\
\text { [NAICS, North American Industrial Classification System code] }\end{array}$}} \\
\hline & \multirow{2}{*}{$\begin{array}{c}\text { Ground- } \\
\text { water }\end{array}$} & \multirow{2}{*}{$\begin{array}{c}\text { Surface } \\
\text { water }\end{array}$} & \multirow[b]{2}{*}{ Total } & & & & & \\
\hline & & & & & & NAICS & Groundwater & Surface water \\
\hline Public supply & 0.18 & 1.19 & 1.37 & - & - & None & - & - \\
\hline Domestic & 0.05 & 0.00 & 0.05 & 1.05 & 0.00 & None & - & \\
\hline Commercial/public use & 0.00 & 0.00 & 0.00 & 0.14 & 0.00 & & & \\
\hline Industrial & 0.00 & 0.00 & 0.00 & 0.01 & 0.00 & & & \\
\hline Public-supply losses & - & - & - & 0.22 & - & & & \\
\hline Public wastewater treatment & - & - & - & - & 0.29 & Withdrawals by Ma & r Public Supp & \\
\hline Mining & 0.01 & 0.00 & 0.01 & $\begin{array}{l}0.01 \\
0.02\end{array}$ & 0.00 & Name & Groundwater & Surface water \\
\hline Irrigation-Crop & 0.02 & 0.00 & $\begin{array}{l}0.02 \\
0.15\end{array}$ & $\begin{array}{l}0.02 \\
0.15\end{array}$ & $\begin{array}{l}0.00 \\
0.00\end{array}$ & & & \\
\hline Irrigation-Golf course & 0.00 & 0.15 & 0.15 & 0.15 & $\begin{array}{l}0.00 \\
0.00\end{array}$ & City of Hiawassee & 0.00 & 1.19 \\
\hline Livestock/aquaculture & 0.00 & 2.88 & 2.88 & 2.88 & 0.00 & City of Young Harris & 0.16 & 0.00 \\
\hline
\end{tabular}

$0.26 \quad 4.22$

4.48

4.48

0.29

'Total use is total withdrawal plus public supply deliveries and losses

\section{Withdrawals by Source}

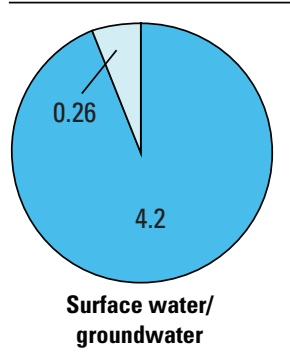

Withdrawals, in million gallons per day

Surface water

Groundwater

Crystalline-rock aquifer

Surface water/

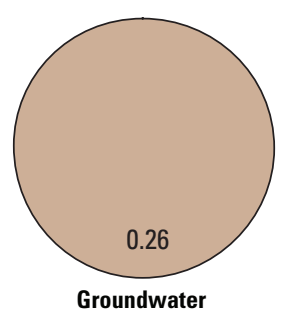

Public-Supply Deliveries by Use Category

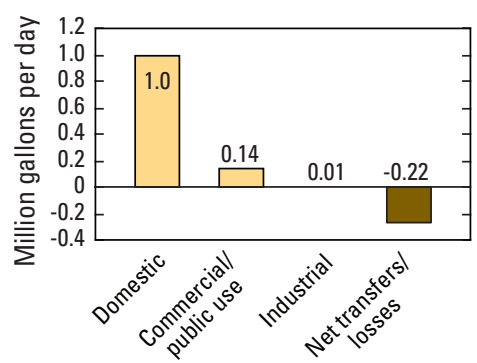

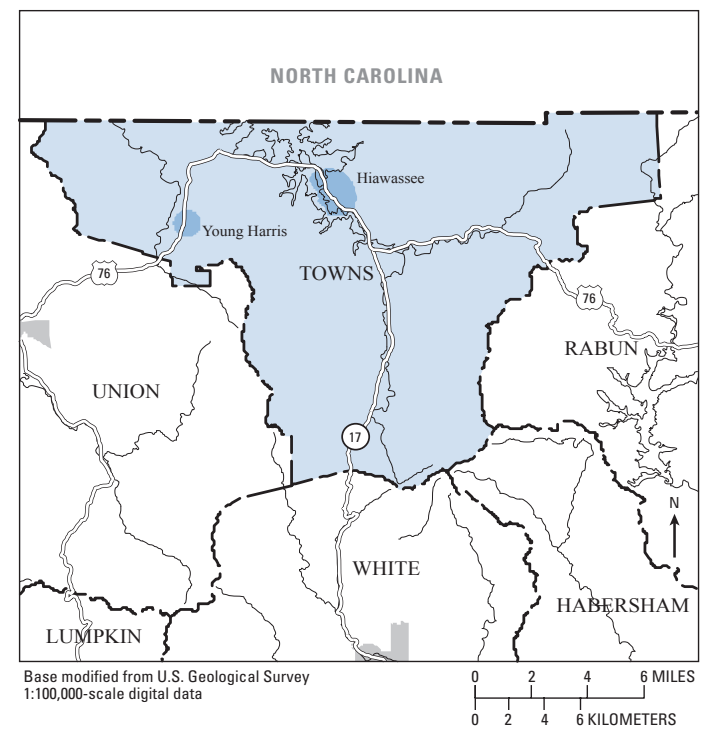

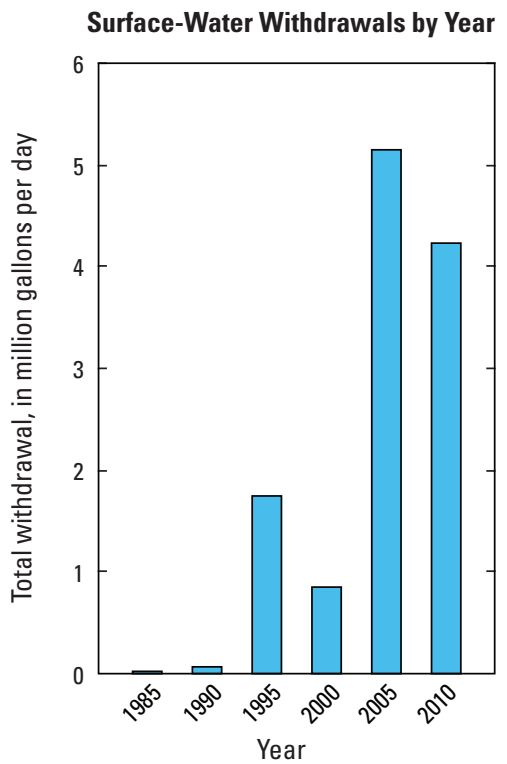

Groundwater Withdrawals by Year

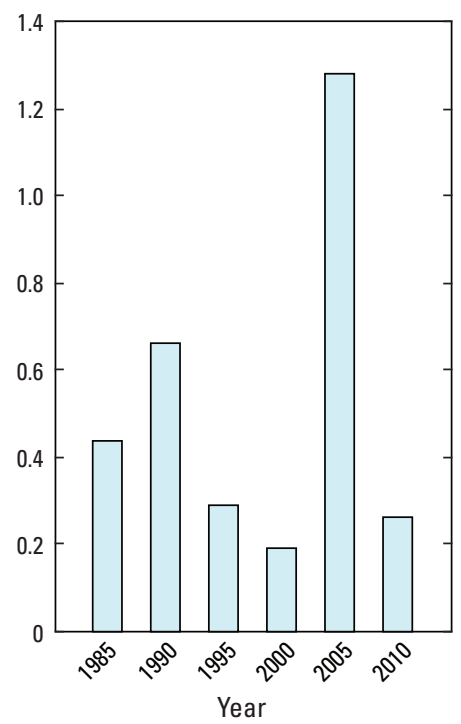




\section{TREUTLEN COUNTY}

Population

6,885

Population served by public supply-Groundwater

Population served by public supply-Surface water Acres irrigated
2,480

0

1,140

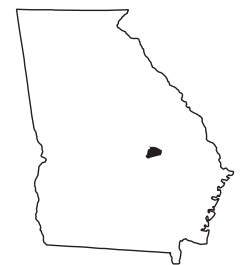

2010 WATER WITHDRAWALS AND ESTIMATED USE, IN MILLION GALLONS PER DAY

$[-$, not applicable; Mgal/d, million gallons per day $]$

\begin{tabular}{|c|c|c|c|c|c|}
\hline \multirow[b]{2}{*}{ Category } & \multicolumn{3}{|c|}{ Withdrawals } & \multirow[b]{2}{*}{$\begin{array}{l}\text { Total } \\
\text { use }^{1}\end{array}$} & \multirow{2}{*}{$\begin{array}{c}\text { Surface- } \\
\text { water } \\
\text { returns }\end{array}$} \\
\hline & $\begin{array}{c}\text { Ground- } \\
\text { water }\end{array}$ & $\begin{array}{c}\text { Surface } \\
\text { water }\end{array}$ & Total & & \\
\hline Public supply & 0.45 & 0.00 & 0.45 & - & - \\
\hline Domestic & 0.33 & 0.00 & 0.33 & 0.59 & 0.00 \\
\hline Commercial/public use & 0.00 & 0.00 & 0.00 & 0.07 & 0.00 \\
\hline Industrial & 0.19 & 0.00 & 0.19 & 0.19 & 0.00 \\
\hline Public-supply losses & - & - & - & 0.07 & - \\
\hline Public wastewater treatment & - & - & - & - & 0.43 \\
\hline Mining & 0.00 & 0.00 & 0.00 & 0.00 & 0.00 \\
\hline Irrigation-Crop & 0.50 & 0.53 & 1.03 & 1.03 & 0.00 \\
\hline Irrigation-Golf course & 0.00 & 0.00 & 0.00 & 0.00 & 0.00 \\
\hline Livestock/aquaculture & 0.01 & 0.01 & 0.02 & 0.03 & 0.00 \\
\hline Thermoelectric power & 0.00 & 0.00 & 0.00 & 0.00 & 0.00 \\
\hline TOTAL & 1.48 & 0.54 & 2.02 & 1.98 & 0.43 \\
\hline
\end{tabular}

Withdrawals by Major Industrial Groups

[NAICS, North American Industrial Classification System code] NAICS Groundwater Surface water

$\begin{array}{lll}314-\text { Textiles } & 0.19 & 0.00\end{array}$

Withdrawals by Major Public Suppliers

Name Groundwater Surface water

City of Soperton $\quad 0.45 \quad 0.00$

${ }^{1}$ Total use is total withdrawal plus public supply deliveries and losses. An average of $0.04 \mathrm{Mgal} / \mathrm{d}$ was delivered to Montgomery County, Georgia in 2010

\section{Withdrawals by Source}
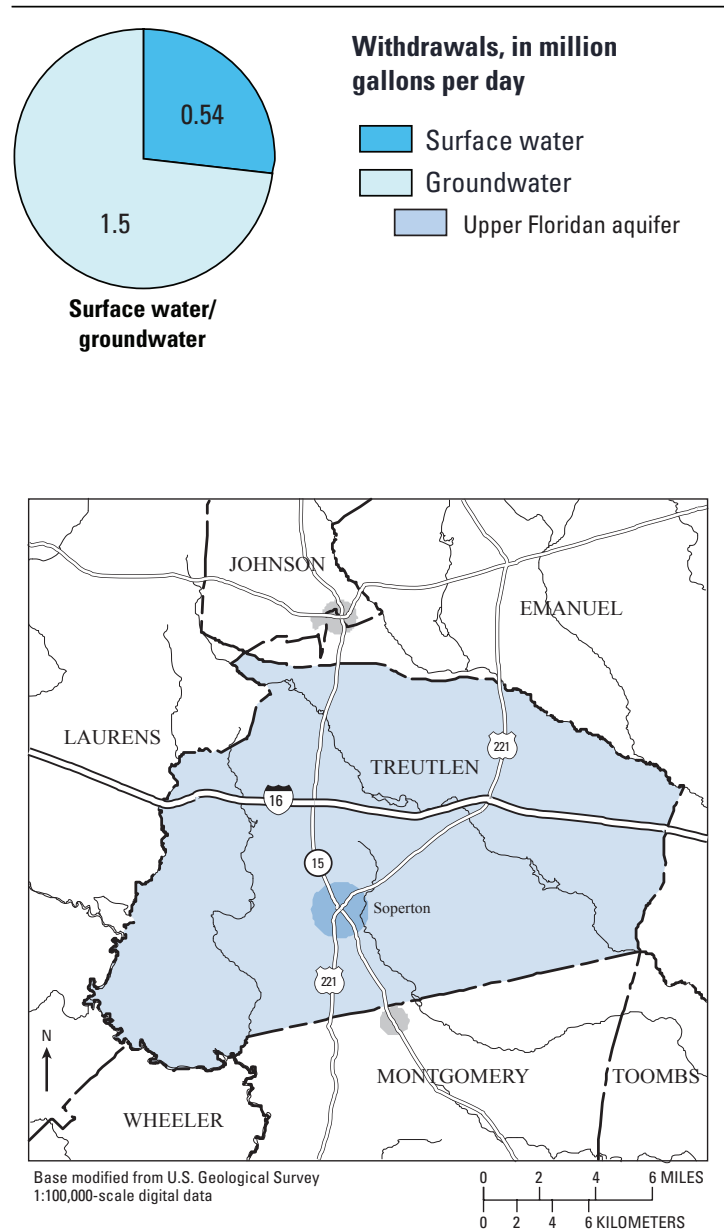

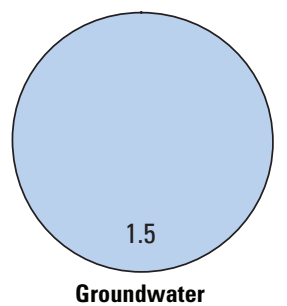

Public-Supply Deliveries' by Use Category

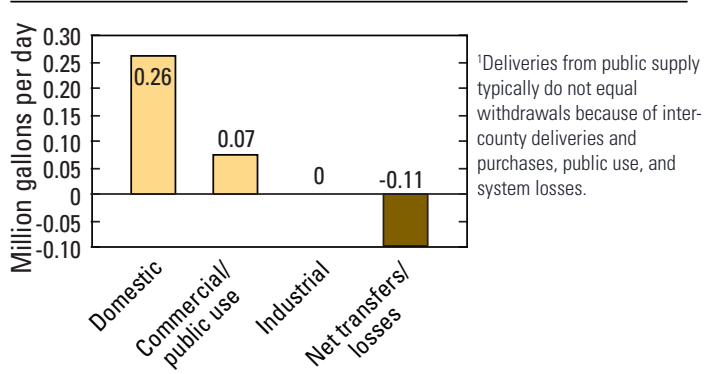

Surface-Water Withdrawals by Year

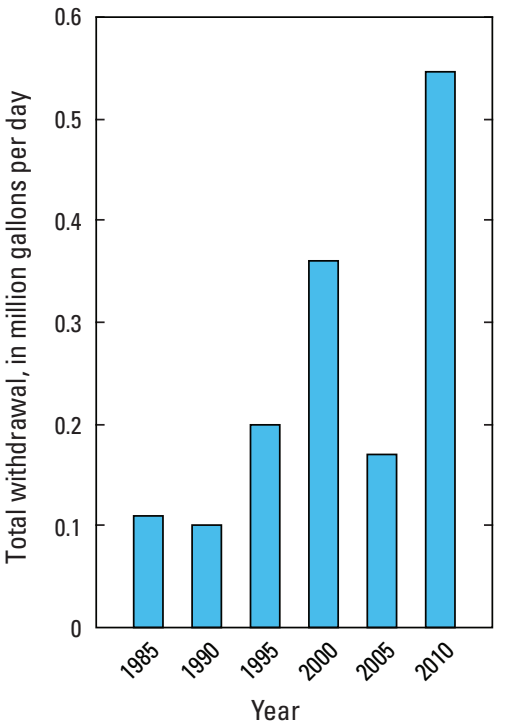

Groundwater Withdrawals by Year

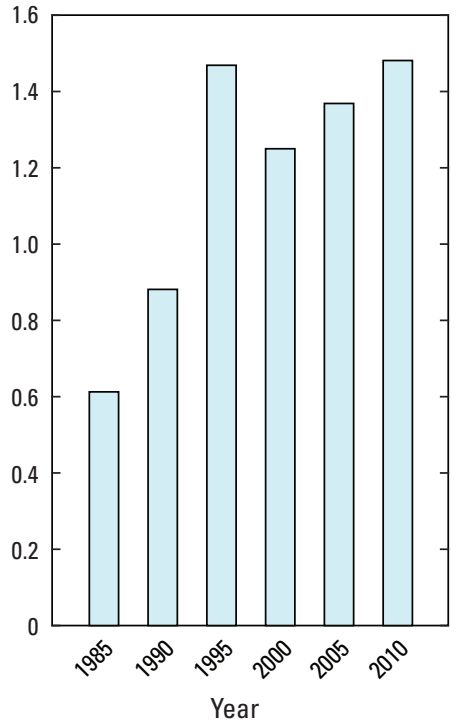




\section{TROUP COUNTY}

Population

Population served by public supply-Groundwater

Population served by public supply-Surface water Acres irrigated
67,044

52,560

85

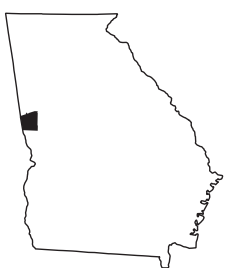

2010 WATER WITHDRAWALS AND ESTIMATED USE, IN MILLION GALLONS PER DAY

$[-$, not applicable; Mgal/d, million gallons per day $]$

\begin{tabular}{|c|c|c|c|c|c|c|c|c|}
\hline \multirow{3}{*}{ Category } & \multicolumn{3}{|c|}{ Withdrawals } & \multirow{3}{*}{$\begin{array}{l}\text { Total } \\
\text { use }^{1}\end{array}$} & \multirow{3}{*}{$\begin{array}{l}\text { Surface- } \\
\text { water } \\
\text { returns } \\
\end{array}$} & \multirow{2}{*}{\multicolumn{3}{|c|}{$\begin{array}{l}\text { Withdrawals by Major Industrial Groups } \\
\text { [NAICS, North American Industrial Classification System code] }\end{array}$}} \\
\hline & \multirow{2}{*}{$\begin{array}{c}\text { Ground- } \\
\text { water }\end{array}$} & \multirow{2}{*}{$\begin{array}{c}\text { Surface } \\
\text { water }\end{array}$} & \multirow[b]{2}{*}{ Total } & & & & & \\
\hline & & & & & & NAICS & Groundwater & Surface water \\
\hline Public supply & 0.00 & 10.52 & 10.52 & - & - & & & \\
\hline Domestic & 1.08 & 0.00 & 1.08 & 5.41 & 0.00 & 314-Textiles & 0.02 & 0.00 \\
\hline Commercial/public use & 0.01 & 0.00 & 0.01 & 2.48 & 0.00 & & & \\
\hline Industrial & 0.02 & 0.00 & 0.02 & 2.17 & 0.00 & & & \\
\hline Public-supply losses & - & - & - & 1.57 & - & & & \\
\hline Public wastewater treatment & - & - & - & - & 5.87 & Withdrawals by I & r Public Supp & \\
\hline Mining & 0.11 & 0.00 & 0.11 & 0.11 & 0.00 & Name & Groundwater & Surface water \\
\hline Irrigation-Crop & 0.01 & 0.00 & 0.01 & 0.01 & 0.00 & Naine & & \\
\hline Irrigation-Golf course & 0.00 & 0.12 & 0.12 & 0.12 & 0.00 & City of Lagrange & 0.00 & 9.58 \\
\hline Livestock/aquaculture & 0.00 & 0.10 & 0.10 & 0.10 & 0.00 & City of West Point & 0.00 & 0.94 \\
\hline Thermoelectric power & 0.00 & 0.00 & 0.00 & 0.00 & 0.00 & & & \\
\hline
\end{tabular}

$1.23 \quad 10.74$

11.97

11.97

5.87

'Total use is total withdrawal plus public supply deliveries and losses. An average of $0.25 \mathrm{Mgal} / \mathrm{d}$ was delivered from Coweta County, Georgi to Troup County; $0.25 \mathrm{Mgal} / \mathrm{d}$ was delivered from Troup County to Meriwether County, Georgia in 2010.

\section{Withdrawals by Source}

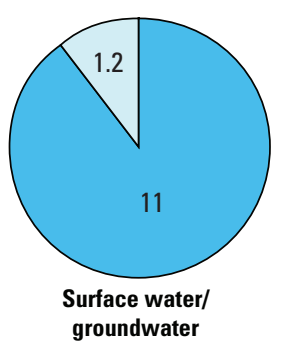

Withdrawals, in million gallons per day

Surface water
Groundwater
$\square$ Crystalline-rock aquifer

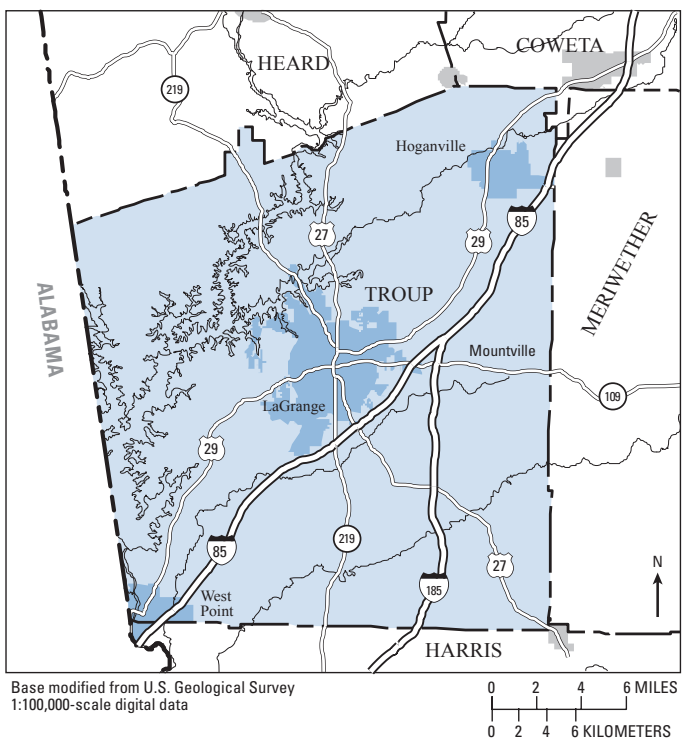

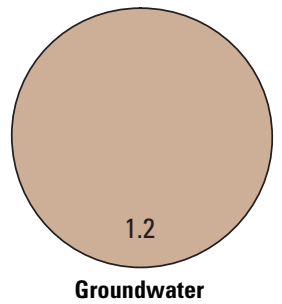

Public-Supply Deliveries by Use Category

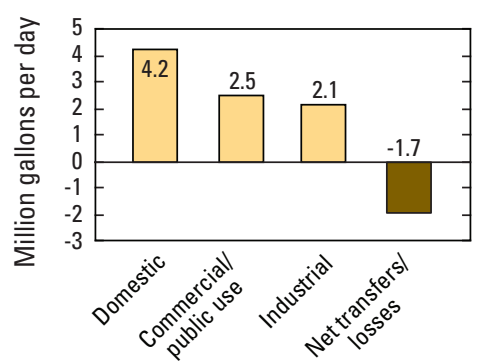

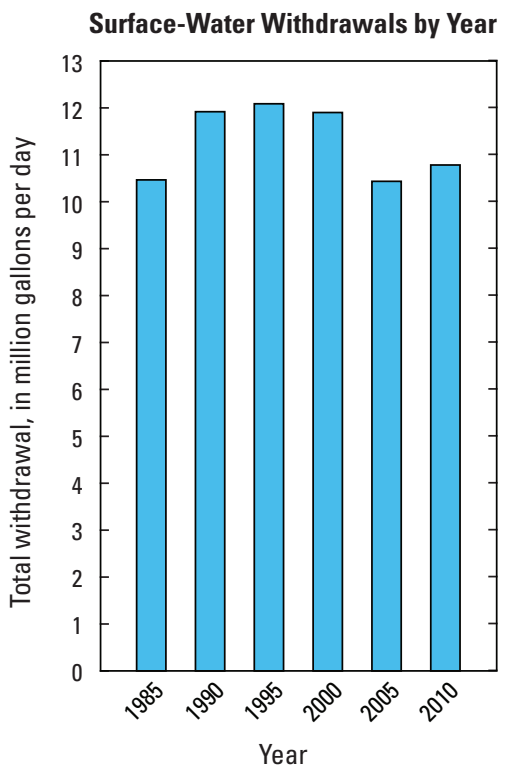

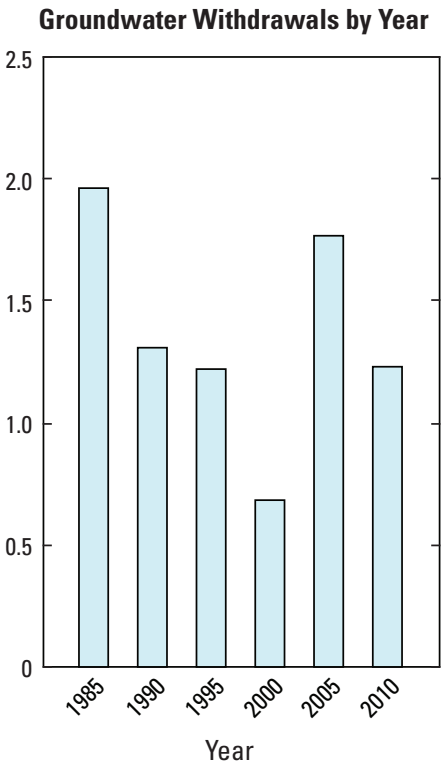




\section{TURNER COUNTY}

Population

8,930

Population served by public supply-Groundwater $\quad 5,670$

Population served by public supply-Surface water 0

Acres irrigated

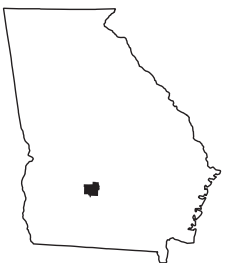

2010 WATER WITHDRAWALS AND ESTIMATED USE, IN MILLION GALLONS PER DAY

$[-$, not applicable $]$

\begin{tabular}{|c|c|c|c|c|c|c|c|c|}
\hline \multirow{3}{*}{ Category } & \multicolumn{3}{|c|}{ Withdrawals } & \multirow{3}{*}{$\begin{array}{l}\text { Total } \\
\text { use }^{1}\end{array}$} & \multirow{3}{*}{$\begin{array}{l}\text { Surface- } \\
\text { water } \\
\text { returns } \\
\end{array}$} & \multirow{2}{*}{\multicolumn{3}{|c|}{$\begin{array}{l}\text { Withdrawals by Major Industrial Groups } \\
\text { [NAICS, North American Industrial Classification System code] }\end{array}$}} \\
\hline & \multirow{2}{*}{$\begin{array}{c}\text { Ground- } \\
\text { water }\end{array}$} & \multirow{2}{*}{$\begin{array}{c}\text { Surface } \\
\text { water }\end{array}$} & \multirow[b]{2}{*}{ Total } & & & & & \\
\hline & & & & & & NAICS & Groundwater & Surface water \\
\hline \multirow{2}{*}{$\begin{array}{l}\text { Public supply } \\
\text { Domestic }\end{array}$} & 0.70 & 0.00 & 0.70 & \multirow{2}{*}{$\begin{array}{l}- \\
0.68\end{array}$} & \multirow{2}{*}{$\begin{array}{c}- \\
0.00\end{array}$} & \multirow{2}{*}{ None } & \multirow{2}{*}{-} & \multirow{2}{*}{-} \\
\hline & 0.24 & 0.00 & 0.24 & & & & & \\
\hline Commercial/public use & 0.02 & 0.00 & 0.02 & 0.14 & 0.00 & & & \\
\hline Industrial & 0.00 & 0.00 & 0.00 & 0.02 & 0.00 & & & \\
\hline Public-supply losses & - & - & - & 0.12 & - & & & \\
\hline \multirow{2}{*}{$\begin{array}{l}\text { Public wastewater treatment } \\
\text { Mining }\end{array}$} & \multirow{2}{*}{$\begin{array}{c}- \\
0.00\end{array}$} & \multirow{2}{*}{$\begin{array}{c}- \\
0.00\end{array}$} & \multirow{2}{*}{$\begin{array}{c}- \\
0.00\end{array}$} & \multirow{2}{*}{$\begin{array}{l}- \\
0.00\end{array}$} & 1.03 & \multicolumn{3}{|c|}{ Withdrawals by Major Public Suppliers } \\
\hline & & & & & $\begin{array}{l}0.00 \\
0.00\end{array}$ & Name & Groundwater & Surface water \\
\hline $\begin{array}{l}\text { Irrigation-Crop } \\
\text { Irrigation-Golf course }\end{array}$ & $\begin{array}{l}4.72 \\
0.00\end{array}$ & $\begin{array}{l}3.38 \\
0.05\end{array}$ & $\begin{array}{l}8.10 \\
0.05\end{array}$ & $\begin{array}{l}8.10 \\
0.05\end{array}$ & 0.00 & City of Ashburn & 0.57 & 0.00 \\
\hline Livestock/aquaculture & 0.06 & 0.44 & 0.50 & 0.50 & 0.00 & City of Sycamore & 0.12 & 0.00 \\
\hline Thermoelectric power & 0.00 & 0.00 & 0.00 & 0.00 & 0.00 & & & \\
\hline
\end{tabular}

$\mathbf{5 . 7 4} \quad \mathbf{3 . 8}$

'Total use is total withdrawal plus public supply deliveries and losses.

\section{Withdrawals by Source}
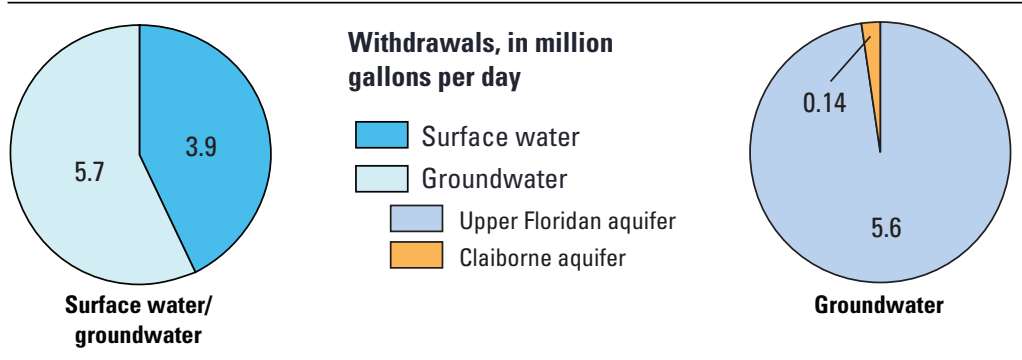

Public-Supply Deliveries by Use Category
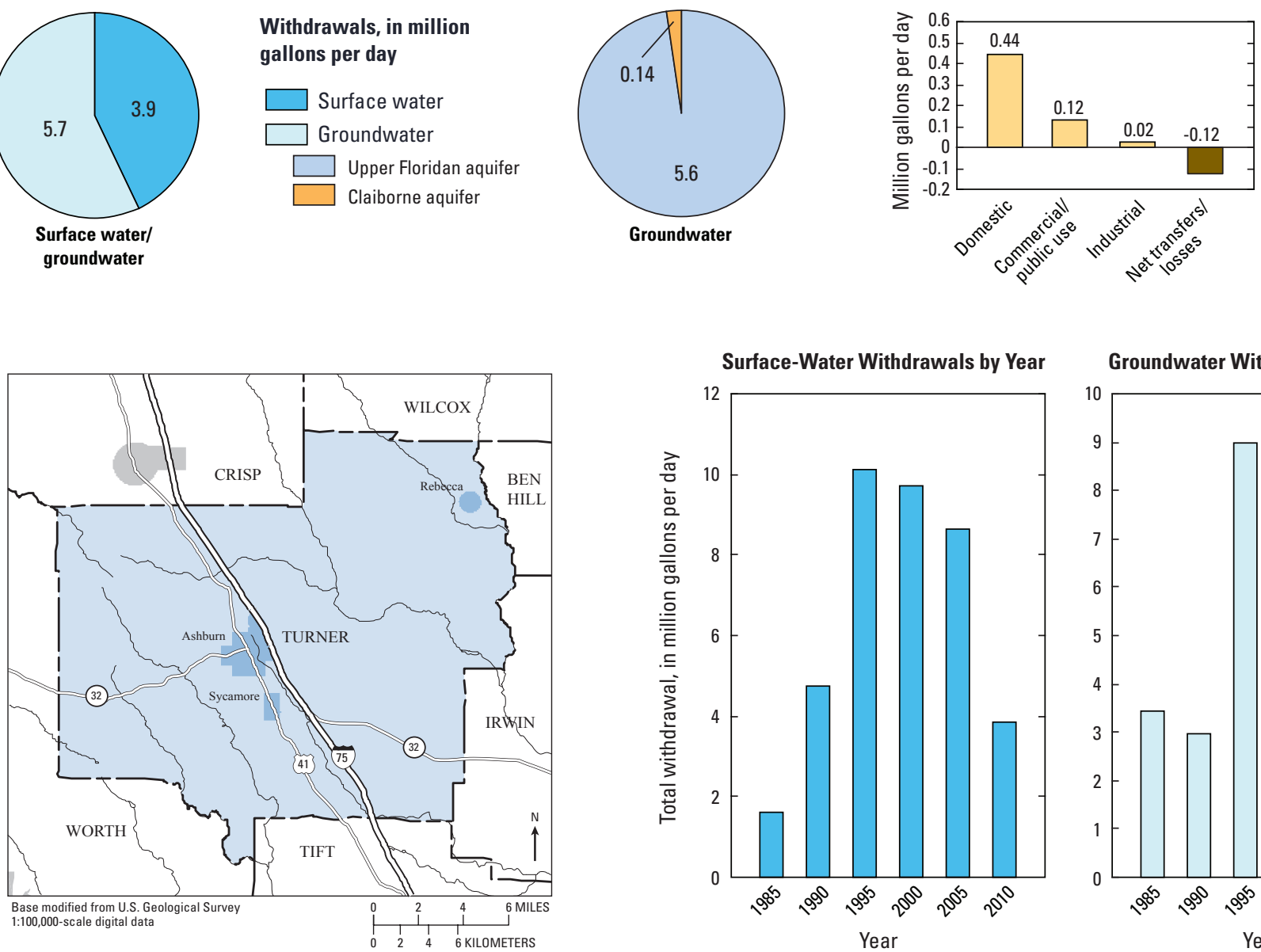

Groundwater Withdrawals by Year

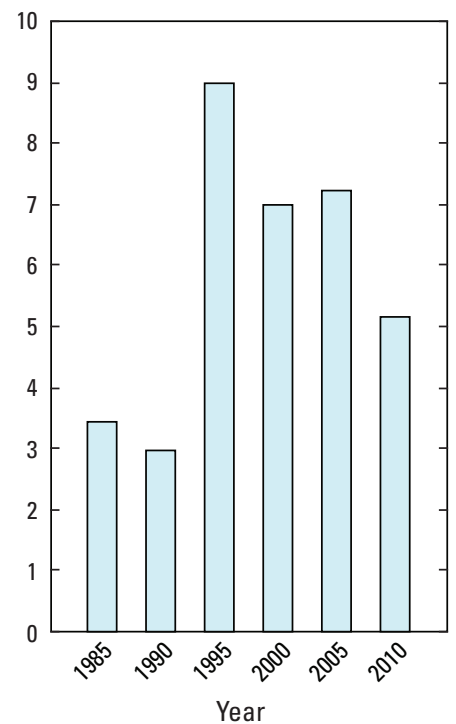


TWIGGS COUNTY

Population

Population served by public supply-Groundwater $\quad 2,500$

Population served by public supply-Surface water $\quad 70$

Acres irrigated

1,470

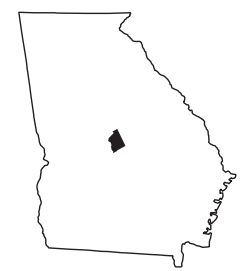

2010 WATER WITHDRAWALS AND ESTIMATED USE, IN MILLION GALLONS PER DAY

$[-$, not applicable $]$

\begin{tabular}{|c|c|c|c|c|c|c|c|c|}
\hline \multirow{3}{*}{ Category } & \multicolumn{3}{|c|}{ Withdrawals } & \multirow{3}{*}{$\begin{array}{l}\text { Total } \\
\text { use }^{1}\end{array}$} & \multirow{3}{*}{$\begin{array}{c}\text { Surface- } \\
\text { water } \\
\text { returns } \\
\end{array}$} & \multirow{2}{*}{\multicolumn{3}{|c|}{$\begin{array}{l}\text { Withdrawals by Major Industrial Groups } \\
\text { [NAICS, North American Industrial Classification System code] }\end{array}$}} \\
\hline & \multirow{2}{*}{$\begin{array}{l}\text { Ground- } \\
\text { water }\end{array}$} & \multirow{2}{*}{$\begin{array}{c}\text { Surface } \\
\text { water }\end{array}$} & \multirow[b]{2}{*}{ Total } & & & & & \\
\hline & & & & & & NAICS & Groundwater & Surface water \\
\hline Public supply & 0.39 & 0.00 & 0.39 & - & - & 212 -Kaolin and ball & ay 5.16 & 0.00 \\
\hline Domestic & 0.52 & 0.00 & 0.52 & 0.74 & 0.00 & & & \\
\hline Commercial/public use & 0.00 & 0.00 & 0.00 & 0.12 & 0.00 & & & \\
\hline Industrial & 5.16 & 0.00 & 5.16 & 5.16 & 0.00 & & & \\
\hline Public-supply losses & - & - & - & 0.05 & - & & & \\
\hline Public wastewater treatment & - & - & - & - & 0.00 & Withdrawals by Ma & r Public Supp & \\
\hline $\begin{array}{l}\text { Mining } \\
\text { Irrigation-Crop }\end{array}$ & $\begin{array}{l}0.03 \\
0.35\end{array}$ & $\begin{array}{l}0.00 \\
0.06\end{array}$ & $\begin{array}{l}0.03 \\
0.41\end{array}$ & $\begin{array}{l}0.03 \\
0.41\end{array}$ & $\begin{array}{l}1.93 \\
0.00\end{array}$ & Name & Groundwater & Surface water \\
\hline Irrigation-Golf course & 0.00 & 0.00 & 0.00 & 0.00 & 0.00 & City of Jeffersonville & 0.33 & 0.00 \\
\hline Livestock/aquaculture & 0.03 & 0.03 & 0.06 & 0.06 & 0.00 & Twiggs County & & \\
\hline Thermoelectric power & 0.00 & 0.00 & 0.00 & 0.00 & 0.00 & Water System & 0.06 & 0.00 \\
\hline TOTAL & 6.48 & 0.09 & 6.57 & 6.57 & 1.93 & & & \\
\hline
\end{tabular}

'Total use is total withdrawal plus public supply deliveries and losses.

\section{Withdrawals by Source}
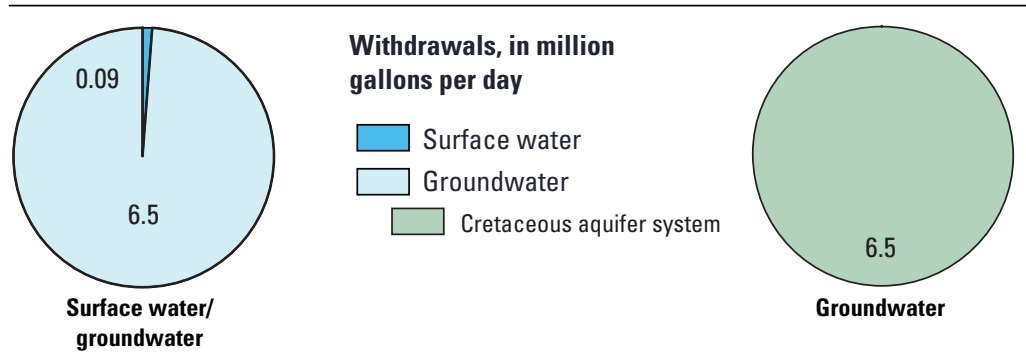

Public-Supply Deliveries by Use Category
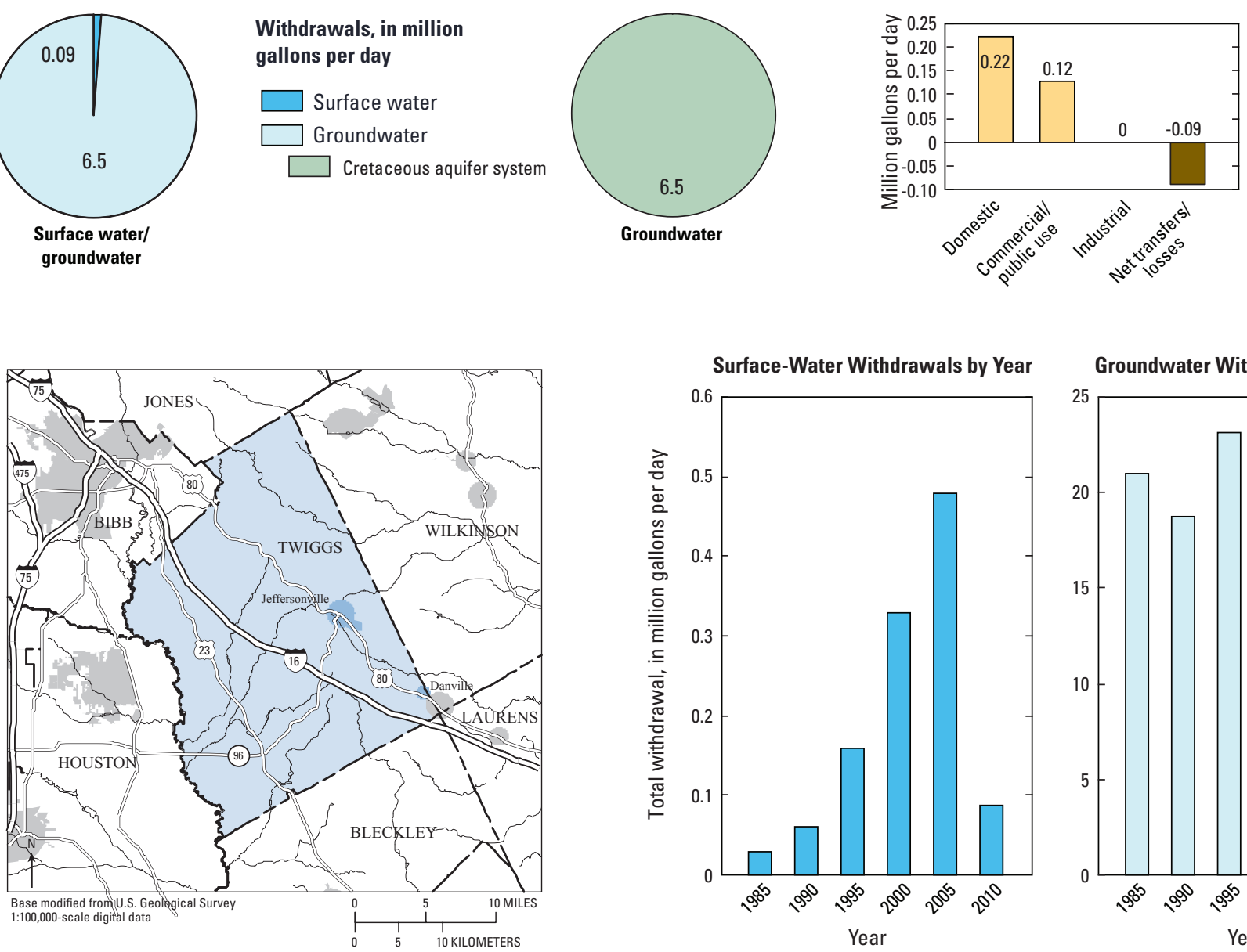

Groundwater Withdrawals by Year

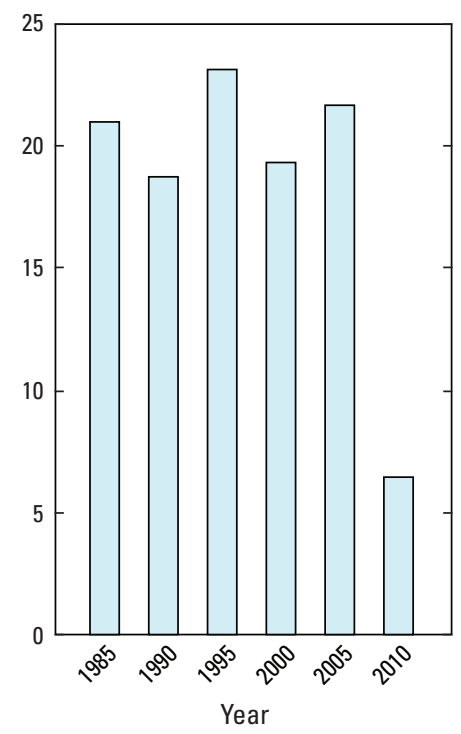




\section{UNION COUNTY}

Population

21,356

Population served by public supply-Groundwater

Population served by public supply-Surface water Acres irrigated
6,980

10,580

255

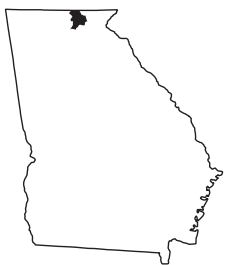

2010 WATER WITHDRAWALS AND ESTIMATED USE, IN MILLION GALLONS PER DAY

$[-$, not applicable $]$

\begin{tabular}{|c|c|c|c|c|c|c|c|c|}
\hline \multirow{3}{*}{ Category } & \multicolumn{3}{|c|}{ Withdrawals } & \multirow{3}{*}{$\begin{array}{l}\text { Total } \\
\text { use }^{1}\end{array}$} & \multirow{3}{*}{$\begin{array}{l}\text { Surface- } \\
\text { water } \\
\text { returns } \\
\end{array}$} & \multirow{2}{*}{\multicolumn{3}{|c|}{$\begin{array}{l}\text { Withdrawals by Major Industrial Groups } \\
\text { [NAICS, North American Industrial Classification System code] }\end{array}$}} \\
\hline & \multirow{2}{*}{$\begin{array}{c}\text { Ground- } \\
\text { water }\end{array}$} & \multirow{2}{*}{$\begin{array}{c}\text { Surface } \\
\text { water }\end{array}$} & \multirow[b]{2}{*}{ Total } & & & & & \\
\hline & & & & & & NAICS & Groundwater & Surface water \\
\hline Public supply & 1.00 & 0.90 & 1.90 & - & - & & & \\
\hline Domestic & 0.28 & 0.00 & 0.28 & 1.40 & 0.00 & None & - & - \\
\hline Commercial/public use & 0.00 & 0.00 & 0.00 & 0.23 & 0.00 & & & \\
\hline Industrial & 0.00 & 0.00 & 0.00 & 0.19 & 0.00 & & & \\
\hline Public-supply losses & - & - & - & 0.36 & - & & & \\
\hline Public wastewater treatment & - & - & - & - & 0.29 & Withdrawals by Majo & r Public Supp & \\
\hline $\begin{array}{l}\text { Mining } \\
\text { Irrigation_-Crop }\end{array}$ & $\begin{array}{l}0.08 \\
0.02\end{array}$ & $\begin{array}{l}0.00 \\
0.07\end{array}$ & $\begin{array}{l}0.08 \\
0.09\end{array}$ & $\begin{array}{l}0.08 \\
0.09\end{array}$ & $\begin{array}{l}0.00 \\
0.00\end{array}$ & Name & Groundwater & Surface water \\
\hline Irrigation-Golf course & 0.00 & 0.30 & 0.30 & 0.30 & 0.00 & Notla Water Authority & 0.36 & 0.65 \\
\hline Livestock/aquaculture & 0.00 & 5.36 & 5.36 & 5.36 & 0.00 & Coosa Water Authority & 0.38 & 0.00 \\
\hline Thermoelectric power & 0.00 & 0.00 & 0.00 & 0.00 & 0.00 & City of Blairsville & 0.21 & 0.25 \\
\hline
\end{tabular}

${ }^{1}$ Total use is total withdrawal plus public supply deliveries and losses.

\section{Withdrawals by Source}

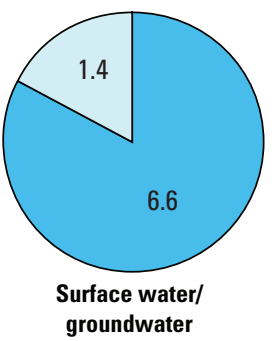

Withdrawals, in million gallons per day

groundwater

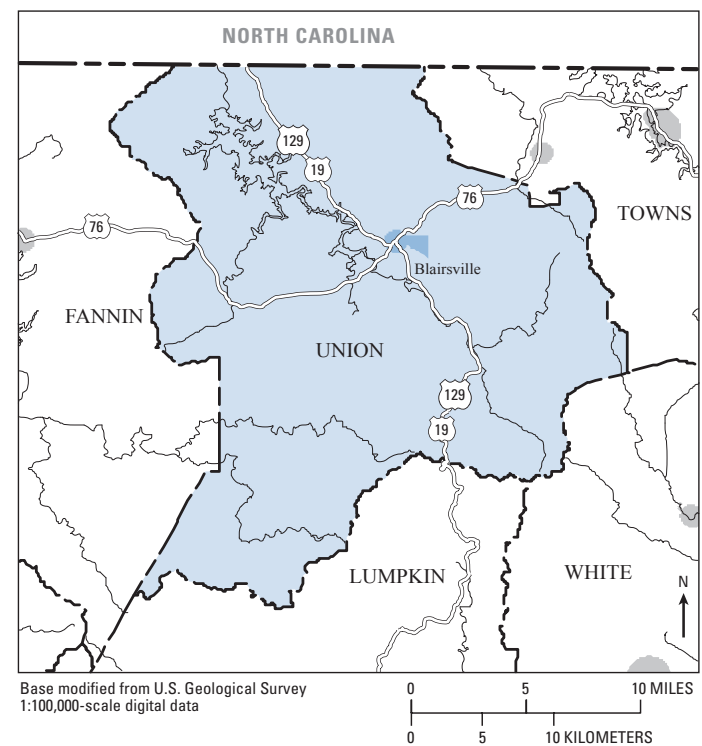

Public-Supply Deliveries by Use Category
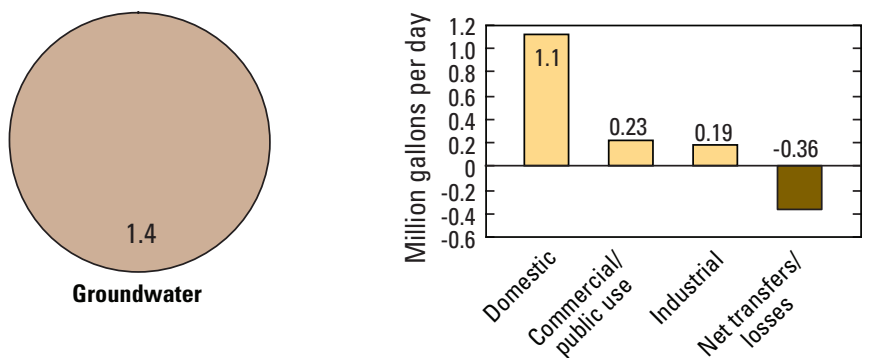

Surface-Water Withdrawals by Year

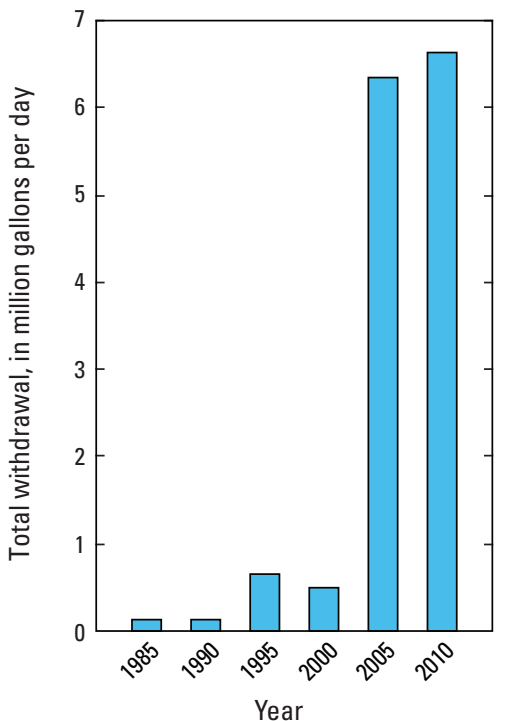

Groundwater Withdrawals by Year

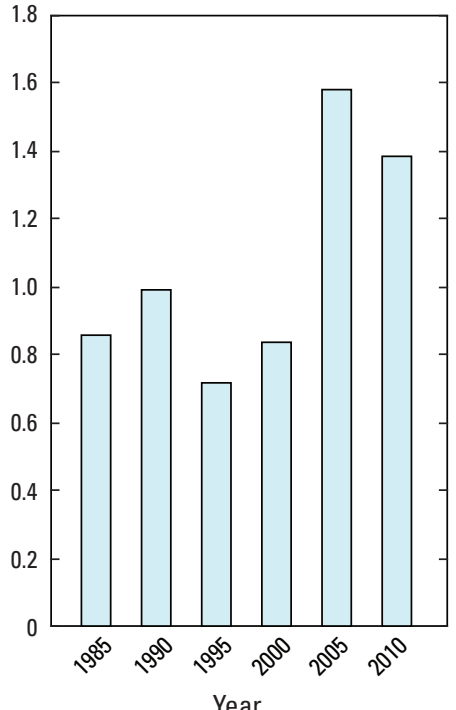




\section{UPSON COUNTY}

Population

Population served by public supply-Groundwater $\quad 3,050$

Population served by public supply-Surface water $\quad 11,540$

Acres irrigated
940

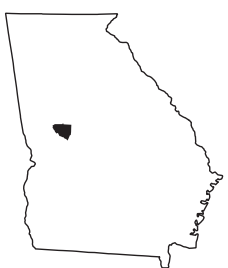

2010 WATER WITHDRAWALS AND ESTIMATED USE, IN MILLION GALLONS PER DAY

$[-$, not applicable $]$

\begin{tabular}{|c|c|c|c|c|c|c|c|c|}
\hline \multirow{3}{*}{ Category } & \multicolumn{3}{|c|}{ Withdrawals } & \multirow{3}{*}{$\begin{array}{l}\text { Total } \\
\text { use }^{1}\end{array}$} & \multirow{3}{*}{$\begin{array}{l}\text { Surface- } \\
\text { water } \\
\text { returns } \\
\end{array}$} & \multirow{2}{*}{\multicolumn{3}{|c|}{$\begin{array}{l}\text { Withdrawals by Major Industrial Groups } \\
\text { [NAICS, North American Industrial Classification System code] }\end{array}$}} \\
\hline & \multirow{2}{*}{$\begin{array}{c}\text { Ground- } \\
\text { water }\end{array}$} & \multirow{2}{*}{$\begin{array}{c}\text { Surface } \\
\text { water }\end{array}$} & \multirow[b]{2}{*}{ Total } & & & & & \\
\hline & & & & & & NAICS & Groundwater & Surface water \\
\hline Public supply & 0.48 & 4.00 & 4.48 & - & - & & & \\
\hline Domestic & 0.94 & 0.00 & 0.94 & 3.61 & 0.00 & 313-Textiles & 0.00 & 0.26 \\
\hline Commercial/public use & 0.00 & 0.00 & 0.00 & 0.65 & 0.00 & & & \\
\hline Industrial & 0.00 & 0.26 & 0.26 & 0.36 & 0.00 & & & \\
\hline Public-supply losses & - & - & - & 1.06 & - & & & \\
\hline Public wastewater treatment & - & - & - & - & 2.71 & Withdrawals by I & r Public Supp & \\
\hline Mining & 0.00 & $\begin{array}{l}0.00 \\
0.40\end{array}$ & $\begin{array}{l}0.00 \\
0.40\end{array}$ & $\begin{array}{l}0.00 \\
0.40\end{array}$ & $\begin{array}{l}0.00 \\
0.00\end{array}$ & Name & Groundwater & Surface water \\
\hline $\begin{array}{l}\text { Irrigation-Crop } \\
\text { Irrigation-Golf course }\end{array}$ & $\begin{array}{l}0.00 \\
0.00\end{array}$ & $\begin{array}{l}0.40 \\
0.00\end{array}$ & $\begin{array}{l}0.40 \\
0.00\end{array}$ & $\begin{array}{l}0.40 \\
0.00\end{array}$ & $\begin{array}{l}0.00 \\
0.00\end{array}$ & City of Thomaston & 0.00 & 4.00 \\
\hline Livestock/aquaculture & 0.02 & 0.23 & 0.25 & 0.25 & 0.00 & Upson County & 0.32 & 0.00 \\
\hline
\end{tabular}

1.44

4.89

6.33

6.33

2.71

'Total use is total withdrawal plus public supply deliveries and losses.

\section{Withdrawals by Source}

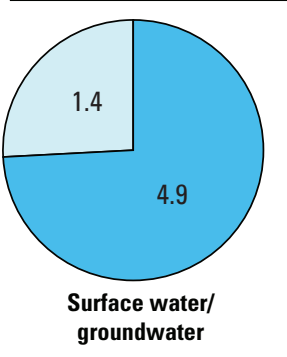

Withdrawals, in million gallons per day

Surface water

Groundwater

$\square$ Crystalline-rock aquifer

groundwater

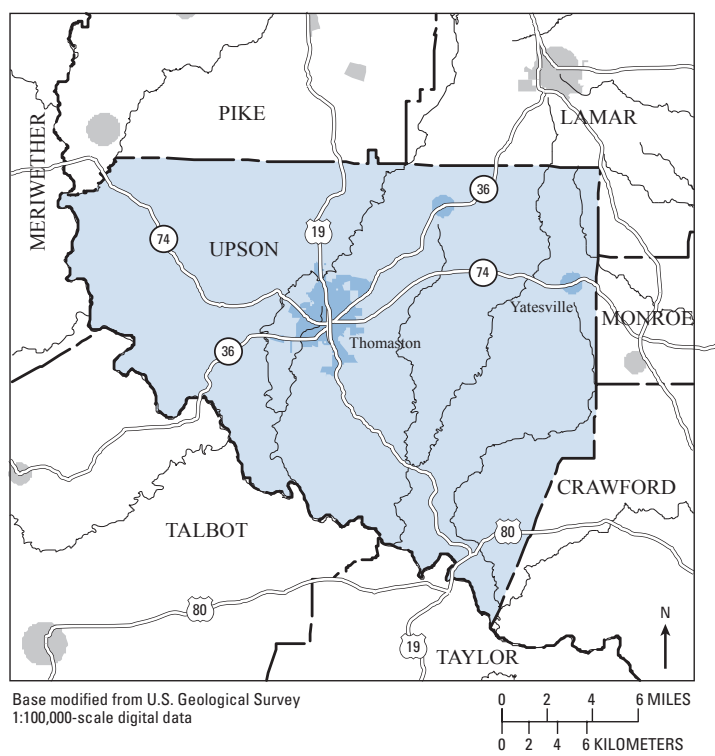

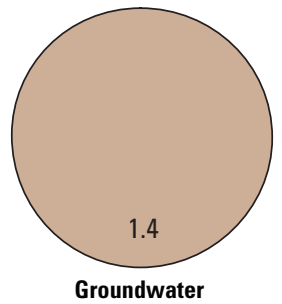

Public-Supply Deliveries by Use Category

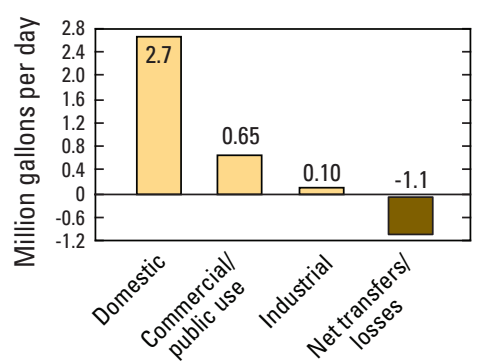

Surface-Water Withdrawals by Year

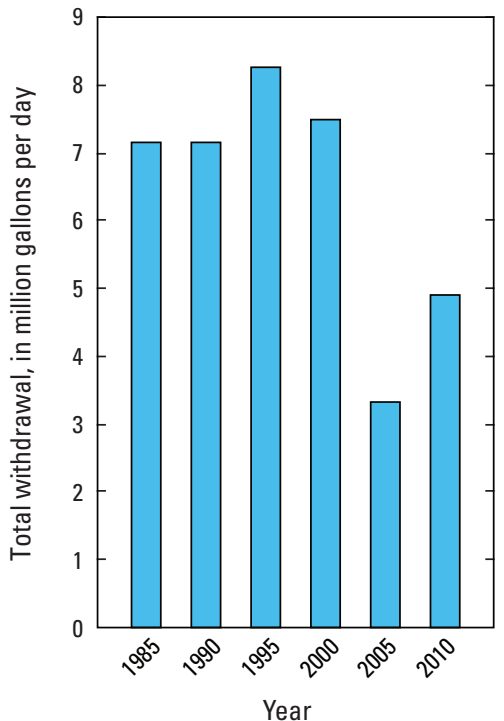

Groundwater Withdrawals by Year

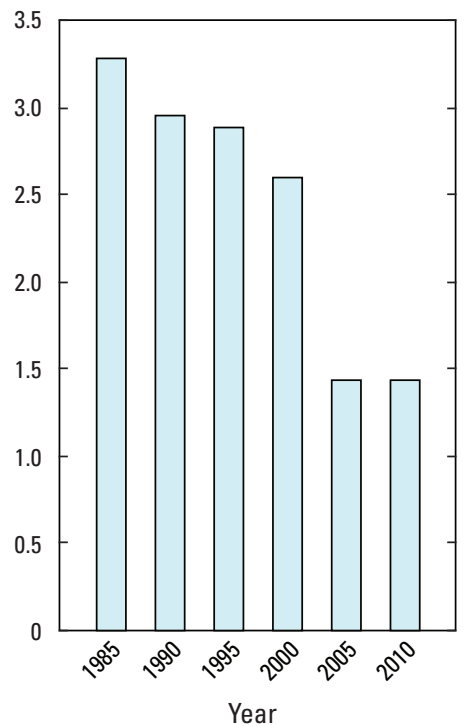




\section{WALKER COUNTY}

Population

68,756

Population served by public supply-Groundwater

Population served by public supply-Surface water Acres irrigated
41,850

22,130

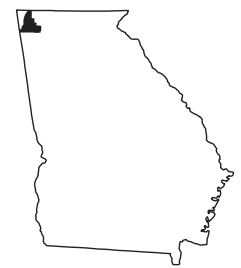

2010 WATER WITHDRAWALS AND ESTIMATED USE, IN MILLION GALLONS PER DAY

$[-$, not applicable; Mgal/d, million gallons per day $]$

\begin{tabular}{|c|c|c|c|c|c|c|c|c|}
\hline \multirow{3}{*}{ Category } & \multicolumn{3}{|c|}{ Withdrawals } & \multirow{3}{*}{$\begin{array}{l}\text { Total } \\
\text { use }^{1}\end{array}$} & \multirow{3}{*}{$\begin{array}{l}\text { Surface- } \\
\text { water } \\
\text { returns }\end{array}$} & \multirow{2}{*}{\multicolumn{3}{|c|}{$\begin{array}{l}\text { Withdrawals by Major Industrial Groups } \\
\text { [NAICS, North American Industrial Classification System code] }\end{array}$}} \\
\hline & \multirow{2}{*}{$\begin{array}{l}\text { Ground- } \\
\text { water }\end{array}$} & \multirow{2}{*}{$\begin{array}{c}\text { Surface } \\
\text { water }\end{array}$} & \multirow[b]{2}{*}{ Total } & & & & & \\
\hline & & & & & & NAICS & Groundwater & Surface water \\
\hline Public supply & 5.23 & 1.10 & 6.33 & - & - & \multirow{3}{*}{$\begin{array}{l}\text { 313-Textiles } \\
325 \text {-Chemical products }\end{array}$} & \multirow{3}{*}{$\begin{array}{l}0.01 \\
0.01\end{array}$} & \multirow{2}{*}{$\begin{array}{l}0.57 \\
0.00\end{array}$} \\
\hline Domestic & 0.36 & 0.00 & 0.36 & 5.03 & 0.00 & & & \\
\hline Commercial/public use & 0.00 & 0.12 & 0.12 & 1.72 & 0.00 & \multirow{2}{*}{\multicolumn{3}{|c|}{ Withdrawals by Major Public Suppliers }} \\
\hline Industrial & 0.02 & 0.57 & 0.59 & 0.99 & 0.00 & & & \\
\hline $\begin{array}{l}\text { Public-supply losses } \\
\text { Public wastewater treatment }\end{array}$ & $\begin{array}{l}- \\
-\end{array}$ & - & - & $\begin{array}{c}1.98 \\
-\end{array}$ & $\begin{array}{c}- \\
3.13\end{array}$ & Name & Groundwater & Surface water \\
\hline Mining & 0.00 & 0.00 & 0.00 & 0.00 & 0.00 & \multirow{2}{*}{$\begin{array}{l}\text { Walker County Water } \\
\text { Authority }\end{array}$} & \multirow{2}{*}{3.22} & \multirow{2}{*}{0.00} \\
\hline Irrigation-Crop & 0.02 & 0.00 & 0.02 & 0.02 & 0.00 & & & \\
\hline Irrigation-Golf course & 0.00 & 0.00 & 0.00 & 0.00 & 0.00 & City of Lafayette & 0.85 & 1.10 \\
\hline Livestock/aquaculture & 0.09 & 1.10 & 1.19 & 1.19 & 0.00 & City of Chickamauga & 0.96 & 0.00 \\
\hline Thermoelectric power & 0.00 & 0.00 & 0.00 & 0.00 & 0.00 & \multirow{2}{*}{$\begin{array}{l}\text { Walker County Rural } \\
\text { Water Authority }\end{array}$} & \multirow[b]{2}{*}{0.20} & \multirow{2}{*}{0.00} \\
\hline TOTAL & 5.72 & 2.89 & 8.61 & 10.93 & 3.13 & & & \\
\hline
\end{tabular}

\section{Withdrawals by Source}
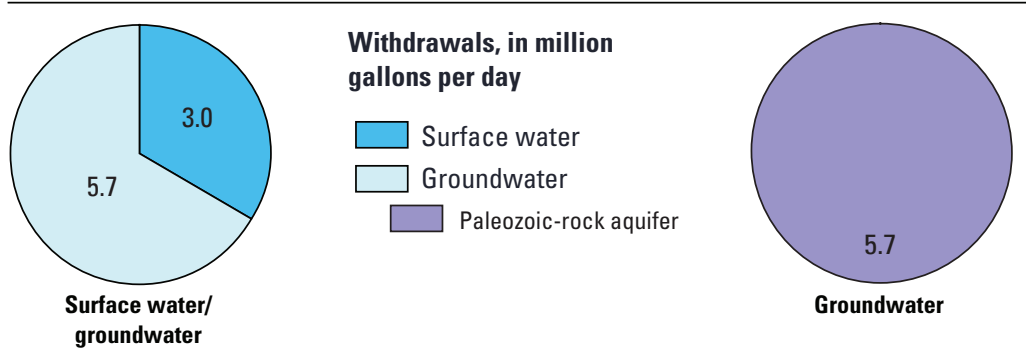

\section{Public-Supply Deliveries' by Use Category}
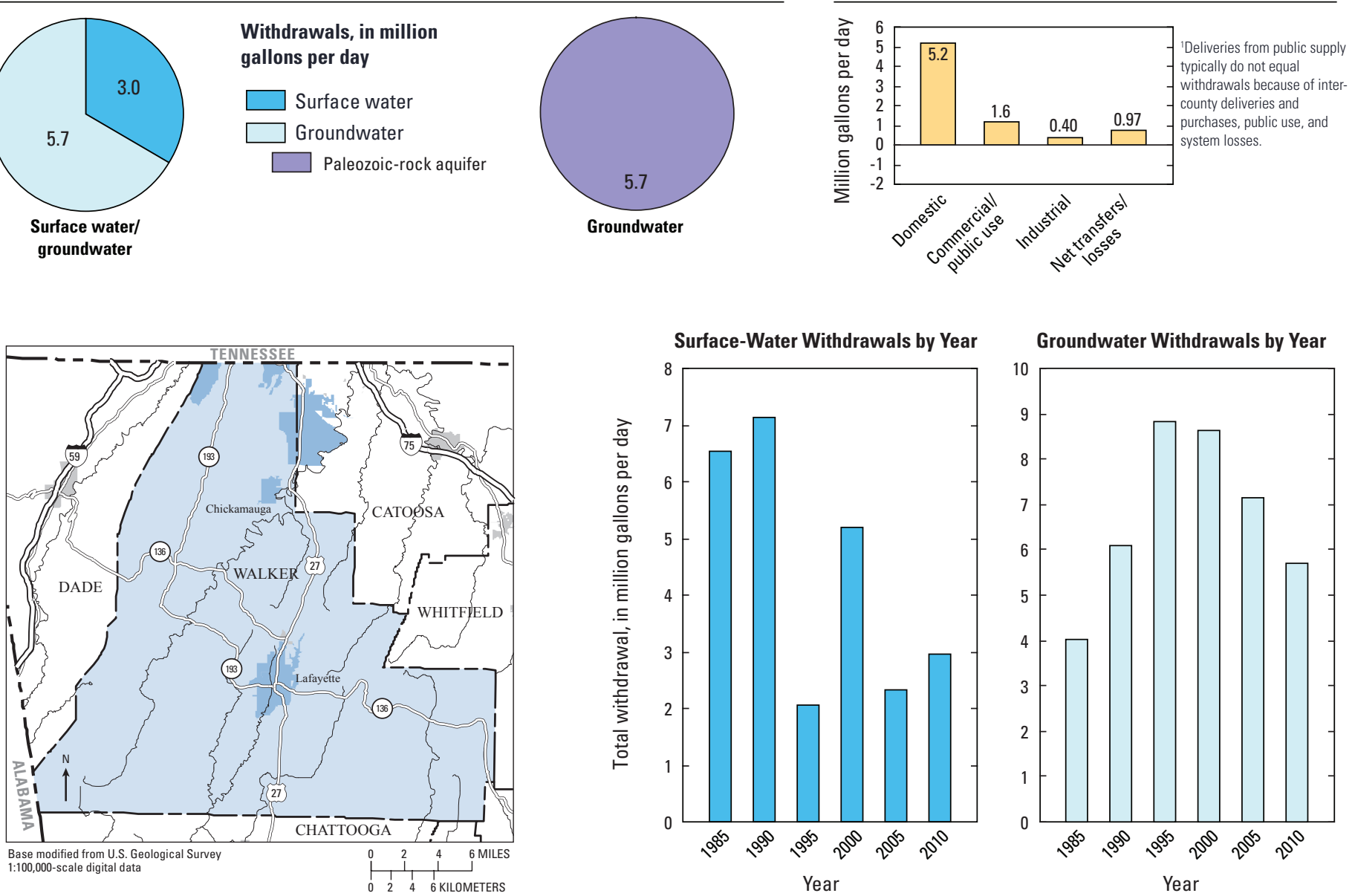

Groundwater Withdrawals by Year

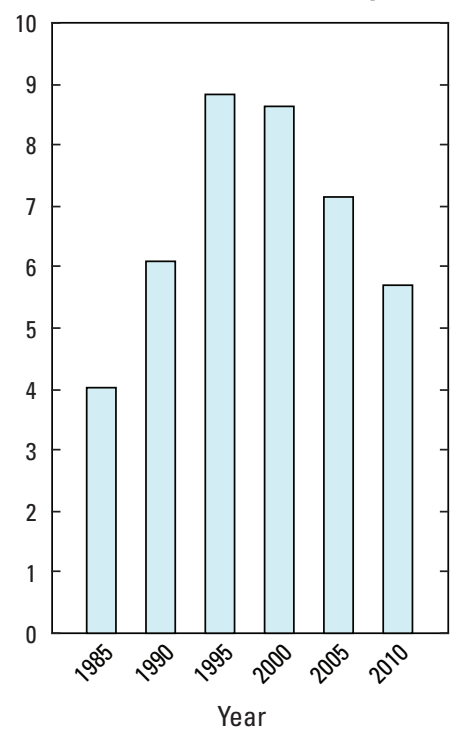




\section{WALTON COUNTY}

Population

83,768

Population served by public supply-Groundwater

Population served by public supply-Surface water $\quad 72,300$

Acres irrigated

1,190

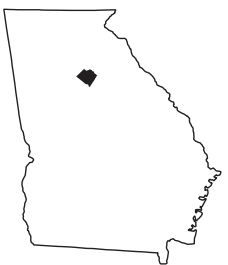

2010 WATER WITHDRAWALS AND ESTIMATED USE, IN MILLION GALLONS PER DAY

$[-$, not applicable; Mgal/d, million gallons per day $]$

\begin{tabular}{|c|c|c|c|c|c|c|c|c|}
\hline \multirow{3}{*}{ Category } & \multicolumn{3}{|c|}{ Withdrawals } & \multirow{3}{*}{$\begin{array}{l}\text { Total } \\
\text { use }^{1}\end{array}$} & \multirow{3}{*}{$\begin{array}{l}\text { Surface- } \\
\text { water } \\
\text { returns } \\
\end{array}$} & \multirow{2}{*}{\multicolumn{3}{|c|}{$\begin{array}{l}\text { Withdrawals by Major Industrial Groups } \\
\text { [NAICS, North American Industrial Classification System code] }\end{array}$}} \\
\hline & \multirow{2}{*}{$\begin{array}{c}\text { Ground- } \\
\text { water }\end{array}$} & \multirow{2}{*}{$\begin{array}{c}\text { Surface } \\
\text { water }\end{array}$} & \multirow[b]{2}{*}{ Total } & & & & & \\
\hline & & & & & & NAICS & Groundwater & Surface water \\
\hline Public supply & 0.04 & 2.78 & 2.82 & - & - & & & \\
\hline Domestic & 0.81 & 0.00 & 0.81 & 4.84 & 0.00 & None & - & - \\
\hline Commercial/public use & 0.00 & 0.00 & 0.00 & 1.10 & 0.00 & & & \\
\hline Industrial & 0.00 & 0.00 & 0.00 & 0.12 & 0.00 & & & \\
\hline Public-supply losses & - & - & - & 1.25 & - & & & \\
\hline Public wastewater treatment & - & - & - & - & 2.87 & Withdrawals by Mc & r Public Supp & \\
\hline $\begin{array}{l}\text { Mining } \\
\text { Irrigation-Crop }\end{array}$ & $\begin{array}{l}0.03 \\
1.98\end{array}$ & $\begin{array}{l}0.00 \\
0.54\end{array}$ & $\begin{array}{l}0.03 \\
2.52\end{array}$ & $\begin{array}{l}0.03 \\
2.52\end{array}$ & $\begin{array}{l}0.00 \\
0.00\end{array}$ & Name & Groundwater & Surface water \\
\hline Irrigation-Golf course & 0.00 & 0.47 & 0.47 & 0.47 & 0.00 & City of Monroe & 0.00 & 2.30 \\
\hline Livestock/aquaculture & 0.00 & 0.16 & 0.16 & 0.16 & 0.00 & City of Social Circle & 0.00 & 0.48 \\
\hline Thermoelectric power & 0.00 & 0.00 & 0.00 & 0.00 & 0.00 & City or social Circie & 0.00 & 0.40 \\
\hline
\end{tabular}

'Total use is total withdrawal plus public supply deliveries and losses. An average of $4.32 \mathrm{Mgal} / \mathrm{d}$ was delivered from Newton County, Georgia and $0.13 \mathrm{Mgal} / \mathrm{d}$ was delivered from Oconee County, Georgia to Walton County in 2010. About 2,400 residential customers of public suppliers in Walton County reside in Gwinnett County; therefore that use is not included for Walton County.

\section{Withdrawals by Source}

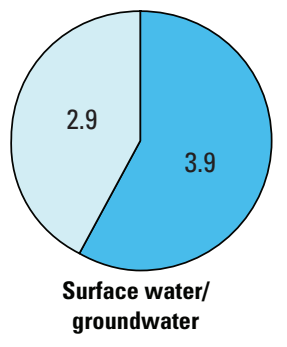

Withdrawals, in million gallons per day
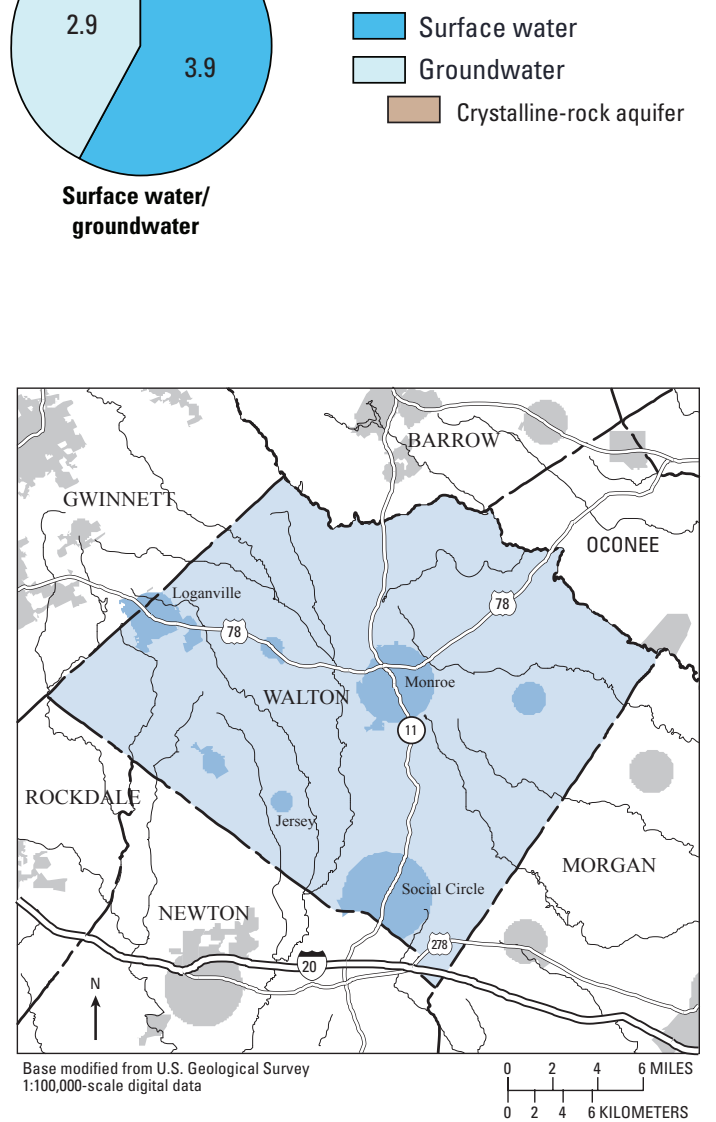

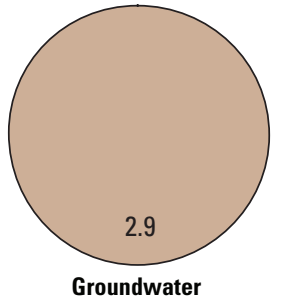

Public-Supply Deliveries' by Use Category

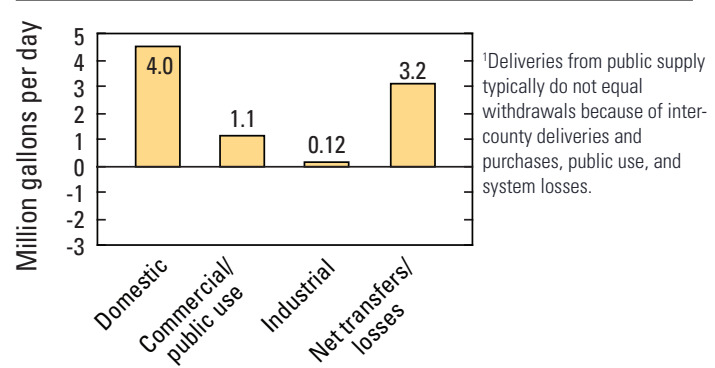

Surface-Water Withdrawals by Year

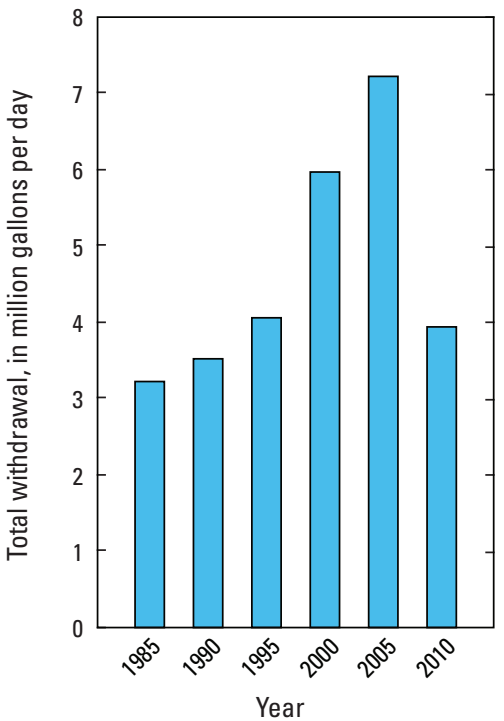

Groundwater Withdrawals by Year

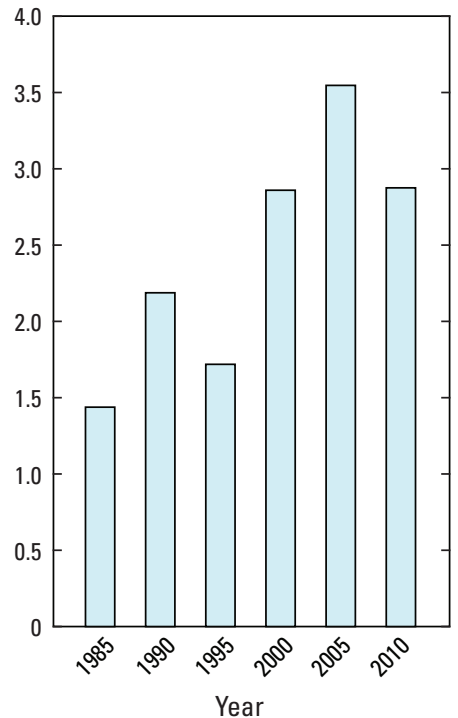


WARE COUNTY

Population

36,312

Population served by public supply-Groundwater

Population served by public supply-Surface water Acres irrigated
31,160

0

4,140

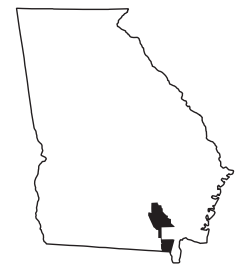

2010 WATER WITHDRAWALS AND ESTIMATED USE, IN MILLION GALLONS PER DAY

$[-$, not applicable $]$

\begin{tabular}{|c|c|c|c|c|c|c|c|c|}
\hline \multirow{3}{*}{ Category } & \multicolumn{3}{|c|}{ Withdrawals } & \multirow{3}{*}{$\begin{array}{l}\text { Total } \\
\text { use }^{1}\end{array}$} & \multirow{3}{*}{$\begin{array}{c}\text { Surface- } \\
\text { water } \\
\text { returns } \\
\end{array}$} & \multirow{2}{*}{\multicolumn{3}{|c|}{$\begin{array}{l}\text { Withdrawals by Major Industrial Groups } \\
\text { [NAICS, North American Industrial Classification System code] }\end{array}$}} \\
\hline & \multirow{2}{*}{$\begin{array}{c}\text { Ground- } \\
\text { water }\end{array}$} & \multirow{2}{*}{$\begin{array}{c}\text { Surface } \\
\text { water }\end{array}$} & \multirow[b]{2}{*}{ Total } & & & & & \\
\hline & & & & & & NAICS & Groundwater & Surface water \\
\hline Public supply & 3.37 & 0.00 & 3.37 & - & - & 339-Misc. manufacturing & g $\quad 0.52$ & 0.00 \\
\hline Domestic & 0.39 & 0.00 & 0.39 & 2.39 & 0.00 & 482_-Railroad transport. & 0.10 & 0.00 \\
\hline Commercial/public use & 0.00 & 0.00 & 0.00 & 0.51 & 0.07 & & & \\
\hline Industrial & 0.64 & 0.00 & 0.64 & 1.10 & 0.00 & & & \\
\hline Public-supply losses & - & - & - & 0.40 & - & & & \\
\hline Public wastewater treatment & - & - & - & - & 4.02 & & & \\
\hline Mining & 0.00 & 0.00 & 0.00 & 0.00 & 0.00 & Withdrawals by Major P & Public Supp & \\
\hline Irrigation- - Crop & 0.26 & 0.69 & 0.95 & 0.95 & 0.00 & Name & Groundwater & Surface water \\
\hline Irrigation-Golf course & 0.33 & 0.00 & 0.33 & 0.33 & 0.00 & & & \\
\hline Livestock/aquaculture & 0.01 & 0.08 & 0.09 & 0.09 & 0.00 & City of Waycross & 2.09 & 0.00 \\
\hline Thermoelectric power & 0.00 & 0.00 & 0.00 & 0.00 & 0.00 & Satilla Regional Water & 1.17 & 0.00 \\
\hline TOTAL & 5.00 & 0.77 & 5.77 & 5.77 & 4.09 & and Sewer Authority & & \\
\hline
\end{tabular}

${ }^{1}$ Total use is total withdrawal plus public supply deliveries and losses.

2010 Withdrawals by Source
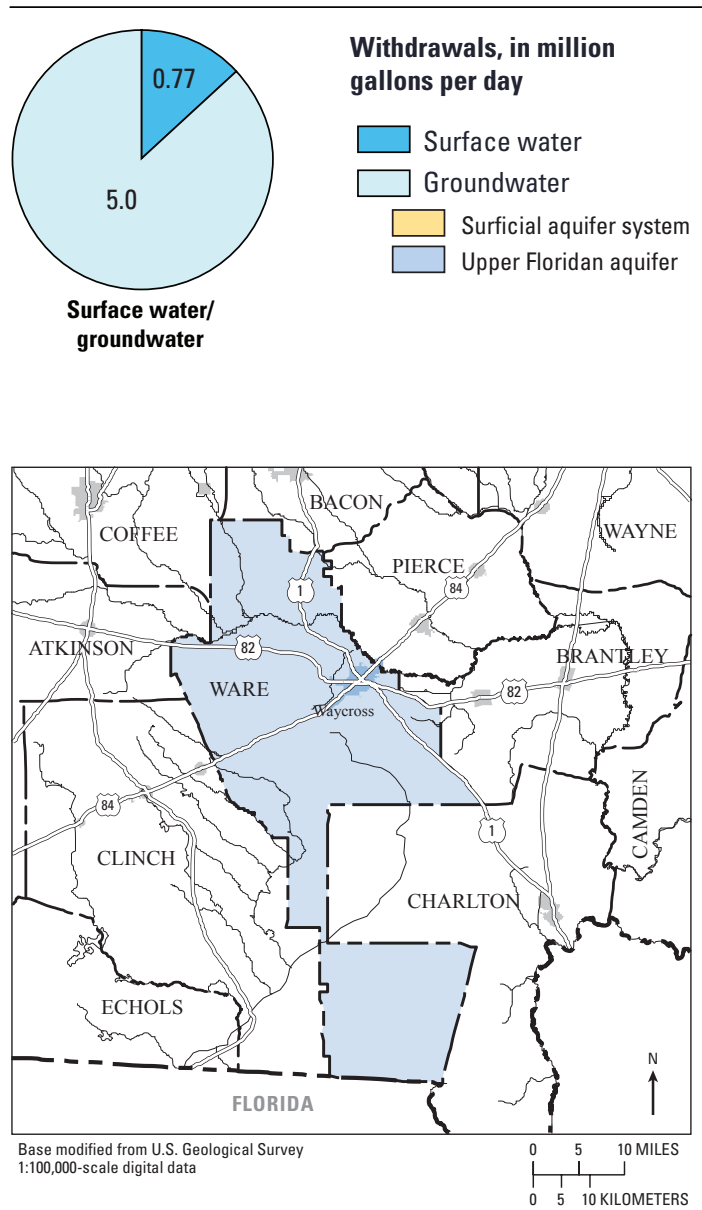

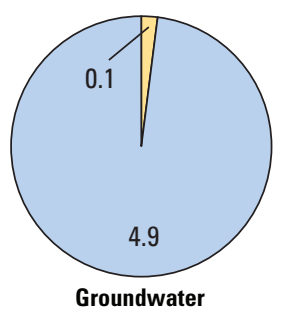

Public-Supply Deliveries by Use Category
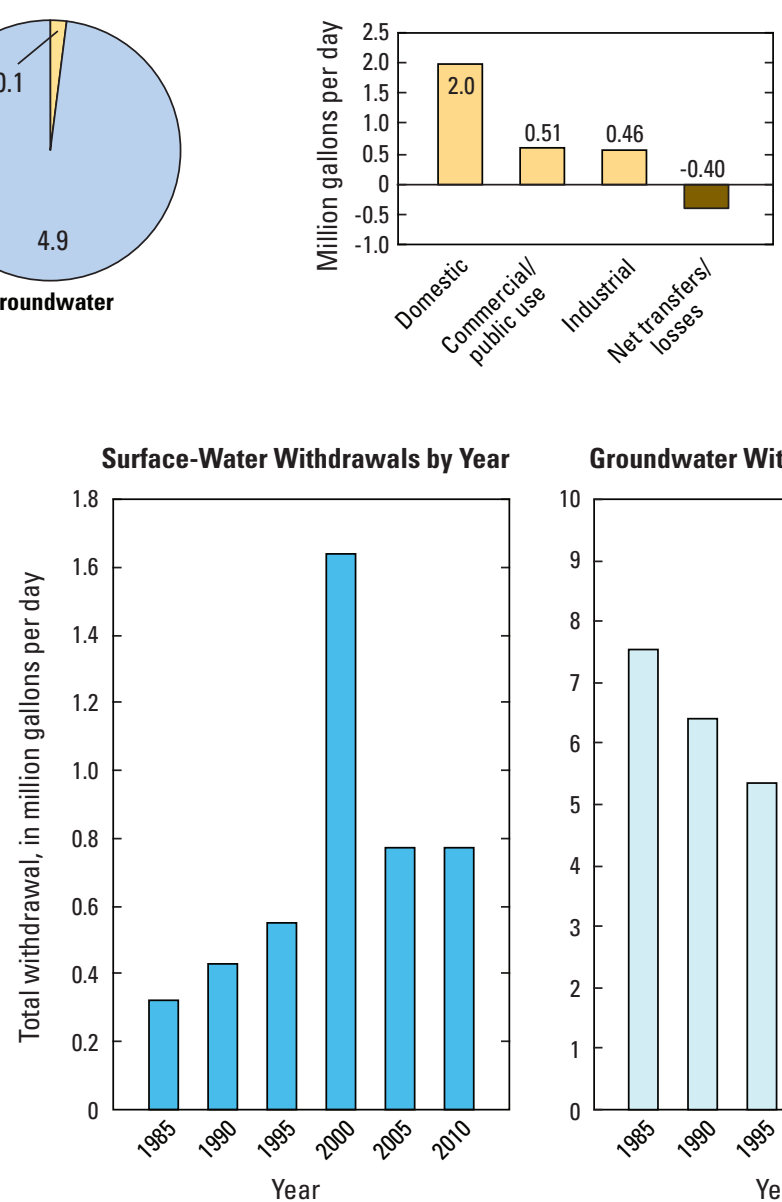

Groundwater Withdrawals by Year

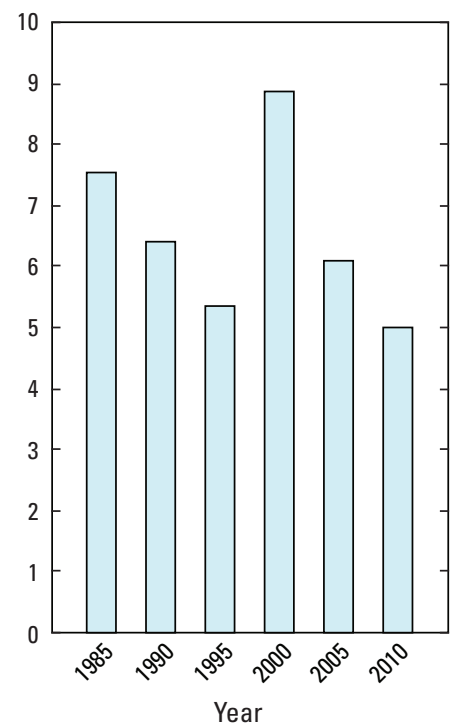




\section{WARREN COUNTY}

Population

Population served by public supply-Groundwater

Population served by public supply-Surface water $\quad 2,260$

Acres irrigated

0

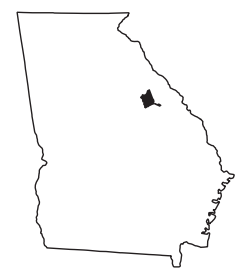

2010 WATER WITHDRAWALS AND ESTIMATED USE, IN MILLION GALLONS PER DAY

$[-$, not applicable $]$

\begin{tabular}{|c|c|c|c|c|c|c|c|c|}
\hline \multirow{3}{*}{ Category } & \multicolumn{3}{|c|}{ Withdrawals } & \multirow{3}{*}{$\begin{array}{l}\text { Total } \\
\text { use }^{1}\end{array}$} & \multirow{3}{*}{$\begin{array}{c}\text { Surface- } \\
\text { water } \\
\text { returns } \\
\end{array}$} & \multirow{2}{*}{\multicolumn{3}{|c|}{$\begin{array}{l}\text { Withdrawals by Major Industrial Groups } \\
\text { [NAICS, North American Industrial Classification System code] }\end{array}$}} \\
\hline & \multirow{2}{*}{$\begin{array}{c}\text { Ground- } \\
\text { water }\end{array}$} & \multirow{2}{*}{$\begin{array}{c}\text { Surface } \\
\text { water }\end{array}$} & \multirow[b]{2}{*}{ Total } & & & & & \\
\hline & & & & & & NAICS & Groundwater & Surface water \\
\hline Public supply & 0.02 & 0.32 & 0.34 & - & - & $221-$ Kaolin and ball clay & 0.64 & 0.40 \\
\hline Domestic & 0.24 & 0.00 & 0.24 & 0.47 & 0.00 & & & \\
\hline Commercial/public use & 0.00 & 0.00 & 0.00 & 0.04 & 0.00 & & & \\
\hline Industrial & 0.64 & 0.40 & 1.04 & 1.06 & 0.00 & & & \\
\hline Public-supply losses & - & - & - & 0.05 & - & & & \\
\hline Public wastewater treatment & - & - & - & - & 0.17 & Withdrawals by Major P & Public Supp & \\
\hline Mining & 0.39 & 0.00 & 0.39 & 0.39 & 2.19 & Name & Groundwater & Surface water \\
\hline $\begin{array}{l}\text { Irrigation-Crop } \\
\text { Irrigation-Golf course }\end{array}$ & $\begin{array}{l}0.00 \\
0.00\end{array}$ & $\begin{array}{l}0.00 \\
0.00\end{array}$ & $\begin{array}{l}0.00 \\
0.00\end{array}$ & $\begin{array}{l}0.00 \\
0.00\end{array}$ & $\begin{array}{l}0.00 \\
0.00\end{array}$ & City of Warrenton & 0.00 & 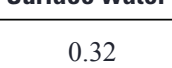 \\
\hline Livestock/aquaculture & 0.00 & 0.13 & 0.13 & 0.13 & 0.00 & City of Norwood & 0.02 & 0.00 \\
\hline Thermoelectric power & 0.00 & 0.00 & 0.00 & 0.00 & 0.00 & & & \\
\hline
\end{tabular}

$1.29 \quad 0.85$

2.14

2.14

2.36

'Total use is total withdrawal plus public supply deliveries and losses.

\section{Withdrawals by Source}
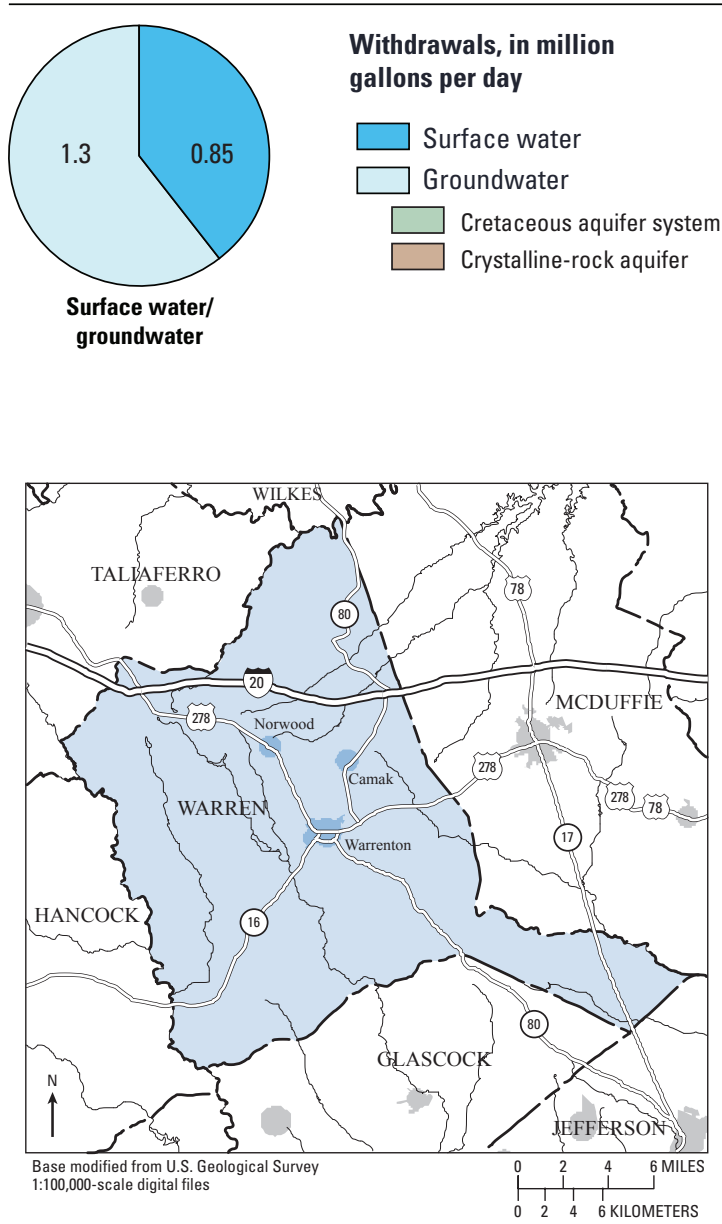

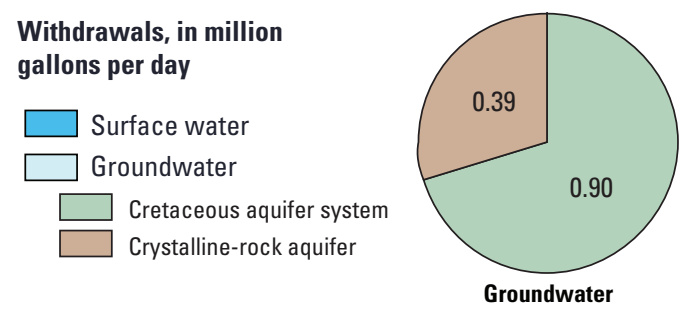

Groundwate

\section{Public-Supply Deliveries by Use Category}

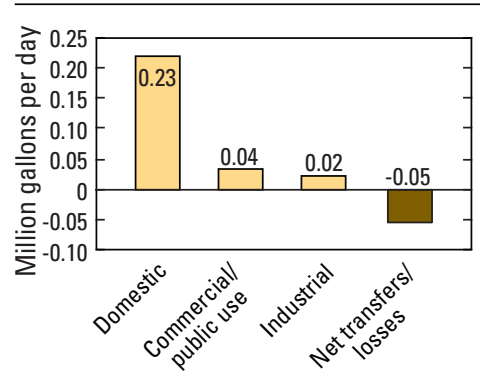

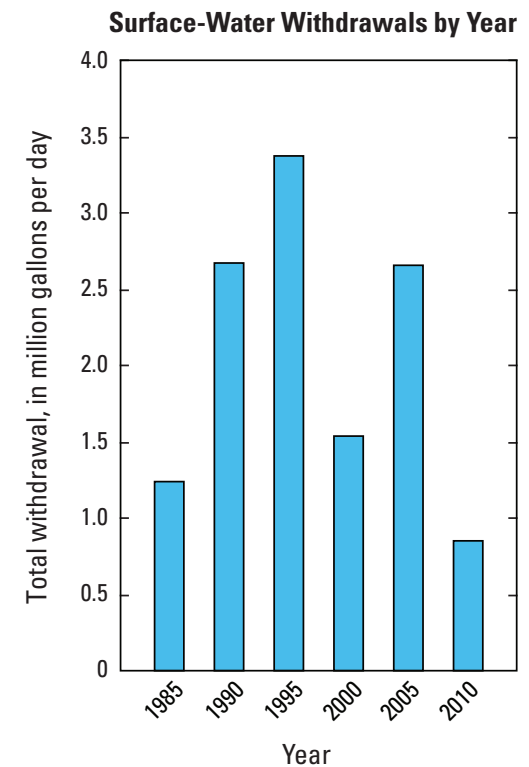

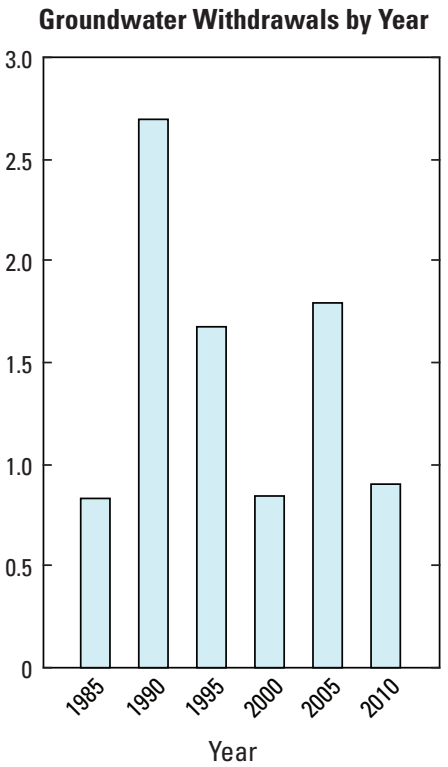




\section{WASHINGTON COUNTY}

Population

21,187

$\begin{array}{lr}\text { Population served by public supply-Groundwater } & 9,830 \\ \text { Population served by public supply-Surface water } & 0\end{array}$

Acres irrigated

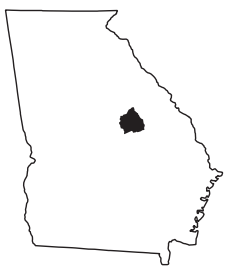

2010 WATER WITHDRAWALS AND ESTIMATED USE, IN MILLION GALLONS PER DAY

$[-$, not applicable $]$

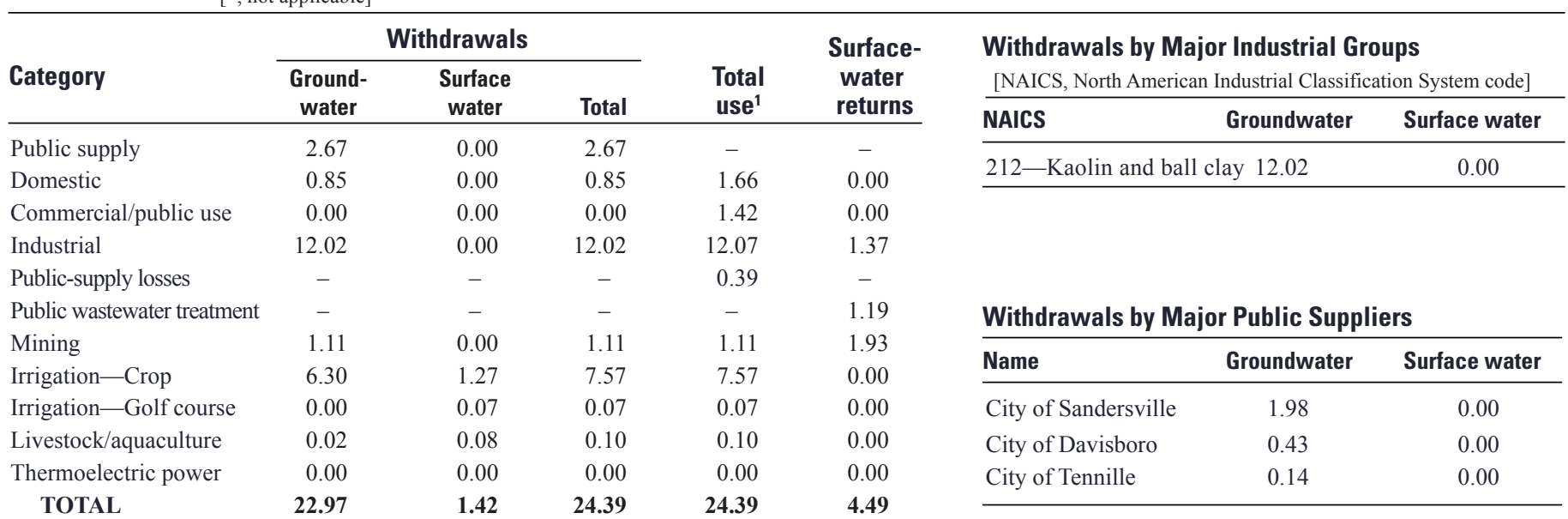

'Total use is total withdrawal plus public supply deliveries and losses.

2010 Withdrawals by Source
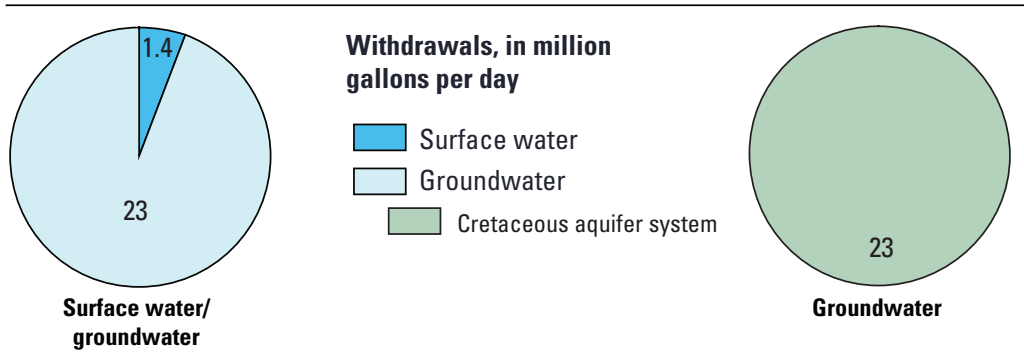

Public-Supply Deliveries by Use Category

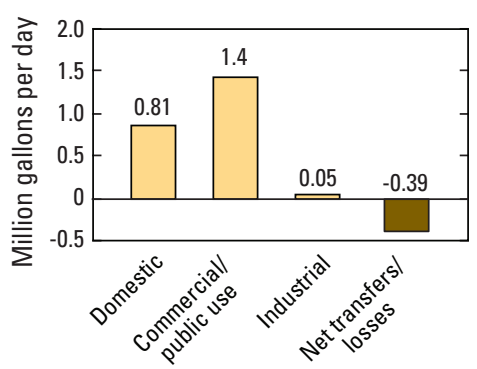

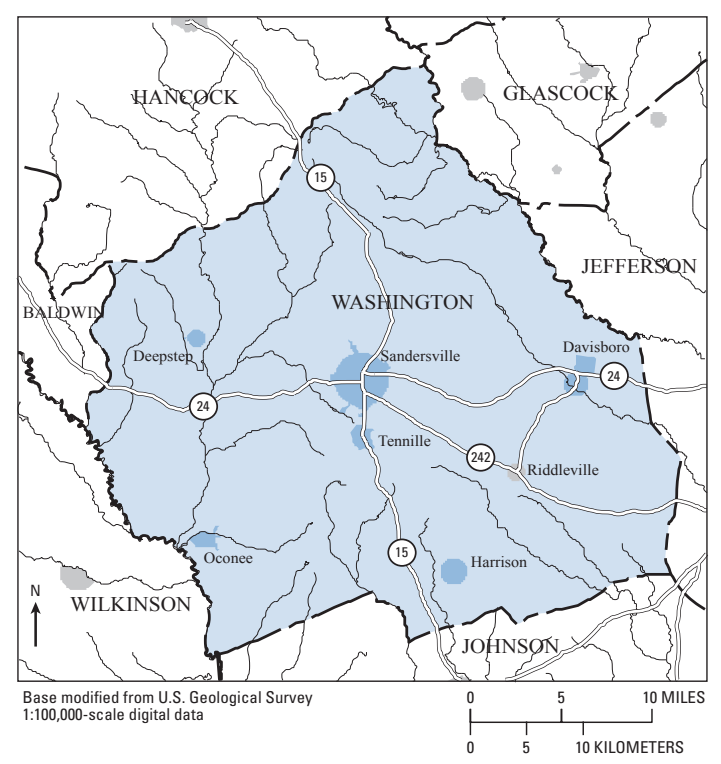

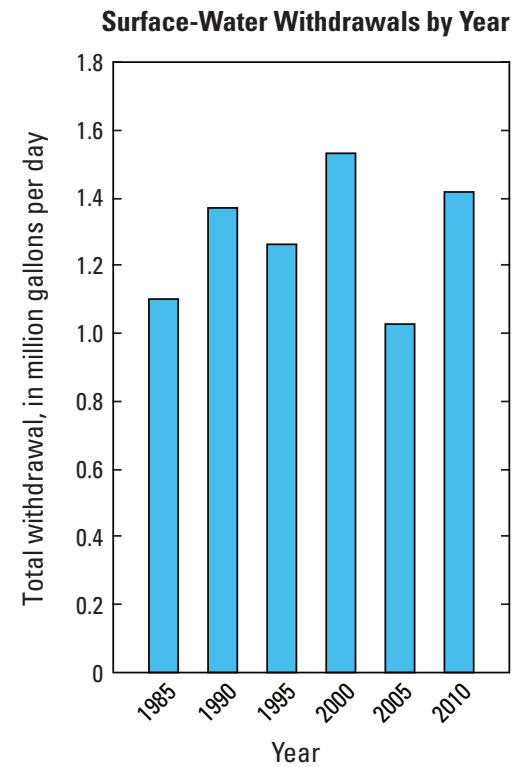

Groundwater Withdrawals by Year

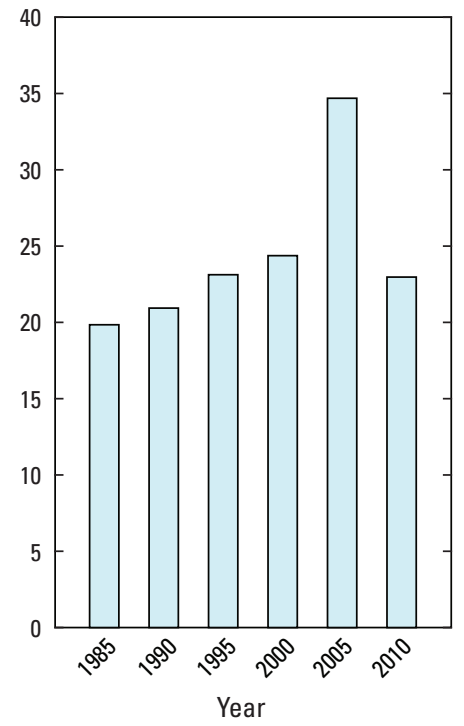




\section{WAYNE COUNTY}

Population

Population served by public supply-Groundwater

Population served by public supply-Surface water

Acres irrigated

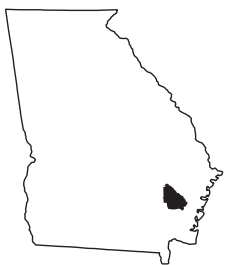

2010 WATER WITHDRAWALS AND ESTIMATED USE, IN MILLION GALLONS PER DAY

$[-$, not applicable]

\begin{tabular}{|c|c|c|c|c|c|c|c|c|}
\hline \multirow{3}{*}{ Category } & \multicolumn{3}{|c|}{ Withdrawals } & \multirow{3}{*}{$\begin{array}{l}\text { Total } \\
\text { use }^{1}\end{array}$} & \multirow{3}{*}{$\begin{array}{l}\text { Surface- } \\
\text { water } \\
\text { returns } \\
\end{array}$} & \multirow{2}{*}{\multicolumn{3}{|c|}{$\begin{array}{l}\text { Withdrawals by Major Industrial Groups } \\
\text { [NAICS, North American Industrial Classification System code] }\end{array}$}} \\
\hline & \multirow{2}{*}{$\begin{array}{c}\text { Ground- } \\
\text { water }\end{array}$} & \multirow{2}{*}{$\begin{array}{c}\text { Surface } \\
\text { water }\end{array}$} & \multirow[b]{2}{*}{ Total } & & & & & \\
\hline & & & & & & NAICS & Groundwater & Surface water \\
\hline Public supply & 1.73 & 0.00 & 1.73 & - & - & \multirow{2}{*}{322 -Paper, pulp } & \multirow{2}{*}{57.84} & \multirow{2}{*}{0.00} \\
\hline Domestic & 1.18 & 0.00 & 1.18 & 2.21 & 0.00 & & & \\
\hline Commercial/public use & 0.02 & 0.00 & 0.02 & 0.51 & 0.00 & & & \\
\hline Industrial & 57.84 & 0.00 & 57.84 & 57.84 & 53.88 & & & \\
\hline Public-supply losses & - & - & - & 0.21 & - & & & \\
\hline Public wastewater treatment & - & - & - & - & 2.03 & \multicolumn{3}{|c|}{ Withdrawals by Major Public Suppliers } \\
\hline Mining & 0.00 & 0.00 & 0.00 & 0.00 & 0.00 & \multirow{2}{*}{ Name } & \multirow[t]{2}{*}{ Groundwater } & \multirow{2}{*}{ Surface water } \\
\hline Irrigation-Crop & 1.01 & 0.01 & 1.02 & 1.02 & 0.00 & & & \\
\hline Irrigation-Golf course & 0.07 & 0.00 & 0.07 & 0.07 & 0.00 & City of Jesup & 1.44 & 0.00 \\
\hline Livestock/aquaculture & 0.01 & 0.08 & 0.09 & 0.09 & 0.00 & City of Screven & 0.07 & 0.00 \\
\hline Thermoelectric power & 0.00 & 0.00 & 0.00 & 0.00 & 0.00 & Water Utility Management & 0.06 & 0.00 \\
\hline TOTAL & 61.86 & 0.09 & 61.95 & 61.95 & 55.91 & & & \\
\hline
\end{tabular}

Total use is total withdrawal plus public supply deliveries and losses.

2010 Withdrawals by Source
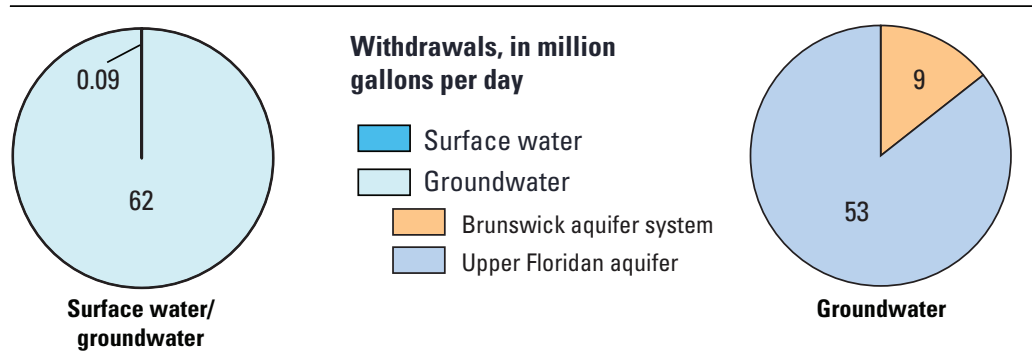

Public-Supply Deliveries by Use Category

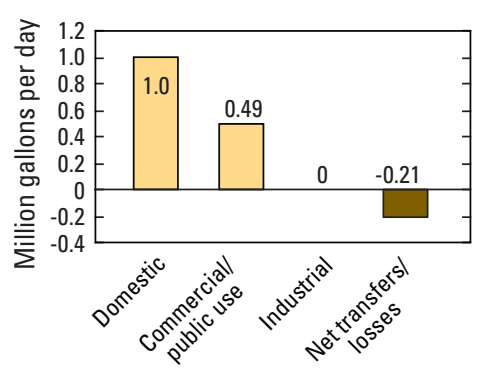

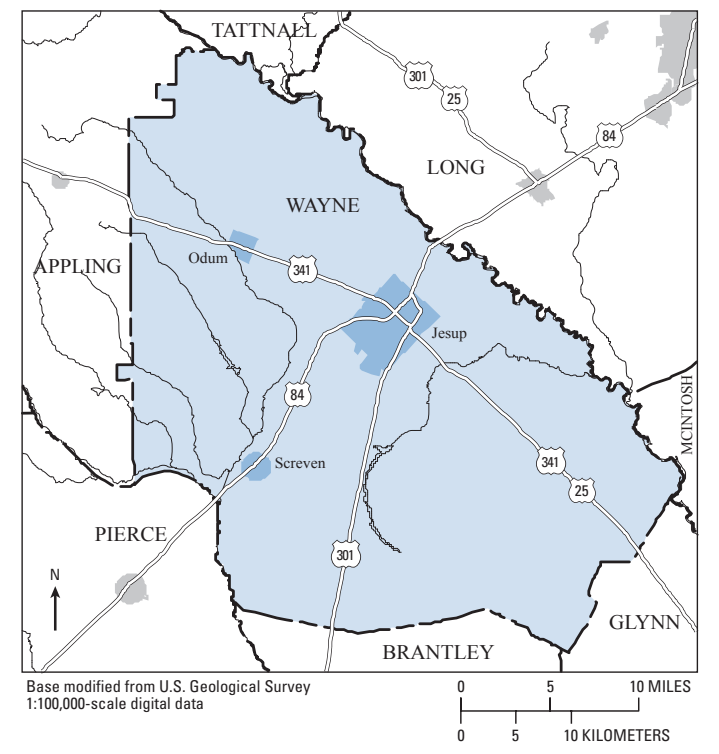

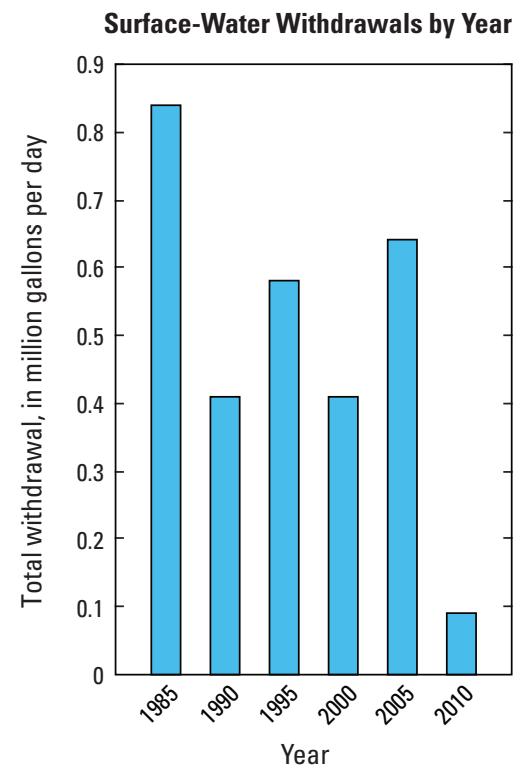

Groundwater Withdrawals by Year

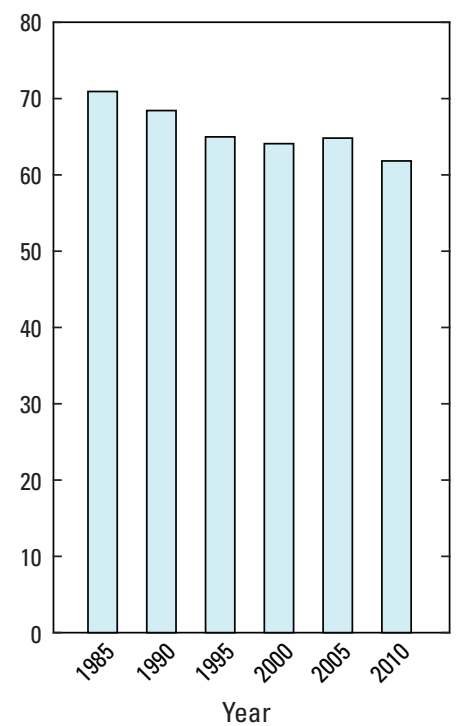




\section{WEBSTER COUNTY}

Population

Population served by public supply-Groundwater Population served by public supply-Surface water Acres irrigated
12,020

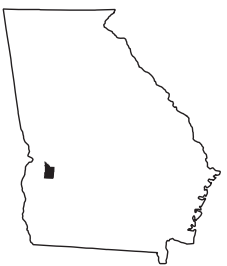

2010 WATER WITHDRAWALS AND ESTIMATED USE, IN MILLION GALLONS PER DAY

$[-$, not applicable $]$

\begin{tabular}{|c|c|c|c|c|c|c|c|c|}
\hline \multirow{3}{*}{ Category } & \multicolumn{3}{|c|}{ Withdrawals } & \multirow{3}{*}{$\begin{array}{l}\text { Total } \\
\text { use }^{1}\end{array}$} & \multirow{3}{*}{$\begin{array}{l}\text { Surface- } \\
\text { water } \\
\text { returns } \\
\end{array}$} & \multirow{2}{*}{\multicolumn{3}{|c|}{$\begin{array}{l}\text { Withdrawals by Major Industrial Groups } \\
\text { [NAICS, North American Industrial Classification System code] }\end{array}$}} \\
\hline & \multirow{2}{*}{$\begin{array}{l}\text { Ground- } \\
\text { water }\end{array}$} & \multirow{2}{*}{$\begin{array}{c}\text { Surface } \\
\text { water }\end{array}$} & \multirow[b]{2}{*}{ Total } & & & & & \\
\hline & & & & & & NAICS & Groundwater & Surface water \\
\hline Public supply & 0.13 & 0.00 & 0.13 & \multirow{2}{*}{$\begin{array}{l}- \\
0.26\end{array}$} & \multirow{2}{*}{0.00} & \multirow{2}{*}{ None } & \multirow{2}{*}{-} & \multirow{2}{*}{-} \\
\hline Domestic & 0.15 & 0.00 & 0.15 & & & & & \\
\hline Commercial/public use & 0.00 & 0.00 & 0.00 & 0.00 & 0.00 & & & \\
\hline Industrial & 0.00 & 0.00 & 0.00 & 0.00 & 0.00 & & & \\
\hline Public-supply losses & - & - & - & 0.02 & - & & & \\
\hline \multirow{2}{*}{$\begin{array}{l}\text { Public wastewater treatment } \\
\text { Mining }\end{array}$} & \multirow{2}{*}{$\begin{array}{c}- \\
0.00\end{array}$} & \multirow{2}{*}{$\begin{array}{c}- \\
0.00\end{array}$} & \multirow{2}{*}{$\begin{array}{c}- \\
0.00\end{array}$} & \multirow{2}{*}{$\begin{array}{l}- \\
0.00\end{array}$} & 0.00 & \multicolumn{3}{|c|}{ Withdrawals by Major Public Suppliers } \\
\hline & & & & & 0.00 & \multirow{2}{*}{ Name } & \multirow{2}{*}{ Groundwater } & \multirow{2}{*}{ Surface water } \\
\hline Irrigation-Crop & 1.37 & 6.18 & 7.55 & 7.55 & 0.00 & & & \\
\hline Irrigation-Golf course & 0.00 & 0.00 & 0.00 & 0.00 & 0.00 & \multirow{2}{*}{$\begin{array}{l}\text { Unified Government } \\
\text { of Webster County }\end{array}$} & \multirow{2}{*}{0.13} & \multirow{2}{*}{0.00} \\
\hline Livestock/aquaculture & 0.09 & 1.02 & 1.11 & 1.11 & 0.00 & & & \\
\hline Thermoelectric power & 0.00 & 0.00 & 0.00 & 0.00 & 0.00 & & & \\
\hline
\end{tabular}

$\begin{array}{llllll}\text { TOTAL } & 1.74 & 7.20 & 8.94 & 8.94 & 0.00\end{array}$

'Total use is total withdrawal plus public supply deliveries and losses.

\section{Withdrawals by Source}
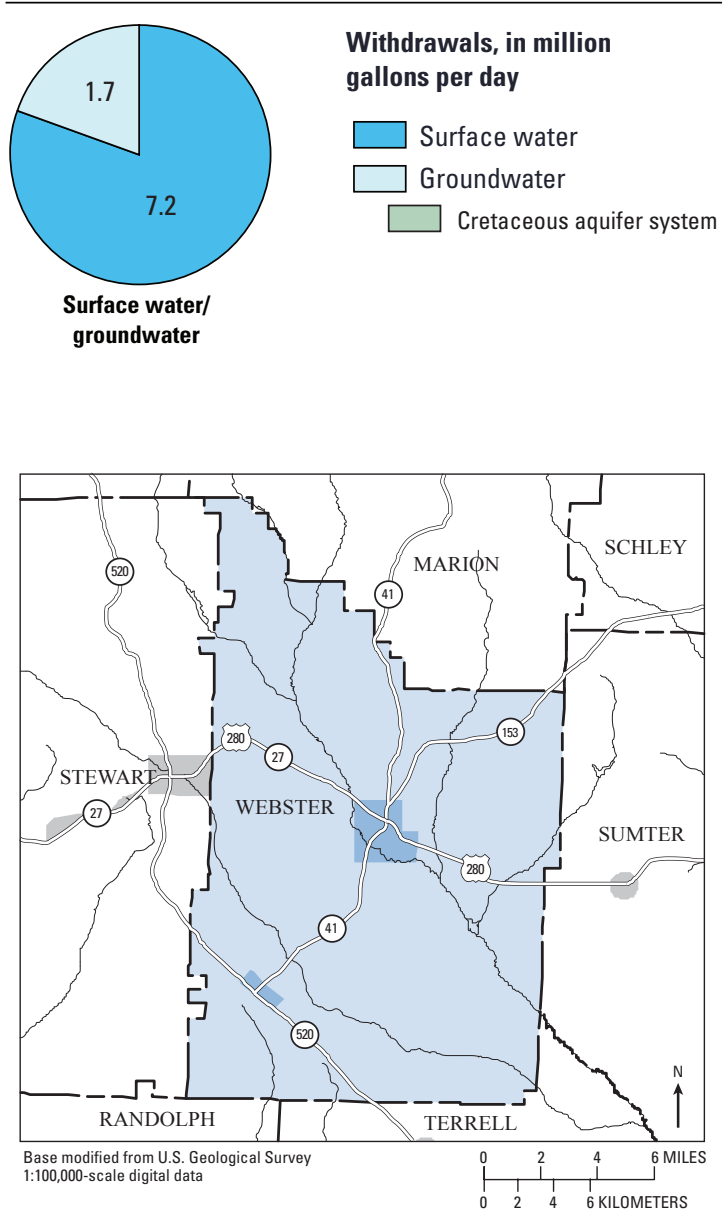

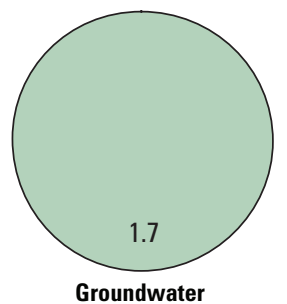

Public-Supply Deliveries by Use Category

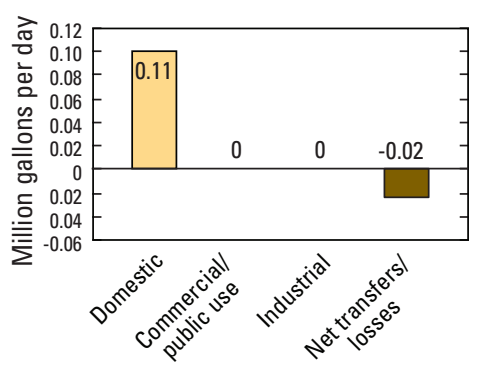

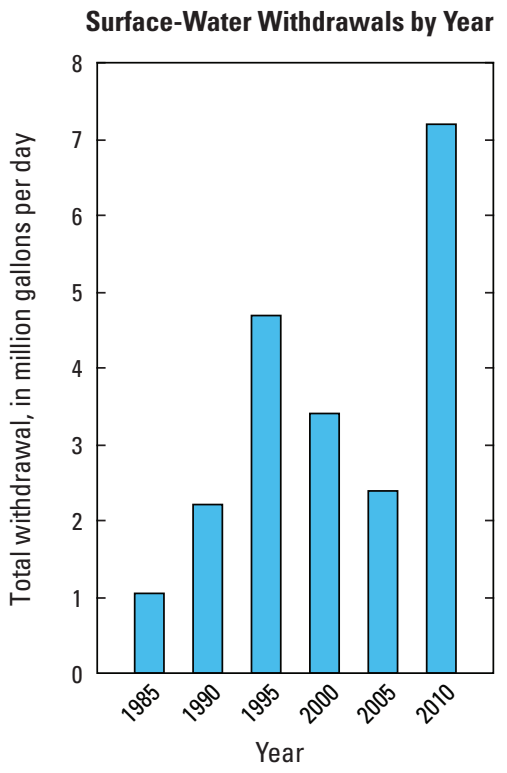

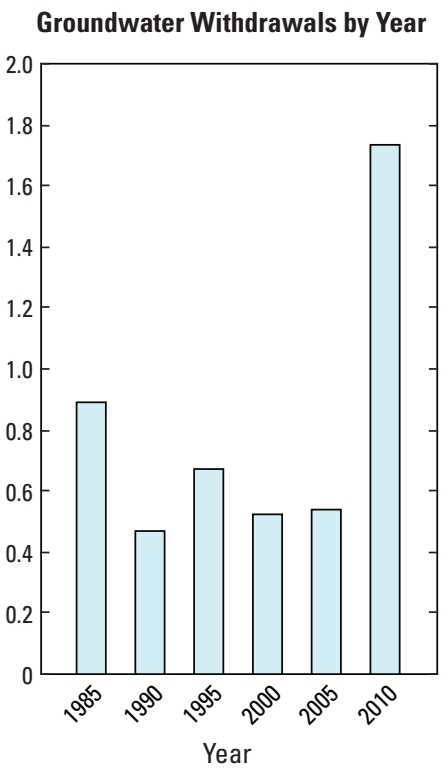




\section{WHEELER COUNTY}

Population

Population served by public supply-Groundwater $\quad 1,770$

Population served by public supply-Surface water 0

Acres irrigated

4,250

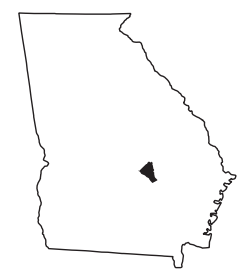

2010 WATER WITHDRAWALS AND ESTIMATED USE, IN MILLION GALLONS PER DAY

$[-$, not applicable $]$

\begin{tabular}{|c|c|c|c|c|c|c|c|c|}
\hline \multirow{3}{*}{ Category } & \multicolumn{3}{|c|}{ Withdrawals } & \multirow{3}{*}{$\begin{array}{l}\text { Total } \\
\text { use }^{1}\end{array}$} & \multirow{3}{*}{$\begin{array}{l}\text { Surface- } \\
\text { water } \\
\text { returns } \\
\end{array}$} & \multirow{2}{*}{\multicolumn{3}{|c|}{$\begin{array}{l}\text { Withdrawals by Major Industrial Groups } \\
\text { [NAICS, North American Industrial Classification System code] }\end{array}$}} \\
\hline & \multirow{2}{*}{$\begin{array}{c}\text { Ground- } \\
\text { water }\end{array}$} & \multirow{2}{*}{$\begin{array}{c}\text { Surface } \\
\text { water }\end{array}$} & \multirow[b]{2}{*}{ Total } & & & & & \\
\hline & & & & & & NAICS & Groundwater & Surface water \\
\hline Public supply & 0.18 & 0.00 & 0.18 & \multirow{2}{*}{$\begin{array}{c}- \\
0.54\end{array}$} & \multirow{2}{*}{$\begin{array}{c}- \\
0.00\end{array}$} & \multirow{2}{*}{ None } & \multirow{2}{*}{-} & \multirow{2}{*}{-} \\
\hline Domestic & 0.42 & 0.00 & 0.42 & & & & & \\
\hline Commercial/public use & 0.00 & 0.00 & 0.00 & 0.03 & 0.00 & \multirow{4}{*}{\multicolumn{3}{|c|}{ Withdrawals by Major Public Suppliers }} \\
\hline Industrial & 0.00 & 0.00 & 0.00 & 0.00 & 0.00 & & & \\
\hline Public-supply losses & - & - & - & 0.03 & - & & & \\
\hline Public wastewater treatment & - & - & - & \multirow{2}{*}{0.00} & \multirow{2}{*}{$\begin{array}{l}0.37 \\
0.00\end{array}$} & & & \\
\hline \multirow{2}{*}{$\begin{array}{l}\text { Mining } \\
\text { Irrigation-Crop }\end{array}$} & 0.00 & 0.00 & 0.00 & & & \multirow{2}{*}{ Name } & \multirow{2}{*}{ Groundwater } & \multirow{2}{*}{ Surface water } \\
\hline & 1.28 & 0.45 & 1.73 & 1.73 & 0.00 & & & \\
\hline Irrigation-Golf course & 0.29 & 0.00 & 0.29 & 0.29 & 0.00 & City of Alamo & 0.09 & 0.00 \\
\hline Livestock/aquaculture & 0.05 & 0.05 & 0.10 & 0.10 & 0.00 & City of Glenwood & 0.09 & 0.00 \\
\hline Thermoelectric power & 0.00 & 0.00 & 0.00 & 0.00 & 0.00 & & & \\
\hline
\end{tabular}

TOTAL

$2.22 \quad 0.50$

2.72

2.72

$\mathbf{0 . 3 7}$

${ }^{1}$ Total use is total withdrawal plus public supply deliveries and losses.

\section{Withdrawals by Source}
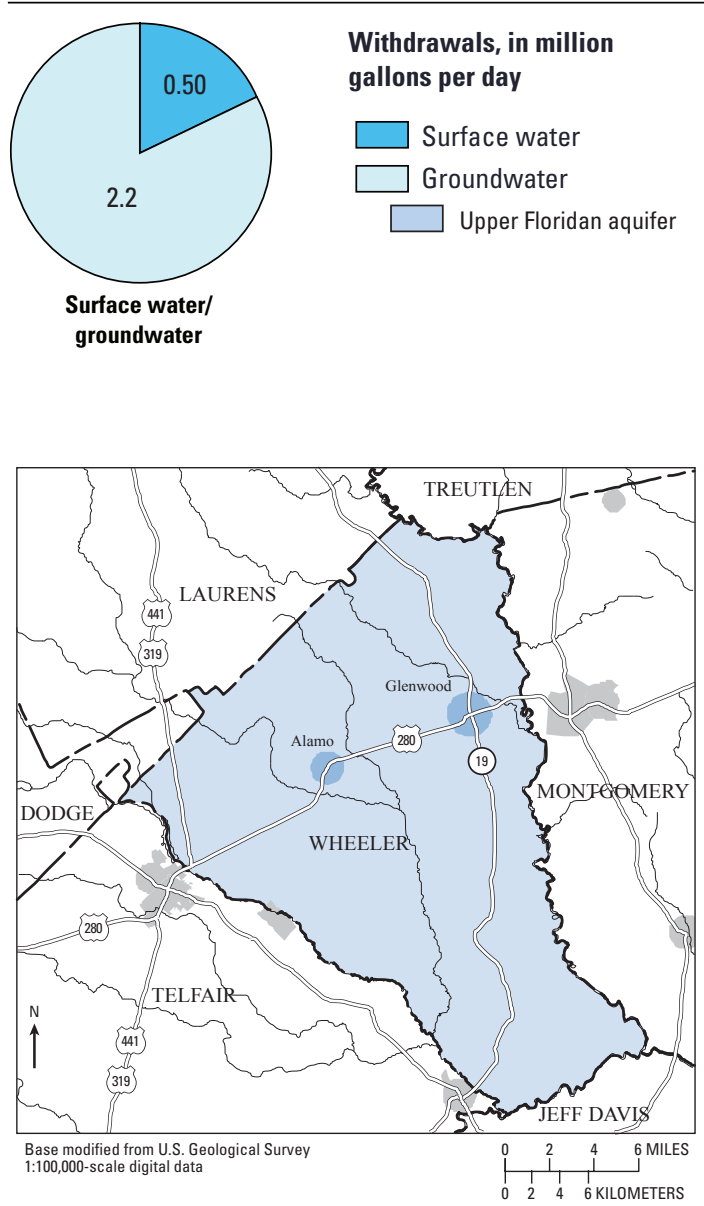

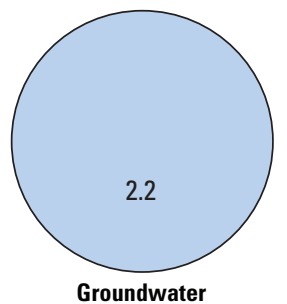

Public-Supply Deliveries by Use Category

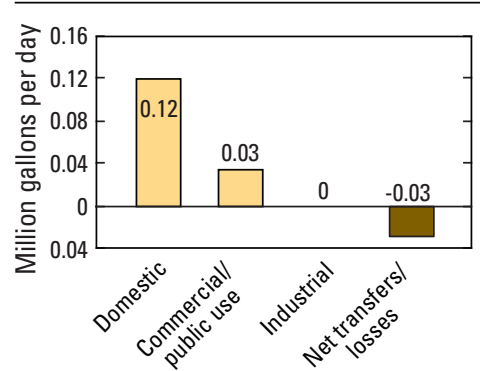

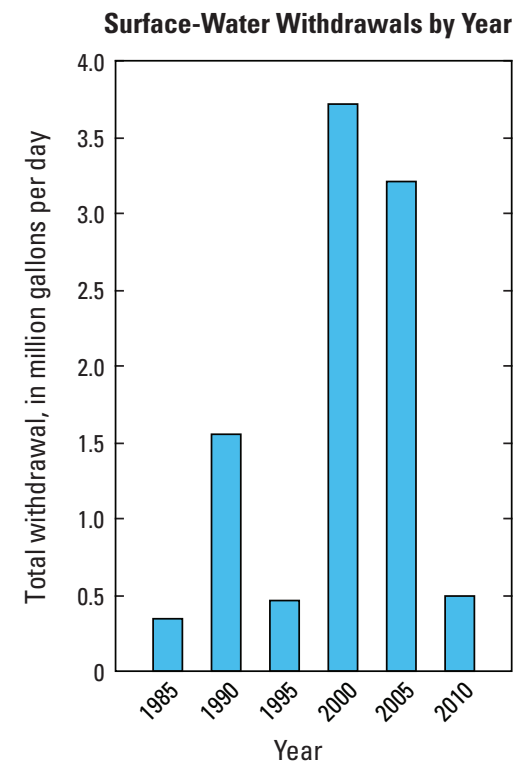

Groundwater Withdrawals by Year

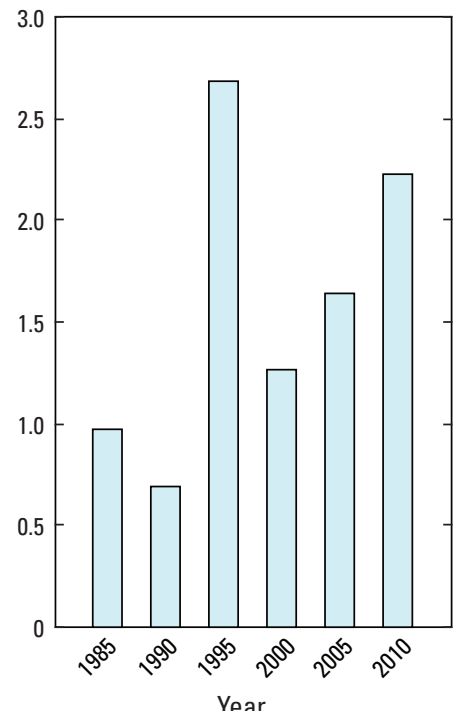

Year 


\section{WHITE COUNTY}

Population

27,144

Population served by public supply-Groundwater

Population served by public supply-Surface water Acres irrigated
8,240

5,760

40

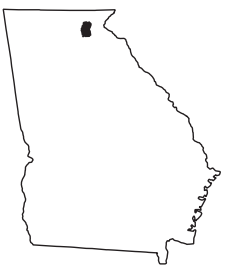

2010 WATER WITHDRAWALS AND ESTIMATED USE, IN MILLION GALLONS PER DAY

$[-$, not applicable $]$

\begin{tabular}{|c|c|c|c|c|c|c|c|c|}
\hline \multirow{3}{*}{ Category } & \multicolumn{3}{|c|}{ Withdrawals } & \multirow{3}{*}{$\begin{array}{l}\text { Total } \\
\text { use }^{1}\end{array}$} & \multirow{3}{*}{$\begin{array}{c}\text { Surface- } \\
\text { water } \\
\text { returns } \\
\end{array}$} & \multirow{2}{*}{\multicolumn{3}{|c|}{$\begin{array}{l}\text { Withdrawals by Major Industrial Groups } \\
\text { [NAICS, North American Industrial Classification System code] }\end{array}$}} \\
\hline & \multirow{2}{*}{$\begin{array}{c}\text { Ground- } \\
\text { water }\end{array}$} & \multirow{2}{*}{$\begin{array}{c}\text { Surface } \\
\text { water }\end{array}$} & \multirow[b]{2}{*}{ Total } & & & & & \\
\hline & & & & & & NAICS & Groundwater & Surface water \\
\hline Public supply & 0.92 & 0.58 & 1.50 & - & - & None & - & - \\
\hline Domestic & 0.99 & 0.00 & 0.99 & 1.82 & 0.00 & None & - & \\
\hline Commercial/public use & 0.00 & 0.00 & 0.00 & 0.48 & 0.00 & & & \\
\hline Industrial & 0.00 & 0.00 & 0.00 & 0.03 & 0.00 & & & \\
\hline Public-supply losses & - & - & - & 0.16 & - & & & \\
\hline Public wastewater treatment & - & - & - & - & 0.32 & Withdrawals by Major & Public Supp & \\
\hline Mining & 0.00 & 0.01 & 0.01 & 0.01 & 0.00 & Name & Groundwater & Surface water \\
\hline Irrigation-Crop & 0.03 & 0.02 & 0.05 & 0.05 & 0.00 & & & \\
\hline Irrigation-Golf course & 0.00 & 0.00 & 0.00 & 0.00 & 0.00 & White County Water and & 0.00 & 0.58 \\
\hline Livestock/aquaculture & 0.00 & 0.34 & 0.34 & 0.34 & 0.00 & Sewerage Authority & 0.00 & 0.50 \\
\hline Thermoelectric power & 0.00 & 0.00 & 0.00 & 0.00 & 0.00 & Cleveland Waterworks & 0.46 & 0.00 \\
\hline TOTAL & 1.94 & 0.95 & 2.89 & 2.89 & 0.32 & City of Helen & 0.31 & 0.00 \\
\hline
\end{tabular}

'Total use is total withdrawal plus public supply deliveries and losses.

\section{Withdrawals by Source}
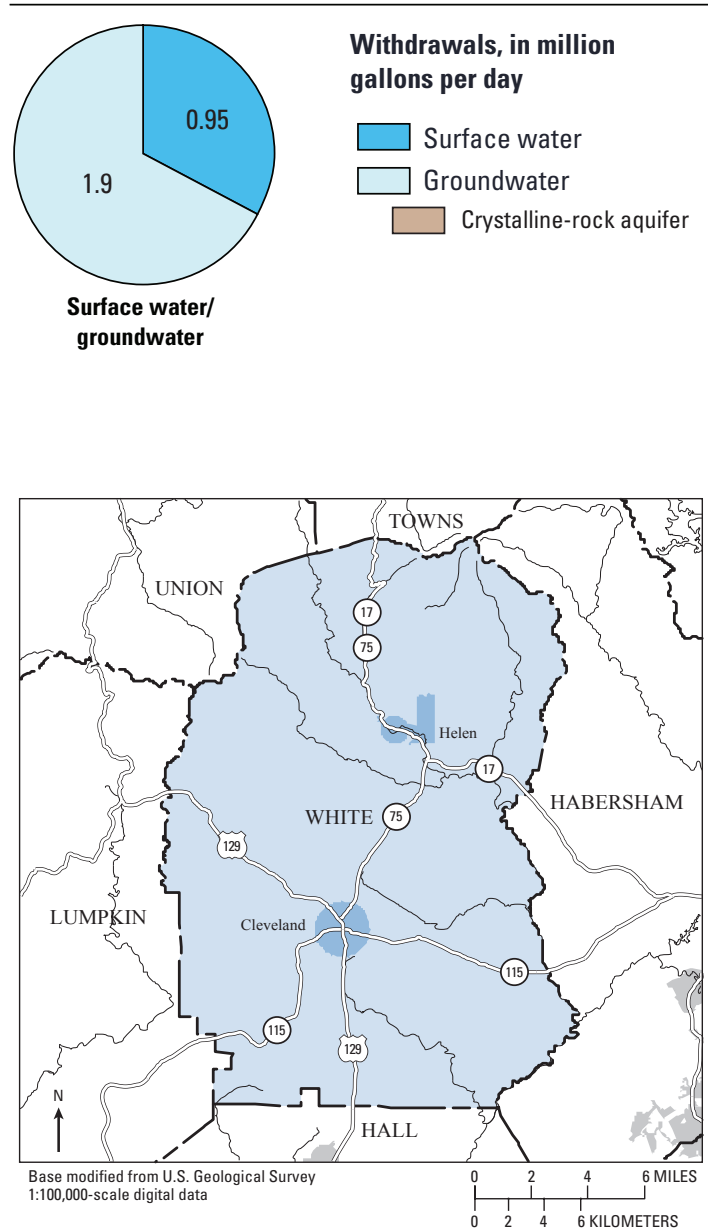

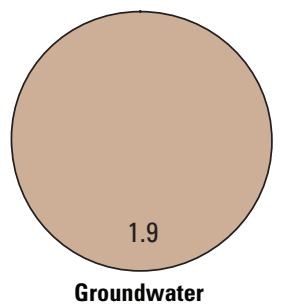

Public-Supply Deliveries by Use Category

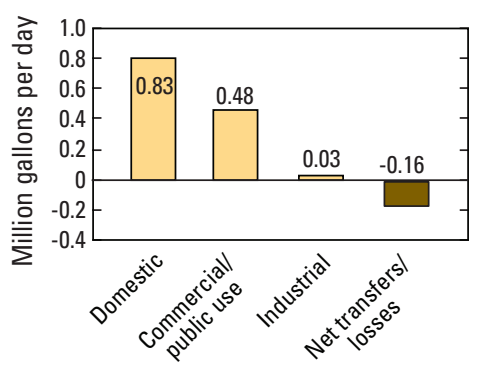

Surface-Water Withdrawals by Year

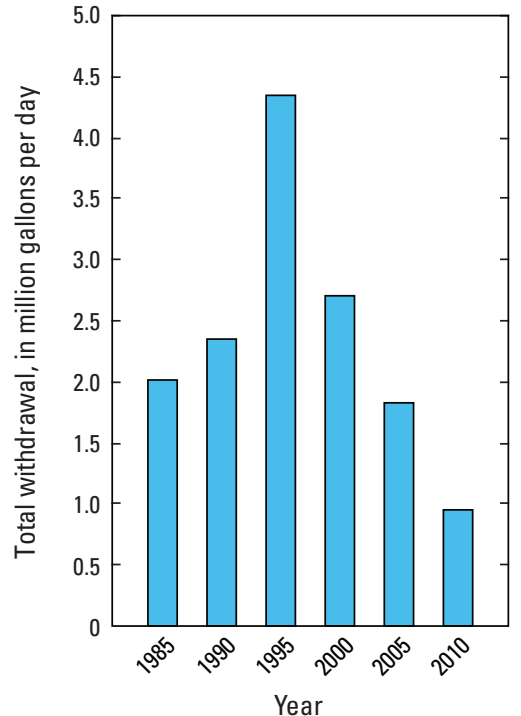

Groundwater Withdrawals by Year

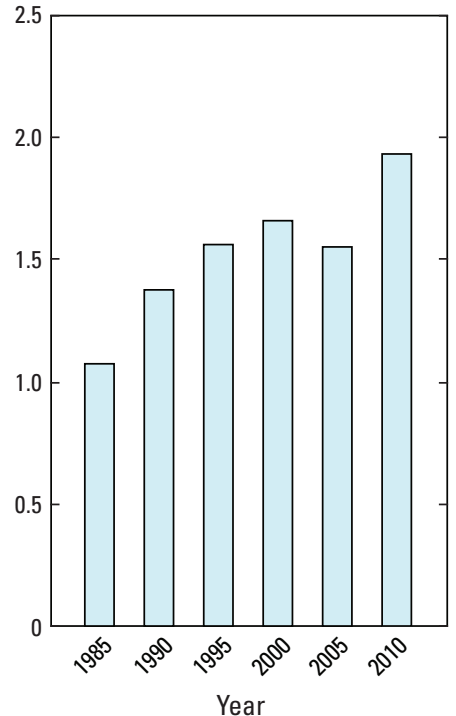




\section{WHITFIELD COUNTY}

Population

102,599

Population served by public supply-Groundwater

Population served by public supply-Surface water

Acres irrigated

97,180

326

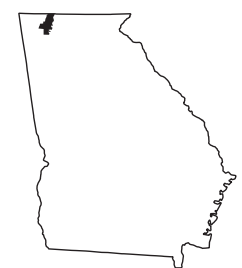

2010 WATER WITHDRAWALS AND ESTIMATED USE, IN MILLION GALLONS PER DAY

$[-$, not applicable; Mgal/d, million gallons per day $]$

\begin{tabular}{|c|c|c|c|c|c|c|c|c|}
\hline \multirow{3}{*}{ Category } & \multicolumn{3}{|c|}{ Withdrawals } & \multirow{3}{*}{$\begin{array}{l}\text { Total } \\
\text { use }^{1}\end{array}$} & \multirow{3}{*}{$\begin{array}{l}\text { Surface- } \\
\text { water } \\
\text { returns }\end{array}$} & \multirow{2}{*}{\multicolumn{3}{|c|}{$\begin{array}{l}\text { Withdrawals by Major Industrial Groups } \\
\text { [NAICS, North American Industrial Classification System code] }\end{array}$}} \\
\hline & \multirow{2}{*}{$\begin{array}{l}\text { Ground- } \\
\text { water }\end{array}$} & \multirow{2}{*}{$\begin{array}{c}\text { Surface } \\
\text { water }\end{array}$} & \multirow[b]{2}{*}{ Total } & & & & & \\
\hline & & & & & & NAICS & Groundwater & Surface water \\
\hline \multirow{2}{*}{$\begin{array}{l}\text { Public supply } \\
\text { Domestic }\end{array}$} & 0.00 & 20.68 & 20.68 & \multirow{2}{*}{$\begin{array}{c}- \\
7.81\end{array}$} & \multirow{2}{*}{$\begin{array}{c}- \\
0.00\end{array}$} & \multirow{2}{*}{ None } & \multirow{2}{*}{-} & \multirow{2}{*}{-} \\
\hline & 0.41 & 0.00 & 0.41 & & & & & \\
\hline Commercial/public use & 0.00 & 0.00 & 0.00 & 6.17 & 0.05 & \multirow{4}{*}{\multicolumn{3}{|c|}{ Withdrawals by Major Public Suppliers }} \\
\hline Industrial & 0.00 & 0.00 & 0.00 & 8.14 & 87.59 & & & \\
\hline Public-supply losses & - & - & - & 2.86 & - & & & \\
\hline Public wastewater treatment & - & - & - & \multirow{2}{*}{0.21} & \multirow{2}{*}{$\begin{array}{l}0.15 \\
0.00\end{array}$} & & & \\
\hline \multirow{2}{*}{$\begin{array}{l}\text { Mining } \\
\text { Irrigation-Crop }\end{array}$} & 0.21 & 0.00 & 0.21 & & & \multirow{2}{*}{ Name } & \multirow{2}{*}{ Groundwater } & \multirow{2}{*}{ Surface water } \\
\hline & 0.07 & 0.00 & 0.07 & 0.07 & 0.00 & & & \\
\hline Irrigation-Golf course & 0.00 & 0.52 & 0.52 & 0.52 & 0.00 & Dalton Utilities & 0.00 & 20.68 \\
\hline Livestock/aquaculture & 0.05 & 0.64 & 0.69 & 0.69 & 0.00 & & & \\
\hline Thermoelectric power & 0.00 & 0.00 & 0.00 & 0.00 & 0.00 & & & \\
\hline TOTAL & 0.74 & 21.84 & 22.58 & 26.47 & 87.79 & & & \\
\hline
\end{tabular}

Total use is total withdrawal plus public supply deliveries and losses. An average of $4 \mathrm{Mgal} / \mathrm{d}$ was delivered from Hamilton County,

Tennessee to Whitfield County, Georgia in 2010.

\section{Withdrawals by Source}
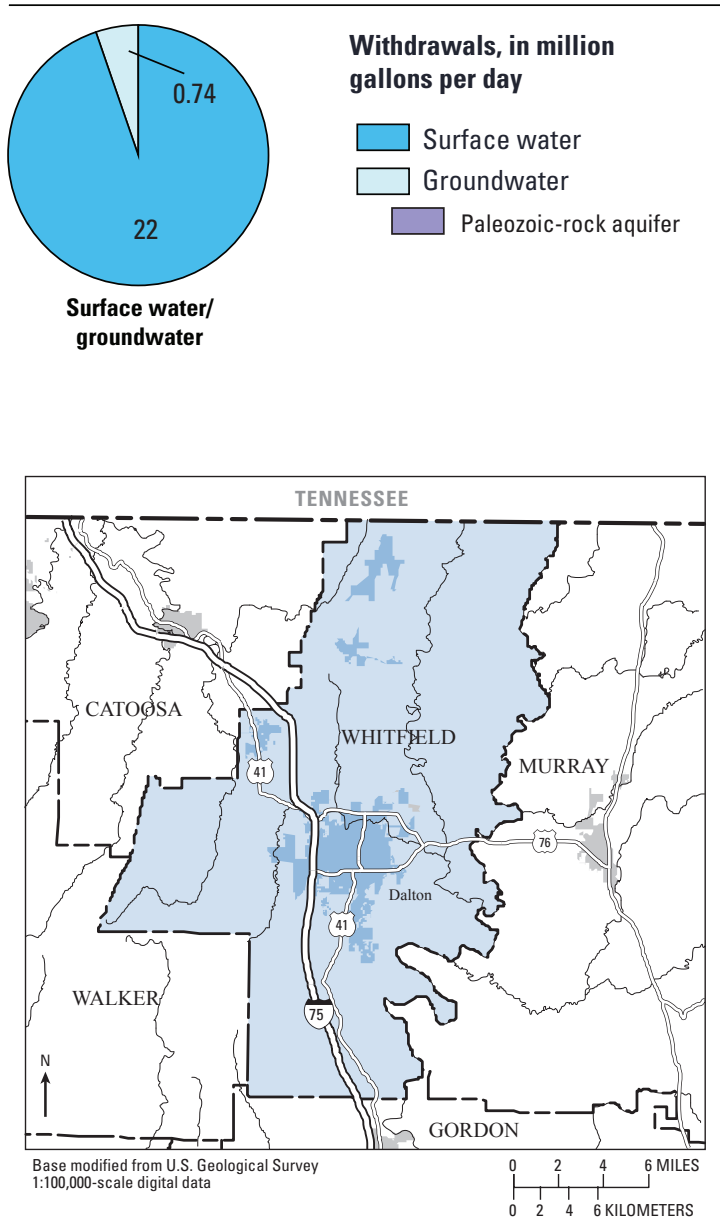

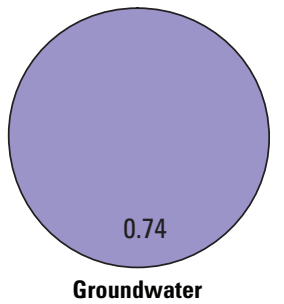

Public-Supply Deliveries ${ }^{1}$ by Use Category

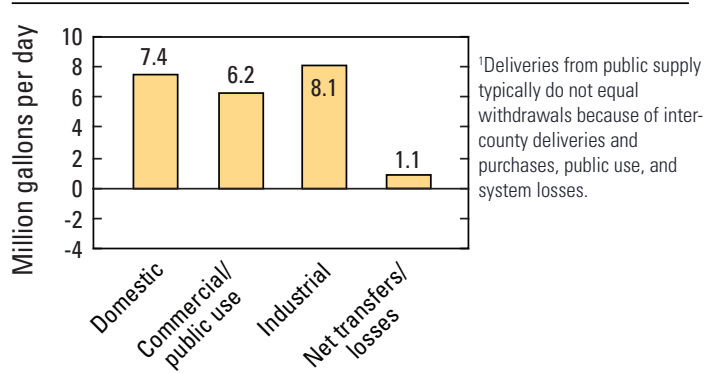

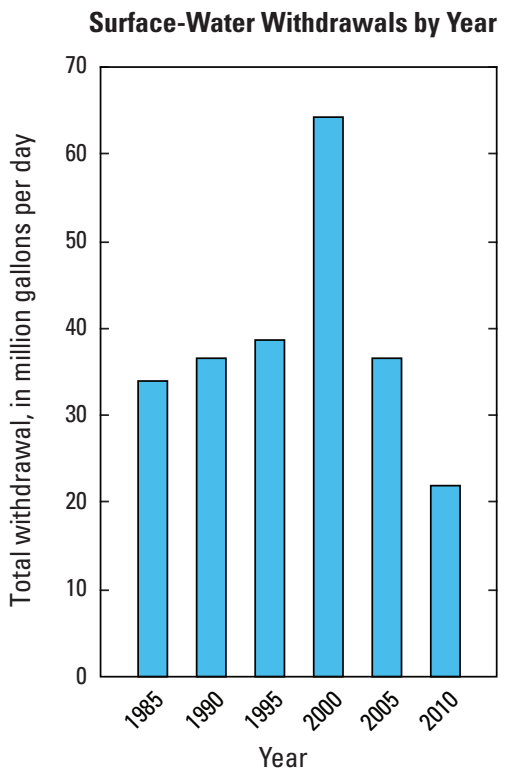

Groundwater Withdrawals by Year

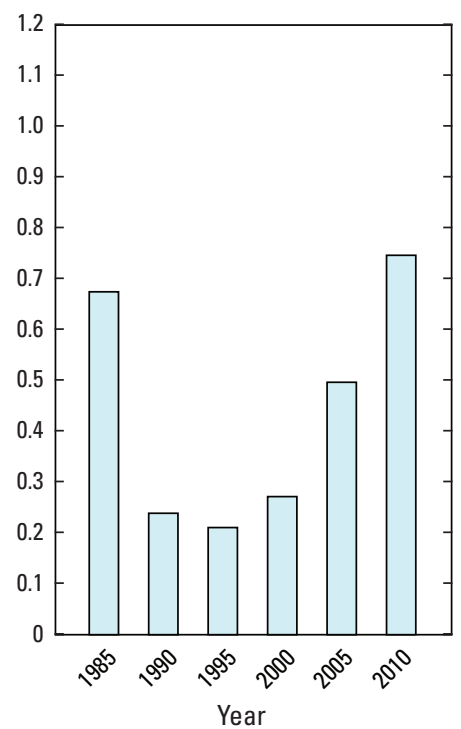




\section{WILCOX COUNTY}

Population

Population served by public supply-Groundwater Population served by public supply-Surface water Acres irrigated
3,190

0

14,290

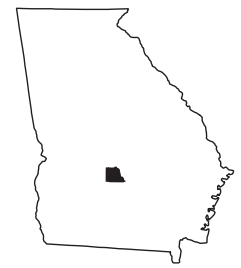

2010 WATER WITHDRAWALS AND ESTIMATED USE, IN MILLION GALLONS PER DAY

$[-$, not applicable $]$

\begin{tabular}{|c|c|c|c|c|c|c|c|c|}
\hline \multirow{3}{*}{ Category } & \multicolumn{3}{|c|}{ Withdrawals } & \multirow{3}{*}{$\begin{array}{l}\text { Total } \\
\text { use }^{1}\end{array}$} & \multirow{3}{*}{$\begin{array}{l}\text { Surface- } \\
\text { water } \\
\text { returns } \\
\end{array}$} & \multirow{2}{*}{\multicolumn{3}{|c|}{$\begin{array}{l}\text { Withdrawals by Major Industrial Groups } \\
\text { [NAICS, North American Industrial Classification System code] }\end{array}$}} \\
\hline & \multirow{2}{*}{$\begin{array}{c}\text { Ground- } \\
\text { water }\end{array}$} & \multirow{2}{*}{$\begin{array}{c}\text { Surface } \\
\text { water }\end{array}$} & \multirow[b]{2}{*}{ Total } & & & & & \\
\hline & & & & & & NAICS & Groundwater & Surface water \\
\hline Public supply & 0.27 & 0.00 & 0.27 & \multirow{2}{*}{$\begin{array}{l}- \\
0.67\end{array}$} & \multirow{2}{*}{$\begin{array}{c}- \\
0.00\end{array}$} & \multirow{2}{*}{ None } & \multirow{2}{*}{-} & \multirow{2}{*}{-} \\
\hline Domestic & 0.45 & 0.00 & 0.45 & & & & & \\
\hline Commercial/public use & 0.02 & 0.00 & 0.02 & 0.05 & 0.00 & \multirow{4}{*}{\multicolumn{3}{|c|}{ Withdrawals by Major Public Suppliers }} \\
\hline Industrial & 0.00 & 0.00 & 0.00 & 0.00 & 0.00 & & & \\
\hline Public-supply losses & - & - & - & 0.03 & - & & & \\
\hline Public wastewater treatment & - & - & - & - & 1.99 & & & \\
\hline \multirow{2}{*}{$\begin{array}{l}\text { Mining } \\
\text { Irrigation-Crop }\end{array}$} & 0.00 & 0.00 & 0.00 & 0.00 & 0.00 & \multirow{2}{*}{ Name } & \multirow{2}{*}{ Groundwater } & \multirow{2}{*}{ Surface water } \\
\hline & 5.08 & 1.25 & 6.33 & 6.32 & 0.00 & & & \\
\hline Irrigation-Golf course & 0.00 & 0.00 & 0.00 & 0.00 & 0.00 & City of Abbeville & 0.09 & 0.00 \\
\hline Livestock/aquaculture & 0.06 & 0.50 & 0.56 & 0.56 & 0.00 & City of Rochelle & 0.10 & 0.00 \\
\hline Thermoelectric power & 0.00 & 0.00 & 0.00 & 0.00 & 0.00 & Town of Pineview & 0.04 & 0.00 \\
\hline TOTAL & 5.88 & 1.75 & 7.63 & 7.63 & 1.99 & & & \\
\hline
\end{tabular}

${ }^{1}$ Total use is total withdrawal plus public supply deliveries and losses.

\section{Withdrawals by Source}
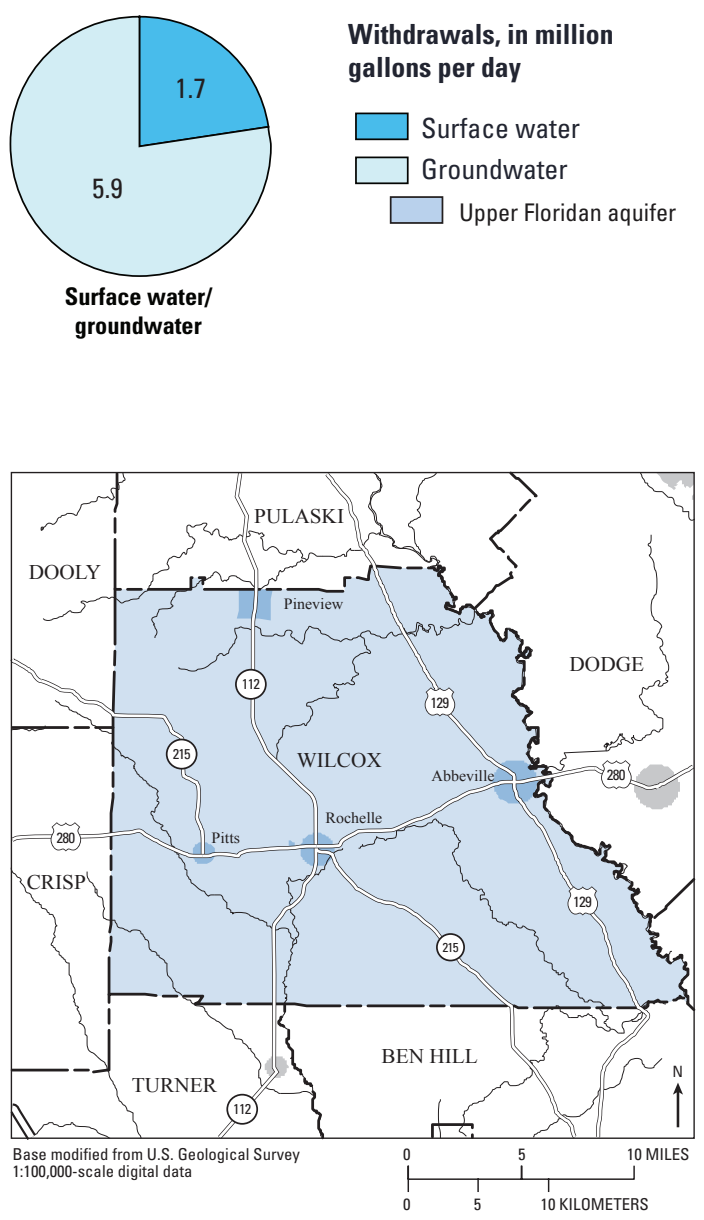

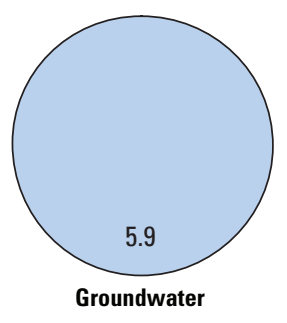

Public-Supply Deliveries ${ }^{1}$ by Use Category

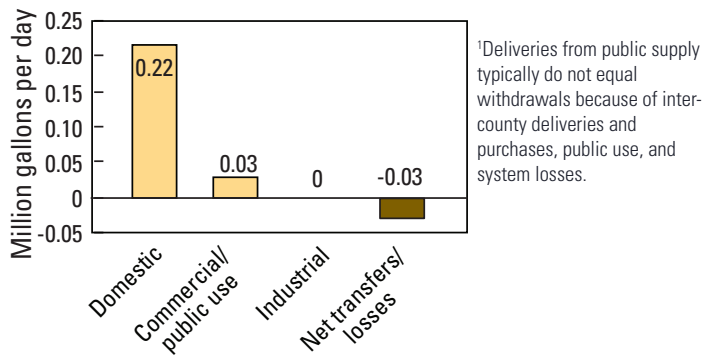

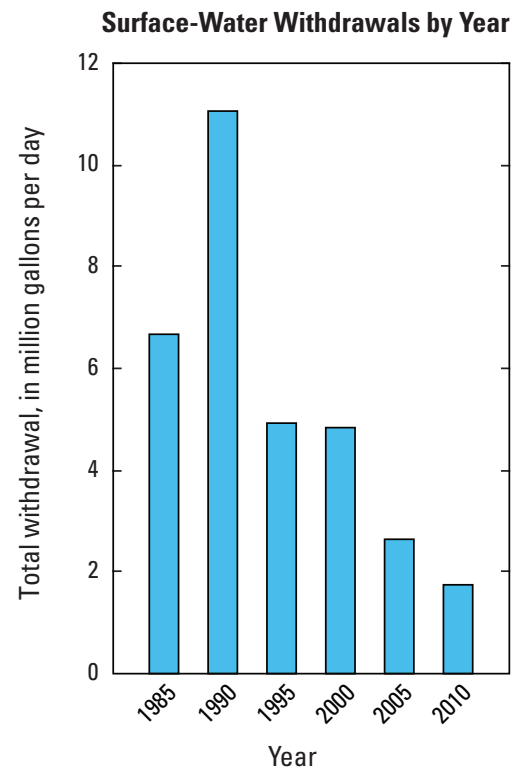

Groundwater Withdrawals by Year

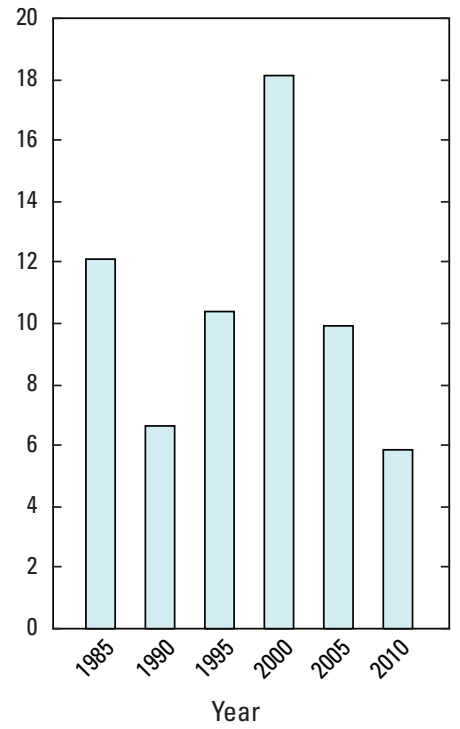




\section{WILKES COUNTY}

Population

Population served by public supply-Groundwater $\quad 750$

Population served by public supply-Surface water $\quad 4,860$

Acres irrigated

30

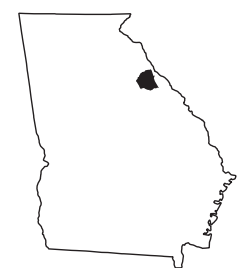

2010 WATER WITHDRAWALS AND ESTIMATED USE, IN MILLION GALLONS PER DAY

$[-$, not applicable $]$

\begin{tabular}{|c|c|c|c|c|c|c|c|c|}
\hline \multirow{3}{*}{ Category } & \multicolumn{3}{|c|}{ Withdrawals } & \multirow{3}{*}{$\begin{array}{l}\text { Total } \\
\text { use }^{1}\end{array}$} & \multirow{3}{*}{$\begin{array}{l}\text { Surface- } \\
\text { water } \\
\text { returns }\end{array}$} & \multirow{2}{*}{\multicolumn{3}{|c|}{$\begin{array}{l}\text { Withdrawals by Major Industrial Groups } \\
\text { [NAICS, North American Industrial Classification System code] }\end{array}$}} \\
\hline & \multirow{2}{*}{$\begin{array}{c}\text { Ground- } \\
\text { water }\end{array}$} & \multirow{2}{*}{$\begin{array}{c}\text { Surface } \\
\text { water }\end{array}$} & \multirow[b]{2}{*}{ Total } & & & & & \\
\hline & & & & & & NAICS & Groundwater & Surface water \\
\hline \multirow{2}{*}{ Public supply } & 0.06 & 0.96 & 1.02 & \multirow{2}{*}{$\begin{array}{l}- \\
0.85\end{array}$} & \multirow{2}{*}{$\begin{array}{c}- \\
0.00\end{array}$} & \multirow{2}{*}{ None } & \multirow{2}{*}{-} & \multirow{2}{*}{-} \\
\hline & 0.37 & 0.00 & 0.37 & & & & & \\
\hline Commercial/public use & 0.00 & 0.00 & 0.00 & 0.11 & 0.00 & \multirow{4}{*}{\multicolumn{3}{|c|}{ Withdrawals by Major Public Suppliers }} \\
\hline Industrial & 0.00 & 0.00 & 0.00 & 0.29 & 0.00 & & & \\
\hline Public-supply losses & - & - & - & 0.14 & - & & & \\
\hline Public wastewater treatment & - & - & - & - & \multirow{2}{*}{0.00} & & & \\
\hline $\begin{array}{l}\text { Mining } \\
\text { Irrigation-Crop }\end{array}$ & 0.00 & 0.00 & 0.00 & 0.00 & & Name & Groundwater & Surface water \\
\hline $\begin{array}{l}\text { Irrigation-Crop } \\
\text { Irrigation-Golf course }\end{array}$ & $\begin{array}{l}0.06 \\
0.00\end{array}$ & $\begin{array}{l}0.02 \\
0.00\end{array}$ & $\begin{array}{l}0.08 \\
0.00\end{array}$ & $\begin{array}{l}0.08 \\
0.00\end{array}$ & $\begin{array}{l}0.00 \\
0.00\end{array}$ & City of Washington & 0.00 & 0.96 \\
\hline Livestock/aquaculture & 0.00 & 0.43 & 0.43 & 0.43 & 0.00 & City of Tignall & 0.04 & 0.00 \\
\hline Thermoelectric power & 0.00 & 0.00 & 0.00 & 0.00 & 0.00 & & & \\
\hline
\end{tabular}

TOTAL

0.49

1.41

1.90

1.90

0.62

${ }^{1}$ Total use is total withdrawal plus public supply deliveries and losses.

\section{Withdrawals by Source}

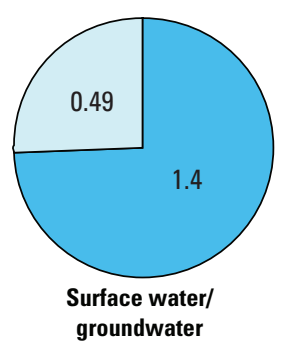

Withdrawals, in million gallons per day

$\square$ Surface water
$\square$ Groundwater
$\square \quad$ Crystalline-rock aquifer

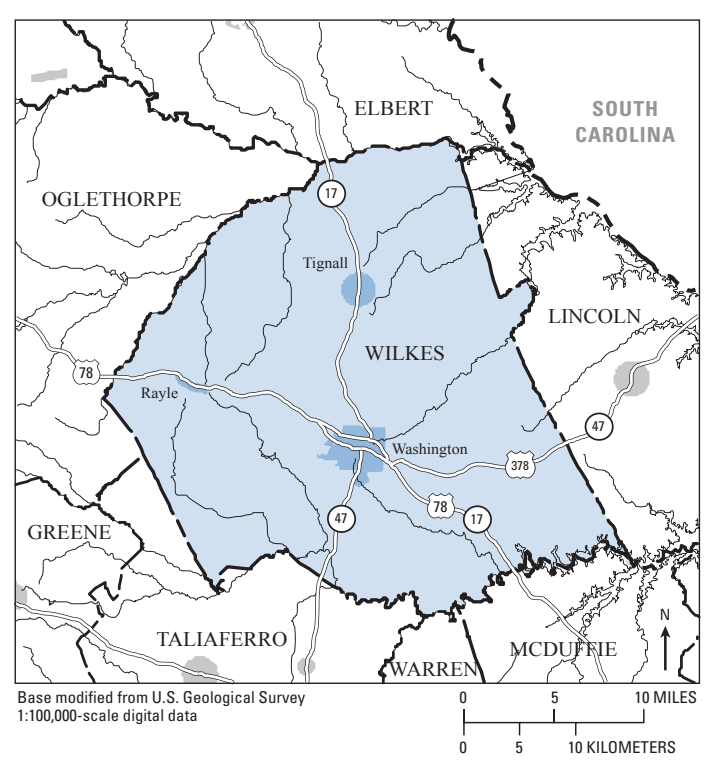

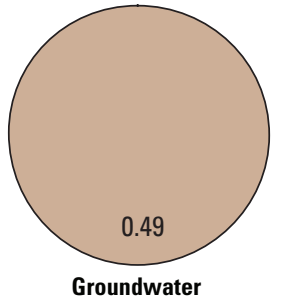

Public-Supply Deliveries by Use Category

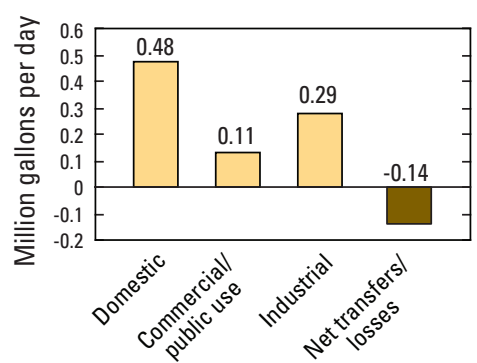

Surface-Water Withdrawals by Year

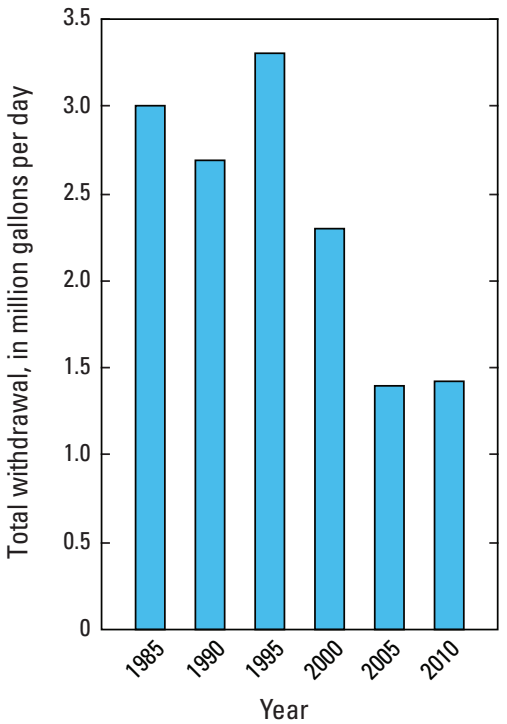

Groundwater Withdrawals by Year

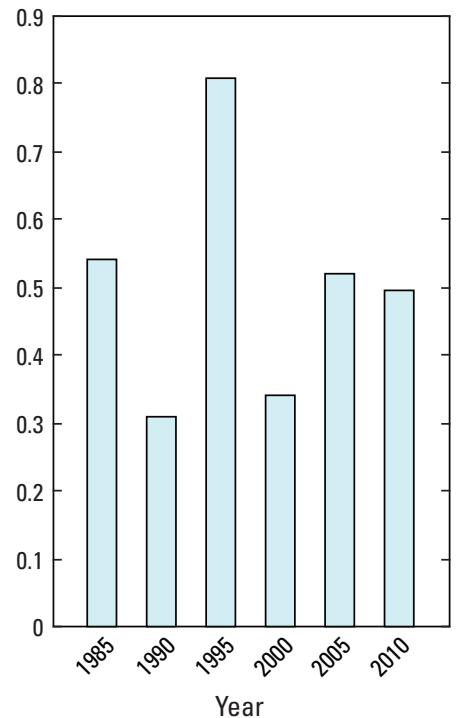




\section{WILKINSON COUNTY}

Population

Population served by public supply-Groundwater

Population served by public supply-Surface water Acres irrigated
5,470

100

80

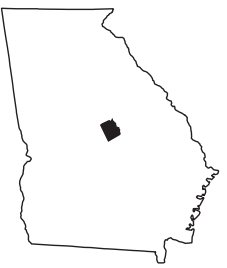

2010 WATER WITHDRAWALS AND ESTIMATED USE, IN MILLION GALLONS PER DAY

$[-$, not applicable $]$

\begin{tabular}{|c|c|c|c|c|c|c|c|c|}
\hline \multirow{3}{*}{ Category } & \multicolumn{3}{|c|}{ Withdrawals } & \multirow{3}{*}{$\begin{array}{l}\text { Total } \\
\text { use }^{1}\end{array}$} & \multirow{3}{*}{$\begin{array}{c}\text { Surface- } \\
\text { water } \\
\text { returns } \\
\end{array}$} & \multirow{2}{*}{\multicolumn{3}{|c|}{$\begin{array}{l}\text { Withdrawals by Major Industrial Groups } \\
\text { [NAICS, North American Industrial Classification System code] }\end{array}$}} \\
\hline & \multirow{2}{*}{$\begin{array}{c}\text { Ground- } \\
\text { water }\end{array}$} & \multirow{2}{*}{$\begin{array}{c}\text { Surface } \\
\text { water }\end{array}$} & \multirow[b]{2}{*}{ Total } & & & & & \\
\hline & & & & & & NAICS & Groundwater & Surface water \\
\hline Public supply & 0.67 & 0.00 & 0.67 & - & - & 212 - Kaolin and ball clay & y 12.24 & 1.18 \\
\hline Domestic & 0.30 & 0.00 & 0.30 & 0.76 & 0.00 & 327 - Stone, clay & 032 & 000 \\
\hline Commercial/public use & 0.00 & 0.00 & 0.00 & 0.09 & 0.00 & $\begin{array}{l}321 \text {-Stone, clay } \\
322 \text {-Paner puln }\end{array}$ & 0.32 & $\begin{array}{l}0.00 \\
0.00\end{array}$ \\
\hline Industrial & 12.60 & 1.18 & 13.78 & 13.84 & 1.40 & 322-Paper, pulp & 0.04 & 0.00 \\
\hline Public-supply losses & - & - & - & 0.06 & - & & & \\
\hline Public wastewater treatment & - & - & - & - & 0.30 & Withdrawals by Major & Public Supp & \\
\hline Mining & 0.01 & 0.00 & 0.01 & 0.01 & 1.17 & Name & Groundwater & Surface water \\
\hline Irrigation-Crop & 0.04 & 0.01 & 0.05 & 0.05 & 0.00 & City of Gordon & 0.25 & 0.00 \\
\hline $\begin{array}{l}\text { Irrigation-Golf course } \\
\text { Livestock/aquaculture }\end{array}$ & $\begin{array}{l}0.00 \\
0.01\end{array}$ & $\begin{array}{l}0.00 \\
0.01\end{array}$ & $\begin{array}{l}0.00 \\
0.02\end{array}$ & $\begin{array}{l}0.00 \\
0.02\end{array}$ & $\begin{array}{l}0.00 \\
0.00\end{array}$ & City of Irwinton & 0.20 & 0.00 \\
\hline Thermoelectric power & 0.00 & 0.00 & 0.00 & 0.00 & 0.00 & City of McIntyre & 0.10 & 0.00 \\
\hline TOTAL & 13.63 & 1.20 & 14.83 & 14.83 & 2.87 & Town of Ivy & 0.07 & 0.00 \\
\hline
\end{tabular}

Total use is total withdrawal plus public supply deliveries and losses.

2010 Withdrawals by Source
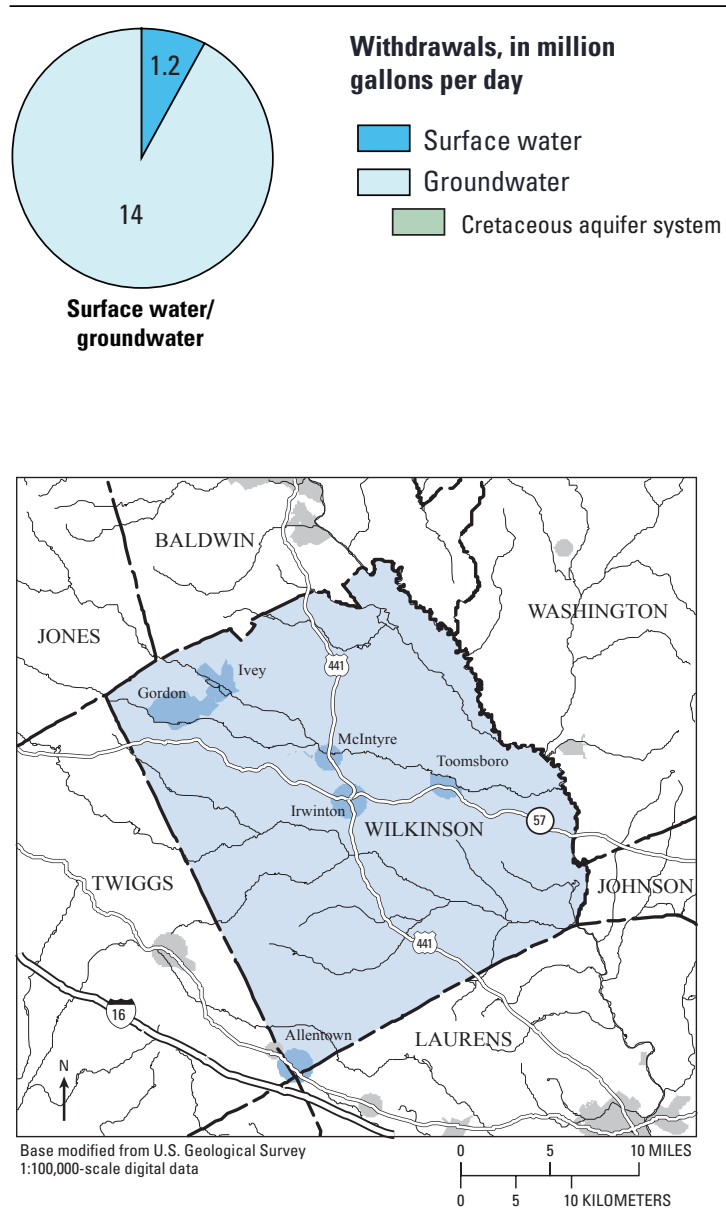

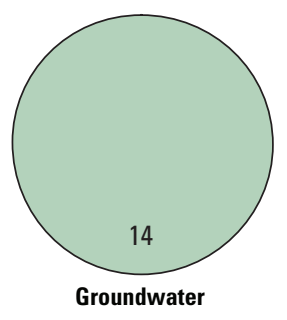

Public-Supply Deliveries by Use Category

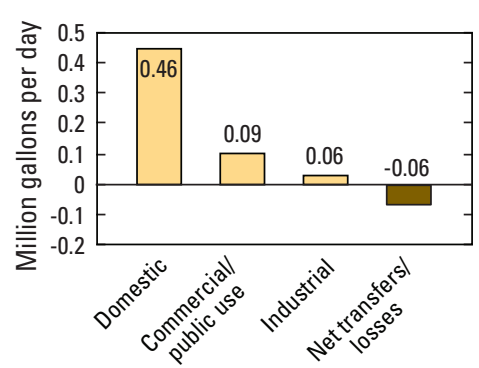

Surface-Water Withdrawals by Year

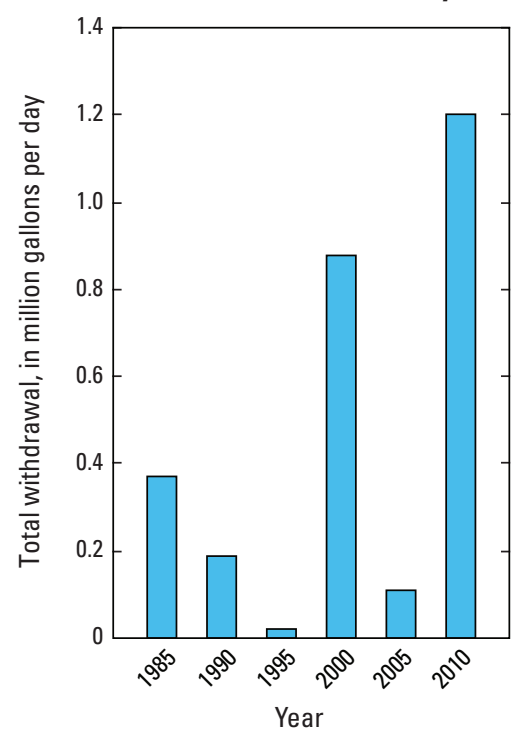

Groundwater Withdrawals by Year

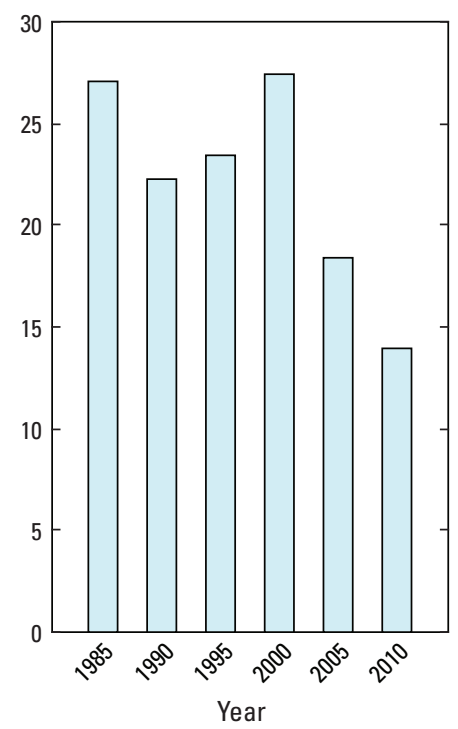




\section{WORTH COUNTY}

Population

Population served by public supply-Groundwater $\quad 9,540$

Population served by public supply-Surface water 0

Acres irrigated

44,275

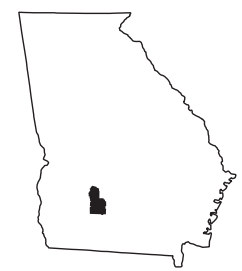

2010 WATER WITHDRAWALS AND ESTIMATED USE, IN MILLION GALLONS PER DAY

$[-$, not applicable $]$

\begin{tabular}{|c|c|c|c|c|c|c|c|c|}
\hline \multirow{3}{*}{ Category } & \multicolumn{3}{|c|}{ Withdrawals } & \multirow{3}{*}{$\begin{array}{l}\text { Total } \\
\text { use }^{1}\end{array}$} & \multirow{3}{*}{$\begin{array}{l}\text { Surface- } \\
\text { water } \\
\text { returns } \\
\end{array}$} & \multirow{2}{*}{\multicolumn{3}{|c|}{$\begin{array}{l}\text { Withdrawals by Major Industrial Groups } \\
\text { [NAICS, North American Industrial Classification System code] }\end{array}$}} \\
\hline & \multirow{2}{*}{$\begin{array}{c}\text { Ground- } \\
\text { water }\end{array}$} & \multirow{2}{*}{$\begin{array}{c}\text { Surface } \\
\text { water }\end{array}$} & \multirow[b]{2}{*}{ Total } & & & & & \\
\hline & & & & & & NAICS & Groundwater & Surface water \\
\hline Public supply & 1.21 & 0.00 & 1.21 & - & - & & & \\
\hline Domestic & 0.91 & 0.00 & 0.91 & 1.62 & 0.00 & None & - & - \\
\hline Commercial/public use & 0.00 & 0.00 & 0.00 & 0.18 & 0.00 & & & \\
\hline Industrial & 0.00 & 0.00 & 0.00 & 0.04 & 0.00 & & & \\
\hline Public-supply losses & - & - & - & 0.28 & - & & & \\
\hline Public wastewater treatment & - & - & - & - & 3.96 & Withdrawals by & r Public Supp & \\
\hline Mining & 0.00 & 0.00 & 0.00 & 0.00 & 0.00 & Name & Groundwater & Surface water \\
\hline Irrigation-Crop & 11.80 & 3.78 & 15.58 & 15.58 & 0.00 & & & \\
\hline Irrigation-Golf course & 0.06 & 0.00 & 0.06 & 0.06 & 0.00 & City of Sylvester & 1.03 & 0.00 \\
\hline Livestock/aquaculture & 0.06 & 0.15 & 0.21 & 0.21 & 0.00 & City of Poulan & 0.06 & 0.00 \\
\hline Thermoelectric power & 0.00 & 0.20 & 0.20 & 0.20 & 0.00 & Town of Sumner & 0.03 & 0.00 \\
\hline TOTAL & 14.04 & 4.13 & 18.17 & 18.17 & 3.96 & City of Warwick & 0.03 & 0.00 \\
\hline
\end{tabular}

Total use is total withdrawal plus public supply deliveries and losses.

\section{Withdrawals by Source}
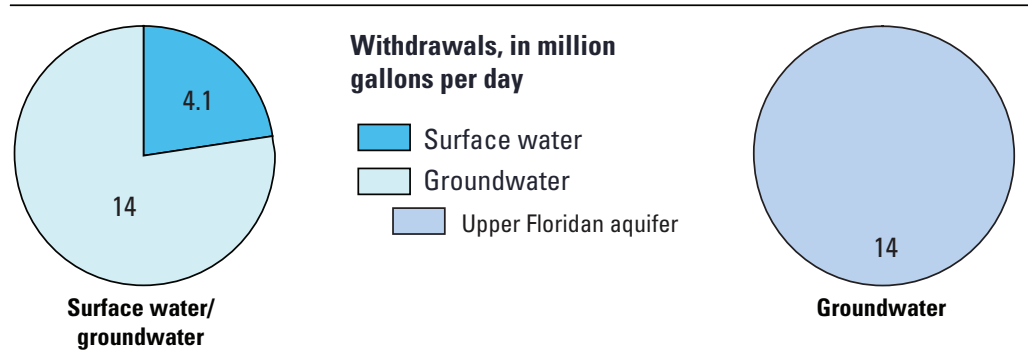

Public-Supply Deliveries by Use Category
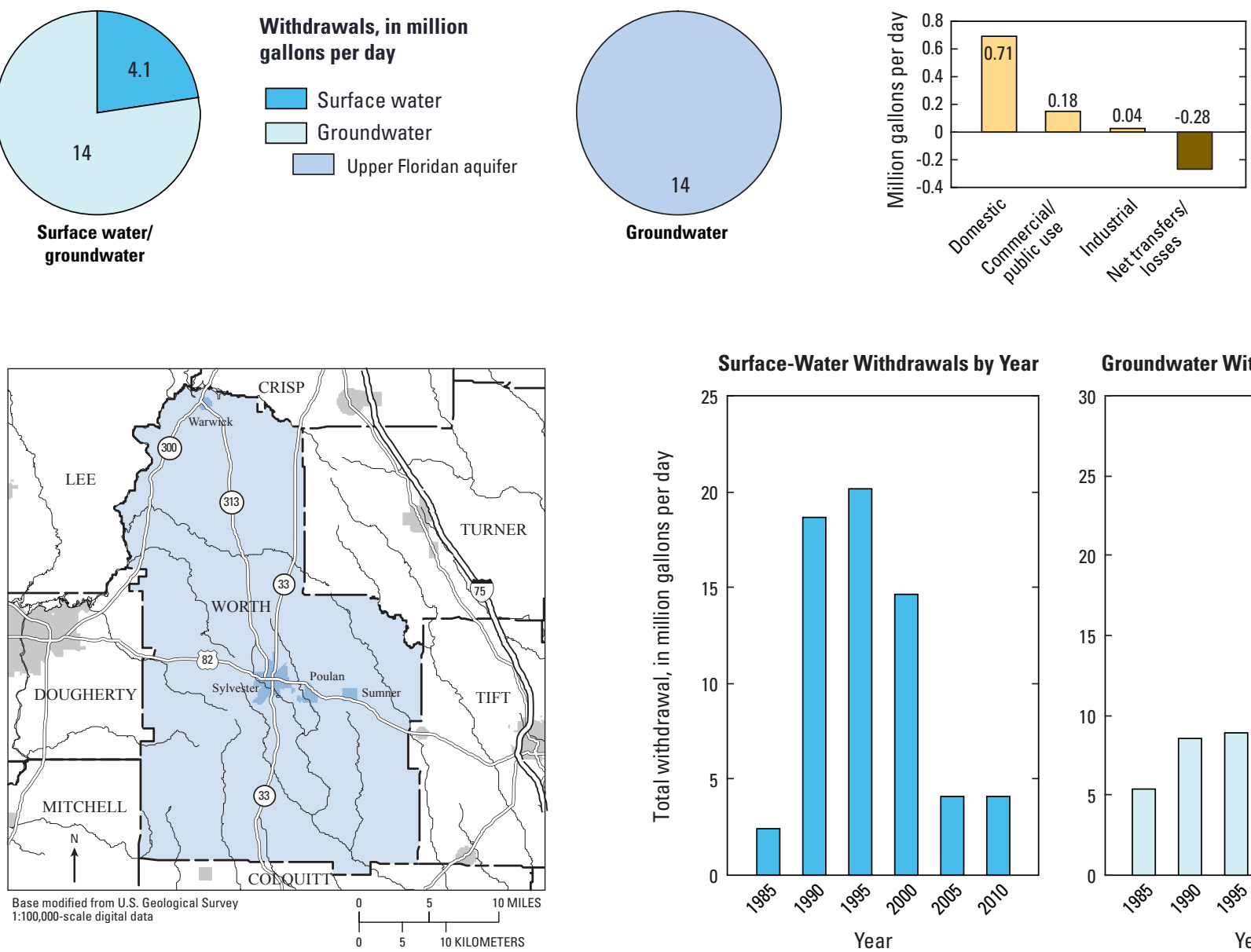

Groundwater Withdrawals by Year

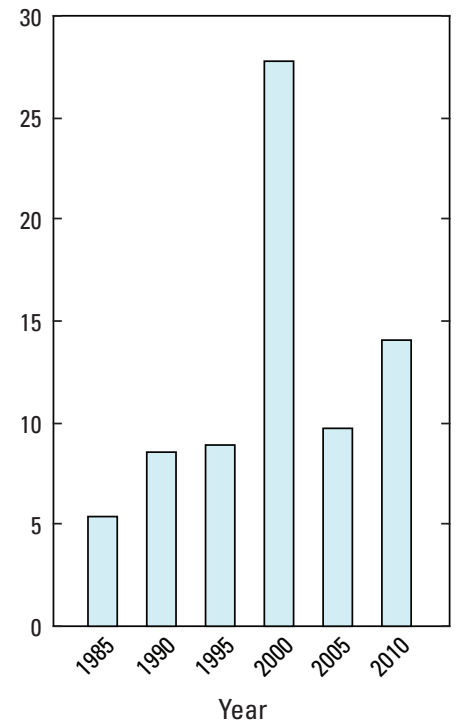


Manuscript approved on December 1, 2015

Prepared by the USGS Science Publishing Network Edited by Rolla PSC

Illustrations and layout by Caryl J. Wipperfurth, Raleigh PSC

For more information about this publication contact:

Director

U.S. Geological Survey

South Atlantic Water Science Center

720 Gracern Road

Columbia, SC 29210

http://www.usgs.gov/water/southatlantic/ 
